University of Louisville

ThinkIR: The University of Louisville's Institutional Repository

$5-2009$

\title{
A comparison of the structural response to synthetic earthquake ground motions compatible with central eastern United States attenuation models.
}

Troy W. Milburn

University of Louisville

Follow this and additional works at: https://ir.library.louisville.edu/etd

\section{Recommended Citation}

Milburn, Troy W., "A comparison of the structural response to synthetic earthquake ground motions compatible with central eastern United States attenuation models." (2009). Electronic Theses and Dissertations. Paper 972.

https://doi.org/10.18297/etd/972

This Doctoral Dissertation is brought to you for free and open access by ThinkIR: The University of Louisville's Institutional Repository. It has been accepted for inclusion in Electronic Theses and Dissertations by an authorized administrator of ThinkIR: The University of Louisville's Institutional Repository. This title appears here courtesy of the author, who has retained all other copyrights. For more information, please contact thinkir@louisville.edu. 


\title{
A COMPARISON OF THE STRUCTURAL RESPONSE TO SYNTHETIC EARTHQUAKE GROUND MOTIONS COMPATIBLE WITH CENTRAL EASTERN UNITED STATES ATTENUATION MODELS
}

\author{
By \\ Troy W. Milburn \\ B.S.C.E., University of Louisville \\ M.Eng. C.E., University of Louisville \\ A Dissertation \\ Submitted to the Faculty of the \\ Graduate School of the University of Louisville \\ in Partial Fulfillment of the Requirements \\ for the Degree of \\ Doctor of Philosophy \\ Department of Civil and Environmental Engineering \\ University of Louisville \\ Louisville, Kentucky
}

May 2009 
Copyright 2009 by Troy W. Milburn

All Rights Reserved 



\title{
A COMPARISON OF THE STRUCTURAL RESPONSE TO SYNTHETIC GROUND MOTIONS COMPATIBLE WITH CENTRAL EASTERN \\ UNITED STATES ATTENUATION MODELS
}

\author{
By \\ Troy W. Milburn \\ B.S.C.E., University of Louisville, 2002 \\ M.Eng.C.E., University of Louisville, 2003
}

A Dissertation approved on

September 25, 2008

By the following Dissertation Committee:

Dr. Th Joseph Hagerty, Dißertation/Director

Dr. Mark N. French

Dr. Thomas D. Rockaway

Dr. Julius P. Wóng

Dr. Gail W. DePty 


\section{DEDICATION}

I dedicate this dissertation to my wife Lori whom I love deeply (thanks for suggesting that I "think about going back to school") and to our children Hannah, Benjamin, and Callie. You four have always provided me with love, strength, and encouragement (literally all the way around the world). You are truly the greatest blessings I've been given in this life. May the sacrifices we have made benefit us all. 


\section{ACKNOWLEDGEMENTS}

I would like to thank my Father in heaven for it is only by His grace that I was able to accomplish this. I would also like to thank my Mom and Dad here on earth for their constant and unyielding support throughout my life.

I want to express thanks to my original mentor Dr. M. A. Cassaro for starting me on this journey. Not only have you helped me become a better structural engineer, but you have helped me become a better person. I would also like to thank Dr. Mario Paz for inspiring me to do my best.

I wish to thank Mr. Steve Durrett of the U. S. Army Corps of Engineers Louisville District for providing me with the time to complete this endeavor and I would like to thank Ms. Jill McCarthy of U. S. Geological Survey Geologic Hazards Team for also providing time and incentive to finish. I also want to express my heartfelt thanks to Ms. Courtney Kerr at the University of Louisville Graduate School for your amazing show of patience towards me.

I also appreciate the support from my committee members, especially Dr. DePuy for accommodating and tolerating my office visits. Finally, I can not express 
adequately my appreciation to my advisor and mentor Dr. D. J. Hagerty for your enduring support and encouragement to see this through to the end. I will never forget you volunteering to take this on. I hope you are pleased. 


\section{ABSTRACT \\ A COMPARISON OF THE STRUTURAL RESPONSE TO SYNTHETIC EARTHQUAKE GROUND MOTIONS COMPATILE WITH CENTRAL EASTERN UNITED STATES ATTENUATION MODELS}

Troy W. Milburn

September 25, 2008

This study focuses on the variation in structural response to synthetic ground motions derived from the latest attenuation models developed for the Central Eastern United States (CEUS) and used by the USGS to develop the latest edition (2008) of the National Seismic Hazard Maps. Specifically, it compares the ductility demands induced by these synthetic earthquake records via nonlinear time history analysis conducted on single degree of freedom systems of various natural frequencies. The synthetic ground motions generated for the study are compatible with target response spectra from these eight CEUS attenuation models and representative of various magnitude and distance combinations. The goal was to investigate the amount of variation in the structural response due to ground motions described by these differing attenuation models. In particular, the study determines whether these discrepancies are too great to permit this approach for developing synthetic records to be used as input in time history analysis for the seismic design of structures in the CEUS.

Results from the study do indeed show that the variation is great with many scenarios indicating conflicting predictions of ductility demand. In many cases there are contradictory results indicating whether damage would or would not occur (i.e., if the structure would reach yielding or not). Prime examples include the earthquake 
scenarios of magnitude 5.5 at $10 \mathrm{~km}, 6.5$ at $25 \mathrm{~km}$, and 7.5 at $50 \mathrm{~km}$. The synthetic time histories compatible with nearly half of the attenuation models representing these scenarios produced ductility demands that indicate significant yielding will occur in most structures while time histories compatible with the remaining attenuation models for these same scenarios indicate that no yielding will occur in any type of structure. In those cases where there is agreement that the structure will yield, there are discrepancies in the level of ductility demand that will be required. Also, a procedure is introduced which uses time history analysis to estimate the magnitude of the synthetic ground motions. This procedure is then applied to the results obtained from this study to infer the over prediction or under prediction of structural response to synthetic motions derived from the eight CEUS attenuation models. 


\section{TABLE OF CONTENTS}

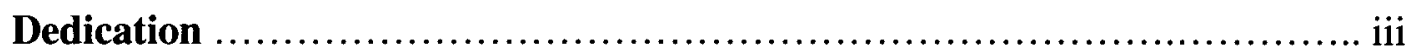

Acknowledgements ........................................................

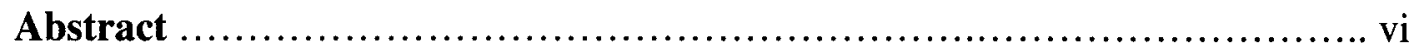

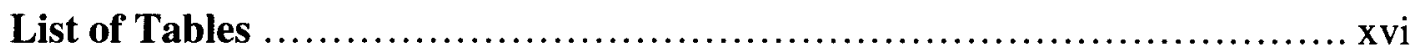

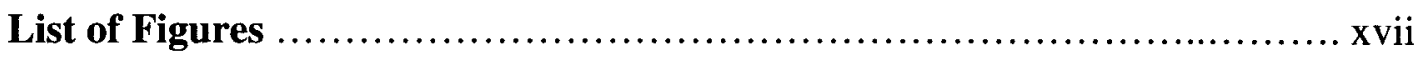

Chapter 1 Introduction .................................................. 1

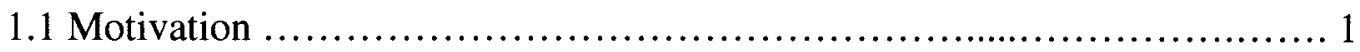

1.1.1 Seismic Hazard in the CEUS ............................... 2

1.1.2 New Madrid Seismic Zone ................................... 3

1.1.2.1 New Madrid History ....................................... 3

1.1.2.2 Current Activity $\ldots \ldots \ldots \ldots \ldots \ldots \ldots \ldots \ldots \ldots \ldots \ldots \ldots$

1.1.3 Other CEUS Seismic Zones .................................. 10

1.1.4 Probability of Recurrence ................................. 12

1.1.4.1 Paleoseismology ...................................... 12

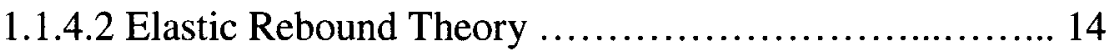


1.1.4.3 Probability Estimates ........................... 14

1.1.5 Repercussions and Possible Losses ........................ 15

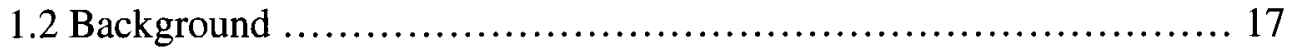

1.2.1 Analysis Methods ....................................... 17

1.2.2 CEUS vs. WUS Earthquakes ................................. 19

1.2.2.1 Frequency of Occurrence ......................... 20

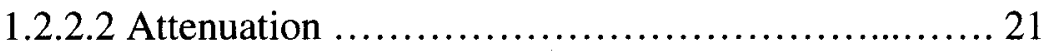

1.2.2.3 Source ......................................... 22

1.2.2.4 Frequency Content ............................. 23

1.2.3 CEUS Attenuation Relationships ......................... 26

1.2.4 Synthetic Ground Motions ........................................ 27

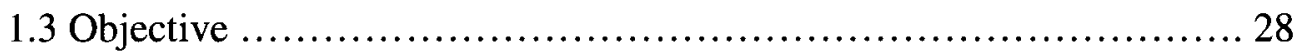

1.4 Organization and Scope ......................................... 34

Chapter 2 Attenuation Models ...................................... 37

2.1 Overview .................................................... 37

2.1.1 Definition .............................................. 37

2.1.2 Ground Motion Modeling Procedures ..................... 41

2.1.2.1 Source Models .................................... 43

2.1.2.2 Wave Propagation ............................. 43

2.1.2.3 Stochastic Method ................................ 44

2.1.2.4 Finite Fault Model ................................ 49

2.2 CEUS Attenuation Models ...................................... 51

2.2.1 CEUS vs. WUS Attenuation Models ......................... 51 
2.2.2 USGS National Seismic Hazard Maps ........................ 51

2.2.3 CEUS Seismological Parameters ............................ 52

2.2.3.1 Stress Drop ..................................... 53

2.2.3.2 Corner Frequency $\ldots \ldots \ldots \ldots \ldots \ldots \ldots \ldots \ldots \ldots \ldots . \ldots \ldots$

2.2.3.3 Kappa ...................................... 54

2.2.4 Previous CEUS Attenuation Models ......................... 54

2.32008 USGS CEUS Attenuation Models .............................. 55

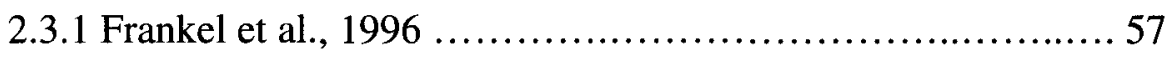

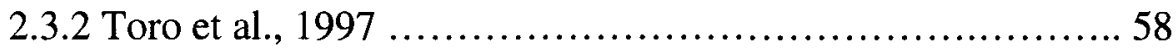

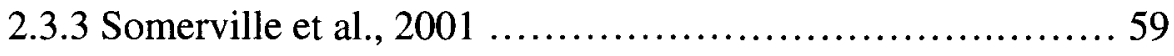

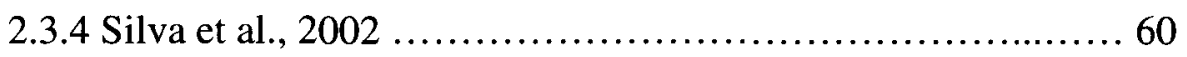

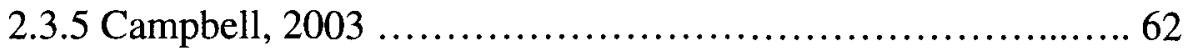

2.3.6 Tavakoli and Pezeshk, 2005 ................................ 63

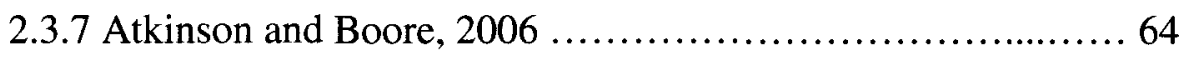

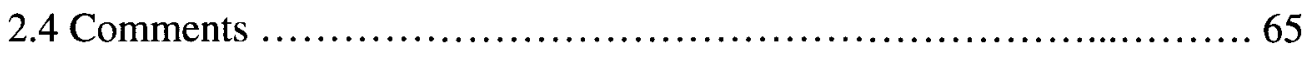

Chapter 3 Generation of Synthetic Ground Motions ..................... 67

3.1 Introduction .................................................... 67

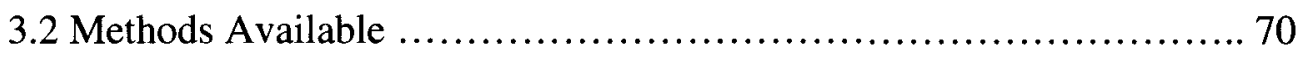

3.2.1 Record Modification .................................... 71

3.2.2 Record Generation ....................................... 73

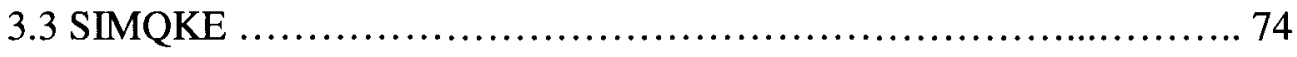

3.3.1 Approach ................................................ 74 
3.3.2 Development ............................................. 77

3.3.3 Procedure ................................................. 80

3.3.3.1 Input Parameters ................................ 81

3.4 Duration Model ...................................................... 83

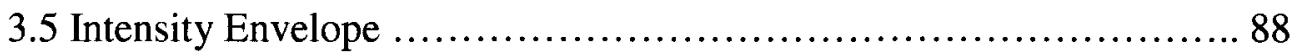

Chapter 4 Structural Response .............................................. 93

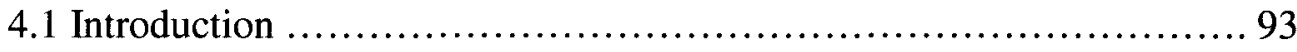

4.2 Time History Analysis ............................................ 94

4.2.1 Equations of Motion ........................................ 94

4.2.2 Nonlinear Response ....................................... 96

4.2.2.1 Hysteretic Behavior .............................. 100

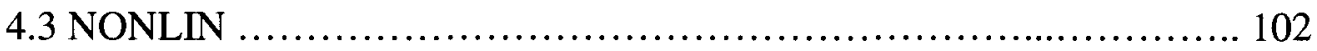

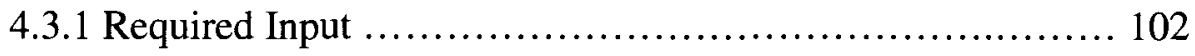

4.3.2 Available Output .......................................... 104

4.4 Structural Parameters .................................................. 104

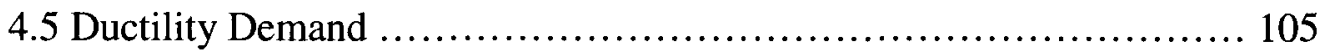

Chapter 5 Magnitude Estimation via Time History Analysis ............... 107

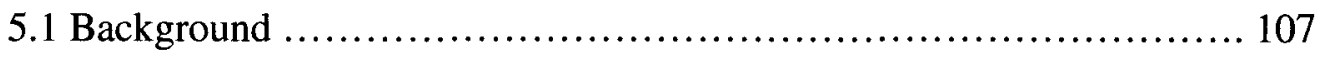

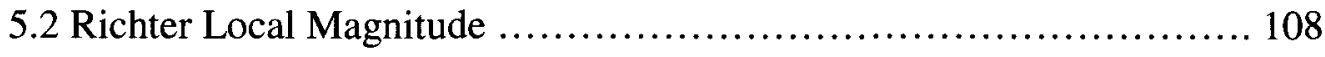

5.2.1 Definition ............................................... 109

5.2.2 Modern Recordings ...................................... 110

5.2.3 ENA Correction ........................................... 112 


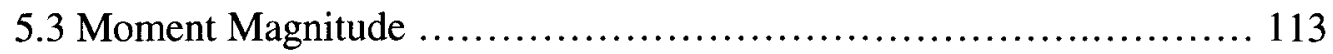

5.3.1 Definition ............................................. 113

5.3.2 Conversion ................................................. 114

5.4 Procedure ......................................................... 115

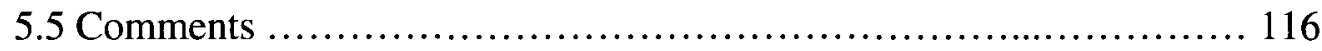

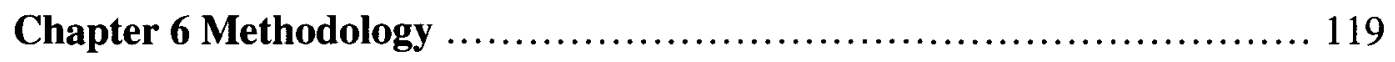

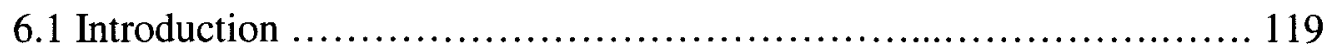

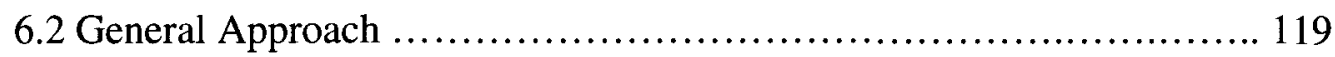

6.2.1 Automation Code ..................................... 120

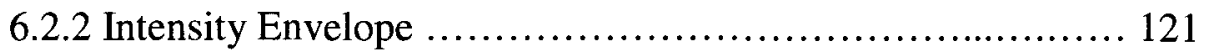

6.2.3 Structural Parameters ....................................... 126

6.2.4 Magnitude Estimation .................................... 130

6.3 Pilot Study ....................................................... 131

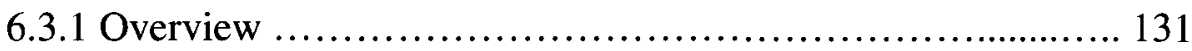

6.3.2 Procedure .................................................. 136

6.3.2.1 Duration and Intensity Envelope ................... 136

6.3.2.2 Input Spectral Range ............................. 137

6.3.2.3 Discretization Interval ............................... 137

6.3.2.4 Seed Numbers .................................... 138

6.3.2.5 Damping ....................................... 138

6.3.2.6 SDOF System Parameters ............................ 139

6.3.2.7 Input Spectra and Record Selection .................. 139

6.3.3 Results ................................................... 143 
6.3.3.2 Statistical Analysis of the Spectra Comparisons ........147

6.3.3.3 Statistical Analysis of the Response Comparisons .......149

\subsubsection{Statistical Analysis of the Estimated Magnitude} Comparisons .................................. 151

6.3.4 Conclusions Regarding the Pilot Study ..................... 153

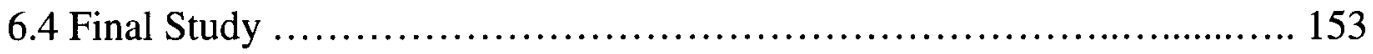

6.4.1 Methodology ............................................ 153

6.4.2 Attenuation Models ......................................... 157

6.4.2.1 Fault Model ...................................... 158

6.4.3 Synthetic Ground Motion Generation ......................... 162

6.4.3.1 Damping ....................................... 163

6.4.3.2 Maximum Ground Acceleration ..................... 164

6.4.3.3 Discretization Interval ......................... 164

6.4.4 Structural Response .................................... 165

6.4.5 Managing, Collecting, and Analyzing the Data .............. 165

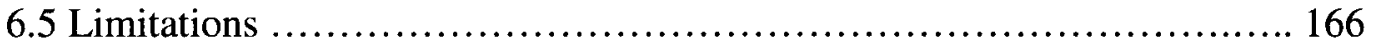

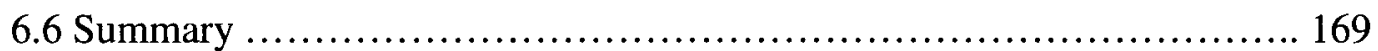

Chapter 7 Analysis of Results ....................................... 172

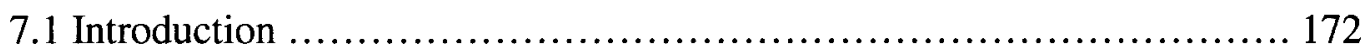

7.2 Organization or Results ..................................... 172

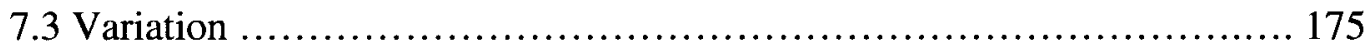

7.3.1 General Observations .................................. 183 
7.3.2 Overall Trends ............................................. 192

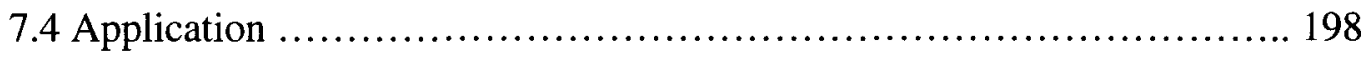

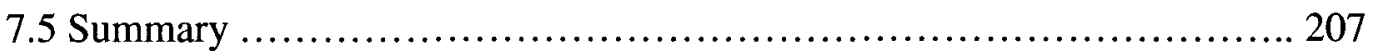

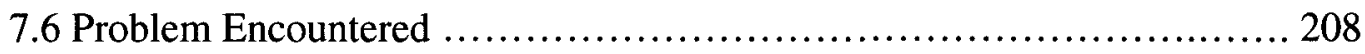

7.6.1 Truncation Study ........................................... 210

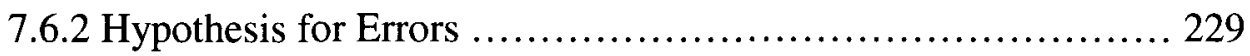

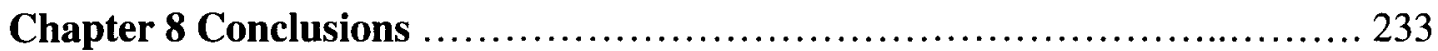

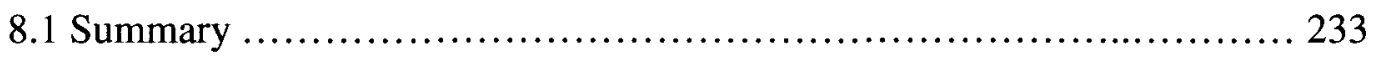

8.2 Further Research ................................................. 236

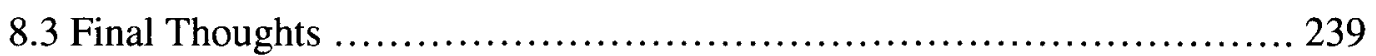

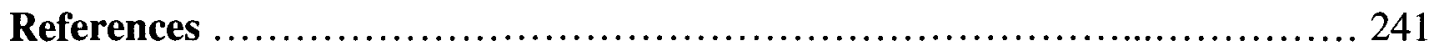

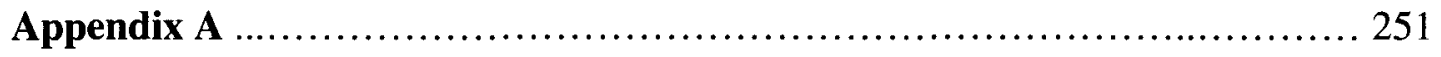

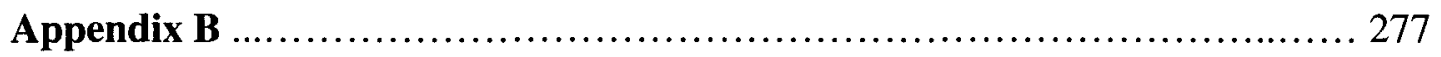

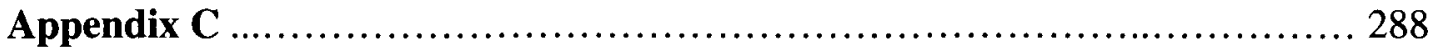

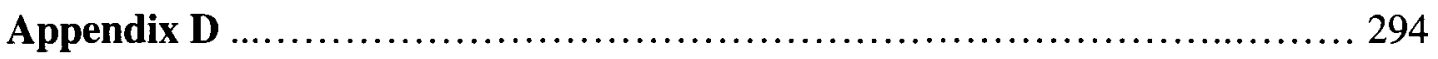

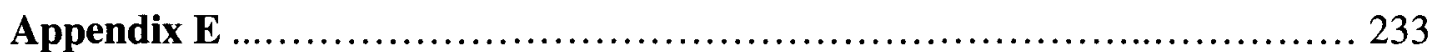

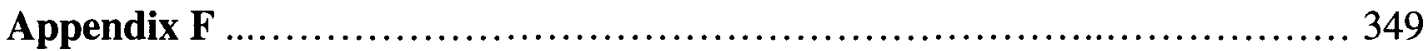

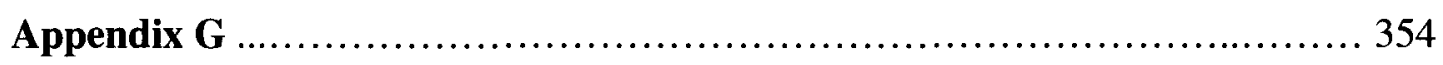




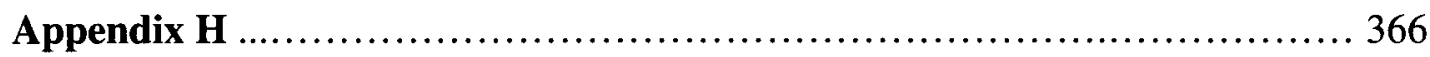

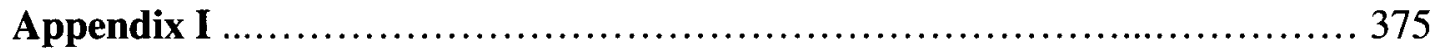

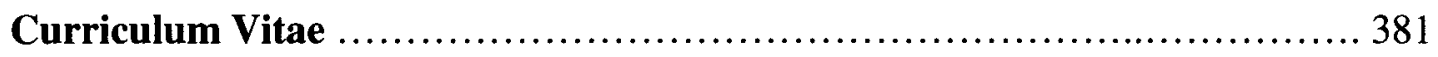




\section{LIST OF TABLES}

Table 6.1 Structural Parameters Used to Define the SDOF Systems Employed in this Study

Table 6.2 Parameters of the Actual Record Used in the Pilot Study 142

Table 6.3 Intensity Envelope Parameters Used in the Pilot Study to Generate Synthetic

Ground Motions from the Actual Records

Table 6.4 Peak Ground Accelerations and Discretization Intervals Used in the Pilot Study to Generate Synthetic Ground Motions from the Actual Records

Table 6.5 Sample Size Output Based on Ductility Demand from Minitab Analysis

Table 6.6 Sample Size Output Based on Estimated Magnitude from Minitab Analysis 146 


\section{LIST OF FIGURES}

Figure 1.1 Seismic Hazard in the Continental U. S. ............................... 2

Figure 1.2 Seismic Design Map ............................................. 3

Figure 1.3 The New Madrid and Wabash Valley Seismic Zones ................... 8

Figure 1.4 Aerial Photos and Trenching Exhibiting Sand Boils ..................... 13

Figure 1.5 Boundary that Delineates WUS Earthquakes from CEUS Earthquakes

Figure 1.6 Affected areas of the 1895 Charleston, Missouri and 1994 Northridge,

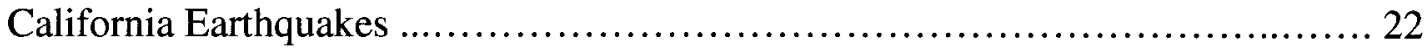

Figure 1.7 Concept of Response Spectrum ................................ 25

Figure 1.8 Sample Size Output Response Spectra Obtained from the Eight CEUS Attenuation Models Applied in this Study for a Magnitude 5.5 at $10 \mathrm{~km}$........... 29

Figure 1.9 Response Spectra Obtained from the Eight CEUS Attenuation Models Applied in this Study for a Magnitude 6.5 at $25 \mathrm{~km}$............................... 30

Figure 1.10 Sample Figure 1.10 Response Spectra Obtained from the Eight CEUS Attenuation Models Applied in this Study for a Magnitude 7.5 at $50 \mathrm{~km}$........... 31

Figure 2.1 Typical Fourier Amplitude Plot Showing Corner and Cutoff Frequencies 46

Figure 2.2 Characterization of a Finite Fault Source ….......................... 50

Figure 3.1Typical Narrow Band and Wide Band Processes ......................... 75

Figure 3.2 Intensity Envelope Options Available in SIMQKE ..................... 85

Figure 3.3 Saragoni and Hart (1974) Intensity Envelope from Boore (2003) ........ 86 
Figure 3.4 Example of an Intensity Envelope and Corresponding Synthetic Record

Figure 3.5 Example of an Intensity Envelope and Corresponding Constrained Synthetic Record

Figure 3.6 Actual Record Compared to the Exponential Envelope in SIMQKE Derived Using the Novikova and Trifunac (1994) Duration Model and the Sargoni and Hart (1974) Intensity Envelope Function Size ................................ 92

Figure 4.1 Elasto-plastic Behavior Assumed in this Study ......................... 101

Figure 6.1 Example of Matching the SIMQKE Function to the Saragoni and Hart Function ................................................................... 123

Figure 6.2 Regression Analysis Fit to Data Relating the $\alpha_{0}$ Parameter in SIMQKE to the Saragoni and Hart Model ....................................................... 124

Figure 6.3 Schematic Illustrating the Validation Methodology Applied in the Pilot

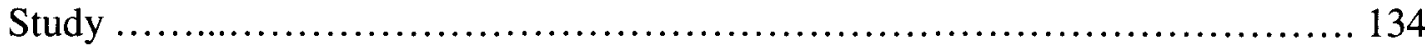

Figure 6.4 Comparisons of Response Spectra from the Loma Prieta Record Obtained

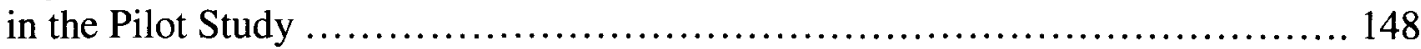

Figure 6.5 Comparisons of Response Spectra from the NE Ohio Record Obtained in

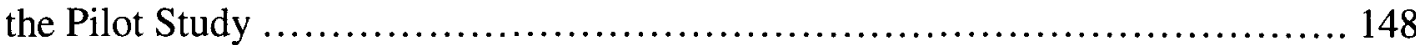

Figure 6.6 Ductility Demands for Actual Loma Prieta Record and Corresponding Synthetic Motions ........................................................... 150

Figure 6.7 Ductility Demands for Actual NE Ohio Record and Corresponding

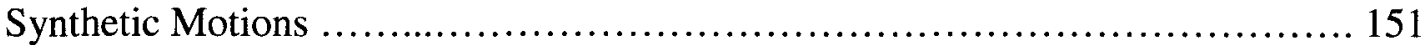

Figure 6.8 Calculated Moment Magnitudes from Actual Records and their Corresponding Synthetic Motions

Figure 6.9 Schematic Illustrating the Methodology Employed in the Final Study . 156

Figure 6.10 Definition Terms for Fault to Site Distances

Figure 6.11 Fault Model Assumed for Final Study to Provide a Consistent Definition of Distance 160

Figure 7.1 Sample Variation of Spectra from CEUS Attenuation Models for Magnitude 5.5 at $10 \mathrm{~km}$ 
Figure 7.2 Sample Ductility Demands from Synthetic Ground Motions Generated from CEUS Attenuation Models for Magnitude 5.5 at $10 \mathrm{~km}$

Figure 7.3 Sample Variation in Response for Magnitude 5.5 and 0.2 second Period

Figure 7.1 Variation of Spectra from CEUS Attenuation Models for Magnitude 6.0 at

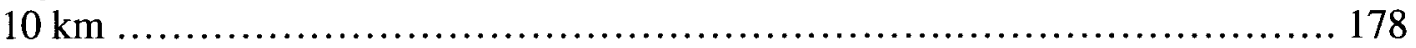

Figure 7.2 Ductility Demands from Synthetic Ground Motions Generated from CEUS Attenuation Models for Magnitude 6.0 at $10 \mathrm{~km}$............................. 179

Figure 7.3 Variation in Ductility Demands for Synthetic Ground Motions Generated from CEUS Attenuation Models for Magnitude 6.5 at $10 \mathrm{~km}$.................. 180

Figure 7.4 Variation in Ductility Demands for Synthetic Ground Motions Generated from CEUS Attenuation Models for Magnitude 6.5 at $25 \mathrm{~km}$..................... 181

Figure 7.8 Variation in Ductility Demands for Synthetic Ground Motions Generated from CEUS Attenuation Models for Magnitude 7.5 at $25 \mathrm{~km}$................... 192

Figure 7.9 Sample Variation in Ductility Demands for Synthetic Ground Motions Generated from CEUS Attenuation Models for Magnitude 7.0 and $0.2 \mathrm{sec}$ Response Period

Figure 7.10 Sample Variation in Ductility Demands for Synthetic Ground Motions Generated from CEUS Attenuation Models for Magnitude 7.5 and $0.6 \mathrm{sec}$ Response

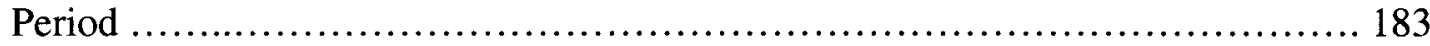

Figure 7.11 Sample Variations in Mean Ductility Demand from Magnitude 6.5 ... 193

Figure 7.12 Sample Variation in Standard Deviation of Ductility Demand from

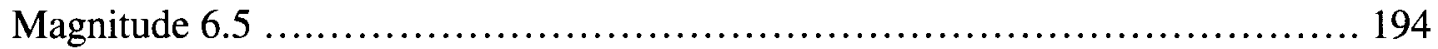

Figure 7.13 Sample Coefficient of Variation in Ductility Demand from Magnitude 6.5 195

Figure 7.14 Coefficient of Variation in Ductility Demand from Magnitude 7.5 ... 196

Figure 7.15 Mean Calculated Moment Magnitudes of Synthetic Ground Motions from CEUS Attenuation Models for Magnitude 5.5 197

Figure 7.16 Mean Calculated Moment Magnitudes of Synthetic Ground Motions from CEUS Attenuation Models for Magnitude 7.5 198 
Figure 7.17 Sample Confidence Intervals for Ductility Demand from Magnitude 6.5

Figure 7.18 Confidence Intervals for Calculated Magnitudes with Target Magnitude of 6.5 200

Figure 7.19 Comparison of Confidence Intervals for Ductility Demand and Calculated Magnitude for Magnitude 6.5

Figure 7.20 Ductility Demands from Synthetic Ground Motions Generated from CEUS Attenuation Models for Magnitude 6.5 at $25 \mathrm{~km}$........................ 202

Figure 7.21 Confidence Intervals for Ductility Demand from Magnitude 7.5 ....... 203

Figure 7.22 Comparison of Confidence Intervals for Ductility Demand and Calculated Magnitude for Magnitude of 7.5 204

Figure 7.23 Ductility Demands from Synthetic Ground Motions Generated from CEUS Attenuation Models for Magnitude 7.5 at $25 \mathrm{~km}$....................... 205

Figure 7.24 Ductility Demands from Synthetic Ground Motions Generated from CEUS Attenuation Models for Magnitude 7.5 at $50 \mathrm{~km}$........................ 206

Figure 7.25 Mean Calculated Moment Magnitudes of Synthetic Ground Motions from CEUS Attenuation Models for Magnitude 7.5 ................................. 206

Figure 7.26 Selection of Models for Magnitude 5.5 at $10 \mathrm{~km}$ to be used in the

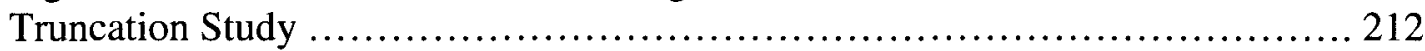

Figure 7.27 Comparison of Magnitude 5.5 at $10 \mathrm{~km}$ Target Spectrum from Somerville Model and Mean Spectra of Synthetics after Truncation 213

Figure 7.28 Comparison of Magnitude 5.5 at $10 \mathrm{~km}$ Target Spectrum from TavakoliPezeshk Model and Mean Spectra of Synthetics After Truncation ................. 215

Figure 7.29 Change in Ductility Demands for Magnitude 5.5 at $10 \mathrm{~km}$ after Truncation of Target Spectra ................................................ 216

Figure 7.30 Sample Change in Estimated Moment Magnitude after Truncation for

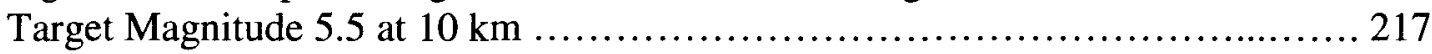

Figure 7.31 Selection of Models for Magnitude 5.5 at $10 \mathrm{~km}$ to be used in the

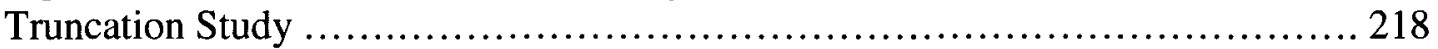


Figure 7.32 Comparison of Magnitude 7.5 at $50 \mathrm{~km}$ Target Spectrum from AtkinsonBoore Model with 140 bar Stress Drop and Mean Spectra of Synthetics after Truncation

Figure 7.33 Comparison of Magnitude 7.5 at $50 \mathrm{~km}$ Target Spectrum from Somerville Model and Mean Spectra of Synthetics after Truncation 220

Figure 7.34 Change in Ductility Demands for Magnitude 7.5 at $50 \mathrm{~km}$ after Truncation of Target Spectra

Figure 7.35 Change in Estimated Moment Magnitude after Truncation for Target Magnitude 7.5 at $50 \mathrm{~km}$

Figure 7.36 Selection of All Eight Models for Magnitude 6.5 at $25 \mathrm{~km}$ to be used in the Truncation Study 223

Figure 7.37 Comparison of Magnitude 6.5 at $25 \mathrm{~km}$ Target Spectrum from AtkinsBoore Model with 140 bar Stress Drop and Mean Spectra of Synthetics after Truncation 224

Figure 7.38 Comparison of Magnitude 6.5 at $25 \mathrm{~km}$ Target Spectrum from TavakoliPezeshk Model and Mean Spectra of Synthetics after Truncation 225

Figure 7.39 Change in Ductility Demands for Magnitude 6.5 at $25 \mathrm{~km}$ after Truncation of Target Spectra. 226

Figure 7.40 Ductility Demands from Magnitude 6.5 at $25 \mathrm{~km}$ after Truncation .... 227 Figure 7.41 Change in Estimated Moment Magnitudes for Magnitude 6.5 at $25 \mathrm{~km}$ after Truncation ... 


\section{Chapter 1}

\section{INTRODUCTION}

\subsection{Motivation}

The seismic threat in the Central and Eastern United States (CEUS) is credible. The region has a well-documented seismological history. This history requires that measures be taken to mitigate the impending risk of further seismic activity in the region. In addition to the inherent challenges of seismic design, the design of structures to withstand the shaking from earthquakes in the CEUS presents unique challenges associated with characterizing the anticipated ground motions that are specific to the region.

This study explores the possibility of the combined use of models proposed to represent the unique characteristics of earthquake ground motions in the CEUS and the most sophisticated method of structural dynamic analysis. The first half of this chapter focuses on the seismic threat in the CEUS as the motivation for conducting the study. The second half introduces some basic background information to help understand the problem statement and questions to be answered by conducting the study. 


\subsubsection{Seismic Hazard in the CEUS}

Seismic hazard refers to the probability of exceeding a specified level of ground shaking at a particular location. One only needs to look at the U. S. National Seismic Hazard Maps such as shown in Figure 1.1 to be convinced that substantial seismic hazard does exist in the CEUS. Areas of high hazard coincide with the locations where strong and devastating earthquakes have occurred in the past. In the CEUS, these areas include the Mississippi Valley, coastal South Carolina, and areas in the Northeast. These areas of high hazard are also conveyed by seismic design maps such as shown in Figure 1.2 and included in U. S. building codes.

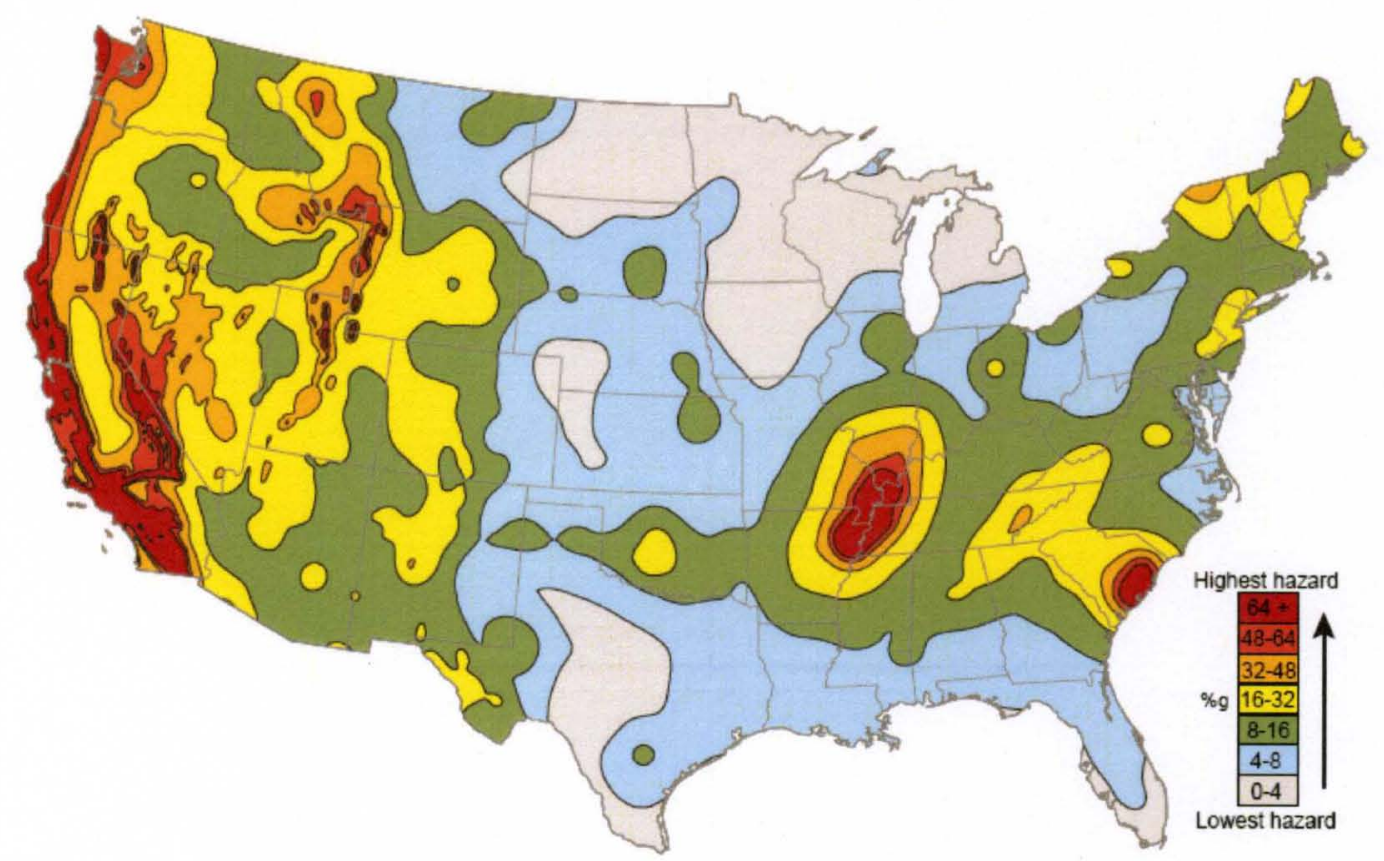

Figure 5.1 Seismic Hazard in the Continental U. S. (U.S. Geological Survey Fact Sheet FS-131-02, October 2002) 


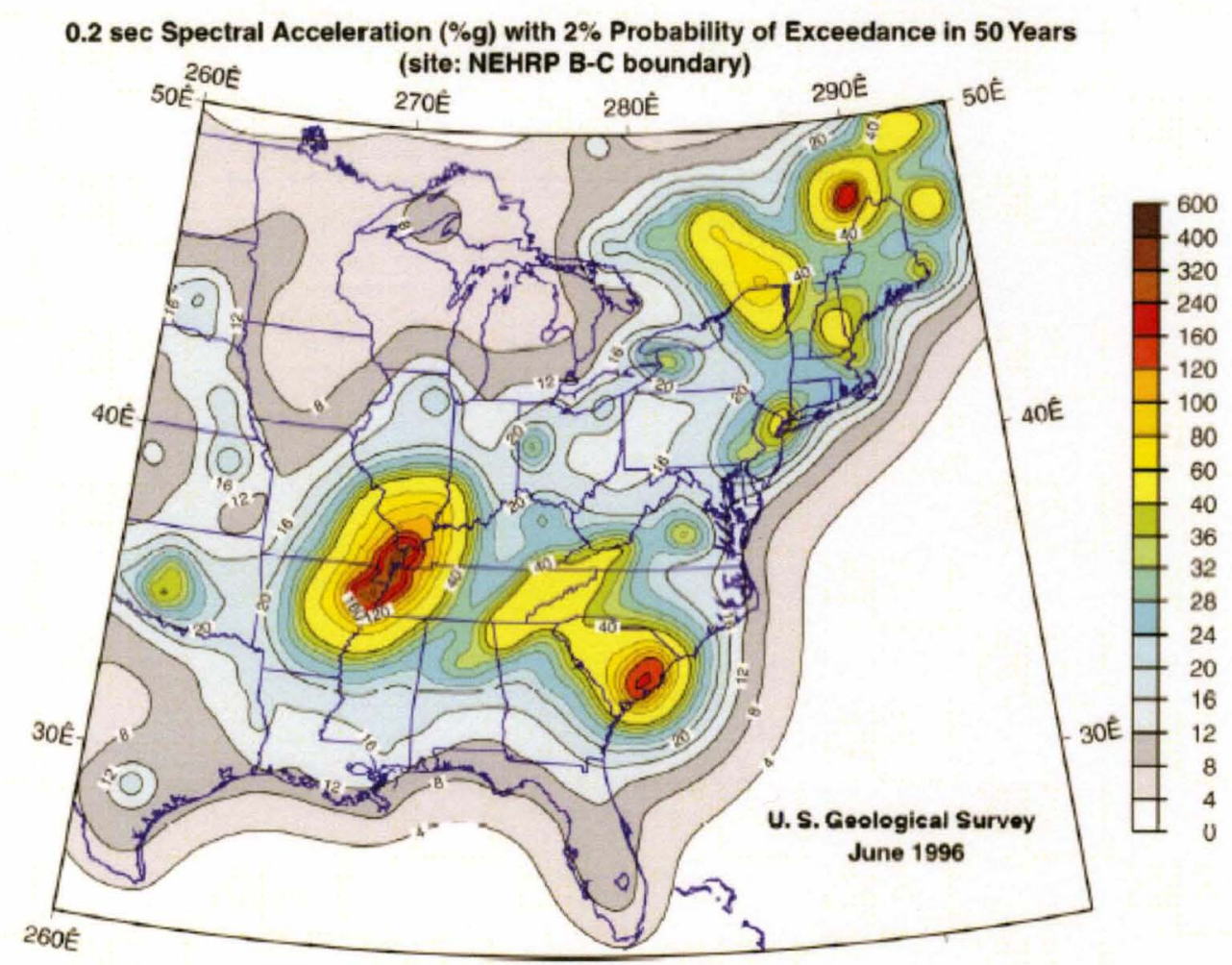

Figure 1.2 Seismic Design Map (U.S. Geological Survey, 1996)

\subsubsection{New Madrid Seismic Zone}

\subsubsection{New Madrid History}

In the winter of 1811-1812, at least four catastrophic earthquakes occurred in the Mississippi Valley of the central United States. These were the most powerful earthquakes to occur within the continental United States in the nation's history. They devastated the area which is now the southeast part of Missouri, the northeast part of Arkansas, the southwest part of Kentucky, and the northwest part of Tennessee (Nuttli, 1974). They were also felt over most of the nation. 
On December 11,1811, the first of these two earthquakes, both estimated at magnitude 8, occurred in Northeast Arkansas. The first shock was violent and followed by a heavy and destructive second event about 6 hours later. Damage was recorded as far distant as Cincinnati, Ohio; St. Louis, Missouri; and in many places in Kentucky, Missouri, and Tennessee (USGS, 2009).

On January 23, 1812 another earthquake of estimated magnitude 7.8 occurred and was then followed by another magnitude 8 event on February 7 . These last two earthquakes were centered about the town of New Madrid, Missouri. Their main shocks were also accompanied by hundreds of aftershocks that continued to be felt through the year 1817, some of which were severely damaging themselves (Nuttli, 1974). The fourth earthquake of the 1811-1812 series on February 7 was reported to have several destructive shocks, the last of which equaled or surpassed the magnitude of any previous event. The town of New Madrid was destroyed and many houses were severely damaged at St. Louis (USGS, 2009).

Extremely severe shaking was generated by these events. This area of the country was sparsely settled at the time; however most structures that did exist within 250 miles of New Madrid were damaged. It was reported by those who survived that great cracks opened in the earth's surface and the ground was seen to roll in waves. Vast areas of the land were reported to have sunk and risen. There were also reports of river traffic on the Mississippi being disrupted, and boatmen being killed. Perhaps the most noted of these disruptions was the local change in course of the Mississippi River creating Reelfoot Lake that covers more than ten square miles on the border of Kentucky and Tennessee (Hildenbrand et al, 1996). The earthquakes were reported to 
have caused church bells to ring 1,000 miles away in Boston, Massachusetts and Quebec, Canada. Damage was reported as far away as Washington, D.C. and Charlestown, South Carolina.

Some of the most dramatic effects of the earthquakes occurred along rivers. Entire islands disappeared and the banks of rivers collapsed. New sections of river channel were formed and old channels were cut off (Nuttli, 1974). Huge waves on the Mississippi River overwhelmed many boats and washed others high on the shore (USGS, 2009). Many boats were capsized and an unknown number of people were drowned.

Dramatic changes in the landscape also took place. Uplift of ten feet was reported at some places and lakes were both created and replaced with sand. Some openings in the ground were reported to be so wide that they could not be crossed on horseback. Previously rich land was left unfit for farming due to the formation of large fissures and swamps where the ground had subsided. In many places, fields were covered with sand and mud. The heavy damage caused by these earthquakes to these lands led the U. S. Congress to pass the first-ever disaster relief act in 1815 (Nuttli, 1974).

The felt areas of the four largest earthquakes were extremely large. They extended south to the gulf coast, southeast to the Atlantic coast, and northeast to Quebec, Canada. The western boundary cannot be established owing to a lack of population (Nuttli, 1974). However, recently published research indicates that the magnitudes and damage areas for the 1811-1812 earthquakes have been underestimated (Street et 
al, 2008). The study also indicates that the epicenter for the January 23 event has been located incorrectly.

An excellent composition of historical accounts that provide a good perspective of the intensity, effects, and damage encountered during the New Madrid events of 1811-1812 is provided by Bagnall (1996). This source also provides an excellent list of references for additional reading on the subject.

The area in which these historic earthquakes occurred is now known as the New Madrid Seismic zone (NMSZ) which reaches from Northeast Arkansas, through Southeast Missouri, Southern Illinois and into Western Tennessee and Kentucky (see Figure 1.3). The largest earthquakes to occur in the area since the 1811-1812 series of earthquakes had estimated magnitudes of 6.3 and 6.6 in 1843 near Marked Tree, Arkansas and in 1895 near Charleston, Missouri respectively. Other events of magnitude 5 or greater have occurred also within the zone. Overall, more than 4000 earthquakes have occurred in the NMSZ since the mid 1970s when monitoring equipment was installed.

The Charleston, Missouri event was the strongest earthquake in the CEUS since 1812 and was felt as far away as Indianapolis, Pittsburgh, New Orleans and Topeka (ISGS, 1995). All 12,000 telephone switches on the main Chicago exchange lit simultaneously and even stronger shaking was felt farther south in St Louis. The most serious damage occurred at the far southern tip of Illinois and Missouri. Hundreds of chimneys and glass windows were broken and every building in 
Charleston's commercial block suffered some form of damage. Damage was also reported in Indiana and Kentucky.

The magnitude 5.2 earthquake that occurred on April 18, 2008 was the largest earthquake to strike the CEUS in forty years. This event was centered at Mt. Carmel, Illinois and was actually located in the neighboring Wabash Valley Seismic Zone (see Figure 1.3). It was widely felt up to $500 \mathrm{~km}$ from the epicenter, an area including the cities of Chicago, Indianapolis, Lexington [Kentucky], Atlanta, Memphis, and St.

Louis. This recent event also serves as a reminder that there is still a seismic threat in the CEUS even today. 


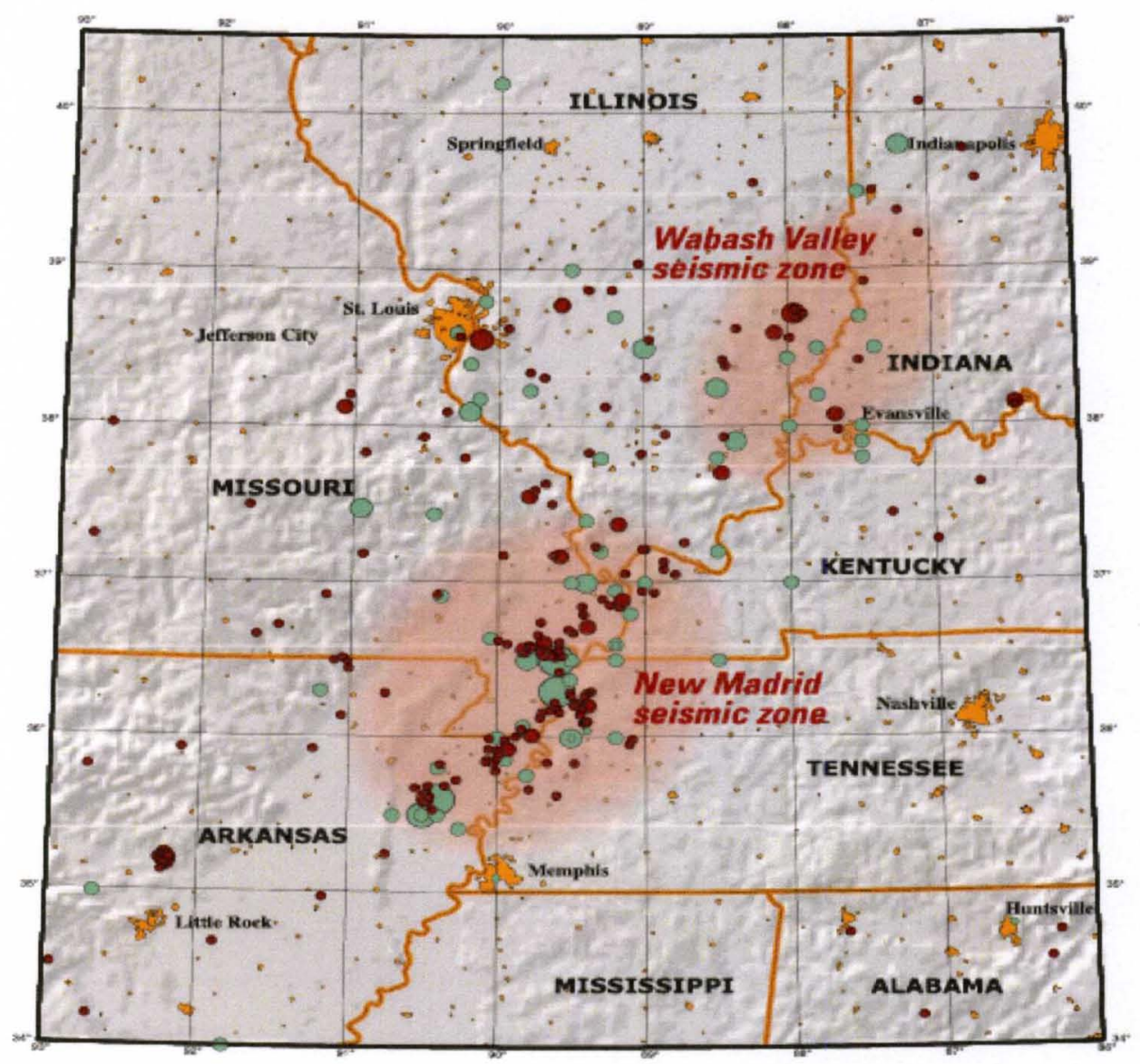

Figure 1.3 The New Madrid and Wabash Valley Seismic Zones (U.S. Geological Survey Fact Sheet FS-131-02, October 2002)

\subsubsection{Current Activity}

Even today, the central Mississippi Valley is more earthquake-prone and seismically active than any other area east of the Rocky Mountains in North America. Smaller earthquakes currently occur frequently in the CEUS and most of this activity takes place in the NMSZ. Throughout the region, between 150 and 200 earthquakes are recorded annually, although most are too small to be felt by people (Gomberg and Schweig, 2007). Earthquakes that are large enough to be felt occur about twice a year 
on average. Since 1900 , moderately damaging earthquakes have struck the seismic zone every few decades.

A large network of faults exists within the NMSZ. However, efforts to understand the cause of earthquakes in this region are hampered by the fact that the faults on which they occur are buried under deep layers of soil. Some of the largest faults have been identified by locating several small earthquakes that have occurred in the region. It was along two of these faults that the 1811-1812 earthquakes occurred. Other small faults have also been located within the zone but many small earthquakes have occurred away from these faults, indicating that there are several unknown faults capable of producing earthquakes.

A large network of faults in the NMSZ is located within the Reelfoot rift. The rift was formed when the region was being stretched through geologic processes and rock in the rift was dropped down about one mile (Hildenbrand et al., 1996). The tectonic movements have reversed and the region is now in compression reactivating the ancient faults of the Reelfoot rift, to generate earthquakes. As a result, the earthquakes that do occur within the NMSZ can strike anywhere within the region at irregular intervals. 


\subsubsection{Other CEUS Seismic Zones}

Other active seismic zones in the CEUS in addition to the NMSZ and Wabash Valley Seismic Zone are those located in South Carolina, Eastern Tennessee, and New England.

Charleston, South Carolina and its surroundings were devastated in 1886 by a very large earthquake of estimated magnitude 7.3. Aftershocks continued for years and some of them were large enough to be damaging by themselves. The 1886 event was the most damaging earthquake to occur in the Southeast United States and one of the largest historic shocks in Eastern North America (USGS, 2009).

As in the New Madrid events, structural damage was reported several hundred miles from the epicenter. At many places, large spaces were filled with sand, and, at other places, large openings were formed. In addition, in light of the types of structural damage reported, it has been speculated that the vertical component was actually dominant for the ground motion. Reports for this earthquake came from places as far away as Boston, Milwaukee, Chicago, Cuba, and Bermuda.

The Eastern Tennessee seismic zone extends across Tennessee and northwestern Georgia into northeastern Alabama. It is one of the most active earthquake areas in the Southeast although the largest earthquake known to have occurred in the zone was of magnitude 4.6 in 2003. 
Since colonial times, several earthquakes have occurred in the Northeastern United States (Wheeler et al, 2001). All of these events were felt at large distances; most were accompanied by several aftershocks and caused local changes to the landscape such as landslides, and sand boils. These events include a magnitude 6.5 earthquake in 1638 in central New Hampshire that was felt throughout New England and on ships near the coast. The largest event reported in the area was a magnitude 7.0 earthquake which occurred in 1663 about 60 miles northwest of northern Maine. This is the largest earthquake known in the northeastern U.S.

Two magnitude 5.2 earthquakes have struck New York City in the past. One occurred in 1737 and the other in 1884. Damage was reported from both events. Three strong shocks occurred with this earthquake with the second being the strongest. A magnitude 6.0 earthquake also struck Cape Ann, Massachusetts in 1755, about 35 miles northeast of Boston. Damage was reported along with reports of shaking having been felt on a ship 200 miles at sea. Those aboard the ship thought it had run aground (Wheeler et al, 2001).

Damage was also reported with a magnitude 5.9 earthquake that occurred in 1904 in easternmost Maine. The most recent event to have occurred in the northeast was the magnitude 5.2 earthquake in 1998 that was centered in northwestern Pennsylvania. It was accompanied by an explosion-like noise (Wheeler et al, 2001). Damage was also reported and more than 100 residential water wells lost water and many of them had to be deepened. 


\subsubsection{Probability of Recurrence}

\subsubsection{Paleoseismology}

Paleoseismology is the study of ancient earthquakes (Gomberg and Schweig, 2007) and scientists working in this discipline have discovered evidence that earthquakes have repeatedly occurred throughout history in the CEUS. An accompanying phenomenon associated with strong earthquakes in the CEUS is the formation of sand blows or sand boils. These features are formed during intense shaking that causes buried saturated sand layers to liquefy and be forced through cracks to the surface due to the overburden weight. The sand erupts at the ground surface in mounds known as sand boils (see Figure 1.4). Even today, an abundant supply of sand boils can be seen in portions of the NMSZ. Often crops do not grow well in these sand deposits making them conspicuous in the corn and soybean fields of the Midwest.

When sand blows bury artifacts or are buried themselves by material that can be dated (usually through carbon dating), the date at which the earthquake that caused them can be estimated. These types of studies have indicated that the 1811-1812 earthquakes were not isolated incidents but a repetition of events that had occurred twice before at roughly 500-year intervals. There are further indications that these events were actually sequences of events similar to those of the 1811-1812 events. 

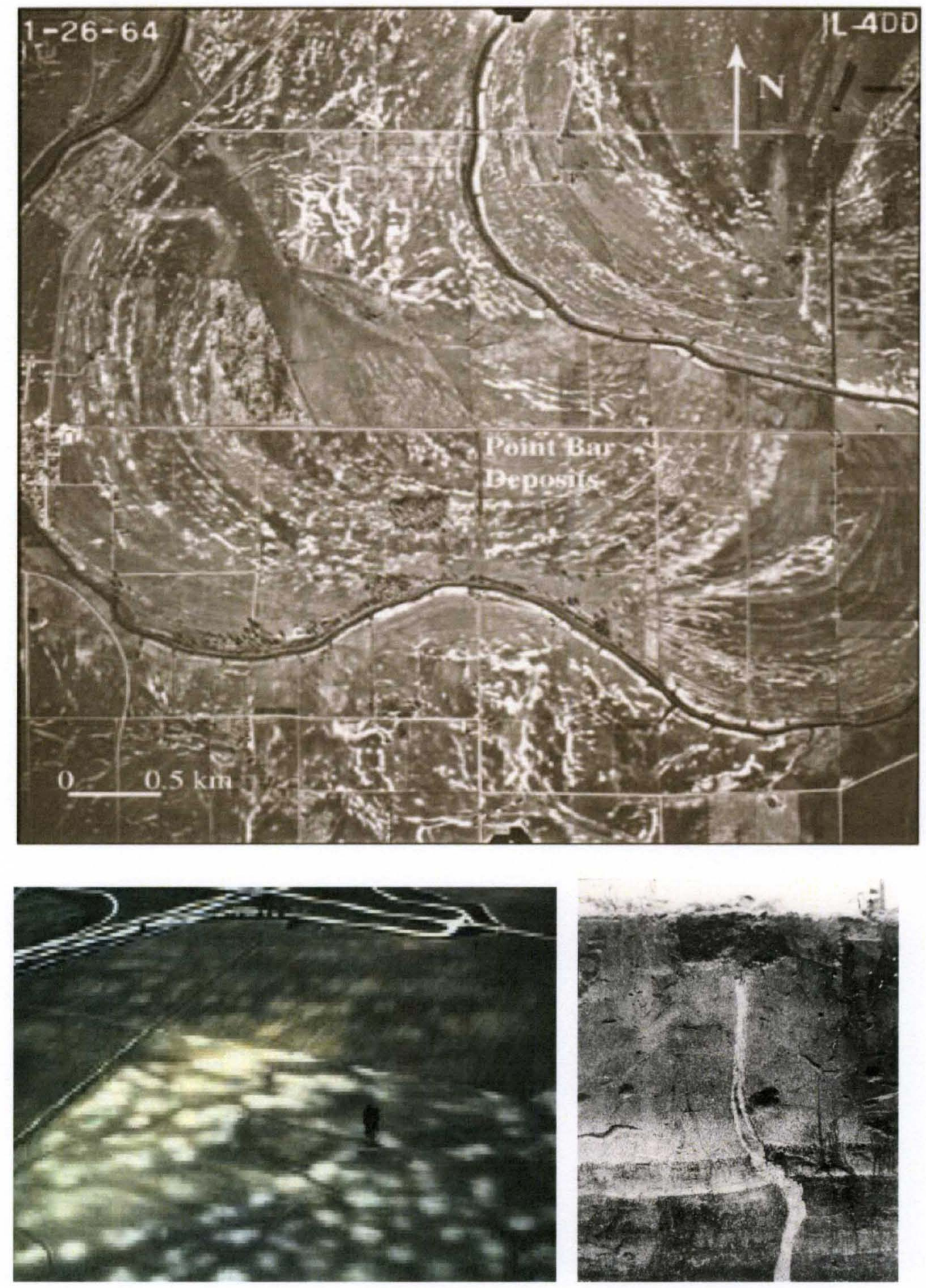

Figure 1.4 Aerial Photos and Trenching Exhibiting Sand Boils (U.S. Department of the Interior, U.S. Geological Survey) 


\subsubsection{Elastic Rebound Theory}

The elastic rebound theory was developed by Reid (1911) after his study of the 1906 San Francisco earthquake and is used to explain the occurrence of earthquakes still today. The theory basically states that strain energy is stored along faults due to the movements associated with plate tectonics. Frictional forces can cause the strain energy to build over several years. At some point, the stored energy generates forces so great that they overcome the frictional resistance and a rupture occurs in the form of a sudden slip along a fault; we refer to the sudden release of energy as an earthquake.

Although the time of occurrence is indeterminate, this explanation of why earthquakes occur implies that the recurrence of a large CEUS earthquake appears likely. Furthermore, this theory could lead one to believe that because large CEUS earthquakes occur infrequently, more strain energy is being stored that will lead to a greater release when an earthquake does occur.

\subsubsection{Probability Estimates}

Scientists with the U. S. Geological Survey (USGS) have established probability estimates of earthquake intensity for the region that were used in the development of the National Seismic Hazard Maps (Gomberg and Schweig, 2007) which serve as a basis for seismic design maps implemented in most U. S. building codes. These estimates give the probability of an earthquake with a magnitude greater than 6 happening in the NMSZ within the next 50 years as between $25 \%$ and $40 \%$. The 
estimates also give the likelihood of a repeat event with similar magnitude as those of the 1811-1812 earthquakes (i.e. magnitude 7.5 to 8 ) occurring within the next 50 years as between $7 \%$ and $10 \%$. Previous estimates have placed the probability of a magnitude 6 to 7 earthquake occurring in the New Madrid Seismic Zone within the next 50 years as higher than $90 \%$ (Schweig et al, 1995). An earthquake of magnitude 6 could cause serious damage to structures and facilities in areas near the epicenter and those in the 7.5 to 8.0 magnitude range could cause widespread damage over a large area resulting in loss of life and property damage in the hundreds of billions of dollars (Elnashai, 2008).

\subsubsection{Repercussions and Possible Losses}

In 1811 , the central Mississippi Valley was sparsely populated and there were few man-made structures. Today, this region is home to millions of people, including the populations of large cities, such as St. Louis, Missouri, and Memphis, Tennessee. Adding to the danger, most structures in the region were not built to withstand earthquake shaking, as they have been in California.

The seismic threat in the CEUS is underscored by an initiative underway by the Federal Emergency Management Agency (FEMA) to develop catastrophic earthquake disaster response plans for the eight states of the NMSZ (FEMA, 2008). The initiative, known as the NMSZ Catastrophic Earthquake Disaster Response Planning Initiative, involves partnerships and collaboration with hundreds of government agencies, business, industry and voluntary organizations, and scientific and academic 
institutions. As part of this initiative, a study was conducted at the Mid-America Earthquake (MAE) Center to assess the potential impacts from future moderate to severe earthquakes in the CEUS.

That study has determined that tens of thousands of homes will be damaged with nearly 400,000 people being displaced. The study also estimates that there will be over 75,000 casualties and over $\$ 200$ billion in direct economic losses. It predicts that Memphis, Tennessee, will be heavily damaged with significant damage and losses extending into surrounding states (Elnashai, 2008).

Critical infrastructure and lifelines also will be heavily damaged and out of service for a considerable period of time severely hampering evacuation and relief efforts. Such mass outages are likely to affect a region much larger than the eight surrounding states that suffer direct losses. Many hospitals as well as police and fire services will be severely impaired. Many schools that serve as public shelters also will be damaged and unusable after the earthquake.

Utility services will be severely disrupted for hundreds of thousands of customers because of extensive facility and pipeline damage. Extended service outages will be highly likely for tens of thousands of customers, making it difficult for them to remain in their homes, even if those homes are structurally sound after the earthquake. Damage to major natural gas and oil transmission lines will lead to service interruptions that will affect areas as far away as the east coast and New 
England. A series of successive earthquakes, as in 1811-1812, would generate even more catastrophic impacts.

The MAE Center study notes that economic losses due to indirect distress are at least as high if not much higher than the direct economic losses and that the total economic impact of a series of NMSZ earthquakes is likely to constitute by far the highest economic loss due to a natural disaster in the U. S. (Elnashai, 2008).

\subsection{Background}

\subsubsection{Analysis Methods}

The threat of a major earthquake occurring in the Central Eastern United States is real. In order to mitigate this threat, structural engineers need the ability to conduct analyses that accurately model building performance during an earthquake. Today there are several methods available to analyze the response of structures to seismic loading. Examples include: equivalent lateral force procedures, modal analysis, capacity-spectrum methods, push-over analysis, and time history analysis.

Simplified equivalent lateral force procedures dominate the design landscape for most practicing engineers. However, it is generally agreed that nonlinear time history analysis provides the best representation of how structures respond during an earthquake. The widespread availability of high speed computers and structural 
analysis software has led to its use becoming increasingly more common. We now have the means with computing capabilities and modern software to build virtual structural models using finite element methods and subject them to earthquake loading to investigate their response.

The main emphasis in earthquake engineering is to introduce ductility into the structure. The reason for introducing ductility is that while failure often cannot be prevented, it can be controlled. Prevention often is done by incorporating "fuses" within the structure that are sacrificed to yielding in order to develop an overall failure mechanism that is designed to absorb energy but resist collapse. These types of designs often require the use of nonlinear time history analyses to estimate the ductility demands that will be placed on the structure during an earthquake. This method allows the actual strength properties of the various elements of a structure to be explicitly considered so that the sequence in which they will yield can be predicted. Engineers then are able to determine those members that require strength adjustments to remain within required ductility limits.

Time history analysis looks at the structural response in very small time increments. Basically, the loading condition at the beginning of a time increment is applied to the structure, which is characterized by known or assumed mechanical properties, etc. The structure then is analyzed using the equations of motions under these conditions and the resulting displacements, changes in stiffness, etc. are then used as the initial conditions for the next time step. To determine the seismic 
response of a structure, the input loading is often an earthquake acceleration record as recorded on a seismograph or accelerometer.

If there is a drawback to the use of time history analysis (especially in the CEUS), it is that as the name implies, this method requires the input of a time history as loading. Although large earthquakes have occurred in the CEUS and are expected to occur again, those that have occurred did so at times that predated the invention of seismographic instruments now used to record such events. Consequently, there are no actual strong motion records available for the CEUS. To complicate the situation further, as will be described in the next section, there are documented differences in the ground motions expected to occur in the CEUS and those anticipated from the west coast of the US where strong motion recordings are available.

\subsubsection{CEUS vs. WUS Earthquakes}

There are at least four major differences between earthquakes that occur in the CEUS and those that occur in the Western United States (WUS) (Nuttli and Herrman, 1987). These differences include frequency of occurrence, attenuation, source and frequency content. These general differences are presented in following paragraphs and some specific differences will be described in the following chapter. Figure 1.5 shows the boundary differentiating WUS earthquakes and CEUS earthquakes. 


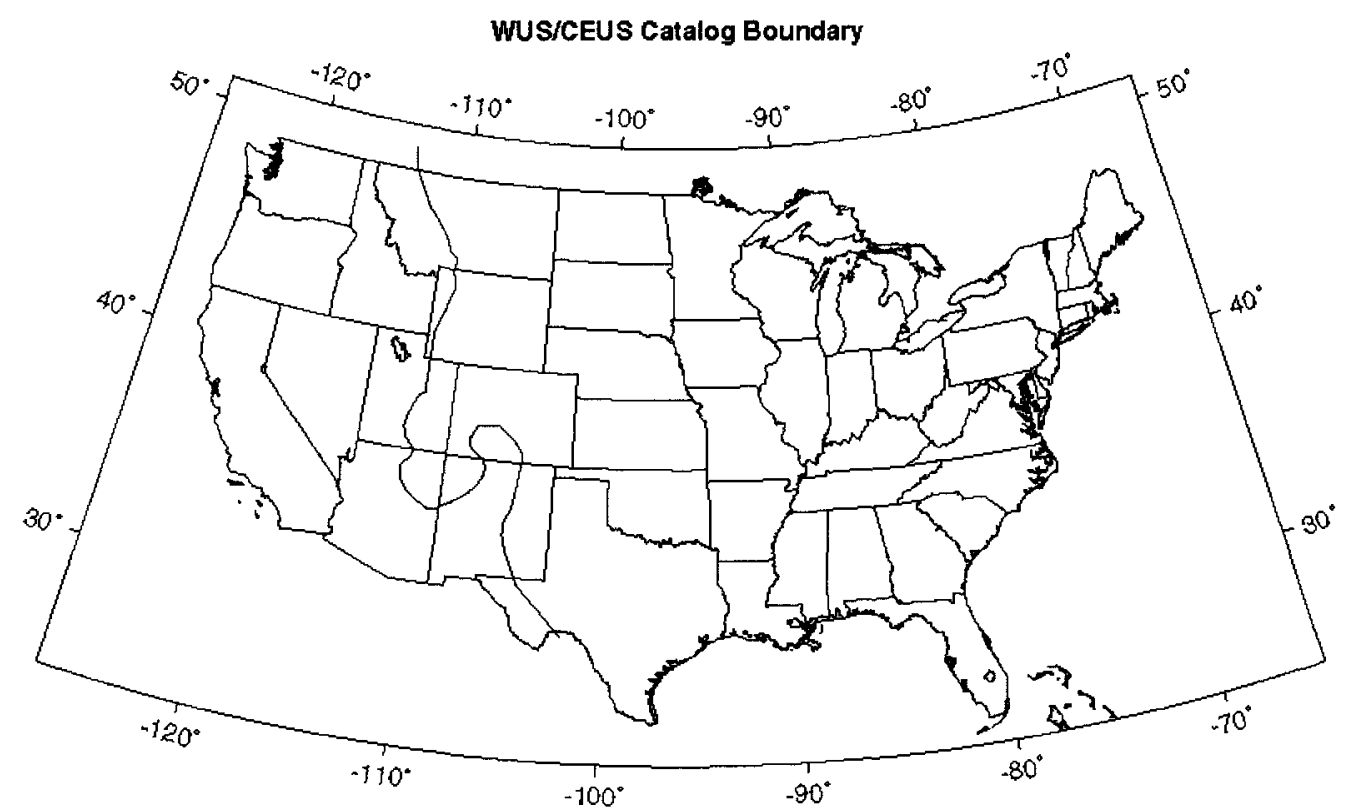

Figure 1.5 Boundary that Delineates WUS Earthquakes from CEUS Earthquakes (U.S. Geological Survey, Open-File Report 97-464)

\subsubsection{Frequency of Occurrence}

Earthquakes of the same magnitude occur about ten times more frequently west of the Rocky Mountains than they do east of them (Nuttli and Herrman, 1987). As cited in the previous section, this lower frequency of occurrence has led to a paucity of actual strong earthquake records for the CEUS region. Despite this lack of data, the interval between large earthquakes in the CEUS is much shorter than expected based on current activity. In other regions, the estimated likelihood of large events is based on statistical analysis of the occurrence of small events; however, this approach does not seem to apply to the CEUS. This nonconformity is shown in hazard estimates for the New Madrid region being similar to those for California even though large earthquakes occur much less frequently in the NMSZ. 


\subsubsection{Attenuation}

Perhaps the largest difference between earthquakes that occur in the CEUS and those that occur in the WUS is that the attenuation of seismic waves at frequencies usually associated with damaging ground motions is significantly smaller in the CEUS. That is, seismic waves of given intensity tend to travel farther in the CEUS than in the WUS. An earthquake in the CEUS can be felt over an area as much as ten times larger than the area affected by a similar magnitude earthquake on the west coast. For example, the San Francisco, California, earthquake of 1906 of magnitude 7.8 was felt some 350 miles away, whereas the New Madrid earthquake of December 1811 of magnitude 8.0 rang church bells in Boston, Massachusetts 1,000 miles away. Another example of the differences between earthquake characteristics in the CEUS and the WUS is shown in Figure 1.6 by comparing the areas affected by two other earthquakes of similar magnitude. The map shows the affected areas of the 1895 Charleston, Missouri earthquake in the New Madrid seismic zone, and of the 1994 Northridge, California earthquake. Red indicates minor to major damage to buildings and their contents. Yellow indicates the area in which shaking was felt but with little or no damage. 


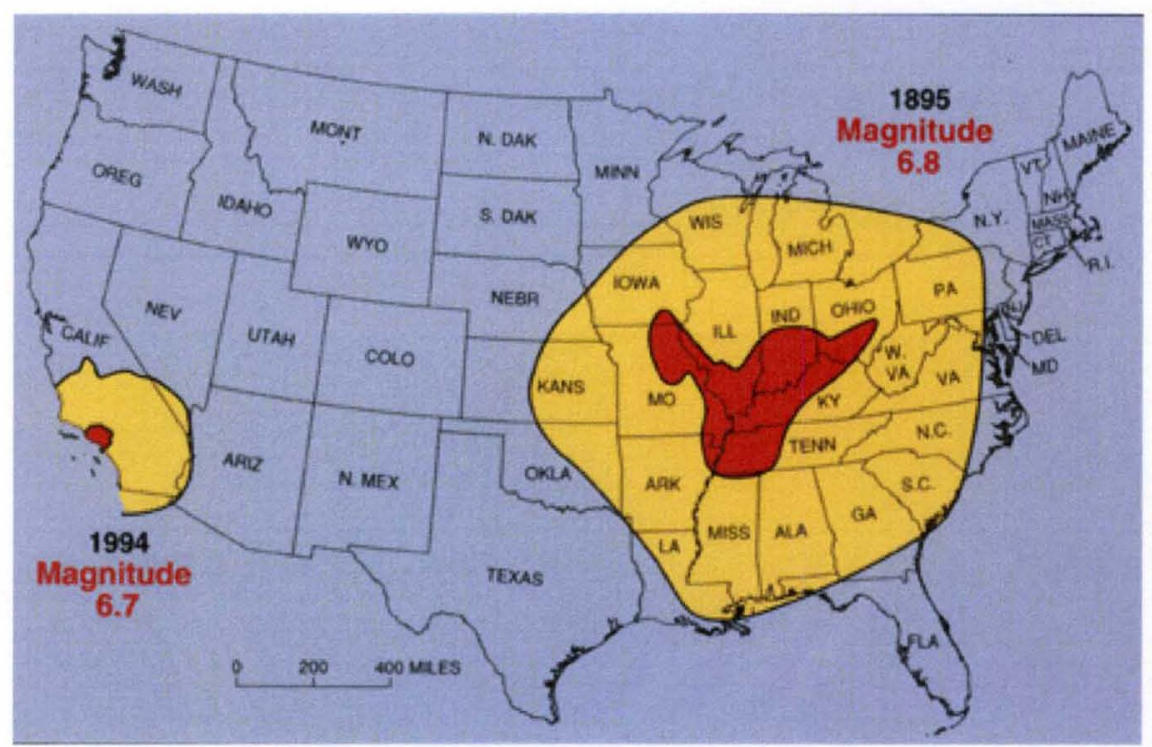

Figure 1.6 Affected areas of the 1895 Charleston, Missouri and 1994 Northridge, California Earthquakes (U.S. Geological Survey Fact Sheet-168-95, 1995)

These differences in attenuation can be attributed to the earth's crust being more intact east of the Rockies whereas it is more fractured west of them. This fracturing causes a tendency in the crust of the WUS to "soak up" earthquake energy, whereas in the CEUS regions of the country the seismic energy is absorbed much less strongly with distance (Nuttli, 1974). It is these differences in geology east and west of the Rocky Mountains that cause this strong contrast in propagation of earthquake effects.

\subsubsection{Source}

The majority of the world's earthquake activity takes place along plate boundaries, an example of a plate boundary being the San Andreas Fault in California. The New Madrid region, however, is located far from any plate boundary. In fact, the NMSZ is closer to being in the middle of the North American plate; the nearest plate boundary is about 12,000 miles away in the Caribbean Sea. As a result, 
there appears to be fundamental differences in the geologic processes, and source mechanisms for earthquakes in the CEUS compared to those occurring along plate boundaries (Gomberg and Schweig, 2007).

In addition, there is a large depth of soil down to rock in the major source region of the NMSZ that exacerbates the difficulty in investigating the source mechanism for earthquakes in the region. Consequently, the source mechanisms for CEUS earthquakes are not well defined or understood. In fact, it is this lack of understanding that has led to variation in the development of the several different attenuation models proposed for the CEUS and applied in this study.

\subsubsection{Frequency Content}

Another major difference between WUS and CEUS earthquakes is their frequency content. This difference can be attributed to the attenuation rate not being as great in the CEUS. As seismic waves travel away from their source, the higher frequency components of the ground motion are scattered and absorbed more rapidly than the lower frequency components. As a result, the frequency content also changes with distance (Kramer, 1996). Because of the attenuation difference in the CEUS, this change in frequency content is also different. However, because of the differences in source conditions, intraplate earthquakes have initially higher frequency components that persist at distances close to the epicenter. It should be noted that the frequency content of the ground motion is one of the most influential factors in how a structure will respond to an earthquake. 
The single degree of freedom (SDOF) displacement response to a harmonic loading is given by Equation 1.1.

$$
y(t)=\frac{F_{0} / k}{1-r^{2}}(\sin \bar{\omega} t-r \sin \omega t) \quad \text { (Equation 1.1) }
$$

As shown in Equation 1.2, $r$ is the frequency ratio defining the ratio of the earthquake frequency $\bar{\omega}$, to the natural frequency of the system, $\omega$.

$$
r=\frac{\bar{\omega}}{\omega} \quad \text { (Equation 1.2) }
$$

Every structure has a frequency at which it will vibrate naturally; that frequency is determined by its mass and stiffness. It can be seen from Equation 1.1 that as the earthquake frequency approaches a system's natural frequency, $r$ tends to one, and the displacement approaches infinity. Such a system is said to be at resonance. However, for most structures, as the frequency content of the ground motion approaches that of the natural frequency of the structure, material strength limitations actually cause failure of the structure (Paz, 1997).

The inverse of a system's natural frequency is known as its natural period of vibration. As just mentioned, SDOFs with different physical properties such as height, weight, and material properties will have different natural periods of vibration. 
Therefore, they will also have different maximum response amplitudes due to the same input ground motion. As shown in Figure 1.7, the maximum amplitudes for each system to a specific ground motion represent one point that makes up the response spectrum for that particular earthquake ground motion. The response spectrum is a concise way to represent the frequency content of an earthquake ground motion (Newmark and Hall, 1982).

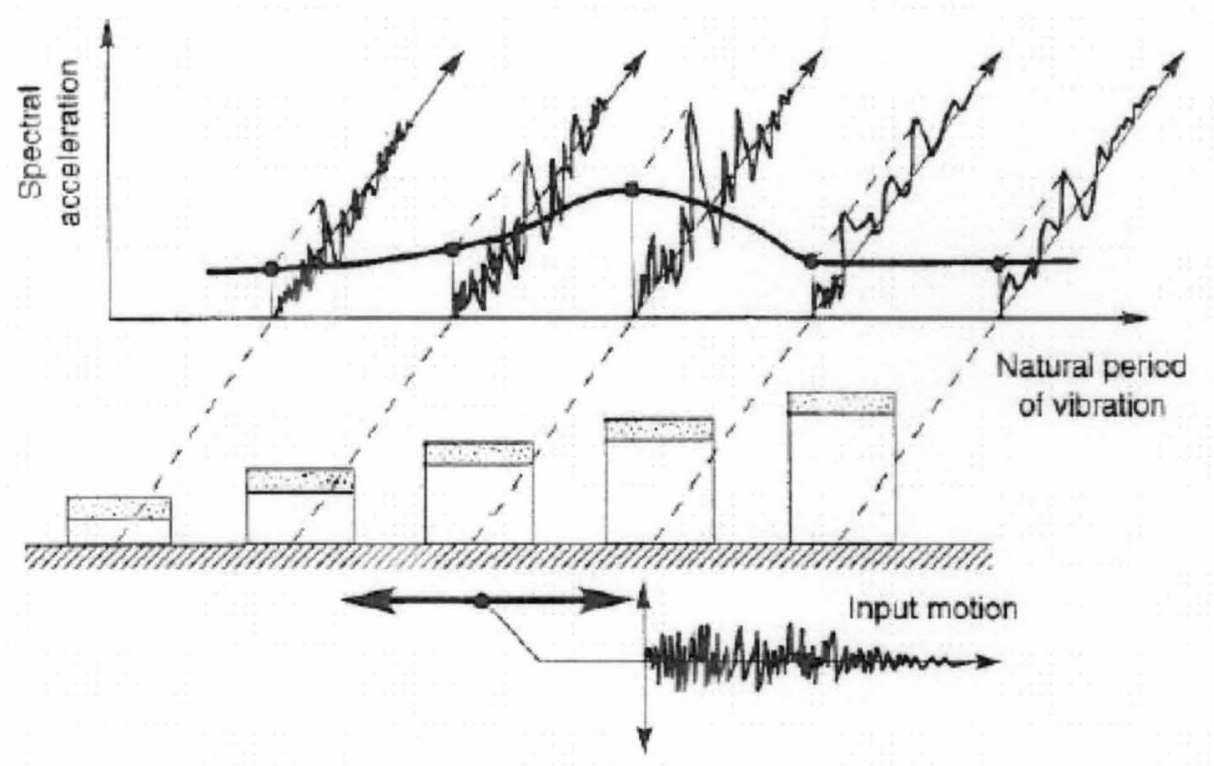

Figure 1.7 Concept of Response Spectrum (Kramer, 1996) 


\subsubsection{CEUS Attenuation Relationships}

Since the frequency content of earthquakes is different for different regions, regionally specific attenuation models have been developed for the CEUS. Those models attempt to describe the proposed frequency content of the ground motion for an earthquake as a function of magnitude and distance in the form of a response spectrum.

Seismologists have made several attempts to develop attenuation relationships that they believe represent the characteristics of earthquake ground motions in the central eastern United States. Each of these models gives different spectral values that provide differing frequency contents proposed for these ground motions. This variation is produced from significant differences in the choice of model parameters as well as assumptions about the radiation of energy from the earthquake source.

There were seven different models selected for use in the development of the 2008 USGS National Seismic Hazard Maps (Petersen et al., 2008). These attenuation models were used to arrive at acceleration values for specific sites within the U. S. via probabilistic seismic hazard analysis. These maps are the basis for many national, state, and local seismic safety regulations and design standards. 


\subsubsection{Synthetic Ground Motions}

The scarcity of actual earthquake strong ground motion recordings has driven the development of methods to generate synthetic earthquake ground motions. In addition, methods have been developed to use input spectra as target spectra to define the frequency content of these synthetic ground motions.

The generation of synthetic ground motions has been used quite extensively for the development of attenuation models used in probabilistic seismic hazard analysis. This is especially true for the CEUS; however, the processes to generate these ground motions are inherently grounded in relatively complex seismological theory. As a result, there is a gap between the need for representative ground motions that can be utilized in structural analyses and the ability of most practicing structural engineers to be able effectively to obtain them because the required knowledge is outside the realm of most structural engineers. There is a need for robust processes that structural engineers can utilize to generate synthetic ground motions for design that are plausible representations of the motions expected to occur in the CEUS.

This study investigates the feasibility of using the CEUS attenuation models used in the development of the most current USGS National Seismic Hazard Maps to develop target spectra for the generation of synthetic accelerograms that are plausible representations of ground motions that can be expected to occur in the CEUS and can be used as input time histories in nonlinear time history analyses to investigate structural response. 


\subsection{Objective}

As described in the previous sections, the seismic threat in the Central Eastern United States (CEUS) is credible. The region has an inescapable seismic hazard possessing an obligatory consideration in structural design requiring that measures be taken to mitigate the impending risk of further seismic activity in the region. These measures further require that structures in the region be designed to withstand the loadings from future earthquakes. An accurate portrayal of a structure's response to ground motions is required to produce an effective seismic design.

Time history analysis methods provide this capability; however, they require earthquake time histories as input and unfortunately, actual recordings of strong ground motion are scarce in the CEUS. To make matters worse, the frequency content of ground motions in the CEUS is different from that in other parts of the world and frequency content may be the single most important factor in determining how a structure will respond to an earthquake. In their final form, attenuation models which are functions of magnitude and distance describe the frequency content of ground motions as response spectra.

Region-specific attenuation models have been developed by several researchers to predict the frequency contents expected for earthquake ground motions in the CEUS. In fact, seven of these attenuation models were employed in the development of the latest edition of the National Seismic Hazard Maps which are used to establish design maps applied in nearly all U. S. building codes. The latest edition of these maps was 
released in April 2008. As seen in Figures 1.8, 1.9, and 1.10, it is obvious by viewing the spectra from the attenuation models used to develop these maps that there is variation in the ground motions they predict. The figures show spectra for example scenarios obtained from the eight attenuation models.

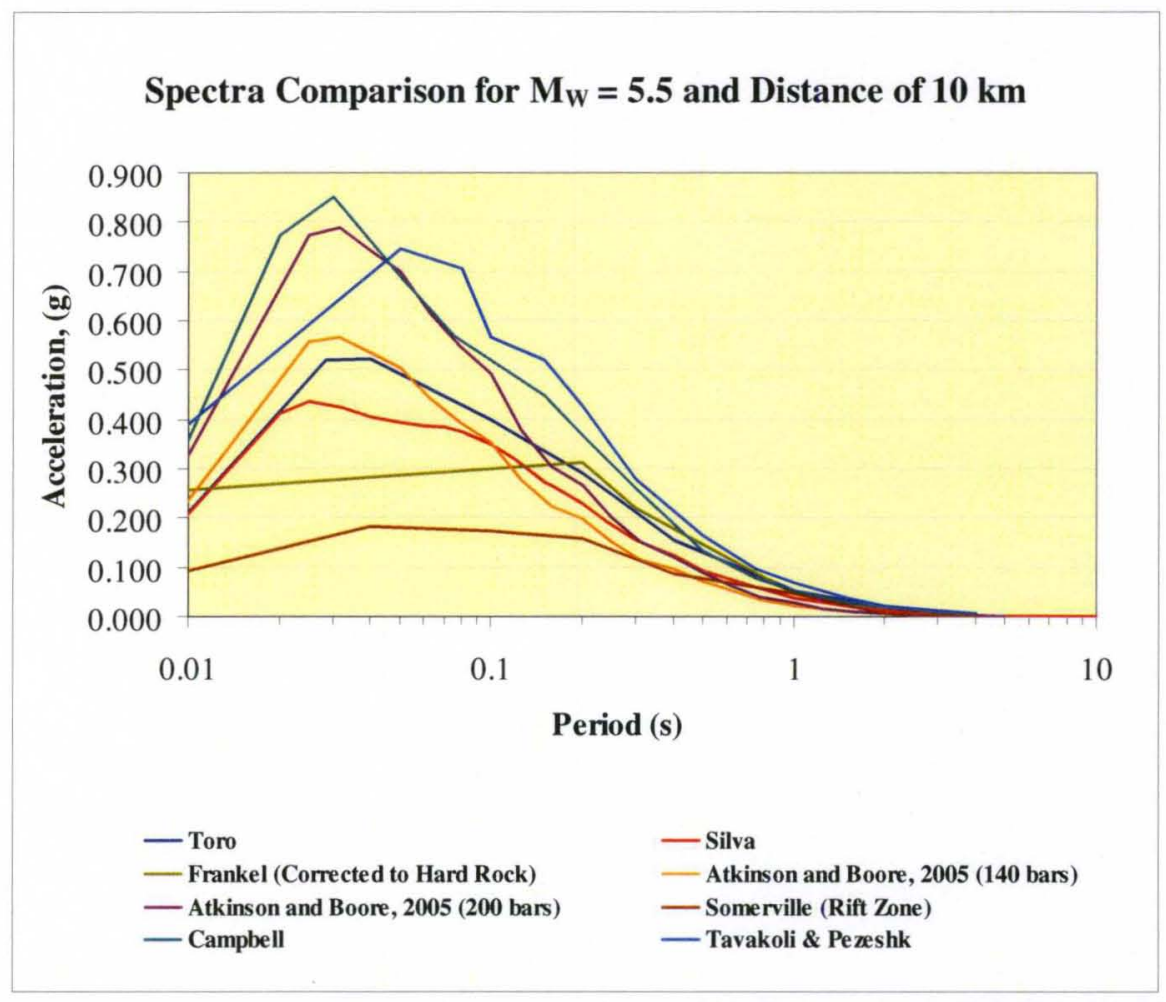

Figure 1.8 Response Spectra Obtained from the Eight CEUS Attenuation Models Applied in this Study for a Magnitude 5.5 at $10 \mathrm{~km}$ 


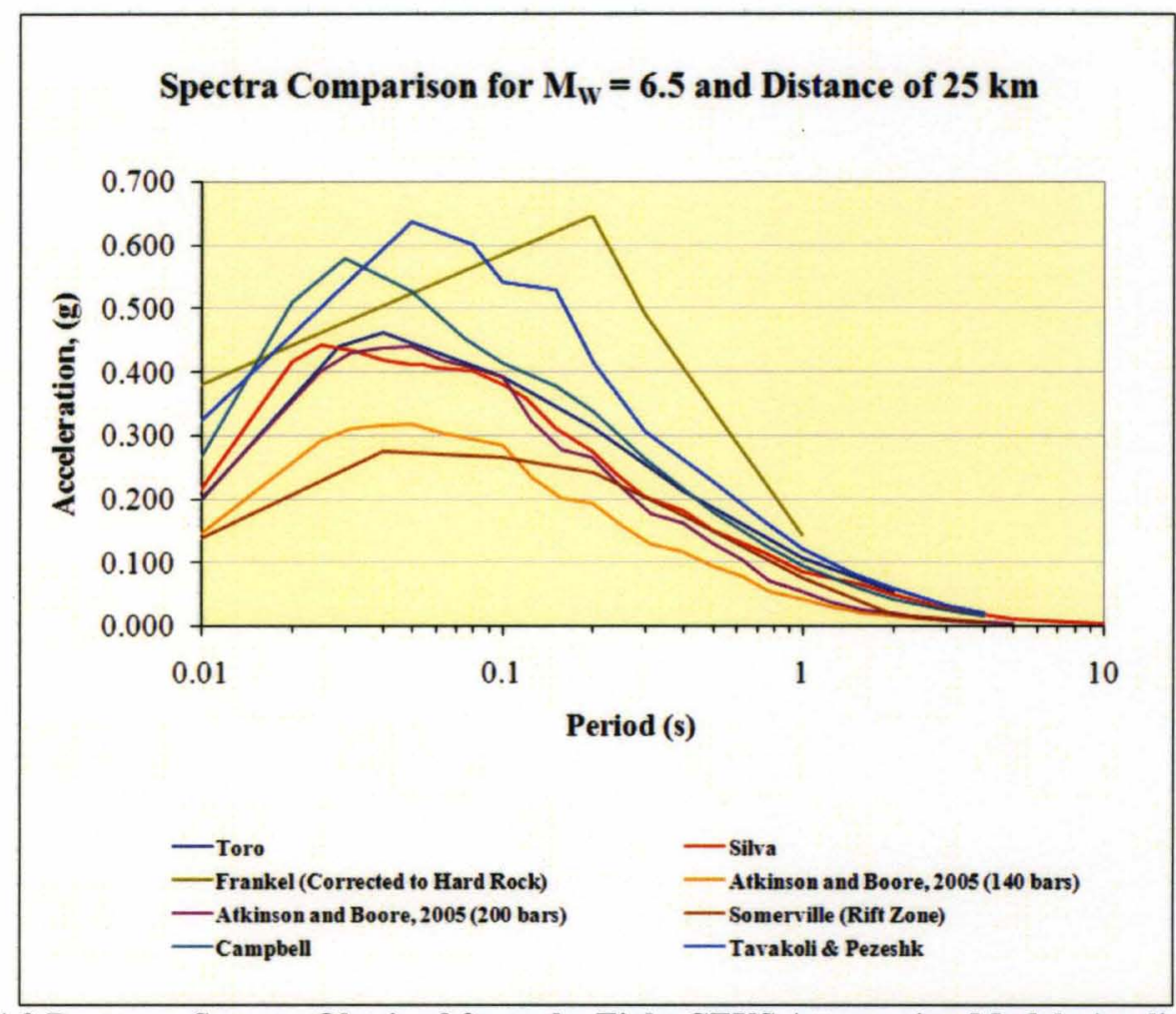

Figure 1.9 Response Spectra Obtained from the Eight CEUS Attenuation Models Applied in this Study for a Magnitude 6.5 at $25 \mathrm{~km}$ 


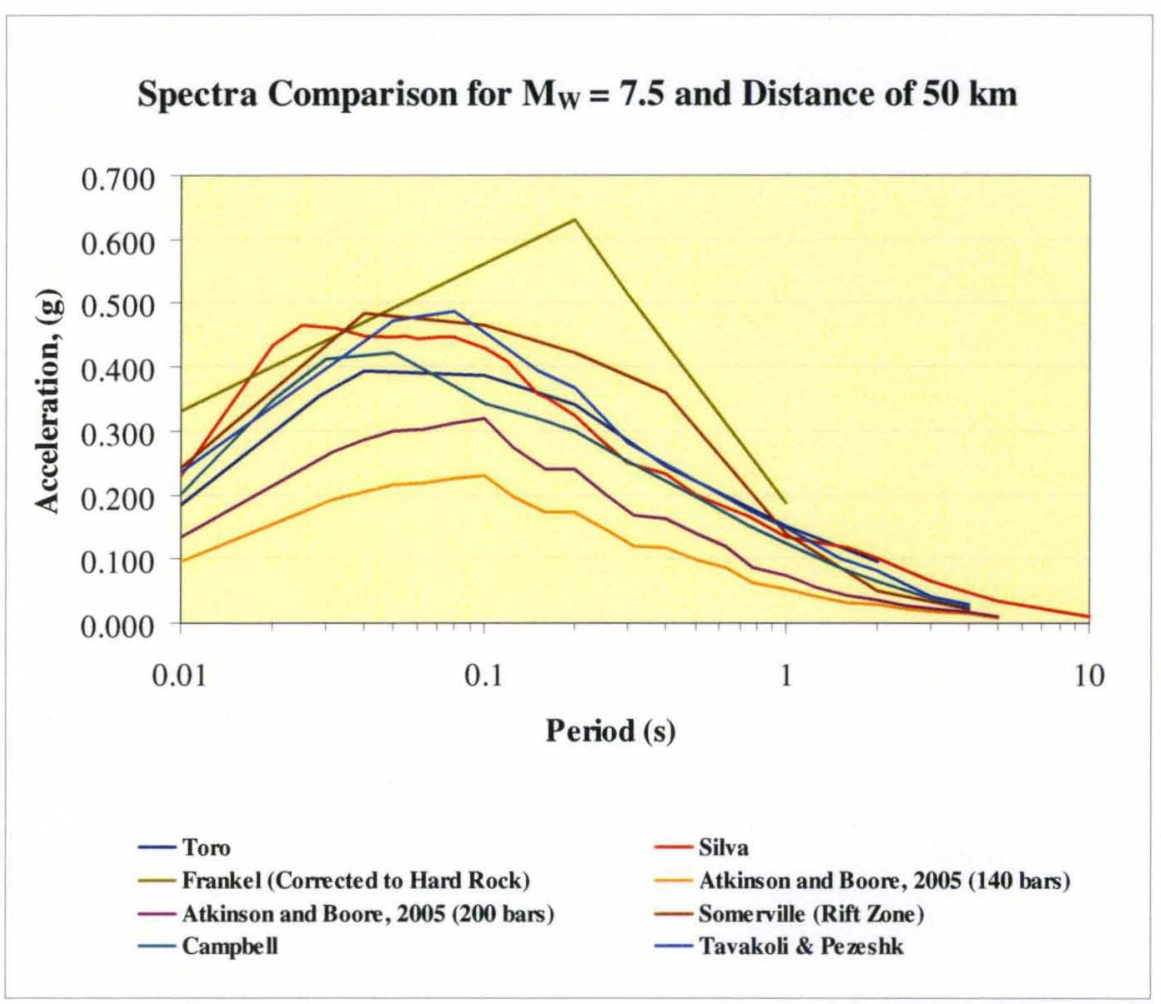

Figure 1.10 Response Spectra Obtained from the Eight CEUS Attenuation Models Applied in this Study for a Magnitude 7.5 at $50 \mathrm{~km}$

What is not apparent in these figures is how the variation in these spectra translates into the variation of how structures would respond to the ground motions that these spectra represent. As an alternative to the use of actual earthquake time histories, synthetic ground motions can be generated and used as input loading for time history analyses used in the seismic design of structures. There are methods available to generate synthetic ground motion time histories whose frequency contents are compatible with those prescribed by the input of response spectra. One such method is the one embodied in the SIMQKE computer program (Gasparini and Vanmarke, 1976). 
It is a logical extension from the previous description that the latest attenuation models developed for the CEUS should be used to produce target response spectra for use in conjunction with these methods to generate synthetic ground motions to be applied as loading in time history analyses for the seismic design of structures in the region.

For the practicing structural engineer, a robust and somewhat simplified method is sought to develop earthquake ground motion time histories that can be used for design purposes. There is a need within the structural engineering community for a procedure that does not require advanced knowledge of seismology. Software that utilizes methods to generate synthetic time histories that are compatible with input target response spectra such as the SIMQKE program seems attractive in this regard. This conclusion is especially true in the CEUS where there is an absence of actual recordings and a large amount of uncertainty in values describing the geophysical parameters associated with the region.

Choosing applicable values for geophysical parameters required in the development of attenuation models and synthetic seismograms is outside the "comfort zone" of most structural engineers. Fortunately, the input target spectra required by the software just mentioned to generate synthetic ground motions can be taken simply as the output response spectra from the attenuation models developed for the CEUS. In this way, the structural engineer attempting to generate plausible synthetic ground motions for the CEUS is relying solely on the knowledge and judgment of the 
geophysicists and seismologists who developed the attenuation models being utilized. By using CEUS attenuation models, as presented, to generate target spectra describing the frequency content for the synthetic ground motions, the engineers are applying the intrinsic assumptions and accepting the selection of parameter values made by the scientists who developed the individual models to represent ground motions for the region based on their expertise and available data.

The purpose of this study is to determine if the variation among the structural response in the form of the displacement ductility demand as determined via nonlinear time history analysis using synthetic earthquake ground motions simulated to be compatible with attenuation models proposed for the CEUS is too great to be a viable approach in the seismic design of structures within the region.

To explain how a variation can be "too great" in this context, a hypothetical case can be considered. In this case, synthetic ground motions compatible with one of the CEUS attenuation models for a particular scenario (i.e. magnitude and distance) applied as input loading in a time history analysis indicate little or no ductility demand on a particular structure (i.e. produces no damage). For this very same scenario however, synthetics compatible with another CEUS attenuation model predicts significant ductility demand on the same structure. The analysis results from such a case may offer more confusion than answers to the design engineer and the question may now be shifted to ask which of the attenuation models is producing the correct synthetic ground motions. 
Traditional design methods in structural engineering and due diligence requires conservatism favoring the "worst case" scenario. However, cost is always an issue in any design and also demands consideration. Furthermore, in order to achieve proper performance, the seismic design of structures requires a reasonably good estimate of seismic ductility demand. A variation in ductility demand that is "too great" in this context impedes reaching conclusions to meet these goals. Specifically, this study concentrates on the amount of variation in the structural response predicted from synthetic ground motions compatible with the eight CEUS attenuation models used to develop the 2008 USGS National Seismic Hazard Maps.

Again, Figures 1.8 through 1.10 show that there are differences among these attenuation relationships. The question posed in this study is how do these differences carry over into synthetic ground motions [generated to be compatible with these attenuation models] and cause variation in the structural response they induce. Specifically this study asks, is this variation in structural response within an acceptable range to permit their use as a feasible approach to the seismic design of structures in the CEUS.

\subsection{Organization and Scope}

Chapters 2 through 5 of this dissertation essentially provide a basic literature review of the topics relevant to this study. Their purpose is to provide the reader with an overview of the general concepts and models used in the study. As such, they are not intended to be a complete review of the subtopic areas but rather focus directly on 
those aspects that are of importance to this particular study and necessary to understand its intent.

Chapter 2 looks at attenuation models providing an overview beginning with a definition and brief presentation of various procedures used to develop them. It also introduces attenuation models specific to the CEUS and provides a brief description of the seven CEUS models used to develop the 2008 version of the National Seismic Hazard Maps investigated in this study.

Chapter 3 introduces the topic of generating synthetic seismograms (also known as records, ground motions, or time histories) with a focus on the approach used in the SIMQKE computer program. The duration model and development of the intensity envelope used in the study also are presented.

The fourth chapter presents structural response as it applies to this study and specifically as achieved through time history analyses. It also describes the NONLIN software (FEMA, 1996) used in this study to conduct these analyses, as well as the important input structural parameters and a description of displacement ductility demand which was captured as the primary output parameter for this study.

The fifth chapter explains the development and use of a procedure for estimating the magnitude of synthetic earthquakes using time history analysis. The procedure is applied in this study to confirm if the synthetic earthquakes produced are indeed of 
the magnitude specified and to discern whether the synthetic ground motions may be over predicting or under predicting the structural response.

Chapter 6 presents the methodology employed to conduct the study providing the general approach taken including specific input parameter values used. It also describes the procedure used and results obtained from a pilot study conducted prior to the final study. The methodology used to conduct the final study is also presented and some limitations to the study results are also indicated.

Chapter 7 provides an analysis of the results obtained from the study including general observations and overall trends. It also provides a description of some problems encountered during the study and their resolution. Finally, the last chapter contains the conclusions drawn from the results of the study and offers some suggestions for further research.

The problem statement and research questions posed for this study were presented in the previous section. The following chapters provide basic information for an understanding of how attenuation models proposed for the CEUS, the generation of synthetic earthquake ground motion compatible with these attenuation models, and time history analysis were combined in an effort to address this problem statement and to answer the questions stemming from it. 


\section{Chapter 2}

\section{ATTENUATION MODELS}

\subsection{Overview}

Seismology is beyond the field of expertise of most structural engineers. In fact, as will become clear later in this chapter, it is for this reason that the SIMQKE program was employed for the generation of the synthetic ground motions used in this study. This program allows the use of response spectra which can be obtained from attenuation models developed by experts in the field of seismology to describe the frequency contents of the ground motions expected to occur in the CEUS.

\subsubsection{Definition}

The word attenuation as it applies to seismic waves refers to their decrease in amplitude as they propagate away from their source. That is, seismic waves "die off" with distance similar to the way ripples diminish on the surface of a pond as they radiate from the point where a stone is dropped. The dispersion of seismic waves is due to many things including the natural dissipation of energy, and internal friction, that occur as the waves travel within the earth (Stein and Wysession, 2003). Attenuation models attempt to provide mathematical representations of this behavior. 
Several approaches are used to model this attenuation behavior and it is the topic of much ongoing research. It is beyond the scope of this dissertation to compare, evaluate, critique, or even to investigate the validity of these different approaches to solving a common problem. Rather this study sought to utilize the most recently available attenuation models that have been developed for the CEUS to describe the frequency content required to generate synthetic ground motions that could be used for the design of structures in this region. Specifically, this study seeks to investigate the variation in the structural response due to synthetic ground motions generated to be compatible with current CEUS attenuation models.

The development of these attenuation models involves the input of many parameters attempting to describe the complicated physical process of seismic waves being generated from the sudden release of strain energy resulting from an earthquake rupture along a geologic fault. Fortunately, in their final form, attenuation models only require the input of a magnitude and distance as well as a site classification to produce response spectra describing the ground motions for a particular event.

Moment magnitude is used in all of the attenuation relations employed in this study. However, the developers of the models do not use a single consistent definition of the distance from site to source. Distance measures used by the attenuation models in this study include defining this distance as the closest horizontal distance to the vertical projection of the rupture (the "Joyner-Boore" distance), the closest distance to the rupture surface, and the hypocentral distance. A 
more thorough description of these distance measures and a detailed explanation of the distance model assumptions used in this study will be provided in Chapter 6. Furthermore, many models offer the option of developing attenuation relations for differing site conditions. To alleviate complications and establish a starting point for future work, only hard rock conditions (e.g. only structures founded on rock) were considered for this study.

The output from these attenuation relationships is provided in the form of a response spectrum. These response spectra provide a representation of the frequency content for the earthquake ground motion from a specified magnitude event felt at a specified distance. As was explained in the previous chapter, the frequency content is perhaps the most important parameter to influence how a particular structure responds to an earthquake. The parameters used to develop attenuation models attempt to characterize the mechanics associated with the earthquake source, the propagation of its waves, and conditions at the site for which the response spectra are being estimated.

These processes vary with location, however, and are influenced by characteristics of the earthquake source such as the type of faulting as well as the by the geologic setting where they are modeled to occur. As was pointed out in the previous chapter, there are distinct differences in the frequency contents of ground motions from earthquakes occurring in the CEUS and WUS. The frequency content of a ground motion is related to the magnitude of the earthquake that produced the 
motion. Higher magnitude events produce lower frequency ground motions. In addition, the high frequency components of ground motion are absorbed and scattered with distance more quickly than lower frequency components. As a result, the frequency content of ground motions also changes with distance (Kramer, 1996).

Attenuation relationships attempt to model these changes in frequency content with variations in magnitude and distance and the variation in different geographic and tectonic environments. As mentioned in the previous chapter, the differences in the frequency content between strong ground motions in the WUS and those in the CEUS is firmly recognized and reasonably well understood (Boore and Atkinson, 1987; Boore et al., 1992; EPRI, 1993; Silva and Darragh, 1995; Atkinson,1996).

It has been known for some time that ground motions for the CEUS attenuate less rapidly with distance than ground motions in the WUS for events of similar moment magnitudes and source depths (Nuttli, 1981; EPRI, 1993; Atkinson and Boore 1995). The difference in attenuation rate has been attributed to the higher absorptive capability that is generally present in the crust beneath the WUS compared to the CEUS (Nuttli, 1981; Herrmann and Nuttli, 1982; Singh and Herrmann, 1983; Boore and Atkinson, 1987; Toro and McGuire, 1987; Frankel et al., 1990; Hanks and Johnston, 1992; EPRI, 1993; Frankel, 1994; Benz et al., 1997). In addition, CEUS ground motions recorded at rock or very shallow soil sites are significantly richer in high-frequency relative to comparable WUS ground motions (Stewart et al., 2001). 
As a result of these differences, regionally specific attenuation models have been developed for the CEUS.

\subsubsection{Ground Motion Modeling Procedures}

The development of all earthquake ground motion models involves the approximation of the effects of three general physical processes observed in ground motions (Stewart et al., 2001). These three processes are modeled in separate parts representing the seismic source, wave propagation, and site response effects. Various modeling procedures have been developed and applied to represent these processes. A general description of the main types of these procedures is presented here. A complete treatment of the many techniques used to develop attenuation relationships is beyond the scope of this review. Rather this presentation will focus only on the procedures used to develop the models applied in this study. An excellent and concise explanation of the various methods used in ground motion simulation is available online through the Pacific Earthquake Engineering Research Center (Stewart et al., 2001).

For this study, the treatment of the processes outlined in this chapter refers to the development of attenuation models. The next chapter will introduce procedures used to generate synthetic ground motions for time history analyses and specifically the SIMQKE program used in this study. 
It is important at this point to distinguish between the two primary reasons for producing simulated earthquake ground motions (Stewart et al., 2001). The first reason to use simulated earthquakes is to generate simulated data to supplement the database of actual recorded ground motions when empirical data for a region, magnitude, or distance of interest is absent. Attenuation models are then developed from the application of regression analyses conducted on these databases including the simulated data (Kramer, 1996). This is the approach used to develop the attenuation models for the CEUS applied in this study. The second reason to generate synthetic ground motions is for use as input loading in time history analysis for the seismic design of a particular structure, again when actual records are scarce or unavailable.

To circumvent what may appear to be circularity and to alleviate possible confusion, the presentation in this chapter refers to the development of geophysical models used to estimate and describe the frequency content of the ground motion (i.e., the development of attenuation models). The presentation in the next chapter will refer to procedures for the generation of synthetic ground motion time histories, some of which utilize the description of the frequency content estimated from attenuation models. In simplest terms, attenuation models can be defined as the product of functions describing earthquake source processes and their wave propagation. 


\subsubsection{Source Models}

The source for earthquakes can be modeled as either a simple point or as a fault having a finite length. At far distances from a fault, the earthquake source can be reasonably assumed as a point in space for the purpose of ground motion modeling. This assumption simplifies the computational effort and is frequently used whenever it is thought to be appropriate. However, when the receiving site of interest is close to the fault being considered, a finite source model needs to be used to account for near field effects such as the constructive interference of the earthquake waves.

\subsubsection{Wave Propagation}

Modeling the propagation of earthquake waves as they travel through the crust of the earth attempts to account for the attenuation of the waves as defined earlier. This effort involves modeling the radial spreading with distance from the source as well as the absorption, reflection, and refraction of the energy contained within the waves. All of these effects are jointly represented by using Green's functions. Green's functions represent the effects from the earth's structure on the signal that the earthquake puts into the ground (Stein and Wysession, 2003). The functions may be calculated analytically or obtained empirically (Hartzell, 1978, 1985).

The similarity of earthquake time histories to random processes that can be described using statistical methods has led to the development of stochastic methods in ground motion modeling. 


\subsubsection{Stochastic Method}

All of the CEUS attenuation relations applied in this study have their basis in the stochastic method and point source model with the exception of the Somerville model.

In general, the sources of variation among the models lies in the choice, derivation, distribution, or application of parameter values associated with the source model (Atkinson and Boore, 1998). Again, all of the models applied in this study use a point source model except for the Somerville model in which the earthquake source is represented as a shear dislocation over an extended fault plane. This latter representation is based on a finite fault model and the application of Green's functions. Since all but one of the models used in this study also rely on the stochastic method for their development, a more detailed description of this method will be presented here.

An excellent and concise explanation of the stochastic method is provided by Boore and Joyner in the Earthquake Engineering Research Institute's monograph on Seismic Hazard and Risk Analysis (McGuire, 2004). Further background on the origins of the stochastic method in ground motion prediction is found in Hanks and McGuire (1981), Boore (1983), and Boore (2003); the latter includes a useful list of references and comparisons of assumptions. 
Stochastic methods of estimating ground motion use a set of assumptions about the earthquake source spectrum and about the effects of path and site conditions. The ground motion is then transformed into spectral response by using the transfer function of a linear SDOF oscillator. These assumptions are described below. Again, the majority of the attenuation relationships developed most recently for the CEUS and used to produce the National Seismic Hazard Maps have been developed using the stochastic method.

In their simplest form, stochastic models are products of the general processes just mentioned as functions of magnitude, distance and frequency as shown in Equation 2.1.

$A\left(M_{0}, R, f\right)=C S\left(M_{0}, f\right) D(R, f) \quad($ Equation 2.1)

In this equation, $C$ is a constant scaling factor, $S\left(M_{0}, f\right)$ is the function for the source model, and $D(R, f)$ is termed the diminution function which describes the effects of wave propagation.

The source function used by all but one of the models used in this study is that proposed by Brune $(1970,1971)$ for an instantaneous shear dislocation at a point. It is a function of two parameters known as the corner frequency and the seismic moment. The corner frequency can be defined as the frequency where the high and 
low frequency trends intersect (Hays, 1980). Most frequencies contributing to the ground motion occur over an intermediate range of frequencies bounded by the corner frequency $f_{c}$ on the low side and a frequency known as the cutoff frequency $f_{\max }$ on the high side (see Figure 2.1).

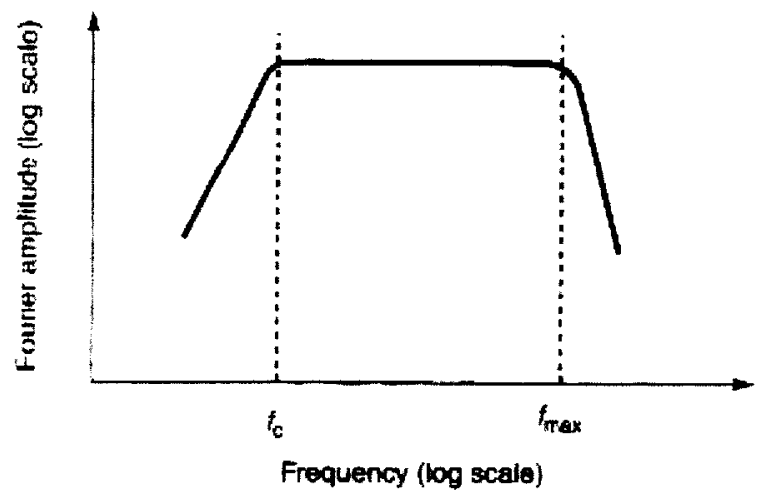

Figure 2.1 Typical Fourier Amplitude Plot Showing Corner and Cutoff Frequencies (Kramer, 1996)

The seismic moment (Hanks and Kanamori, 1979) gives an estimate of the energy released during an earthquake in terms of the size of the fault and the forces developed across it during the rupture process. This moment is also used to estimate the moment magnitude of an event as will be described further in Chapter 5.

As mentioned in the previous section, the magnitude of an earthquake plays a key role in determining its frequency content. As the magnitude of an earthquake increases, its frequency bandwidth also increases, forcing the corner frequency to decrease (Kramer, 1996). This relation implies that lower frequency ground motions will accompany larger magnitude events. This relationship is modeled within the 
source function by relating the corner frequency to a parameter known as the stress drop. The stress drop is a measure of the difference in stress acting to resist movement along a fault before and after an earthquake occurs; the stress drop controls the high frequency content of earthquake ground motions (Hayes, 1980).

The constant factor $C$ in equation 2.1 used to scale the source spectrum is made up of parameters representing the radiation pattern, the partitioning of energy into two horizontal components, amplification at the free surface, the distance from the source, the density of the crust, and the shear wave velocity near the source. The source models themselves also contain parameters that shape the source spectra (e.g., one or two corner frequency models).

With these parameters having been taken into account, the source function represents all of the effects on the frequency content of the ground motion caused by the earthquake source. The source function produces constant acceleration energy above the corner frequency. The attenuation of energy from an earthquake is then modeled by multiplying this source function by the inverse of the distance to the site being considered. By applying the stochastic method, the frequency content emanating from the source spectrum can be scaled by the two independent parameters of magnitude and stress drop

The diminution function $D(R, f)$ shown in Equation 2.1 accounts for inelastic attenuation and damping in the earth's crust; it accounts for the effects of path and 
site conditions on the estimated motion at the surface and distance of the site being considered. The function contains a quality factor which is a function of frequency and varies for the region being studied. It will be shown in the following section that this factor plays a key role in developing ground motion models for the CEUS. The diminution function also includes a high-cut filter that accounts for sharp decline in accelerations above a cut-off frequency that cannot be attributed to path attenuation but is observed in actual earthquake recordings.

The diminution function is multiplied by a high frequency filter to account for effects on the ground motion from near surface rock or soil properties. The function involves a parameter, the kappa term, which is a function of shear wave velocity, depth of material, and the quality factor mentioned previously.

Finally, these models also include simple functions to account for such things as source and path duration, as well as shaping the spectrum to coincide with the requested type of ground motion measurement (i.e. acceleration, velocity, or displacement). Duration is commonly taken as a function of the source corner frequency and the response function is calculated using the transfer function of a SDOF linear oscillator. By relating all of these parameters, it is possible to estimate the frequency content of an earthquake ground motion at a particular distance as a function of magnitude, stress drop, and the physical properties of the region. 


\subsubsection{Finite Fault Model}

As mentioned earlier, the Somerville model used in this study relies on a finite fault representation of the source model and the use of Green's functions rather than the stochastic method outlined above. Therefore, an explanation in basic terms of the procedure used in this model is presented here.

The approach for this modeling procedure is to lag and sum multiple Green's functions over a two dimensional grid of subfaults as shown in Figure 2.2 (Stewart et al., 2001). The procedure is quite complex but in simple terms, the fault rupture plane is discretized into a grid of equal size subfault regions, and different values of slip are assigned to each subfault element. Empirical source functions are used for each subevent to build up a larger finite source. Each subevent ${ }_{2}$ from which the empirical source functions are derived, is estimated independently. The source process is randomized through the selection and location of the empirical source function for the subevents. Wave propagation to the site is represented by analytical Green's functions. Additional details on this source characterization procedure are described in Somerville et al. (1999). 


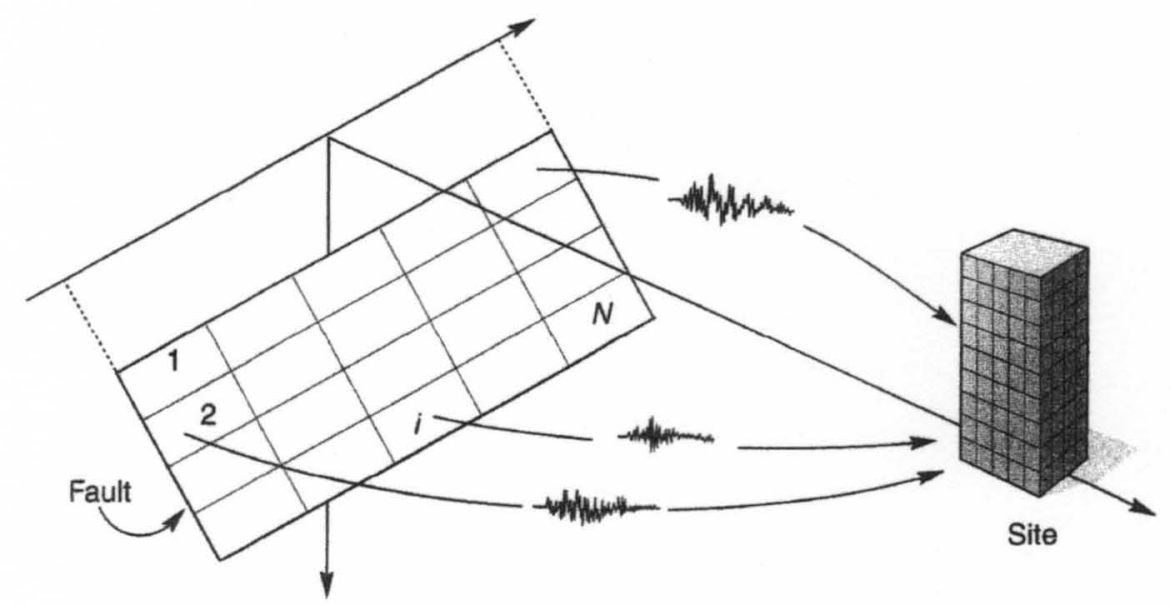

Figure 2.2 Characterization of a Finite Fault Source (Kramer, 1996)

As mentioned earlier, there has been a trend toward developing regionally specific ground motion attenuation models, especially in the CEUS due to the differences in the ground motions expected in this region.

One controversial issue with the development of ground motion relations for the CEUS is the lack of availability of actual strong ground motion recordings. That lack does not allow the development of models to be derived directly from empirical data. Rather, the most utilized approach has been to use modeling to generate synthetic ground motions and then use these motions in the development of attenuation models. That is, the sample space for regression analyses is populated with synthetic earthquakes generated from data of analogous regions or extrapolated from smaller events. 


\subsection{CEUS Attenuation Models}

\subsubsection{WUS vs. CEUS}

The differences between ground motions from stable continental regions such as the CEUS and those from other active tectonic regions such as the WUS have been alluded to in the previous chapter and in the previous section of this chapter. These differences have lead to the development and evolution of models specifically intended to describe ground motions expected to occur in the CEUS. The most significant difference for stable continental regions such as the CEUS is that much larger high-end frequencies are expected in the ground motions and yet the attenuation of seismic waves is less rapid than in other regions; these trends contribute to different changes in frequency content.

In addition to estimating the recurrence of earthquakes, seismic hazard analysis also depends on estimating the area that will be affected. For this reason, attenuation models proposed or the CEUS have been selected for use in the development of the National Seismic Hazard Maps.

\subsubsection{USGS National Seismic Hazard Maps}

The National Seismic Hazard Maps are developed from probabilistic seismic hazard analysis which relies on the characterization of site response at various distances from prospective sources. Attenuation models play a key role in conducting 
these analyses. These maps are the basis for national, state, and local seismic safety regulations and design standards, such as the NEHRP Recommended Provisions for Seismic Regulations for New Buildings and Other Structures (2003), the International Building Code (2006), and the American Society of Civil Engineers Minimum Design Loads for Buildings and Other Structures (2005).

Building officials in cities and counties rely on the seismic design provisions in building codes to ensure that structures can resist earthquakes (Brown et al, 2001). These codes include design maps that are based on hazard maps prepared by the U.S. Geological Survey (USGS) as part of the National Earthquake Hazard Reduction Program (NEHRP). These types of maps have been produced for the United States since 1948. The need for attenuation models in probabilistic seismic hazard analysis necessary for the development of the National Seismic Hazard maps has been a key impetus for the regional specific development of attenuation models representative of ground motions in the CEUS.

\subsubsection{CEUS Seismological Parameters}

Certain parameters in the source model are especially important in developing ground motion simulations representative of those expected for CEUS and strongly influence the models developed for the region. 


\subsubsection{Stress Drop}

One such parameter is the stress drop as described previously relative to the stochastic method. For the CEUS, the simple point-source model with a stress drop of about 100 bars, which is about double that for earthquakes in the WUS, was in good agreement with actual data (Atkinson, 1984; Boore and Atkinson, 1987; Toro and McGuire, 1987) until the Saguenay earthquake occurred in1988. The stress drop required to match the high frequency for this earthquake exceeded 500 bars, and yet the intermediate frequency levels are overestimated by a factor of two or more and required a significantly lower stress drop (Boore and Atkinson, 1992). This anomaly led to the development an empirical two-corner source model for CEUS earthquakes (Boatwright and Choy, 1992; Atkinson, 1993).

\subsubsection{Corner Frequency}

An alternative source model that has been produced for the CEUS (Atkinson and Silva 2000) involves two corner frequencies of the source, with varying energy across the band. This source spectrum can be incorporated in the stochastic model by substitution in Equation 2.1. This source model produces a sag in spectral amplitudes at intermediate frequencies and the two corner frequencies provided by this model lie on either side of the corner frequency produced by the Brune source model previously mentioned. 


\subsubsection{Kappa}

As mentioned earlier, the kappa parameter controls the damping that occurs in the shallow portions of the earth near the surface (Anderson and Hough, 1984). It is dependent on the thickness over which this damping takes place and the average seismic propagation velocity in the damping zone. Values of kappa are greater in soft material resulting in a dramatic loss in high frequency energy content. Conversely, it has lower values for hard rock conditions such as are prevalent in the CEUS. On average, kappa values for the WUS are about 5 times larger than kappa values for the CEUS.

Values for kappa used to develop CEUS attenuation models are determined from instrumental analysis of actual small and moderate events that have occurred in the region and from isoseismals estimated for large historic events that have occurred in the region.

\subsubsection{Previous CEUS Models}

As new data and understanding has become available, previous versions of CEUS attenuation models have evolved into more up to date versions. The development of the 1996 National Seismic Hazard Maps used a compilation of two CEUS attenuation models available at the time (Toro et al., 1997; Frankel et al., 1996). The 2002 National Seismic Hazard Maps saw the addition of even more proposed CEUS models (Atkinson and Boore, 1995; Somerville et al., 2001; Campbell, 2002). As 
will be shown later in the next section, the current 2008 version of the maps have even added more new and updated CEUS attenuation models.

The uncertainties described earlier associated with ground motions in the CEUS have led to an evolution of the models attempting to depict those motions. This study was focused on the structural response to synthetic records depicting ground motions expected in the CEUS. As such, it used the eight CEUS attenuation models used by the USGS to develop the most current 2008 National Seismic Hazard Maps (Petersen et al.,, 2008) to develop the synthetic records applied in the study.

\subsection{USGS CEUS Attenuation Models}

The CEUS attenuation models applied in this study are the following: Frankel et al. (1996), Toro et al. (1997), Somerville et al. (2001), Silva et al (2002), Atkinson and Boore (2006) with both 140 and 200 bar stress drops, Campbell (2003), and Tavakoli and Pezeshk (2005). The US Geological Survey used these seven attenuation models for the CEUS to produce the latest edition of the seismic hazard maps (Petersen et al., 2008). The Atkinson and Boore model was used at two different stress parameter levels so that there were actually eight different attenuation relationships used to develop the maps.

The variation in the response spectra produced from these attenuation models can be attributed to differences in the choice of model parameters as well as assumptions about the radiation of energy from the earthquake source (Atkinson and Boore, 1998). 
A brief description of each of these seven attenuation models used in this study and by the USGS is provided below. The characteristics for each model are also presented in tabular format below each description. Only a brief description of each model is presented here. The reader is referred to each reference for a more detailed explanation of the development and parameters selected for each individual model. 


\subsubsection{Frankel et al. (1996)}

This attenuation relationship is also developed from equations based on a point source model similar to a Brune point source model. A constant stress drop of 150 bars was used and site conditions were assumed to be at the NEHRP B-C boundary (i.e. shear wave velocity of $760 \mathrm{~m} / \mathrm{s}$ in the top $30 \mathrm{~m}$ ). Frequency dependent amplification factors were applied to correct for hard rock in this study.

\begin{tabular}{|c|l|}
\hline Region & Frankel et al. (1996) \\
\hline Model Type & $\begin{array}{l}\text { Brune point source model with a single corner frequency } \\
\text { and developed using the stochastic method. }\end{array}$ \\
\hline $\begin{array}{c}\text { Magnitude } \\
\text { Range }\end{array}$ & $\mathrm{M}_{\mathrm{W}} 4.4$ to 8.2 \\
\hline $\begin{array}{c}\text { Distance } \\
\text { Range }\end{array}$ & 10 to $1000 \mathrm{~km}$ \\
\hline $\begin{array}{c}\text { Distance } \\
\text { Measure and } \\
\text { Focal Depth }\end{array}$ & $\begin{array}{l}\text { The hypocentral distance is used and therefore focal } \\
\text { depth is implied in its input }\end{array}$ \\
\hline $\begin{array}{c}\text { Soil/Rock } \\
\text { Damping }\end{array}$ & $\begin{array}{l}\text { Defined for the NEHRP B-C boundary (firm rock) } \\
\text { however conversion factors for hard rock are provided }\end{array}$ \\
\hline $\begin{array}{c}\text { Spectral } \\
\text { Periods }\end{array}$ & $0.2,0.3$, and 1.0 seconds \\
\hline PGA & Explicitly provided \\
\hline Stress Drop & 150 bars \\
\hline $\begin{array}{c}\text { Site } \\
\text { Attenuation } \\
\text { kappa) }\end{array}$ & 0.01 for firm rock \\
\hline Notes & $\begin{array}{l}\text { One of the two original models used to develop the 1996 } \\
\text { USGS National Seismic Hazard maps }\end{array}$ \\
\hline
\end{tabular}




\subsubsection{Toro et al. (1997)}

This attenuation relationship is based on equations from a Brune $(1970,1971)$ point source model for instantaneous slip on a circular rupture surface. This source model is thought to be conservative (and somewhat controversial) but has been consistently used in recent practice. This attenuation model has also been updated to include extended source effects to account for the potentially large dimension of the earthquake rupture that is particularly important at close distances.

\begin{tabular}{|c|c|}
\hline \multicolumn{2}{|r|}{ Toro et al. (1997) } \\
\hline Region & Central and Eastern North America \\
\hline Model Type & Stochastic method with a Brune point source model \\
\hline $\begin{array}{l}\text { Magnitude } \\
\text { Range }\end{array}$ & $\mathrm{M}_{\mathrm{w}} 5$ to 8 \\
\hline $\begin{array}{c}\text { Distance } \\
\text { Range }\end{array}$ & $\begin{array}{l}\text { Horizontal Distances } 1 \text { to } 500 \mathrm{~km} \text { (with emphasis on } \\
\text { distances of } 1 \text { to } 100 \mathrm{~km} \text { ) }\end{array}$ \\
\hline $\begin{array}{l}\text { Distance } \\
\text { Measure and } \\
\text { Focal Depth }\end{array}$ & $\begin{array}{l}\text { Closest horizontal distance to the earthquake rupture } \\
\text { (Joyner-Boore distance). Probability distribution from } \\
\text { EPRI, } 1993 \text { and Abrahamson et al., } 1996 .\end{array}$ \\
\hline Soil/Rock & $\begin{array}{l}\text { Directly applicable to Hard Rock (average shear wave } \\
\text { velocities of } 600 \mathrm{ft} / \mathrm{s} \text { at the surface), soil factors are } \\
\text { available }\end{array}$ \\
\hline Damping & $\begin{array}{l}\text { Not explicitly provided but comparisons are made in the } \\
\text { paper to the Atkinson and Boore (1995) model which } \\
\text { does explicitly define its spectral accelerations as being } \\
\text { for } 5 \% \text { damping }\end{array}$ \\
\hline $\begin{array}{l}\text { Spectral } \\
\text { Periods }\end{array}$ & $0.0286,0.04,0.1,0.2,0.4,1.0,2.0$, and 3.0 seconds \\
\hline PGA & Explicitly provided \\
\hline Stress Drop & Median of 120 bars \\
\hline $\begin{array}{c}\text { Site } \\
\text { Attenuation } \\
\text { (kappa) }\end{array}$ & Equally weighted values of $0.003,0.006$ and $0.012 \mathrm{sec}$. \\
\hline Notes & $\begin{array}{l}\text { Provides models for } 2 \text { Crustal Regions (Midcontinent } \\
\text { and Gulf), defines and quantifies uncertainty in model } \\
\text { parameters. Latest modifications account for extended } \\
\text { source effects. }\end{array}$ \\
\hline
\end{tabular}




\subsubsection{Somerville et al. (2001)}

This attenuation relationship was developed using a finite-source model that allows for varying slip distribution along the fault. It incorporates full waveform simulations to model both the source and propagation effects. This simulation is accomplished by using a broadband Green's function method that has a rigorous basis in theoretical and computational seismology. The model also accounts for extended source effects.

\begin{tabular}{|c|c|}
\hline \multicolumn{2}{|r|}{ Somerville et al. (2001) } \\
\hline Region & Central and Eastern United States \\
\hline Model Type & $\begin{array}{l}\text { Finite fault source model with spatially varying slip } \\
\text { distributions and Green's functions }\end{array}$ \\
\hline $\begin{array}{l}\text { Magnitude } \\
\text { Range }\end{array}$ & $\mathrm{M}_{\mathrm{W}} 6.0$ to 7.5 \\
\hline $\begin{array}{c}\text { Distance } \\
\text { Range } \\
\end{array}$ & 0 to $500 \mathrm{~km}$ \\
\hline $\begin{array}{l}\text { Distance } \\
\text { Measure and } \\
\text { Focal Depth }\end{array}$ & $\begin{array}{l}\text { Closest horizontal distance to the surface projection of } \\
\text { the fault. A constant focal depth of } 6 \mathrm{~km} \text { was used. }\end{array}$ \\
\hline Soil/Rock & Hard Rock $\left(\mathrm{V}_{\mathrm{s}}=2.83 \mathrm{~km} / \mathrm{sec}\right)$ \\
\hline Damping & $\begin{array}{l}\text { Not explicitly provided but comparisons are made in the } \\
\text { paper to the Atkinson and Boore (1995) model and } \\
\text { Toro et al. (1997) which do explicitly define their } \\
\text { spectral accelerations as being for 5\% damping }\end{array}$ \\
\hline $\begin{array}{l}\text { Spectral } \\
\text { Periods }\end{array}$ & 0.01 (Taken as PGA), $0.04,0.10,0.20,0.40,1.0,2.0,4.0$ \\
\hline PGA & $\begin{array}{l}\text { Not explicitly given but taken as } 0.01 \text { second spectra } \\
\text { acceleration which is provided }\end{array}$ \\
\hline Stress Drop & N/A for the source model used \\
\hline $\begin{array}{c}\text { Site } \\
\text { Attenuation } \\
\text { (kappa) }\end{array}$ & $0.006 \mathrm{sec}$ \\
\hline Notes & $\begin{array}{l}\text { One function is used if } \mathrm{r}<50 \mathrm{~km} \text { and another is used if } \mathrm{r} \\
>50 \mathrm{~km} \text {. One set of regression coefficients is provided } \\
\text { for rifted domains and another for non-rifted domains. } \\
\text { A model for vertical ground motions is also provided. }\end{array}$ \\
\hline
\end{tabular}




\subsubsection{Silva et al (2002)}

This attenuation relationship was also developed using a point source model. It provides regression coefficients for a single corner model with variable stress drop as a function of moment magnitude, a single corner model with constant stress drop, a single corner model with constant stress drop and saturation, a double corner model, and a double corner model with saturation. The single corner model with constant stress drop and saturation to account for near source effects was used in this study as was applied in the development of the 2008 Seismic Hazard Maps. 


\begin{tabular}{|c|c|}
\hline \multicolumn{2}{|r|}{ Silva et al. (2002) } \\
\hline Region & Central and Eastern North America \\
\hline Model Type & $\begin{array}{l}\text { Stochastic method with point source model. The single } \\
\text { corner model with constant stress drop and saturation } \\
\text { was used in this study }\end{array}$ \\
\hline $\begin{array}{l}\text { Magnitude } \\
\text { Range }\end{array}$ & $\mathrm{M}_{\mathrm{W}} 4.5$ to 8.5 \\
\hline $\begin{array}{l}\text { Distance } \\
\text { Range }\end{array}$ & 1 to $400 \mathrm{~km}$ \\
\hline $\begin{array}{l}\text { Distance } \\
\text { Measure and } \\
\text { Focal Depth }\end{array}$ & $\begin{array}{l}\text { Closest distance to the surface projection of the rupture } \\
\text { surface. } 2 \text { to } 20 \mathrm{~km} \text { with mean depths ranging from } 6 \text { to } \\
10 \mathrm{~km} \text { ) }\end{array}$ \\
\hline Soil/Rock & $\begin{array}{l}\text { Crystalline rock (hard rock) outcrop simulations were } \\
\text { used for this study }\end{array}$ \\
\hline Damping & $\begin{array}{l}\text { The response spectra represent } 5 \% \text { damped pseudo- } \\
\text { acceleration }\end{array}$ \\
\hline $\begin{array}{l}\text { Spectral } \\
\text { Periods }\end{array}$ & $\begin{array}{l}0.01,0.02,0.025,0.032,0.04,0.05,0.055,0.06,0.07 \\
0.08,0.1,0.12,0.15,0.16,0.2,0.24,0.3,0.4,0.5,0.75 \\
1.0,1.6,2.0,3.0,5.0 \text {, and } 10.0 \text { seconds }\end{array}$ \\
\hline PGA & Explicitly provided \\
\hline Stress Drop & $\begin{array}{l}120 \text { bars used in the constant models ( } 160 \text { to } 70 \text { bars } \\
\text { used in the variable models) }\end{array}$ \\
\hline $\begin{array}{c}\text { Site } \\
\text { Attenuation } \\
\text { (kappa) } \\
\end{array}$ & Mean of $0.006 \mathrm{sec}$ \\
\hline Notes & $\begin{array}{l}\text { Single corner model with constant stress drop, single } \\
\text { corner model with constant stress drop and saturation, } \\
\text { single corner model with variable stress drop as a } \\
\text { function of moment magnitude, double corner model, } \\
\text { and a double corner model with saturation are also } \\
\text { provided }\end{array}$ \\
\hline
\end{tabular}




\subsubsection{Campbell (2003)}

The method used to develop this attenuation relationship also relies on a point source and use of the stochastic method. It incorporates a hybrid empirical model that utilizes source spectra from empirical sources in WUS and modifies them to fit parameters assumed for the CEUS.

\begin{tabular}{|c|l|}
\hline \multicolumn{2}{|c|}{ Campbell (2003) } \\
\hline Region & Eastern North America \\
\hline Model Type & $\begin{array}{l}\text { Hybrid-empirical model using stochastic Brune point } \\
\text { source }\end{array}$ \\
\hline $\begin{array}{c}\text { Magnitude } \\
\text { Range }\end{array}$ & $\mathrm{M}_{\mathrm{W}} 5.0$ to 8.2 \\
\hline $\begin{array}{c}\text { Distance } \\
\text { Range }\end{array}$ & 0 to $1000 \mathrm{~km}$ \\
\hline $\begin{array}{c}\text { Distance } \\
\text { Measure and } \\
\text { Focal Depth }\end{array}$ & $\begin{array}{l}\text { Closest distance to the rupture plane. Focal depths used } \\
\text { vary from } 0.2 \mathrm{~km} \text { to } 70 \mathrm{~km} \text { depending on magnitude. }\end{array}$ \\
\hline Soil/Rock & Hard Rock $\left(\mathrm{V}_{\mathrm{s}}=2.80 \mathrm{~km} / \mathrm{sec}\right)$ \\
\hline Damping & $\begin{array}{l}\text { The response spectra represent } 5 \% \text { damped pseudo- } \\
\text { acceleration }\end{array}$ \\
\hline $\begin{array}{c}\text { Spectral } \\
\text { Periods }\end{array}$ & $\begin{array}{l}0.01,0.02,0.03,0.05,0.075,0.1,0.15,0.2,0.3,0.5, \\
0.75,1.0,1.5,2.0,3.0, \text { and } 4.0 \text { seconds }\end{array}$ \\
\hline PGA & $\begin{array}{l}\text { Not explicitly given but taken as } 0.01 \text { second spectra } \\
\text { acceleration which is provided }\end{array}$ \\
\hline Stress Drop & $105,125,150,180$, and 215 bars \\
\hline $\begin{array}{c}\text { Site } \\
\text { Attenuation } \\
\text { (kappa) }\end{array}$ & \begin{tabular}{l}
$0.003,0.006$, and 0.012 sec. \\
\hline Notes
\end{tabular} \\
\hline
\end{tabular}




\subsubsection{Tavakoli \& Pezeshk (2005)}

This attenuation relationship is also developed from a hybrid model that uses the approach of developing modification factors from ground motions in western North America to be used in the development of ground motions for eastern North America. It uses an empirical-stochastic approach where empirical refers to the empirical attenuation models developed in a host region (WNA) and hybrid refers to models that transform attenuation relationships to a target region (ENA) by using seismological parameters.

\begin{tabular}{|c|l|}
\hline Region & Tavakoli \& Pezeshk (2005) \\
\hline Model Type & $\begin{array}{l}\text { Hybrid-empirical model using stochastic Brune single } \\
\text { corner point source and double corner point source }\end{array}$ \\
\hline $\begin{array}{c}\text { Magnitude } \\
\text { Range }\end{array}$ & $\mathrm{M}_{\mathrm{W}} 5.0$ to 8.2 \\
\hline $\begin{array}{c}\text { Distance } \\
\text { Range }\end{array}$ & 0 to $1000 \mathrm{~km}$ \\
\hline $\begin{array}{c}\text { Distance } \\
\text { Measure and } \\
\text { Focal Depth }\end{array}$ & $\begin{array}{l}\text { Closest distance to the fault rupture. Focal depths used } \\
\text { vary from } 4.5 \mathrm{~km} \text { to } 15 \mathrm{~km} \text { depending on magnitude. }\end{array}$ \\
\hline Soil/Rock & Hard rock \\
\hline Damping & $\begin{array}{l}\text { The response spectra represent 5\% damped pseudo- } \\
\text { acceleration }\end{array}$ \\
\hline $\begin{array}{c}\text { Spectral } \\
\text { Periods }\end{array}$ & $\begin{array}{l}0.01,0.05,0.08,0.1,0.15,0.2,0.3,0.5,0.75,1.0,1.5, \\
2.0,3.0, \text { and } 4.0 \text { seconds }\end{array}$ \\
\hline PGA & Explicitly provided \\
\hline Stress Drop & $105,125,150,180$, and 215 bars \\
\hline $\begin{array}{c}\text { Site } \\
\text { Attenuation } \\
\text { (kappa) }\end{array}$ & $0.003,0.006$, and 0.012 sec. \\
\hline Notes & $\begin{array}{l}\text { This approach derives modification factors from ground } \\
\text { motions recorded in WNA used to translate to ground } \\
\text { motions for the ENA. }\end{array}$ \\
\hline
\end{tabular}




\subsubsection{Atkinson and Boore (2006)}

This model is similar to previous versions based on a stochastic point source (Atkinson and Boore, 1995) but it also incorporates a dynamic-corner frequency. The main difference from other models is lower values of high frequency due to slightly lower assumed stress drop and larger attenuation near the source. As was used in the development of the 2008 Seismic Hazard Maps, stress drop values of both 140 bars and 200 bars were used for this model in this study.

\begin{tabular}{|c|c|}
\hline \multicolumn{2}{|r|}{ Atkinson and Boore (2006) } \\
\hline Region & Eastern North America \\
\hline Model Type & $\begin{array}{l}\text { Stochastic finite fault model using a dynamic corner } \\
\text { frequency }\end{array}$ \\
\hline $\begin{array}{l}\text { Magnitude } \\
\text { Range }\end{array}$ & $\mathrm{M}_{\mathrm{W}} 3.5$ to 8.0 \\
\hline $\begin{array}{l}\text { Distance } \\
\text { Range }\end{array}$ & 1 to $1000 \mathrm{~km}$ \\
\hline $\begin{array}{l}\text { Distance } \\
\text { Measure and } \\
\text { Focal Depth }\end{array}$ & $\begin{array}{l}\text { Closest distance to the fault. Mean focal depth of } 13 \mathrm{~km} \\
\text { was used for the simulations. }\end{array}$ \\
\hline Soil/Rock & $\begin{array}{l}\text { Defined for hard rock and soil sites (NEHRP B-C } \\
\text { boundary) }\end{array}$ \\
\hline Damping & $\begin{array}{l}\text { The response spectra represent 5\% damped pseudo- } \\
\text { acceleration }\end{array}$ \\
\hline $\begin{array}{l}\text { Spectral } \\
\text { Periods }\end{array}$ & $\begin{array}{l}0.01,0.025,0.031,0.04,0.05,0.063,0.079,0.1,0.125, \\
0.159,0.2,0.25,0.313,0.4,0.5,0.625,0.769,1.0,1.25 \text {, } \\
1.59,2.0,2.5,3.13,4.0 \text { and } 5.0 \text { seconds }\end{array}$ \\
\hline PGA & Explicitly provided \\
\hline Stress Drop & Median value of 140 bars \\
\hline $\begin{array}{c}\text { Site } \\
\text { Attenuation } \\
\text { (kappa) }\end{array}$ & Median value of $0.005 \mathrm{sec}$. \\
\hline Notes & $\begin{array}{l}\text { Stress drop can be adjusted and values of } 140 \text { and } 200 \\
\text { bars were used in this study as were for the development } \\
\text { of the } 2008 \text { National Seismic Hazard Maps }\end{array}$ \\
\hline
\end{tabular}




\subsection{Comments}

It is evident in the plots provided in Figures 1.8 through 1.10 that there are definite variations among the spectral acceleration values predicted from the eight CEUS models used to develop the 2008 National Seismic Hazard maps. As pointed out earlier, these variations stem from differences in the methods and assumed values for the parameters used to develop the models.

There is very little strong motion data available for the CEUS region, and therefore the development of these attenuation relationships are primarily based on simulated ground motions. Furthermore, regression analyses on these simulated motions are used to develop the attenuation models.

Due to the lack of empirical data, there is ultimately some inherent subjectivity introduced among the models in the approach used in their development and in the selection of parameter values used to describe the indefinite mechanics of the source rupture and wave propagation within the CEUS. Again, this subjectivity has lead to the variation in the response spectra generated from these models as exhibited in examples shown in Figures 1.8 through 1.10. It should be pointed out that the goal of this study is to investigate how this variation translates into variation among the structural responses and if this variation defeats the purpose of using these attenuation models to generate synthetic ground motion records applicable for use in time history analysis and design of structures located in the CEUS. 
It is not the intent of this study to evaluate the geophysical validity of these models.

Rather, this study merely compares the structural response to synthetic ground motions [generated to be compatible with the frequency contents as prescribed by these attenuation models]. Furthermore, this comparison is made strictly from an engineering perspective and not from a strictly seismological perspective. 


\section{Chapter 3}

\section{GENERATION OF SYNTHETIC GROUND MOTIONS}

\subsection{Introduction}

The use of earthquake ground motion time histories in the analysis of structures has increased considerably in recent years. These time histories are used in seismic design as well as the development of hazard and risk analyses. Unfortunately, actual recorded ground motions are rarely available for the specific location of interest and this is true especially in the CEUS. As a result, several models have been developed for the numerical simulation of earthquake ground motions. These models are based on either stochastic processes, geophysical models, or a combination of the two.

Information was provided on methods available for generating synthetic ground motions in the previous chapter. It was also pointed out in the previous chapter that there are two main reasons for generating synthetic ground motions. The information provided in the previous chapter focused on geophysical models and their combination with stochastically based models. This chapter focuses on the latter of the two reasons for use of generating synthetic motions, the generation of time 
histories to be used in the engineering analysis and design of specific structures. That is, the generation of synthetic ground motions to be used as input for time history analysis. A major portion of the next chapter is devoted to time history analysis.

This chapter however, focuses on the generation of artificial time histories and particularly those that are compatible with prescribed target spectra as generated by the computer program SIMQKE (Gasparini and Vanmarcke, 1976). This program relies wholly on stochastic processes to generate synthetic ground motions whose geophysical characteristics are derived from an input target spectrum describing the desired frequency content and as mentioned earlier, the frequency content may be the single most important characteristic of ground motion affecting the structural response. It is because of this program's ability to generate time histories from a target spectrum, such as one determined using attenuation models that it was chosen to be used in this study.

A brief review of other methods available to generate time histories will be presented here. This review will be followed by a more in-depth treatment of the SIMQKE program concluding with an explanation of the incorporation of duration and intensity models into the procedure used in this study to generate synthetic ground motions compatible with the eight CEUS attenuation models.

Current engineering practice and code requirements for the investigation of nonlinear ductility demands requires the selection of appropriate earthquake records 
that can somehow represent those that are plausible for the site being considered and in CEUS practice, synthetic time histories are most commonly used today. According to new requirements outlined in chapter 21 of the American Society of Civil Engineers Standard (ASCE/SEI 7-05, 2005) at least five recorded or simulated horizontal ground motion acceleration time histories shall be selected from events having magnitude and fault distances that are consistent with those that control the maximum considered earthquake. In the CEUS, for larger magnitude events or smaller events at closer distances, there probably will not be five sets of recorded ground motions that are appropriate and simulated ground motions will need to be generated.

Parameters associated with the artificial ground motions and their effects on structural response (not to be confused with the geophysical parameters described in the previous chapter on attenuation) include: amplitude parameters (acceleration, velocity, or displacement), duration, and parameters describing the frequency content of the motion (Fourier or response spectra). Although parameters such as peak ground acceleration and duration of the record have important effects on structural response, the frequency content of the record has perhaps the most important role in determining how a structure will respond to the ground motion. 


\subsection{Methods Available}

A very brief and general treatment of the methods available will be presented here whereas a more detailed explanation of the particular method used in this study will be provided in a later section of this chapter. It is worth mentioning again at this point that there are two main reasons for developing synthetic earthquakes. One is for the development of attenuation models and the other is for the development of time histories to be used in the engineering design of a particular structure located at a particular site. Unfortunately, the separation between the two reasons is not always clear. Usually methods developed by seismologists relying on geophysical models are not made available to the public. However, more and more seismologists are developing computer programs based on their methods and are making them publically available to the engineering and research communities.

Several methods for the generation of artificial ground motions have been proposed and utilized over the past several years. This treatment is not a comprehensive review of all methods available nor does it even provide detailed descriptions of the methods cited. Rather, this description introduces the general approaches and mentions some of the most popular programs that use these approaches. The next section will focus on the computer program SIMQKE that was used to generate the synthetic time histories applied in this study. Concise reviews of the methods available are provided in USACE (2000), Carballo and Cornell (2000), and Kramer (1996). 
The goal in developing artificial time histories is to generate synthetic records that have response spectra as close as possible to a target spectrum (Carballo and Cornell, 2000). Artificially generated ground motion time histories with response spectra whose shapes are equal within a prescribed tolerance to a predetermined target spectrum are considered "compatible" with their target. The process of generating such time histories is often referred to as spectral matching.

\subsubsection{Record Modification}

There are two main approaches to generating artificial earthquake time histories. One involves the modification of actual ground motion recordings and the other involves the generation of synthetic ground motion records. In addition, these two approaches can be conducted in either the time or frequency domain. The modification of existing records can also be carried out by applying linear scaling (Kircher, 1993; Naeim and Bhatia, 2000), in which the entire acceleration time history is scaled by a constant factor. The scaling factor is typically selected to achieve a match to target peak ground acceleration or spectral response acceleration at the fundamental period of the structure. This approach is easy to understand and perform; however, there are no hard rules for the application of scaling factors and care has to be taken to avoid possible unrealistic distortions in relative amplitudes, frequency content, and duration.

There are also algorithms available to modify an existing record either in the time domain (e.g. RSPMATCH, Abrahamson, 1993), or in the frequency domain (e.g. 
RASCAL, Silva, 1987). Time domain techniques involve adding or subtracting "wavelets" of finite duration to or from the original time history. The wavelets are selected to provide a match to the target spectrum at specific periods. RSPMATCH (Abrahamson, 1998) is an example of software that is based on a time domain approach. Several iterations are usually required to achieve a match to a target spectrum. Currently, time domain scaling is the generally preferred frequency modification approach for matching to a target spectrum.

Frequency domain techniques generally involve adjusting Fourier amplitudes while maintaining Fourier phases of the time histories. In simple terms, this is similar to the addition or subtraction of sinusoidal waves of different periods to the full length of the original time history. The RASCAL (Silva and Lee, 1987) program is an example of the frequency domain approach. As in time domain techniques, several iterations can be required to achieve a match. In most cases, attempts to match the target spectrum exactly are usually useless. Care must be taken also so that modifications do not produce unrealistic time histories that significantly differ in appearance from the original time histories. Also in some cases, the acceleration time histories may appear visually similar to the original, but the velocity and displacement histories may exhibit significant changes. 


\subsubsection{Record Generation}

As described in the previous chapter, artificial time histories can be generated based on numerical modeling of the fault rupture process and the source-to-site propagation of seismic waves. Several theoretical approaches are available and are best left to qualified seismologists.

As an alternative, many programs have been developed to generate synthetic ground motion time histories in the time domain. Many of these programs employ the geophysical models presented in the previous chapter which are also used in the development of attenuation models. The stochastic method is the predominant method among these procedures. Computer programs using time domain procedures to generate artificial time histories include SMSIM (Boore), RCTH and SGMS (Haldorsson and Papageorgiou, 2004),CPS (Hermann, 2005), , FINSIM (Beresnev and Atkinson, 1998), and EXSIM (Motazedian \& Atkinson, 2005).

Again, the procedures for generating time histories can be separated into two main categories, time domain procedures and frequency domain procedures. Unfortunately, there is confusion as to which methods belong to which of these two categories depending on the reference. Perhaps this confusion was caused because many of the methods implement parts of their procedures in both the time and frequency domains. Again, depending on the reference, SIMQKE has been referenced as being both a time and frequency domain procedure. It has even been grouped with procedures that modify existing ground motions. However, the method 
applied by SIMQKE to generate time histories actually takes place in the frequency domain as will be explained in more detail in the following section.

\subsection{SIMQKE}

An earthquake simulation program was developed by Gasparini and VanMarcke (1976) that has the capability of producing synthetic ground motions that are compatible with prescribed response spectra. A detailed and specific explanation of the development of the method used is provided in a report published by these authors.

\subsubsection{Approach}

Most recent ground motion relations for the CEUS have been developed using a stochastic process in which the motions are modeled as band limited Gaussian white noise. White noise can best be defined using the shape of its spectral density function. The spectral density function describes the frequency content of a particular signal. For example, a signal that has a single dominant frequency (like a sinusoidal wave), would have a spectral density function in the shape of a narrow spike centered about the dominant frequency value. This type of signal is known as a narrow band process. In contrast, motion that is made up of many different frequencies (like a random vibration) would have a spectral density function with a much wider curve to include many frequency values. These are called wide band processes. Figure 3.1 provides examples of a narrow band process and a wide band process both having the same predominant period. 


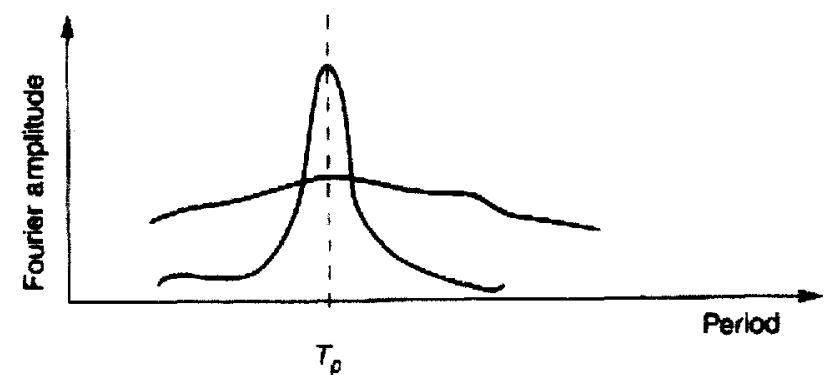

Figure 3.1 Typical Narrow Band and Wide Band Processes (Kramer, 1996)

White noise is really an idealization of a signal made up of an infinite number of frequencies, none of which are alike. Thus, the spectral density function for white noise would simply be a flat, straight and horizontal line extending indefinitely in both directions. To facilitate the use of white noise in the generation of synthetic ground motions, it is band limited or windowed to include only the frequencies of relevance to earthquake ground motions.

The stochastic method of developing synthetic ground motions involves the development of random white noise which is then shaped by a curve that describes an envelope outline of the synthetic record. This enveloped noise is then transformed into the frequency domain and normalized. It is then multiplied by the predicted Fourier spectrum developed from geophysical models and contains the proposed frequency content. Finally, the ground motion is transformed back into the time domain. Because the motion is shaped before it is transformed and multiplied by the frequency content, the shape of the envelope (and hence the final shape of the record) 
does not influence the frequency content and therefore does not affect the structural response induced by the record.

In contrast, the approach used in the SIMQKE program is not directly based on geophysical models of earthquake source processes and seismic wave propagation but rather relies on an input target response spectrum from these models to provide this information describing the synthetic ground motion to be generated.

The two methods are very similar in approach once the procedure reaches the point of calculating the response of SDOF oscillators. The overall difference between methods is that the stochastic approach applies geophysical models to estimate Fourier spectra describing the frequency content of the ground motion. At that point, synthetic ground motions can be generated or random vibration theory can be used to calculate the responses of SDOF oscillators to develop response spectra which are the products of attenuation models. The approach taken by the SIMQKE program works in the opposite direction using random vibration theory to generate Fourier spectra through a relationship to SDOF input in the form of a target response spectra.

As with the attenuation models used in this study, there has been no comparison made in this study of the different methods available to generate synthetic earthquake time histories. Therefore, this study makes no claims on the strengths or weaknesses of any of the many methods available. 


\subsubsection{Development}

The synthetic ground motions used in this study were generated using the SIMQKE software for simulating earthquake motions compatible with prescribed response spectra. The program was developed by Gasparini and Vanmarcke (1976) at the Massachusetts Institute of Technology.

The algorithm used by the program to generate synthetic ground motions relies on a relationship that can be established between a prescribed target response spectrum and its corresponding spectral density function using random vibration analysis. This relationship allows the response spectra to be written as the product of a peak factor and the standard deviation of a single degree of freedom system response. While the concept behind the algorithm may seem straightforward, attempting to grasp the complexity of its derivation is formidable at best. It establishes a relationship between expected response spectral values and the spectral density function for an earthquake ground motion evaluated as a random process. This relationship is derived from analytic random vibration theory techniques (Vanmarcke, 1976).

The derivation is quite complex, but in general it takes advantage of the analogies that exist between a spectral density function and a probability density function and of methods used to describe random vibrations and stochastic processes. In much the same way that a probability density distribution expresses the likelihood of a value occurring, so does the spectral density function convey the contribution of a frequency to the overall ground motion. It is also referred to as a power spectrum 
because the area under the curve represents the power contained in the ground motion. Following the analogy just pointed out, the total area under the spectral density function is equal to one. The spectral density function is derived by using the fast Fourier transform into the frequency domain. In general, a response spectral value can be expressed as a multiple of a peak factor and standard deviation of a onedegree system response. The peak factor is a function of the spectral moments which are analogous to the root mean square and the ratio of the standard deviation to the root mean square of a random variable. The peak factor is also a function of the SDOF response and the equivalent stationary duration.

Once the program computes a power spectral density function from a specified target response spectrum, the generated power spectral density function is then used to develop artificial acceleration time histories whose frequency contents correspond with the specified target spectrum. The method involves the superposition of sinusoids having random phase angles and amplitudes derived from the power spectral density function as just described. The result is an artificial acceleration time history corresponding with the specified target spectrum by containing compatible frequency content. Finally, to simulate the transient character of actual earthquakes, the superposition of sinusoids is multiplied by an intensity envelope function which also prescribes the duration for the motion.

There were many reasons for choosing the SIMQKE program for use in generating the synthetic ground motions used in this study. Perhaps the dominant 
reason is that it does not require the input of seismological parameters and the estimation of appropriate values for these parameters. As mentioned earlier, this is beyond the confidence level of most structural engineers. The only input required to describe the desired frequency content is the input target spectrum. The ability to generate these spectra via attenuation models that only require magnitude and distance as input makes the combination of these two models attractively simple.

Additional incentives for the use of SIMQKE in this study include that it is offered on the University of California at Berkeley's NISEE website and is therefore widely available to practicing engineers. Also, it is totally disconnected from any attenuation model and authors of such models (some of the authors for the attenuation models used in this study have developed their own programs for the generation of simulated ground motions). In addition, the central frequency for the generated ground motion is reported in the output lending itself to the determination of the intensity envelope to be used for the refined generation of the synthetic record. Finally, it was chosen because it is robust and fairly straightforward to use.

The superposition of sinusoids from which the SIMQKE program is derived can be expressed as given in Equation 3.1.

$$
x(t)=\sum_{i=1}^{n} A_{i} \sin \left(\omega_{i} t+\phi_{i}\right) \quad \text { (Equation 3.1) }
$$


In this equation, $\omega_{i}$ represents the natural frequency as determined from the spectral density function derived from the input target spectrum. Also, $\phi_{i}$ represents the random phase angle which is determined from a random number generator and $A_{i}$ represents the amplitude of the $i$ th sinusoid which is initially randomized but then modulated by the intensity envelope function that is prescribed in the input. This function describes the desired shape of the output synthetic record and will be described in detail in later sections of this chapter.

\subsubsection{Procedure}

The SIMQKE program derives the spectral density function from an input prescribed target response spectrum through the following relationship shown in Equation 3.2.

$$
G\left(\omega_{n}\right) \cong \frac{1}{\omega_{n}\left(\frac{\pi}{4 \xi_{s}}-1\right)}\left(\frac{\omega_{n}^{2}\left(S_{V}\right)_{s, p}^{2}}{r_{s, p}^{2}}-\int_{0}^{\omega_{n}} G(\omega) d \omega\right)^{1 / 2}
$$

(Equation 3.2)

In this relationship, $G\left(\omega_{n}\right)$ represents the spectral density function being developed as function of the natural frequency $\omega_{h}$. $\left(S_{V}\right)_{s, p}$ represents the input spectral values from the target spectra. For this study, the target response spectra were obtained from the eight CEUS attenuation models for various magnitude and distance combinations. The parameter $r_{s, p}$ represents the peak factor which is a function of the 
spectral moments (analogous to mean and standard deviation) of the response. It is also a function of the equivalent stationary response duration (VanMarcke, 1976). $\xi_{s}$ represents the input damping ratio for the SDOF used in the random vibration amplification function. Note that this value is located in the denominator and therefore cannot have a value of zero.

Finally, note that the value for the spectral density function is contained within the function to determine the spectral density function. That is, the relationship established between the response spectrum and the spectral density function is not unique. Therefore, an iterative process of subtracting the area under the curve that has been developed up to each point is required. The equation is evaluated numerically and used iteratively within the evaluation. This will be revisited in the Chapter 7 in which the results obtained from this study are analyzed. The capabilities of the SIMQKE program to generate synthetic time histories whose response spectra are compatible with their target input spectra were verified in an extensive pilot study that is described in detail in Chapter 6.

\subsubsection{Input Parameters}

The main input required by the SIMQKE program is a target response spectrum. Other required input includes desired maximum ground acceleration, damping value, discretization interval, input spectral range, and a seed number to initialize the random number generator. Each of these inputs will be explained in the following paragraphs. The SIMQKE program also requires the input of a duration time and an 
intensity envelope to describe the shape and transient character of the synthetic ground motion. Two separate models were used in this study to develop these values which were then adapted for use in the SIMQKE program. An evaluation of these models is provided in the following section. An explanation of the process to incorporate them into SIMQKE is provided in Chapter 6.

The desired maximum ground accelerations used to generate synthetic ground motions for this study were provided from attenuation models that also provided the target response spectrum.

The damping input value is used in the algorithm to determine the spectral density function for the synthetic ground motion from the input target response spectrum. The response spectra used in this study as in most common applications, represent 5\% damped spectral response. Because of this condition and because a damping value is required as input into the SIMQKE program, a value very close to $0 \%$ damping was used in generating the synthetics for this study. Also, because the damping ratio appears in the denominator of Equation 3.2, a value of exactly zero could not be input. However the intent was not to apply more damping "in excess" of that already implied through the response spectral a value representing 5\% damping, and, therefore, an extremely small value relatively corresponding to zero was used as input. 
The discretization interval represents the constant time interval between values describing the synthetic record generated. This parameter proved to be critical in the results of this study and will be evaluated in detail in the analysis of results. For most practical applications, its value is usually taken to be 0.01 seconds. The input spectral range describes the smallest period and the largest period to be used from the input target spectrum.

The input seed number initiates the random phase angle generator in the algorithm. The idea is that by entering a different seed number the algorithm will produce an earthquake that is different in "appearance" but with the same "details" (i.e. the earthquakes look different but contain the same parameters, especially frequency content). In other words, by varying the seed number it is possible to generate different earthquakes that are the same in magnitude, distance, depth, and frequency content. By varying the seed number used to initialize the random phase angle generator, suites of ground motions can be generated that differ in their appearance and intricacies, however are very similar in their seismological characteristics (i.e. frequency content).

\subsection{Duration Model}

As mentioned in the previous treatment of the SIMQKE program, the inputs of duration and an intensity envelope are necessary to describe the transient character of the simulated ground motions. There are three options to describe the intensity

envelope in the SIMQKE program. These three options are shown in Figure 3.2. The 
exponential option was chosen for use in this study. Three parameter values must be input to describe this function in SIMQKE. For this study, these parameters describe the shape of earthquake records proposed for the CEUS. As pointed out in the previous chapters, there is quite a bit of variation and uncertainty associated with earthquakes in the CEUS with their shape and particularly their duration being no exception. 

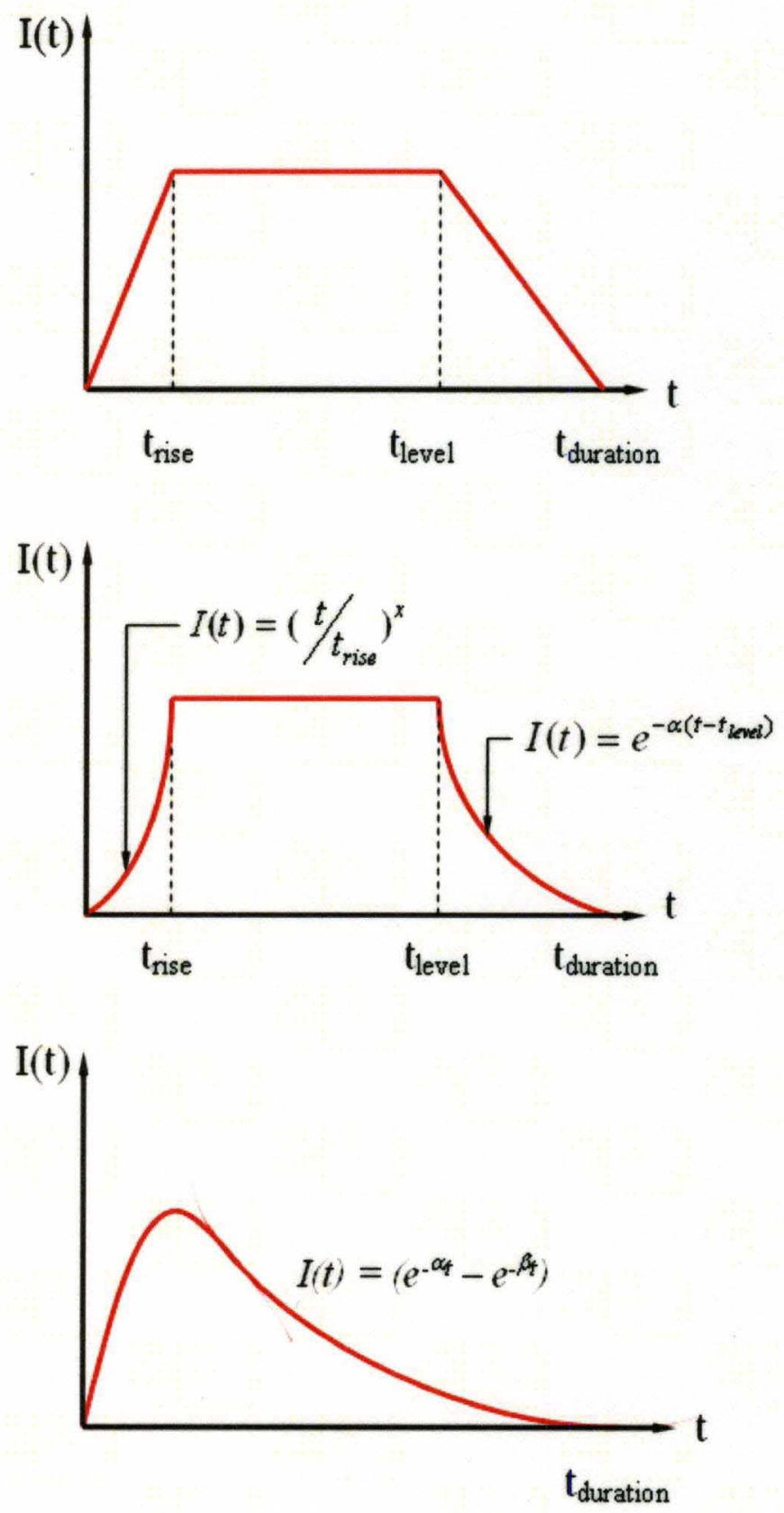

Figure 3.2 Intensity Envelope Options Available in SIMQKE

Fortunately there is an intensity envelope that is available in the literature (Saragoni and Hart, 1974) and is itself an exponential function similar to the one 
available in SIMQKE. An example of this envelope function is shown in Figure 3.3. In addition, the intensity envelopes for this model are a function of the strong motion duration. Fortunately again, there are also earthquake duration models available in the literature. For this study, the duration model of Novikova and Trifunac (1994) was employed. This model provides the duration as a function of magnitude, distance, geological and local soil site conditions, and central frequency of the ground motion. The central frequency can be interpreted as the average rate of zero up crossings in a time history and it also indicates the frequency where most of the power is concentrated in the record (Preumont, 1994).

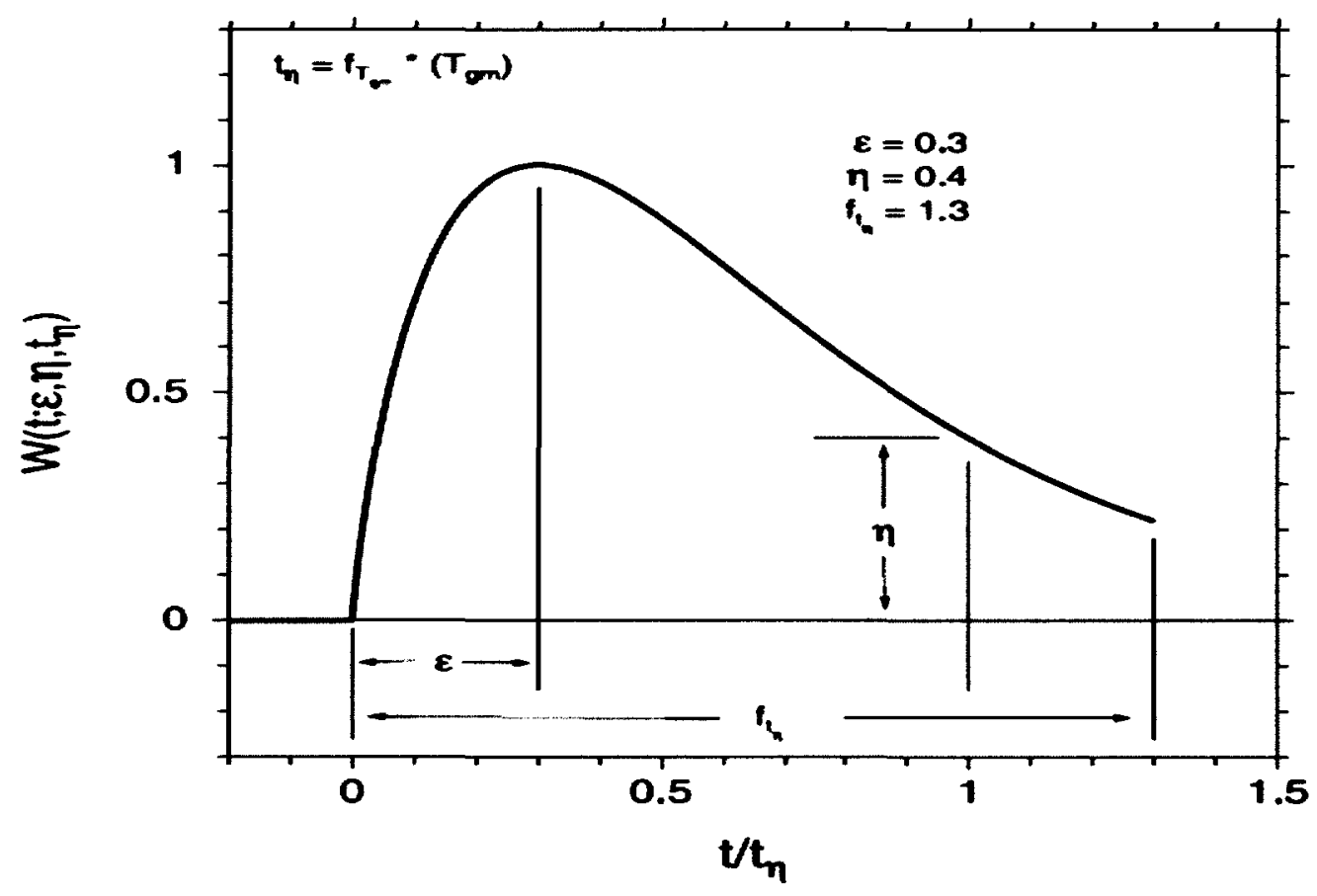

Figure 3.3 Saragoni and Hart (1974) Intensity Envelope from Boore (2003) 
Other models for duration were investigated for use in this study which included those used in the development of attenuation models for the CEUS also used in this study. Unfortunately, those models provide duration values as a function of corner frequency. As pointed out in the previous chapter, the corner frequency is a function of the seismic moment, stress drop, and shear wave velocity. Because simulated ground motions are being generated for this study, parameter values required to determine the seismic moment (e.g. geometrical dimensions of the fault, etc.) are not available.

Because the Novikova and Trifunac model is a function of magnitude, distance, and central frequency only, it was used to determine duration times for this study. Once the duration was determined using the Novikova and Trifunac model for a scenario event in this study, this duration could then be used to determine the intensity envelope using the Saragoni and Hart model. The only problem remaining becomes to relate the parameters describing this model to those that describe the exponential intensity envelope option available in the SIMQKE program. The procedure used to determine the parameter values for the exponential envelope function used by SIMQKE from the duration model of Novakova and Trifunac and Saragoni and Hart will be explained in detail in Chapter 6. 


\subsection{Intensity Envelope}

As mentioned earlier, there are three options available in SIMQKE to describe and shape the intensity function. These three envelope options were shown in Figure 3.2. The first option defines a trapezoidal shape made of straight line segments defining growth, level, and decay portions of the shape. The second option is similar and is called a compound envelope. The only difference between it and the trapezoidal is that concave curves are used to describe the growth and decay portions of the envelope. The third option is an envelope completely defined using an exponential function. An extensive amount of study as well as trial and error went into investigating the use of all three types of intensity functions prior to conducting the final study for this work. Because of the eventual prospect of combined use of a duration model as well as an exponential intensity function defined in the literature, the exponential function option was chosen to be used in this study.

The exponential envelope option in SIMQKE requires three parameters to define the envelope. $\alpha$ controls the curvature of the growth portion, $\beta$ controls the curvature of the decay portion, and $A_{0}$ controls the height. The process of mapping the Saragoni and Hart function onto these parameters is described in detail in Chapter 6.

In the stochastic method treated in the previous chapter, the frequency content is already determined when the Intensity Envelope is applied (and therefore it has very little effect on the response). However in the method employed by the SIMQKE program, the Intensity Envelope is predetermined and the frequency content (and 
associated energy) has to be either "compressed into" or "distributed" over this predefined envelope (and therefore the choice of Intensity Envelope has a pronounced effect on the response).

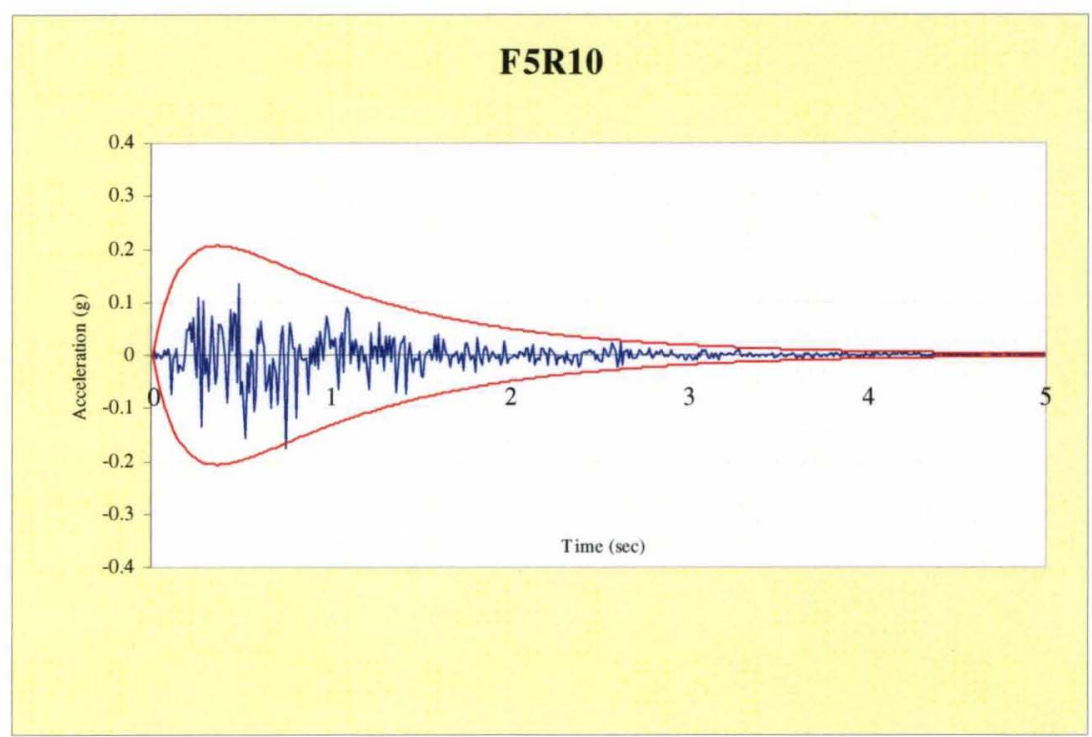

Figure 3.4 Example of an Intensity Envelope and Corresponding Synthetic Record

Figure 3.4 indicates that if the boundaries of an input intensity envelope (i.e. shape) are beyond the capabilities of the other inputs describing the ground motion (e.g. the frequency content from the input target spectrum) to produce amplitudes that will not reach the envelope, the program will not force them to do so. Figure 3.5 shows that, on the other hand, converse to what is shown in Figure 3.4, SIMQKE will force the record generated to remain within the envelope specified even if it is "condensed: to do so. 


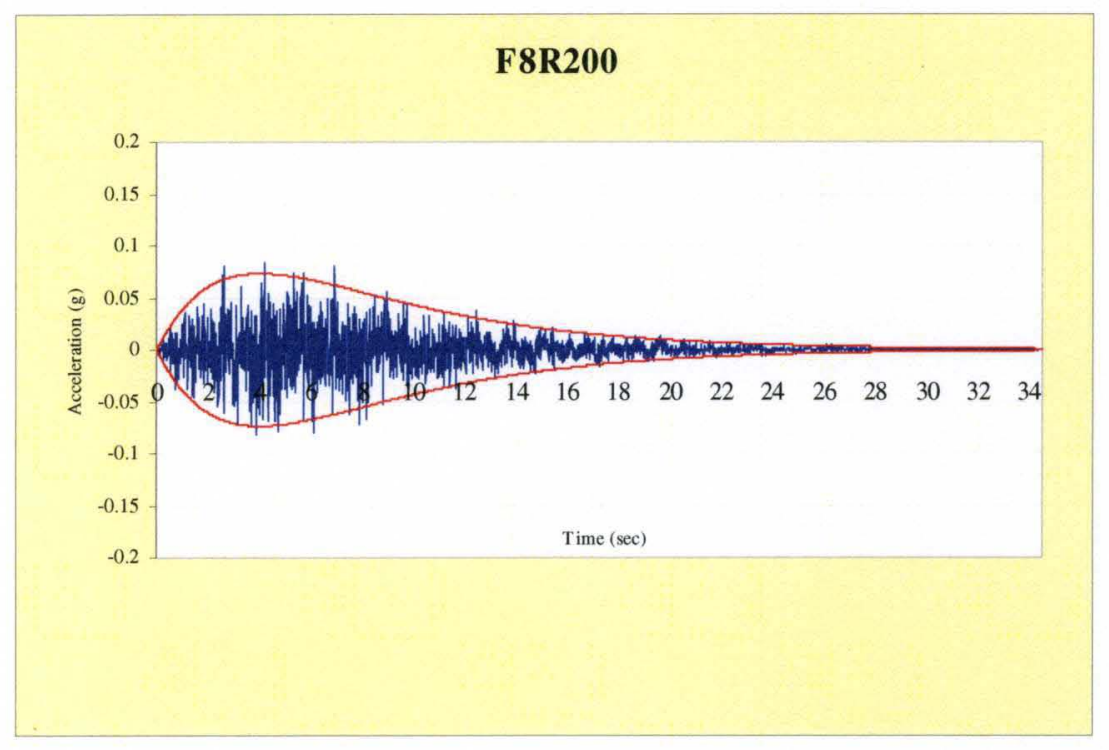

Figure 3.5 Example of an Intensity Envelope and Corresponding Constrained Synthetic Record

These two figures indicate the importance that the intensity envelope has in the generation of synthetic ground motions using the SIMQKE program. For this reason, much care went into the development of the procedure used to develop the duration times and intensity envelopes used to develop the synthetic ground motions used in this study.

A complete study of how the Intensity Envelope affects the structural response to synthetic time histories generated using the SIMQKE program may in itself be worthy of further research but is beyond the scope of this study. 
After applying the intensity envelope, the superposition of sinusoids in the SIMQKE algorithm as described in Equation 3.1 now becomes the relation shown in Equation 3.3.

$$
z(t)=I(t) \sum_{i=1}^{n} A_{i} \sin \left(\omega_{i} t+\phi_{i}\right) \quad(\text { Equation 3.3) }
$$

Figure 3.6 shows, as an example, the Saragoni and Hart model combined with the Novakovia and Trifunac duration model transformed to the SIMQKE model using the parameters (i.e. magnitude, distance, and central frequency) for tan actual Loma Prieta record. This figure confirms that the envelope does provide a good representation for the general shape of the strong motion portion of most actual recordings. Further validation is provided in a pilot study that was conducted prior to the final study as described in detail in Chapter 6. 


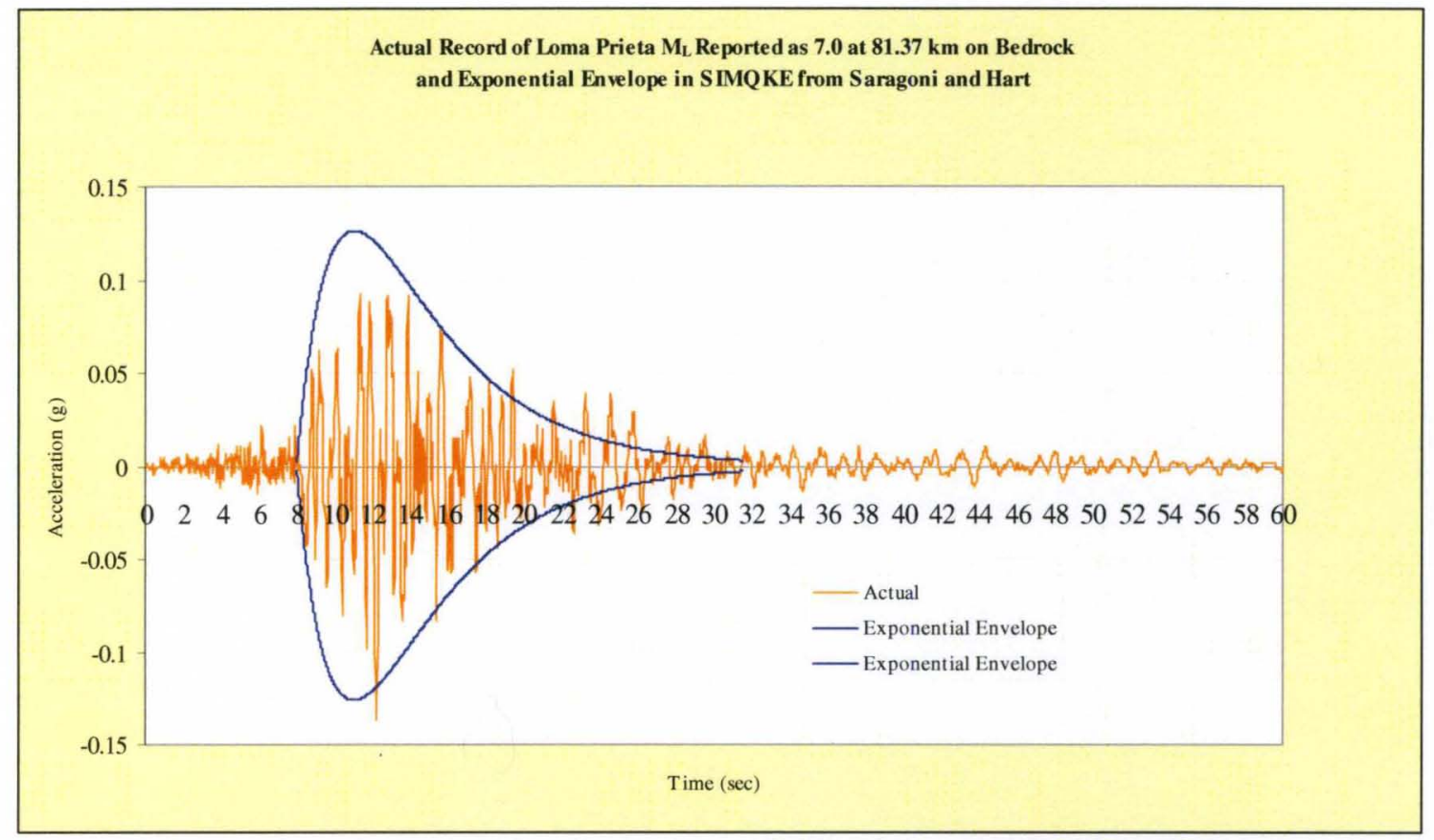

Figure 3.6 Actual Record Compared to the Exponential Envelope in SIMQKE Derived Using the Novikova and Trifunac (1994) Duration Model and the Sargoni and Hart (1974) Intensity Envelope Function 


\section{Chapter 4}

\section{STRUCTURAL RESPONSE}

\subsection{Introduction}

As mentioned in Chapter 1, among all of the seismic analysis methods available, the nonlinear time history analysis of structures provides the best representation of how structures respond to earthquake ground motions. This type of analysis was employed in this study and a brief explanation of the procedure, software, and parameters used in this study is provided in the following sections.

With the abundant availability of high speed computers and relatively robust structural analysis software, the use of nonlinear time history analysis is becoming more common in the seismic design of structures. This approach allows structural computer models to be built using finite element methods and subjected to earthquake loading to investigate their responses. This procedure is used to estimate how the elements within a structure will yield due to seismic loading in order to design adjustments to their strength to meet ductility requirements. As such, this procedure requires a good estimation of the ductility demand placed on the structure during a specified seismic event. 
As always, the quality of the output from a computer analysis depends on the quality of the input information. It is important that not only should the numerical model represent the structure well, but the acceleration time histories applied as loading in the analysis should also represent the design earthquake well.

For this study, time histories were artificially generated using the SIMQKE program to be compatible in frequency content with the eight CEUS attenuation models used to develop the 2008 National Seismic Hazard Maps. The duration and intensity models described in the previous chapter were also used to generate these synthetic records.

\subsection{Time History Analysis}

\subsubsection{Equations of Motion}

The equation of motion describing the linear response of single degree of freedom systems to applied ground acceleration is provided in Equation 4.1. This is a linear second-order ordinary differential equation with constant coefficients.

$$
m \ddot{y}+c \dot{y}+k y=m \ddot{y}_{g} \quad(\text { Equation 4.1) }
$$

In Equation 4.1, $y$ represents relative displacement with its first and second derivatives being velocity and acceleration, respectively. The coefficients of these values are the stiffness $k$, damping $c$, and mass $m$. Input may be stated in matrices; 
however, for a single degree of freedom system scalar operations are adequate. The natural frequency of the system is determined by its mass and stiffness as in Equation 4.2.

$$
\omega=\sqrt{\frac{k}{m}} \quad \text { (Equation 4.2) }
$$

The natural period of the system is the inverse of its natural frequency. The stiffness is a mechanical property of the system and the damping is usually expressed in terms of critical damping. Damping is a naturally occurring dissipation of energy within the structure and is typically caused by internal friction and hysteretic material behavior. Critical damping is defined as the smallest amount of damping required to prevent an oscillatory motion (no zero displacement crossings) after a system is given an initial displacement and then released.

Damping values of two to seven percent of critical damping are usually used for the analysis of structures responding to earthquake ground motions. Five percent of critical damping is commonly used for the analysis of most structures. Damping is also generally incorporated within the response spectra describing the frequency content of an earthquake ground motion. Five percent of critical damping was applied for all SDOFs used in this study. 


\subsubsection{Nonlinear Response}

There are situations for which the linear model given in Equation 4.1 does not adequately represent the dynamic characteristics of the structure being modeled. Such cases require the introduction of a model in which the spring force may not remain proportional, respectively, to the displacement or to the velocity. Consequently, the resulting equation of motion will no longer be linear and its mathematical solution, in general, will have a much greater complexity, often requiring a numerical procedure for its integration (Paz, 1997). The equation of motion expressing the nonlinear response of a single degree of freedom to ground acceleration is given by Equation 4.2. The derivation of this equation is provided in several texts available on structural dynamics. An excellent and concise explanation is offered by Paz (1997) with more detailed explanations and derivations offered by Clough and Penzien (1993),

$$
m \ddot{y}+c \dot{y}+f_{s}(y, \dot{y})=m \ddot{y}_{g} \quad \text { (Equation 4.2) }
$$

As mentioned, analytical closed form solutions for this equation are not usually available especially if the applied force is an arbitrary function of time as in the case of an earthquake time history. Direct integration methods provide numerical solutions for the equations of motion yielding estimates of the dynamic response of structures. As the name implies, these methods directly integrate the equations of motion using numerical step-by-step procedures in which the state of the system is 
determined at small time increments, each state being calculated from its immediate predecessor.

Many numerical integration procedures have been developed; however, only a brief summary of the most common methods is presented here. An overview of the methods available is presented in U. S. Army Corps of Engineer Manual EM1110-26051 and a more detailed description of the available methods is provided in the cited references and elsewhere in the literature.

The procedures described here are often referred to as time history analyses. These methods can be classified into one of two approaches, implicit or explicit. In practice, for the design of real structures that have multiple degrees of freedom (MDOF), implicit methods are preferred over explicit methods; however, for single degree of freedom (SDOF) systems explicit methods are acceptable.

Explicit procedures are carried out by approximating the initial velocity and acceleration using finite difference expressions and then solving for response at the end of the time step. In this way, the response values calculated in each step depend only on quantities obtained in the preceding step. Therefore, the numerical process proceeds directly from one step to the next. Explicit methods are very convenient, but they are only conditionally stable if the time step is very small. 
Implicit methods solve the differential equations at the current time step using the solutions determined in the previous time step. In other words, the solutions for the previous time step are used as initial conditions in the next time step. These equations have endured and been applied to the analysis of many structures over the last half of the twentieth century. Since the inception of the method, improvements have been made to increase its efficiency and applicability (Wilson 1962, Wilson 1973, Hughes 1987).

An approach to solving structural dynamics problems using a step-by-step numerical integration procedure was first introduced by Newmark (1959). He used Taylor series expansions to write the displacement and velocity terms for the equations of motion at the current time step in terms of time derivatives at the next time step. He truncated these expressions, introduced a scalar to the last terms, and used the assumption of linear acceleration within each time step to develop his equations.

The basic Newmark constant acceleration method can be extended to nonlinear dynamic analysis. This extension requires that iteration must be performed at each time step in order to satisfy equilibrium. Also, the incremental stiffness matrix must be formed and triangularized during each iteration or at selective points in time.

The central difference method is a very simple explicit method that uses the finite difference method for solving differential equations to approximate initial velocity 
and acceleration terms (Clough and Penzien, 1993; Bathe and Wilson, 1976). An excellent and concise explanation of the derivation for the method is provided in Paz (1997) with more detailed explanations in Clough and Penzien (1993).

Nonlinear dynamic analysis procedures require the inclusion of a nonlinear forcedisplacement relationship. These relationships are necessary to describe the stiffness degradation that occurs in the structural system under cyclic loading. This degradation of stiffness within the structure is known as hysteretic behavior. The specific values applied to describe the relationships used in this study are provided in Chapter 6.

In addition, to perform time history numerical procedures using analysis software requires input associated with the fundamental coefficients in the equation of motion mentioned earlier. Most software also requires the input of mechanical properties necessary to implement the finite element method. The output from such analyses are usually forces and displacements and can be in the form of more elaborate response histories describing seismic demand (moments, shears, stresses, etc.). As will be described later, the nonlinear time history analyses conducted for this study were conducted using a relatively simple computer program for the analysis of the SDOF systems. 


\subsubsection{Hysteretic Behavior}

For nonlinear time history analysis, the stiffness of a structural system is described by a capacity curve defining the hysteretic behavior of the system. Under heavy seismic loading, the stiffness of the system typically will degrade. This degradation is modeled through a capacity curve.

Hysteretic behavior is a function of the material and type of construction and is usually determined through laboratory testing. For the general purpose of this study, a simple perfectly elasto-plastic capacity curve was assumed such as that shown in Figure 4.1. This is a simplified model whose use is widespread although it represents an idealized system. Its use in this study was based on brevity considering the goal was to determine the variation among structural responses due to the synthetic ground motions from different attenuation models and was not focused on the accurate portrayal of the actual response for a particular structure. The development of capacity models that more accurately portray the hysteretic behavior of specific types of structures is the topic of much ongoing research and is beyond the scope of this study. 


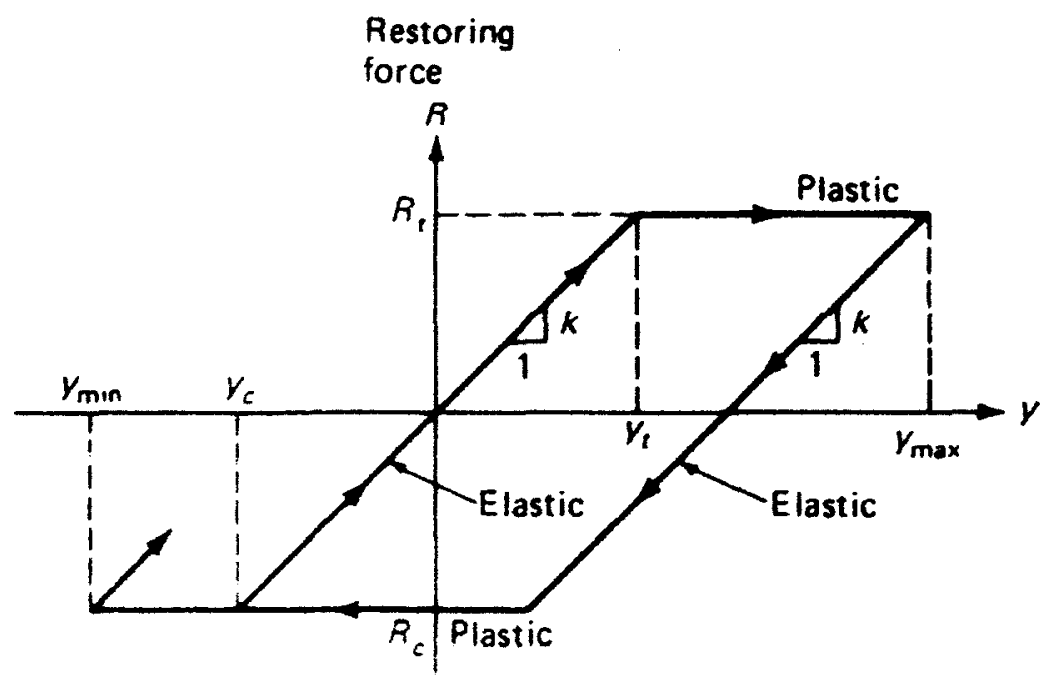

Figure 4.1 Elasto-plastic Behavior Assumed in this Study (Paz, 1997)

The initial slope in the capacity model is defined by the stiffness of the system. The secondary slope can be either positive indicating strain hardening of the system or it can be negative indicating degradation in the system stiffness. As is the case for this study, it can also be flat representing a perfectly elasto-plastic response. The system can also be modeled as multi-linear, with the response graph composed of a combination of increasing and decreasing slopes to give a more realistic model of structural capacity. 


\subsection{NONLIN}

Several programs are available to conduct nonlinear time history analyses of structures: SAP2000 (Computers and Structures, Inc.), OpenSees Pacific Earthquake Engineering Research Center), DRAIN (National Information Service for Earthquake Engineering), IDARC (State University of New York at Buffalo), etc. All of these programs are more sophisticated than the one used in this study but rely on the same or similar approaches to solving the equations of motion described earlier.

The NONLIN program used in this study is an application for the dynamic analysis of single degree of freedom structural systems with the dynamic loading input as an earthquake accelerogram acting at the base of the structure. The NONLIN software is made available free to the public through the Federal Emergency Management Administration (NONLIN, 1996). The program uses a step by step method to solve incrementally the nonlinear equations of motion described earlier and while the procedure used by the NONLIN software is not explicitly defined, its user's manual refers to Clough and Penzien for a theoretical description of the solution technique.

\subsubsection{Required Input}

The constitutive structural properties required as input by the NONLIN software are the structural weight and damping, as well as the initial stiffness, secondary yield stiffness, and yield strength, which define the capacity curve for the SDOF. 
In the NONLIN program, a structure is idealized as a single degree of freedom system, and the mass and damping inputs are as explained earlier in this chapter. For a nonlinear analysis, two additional properties are required, the initial stiffness as illustrated by the slope in Figure 4.1, having units of force/length, and the yield strength of the system. For nonlinear analysis, the unloading stiffness is assumed to be equal to the initial stiffness.

The secondary stiffness is the slope of the post-yielding portion of the force displacement response of a structure and for an elasto-plastic system representing an elastic-perfectly plastic response, is assumed to be zero as shown in Figure 4.1.

The yield strength of the system is also required input for nonlinear analysis. This parameter is given in units of force and defines the point at the top of the slope representing the initial stiffness. This is the point where the structure yields and transfers from elastic to plastic behavior. The three inputs of initial stiffness, secondary stiffness, and yield strength define the capacity curve that describes the structural system.

Of course, an essential input for time history analysis is the time history record. The time history records used in this study were derived to be compatible with CEUS attenuation models as explained in the previous chapters. In order for the NONLIN program to be able to read these time histories, they had to be presented in a specified format given in the program. 


\subsubsection{Available Output}

The output made available by the NONLIN software can be utilized in a number of different ways. Using the plotting options, the user may plot input time history as well as an elastic response spectrum, or a Fourier amplitude spectrum describing the frequency content of the input time history. Also, a summary of numerical results from the analysis are made available including the structural properties and a summary of response maxima.

\subsection{Structural Parameters}

As can be seen from Equation 4.2, the input values for weight and initial stiffness can be calibrated within the NONLIN software to achieve a desired natural period for the SDOF. The natural periods of the SDOFs used in this study are presented in Chapter 6 along with the parameter values used to produce the SDOFs with these specified natural periods. The values used in this study were taken from examples of practical design applications (Chopra, 1995; Naeim, 1985; Williams, 2005). The systems analyzed serve only as single, general, and idealized representations of structures having the natural periods used in this study.

In addition, to accommodate the estimation of the magnitude of the synthetic time histories generated for this study, and as described in detail in the following chapter, the NONLIN software was used to develop a SDOF system that has the parameters described for the Wood-Anderson seismograph. This latter estimation relied on the maximum displacement response reported for this SDOF system and synthetic ground 
motion. Therefore, six natural period values were chosen for this study as well as parameters to simulate a Wood-Anderson seismograph.

\subsection{Ductility Demand}

The displacement ductility demand was the primary output captured from the time history analyses and used for the comparisons in this study. The displacement ductility demand is defined as the ratio of the maximum displacement undergone by the system due to the input time history compared to the displacement at which the system yields. According to this definition, the displacement ductility demand is an indicator of inelastic deformation and system ability to deform beyond its elastic limit. Maximum displacement by itself is not a good measure of performance in this regard.

Much research is being done to develop damage indices in terms of displacement ductility demand. There are many approaches to relating ductility demand to various levels of damage for structures of various types of materials. Despite the sophistication of some of these models, ultimately some degree of subjectivity is required in selecting the values of some of parameters. The primary focus of this study was on the variation in ductility demand calculated as the response to synthetic ground motions generated from the attenuation models used in this study. As such and for brevity, this study focuses on the displacement ductility demand milestone value of "one" representing the interface between linear and plastic response. 
In this study, a ductility demand of one is taken as indicating the onset of yielding and ductility demands below one indicate that the structure has remained elastic under the loading from the synthetic motion. Likewise for this study, a ductility demand greater than one is simply taken to indicate that the structure has yielded and although comparisons between the values of the ductility demands of the synthetics from the various models have been made, there is no direct attempt to relate these values to specific levels of damage. 


\section{Chapter 5}

\section{MAGNITUDE ESTIMATION VIA TIME HISTORY ANALYSIS}

\subsection{Background}

A procedure to estimate the magnitude of synthetic ground motions was developed for this study and is presented in this chapter. The concept was introduced originally to investigate the error introduced by the process used to generate the synthetic motions for this study. The same procedure was used as another way to confirm that the synthetic earthquakes being generated were actually compatible with what was requested [magnitude of event at given distance]. This latter work was part of a pilot study conducted prior to the final study comparing actual ground motion records and synthetic ground motions generated from those same records. This pilot study is described in detail in the next chapter.

This procedure described in the next chapter also was incorporated into the final study to investigate the error between the synthetic ground motions and their respective target magnitudes. It also proved to be beneficial in identifying the ductility demands from the synthetic motions that best represent the true structural 
response. The method utilizes the results from time history analysis. As will be explained in the following sections, it also relies on the Richter magnitude which will also be defined in the following section.

Most seismologists at this writing use a scale known as moment magnitude. This scale also is used as the input target magnitude required by the attenuation models applied in this study. As illustrated in the next section, moment magnitude is a function of the seismic moment and the seismic moment is a function of parameters associated with the source fault geometry. Because this study incorporates the use of synthetic ground motions, this information on source fault geometry is not available. For this reason, and because of the realization that displacements determined from time history analysis can be used to estimate the Richter magnitude, it was decided to use this measure of magnitude for this study and then convert these values to moment magnitude. The overall procedure applied to accomplish this will be explained in the last section of this chapter.

\subsection{Richter Local Magnitude}

The concept of earthquake magnitude was introduced by Richter (1935) in an effort to present a single, standardized, and objective quantity to describe the size [intensity or effect] of an earthquake. This magnitude scale relies on instrumental measurements of earthquake ground motions which are adjusted depending on the distance between the earthquake source and the location of the station where the measurements were made. 


\subsubsection{Definition}

Richter found by plotting the logarithm of maximum horizontal trace amplitudes recorded on Wood-Anderson seismometers that they decreased with distance as essentially parallel curves for different size earthquakes. This result led him to formulate the following definition to quantify the size of an earthquake by its magnitude, " The magnitude of any shock is taken as the logarithm of the maximum trace amplitude, expressed in microns, with which the standard short-period (WoodAnderson) torsion seismometer ... would register that shock at an epicentral distance of $100 \mathrm{~km} "$ (Richter, 1935).

By specifying the use of a Wood-Anderson seismograph in his definition, Richter standardized the instrument type to eliminate the effects of instrumental response on the recordings used in calculating the magnitude value. The Wood-Anderson torsion seismometer had standardized instrument parameters of a natural period of 0.8 seconds, a dynamic magnification factor of 2800 , and a damping ratio of $80 \%$ of critical.

Richter developed his original scale from local shocks measured in southern California. The Richter local magnitude $M_{L}$ is still the best known of all the magnitude scales in use today. Richter's original definition was based on recordings taken at an epicentral distance of $100 \mathrm{~km}$ and he provided a table of attenuation correction factors for other distances between 30 and $600 \mathrm{~km}$. These values were later complemented by including corrections for distances less than $30 \mathrm{~km}$ (Gutenberg and Richter 1942) and they were published all together in tabulated form 
by Richter (1958) as the correction factors $-\log A_{0}$ which are the logarithms of amplitudes with which a standard torsion (Wood-Anderson) seismometer should register an earthquake of magnitude zero. The local magnitude as defined by Richter is given by Equation 5.1 .

$$
M_{L}=\log A_{\max }-\log A_{0} \quad(\text { Equation 5.1) }
$$

$A_{\max }$ is the maximum recorded trace amplitude for a given earthquake at a given distance as recorded on a Wood-Anderson seismograph. Richter (1958) termed $A_{0}$ as the zero level and stated that it can be fixed by naming its value at a particular distance. At a distance of $100 \mathrm{~km}$ it is taken as one thousandth of a millimeter. The values at other distances were determined by setting $A=A_{0}$ which provides a magnitude of zero. This event is therefore referred to as the standard shock or the zero shock. This reference does not imply that no earthquake occurred, however. It is possible that small earthquakes can be recorded whose amplitudes are smaller than the standard shock and therefore produce negative magnitudes.

\subsubsection{Modern Recordings}

In the twenty-first century, many magnitude scales are used to describe earthquakes and moment magnitude is perhaps the most popular in the seismological community. This magnitude is preferred because it is not subject to saturation of the instrument such as can occur for other magnitude scales. Saturation occurs during large events whose wave lengths and trace displacements surpass the physical 
capabilities of the instrument on which they are being recorded. However, in order to determine the moment magnitude, knowledge of the geometry and size of the causative fault is required. Because the ground motions used in this study were synthetic simulations, information on fault characteristics was not available. Therefore, this magnitude could not be determined directly. However, the local Richter magnitude for these simulated ground motions can be determined using the displacements they produce on a simulated instrument. For this reason, in this study a simulated instrument was used to obtain maximum displacements simulating maximum trace recordings from a Wood-Anderson seismograph that could be used to determine the local Richter magnitude of the synthetic motions. In order to make comparisons to their target magnitudes that were expressed as moment magnitudes, a conversion had to be made. The derivation of the conversion used in this study is explained in the following section.

Despite significant advances in instrumentation, current procedures to determine the magnitude of an earthquake are still based on Richter's definition and the response of a Wood-Anderson seismometer. However, today procedures are used to synthesize precisely the response characteristics of the Wood-Anderson seismograph from digital broadband recordings made on sophisticated modern equipment (Uhrhammer et al., 1990). Even though actual physical Wood-Anderson seismometers are no longer required, responses simulated to meet their specifications are still used to determine the local Richter magnitude of current earthquakes. This determination is accomplished through convolution in the frequency domain to 
remove the original instrument response and replace it with the Wood-Anderson response. The synthetic Wood-Anderson traces then are returned to the time domain, where the maximum trace amplitude is determined and the local magnitude can be calculated using Richter's definition (Uhrhammer et al., 1996).

\subsubsection{ENA Correction}

The $-\log A_{0}$ term in Richter's definition accounts for attenuation and station corrections and were developed from the seismicity in southern California. These are regional corrections and their shape and level may be different in other areas of the world with different geologic structure and composition. Accordingly, when determining $M_{L}$ for other regions, calibration functions have to be developed for the specific region to allow proper scaling of the original definition for $M_{L}$ at the $100 \mathrm{~km}$ epicentral distance in order to yield magnitudes compatible with the original definition.

The formula in Equation 5.2 was proposed by Kim (1998) to determine the local magnitude $M_{L}$ for earthquakes in Eastern North America (ENA) from the horizontal maximum trace amplitude as measured on a Wood-Anderson seismogram:

$$
M_{L}=\log A_{\max }+1.55 \log A_{0}-0.22+C, \text { for } 100 \mathrm{~km}<A_{0}>800 \mathrm{~km} \text { (Equation 5.2) }
$$

The corrections in this equation account for the arbitrary constraint in the Richter definition (i.e. the standard shock referred to earlier where $-\log A_{0}$ for $100 \mathrm{~km}$ gives $M_{L}=3$ ) as well as the differences in amplitude attenuation between ENA and 
southern California at the reference distance of $100 \mathrm{~km}$ for a maximum trace

amplitude. This equation calibrated for ENA was used to estimate the magnitudes of the synthetic motions generated in this study for events measured at distances of 100 $\mathrm{km}$ and $200 \mathrm{~km}$.

\subsection{Moment Magnitude}

As referenced earlier, moment magnitude $M_{W}$ is the preferred measure of magnitude among seismologists and is used by all of the attenuation relations employed in this study. Moment magnitude was introduced by Hanks and Kanamori (1979) and is a function of the seismic moment.

\subsubsection{Definition}

The seismic moment provides an estimate of the energy released from an earthquake as it is related to the size of the rupture and the force couples developed across the fault. Specifically the seismic moment is a function of the modulus of rigidity of the ruptured material, the area of the fault, and the amount of displacement occurring along the fault. Again, as mentioned before, this study utilized simulated earthquakes and therefore the physical measurements related to fault dimensions were not available. However, using the procedure described above, estimates of the maximum trace displacement for a Wood-Anderson seismograph could be obtained for the synthetic records. This measurement then could be used to calculate estimates of the local Richter magnitude $M_{L}$. In order to make comparisons of the calculated 
Richter magnitudes for the synthetic motions with their target input moment magnitudes, a conversion had to be made from one magnitude scale to the other.

\subsubsection{Conversion}

By applying the Choy and Boatwright (1995) correction to the Gutenburg-Richter equation for radiated energy as a function of surface wave magnitude $M_{S}$, it is possible to write the energy magnitude in terms of $M_{S}$. Under the Kanamori (1977) condition for the relationship between radiated energy and seismic moment, energy magnitude is equal to moment magnitude yielding Equation 5.3 which reduces to Equation 5.4.

$$
\begin{gathered}
M_{W}=2 / 3\left(1.5 M_{S}+4.4\right)-3.2 \quad(\text { Equation 5.3) } \\
M_{S}=M_{W}+0.27 \text { (Equation 5.4) }
\end{gathered}
$$

This equation provides an estimate of the difference between surface wave magnitude and moment magnitude. Finally, by using Ambraseys' (1990) orthogonal regression relationship between surface wave magnitude and local Richter magnitude $M_{L}$ given as in Equation 5.5, a relationship can be established between $M_{L}$ and $M_{W}$ as shown in Equation 5.6.

$$
0.80 M_{L}-0.60 M_{S}=1.04 \quad(\text { Equation 5.5) }
$$




$$
M_{W}=1.33 M_{L}-2 \quad \text { (Equation 5.6) }
$$

Equation 5.6 is the relationship used in this study to convert the estimated local Richter magnitude to moment magnitude. Prior to this conversion to $M_{W}$, the calculated $M_{L}$ values were corrected for Eastern North America using the equation developed by Kim (1998) as described earlier. In accordance with its recommended application, this correction was only applied to the events simulated at distances of $100 \mathrm{~km}$ and $200 \mathrm{~km}$ in the study.

\subsection{Procedure}

The procedure for estimating the magnitudes of the synthetic ground motions used in this study begins with inserting parameter values for a SDOF system to be used in time history analysis to simulate the Wood-Anderson seismograph. These values include a weight and stiffness to produce the specified natural period of 0.8 seconds. The exact values used in this study are provided in the next chapter. A damping ratio of $80 \%$ of critical is specified to simulate the instrument. The synthetic time histories simulating the prescribed ground motions for this study then were loaded onto the SDOF system and the maximum displacement determined from the analysis was captured. Richter's definition of magnitude was modified with the correction for attenuation in ENA as formulated by Kim and then applied to estimate the local Richter magnitude of the synthetic ground motions. Finally, the Richter magnitudes were converted to moment magnitude using the calculations described in 
the previous section so that the estimated magnitudes for the synthetic motions could be compared to their target input moment magnitudes.

\subsection{Comments}

It is pertinent to note that the objective of this study was not to evaluate or critique one attenuation model versus the others but rather to investigate their use combined with spectral matching as a practical method for engineers to develop design ground motions for the CEUS. In line with this intent, Kanamori (1983) states, "the magnitude scale merely aims at providing a quickly determined and simple parameter which can be used for first-cut reconnaissance analysis of earthquake data for various geophysical and engineering investigations". Kanamori also suggests that special caution should be exercised in using the magnitude beyond this reconnaissance purpose.

Perhaps it should be pointed out also that determining the magnitude of an actual earthquake appears to be somewhat subject to interpretation and requires an understanding of and the application of corrections for the effects caused by the recording process. The main objective of the seismologist trying to assign a magnitude to a record is filtering out the instrument and site effects to uncover the true ground motion (Scherbaum, 1996). Many times the recording is taken by an instrument located within a structure and the device may or may not have been located on the ground level or below. In such cases, the response of the structure itself is going to contribute to the record. Even in those instances where the 
instrument is located at the ground level, local site effects such as soil conditions and distance to the fault often contribute to concealing the true ground motion recording.

Even after the site effects are filtered from the recording and the traces transformed from the type of instrument that made the recording to a Wood-Andersen instrument, one must take into account the fact that often many recordings are reported from many different stations all with varying distances to the epicenter of the earthquake. The calculated magnitudes of individual records can vary significantly. It seems that some subjectivity has to be applied in weighting the contribution of these varying recordings. Recordings made at large distances may not give accurate representations of the event, and, conversely, recordings made within the near field can be expected to overestimate the magnitude of the actual event. Richter himself suggested that several recordings of the same event should be averaged to reach an appropriate magnitude, but again these averages are undoubtedly weighted in some fashion and subjective decisions are made as to their inclusion or exclusion.

Nevertheless, it appears that the procedure used in this study is a relatively straightforward approach to a complicated problem. The main objective for introducing it in the study was to estimate if the synthetic ground motions being generated were representative of the target magnitudes requested. Furthermore, with no physical characteristics such as fault geometry to associate with these synthetic ground motions, it seemed totally appropriate and only made sense to use a simulated instrument to estimate the magnitude of a simulated earthquake. Again, the original 
intent of this procedure was to verify the validity of the synthetic ground motions generated in this study but it has also proved to be a possible tool in evaluating which generated synthetic motions are closer to their intended targets as will be shown in the analysis of results for this study. This procedure itself was also verified in a pilot study that will be described in the following chapter. 


\section{Chapter 6}

\section{METHODOLOGY}

\subsection{Introduction}

The methodology applied to conduct this study will be presented in this chapter. Its presentation will begin with a description of the general approach taken, followed by a description of the development of the intensity envelope function used throughout the study, and a description of the parameters used to define the single degree of freedom systems. The development, application, and results of an extensive pilot study conducted prior to the final study then will be presented. Finally, a description of the specific conditions pertinent to the methodology as it was applied in the final study will be given.

\subsection{General Approach}

The design of this study is based on the combined use of the attenuation models proposed for the CEUS as described in Chapter 2, and the SIMQKE program as explained in Chapter 3 to generate synthetic ground motions to be used as input loading for time history analyses as explained in Chapter 4. Again, the objective for 
doing so was to investigate the validity of this procedure in generating time histories to be used for seismic design of structures in the CEUS. Validity is measured in the amount of variation expressed in the structural response induced by these synthetically generated ground motion time histories.

\subsubsection{Automation Code}

As will be explained later, a total of 30,000 runs were necessary for the final study. Therefore, a computer code serving as an umbrella program that synchronized and operated all of the software used in the study was written and utilized. The umbrella also automatically created all necessary input from an individual root file as well as collected all associated output. The root files were created for each attenuation model and scenario from a template following a precise format. Each root file is essentially the SIMQKE input file appended with additional information necessary to run the automation code. The automation code reads in the necessary information from the root file. The first thing read is the title of the run. In the final study, the input files were named in accordance with a naming convention that will be explained later in this chapter. All associated files are named using the title provided in the root file and each file name is automatically appended with the correct seed number. The code creates all SIMQKE input files and generates the associated synthetic records. Then the NONLIN input files are created by formatting the correct heading associated with each event and by extracting and inserting the record from each SIMQKE output file. Next, NONLIN is opened and the assigned parameters are set for a SDOF system and each NONLIN input file (with each synthetic record for 
each seed number) is run on that SDOF system and all NONLIN output is captured. This procedure is followed for each SDOF specified. Finally, the ductility ratios and maximum displacements for the Wood-Anderson SDOF are extracted from each NONLIN output file and stored in a separate file. This file is then automatically stored in an individual folder along with all of the SIMQKE input/output files and NONLIN input/output files. Each of these folders as well as the root file are stored in a folder for each scenario and attenuation model used and titled according to the naming convention.

\subsubsection{Duration and Intensity Envelope}

The SIMQKE program requires the input of an intensity envelope that defines the shape and duration of the output synthetic record. As explained in the Chapter 3 on the generation of synthetic ground motions, the Sargoni and Hart intensity function and the Novikova and Trifunac duration model were combined for this study to utilize the exponential intensity envelope option available within the SIMQKE program. Using parameter values similar to those suggested by Boore (2003), the Saragoni and Hart model has the following form:

$$
w(t)=15.6\left(\frac{t}{t_{n}}\right) e^{5.74\left(\frac{t}{t_{n}}\right)} \quad \text { (Equation 6.1) }
$$


Where $t_{n}$ was taken as the duration time as determined from the Novikova and Trifunac model for this study. An example of this envelope function was shown in Figure 3.2 of Chapter 3.

Because the expression for the intensity envelope coded in the SIMQKE program is in a different form than the form provided by the Saragoni and Hart model, parameter values for the Saragoni and Hart model had to be mapped onto the parameter values for the function used in SIMQKE. This mapping was accomplished by using parameters for Saragoni and Hart as suggested by Boore (2003) to determine envelope functions at duration times of $5,10,20,30,40,50$, and 60 seconds. These functions were plotted simultaneously with the function available in SIMQKE. The SIMQKE parameter values were adjusted until its function was overlain onto the Saragoni and Hart function as closely as possible. The exponential function available in SIMQKE has the form shown in equation 6.2.

$$
I(t)=A_{0}\left(e^{-\alpha t}-e^{-\beta t}\right) \quad \text { (Equation 6.2) }
$$

$A_{0}$ adjusts the amplitude height and $\alpha$ mainly affects the curvature of the decay portion. The $\beta$ parameter mostly controls the steepness of the growth portion of the curve. Values for these parameters providing very near fits were determined at all of the duration times used to establish the relationship. These parameter values were then fit with curves via regression analyses as functions of the duration time. These functions were used in the study to determine the exponential intensity envelope in 
SIMQKE as a function of the duration time which again, was determined using the Novikova and Trifunac model. A typical plot showing the two envelope functions simultaneously after the SIMQKE function was adjusted to match the Saragoni and Hart function is provided below in Figure 6.1.

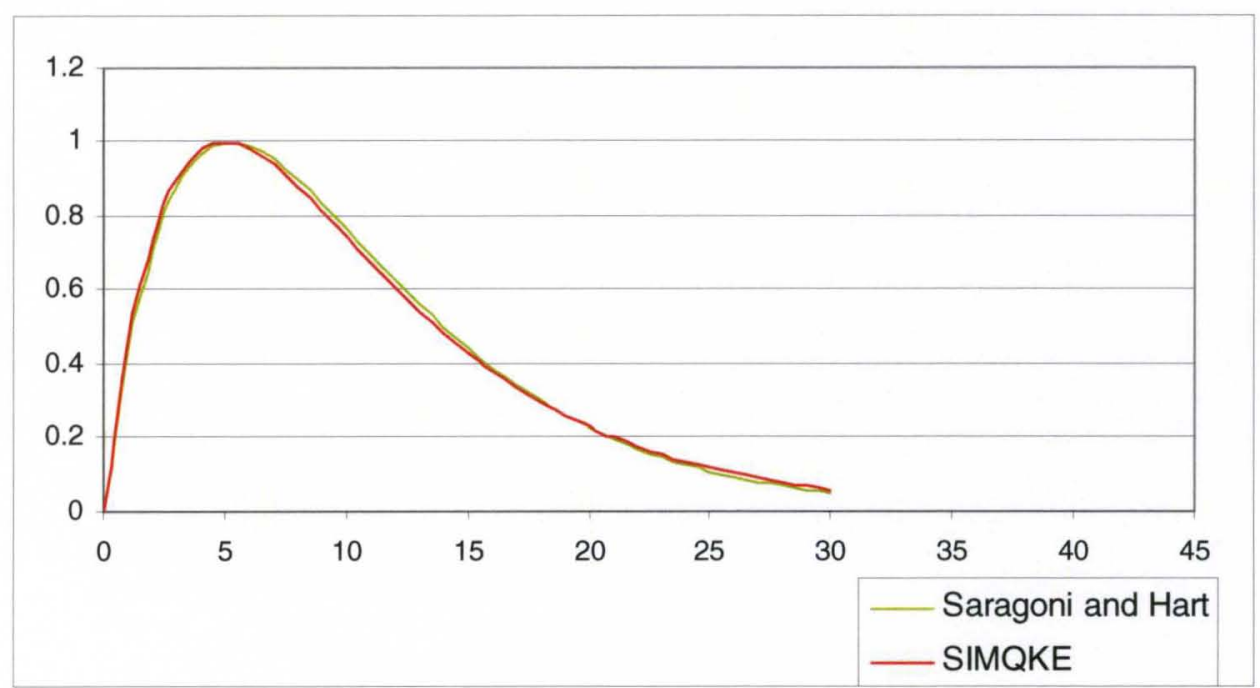

Figure 6.1 Example of Matching the SIMQKE Function to the Saragoni and Hart Function

The correlation coefficients were calculated from the regression analyses and based on the residuals for each of the parameter fits. All coefficients were extremely close to a value of one indicating the curves fit the data very well. As an example, the plot showing the curve fit to the data for the $\alpha_{0}$ parameter is shown below in Figure 6.2. 


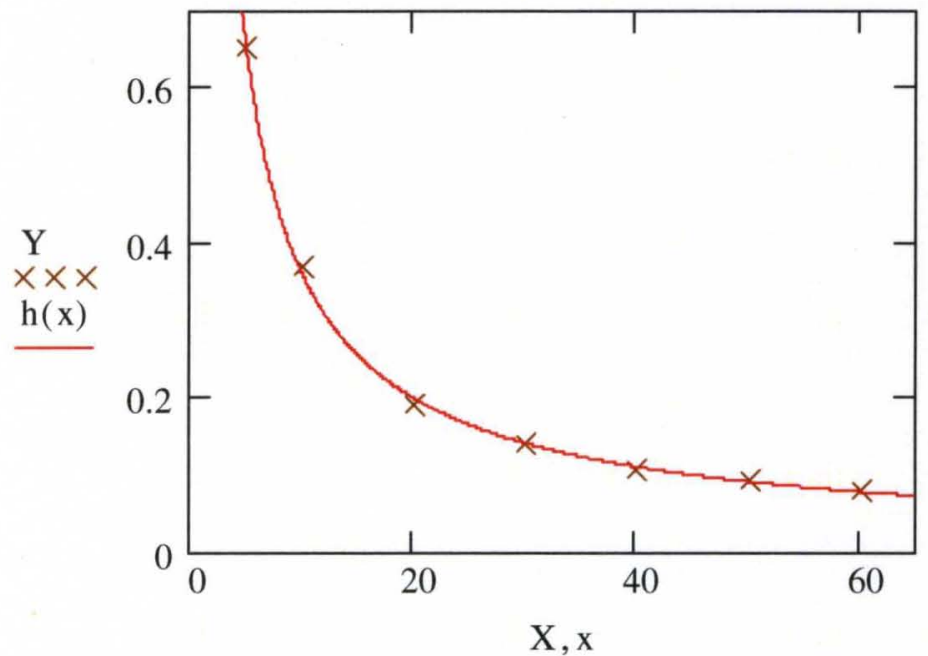

Figure 6.2 Regression Analysis Fit to Data Relating the $\alpha_{0}$ Parameter in SIMQKE to the Saragoni and Hart Model

The remaining plots for the other duration times used to develop these relationships by applying the SIMQKE envelope onto the Saragoni and Hart model are shown in Appendix I. The other two parameter curves used in the SIMQKE program from the fitted data are also provided in Appendix I.

As just mentioned and explained in the previous chapter, strong motion duration times were determined for this study using the Novikova and Trifunac model. This model provides duration as a function of magnitude, distance, and central frequency. The central frequency is analogous to the mean value for the frequency content of the record and is conveniently provided in the SIMQKE output. However, because of the procedure used by SIMQKE to generate the synthetics, the central frequency of the artificial record being generated is dependent on the intensity envelope assigned. As just explained, the intensity envelope parameters used in this study were written as 
functions of duration and the Novikova and Trifunac model provides duration as a function of the central frequency. Therefore, the following iterative procedure was used to determine the duration and intensity envelopes used in the study.

A preliminary run in SIMQKE was made with an arbitrary intensity envelope as input and the central frequency was determined. Using this central frequency with the Novakovia and Trifunac model, the duration time was calculated as a function of magnitude, distance and the central frequency just determined. This duration was then used to determine the parameters defining a new intensity envelope. A revised run in SIMQKE was then made using the newly obtained intensity envelope and a new central frequency was obtained.

This new central frequency was then compared to the one obtained in the previous iteration. If there was no difference, the last duration and intensity envelope function was used for the final generation of synthetic ground motions. If there was a difference, the process of obtaining a new duration value and intensity envelope was repeated using this new central frequency and the iterative process was continued until the central frequency converged to a stable value.

Finally, the application of the Saragoni and Hart model in combination with the duration model of Novikova and Trifunac to determine the intensity envelope used in the SIMQKE program to generate synthetic ground motions was verified in preliminary studies. These preliminary studies compared the structural responses due 
to actual records with those induced by synthetic motions generated from their response spectra and using this method to define their intensity envelopes. The results were promising and an extensive pilot study was undertaken as will be explained in detail in a later section of this chapter.

\subsubsection{Structural Parameters}

The NONLIN program as described in Chapter 4 was used to conduct the nonlinear time history analyses. In order to conduct these time history analyses, parameter values defining the SDOF systems had to be determined. As explained in Chapter 4 on structural analysis, the natural periods of the SDOF systems can be set by adjusting their weight and stiffness. The values determined for these parameters were taken from practical examples as also described in Chapter 4. The yield strengths for the SDOF systems were determined as percentages of their structural weight and were also selected from the practical examples. Descriptions of representative example structures will be provided in following paragraphs. As previously mentioned, bilinear perfectly elasto-plastic systems were assumed in this study and therefore the secondary stiffness was taken as zero for all SDOF systems.

The natural periods of the SDOFs used in this study are presented here with a brief definition and reason why they were included in this study. The specific values used to achieve these natural periods are provided in Table 6.1. Descriptions of representative structure types given for these examples are also provided in the 
following paragraphs. They serve only as single, general, and idealized representations of structures having the natural periods used in this study.

A $0.1 \mathrm{sec}$. natural period could be considered very short for most structures; however, future attenuation models may define the vertical component as a function of the $0.1 \mathrm{sec}$ response. Therefore, a SDOF system with this very short natural period was included in this study.

A natural period of $0.2 \mathrm{sec}$. is the short period response used in the equivalent lateral force procedure of most building codes (NEHRP, IBC, etc.) and acceleration values for this period are provided in seismic design maps.

A natural period of $0.6 \mathrm{sec}$. is midway between the $0.2 \mathrm{sec}$. and $1.0 \mathrm{sec}$. periods used in design procedures of most building codes and provided in seismic design maps. It has been shown by Leyendecker, et al $(1994,1995)$ and Algermissen, et al (1991) that a uniform hazard response spectrum can be approximated by two spectral values at short period and long period spectral response accelerations. In order to verify this, a natural period of 0.6 seconds which lies directly midway between the two spectral values used in seismic design procedures defined in building codes was used.

A $1.0 \mathrm{sec}$. natural period is considered a long period response and as just mentioned is used in equivalent lateral force procedures of most building codes 
(NEHRP, IBC, etc.). Again, the values of acceleration at this period are provided in seismic design maps.

Finally, a structure with a $3.0 \mathrm{sec}$. natural period is at the threshold of being a very long period structure; therefore, it was included in this study to investigate this structural response. Currently, this very long period response is beyond that used in building codes.

The following paragraphs provide values for the input parameters that were used to provide the natural periods and define the SDOF systems used in this study. Examples of the representative structures from which these values were taken are also provided. All of the values chosen to define the SDOF systems for this study are also conveniently provided in Table 6.1. Note that one kip is equal to 1000 pounds.

For the very short period structure with natural period of 0.1 seconds (e.g. reinforced concrete containment structure for a nuclear reactor) a weight of 40,000 kips was used along with an initial stiffness of 400,000 kips /in. The yield strength was estimated as half the structural weight at 20,000 kips.

For the short period structure of 0.2 seconds (e.g. three story concrete shear wall system office building.), weight was taken to be 6,000 kips and the initial stiffness was set as 15,000 kips/in. Like the 0.1 second SDOF system, the yields strength for this system was estimated as half of the structural weight giving a value of 3,000 kips. 
For the SDOF system with the intermediate period of 0.6 seconds (e.g., a reinforced concrete multi-column bent bridge pier), a weight of 7,000 kips and initial stiffness of 2,000 kips/in. were used. The yield strength was estimated at one third of the structural weight at 2,333 kips.

The weight of the SDOF system with a long period of 1.0 seconds (e.g., $30 \mathrm{ft}$. reinforced concrete mono-column bridge pier), was taken as 1,700 kips and its initial stiffness as $175 \mathrm{kips} / \mathrm{in}$. Its yield strength was estimated at one quarter of the structural weight as 425 kips.

Finally, the very long period structure with a natural period of 3.0 seconds (e.g., twenty- five story ductile special steel moment frame office building.) was modeled with a weight of 44,000 kips and initial stiffness of $500 \mathrm{kip} / \mathrm{in}$. As with the long period structure, its yield strength was estimated as one quarter of its weight giving a value of 11,000 kips.

\begin{tabular}{|c|c|c|c|c|}
\hline $\begin{array}{c}\text { Natural } \\
\text { Period } \\
\text { (seconds) }\end{array}$ & $\begin{array}{c}\text { Weight } \\
\text { (kips) }\end{array}$ & $\begin{array}{c}\text { Initial } \\
\text { Stiffness } \\
\text { (kips/in.) }\end{array}$ & $\begin{array}{c}\text { Yield } \\
\text { Strength } \\
\text { (kips) }\end{array}$ & $\begin{array}{c}\text { Strength } \\
\text { Fraction } \\
\text { of Weight }\end{array}$ \\
\hline 0.1 & 40000 & 400000 & 20000 & 0.5 \\
\hline 0.2 & 6000 & 15000 & 3000 & 0.5 \\
\hline 0.6 & 7000 & 2000 & 2333 & 0.33 \\
\hline 1.0 & 1700 & 175 & 425 & 0.25 \\
\hline 3.0 & 44000 & 500 & 11000 & 0.25 \\
\hline
\end{tabular}

Table 6.1 Structural Parameters Used to Define the SDOF Systems Employed in this Study 


\subsubsection{Magnitude Estimation Parameters}

As mentioned in the previous chapter on magnitude estimation, a procedure was proposed to verify the magnitude of the output synthetic ground motions generated and applied within this study. The NONLIN software was used to develop a SDOF system that had parameters as described for the Wood-Anderson seismograph and used in Richter's definition of local magnitude. The synthetic ground motions generated for this study were run on this SDOF system via nonlinear time history analysis to determine the maximum displacement. These maximum displacements were then used to estimate the magnitudes for the output synthetic ground motions.

Therefore, in addition to the SDOF systems representing the various structures as outlined in the previous section, the NONLIN input parameter values also were set for an SDOF system simulating a Wood-Anderson seismograph. To accomplish this, a weight of twenty five pounds was used with an initial stiffness of 4 pounds per inch to give the required natural period of 0.8 seconds. A secondary stiffness input value is required by the NONLIN program and was therefore set at zero, even though the system would always remain elastic. Finally, in accordance with the specifications for the Wood-Anderson instrument, the damping ratio was set to $80 \%$ of critical damping.

Prior to conducting the final study an extensive pilot study was conducted to verify the procedure for estimating the magnitude of the output synthetics as well as the ability of SIMQKE to generate time histories compatible with the prescribed 
response spectra. This pilot study was used to confirm that the structural responses induced from the synthetic motions generated using the SIMQKE program coincided with that of their target spectra. As mentioned earlier, this test also was conducted to verify, and to justify the use of the Saragoni and Hart model with the Novikova and Trifunac duration model combined with the exponential intensity envelope available within the SIMQKE program.

\subsection{Pilot Study}

\subsubsection{Overview}

A thorough and comprehensive pilot study was performed prior to the final study to confirm and validate the ability of the SIMQKE program to produce synthetic ground motions whose mean spectra are compatible with prescribed target spectra. This compatibility test is illustrated by the comparison shown in red in Figure 6.3. This test was done to validate the use of the SIMQKE program for generating synthetic ground motions to be used in the final study.

The pilot study also was conducted to confirm that the structural response induced by synthetic ground motions generated using the SIMQKE program and target response spectra from actual records does coincide with the structural response induced by the actual target records. This confirmation is indicated by the comparison shown in blue in Figure 6.3. 
The pilot study also sought to justify the use of the Saragoni and Hart intensity function combined with the Novikova and Trifunac duration model to determine the parameters used to define the exponential intensity envelope available in the SIMQKE program.

In addition, the pilot study was conducted to calibrate and verify that the best choice of available values had been made to define the input spectral range used to generate the synthetic ground motions. This study also served to investigate the significance of the discretization interval and to verify the use of a value approaching $0 \%$ damping in the generation of the synthetic motions.

The pilot study was used also to calibrate and authenticate the practicality of the parameter values selected to define the SDOF systems for the nonlinear time history analyses used in the study. The study also helped to substantiate the procedure proposed in this study to estimate the magnitude of the output synthetic ground motions via time history analysis.

Finally, the pilot study provided data to determine the sample size and number of replicates necessary to achieve an adequate power and detectible difference in ductility demand for statistical analysis of the results from the final study. The pilot study helped to develop and synchronize the computer automation code written to operate the software and manage the data necessary to conduct the study accurately. 
The main goals in conducting the pilot study were to show that the SIMQKE program can produce synthetic records that are compatible with target spectra and to show that the response from these synthetic ground motions coincides with the response from the actual record that produced the target response spectrum. Again, the general procedures conducted to achieve these goals are illustrated in Figure 6.3.

As illustrated in the top portion of Figure 6.3, actual corrected accelerogram recordings were obtained for five different earthquake events. These actual acceleration records were used as input loading for nonlinear time history analyses run on single degree of freedom systems with input parameter values as described earlier to give natural periods of $0.1,0.2,0.6,1.0$, and 3.0 second systems. The structural response for each of these systems was determined in the form of displacement ductility demand placed on the system. These actual records were also applied as loading to a single degree of freedom system with parameters set to simulate a Wood-Anderson seismograph as also described in the previous section. The simulated seismograph was used to estimate the magnitudes of these individual actual earthquake ground motion records. 


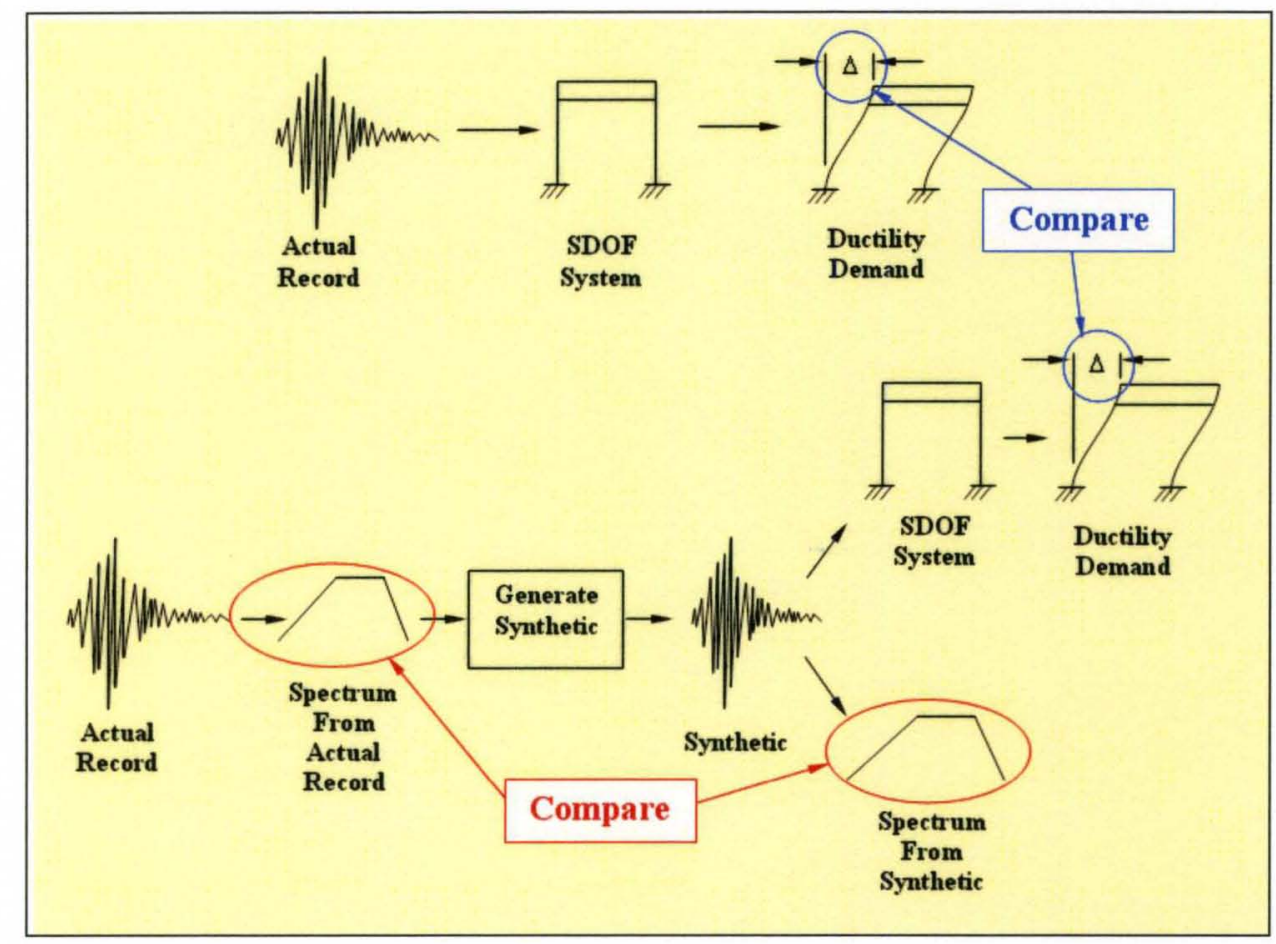

Figure 6.3 Schematic Illustrating the Validation Methodology Applied in the Pilot Study

The bottom portion of Figure 6.3 illustrates that response spectra were generated from these same actual records and used as target spectra to generate synthetic ground motions using the SIMQKE software and methodology to be used in the final study. This methodology included determining the central frequencies of the actual records and using them in the Novikova and Trifunac model to determine their duration times with the Saragoni and Hart function to determine the intensity envelopes for the synthetics using the procedure explained previously.

Twenty-five replicate synthetic ground motions from each of the actual records were generated. Statistical analyses comparing response spectra developed from each 
of the synthetic records with response spectra generated as targets from the actual records were conducted. These statistical comparisons were made at the five natural periods of the single degree of freedom systems used in the study. This comparison was done to demonstrate the ability of the SIMQKE program to generate synthetic ground motions whose response spectra are compatible with their prescribed targets. For the pilot study, the target spectra were taken from the actual records. For the final study, these target spectra were determined from CEUS attenuation models

The synthetic ground motions generated from the actual records were applied as loading in nonlinear time history analyses conducted on the same single degree of freedom systems that their actual parent recordings were applied to as described previously. The ductility demands determined for each of the SDOF systems from the time history analyses using both the actual records and the synthetics generated from them were collected. The ductility demand was captured as the primary output for the pilot study as was to be used in the final overall study. Also the maximum displacement for the Wood-Anderson response was captured for use in the magnitude estimation procedure described earlier. Statistical analyses were conducted to compare the structural response in terms of ductility demand produced by the synthetic records to the structural responses produced by their respective actual target records from which they were generated. Statistical analyses comparing the estimated magnitudes for each of the synthetic ground motions with the estimated magnitudes of their respective parent actual records were conducted. The results 
from these statistical analyses are provided in a later section of this chapter following an explanation of the procedure used to conduct this pilot study.

\subsubsection{Procedure}

The general procedure used to conduct the pilot study has been essentially presented in the previous sections; however, some specific details related to its application are presented here. The input parameter values used to generate the synthetic ground motions and conduct the nonlinear time history analyses are presented in this section. Many of these same values were also used to conduct the final study.

\subsubsection{Duration and Intensity Envelope}

It is important to emphasize again that for the pilot study, the Saragoni and Hart intensity envelope was used with the duration model of Novikova and Trifunac, rather than the actual duration times or envelopes determined by matching the actual records. This was done to test the validity of using these models to determine the duration and shape of the synthetic ground motions used in the final study. In the final study only the frequency content of the ground motions as described by the attenuation models were available to determine duration times and intensity envelopes. The pilot study tested the ability of the combined use of these models to produce structural responses comparable and within accordance to the actual records and ultimately to the prescribed spectra from the CEUS attenuation models applied in the final study. 


\subsubsection{Input Spectral Range}

The input spectral range is an input required by the SIMQKE program as explained in Chapter 3. Several preliminary studies were conducted to determine the sensitivity of synthetic motions generated to the values used to define the input spectral range.

The pilot study was used to calibrate the input spectral range to be used in the final study. An input spectral range of 0.2 to 5.0 seconds provided the best fit of mean synthetic spectra to their target spectra of all of the actual records investigated in the pilot study. However, when using the 0.02 to 5.0 seconds input spectral range the synthetics of the actual records matched better with their target spectra but their magnitudes were estimated to be too high. It was then determined that an input spectral range of 0.01 to 4.0 seconds provides synthetic motions whose magnitudes match much better with the calculated magnitudes of the actual records as well as providing the best fit with their target spectra. Therefore, it was decided to use an input spectral range of 0.01 to 4.0 seconds in both the pilot and final studies. These values also only limited the range made available by one of the attenuation models used in the final study.

\subsubsection{Discretization Interval}

To generate synthetic ground motions for the pilot study the discretization interval used was the same as the discretization interval in which each of the original (actual) records was provided. The discretization interval also plays a role in determining the 
frequency content of the motions being generated. This subject will be revisited in an analysis of the results from the final study.

\subsubsection{Seed Numbers}

As described in the chapter on the generation of synthetic motions, the SIMQKE program requires that the input seed number be odd. It was also explained that a seed number used within the SIMQKE program will produce the same "pattern" for a record regardless of its frequency content. For example, synthetic motions generated using the same seed number but different input target spectra will have very similar "patterns" but will still have frequency contents compatible with their target spectra. For both the pilot study and the final study, twenty-five replicates were generated for each scenario from each attenuation model. In the interest of making fair comparisons between synthetic ground motions compatible with the eight attenuation models used in the final study and the structural responses they induced, the same 25 seed numbers were used for all events and attenuation models. The odd numbers 1 through 49 were used both in the pilot study and in the final study to generate the synthetic ground motions.

\subsubsection{Damping}

The input target response spectra from the actual records used in the pilot study were developed for $0 \%$ damping. As mentioned earlier, the intent was to represent the frequency content of the ground motions as they arrive at the structure. Also as previously explained, the SIMQKE program requires a damping value to be input. 
Again, a value relatively close to zero was applied. The time history analysis also requires a damping value to be input and a value of 5\% damping was applied within the NONLIN program. This $5 \%$ damping was taken to represent the damping contribution to the response due to the material characteristics within the structure. Typically, 5\% of critical damping is used in practice for most common structures.

\subsubsection{SDOF System Parameters}

The primary goal of the pilot study was to verify the procedure used in the final study. Actual earthquake records were used to develop the target spectra used in the pilot study. The specific parameters applied to define the SDOF systems used for both the pilot and final studies were provided in a previous section. As explained earlier in this chapter, SDOFs with natural periods of $0.1,0.2,0.6,1.0$, and 3.0 seconds were employed in the pilot study as well as in the final study. Likewise, the parameters used to simulate the Wood-Anderson seismograph for use in the magnitude estimation procedure as described earlier were also used in both the pilot and final studies.

\subsubsection{Input Spectra and Record Selection}

Individual records for five different events were selected for the pilot study. The main criterion in selecting these earthquakes was to represent strong ground motions in both the eastern and western geologic regimes of the United States. Three records were selected from the WUS and two were selected from the CEUS. 
The WUS records are from events that occurred in California. One of the CEUS records is from an event that occurred in Northeast Ohio and the other is from an event in Southeastern Canada. The eastern events were chosen because the main study of this dissertation concentrates on ground motions for the Central Eastern United States. Given the scarcity of strong ground motion recordings for the CEUS, the two events chosen, although not of great magnitude, were among the highest magnitude events available in the region and were deemed to represent strong ground motions satisfactorily for the area. In addition to examining a variation in magnitude, it was also the intent in choosing the various records to obtain those recorded at both near and far epicentral distances.

One of the WUS records used is from the January 17, 1994 magnitude 6.7 Northridge event and was acquired from the Nation Information Service for Earthquake Engineering at the University of California at Berkeley. The recording station was at an epicentral distance of $21.33 \mathrm{~km}$. The other two WUS records are from the May 18, 1940 magnitude 6.7 Imperial Valley (El Centro) and October 17, 1989 magnitude 7.0 Santa Cruz Mountains (Loma Prieta) earthquakes. These records were obtained from the Consortium of Organizations for Strong-Motion Observation Systems data center. The recordings used in this study were taken at epicentral distances of $12.2 \mathrm{~km}$ and $81.37 \mathrm{~km}$ respectively.

The two CEUS records are from the January 31, 1986 magnitude 4.96 Northeastern Ohio and November 25, 1988 magnitude 5.9 Saguenay events. These 
recordings were collected from the Strongmo Database System offered through the National Center for Earthquake Engineering Research. The two recordings used in this study were taken at $17.7 \mathrm{~km}$ and $64.1 \mathrm{~km}$ respectively. All of the recordings used were corrected and either taken on bedrock or on the ground floor if the instrument was located within a structure.

Table 6.2 provides the events from which these records were selected as well as their magnitudes and distances. The table gives the reported local Richter magnitude $M_{L}$, the calculated $M_{L}$, and the calculated moment magnitude $M_{W}$. The reported $\mathrm{M}_{\mathrm{L}}$ is the official magnitude assigned by the appropriate authorities for the event and was determined from all records available from all recording stations. The calculated $M_{L}$ was determined for each particular record chosen to be used in this study following the magnitude estimation procedure via time history analysis as described in the preceding chapter. The calculated moment magnitude $M_{W}$ is also for each particular record used in this study and was determined using the conversion relationship developed for this study as also described in the previous chapter. The discrepancies between the reported $M_{L}$ values and those calculated for each record are feasible considering that again, the calculated values are for individual records where the reported values are from a compilation of several records for each event. 


\begin{tabular}{|c|c|c|c|c|}
\hline Record & $\begin{array}{c}\text { Reported } \\
\mathbf{M}_{\mathbf{L}}\end{array}$ & $\begin{array}{c}\text { Calculated } \\
\mathbf{M}_{\mathbf{L}}\end{array}$ & $\begin{array}{c}\text { Calculated } \\
\mathbf{M}_{\mathbf{W}}\end{array}$ & $\begin{array}{c}\text { Epicentral } \\
\text { Distance } \\
(\mathbf{k m})\end{array}$ \\
\hline Loma Prieta & 7.00 & 7.37 & 7.80 & 81.37 \\
\hline Northridge & 6.70 & 6.86 & 7.12 & 21.33 \\
\hline El Centro & 6.70 & 6.23 & 6.28 & 12.20 \\
\hline NE Ohio & 4.96 & 4.85 & 4.85 & 17.70 \\
\hline Saguenay & $5.90^{*}$ & 5.98 & 5.98 & 64.09 \\
\hline
\end{tabular}

* This magnitude was reported in $\mathrm{M}_{\mathrm{w}}$.

Table 6.2 Parameters of the Actual Record Used in the Pilot Study

In order to generate synthetic records for the pilot study that were representative and compatible with their parent actual records, the calculated $M_{W}$ and epicentral distance for each actual recording were used as input to determine duration and intensity envelope parameters. The central frequencies, duration times, and envelope parameter values determined for these actual records and used in this study are provided in Table 6.3.

\begin{tabular}{|c|c|c|r|r|r|}
\hline \multicolumn{7}{|c|}{ Intensity Envelope Parameters } \\
\hline \multirow{2}{*}{ Record } & $\begin{array}{c}\text { Central } \\
\text { Frequency (Hz) }\end{array}$ & $\begin{array}{c}\mathbf{t}_{\text {dur }} \\
(\mathbf{s e c})\end{array}$ & $\mathbf{A}_{\mathbf{0}}$ & $\boldsymbol{\alpha}_{\mathbf{0}}$ & $\boldsymbol{\beta}_{\mathbf{0}}$ \\
\hline $\begin{array}{c}\text { Loma } \\
\text { Prieta }\end{array}$ & 10.24 & 22.0 & 2.99 & 0.2248 & 0.5369 \\
\hline Northridge & 3.02 & 14.0 & 2.52 & 0.3361 & 1.0260 \\
\hline El Centro & 5.32 & 14.0 & 2.28 & 0.4712 & 1.7679 \\
\hline NE Ohio & 37.63 & 14.0 & 1.98 & 1.2244 & 8.2280 \\
\hline Saguenay & 26.01 & 14.0 & 2.29 & 0.4591 & 1.6954 \\
\hline
\end{tabular}

Table 6.3 Intensity Envelope Parameters Used in the Pilot Study to Generate Synthetic Ground Motions from the Actual Records 
Also, the peak ground acceleration (PGA) value contained within each of the actual records was provided as input for the maximum ground acceleration value required by the SIMQKE program. Finally, in an attempt to keep the synthetic motions generated as close to their parent actual records as possible, the discretization intervals in which the actual records were provided were used as input to generate the synthetic motions. These interval values are provided in Table 6.4

\begin{tabular}{|c|c|c|}
\hline \multirow{2}{*}{ Record } & Target PGA from Actual Record & Dicretization Int. \\
\hline Loma Prieta & $0.137 \mathrm{~g}$ & $0.02 \mathrm{sec}$. \\
\hline Northridge & $0.419 \mathrm{~g}$ & $0.02 \mathrm{sec}$. \\
\hline El Centro & $0.348 \mathrm{~g}$ & $0.02 \mathrm{sec}$. \\
\hline NE Ohio & $0.180 \mathrm{~g}$ & $0.005 \mathrm{sec}$. \\
\hline Saguenay & $0.156 \mathrm{~g}$ & $0.01 \mathrm{sec}$. \\
\hline
\end{tabular}

Table 6.4 Peak Ground Accelerations and Discretization Intervals Used in the Pilot Study to Generate Synthetic Ground Motions from the Actual Records

\subsubsection{Results}

The results obtained from the pilot study conducted using the procedures described in the previous sections are provided in the following sections. An explanation of the logic applied to these results to determine the sample size for the final study is provided. The outcomes of the comparative statistical analyses conducted on the results of the pilot study also are presented. 


\subsubsection{Final Study Sample Size}

In the pilot study 25 synthetic records were generated from the response spectra of each actual record. These 25 synthetic motions then were run on five SDOF systems with the natural periods and structural parameters as described in the previous section as well as on one SDOF system with parameters set to simulate a Wood-Anderson seismograph used to estimate the magnitudes. The largest standard deviation for ductility demand determined in the pilot study due to loading from the synthetic records was 2.06 for the $1.0 \mathrm{sec}$. SDOF systems. The majority of this variance came from synthetic motions of the Northridge record.

Comparing the ductility demands from the records for the actual various events in the pilot study translates to comparing the event scenarios selected for the final study. The final study includes five magnitudes and five distances for a total of twenty-five scenarios (i.e., magnitude and distance combinations). Using the largest overall standard deviation for ductility demand just mentioned (for the $1.0 \mathrm{sec}$. SDOF system responses) translates to comparing the ductility demands over all five of the structural SDOF systems to be used in the final study. For the twenty-five scenarios, this gives a total of $125 \mathrm{SDOF}$ system responses. If in the final study twenty-five replicates are used as was done for the pilot study, this would have given a total of 3,125 observations per attenuation model.

Power and sample size calculations for $\mathrm{t}$-test confidence intervals using the standard deviation mentioned above with a sample size of 3,125 observations per 
each attenuation model indicates that the maximum detectible difference in ductility demand due to variations in synthetic motions generated from the attenuation models will be slightly more than 0.13 at a power of 0.95 in the final study. This value is a reasonably small detectible difference in the ductility demand and as a result, a sample size of twenty-five synthetics per attenuation model, magnitude, and distance combination was generated in the final study. Table 6.5 shows the maximum detectible differences in ductility demand at various powers calculated using the Minitab statistical software, and displayed in the output format for Minitab.

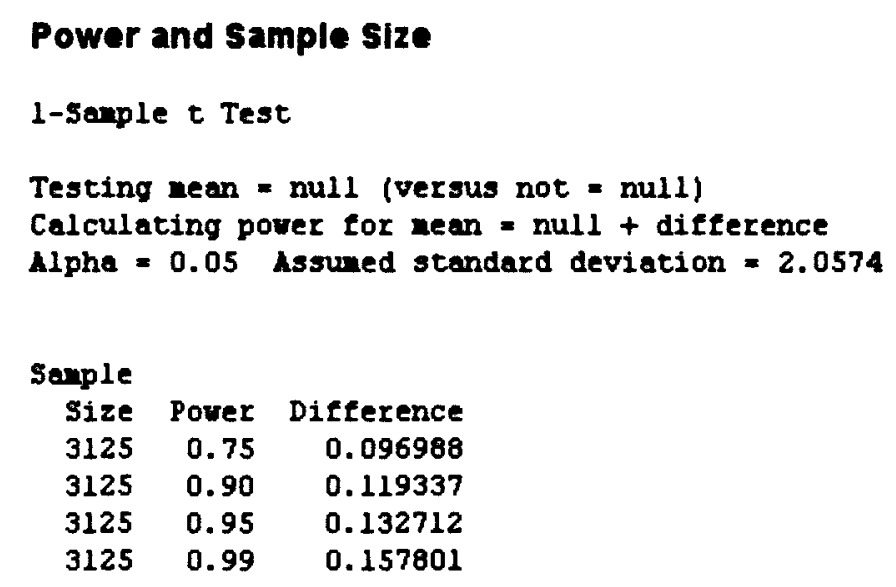

Table 6.5 Sample Size Output Based on Ductility Demand from Minitab Analysis

The standard deviation of the estimated moment magnitudes (i.e., using the simulated Wood-Anderson responses) of the synthetic records was also considered in determining the sample size for the final study. The maximum standard deviation for estimated moment magnitude determined in the pilot study was 0.11 for the synthetics from the Saguenay event. This value is much smaller than the maximum 
standard deviation mentioned earlier for the SDOF system responses. Following the logic explained for the SDOF system responses, the comparison of the magnitude for the various events in the pilot study translates to comparing the magnitudes over all twenty-five scenarios in the final study (i.e., all magnitude and distance combinations). The analyses producing the Wood-Anderson responses used in the magnitude estimation in the final study were done with the other SDOF system analyses and therefore there were twenty-five replicates of them also. This procedure yields a total of 625 observations of estimated moment magnitude per each attenuation model in the final study. Sample size calculations using the largest standard deviation from the Saguenay event and a sample size of 625 indicate that the maximum detectible difference in moment magnitude among the synthetics generated for the various scenarios from the various attenuation models will be as small as about 0.02 at a power of 0.95 . The results of these sample size calculations are shown in Table 6.6.

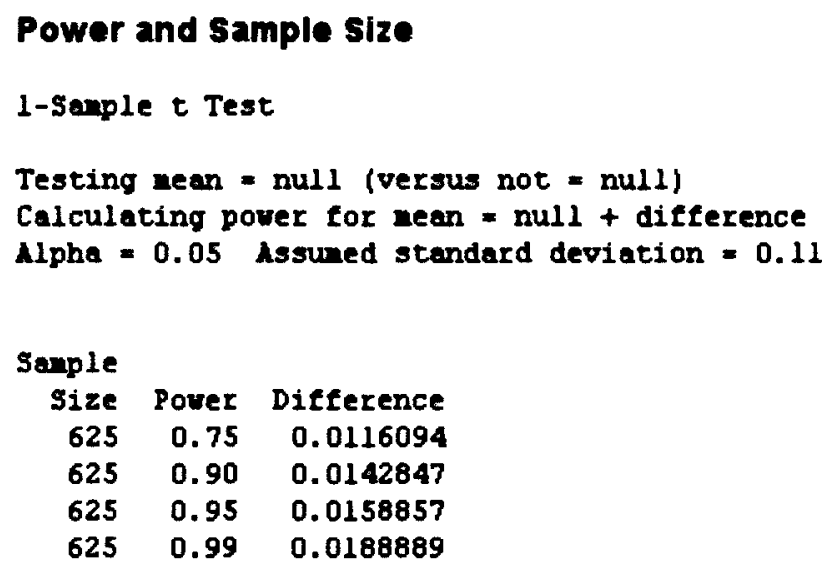

Table 6.6 Sample Size Output Based on Estimated Magnitude from Minitab Analysis 
Such a small detectible difference at this power is well within the expectations for the final study and therefore the sample size for the final study was controlled by the standard deviation of the SDOF system response and as indicated previously, 25 replicates of synthetics per attenuation model, magnitude, distance were generated for use in the final study.

\subsubsection{Statistical Analyses of the Spectra Comparisons}

As stated earlier, the first goal in conducting the pilot study was to verify the ability of the SIMQKE program to generate synthetic records that are compatible with their target spectra. In this effort, statistical analyses comparing the mean spectral accelerations for the synthetic records at the $0.1,0.2,0.6,1.0$, and 3.0 second natural periods to their respective target values from response spectra for the actual records used to generate the synthetics was conducted. Dunnett's method for one-way analysis of variance for multiple comparisons was used to generate $95 \%$ confidence intervals for the differences between the means and their respective target values. These analyses comparing the spectral accelerations at the points stated above for all five events applied to all five single degree of freedom systems indicates that there is no significant difference between any of the mean response spectra of the synthetics and those of their parent actual records at the points evaluated. Plots showing the spectra comparisons for the Loma Prieta and Northeastern Ohio records to the mean of their synthetics are provided below in Figures 6.4 and 6.5. Plots showing the spectral comparisons for the other three records used in the pilot study are provided in Appendix G. 


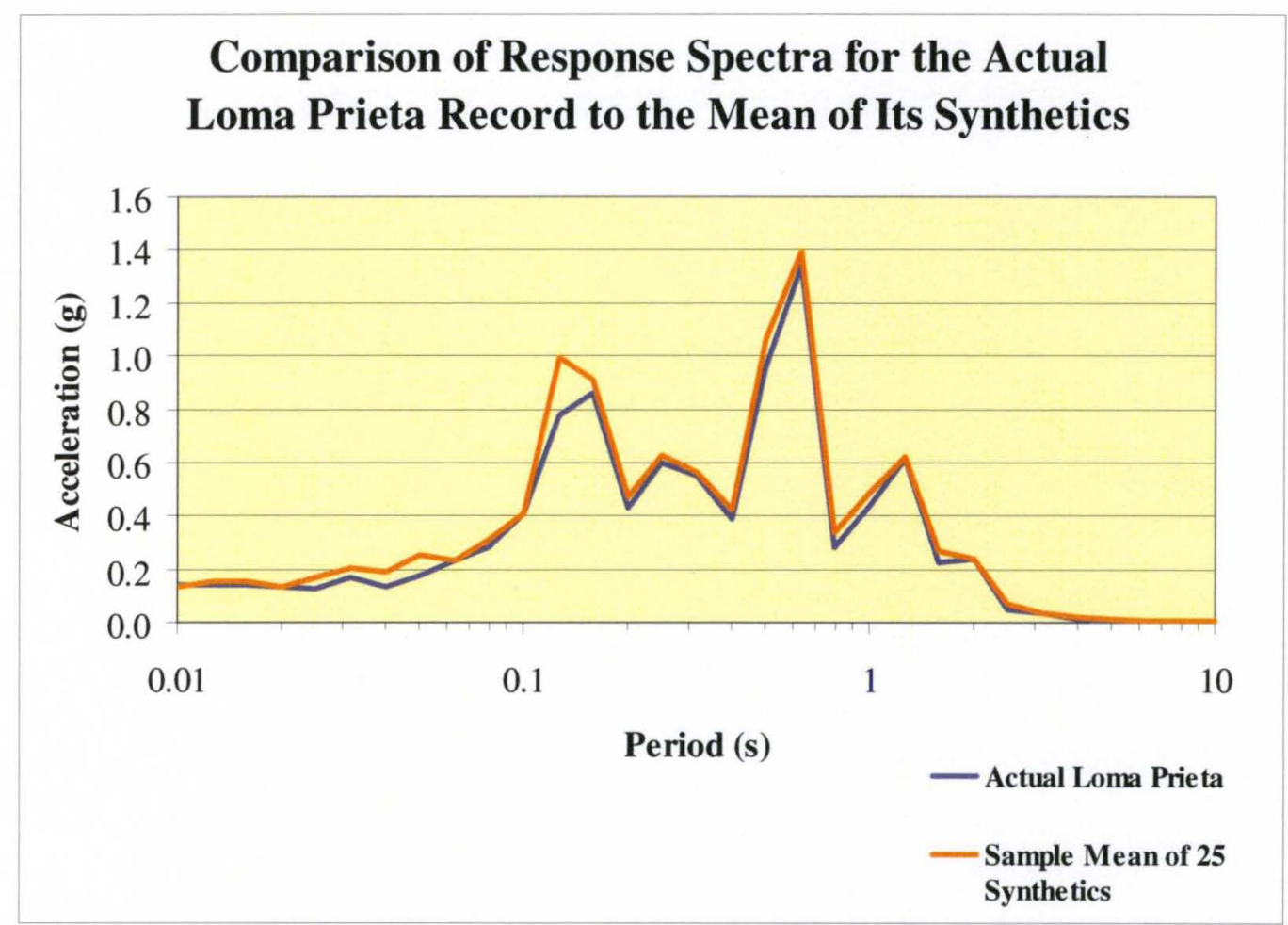

Figure 6.4 Comparisons of Response Spectra from the Loma Prieta Record Obtained in the Pilot Study

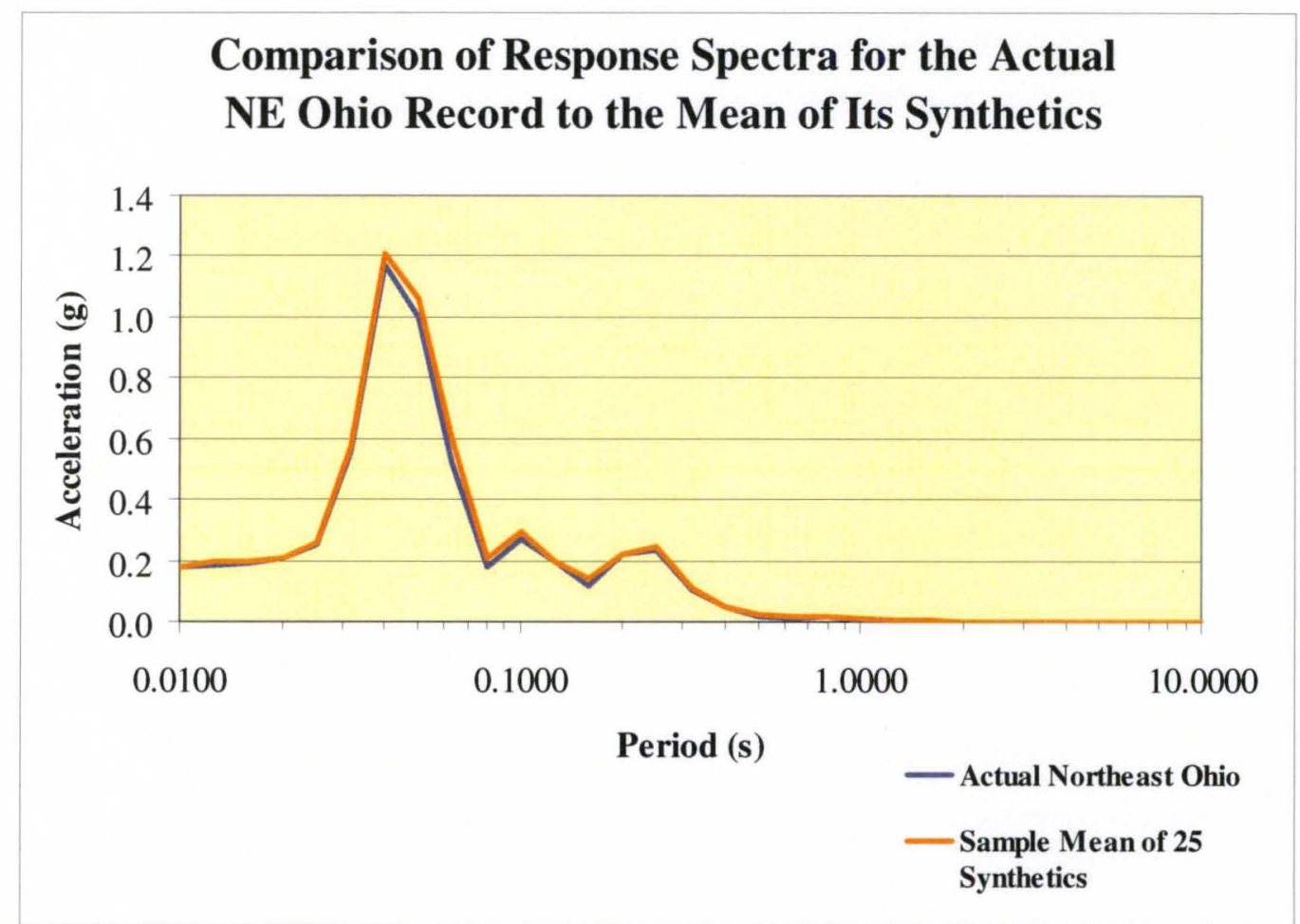

Figure 6.5 Comparisons of Response Spectra from the NE Ohio Record Obtained in the Pilot Study 
It is also interesting that Figures 6.4 and 6.5 show the obvious shift in location along the abscissa for the response spectra of the two events. It is evident that the California earthquake has a frequency content shifted towards the longer period range compared to the Northeastern Ohio event which has a response spectra shifted towards the shorter period/higher frequency range. This distinction in frequency content represents the differences noted in earlier chapters concerning the differences in characteristics of earthquakes that occur in the Western United States versus those that occur in the Central Eastern United States.

\subsubsection{Statistical Analyses of the Response Comparisons}

The second goal of the pilot study was to verify that the structural response to synthetic records generated using the procedure utilized in the final study coincides with the structural response induced by the parent record used to produce the target response spectrum. In this effort, statistical analyses comparing the mean displacement ductility demands imposed by the synthetic motion developed from the actual records on each of the $0.1,0.2,0.6,1.0$, and 3.0 second natural period single degree of freedom systems to those imposed by their actual records were conducted.

As was done for the spectra comparisons, Dunnett's method for one-way multiple comparisons was used to generate $95 \%$ confidence intervals for the differences between the treatment means of the ductility demands of the synthetics with those of the actual records. These statistical comparisons indicate that there is statistically no significant difference between the mean ductility demands for each of 
the SDOF systems and the ductility demands obtained from analysis of the respective actual records.

Plots showing the ductility demands for the five SDOF systems from the actual Loma Prieta and Northeast Ohio records along with the demands from their respective synthetic motions are shown in Figures 6.6 and 6.7. The plots also include the individual ductility demands for each synthetic motion, indicating the variation in their displacement ductility demand. The plots showing comparisons in displacement ductility demand for the other three records used in the pilot study are provided in Appendix G.

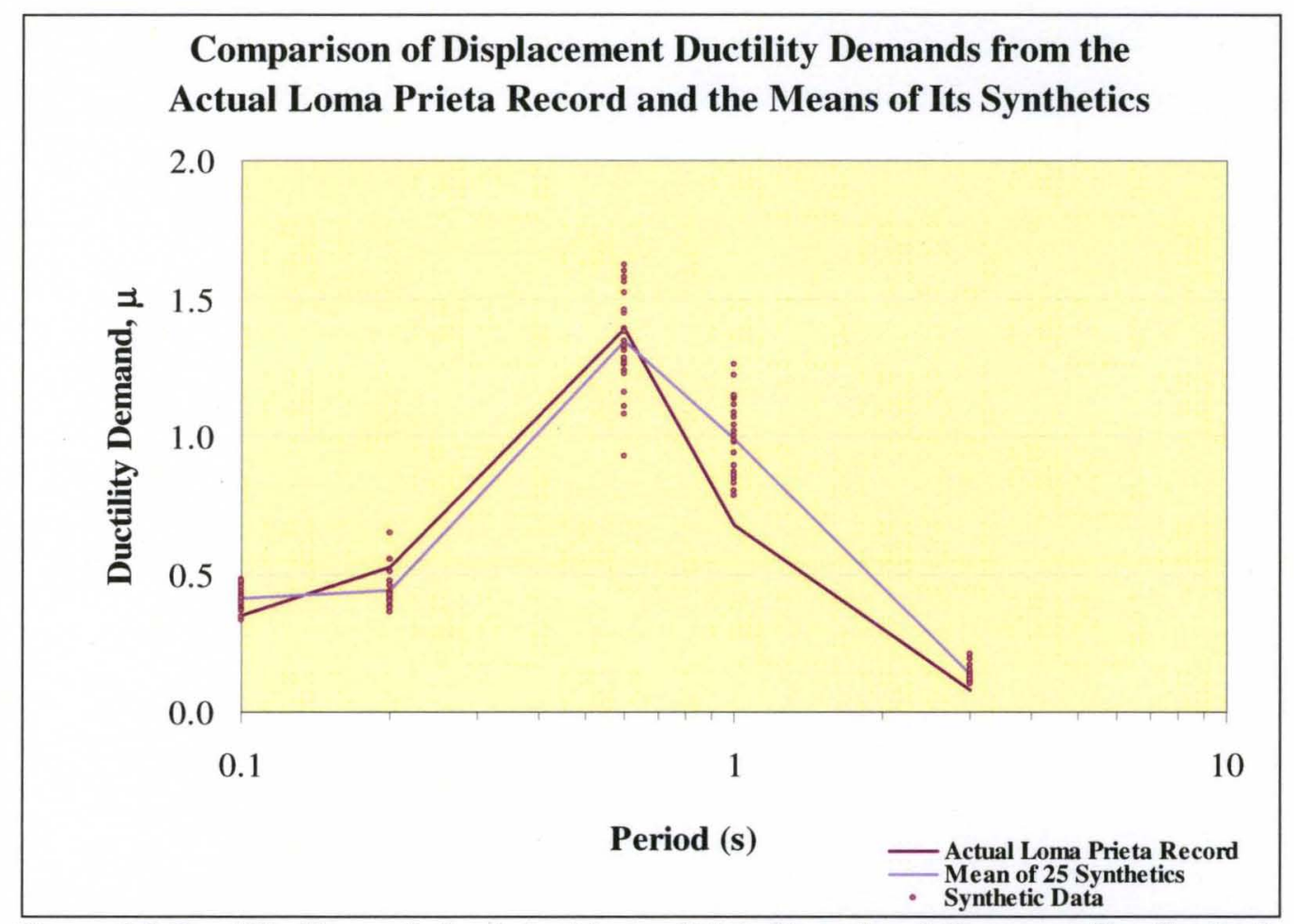

Figure 6.6 Ductility Demands for Actual Loma Prieta Record and Corresponding Synthetic Motions 


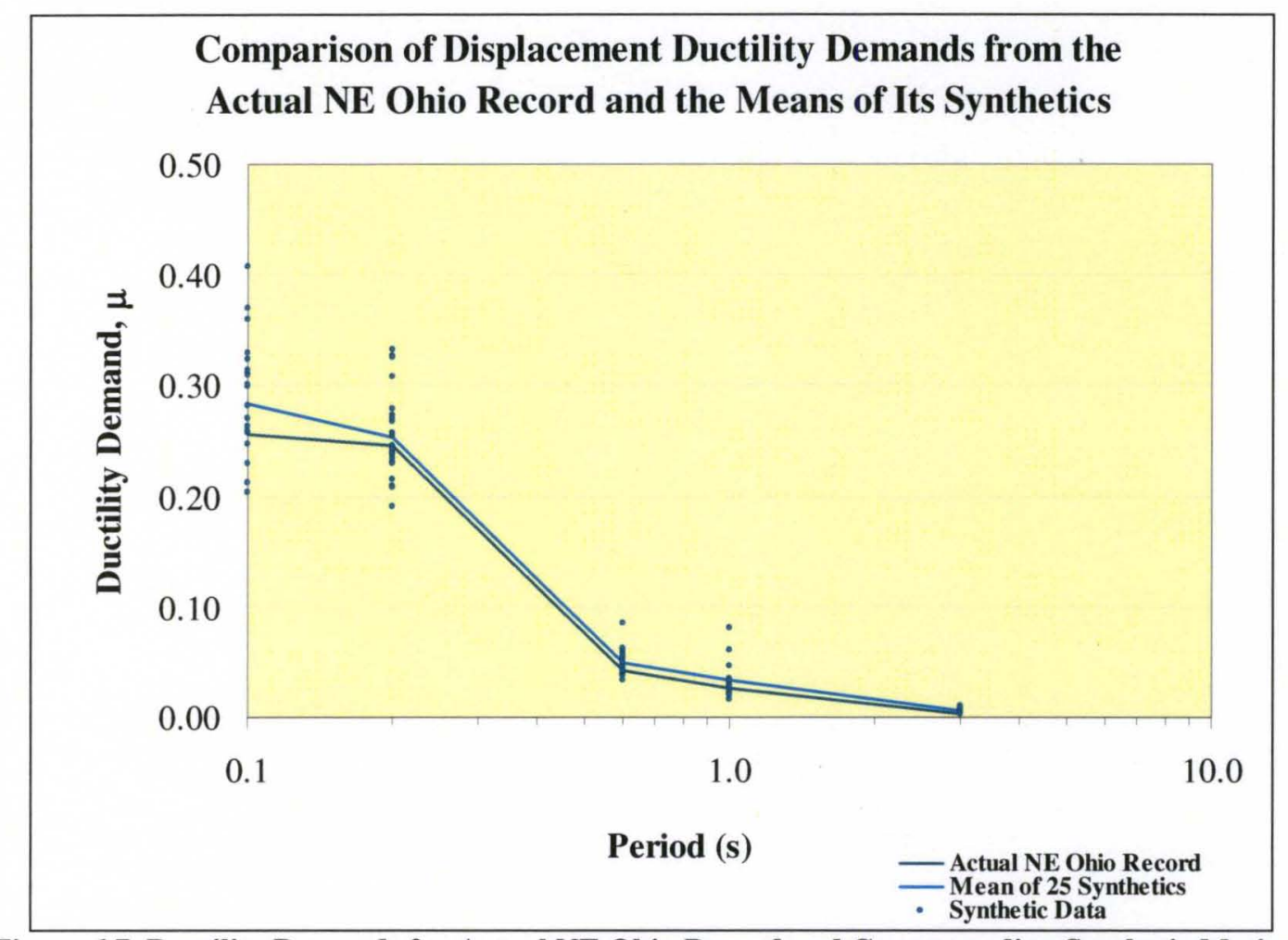

Figure 6.7 Ductility Demands for Actual NE Ohio Record and Corresponding Synthetic Motions

\subsubsection{Statistical Analyses of the Estimated Magnitude Comparisons}

Finally, statistical analyses comparing the mean estimated moment magnitudes of the synthetic records as determined using the procedure developed for this study to those determined for the actual parent records from which they were generated were conducted also. As in the previous comparisons, Dunnett's method for one-way multiple comparisons was used to generate $95 \%$ confidence intervals for the differences between the treatment means of the estimated synthetic moment magnitudes to those estimated for the actual records. 
Again, all statistical comparisons conducted indicate that there is no significant difference between the estimated moment magnitudes of the synthetic records and those of their respective parent actual records. A plot showing the dispersion of the estimated moment magnitudes for the synthetic ground motions along with their respective targets is provided in Figure 6.8

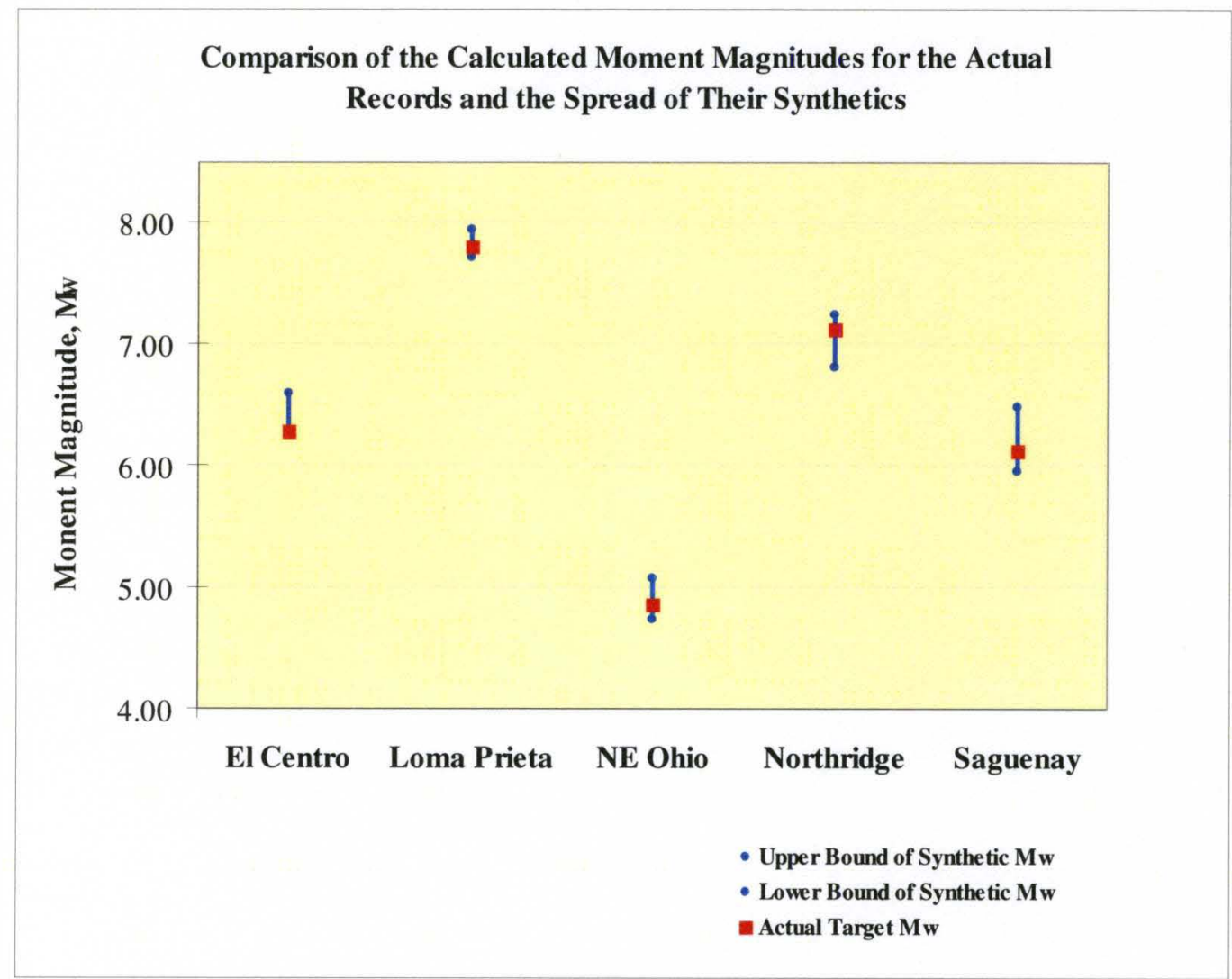

Figure 6.8 Calculated Moment Magnitudes from Actual Records and their Corresponding Synthetic Motions 


\subsubsection{Conclusions Regarding the Pilot Study}

All of the goals for conducting the pilot study were successfully achieved. Furthermore, the ability of the SIMQKE program to generate synthetic records compatible with their target spectra was verified. Likewise, it was verified that these synthetic records do in fact induce structural responses that coincide with those induced by parent records. The ability to estimate the magnitude of synthetic records using the procedure developed for this study was also verified. The sample size and number of replicates required in the final study to achieve the desired level of accuracy was also determined. And finally, the procedure used in this study to determine the duration and intensity envelope was verified. It is worth noting that there was excellent agreement in both the structural response and estimated magnitude from the actual records and the synthetic records generated using the shapes and durations as determined using the procedure developed for this study.

\subsection{Final Study}

\subsubsection{Methodology}

The main difference in the methodology used to conduct the final study from that used in the pilot study is in the development of the target response spectra used to generate the synthetic records. In the pilot study, these spectra were obtained from actual records. In the final study, the target response spectra were obtained from the 
eight CEUS attenuation models employed by the USGS to develop the 2008 National Seismic Hazard Maps.

For completeness, the general methodology used to conduct the final study is presented in the following basic steps. Response spectra were developed from the eight different attenuation models for various event scenarios at the specified magnitudes and distances. These response spectra were then used as input target spectra in the SIMQKE program along with the Novikova and Trifunac model to define the duration and the Saragoni and Hart model to define the intensity envelope to develop synthetic ground motions with frequency contents compatible with the input spectra. These synthetic ground motions developed from the attenuation models were then applied as loadings in the NONLIN software to conduct nonlinear time history analyses on single degree of freedom systems with specified natural periods as described in the first section of this chapter. The structural responses to these synthetic ground motions in the form of displacement ductility demands placed on these single degree of freedom systems were captured. In addition, the maximum displacements from the time history analyses on the Wood-Anderson SDOF system were used to estimate the magnitude of the synthetics applied as loading.

Finally, statistical analyses were conducted to determine the confidence intervals of ductility demand from the synthetic records derived for each model and each scenario loaded on each SDOF. Also, statistical analyses were conducted to determine confidence intervals for the estimated magnitude of the synthetic records 
from each model at each distance for each target magnitude. A schematic illustrating the methodology for the final study is provided in Figure 6.9. 


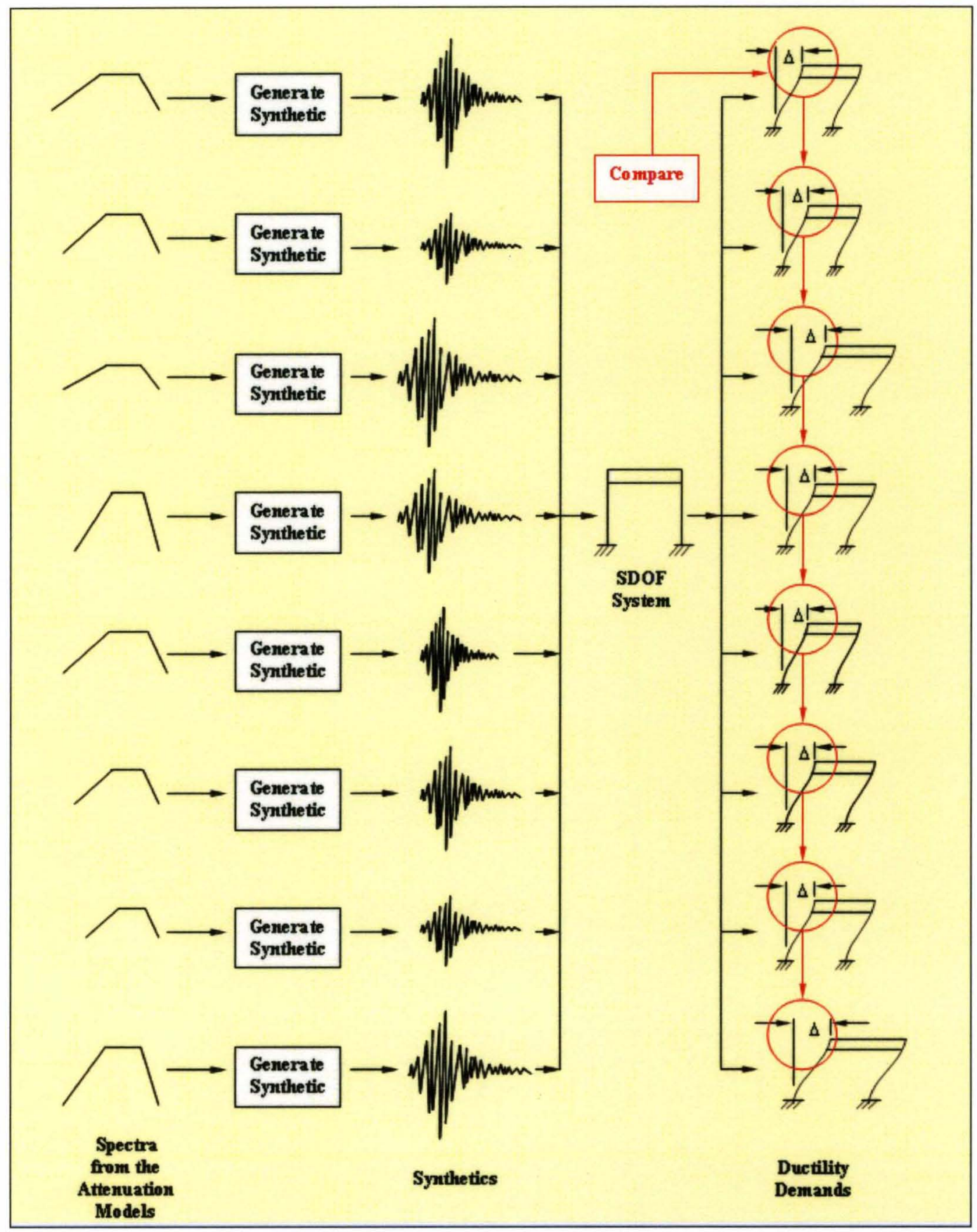

Figure 6.9 Schematic Illustrating the Methodology Employed in the Final Study

As noted earlier, many of the same input parameter values were used in the pilot study and in the final study. These values include the same seed numbers as well as 
values for the input spectral range and damping used to generate the synthetic ground motions. Likewise, the same structural parameter values used to describe the SDOF systems were used in both the pilot and final studies. The methodology used to conduct the final study along with the specifications associated particularly with the final study in regards to generating the synthetic ground motions and conducting the nonlinear time history analyses are presented here.

\subsubsection{Attenuation models}

Again, the eight CEUS attenuation models used by the USGS to develop the 2008 National Seismic Hazard Maps were used in this study. Moment magnitudes of 5.5, $6.0,6.5,7.0$ and 7.5 were used as input. Because of the manner of development of some of the models, model results were indicated to be valid only for moment magnitudes up to 7.5 and therefore this was the maximum moment magnitude used in the study. Likewise, these five magnitudes were combined with distances of 10,25 , 50,100 , and $200 \mathrm{~km}$ to develop the response spectra representing the twenty-five scenarios used as targets to generate the synthetic ground motions for this study. Near-field effects should be considered at distances closer than $10 \mathrm{~km}$ and while many of the models have been developed with this capability, for brevity this study took $10 \mathrm{~km}$ to be the shortest distance from the source considered.

For this study, the attenuation models were applied as they come "off the shelf". In other words, none of the seismological parameters associated with the development of the individual attenuation relationships (as described in Chapter 2) were altered or 
varied for this study. The only possible exception would be of the Atkinson and Boore model whose stress factor was adjusted to give stress drops of 140 and 200 bars to coincide with what was done in the development of the 2008 National Seismic Hazard Maps. The intent of this study was to compare synthetics compatible with these attenuation relationships as presented in their final form and as used to develop the National Seismic Hazard Maps. With this situation in mind, only the input values for magnitude and distance were varied for this study.

In addition, all target spectra were developed from the attenuation models by applying hard rock conditions. All of the models are provided with options for this site condition except for the Frankel model to which the suggested corrections were applied.

\subsubsection{Fault Model}

The point at where an earthquake rupture begins along a fault is termed its focus or hypocenter (Kramer, 1996). Although the rupture can reach the ground, the focus is located at some depth below the surface. As shown in Figure 6.10, the point directly above the focus is known as the epicenter. The distance measured from the epicenter to the site under consideration is called the epicentral distance and likewise, the distance from the hypocenter to the site is called the hypocentral distance. 


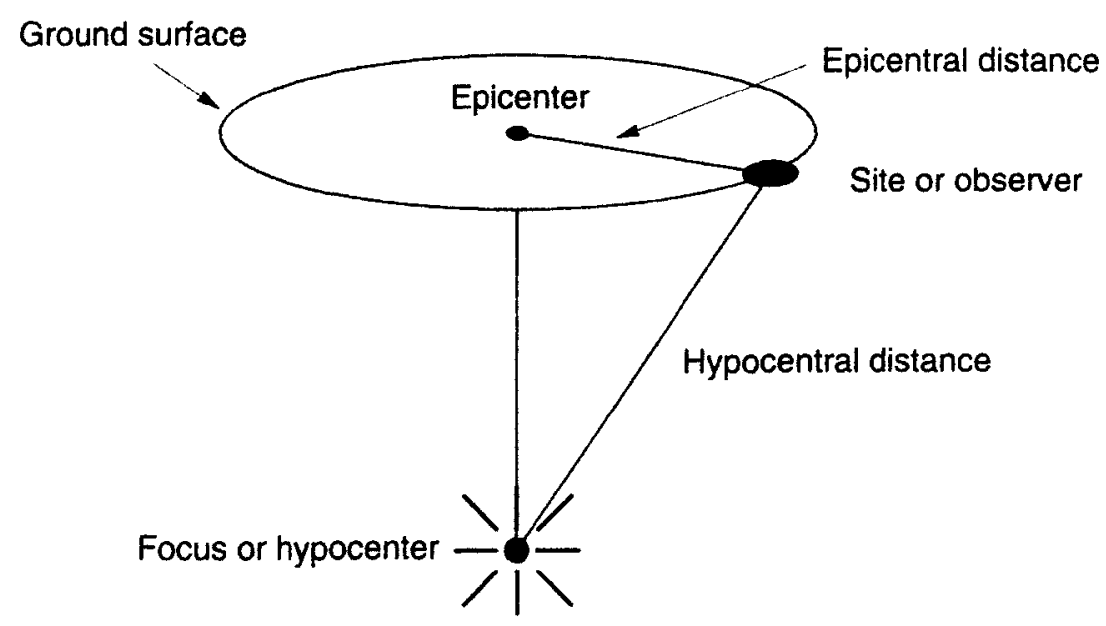

Figure 6.10 Definition Terms for Fault to Site Distances (Kramer, 1996)

As mentioned in Chapter 2, unfortunately there is no consistent distance measure used among seismologists when developing attenuation models. This held true for the CEUS attenuation models used to develop the 2008 National Seismic Hazard Maps; those models were applied in this study. This lack of consistent definition of distance proved to be problematic and required consideration in making comparisons of structural response from synthetic motions generated from model output.

There are other distance measures besides the ones used by seismologists to develop attenuation models. The most common distance measure applied in the CEUS attenuation models used in this study is the rupture distance $r_{\text {rup }}$ which is defined as the closest distance to the rupture surface and is sometimes referred to as the slant distance. For a vertical fault this would be taken as the distance from the top of the rupture to the site of interest. The Joyner-Boore distance $r_{j b}$ is another measure 
used in these models and is defined as the closest horizontal distance to the vertical projection of the rupture.

In order to make the comparisons for this study, a model fault was assumed with orientation and depth idealized to rationalize the different distance measures required by the models. A vertical strike-slip fault with a depth to top of rupture of $6 \mathrm{~km}$ and focal depth of $12 \mathrm{~km}$ was assumed for this study. This fault model, as well as the three distance measures used by the attenuation models applied in this study, is illustrated in Figure 6.11.

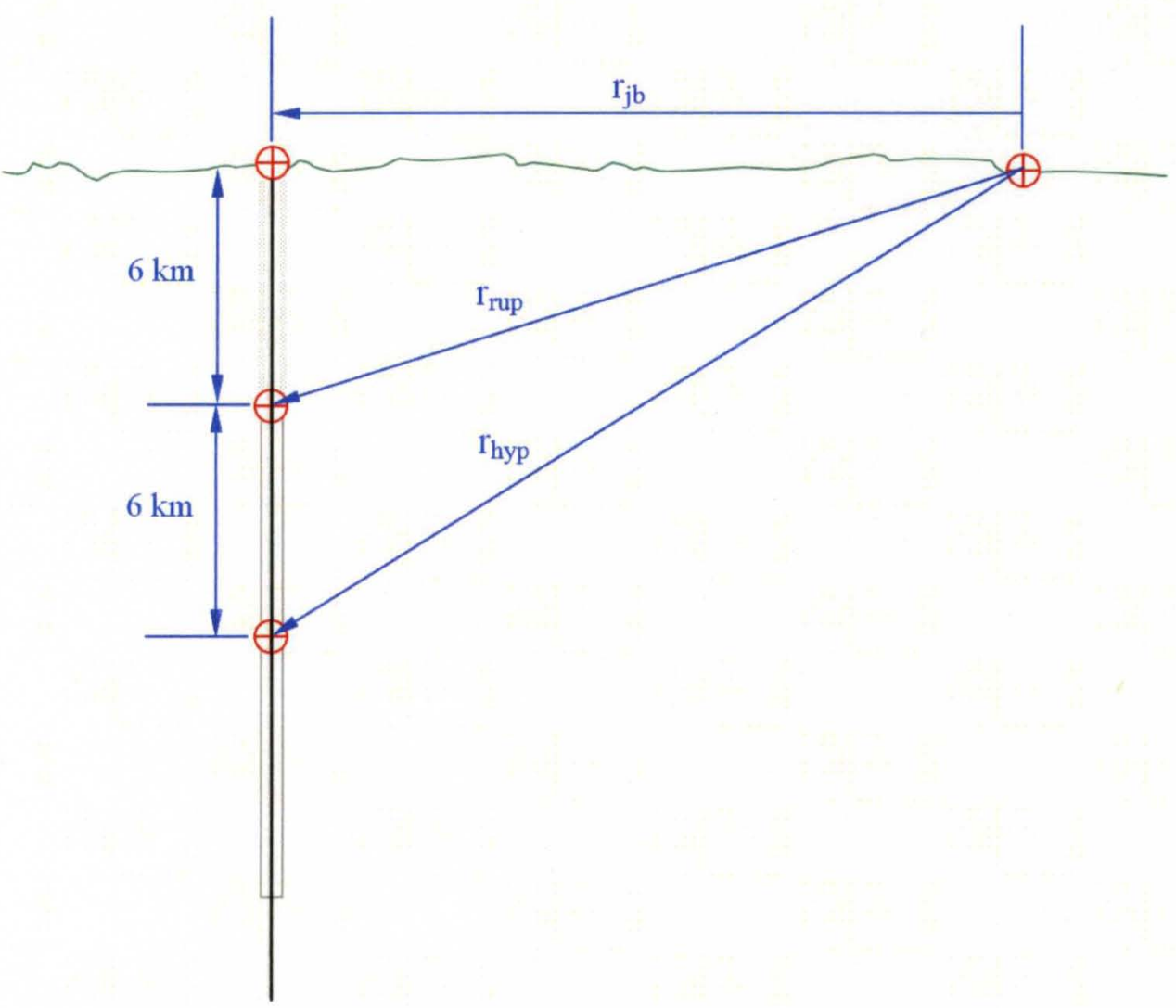

Figure 6.11 Fault Model Assumed for Final Study to Provide a Consistent Definition of Distance 
By assuming that the fault is vertical, the Joyner-Boore distance is taken to be the same as the epicentral distance. The Joyner-Boore distance was used in the Silva et al. model; the Somerville et al. model also uses this distance measure but in combination with a constant rupture depth of $6 \mathrm{~km}$. The Atkinson and Boore model uses the closest distance to the fault which for a vertical fault as assumed for this study is the same as the rupture distance. The Campbell, Toro et al, and Tavakoli and Pezeshk models also use the rupture distance. Finally, the Frankel model uses the hypocentral distance as defined earlier to determine the response spectra for the scenario events.

The $6 \mathrm{~km}$ model depth was chosen for this study since Somerville et al. found it to be constant over their simulations verifying the same value as found by Abrahamson and Silva in 1997. Furthermore Campbell used a minimum depth of $3 \mathrm{~km}$ for his simulations and comments that a depth of rupture this shallow has never been witnessed in Eastern North America nor is expected to occur.

Finally, in order to determine the hypocentral distance required by the Frankel et al. model, the focal depth of the fault was assumed to be at $12 \mathrm{~km}$ which is twice the depth to the top of the assumed rupture. This value coincides well with the recorded focal depths for many events that have occurred in the CEUS (Atkinson and Boore found the average focal depth to be $13 \mathrm{~km}$ ). 


\subsubsection{Synthetic Ground Motion Generation}

To generate the synthetic earthquake ground motions for the final study, the input target spectra generated from the attenuation models used the moment magnitudes and distances just described. The intensity envelopes and duration times were determined as in the pilot study only using moment magnitudes and distances describing the scenarios used in the final study and central frequencies determined according to the attenuation models. The seed numbers used in the final study are the same as those used in the pilot study. Again, twenty-five replicates for each scenario were produced for the final study.

As mentioned in the previous section regarding the parameter values used in the pilot study, an input spectral range in the SIMQKE program of 0.01 to 4.0 seconds was selected after several preliminary studies. With the duration times determined for the scenarios and attenuation models in this study, a decision between using an input spectral range of 0.01 to 4.0 seconds or using a range of 0.02 to 5.0 seconds was required.

Most of the attenuation models used in the final study provide spectral accelerations within the range of 0.01 to 4.0 seconds. The only model that does not provide such a range is the Atkinson and Boore model which provides a spectral range of 0.01 to 5.0 seconds. It was decided not to "shift" up one point to 0.02 to 5.0 seconds after the findings from preliminary studies as explained in the pilot study evaluation. Rather it was deemed more appropriate to "shift" down one point to 0.01 
to 4.0 seconds based on the findings from these preliminary studies and considering the remaining six models to be used in the final study provides values within this range. Again, the only model that was affected by using this input spectral range is the Atkinson and Boore model. The central frequencies of this model will change only slightly, affecting the intensity envelope minutely and having an even smaller impact on structural response.

\subsubsection{Damping}

All of the CEUS attenuation models used to develop the target spectra for the final study produce $5 \%$ damped response spectra. The $5 \%$ value is widely accepted and used to represent the damping contribution for a variety of media. Here, it is assumed to represent the damping in the ground motion as it travels through the various SDOF systems whose natural periods make up the abscissa of the response spectra that describes the frequency content of the ground motion. As mentioned previously, the algorithm used by SIMQKE for the generation of the synthetic ground motions also requires that a damping value be input. Because the input target spectra developed from the attenuation models for the final study already had a value of 5\% damping applied, as was done for the pilot study, a value essentially equal to $0 \%$ damping was used as input for the generation of the synthetic motions. As before, this was done in order not to apply more damping than that which was already implied by the attenuation models. However, again as in the pilot study, 5\% damping was used in developing the input for the nonlinear time history analyses of the 
SDOFs. This 5\% damping was taken to represent the damping contribution applied to the ground motion from the structure as it responds to the ground motion.

\subsubsection{Maximum Ground Acceleration}

The maximum ground acceleration desired for the synthetic motion produced is also required as input into the SIMQKE program. For the final study, this value was taken as the peak ground acceleration as determined from the attenuation models. Some models provide this value explicitly. Those that do not provide this value, provide spectral accelerations at 0.01 second period and this value was taken as the peak ground acceleration. Recalling the explanation of response spectra made earlier, the peak ground acceleration is presented at the lowest (i.e., shortest) period end of the abscissa for the response spectrum. This acceleration can be imagined as the response of an "infinitely stiff" SDOF system, one so stiff that it oscillates at the same period as the ground motion.

\subsubsection{Discretization Interval}

As will be explained later, the results presented in the next chapter were obtained by using a discretization interval of 0.02 seconds to generate the synthetics. A time step interval of 0.01 seconds was used as is customary to conduct the entire study prior to obtaining these results. This study highlighted the importance of the discretization interval and the role that it plays in the generation of the artificial ground motions. A more in depth explication of this importance will be provided during the subsequent analysis and evaluation of results. 


\subsubsection{Structural Response}

All of the same parameter values used to define the SDOF systems in the pilot study were used to conduct the nonlinear time history analysis for the final study. These values included the SDOF systems with natural periods of $0.1,0.2,0.6,1.0$, and 3.0 seconds as well as the Wood-Anderson seismograph simulation for magnitude estimation.

Likewise as in the pilot study, an idealized linear elastic-perfectly-plastic system was assumed for all structures. Also, the same values of yield strength were used to define the systems for the final study as were used in the pilot study and provided in previous sections.

\subsubsection{Managing, Collecting, and Analyzing the Data}

As mentioned earlier in this chapter, an automation code was utilized to generate and collect all necessary input and output for the study. A file naming convention and directory hierarchy was established to manage and organize the large amounts of necessary input and output data generated for this study as well as all output that was made available so that it may be mined later for future work. This naming convention is based on attenuation model, magnitude, distance, and seed number of the replicate. There were twenty-five replicate synthetic motions generated from each root file which were each used to develop NONLIN input files. Each of these files was then run on each of the six SDOF systems generating a total of 30,000 responses. The 
development of the automation code proved essential in managing the large amounts of data used and produced in this study.

The ductility ratios from each analysis were captured as the main output for this study. As outlined in the problem statement, the variation in structural response for this study was investigated in terms of displacement ductility demand. Also, the "maximum displacement for the Wood-Anderson response was captured as an additional output to accomplish the magnitude estimation of the synthetics.

Statistical analyses were used to analyze the data collected from the final study. Two sided t-tests were conducted for each scenario to determine confidence intervals for the mean ductility demands as well as the mean estimated magnitudes. For these analyses, the hypothesis was taken as the mean ductility demands equaling one and the mean estimated moment magnitude equaling their target magnitudes.

\subsection{Limitations}

While every effort was made during the design of this study to minimize as many issues as possible, some limitations were inevitable in this study.

The synthetic ground motions generated for this study are for bedrock conditions only. This study was intended as a starting point for future work and to alleviate complications from considering differing site conditions, only hard rock conditions were considered for this study. Some of the latest models used in this study provide 
regression coefficients for site specific responses. Also, these motions could be run easily through a site-specific soil column using any of the many nonlinear analysis software packages available.

Furthermore, this study only considers the horizontal component of ground motion and not the vertical component. Again, this constraint was done to establish a possible starting point for future work which could include additional consideration of the vertical component.

For simplicity, an elasto-plastic response was assumed for all SDOF systems used in the study. By applying this model, it is assumed that once the yield strength is achieved, the stiffness will remain constant at that point under increasing load. Undoubtedly, once yield is reached in actual structures, some softening in the stiffness will occur. However, again for simplicity and in an effort not to cloud the results of comparisons among responses from the varying input synthetic ground motions, an idealized linear elastic and perfectly plastic system was assumed for this study. During a significant event, stiffness degradation can realistically be expected to occur for all systems at some point. The elasto-plastic model obviously does not account for this degradation and therefore leads to an overestimation of the response. Despite this discrepancy, in this study all of the synthetic ground motions were applied to the same SDOF systems with the same elasto-plastic nonlinear hysteretic behavior being assumed. In other words, it was consistently applied throughout the study. Therefore, even though this model does not provide the most realistic 
response, for the purpose of this study which was to investigate the variation among the structural response due to the synthetic motions it seems appropriate.

As noted earlier, a value approaching $0 \%$ damping was used as input in the SIMQKE program to generate the synthetics used in this study. As was shown in Equation 3.2, the SIMQKE algorithm contains the damping term in the denominator. It is unclear as to what effect allowing this value to approach zero has on the synthetic motions generated. As explained earlier, it is suspected to have some effect, but it was decided for this study that it was more important not to apply more damping over that already prescribed by the target spectra determined form the attenuation models. Again, this application of damping was consistently applied while generating the synthetic ground motions to be compatible with all of the attenuation models

It has been emphasized that the attenuation models used in this study provide elastic response spectra; however, many of the ductility demands induced from the synthetic motions analyzed in this study coincide with nonlinear behavior. In fact, nonlinear time history analyses were conducted for this study. This circumstance is not a contradiction, however, because the linear response spectra in this application are merely used to describe the ground motion and not the structural response they induce. The spectra describe the ground motion predicted for each scenario and specifically its frequency content. The frequency content for the particular scenario "is what it is" whether or not a linear or nonlinear response spectrum is used to describe it. 
The Novikova and Trifunac duration model and to some degree the Saragoni and Hart intensity function used to define the intensity envelopes used to generate the synthetic motions were both developed as described earlier from databases comprised predominantly of WUS earthquakes. Unfortunately, this development from WUS data is unavoidable given the lack of strong ground motion recordings for the CEUS. It is not clear, however, what the differences are if any in shape and duration between WUS and CEUS earthquakes in this regard. Furthermore, it is not clear how these differences would affect the structural response.

Finally, problems with the results from the final study were discovered as will be explained in detail later in the next chapter. An efficient resolution to these problems was applied, as will be explained. A thorough explication of the causes of the problem also is provided in the next chapter.

\subsection{Summary}

In summary, twenty-five replicate synthetic ground motions of the twenty-five magnitude-distance scenarios were generated to be compatible with the response spectra from the eight CEUS attenuation models to give a total of 5,000 synthetic earthquakes. These 5,000 simulated ground motions were then applied as input loading for nonlinear time history analyses conducted on SDOF systems with parameters set to represent five different structures as well as a simulated WoodAnderson seismograph to capture a total of 30,000 responses. 
The data necessary to conduct the analyses for the final study were generated and compiled successfully using the methodology described in this chapter. The displacement ductility demands placed on each structural SDOF system due to the synthetic ground motions compatible with the scenarios described by each attenuation model were captured and statistical analyses were conducted to identify the amount of variability in the structural response.

Likewise, the maximum displacements obtained from the simulated seismograph time history analyses were used to estimate the moment magnitudes of each synthetic earthquake. These magnitudes then were used in statistical analyses to make comparisons with the target magnitudes input into the attenuation models that provided the response spectra used to generate the synthetic motions.

The mean values of displacement ductility demands for each synthetic from each attenuation model representing each scenario on each SDOF are provided in plots and shown as a function of period for each scenario distance for each magnitude and SDOF system. In addition, charts providing the $95 \%$ confidence intervals of ductility demand for each model, distance, and SDOF are provided for each target magnitude. Also, plots showing the estimated magnitudes as a function of distance for each target magnitude were developed as were charts showing the $95 \%$ confidence intervals of estimated magnitude for each model at each distance for each target magnitude. Finally, surface plots indicating the standard deviation and coefficients of variation 
for displacement ductility demand as functions of SDOF periods and distance also were developed.

All of these plots are provided in the appendices and a detailed analysis and explanation of the results gathered from the final study and shown in these plots and charts are presented in the next chapter. 


\section{Chapter 7}

\section{ANALYSIS OF RESULTS}

\subsection{Introduction}

The results obtained from this study are presented in the form of charts and graphs which are provided in the appendices. Analyses of these results are presented in this chapter. Explanations of these charts and graphs presenting the data are also presented here. An evaluation of the variation in ductility demand using example graphs to clarify the analysis is presented with some general observations. Interpretations of overall trends in the results are indicated and examples of the application of the results obtained are suggested. Finally, a summary of the results is given with an evaluation of problems encountered while conducting the study.

\subsection{Organization of Results}

The displacement ductility demand is defined as the ratio of the maximum displacement encountered during shaking to the displacement that causes yielding. Therefore, a ductility demand of one indicates the onset of yielding in the structure and consequently for structures designed using elastic design principals, the onset of 
failure. For this reason, the plots in the appendix showing ductility demands also have the ductility value at one highlighted for comparison if it is relevant to the values being shown.

Appendix A provides plots of the mean displacement ductility demands as a function of natural period for the single degree of freedom (SDOF) systems investigated in this study. These SDOF systems have natural periods of $0.1,0.2,0.6$, 1.0 and 3.0 seconds. The curves shown are made up of the mean ductility demands from 25 synthetic motions from each of the attenuation models for each of the magnitude and distance combinations considered in this study.

Appendix B provides charts containing 95\% confidence intervals for displacement ductility demand from nonlinear time history analyses with 25 synthetic motions for each attenuation model, each magnitude and distance combination, and each SDOF system. These confidence intervals were obtained from individual onesample two-sided t-tests on the 25 observations from each model at a $95 \%$ confidence level.

This appendix also provides charts containing the $95 \%$ confidence intervals of the estimated moment magnitudes calculated for each of the attenuation models at each of the five distances investigated in this study. The moment magnitudes were calculated using the maximum displacements as determined from the nonlinear time history analyses with the SDOF parameters set to simulate those of the Wood- 
Anderson seismograph. The confidence intervals are based upon 25 observations and individual one-sample t-tests on each model at each magnitude for each of the five distances investigated in the study.

Appendix $\mathrm{C}$ contains plots showing comparisons of the mean calculated moment magnitudes for 25 synthetics from each of the attenuation models and their target moment magnitudes at the five distances investigated in this study. Again, the moment magnitudes were calculated using the maximum displacements as determined from the nonlinear time history analyses with parameters set to simulate those of the Wood-Anderson seismograph.

Appendix D provides plots of the mean displacement ductility demands for each moment magnitude and single degree of freedom (SDOF) system as a function of distance. The distances investigated in this study are $10 \mathrm{~km}, 25 \mathrm{~km}, 50 \mathrm{~km}, 100$ $\mathrm{km}$ and $200 \mathrm{~km}$. The curves shown are made up of the mean ductility demands from 25 synthetics from each of the attenuation models for each of the magnitude and distance combinations applied as loading on each of the SDOF systems.

Appendix E contains 2-dimensional surface plots showing the trends in the standard deviations of the displacement ductility demands for each moment magnitude as a function of natural period and distance. This appendix also contains the same surface plots for trends in the coefficient of variation at each moment magnitude taken as the ratio of the standard deviation to the mean. Finally, this 
appendix also contains these same surface plots showing the trends in the mean displacement ductility demands at each moment magnitude as a function of natural period and distance.

\subsection{Variation}

The original question posed by this study asked how significant is the variation seen among the response spectra predicted by the eight attenuation models for the CEUS in terms of the variation produced in the structural response to synthetic ground motions generated to be compatible with these models. Specifically, this study looked at this variation in terms of displacement ductility demands for SDOF systems of different natural period.

An example of the variation in the response spectra can be seen for the moment magnitude 5.5 at $10 \mathrm{~km}$ event as shown in Figure 7.1. Figure 7.2 shows the variation in ductility demand from synthetic ground motions generated for this event at this distance using the attenuation models. Note that the mean ductility demands for the $0.2,0.6$, and 1.0 second period SDOF systems are very close to one and yet there is a wide variation among the ductility demands at these periods for synthetic motions generated from the various models. The periods of these SDOF systems represent the natural period range containing most civil engineering structures. Response to this event is almost perfectly split among the eight models with nearly half producing 
ductility demands less than one indicating that all structures will remain elastic with the other half producing ductility demands above one indicating yielding in most

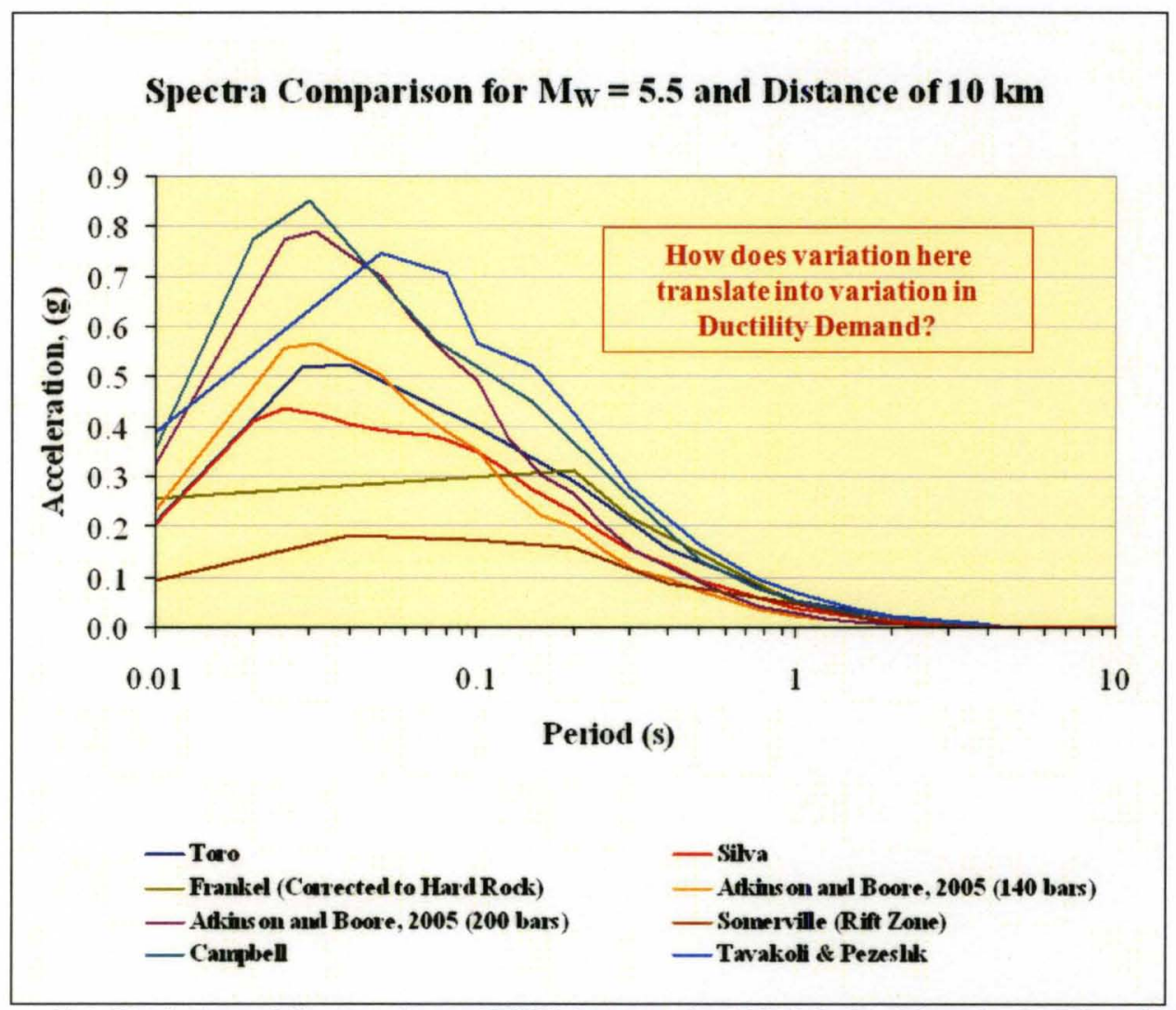

Figure 7.6 Variation of Spectra from CEUS Attenuation Models for Magnitude 5.5 at $10 \mathrm{~km}$ 


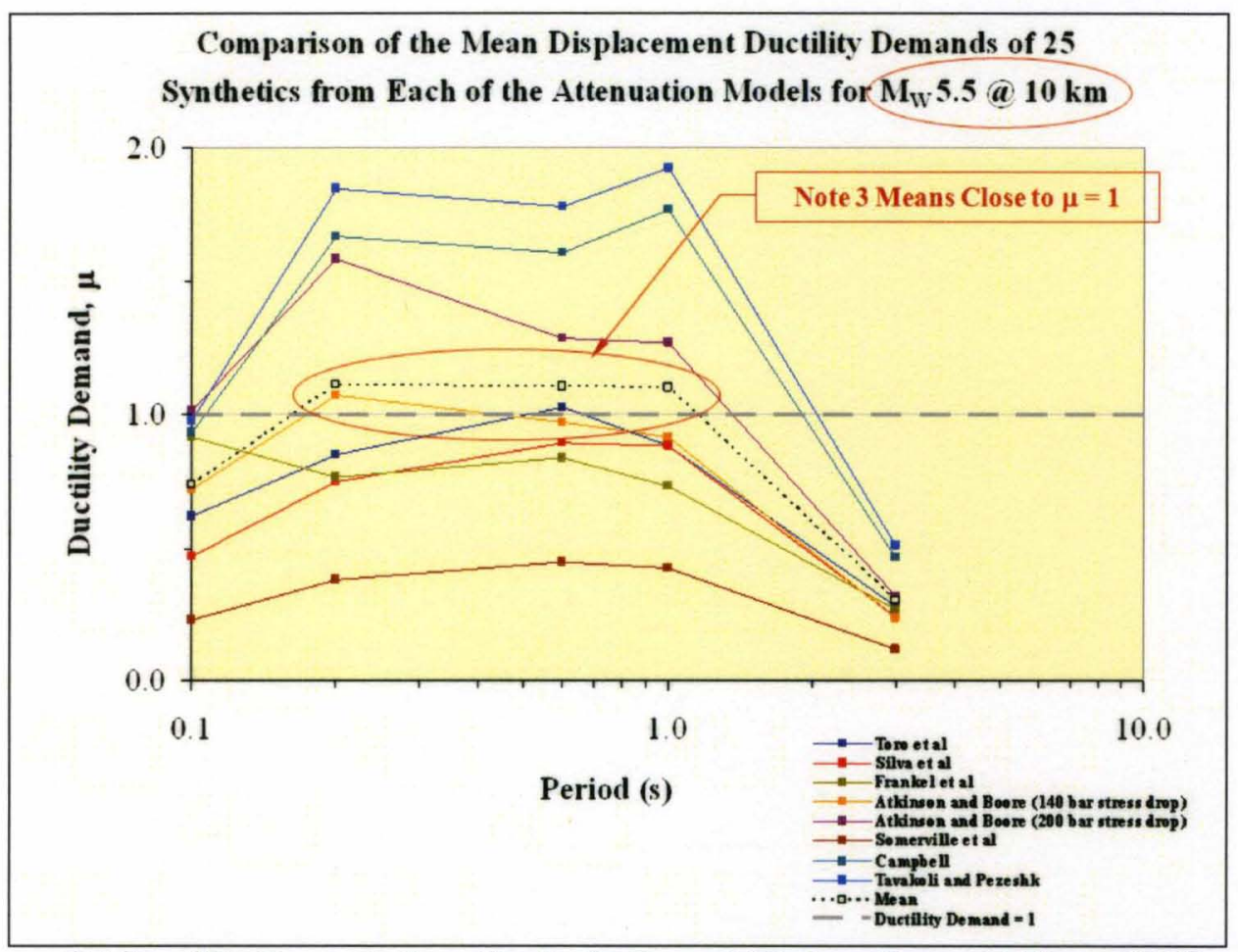

Figure 7.7 Ductility Demands from Synthetic Ground Motions Generated from CEUS Attenuation Models for Magnitude 5.5 at $10 \mathrm{~km}$

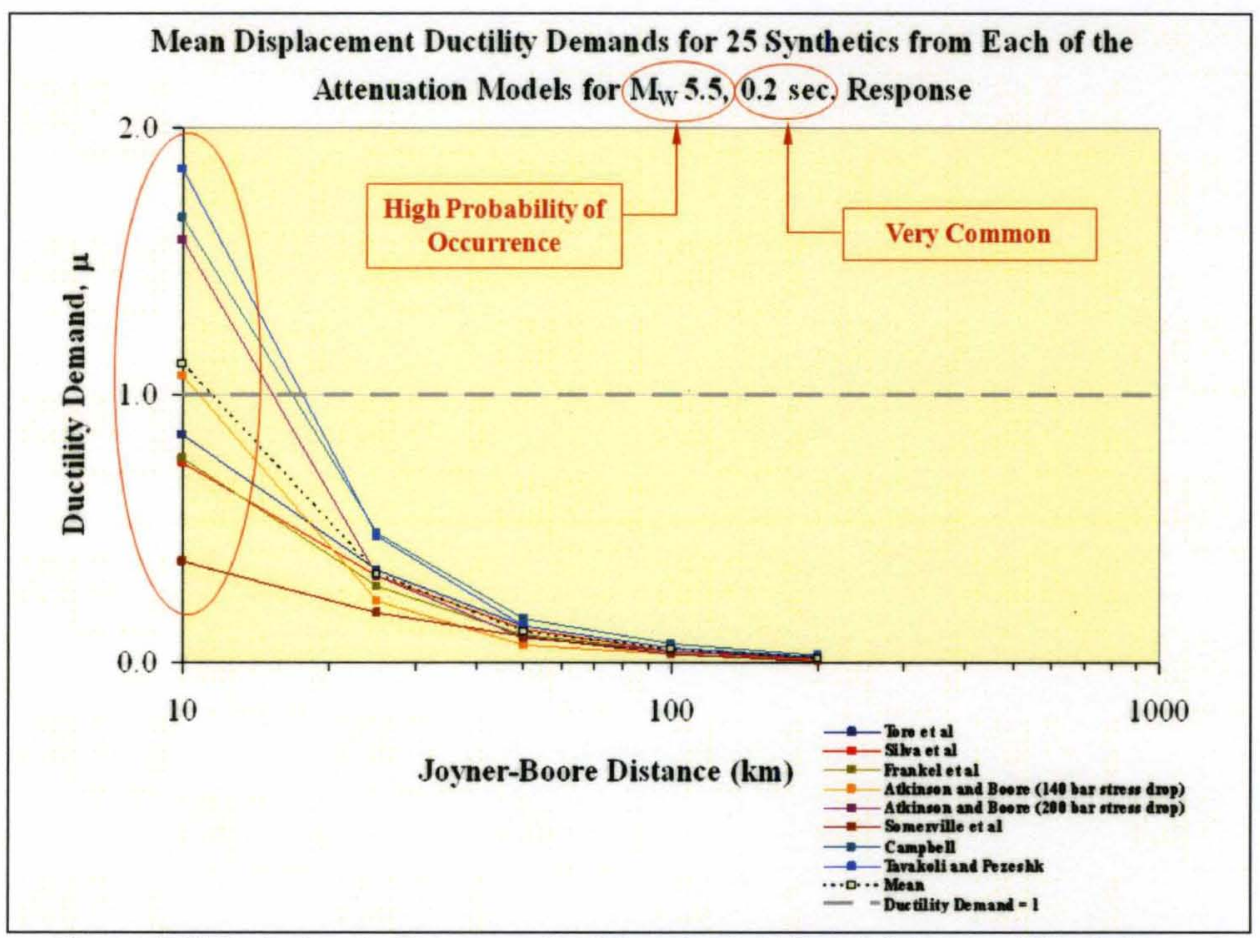

Figure 7.8 Variation in Response for Magnitude 5.5 and 0.2 second Period 
structures. The results for this event were among some of the most divisive that were obtained in this study. Results for magnitude 5.5 events with 0.2 second response period are shown in Figure 7.3. Figure 7.3 shows the wide variation in the 0.2 second response to synthetic motions generated from the attenuation models for this event at this distance. Note that this magnitude of event has a high probability of occurrence and this period represents the natural period of very common structures. Another scenario that shows variation among the response spectra from the CEUS attenuation models is the $M_{W} 6.0$ at $10 \mathrm{~km}$ event as shown in Figure 7.4. This

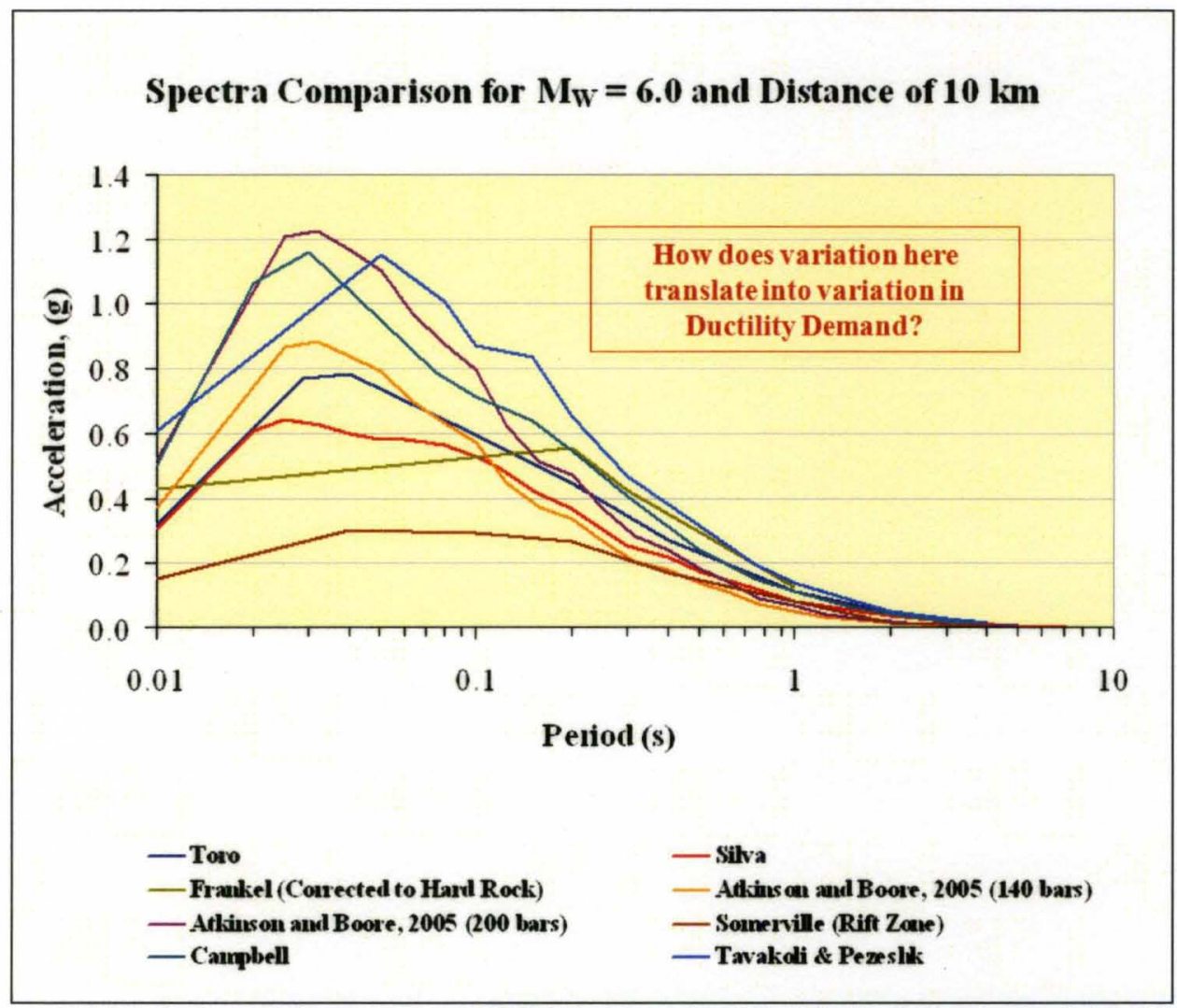

Figure 7.9 Variation of Spectra from CEUS Attenuation Models for Magnitude 6.0 at $10 \mathrm{~km}$ 
scenario also provided divisive responses from the synthetic ground motions compatible with the eight attenuation models. It can be seen from Figure 7.5 that one model indicates that all structures will remain elastic during this event while the responses to the synthetic motions generated from the remaining models indicate a wide variation in ductility demands indicating yielding will be required in all structures. The figure shows that the wide variations in ductility demands are indicated in nearly all period structures. As seen in Figure 7.6, the scenario for

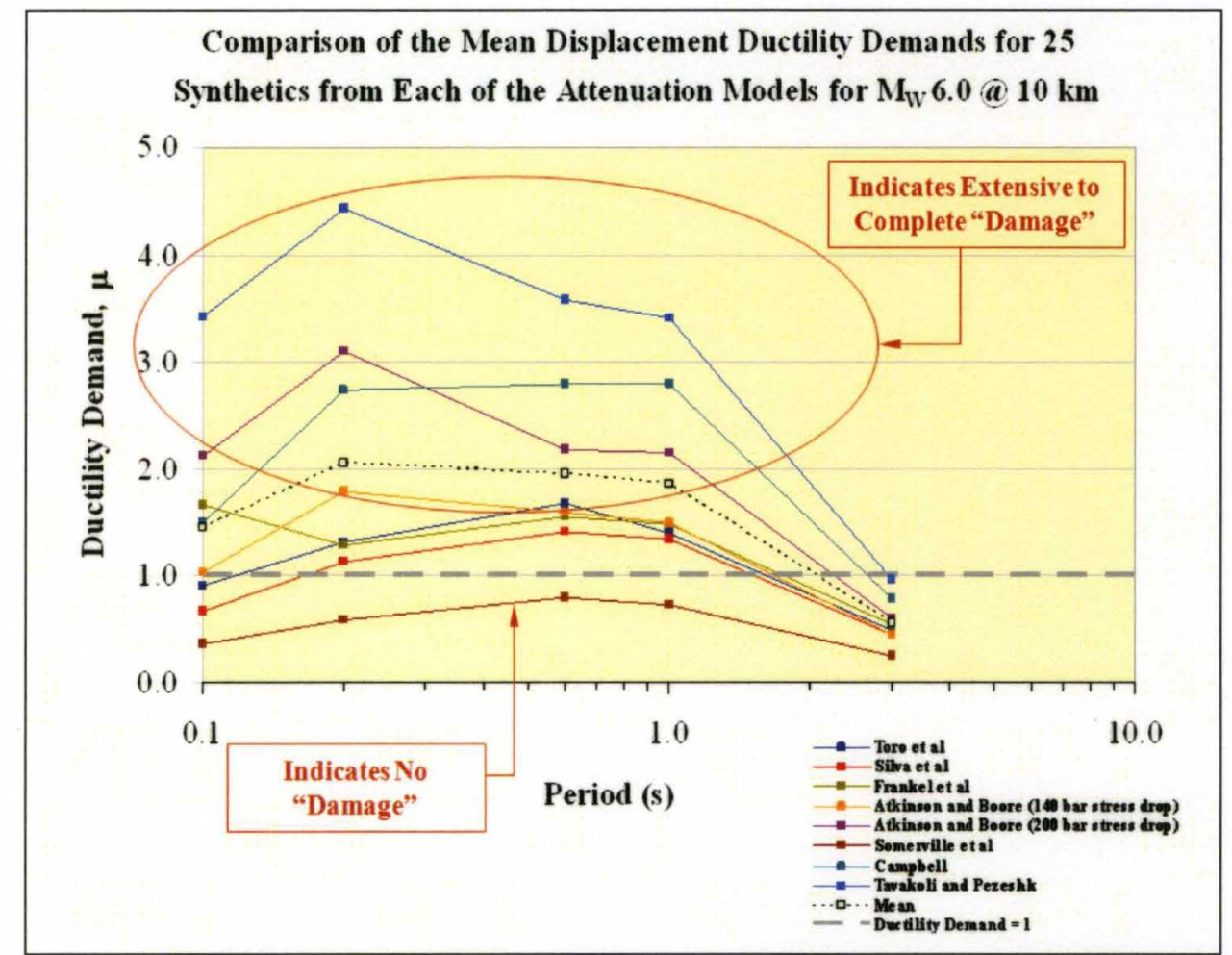

Figure 7.10 Ductility Demands from Synthetic Ground Motions Generated from CEUS Attenuation Models for Magnitude 6.0 at 10 km 


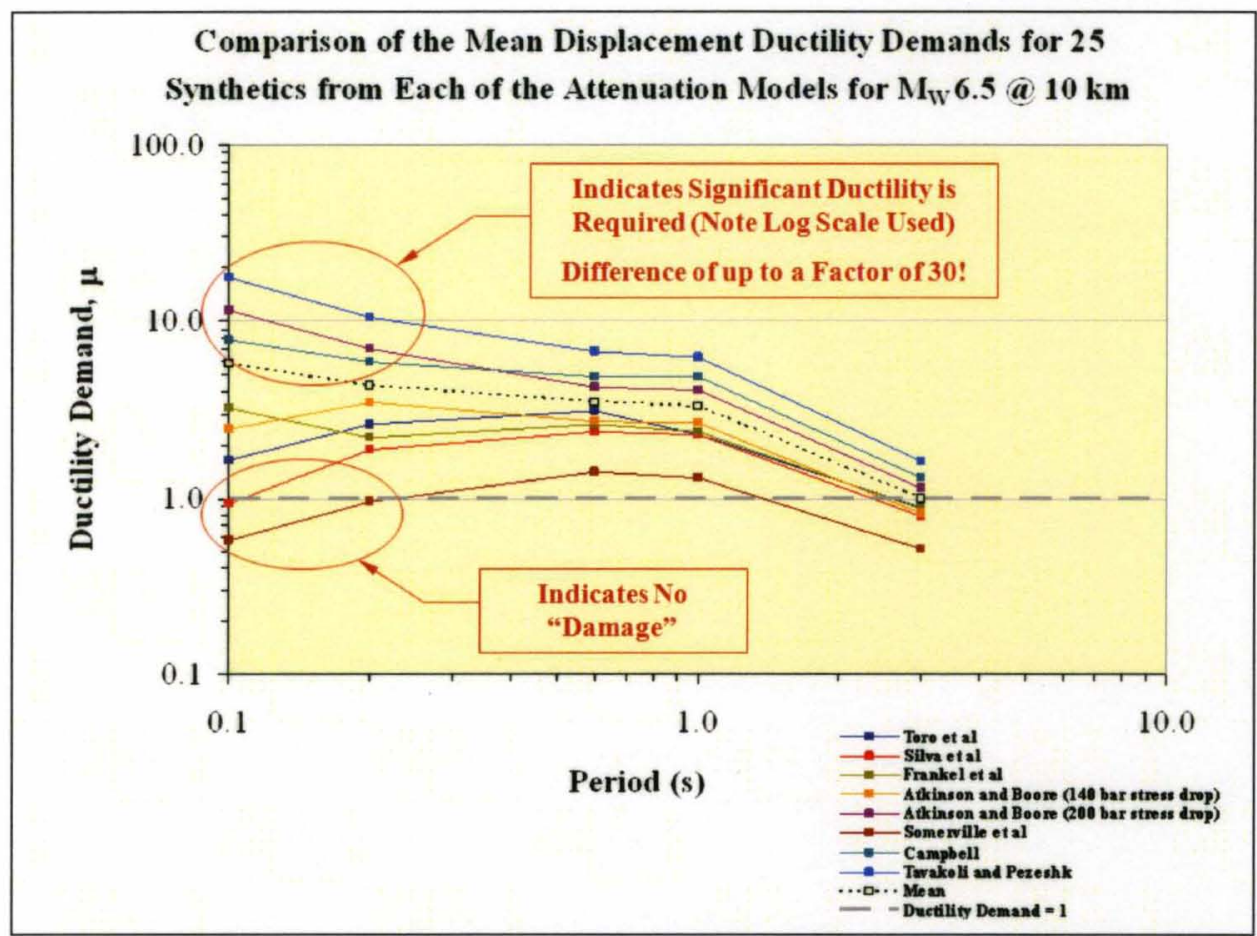

Figure 7.11 Variation in Ductility Demands for Synthetic Ground Motions Generated from CEUS Attenuation Models for Magnitude 6.5 at $10 \mathrm{~km}$

magnitude 6.5 at $10 \mathrm{~km}$ has synthetic ground motions from at least one model showing no damage to short period structures while the others indicate significant ductility demands of as high as 10 and 20 being placed on short period structures by this same scenario.

Synthetic ground motions representing the $M_{W} 6.5$ at $25 \mathrm{~km}$ and the $M_{W} 7.5$ at 50 $\mathrm{km}$ events from the eight attenuation models also reveal discrepancies in the ductility demands required. These events are shown in Figures 7.7 and 7.8 respectively. The synthetic motions from at least two of the models indicate no damage to any types of structures while the synthetic motions from at least two of the other models indicate most structures will yield due to these events at these distances. 
Finally, Figures 7.9 and 7.10 provide examples of large variations in ductility demand in 0.2 second period SDOF systems from the moment magnitude 7.0 at 25 $\mathrm{km}$ scenario and in 0.6 second period SDOF systems from the moment magnitude 7.5 at $50 \mathrm{~km}$ scenario, respectively.

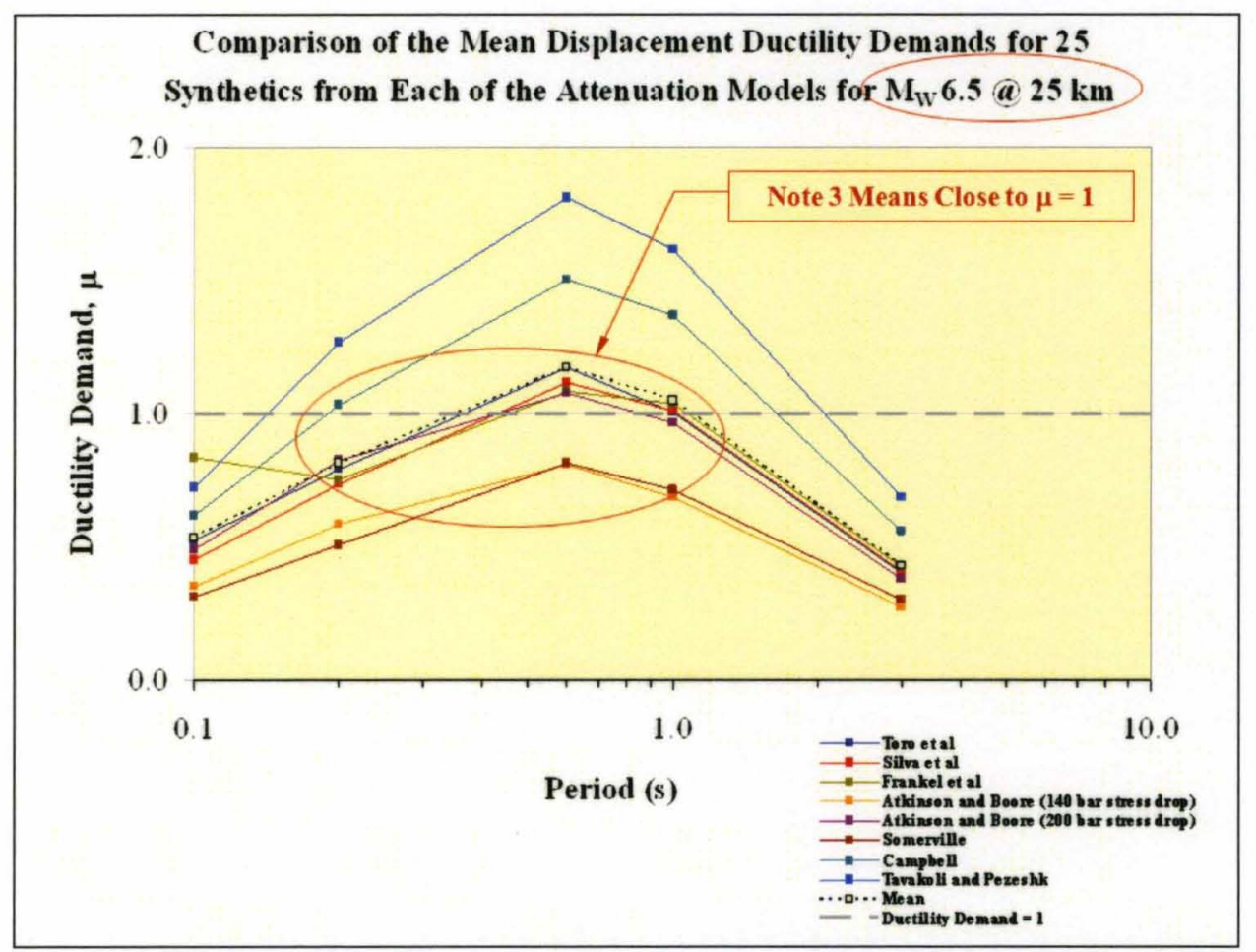

Figure 7.12 Variation in Ductility Demands for Synthetic Ground Motions Generated from CEUS Attenuation Models for Magnitude 6.5 at $25 \mathrm{~km}$ 


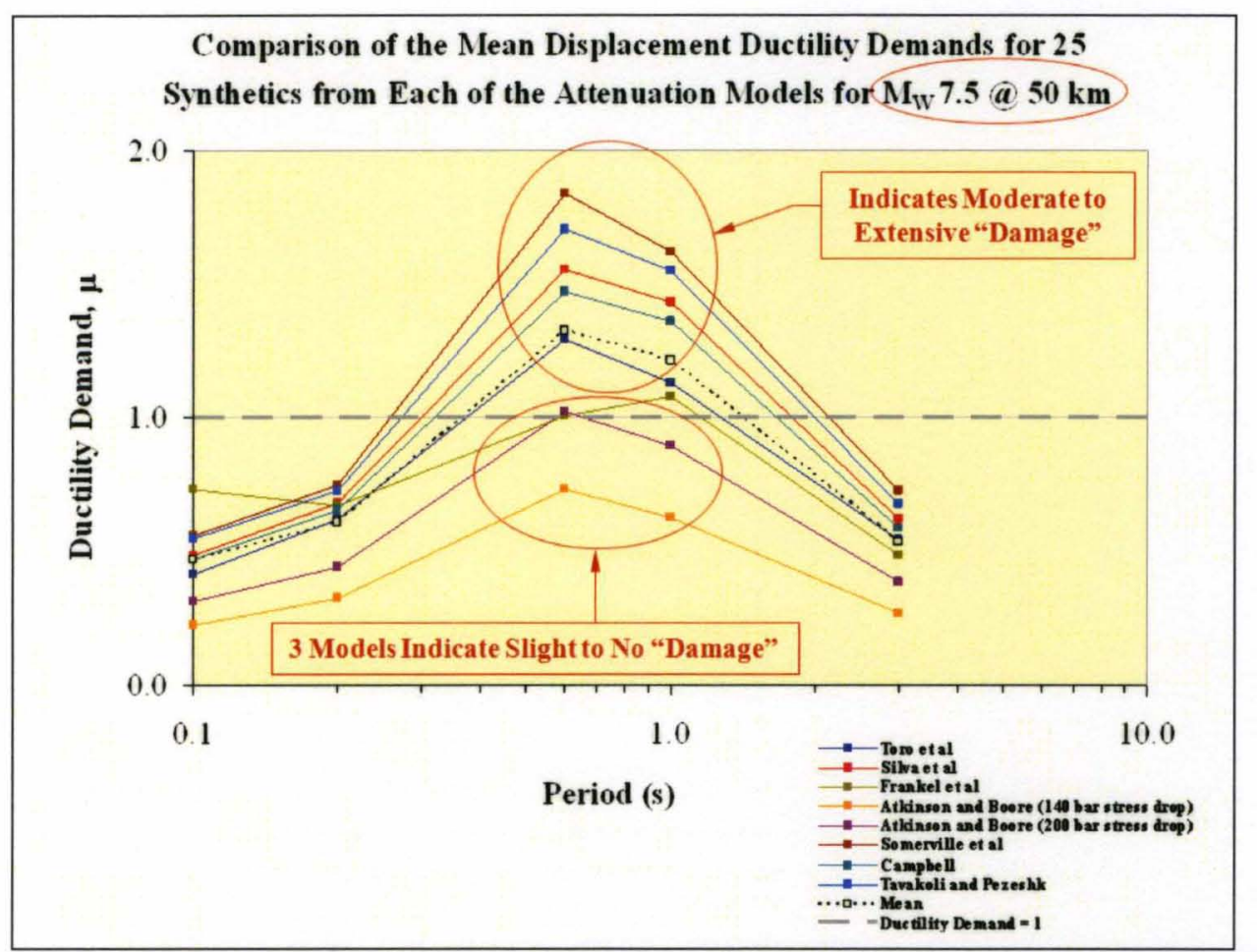

Figure 7.13 Variation in Ductility Demands for Synthetic Ground Motions Generated from CEUS Attenuation Models for Magnitude 7.5 at $25 \mathrm{~km}$

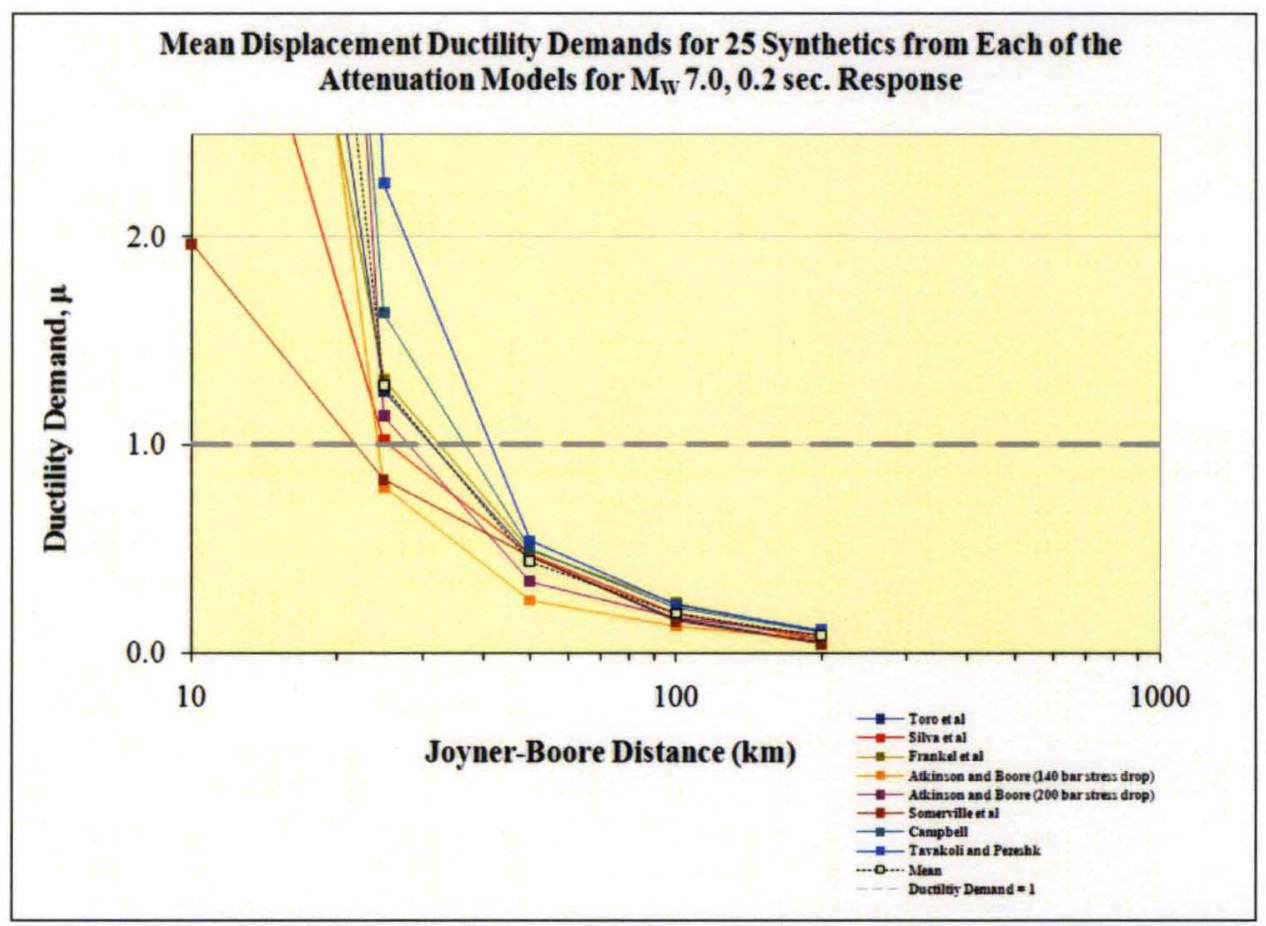

Figure 7.14 Variation in Ductility Demands for Synthetic Ground Motions Generated from CEUS Attenuation Models for Magnitude 7.0 and 0.2 sec Response Period 


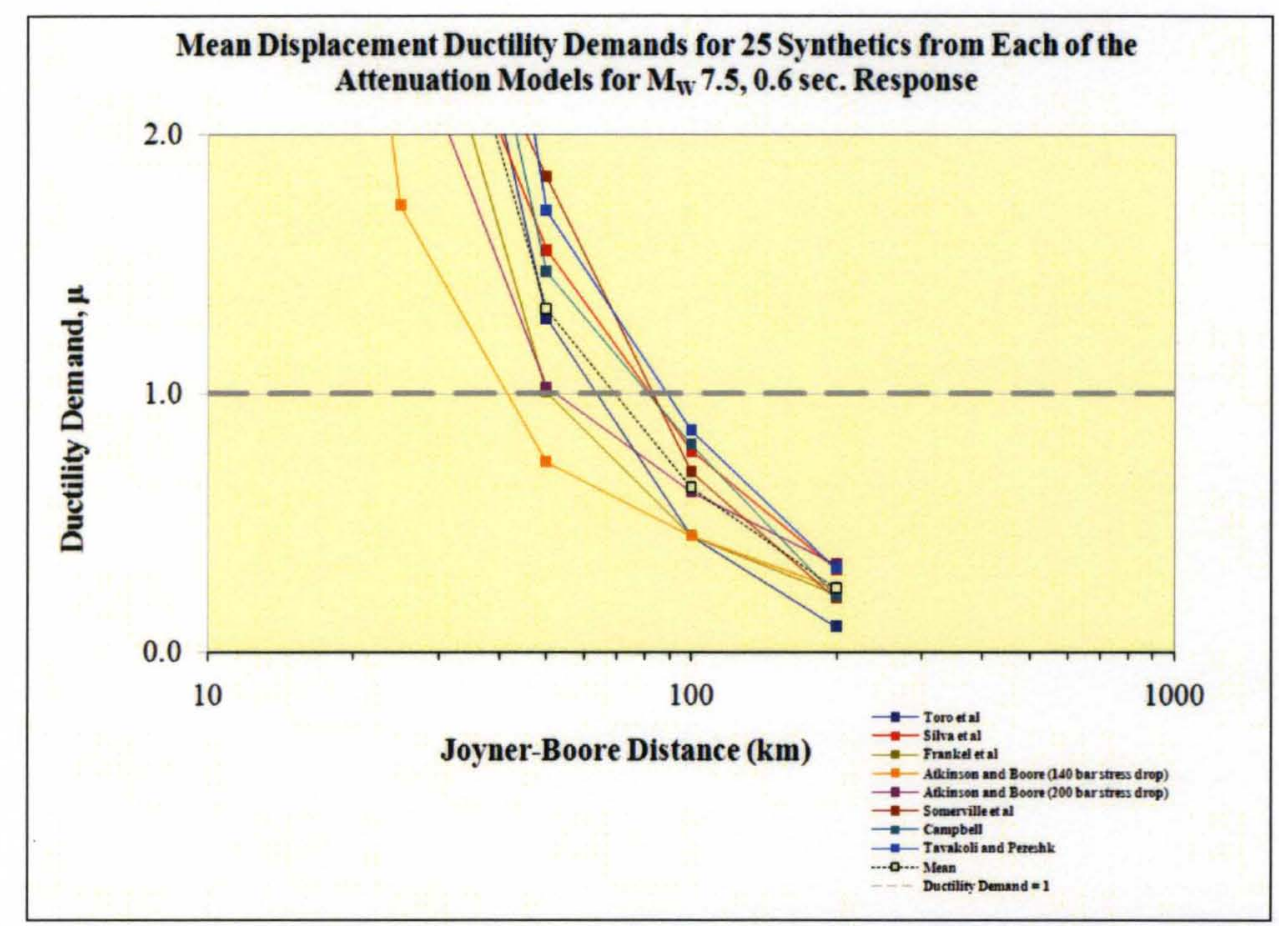

Figure 7.15 Variation in Ductility Demands for Synthetic Ground Motions Generated from CEUS Attenuation Models for Magnitude 7.5 and 0.6 sec Response Period

\subsubsection{General Observations}

Further observations taken from the plots provided in the appendices of the variation in ductility demand from synthetic ground motions compatible with the CEUS attenuation models are provided in this section.

The plots contained in Appendices A and D report the same data but in different formats. 
The ductility demands from the magnitude 6.5 at $25 \mathrm{~km}$ scenario has synthetic ground motions from two models indicating no damage to any type of structure while those from the other models indicate yielding in a large amount of most structures.

The magnitude 7.0 at $10 \mathrm{~km}$ scenario shows ductility demands from some models up to 40 times greater than those of other models for higher frequency structures. Even though all models indicate damage, seismic design is based upon the required ductility demand. Ductility demands at this wide variation could lead to extremely different approaches to seismic designs.

For the magnitude 7.0 at $25 \mathrm{~km}$ scenario synthetic ground motions from two models indicate yielding in very high frequency structures while the other models do not. Also for this scenario, synthetic motions for two models indicate no damage to 0.2 second period structures while those from the remaining models do. Also while synthetics from all models indicate yielding in mid- to low-period structures, the actual values of ductility demand vary greatly. Finally, the responses to synthetic motions generated from one model also indicate yielding for this scenario to very long-period structures while the motions generated from other models do not.

The ductility demands from the magnitude 7.5 at $10 \mathrm{~km}$ scenario show that even though all models indicated yielding there is a wide variation at the shorter periods. This variation is also evident at the magnitude 7.5 at $25 \mathrm{~km}$ scenario where some models predict no damage at the shorter periods while the others indicate significant 
ductility demands. Again, there is wide variation at the mid- to long-periods with the synthetic motions from a couple of models showing no damage at very long periods while the other models indicate yielding. Finally, for the magnitude 7.5 at $50 \mathrm{~km}$ scenario synthetic motions from three models show no damage or only slight yielding at the mid- to long-period structures while those from the others all show ductility demands greater than one.

The following observations were made using the plots available in Appendix D.

The magnitude 5.5 event has a response from structures with a 0.1 second response period to synthetic motions for one model at $10 \mathrm{~km}$ indicating yielding while all of the others indicate no damage. Also approximately half of the model's synthetic motions indicate yielding in short- to mid-period structures from this event at $10 \mathrm{~km}$ while those for the other half do not.

For the magnitude 6.0 event, approximately half of the models produced synthetic motions that indicate yielding in very short-period structures at $10 \mathrm{~km}$ while those produced by the others indicate only slight or no damage to these structures at this distance. The synthetic motions from one model indicate no damage to any type of structure at $10 \mathrm{~km}$ while those from all of the other models do. There are two models whose synthetic motions indicate yielding or the onset of yielding from this event at $25 \mathrm{~km}$ for mid- to long-period structures while those from the others do not. 
For the magnitude 6.5 event, two models indicate only slight or no damage at 10 $\mathrm{km}$ to very short-period structures while all others indicate significant yielding of these types of structures. One model indicates none to relatively slight damage for most structures at $10 \mathrm{~km}$ while all other models indicate significant ductility demands. For most structures, the synthetic motions from at least two models predict no damage at $25 \mathrm{~km}$ from this event while synthetic ground motions compatible with the other models do. Finally for this event, the synthetic motions from three of the models indicate yielding in very long-period structures at $10 \mathrm{~km}$ while the others do not.

For the magnitude 7.0 event, there is one model whose synthetic motions does not indicate yielding of very short-period structures at $10 \mathrm{~km}$ while the others do. Also for this event, there are two models with synthetics that indicate yielding of very short- period structures at $25 \mathrm{~km}$ and two models with synthetics indicating no damage to short-period structures at this distance. However, synthetic motions from all of the models indicate damage for mid- to long-period structures and none to only slight yielding for very long period structures at this distance. Also for this event, the synthetic motions from one model indicate yielding with those from three other models at the onset of yielding for mid- to long-period structures at $50 \mathrm{~km}$ while synthetic motions from the remaining models indicate no damage. Finally for the magnitude 7.0 event, the synthetic motions from one model indicate only slight yielding in very long-period structures at $25 \mathrm{~km}$ while the synthetic motions from the 
remaining models indicate that yielding will not occur in these types of structures from this magnitude of event at this distance.

For the magnitude 7.5 event, synthetic motions from half of models indicate only slight to no damage to very short-period structures at $25 \mathrm{~km}$ and those from the others indicate significant ductility demands. Also for this event at this distance, the synthetic ground motions from one model indicate only the onset of damage to short period structures while the others indicate significant yielding in these structures. Also for this event, the synthetic motions from one model indicate no damage and those from two others indicate only the onset of damage to mid-period structures at $25 \mathrm{~km}$ while the synthetic motions from all of the other models for these structures at these distances indicate ductility demands greater than one. Also, the synthetic motions from one model indicate ductility demands approaching the onset of yielding in mid- period structures at $100 \mathrm{~km}$. Finally for this event, synthetic motions from two of the models indicate no damage to long-period structures at $50 \mathrm{~km}$ or very long-period structures at $25 \mathrm{~km}$ while synthetic motions from all of the other models indicate yielding in these structures at these distances.

The following observations were made from the plots for estimated magnitude provided in Appendix C. 
For the synthetic motions of the magnitude 5.5 event, all calculated magnitudes are well above the target magnitude with only a few within a half magnitude at $10 \mathrm{~km}$ and $25 \mathrm{~km}$.

For the magnitude 6.0 event, all of the calculated magnitudes from the synthetic motions are above the target magnitude except for one slightly below at $10 \mathrm{~km}$. Several values are within a half magnitude of the target at $10 \mathrm{~km}$ and $25 \mathrm{~km}$ and one or two at $50 \mathrm{~km}, 100 \mathrm{~km}$, and $200 \mathrm{~km}$.

Estimated magnitudes calculated for the synthetic motions of the magnitude 6.5 event are all above the target magnitude except for one below the target at $10 \mathrm{~km}$ and two only slightly below the target at $25 \mathrm{~km}$. All calculated magnitudes are within a half magnitude at $10 \mathrm{~km}$ and $25 \mathrm{~km}$ with three within a half magnitude at $50 \mathrm{~km}$, five at $100 \mathrm{~km}$, and two at $200 \mathrm{~km}$.

All calculated magnitudes for the synthetic motions of the magnitude 7.0 event are below the target at $10 \mathrm{~km}$ except three that are slightly above at this distance. All magnitudes but from two attenuation models are below the target magnitude at 25 $\mathrm{km}$. All calculated magnitudes are above the target at distances of $50 \mathrm{~km}, 100 \mathrm{~km}$, and $200 \mathrm{~km}$ with one only slightly above at $50 \mathrm{~km}, 100 \mathrm{~km}$, and $200 \mathrm{~km}$. All calculated magnitudes are within a half magnitude from the target except for one at $50 \mathrm{~km}$ and two at $200 \mathrm{~km}$. 
The calculated magnitudes for the synthetic motions of the magnitude 7.5 event are all below the target magnitude at $10 \mathrm{~km}$ and $25 \mathrm{~km}$ while all are above at $50 \mathrm{~km}$, $100 \mathrm{~km}$, and $200 \mathrm{~km}$ except for two below at $50 \mathrm{~km}$, three at $100 \mathrm{~km}$, and one at 200 $\mathrm{km}$. One of these last predicted magnitudes is only slightly below the target magnitude. Only two are slightly above target at $100 \mathrm{~km}$ and one slightly below at $200 \mathrm{~km}$. Only a few of the estimated magnitudes stray slightly more than plus or minus a half magnitude from this target magnitude.

As will be explained later in this chapter, the inclusion of the estimated magnitude values in this appendix was intended to provide a logical reference to whether a model may be over predicting or under predicting the ground motions.

Appendix B contains the actual limit values for the confidence intervals portrayed in the plots provided in Appendices A, C, and D. Observations made from the 2dimensional surface plots contained in Appendix E are provided in the next section.

As pointed out in these examples and in the plots provided in the appendices there are many discrepancies in the ductility demand reported from the nonlinear time history analyses using the synthetic motions generated from the eight attenuation models. It is not possible to dissect every discrepancy but some of the most obvious cases where the synthetic motions from at least one model indicated a ductility demand below one and synthetic motions from at least one of the other models produced ductility 
demand above one will be evaluated here. For conciseness, only the extremes will be mentioned.

The largest discrepancy for the $M_{W} 5.5$ event was at the $10 \mathrm{~km}$ distance. The largest differences were shown for the 0.2 and 1.0 second responses. Synthetic motions from the Somerville model gave mean ductility demands of about 0.4 and synthetics from the Tavakoli and Pezeshk model produced mean ductility demands of about 1.9. This is a considerable difference.

For the $M_{W} 6.0$ at $10 \mathrm{~km}$ scenario, every SDOF system had considerable discrepancies. Again the extremes were between synthetic motions from the Somerville model on the low end and those from the Tavakoli and Pezeshk model on the high end. The largest difference was in the 0.2 second response with the Somerville synthetic motions producing a mean ductility demand of about 0.6 and those from the Tavakoli and Pezeshk model producing ductility demands of about 4.4. Again this is a significant difference.

The largest discrepancy for the $M_{W} 6.5$ event at $10 \mathrm{~km}$ was again between synthetic motions from the Somerville model on the low end and from the Tavakoli and Pezeshk model on the high end. The largest difference was in the 0.1 second response with the extremes in ductility demand of 0.58 and 17.8 respectively. There was also a significant difference in the 0.6 second response from the $M_{W} 6.5$ at $25 \mathrm{~km}$ scenario. The largest ductility demand was by synthetic motions from the Tavakoli 
and Pezeshk model of 1.8 and the lowest from synthetics of the Atkinson and Boore model with a 140 bar stress drop. Synthetics from this model produced a mean ductility demand of about 0.81 for this event.

There were large discrepancies for every SDOF system response from the $M_{W} 7.0$ at $10 \mathrm{~km}$ scenario with a difference as large as nearly 40 in ductility demand at the 0.1 second response. However, the largest difference above and below a ductility demand of one was for the 0.2 second response from synthetic motions for this event at a distance of $25 \mathrm{~km}$. Synthetic motions from the Atkinson and Boore model with a stress drop of 140 bars produced ductility demands of about 0.8 and those from the Tavakoli and Pezeshk model produced mean ductility demands of about 2.3.

Severe differences in the mean ductility demands for all of the SDOF systems were produced by synthetic motions for the $M_{W} 7.5$ event at $10 \mathrm{~km}$; however, the synthetic motions from all models indicated large ductility demand. For the $M_{W} 7.5$ at $25 \mathrm{~km}$ scenario, the largest difference was for the 0.1 second response by synthetic motions from the Atkinson and Boore model with a stress drop of 140 bars producing a mean ductility demand of about 0.7 and the synthetic motions from the Tavakoli and Pezeshk model producing about 4.5. There was also a large difference in the 0.6 and 1.0 second response from synthetic motions for the $M_{W} 7.5$ at $50 \mathrm{~km}$ scenario. Synthetic motions from the Atkinson and Boore model with a 140 bar stress drop produced ductility demands of about 0.6 while those from the Somerville model produced ductility demands of about 1.8 
As these examples testify, large discrepancies in the amount of ductility demanded of structures by synthetic ground motions from these attenuation models is indicated by the results of this study.

\subsubsection{Overall Trends}

Considering that a ductility demand of one indicates the onset of yielding, a standard deviation in the ductility demand above 0.5 would probably be considered high by most standards. The coefficient of variation provides an estimate of the standard deviation relevant to the mean of the sample. As mentioned earlier, Appendix D provides the standard deviation and coefficient of variation, as well as the means for the displacement ductility demands. All three of the plots are placed in the same appendix so that they may be viewed together. As an example of how they work together to provide information, it can be seen that the coefficient of variation for the magnitude 5.5 event indicates high values for very short-period structures at a distance of $200 \mathrm{~km}$. However, the plot of the mean ductility demand for this same event shows that these structures at this distance have extremely low ductility demands and therefore the high values of coefficient of variation are of no practical concern. Furthermore, it can be seen that ductility demands greater than one at very close distances are indicated for short- to long- period structures for this scenario.

The following general trends were recognized. The highest ductility demands as well as the highest standard deviations at each magnitude occur in the shorter period structures at the closest distances. These trends, shown in Figure 7.11, also indicate 
the standard deviations increase with the mean ductility demands in these structures at these distances as magnitude increases. Finally, the general trends indicate that the maximum coefficients of variation increase at the closest distances for increasingly shorter periods as magnitudes increase until reaching a maximum for the magnitude 6.5 events and then gradually decreasing for the magnitude 7.0 and magnitude 7.5 events.

\section{Trends in the Means of Ductility Demands from a $M_{W} 6.5$}

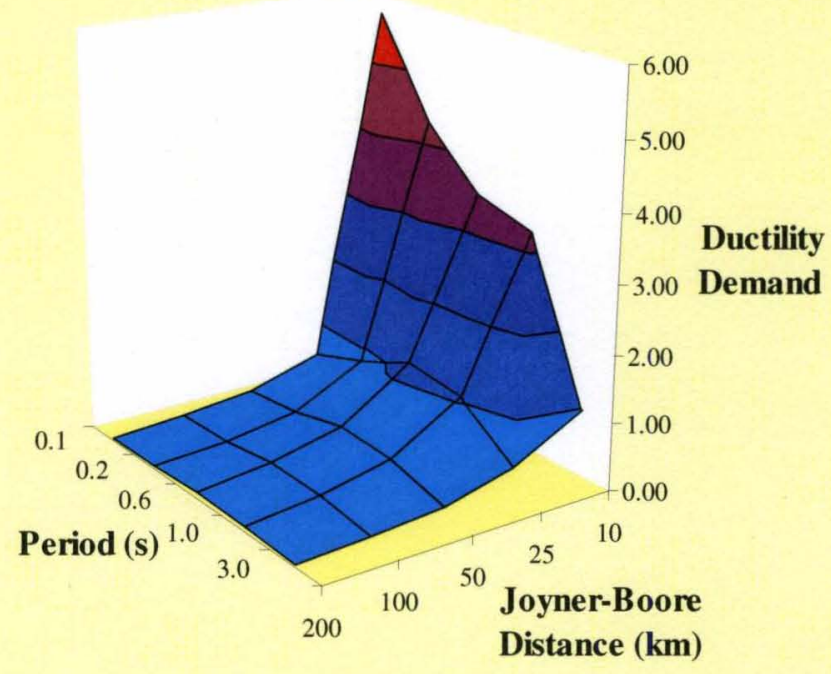

Figure 7.16 Variations in Mean Ductility Demand from Magnitude 6.5

The standard deviation plots of ductility demand for the magnitude 6.0 event show high standard deviations at very close distances for all structures and very high standard deviations for short to medium structures at these distances. The coefficients of variation for ductility demands from this event indicate standard 
deviations of approximately $70 \%$ of the mean for short-period structures at very close distances, as shown in Figure 7.12. The trends in the means of ductility demands for this event indicates that there are high ductility demand for most structures at close distances which is also where there are high standard deviations.

\section{Trends in Standard Deviation for Ductility Demands from a $M_{W} 6.5$}

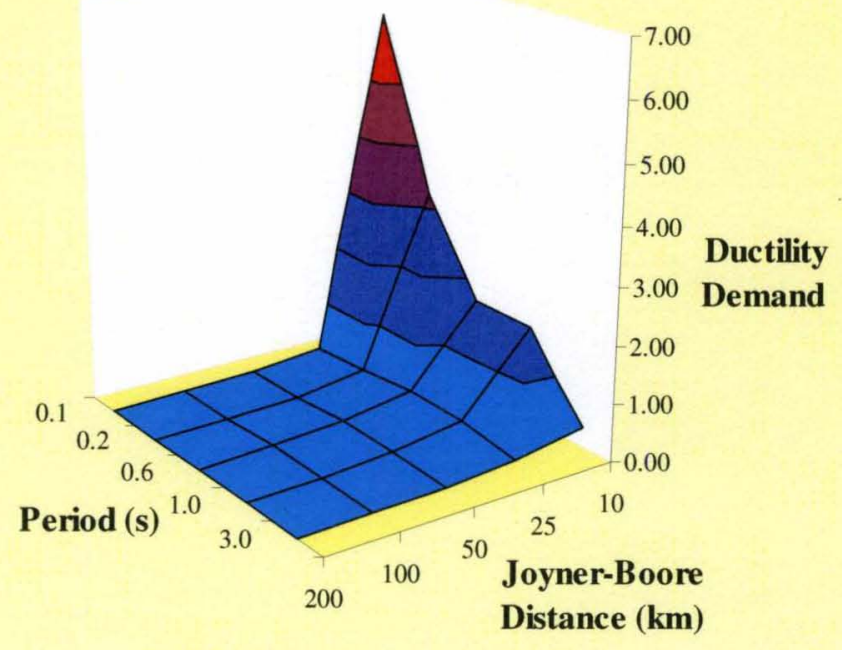

Figure 7.17 Variation in Standard Deviation of Ductility Demand from Magnitude 6.5

The trends in standard deviation and coefficient of variation for all scenarios coincide with the trends in the mean ductility demand from the magnitude 6.5 event. The plots of mean ductility demand, standard deviation in demand and coefficient of variation for ductility demand are shown in Figures 7.11, 7.12, and 7.13. High standard deviations for all structures are indicated at close distances with very high standard deviations at the shorter periods. This magnitude 6.5 event provides the 
highest coefficient of variation with values greater than one indicating that the standard deviation is greater than the mean response at very short periods and short distances. Figure 7.14 shows the highest coefficient of variation obtained in the study which was for an event with $M_{W}$ 7.5. The highest coefficient of variation for this event also occurs at the very short period responses but at a distance of $25 \mathrm{~km}$.

\section{Trends in Coeffecient of Variation for Ductility Demands from a $M_{W} 6.5$}

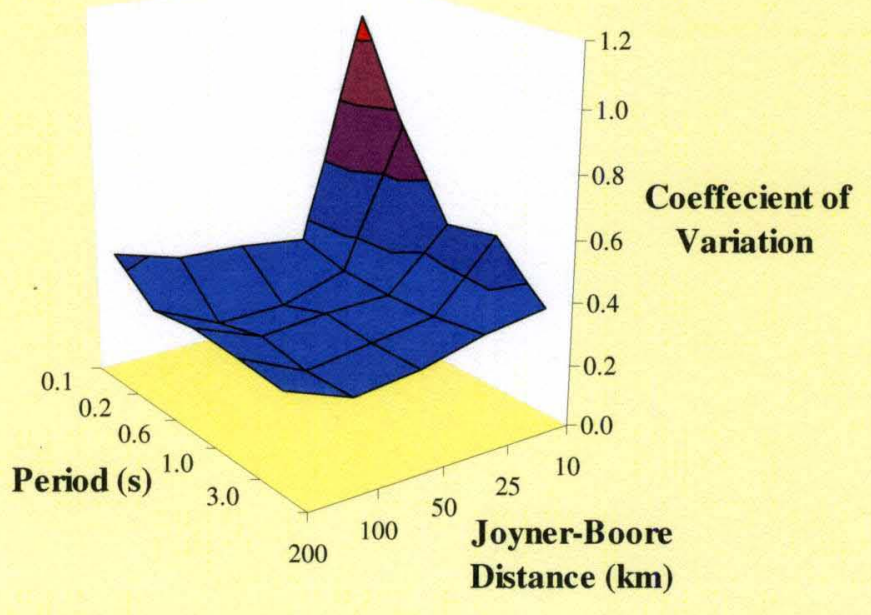

Figure 7.18 Coefficient of Variation in Ductility Demand from Magnitude 6.5

The overall trends show coincidence among high standard deviation values and those of high ductility demand. Extremely high standard deviations are indicated for very short-period structures at very close distances to the source. These same structures also exhibit extremely high ductility demands at these distances. Relatively 
high ductility demands are also indicated at $25 \mathrm{~km}$ to $50 \mathrm{~km}$ from the source for most structures, conditions where high standard deviations are indicated.

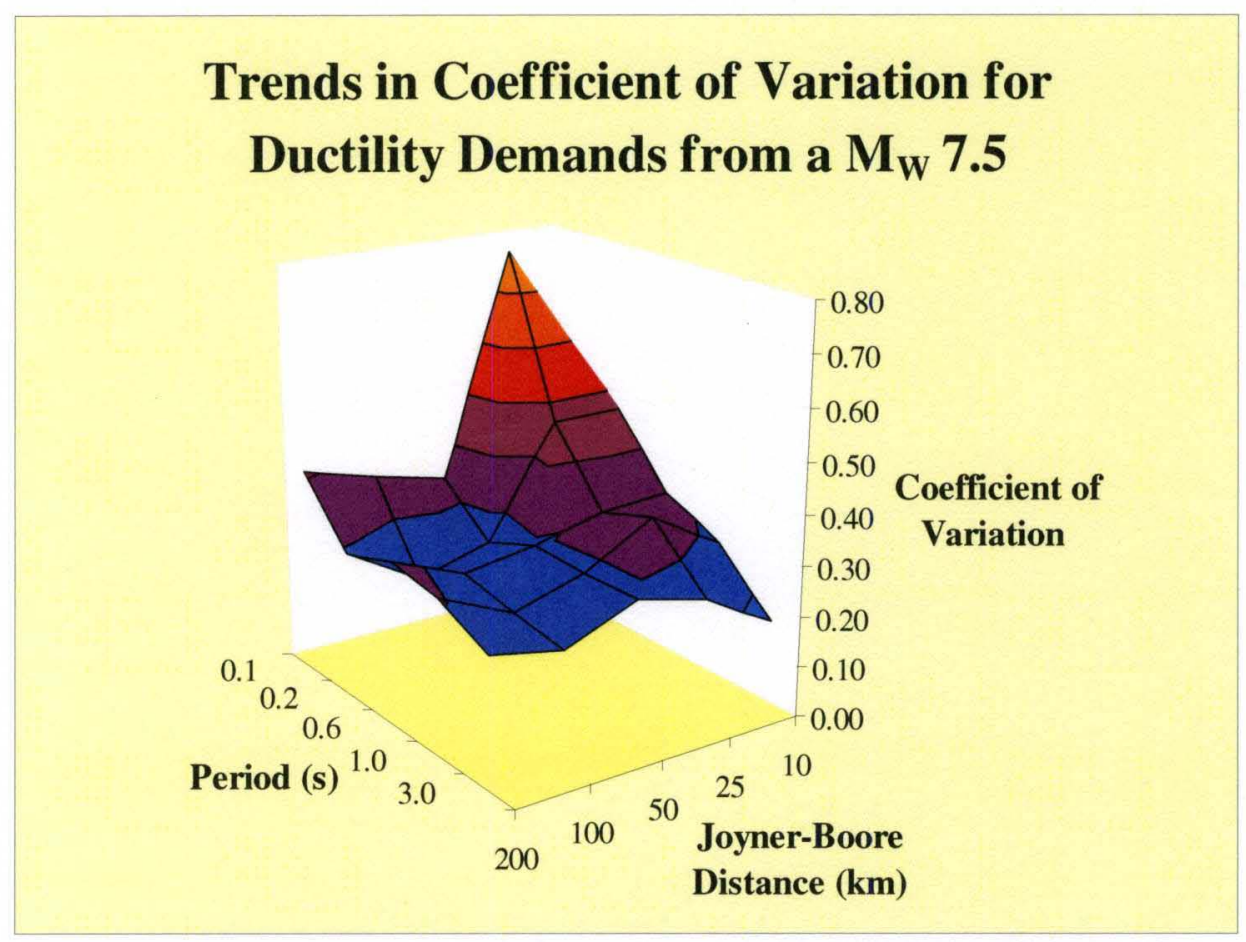

Figure 7.19 Coefficient of Variation in Ductility Demand from Magnitude 7.5

From the surface plots showing mean ductility demands it can be seen that high ductility demands are indicated for most structures at distances out to about $50 \mathrm{~km}$ while extremely high ductility demands are indicated for short-period structures located close to the source. Again, these areas indicating high ductility demands coincide with those where high standard deviations in the ductility demand are also indicated. 
Finally, for conciseness, only two of the plots showing the estimated magnitudes are shown here. The remaining plots for estimated magnitude are provided in Appendix C. Figures 7.15 and 7.16 shown here represent the low and high magnitudes included in this study. They represent the bookends for the trends seen in the estimated magnitudes. There is an overall trend at all magnitudes for the estimated values calculated to increase with distance.

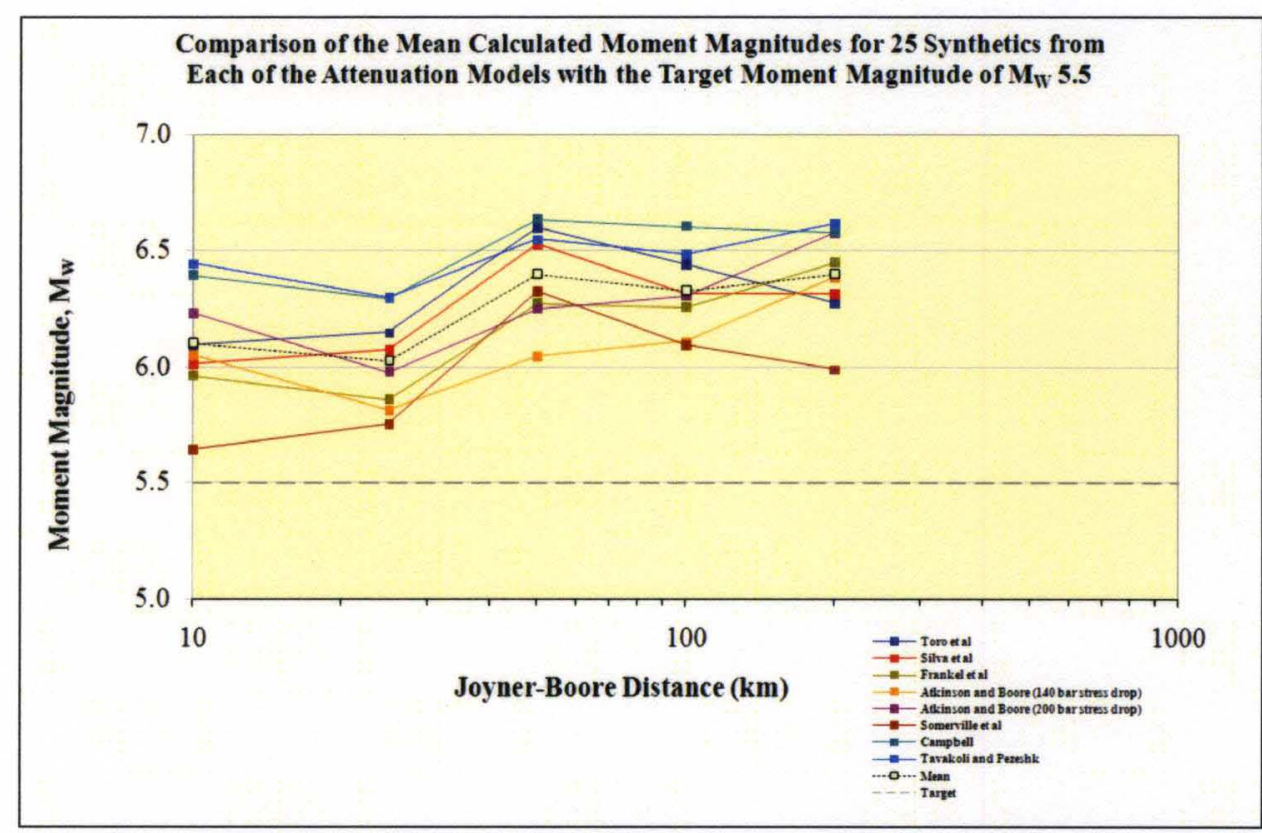

Figure 7.20 Mean Calculated Moment Magnitudes of Synthetic Ground Motions from CEUS Attenuation Models for Magnitude 5.5 


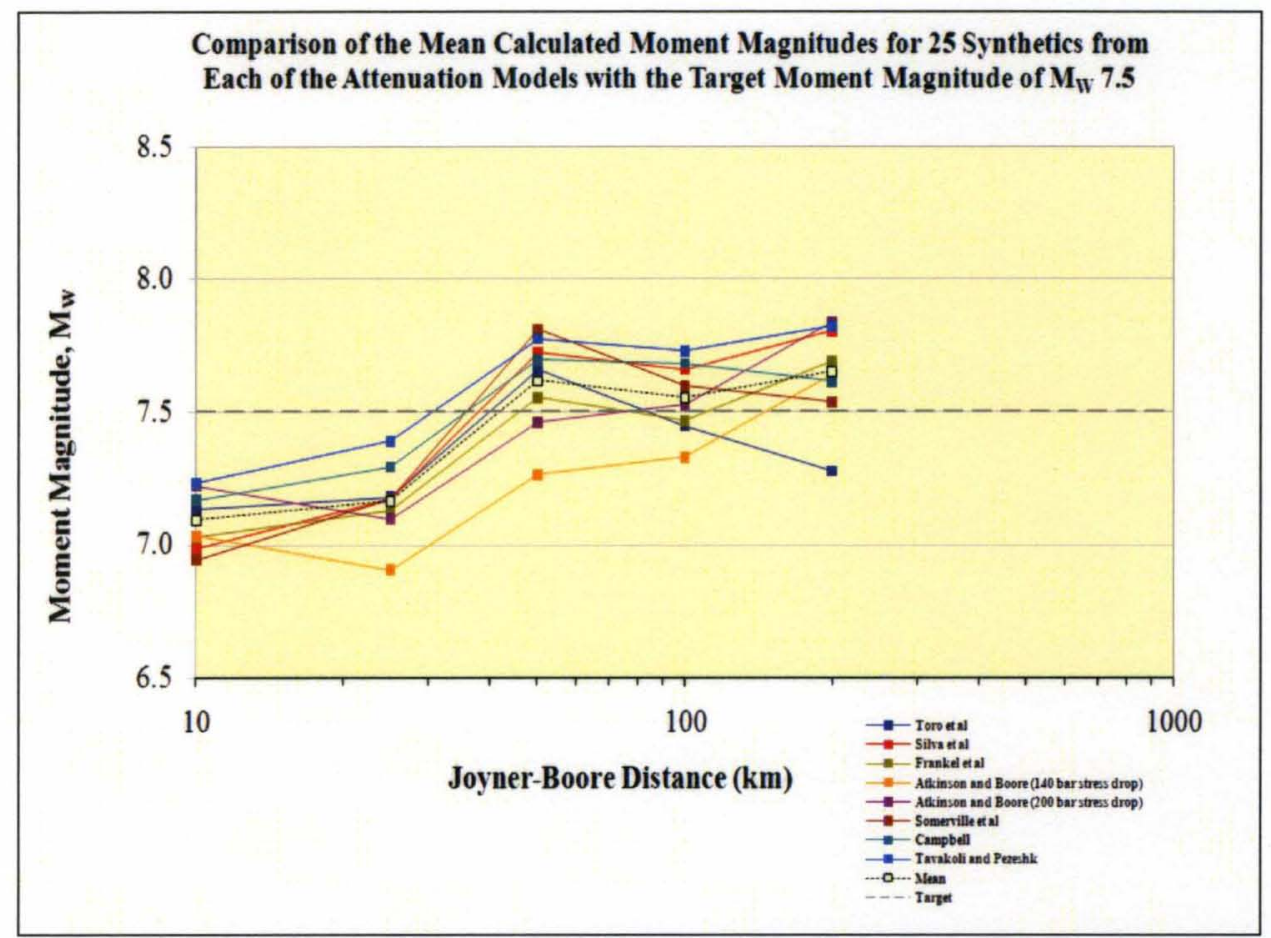

Figure 7.21 Mean Calculated Moment Magnitudes of Synthetic Ground Motions from CEUS Attenuation Models for Magnitude 7.5

\subsection{Application}

Even though it is not the intent of this study to test the validity of one model over the other, the variation among the structural responses begs the practicing engineer to ask which synthetic ground motions best represent the target scenario event. The magnitude estimation procedure developed in this study can be applied to help make decisions in this regard. The following figures help to illustrate how inferences can be made.

As an example of how the estimated moment magnitude may assist the practicing design engineer, it is pertinent to note that the ductility demands from the synthetic 
motions for the moment magnitude 6.5 at $25 \mathrm{~km}$ scenario showed the greatest

deviation in results. Confidence intervals for ductility demands coinciding with this event are shown in Figure 7.17. It is clearly shown from the chart that synthetics

\begin{tabular}{|c|c|c|c|c|c|c|c|c|c|}
\hline \multicolumn{10}{|c|}{ 95\% Confidence Intervals for Ductility Demand from $\mathbf{M}_{W} 6.5$} \\
\hline & & Toro et al & Silva et al & Frankel et al & $\begin{array}{l}\text { Atkinson and } \\
\text { Boore } \\
\text { (140 bar stress drop) }\end{array}$ & $\begin{array}{c}\text { Atkinson and } \\
\text { Beore } \\
\text { (200 barstress drop) }\end{array}$ & Somerville et al & Campbell & $\begin{array}{c}\text { Tavakoli and } \\
\text { Pezeshk }\end{array}$ \\
\hline \multirow{5}{*}{$10 \mathrm{~km}$} & 0.1 & $(1.370,1.903)$ & $(0.9063,0.9828)$ & $(2.734,3.653)$ & $(2.155,2.738)$ & $(9.493,13.342)$ & $(0.56518,0.60123)$ & $(6.752,8.829)$ & $(15.67,19.88)$ \\
\hline & 0.2 & $(2.251,3.007)$ & $(1.603,2.174)$ & $(1899,2478)$ & $(3.070,3.929)$ & $(5.935,8.005)$ & $(0.8720,1.0440)$ & $(4.857,6.886)$ & $(9.029,11.982)$ \\
\hline & 0.6 & $(2.626,3.595)$ & $(2.038,2.742)$ & $(2.227,2997)$ & $(2.306,3.158)$ & $(3.586,4.863)$ & $(1.2500,1.5653)$ & $(4.062,5.596)$ & $(5.714,7.646)$ \\
\hline & 1.0 & $(1.958,2626)$ & $(182622000$ & - 2091,270$)$ & (2.214, JITY) & Trat, & (46) & $(3.946,5.714)$ & $(5.184,7.294)$ \\
\hline & 3.0 & Comev, TU1U9) & $(0.6737,0.9017)$ & $(0.7533,0.9848)$ & $(0.6971,0.9355)$ & $(0.9600,1.3136)$ & $(0.4393,0.5887)$ & $(1.0999,7904)$ & $(1.337,1.871)$ \\
\hline \multirow{10}{*}{$50 \mathrm{~km}$} & 0.1 & $(0.4898,0.5601)$ & $(0.43419,0.46573)$ & $(0.7678,0.9029)$ & $(0.33820,0.36945)$ & $(0.4673,0.5111)$ & $(0.30123,0.32523)$ & $(0.5961,0.6389)$ & $(0.7049,0.021)$ \\
\hline & 0.2 & $(0.7290,0.8628)$ & $(0.6732,0.8034)$ & $(0.7026,0.8104)$ & $(0.5398,0.6376)$ & $(0.7574,0.8993)$ & $(0.4614,0.5503)$ & $(0.9316,1.1337)$ & $(1.1184,1.4217)$ \\
\hline & 0.6 & $(1.0693,12790)$ & $(1.0004,1.2350)$ & $(0.9474,1.2213)$ & $(0.7181,0.9056)$ & $(0.9782,11787)$ & $(0.7211,0.9126)$ & $(1.3358,1.6735)$ & $(1.6101,2.0045)$ \\
\hline & 1.0 & $(0.9077,1.1068)$ & $(0.8929,1.1300)$ & $(0.9269,1.1510)$ & $(0.6120,0.7663)$ & $(0.8389,1.0994)$ & $(0.6389,0.7860)$ & (1. 1968, 1.5373) & $(1.4434,1,791$ \\
\hline & 90 & $(0.3436,0.4656)$ & $(0.3559,0.4648)$ & $(0.3683,0.4821)$ & $(0.2326,0.3123)$ & $(0.3236,0.4312)$ & $(0.2594,0.3428)$ & $(0.4832,0.6382)$ & (69074, 0.7843) \\
\hline & 0.1 & $(0.18827,0.20305)$ & Nomowe 0.21628 & $(0.3275,0.3880)$ & $(0.10062,0.10781)$ & $(0.14031,0.15005)$ & (1.1.17640 0.18009) & (0.21851.0.23398) & $(0.23473,0.25432)$ \\
\hline & 0.2 & $(0.2924,0.3508)$ & $(0.3006,0.3567)$ & $(0.2645,0.3128)$ & $(0.15569,0.18339)$ & $(0.2145,0.2569)$ & $(0.2444,0.2910)$ & $(0.3299,0.3972)$ & $(0.3425,0.4015)$ \\
\hline & 0.6 & $(0.4784,0.5843)$ & $(0.5056,0.6378)$ & $(0.2897,0.3698)$ & $(0.2438,0.3092)$ & $(0.3449,0.4433)$ & $(0.4152,0.5303)$ & $(0.5380,0.6739)$ & $(0.5704,0.7273)$ \\
\hline & 1.0 & $(0.4175,0.5117)$ & $(0.4490,0.5489)$ & $(0.2812,0.3729)$ & $(0.2167,0.2624)$ & $(0.3068,0.3729)$ & $(0.3614,0.4327)$ & $(0.4690,0.5827)$ & $(0.4979,0.6062)$ \\
\hline & 3.0 & $(0.1655,0.2260)$ & $(0.1868,0.2432)$ & $(0.1221,0.1656)$ & $(0.08736,0.11570)$ & $(0.12569,0.16457)$ & $(0.1509,0.1969)$ & $(0.1966,0.2536)$ & $(0.2101,0.2755)$ \\
\hline \multirow{5}{*}{$100 \mathrm{~km}$} & 0.1 & $(0.07259,0.07795)$ & $(0.078173,0.082027)$ & $(0.14714,0.17637)$ & $(0.055716,0.059068)$ & $(0.07741,0.08205)$ & $(0.06305,0.06722)$ & $(0.10712,0.11435)$ & $(0.11624,0.12649)$ \\
\hline & 0.2 & $(0.10162,0.12014)$ & $(0.10932,0.12560)$ & $(0.12841,0.15321)$ & $(0.07898,0.09364)$ & $(0.10958,0.12855)$ & $(0.08168,0.09665)$ & $(0.14078,0.16619)$ & $(0.14476,0.17103)$ \\
\hline & 0.6 & $(0.18099,0.21978)$ & $(0.2192,0.2748)$ & $(0.1604,0.2025)$ & $(0.15081,0.19016)$ & $(0.2122,0.2651)$ & (0.16052, 0.19972) & $(0.2815,0.3463)$ & $(0.2905,0.3548)$ \\
\hline & 1.0 & $(0.15880,0.19869)$ & $(0.19571,0.23656)$ & $(0.1610,0.2084)$ & $(0.13186,0.15501)$ & $(0.18480,0.21951)$ & $(0.13779,0.16166)$ & $(0.2408,0.2846)$ & $(0.2485,0.2912)$ \\
\hline & 3.0 & $(0.06533,0.08650)$ & \begin{tabular}{|c|}
$(0.08327,0.10914)$ \\
\end{tabular} & $(0.06913,0.09342)$ & $(0.05516,0.07305)$ & $(0.07704,0.10090)$ & $(0.05791,0.07668)$ & $(0.10139,0.13236)$ & $(0.10533,0.13704)$ \\
\hline \multirow{5}{*}{$200 \mathrm{~km}$} & 0.1 & $(0.03739,0.04346)$ & $(0.026876,0.028940)$ & $(0.05860,0.06731)$ & $(0.032021,0.034643)$ & $(0.044877,0.048195)$ & $(0.017268,0.018588)$ & $(0.06919,0.07791)$ & $(0.06248,0.07077)$ \\
\hline & 0.2 & $(0.03369,0.03793)$ & $(0.03550,0.04014)$ & $(0.05407,0.06064)$ & $(0.03791,0.04233)$ & $(0.05332,0.05921)$ & $(0.021874,0.024654)$ & $(0.05880,0.07046)$ & $(0.06169,0.07014)$ \\
\hline & 0.6 & $(0.04368,0.05450)$ & $(0.09204,0.10667)$ & $(0.08029,0.09482)$ & $(0.09332,0.10632)$ & $(0.13140,0.14918)$ & $(0.05150,0.05904)$ & $(0.08773,0.10290)$ & \begin{tabular}{|l|}
$(0.12802,0.14597)$ \\
\end{tabular} \\
\hline & 1.0 & $(0.03845,0.04561)$ & $(0.07814,0.09579)$ & $(0.07860,0.09917)$ & $(0.07668,0.09443)$ & $(0.10725,0.13272)$ & $(0.04218,0.05194)$ & $(0.07596,0.09527)$ & $(0.10440,0.13013)$ \\
\hline & 3.0 & $(0.01877,0.02306)$ & $(0.03299,0.04371)$ & $(0.03555,0.04710)$ & $(0.03338,0.04246)$ & $(0.04656,0.05981)$ & $(0.01817,0.02348)$ & $(0.03390,0.04293)$ & $(0.04628,0.05831)$ \\
\hline
\end{tabular}

Figure 7.22 Confidence Intervals for Ductility Demand from Magnitude 6.5

from all of the models except two indicate ductility demands greater than one for the 0.6 and 1.0 second SDOF systems. Figure 7.18 also provides a chart showing the $95 \%$ confidence intervals for estimated moment magnitude of the synthetics for this event. As is shown in Figure 7.19, the synthetic motions from the only two attenuation models whose confidence intervals contain the target moment magnitude of 6.5 are also the only two models whose synthetic motions indicate no damage. 
It could be logically inferred that the synthetic motions compatible with the other attenuation models as shown in Figure 7.20, are perhaps over predicting the response of these SDOF systems.

\begin{tabular}{|c|c|c|c|c|c|c|c|c|}
\hline \multicolumn{9}{|c|}{$95 \%$ Confidence Intervals for $M_{W}$ with a Target of $M_{W} 6.5$} \\
\hline & Toro et al & Silva et al & Frankel et al & $\begin{array}{l}\text { Atkinson and } \\
\text { Boore } \\
\text { (140 bar stress drop) }\end{array}$ & $\begin{array}{c}\text { Atkinson and } \\
\text { Boore } \\
\text { (200 bar stress drap) }\end{array}$ & Somerville et al & Campbell & $\begin{array}{l}\text { Tavakoli and } \\
\text { Pezeshk }\end{array}$ \\
\hline $10 \mathrm{~km}$ & $(6.5685,6.6494)$ & $(6.5010,6.5779)$ & $(6.4955,6.6035)$ & $(65557,6.6642)$ & $(6.7455,6.8534)$ & $(6.2330,63162)$ & $(6.7991,6.8786)$ & $(6.9240,69984)$ \\
\hline $25 \mathrm{~km}$ & $(6.6361,6.7060)$ & $(6.6205,6.6971)$ & $(6.5940,6.6963)$ & $(6.3983,6.4860)$ & $(6.5923,6.6746)$ & $(6.4260,6.5002)$ & $(6.8003,6.8843)$ & $(6.9109,6.9902)$ \\
\hline $50 \mathrm{~km}$ & $(7.0972,7.1705)$ & $(7.1406,7.2148)$ & $(68404,69630)$ & $(6.7233,6.7957)$ & $(69221,69970)$ & $(7.0248,7.0935)$ & $(7.1758,72482)$ & $(72114,72789)$ \\
\hline $100 \mathrm{~km}$ & $(6.8975,6.9798)$ & $(7.0184,7.0744)$ & $(6.8681,6.9781)$ & $(6.7926,6.8589)$ & $(6.9862,7.0449)$ & $(6.8173,6.8934)$ & $(7.1334,7.1987)$ & $(7.1411,7.2212)$ \\
\hline $200 \mathrm{~km}$ & $(6.7017,6.7900)$ & $(7.0575,7.1278)$ & $(7.0928,7.1529)$ & $(7.0592,7.1364)$ & $(7.2508,7.3294)$ & $(6.7185,6.7918)$ & $(0.0403,7.1539)$ & $(7.2481,7.3358)$ \\
\hline \multicolumn{9}{|c|}{ Based on 25 Observations per Model and a One-Sample t-Test vith a 95\% Confidence Level. } \\
\hline & \multicolumn{8}{|c|}{ Indicates that the Confidence Interval Contains the Target $\mathbf{M}_{\mathrm{w}}$. } \\
\hline & \multicolumn{8}{|c|}{ Indicates that the Confidence Interval is Greater Than the Target $\mathrm{M}_{\mathrm{w}}$. } \\
\hline & Indicates that the $\mathrm{C}$ & ence Interval & ss Than the Ta: & & & & & \\
\hline
\end{tabular}

Figure 7.23 Confidence Intervals for Calculated Magnitudes with Target Magnitude of 6.5 


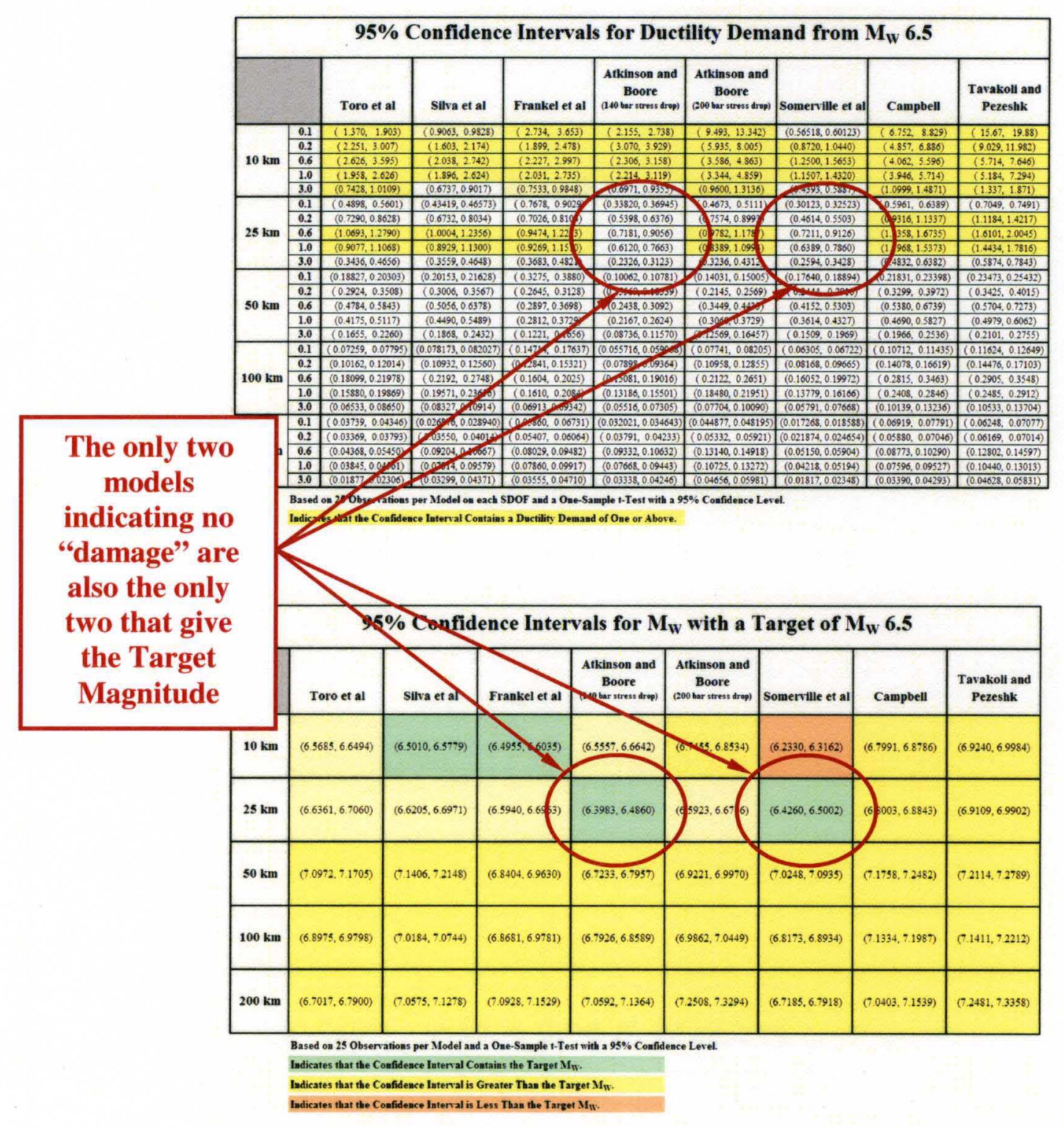

Figure 7.24 Comparison of Confidence Intervals for Ductility Demand and Calculated Magnitude for Magnitude 6.5 


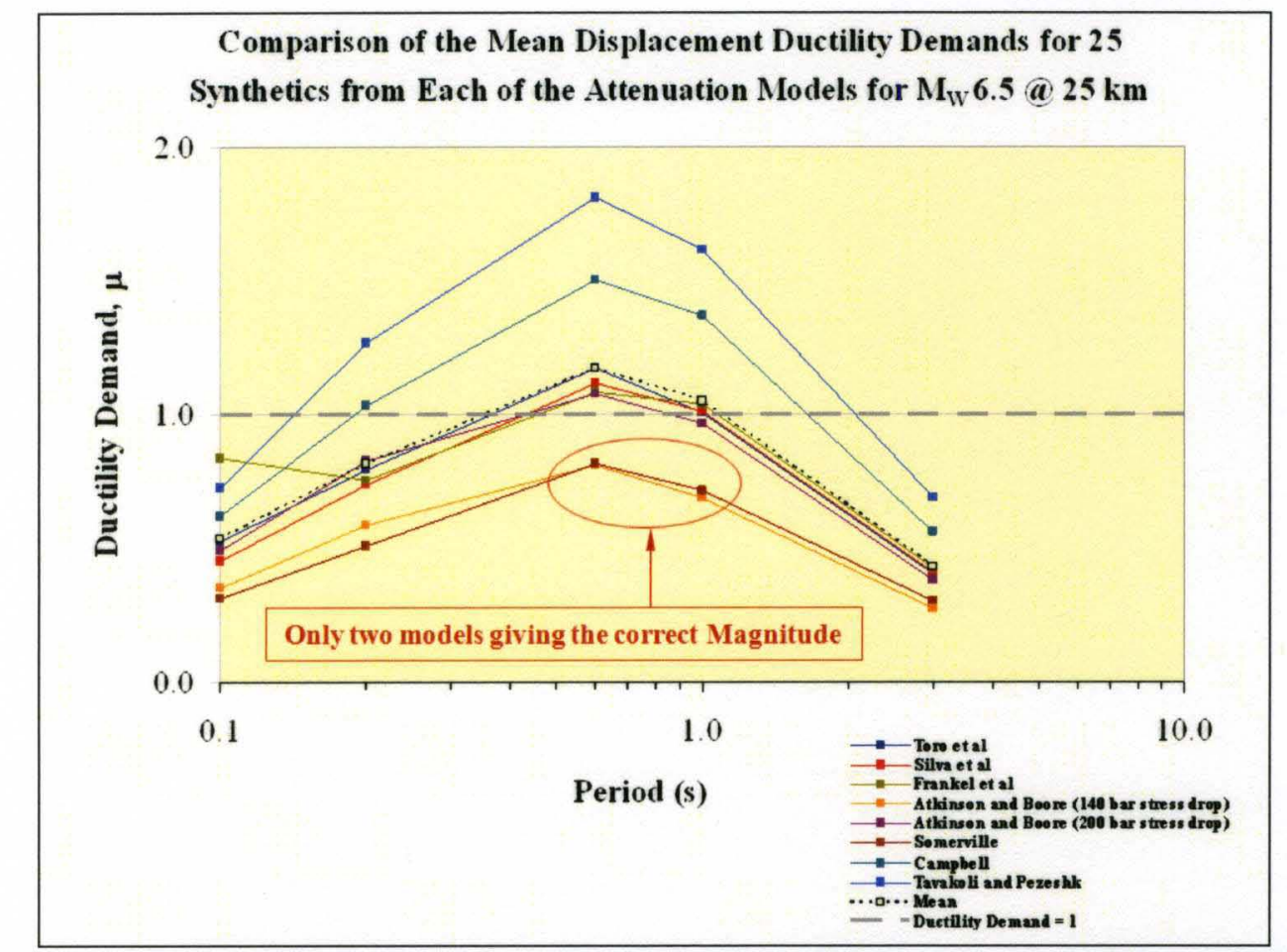

Figure 7.25 Ductility Demands from Synthetic Ground Motions Generated from CEUS Attenuation Models for Magnitude 6.5 at $25 \mathrm{~km}$

In addition, as an example of how the estimated moment magnitude may assist the practicing design engineer in recognizing discrepancies in ductility demand, consider the moment magnitude 7.5 event simulated at distances of 10 and $25 \mathrm{~km}$. On first glance at Figure 7.21 it appears that the confidence intervals for these scenarios appear to be in good agreement on high ductility demand. However, as shown in Figure 7.22, estimated moment magnitudes for synthetic motions from all of the models are below the target magnitude. This result implies that although responses to all synthetic motions indicate yielding, the analyses may be under predicting the amount of ductility required by this scenario. It is important to have a good measure 


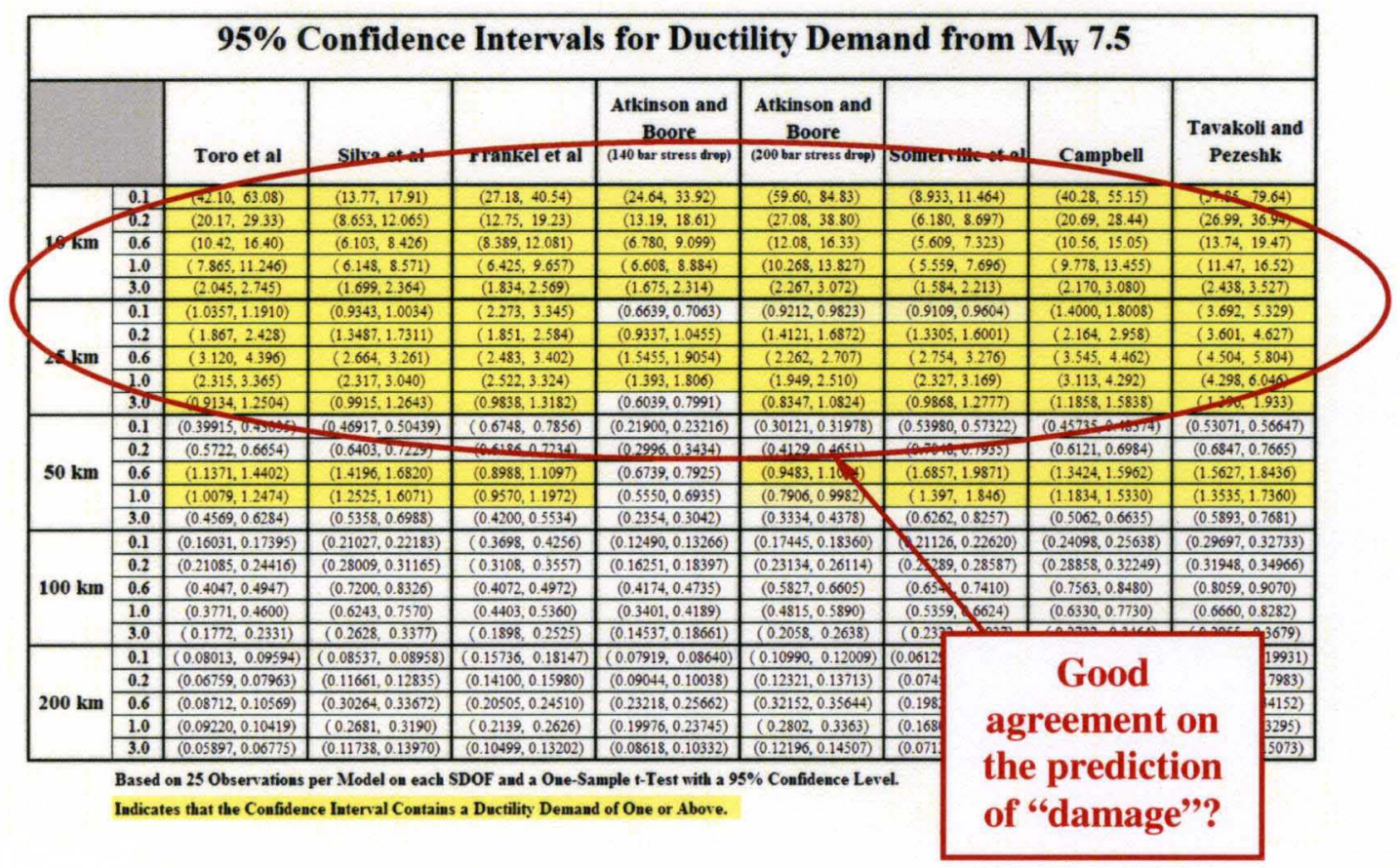

Figure 7.26 Confidence Intervals for Ductility Demand from Magnitude 7.5

of ductility demand to produce an effective seismic design. As shown in Figure 7.23, even though synthetic motions from nearly all of the attenuation models produce ductility demands greater than one for a moment magnitude 7.5 event at $25 \mathrm{~km}$, their large variations in value could also lead to large variations in design effort, approach, and cost. 


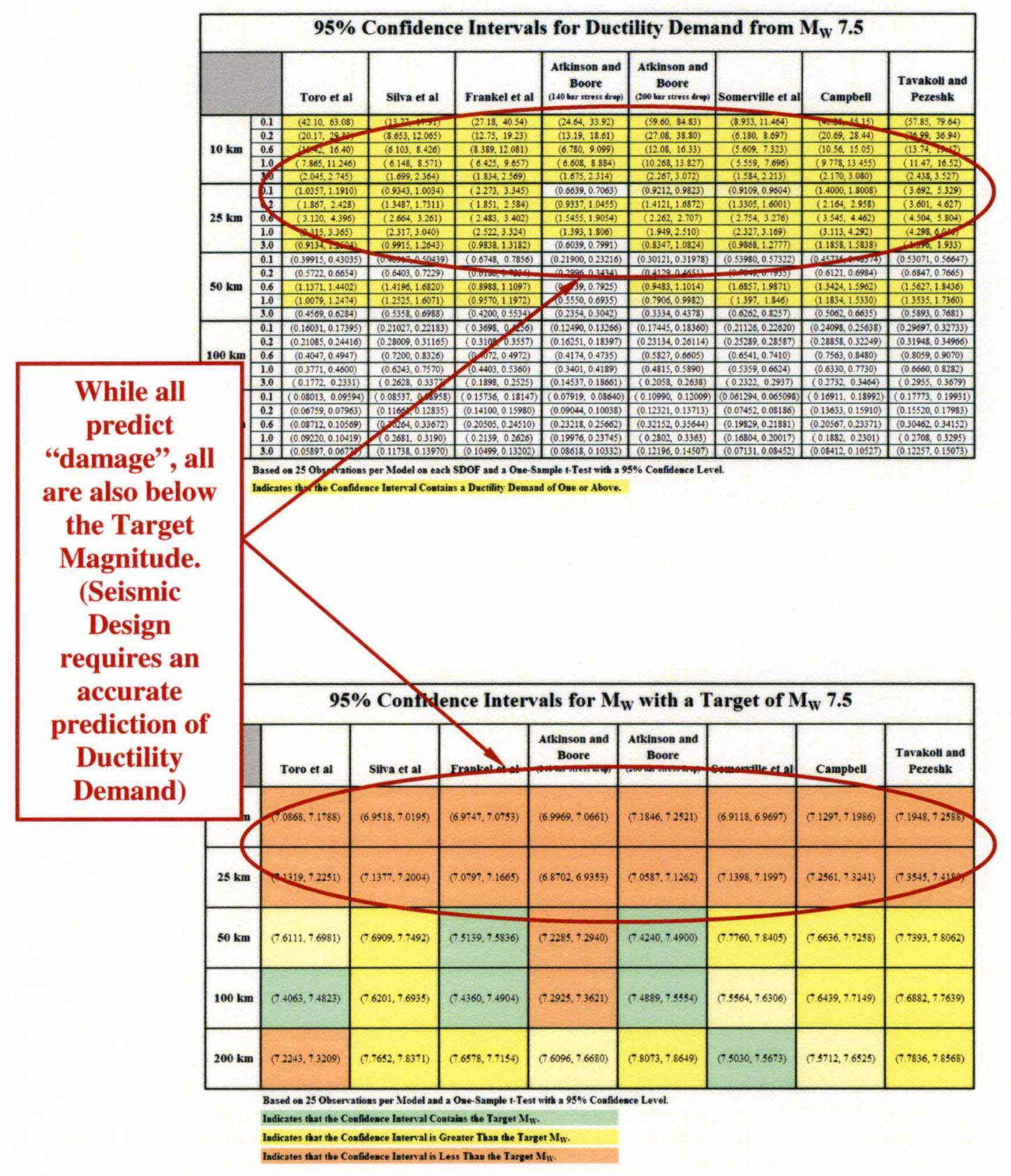

Figure 7.27 Comparison of Confidence Intervals for Ductility Demand and Calculated Magnitude for Magnitude of 7.5 


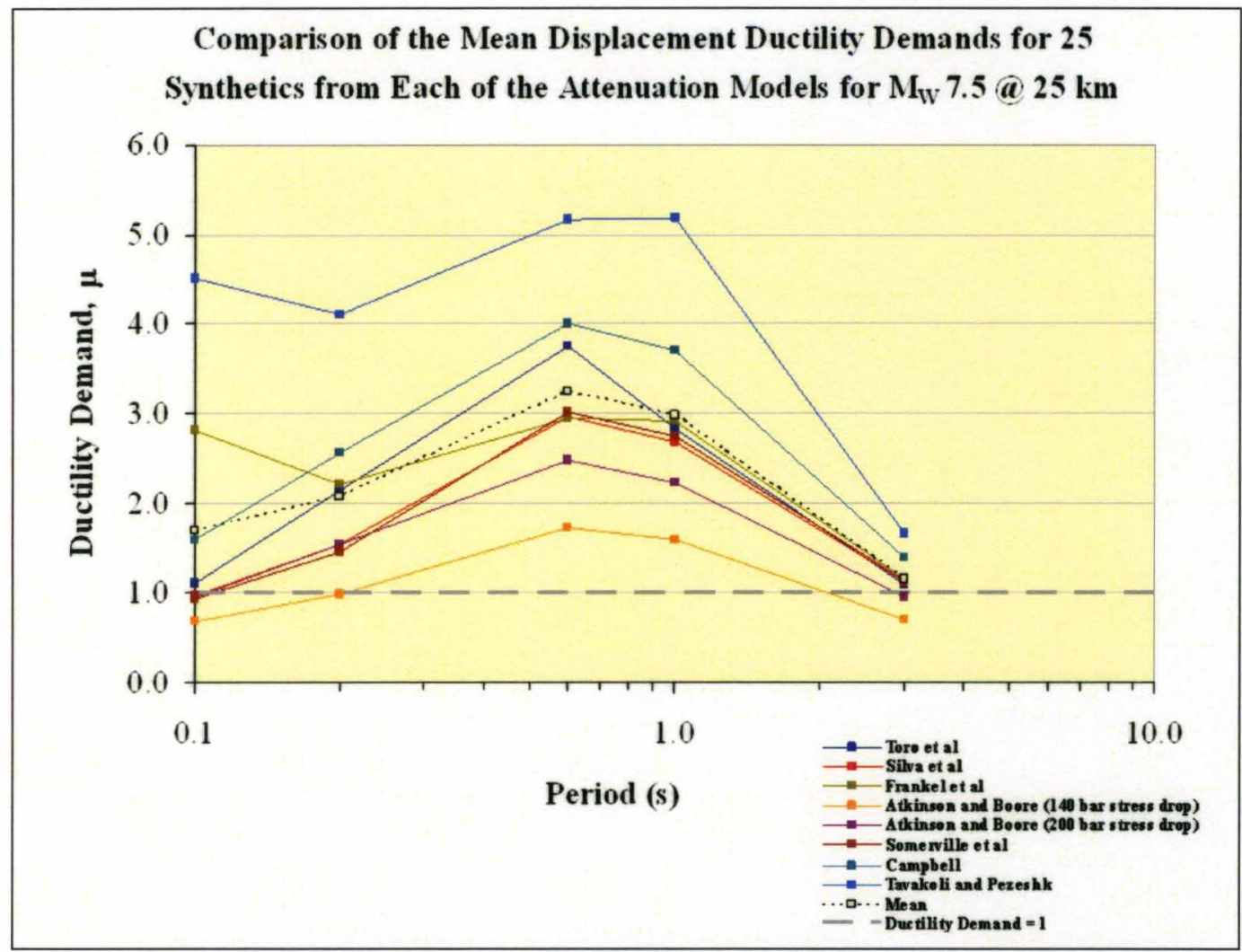

Figure 7.28 Ductility Demands from Synthetic Ground Motions Generated from CEUS Attenuation Models for Magnitude 7.5 at $25 \mathrm{~km}$

As a final example of how the estimated moment magnitude could be applied, note that in Figure 7.24, the Atkinson and Boore Model with a stress drop of 140 bars indicates no damage for structures with natural periods of 0.6 and 1.0 seconds from a magnitude 7.5 event at $50 \mathrm{~km}$. However, Figure 7.25 shows that the for magnitude 7.5 events this model is producing synthetic motions whose mean estimated moment magnitude is below the target magnitude. This result indicates that if the synthetic ground motions from this attenuation model were taken to represent this magnitude and distance scenario event, they would under predict the structural response. 


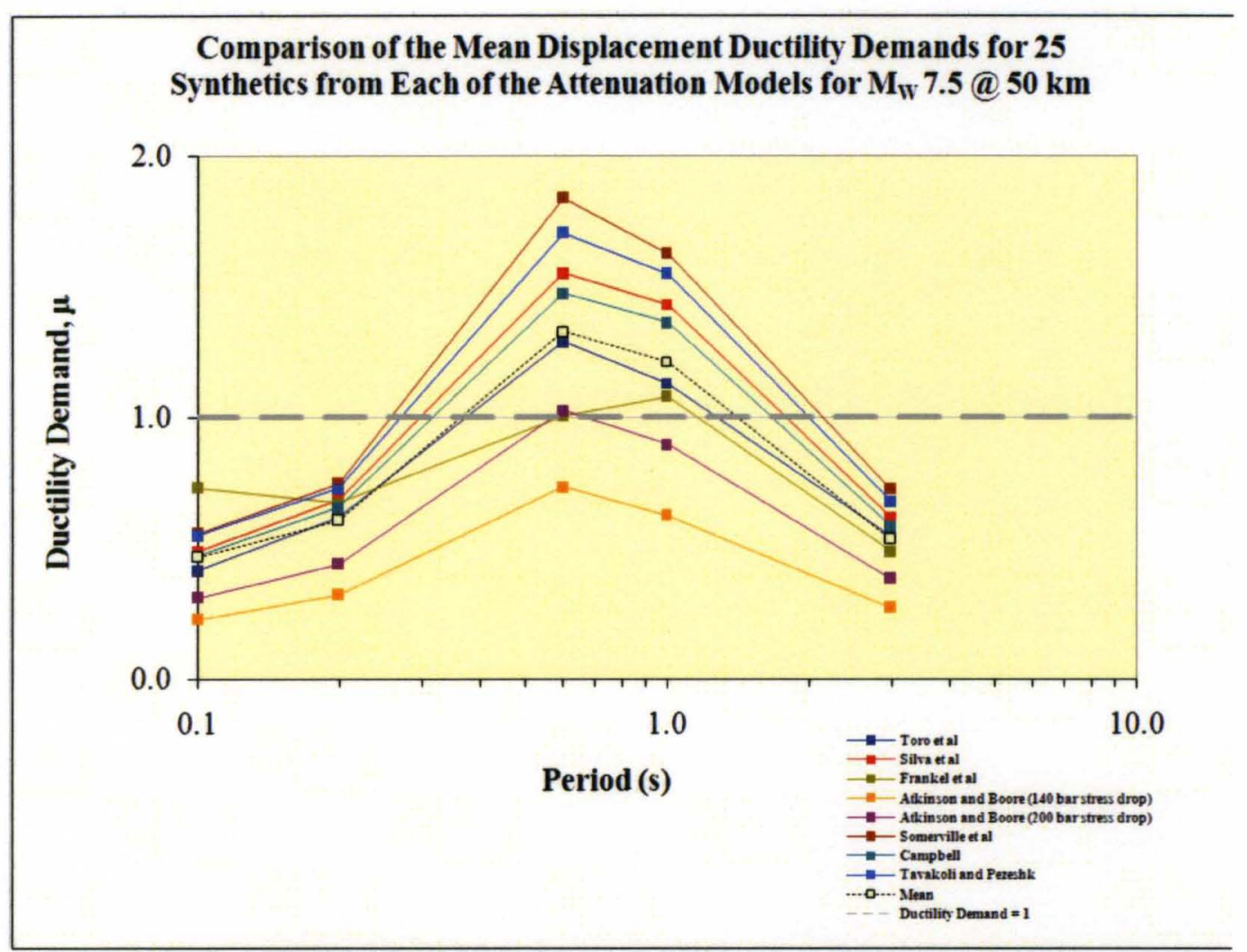

Figure 7.29 Ductility Demands from Synthetic Ground Motions Generated from CEUS Attenuation Models for Magnitude 7.5 at $50 \mathrm{~km}$

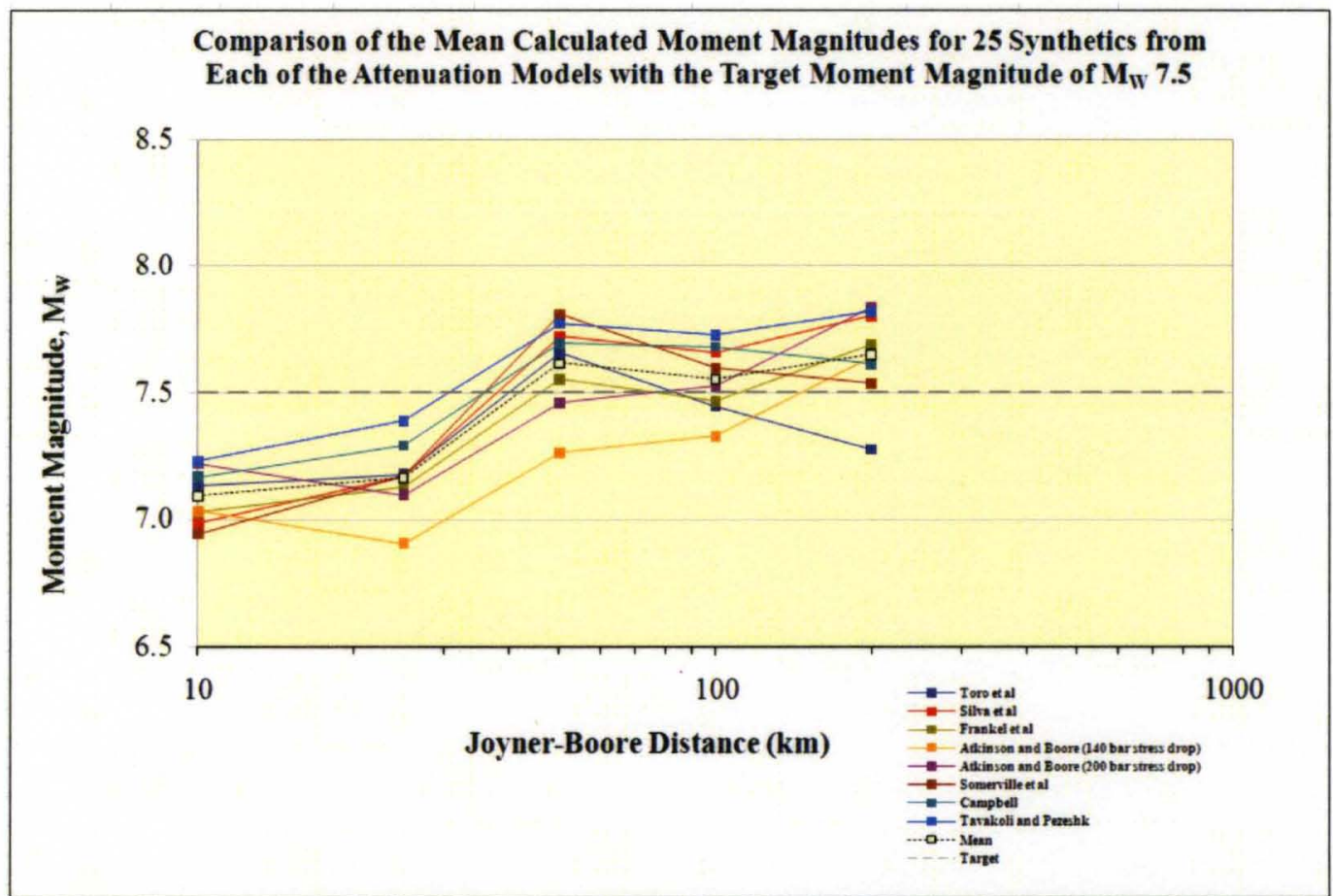

Figure 7.30 Mean Calculated Moment Magnitudes of Synthetic Ground Motions from CEUS Attenuation Models for Magnitude 7.5 


\subsection{Summary}

In summary, the results from this study indicate that there is a wide variation among the predicted ductility demands from nonlinear time history analysis using the synthetic ground motions compatible with the CEUS attenuation models. It has been shown that the estimated magnitude of the synthetic ground motions may be used to infer the quality of prediction of ductility demand. Based on this application of the estimated magnitude, the results indicate that the over prediction of magnitude for these synthetic ground motions increases with distance up to $50 \mathrm{~km}$ ( corrections for ENA were made at distances of $100 \mathrm{~km}$ and $200 \mathrm{~km}$ ). Furthermore, the results of this application indicate that the under prediction of magnitude increases with magnitude at distances of $10 \mathrm{~km}$ and $25 \mathrm{~km}$. A significant increase in ductility demand from the synthetic ground motions at increased distances with increasing magnitude was not seen as might have been expected. However, a dramatic increase in ductility demand at close distances was seen with increasing magnitude. Finally, the results of the study indicate that high standard deviations coincide with high ductility demands produced by synthetic motions compatible with the attenuation models. 


\subsection{Problem Encountered}

The first attempt at this study was conducted using a 0.01 second discretization interval in the process of generating the synthetic ground motions as is common practice. The initial results of this study gave unrealistically benign structural responses. For example, in the results from the first study there were cases in which the ductility demands for all structures were below a value of one (i.e. indicating no damage) from a moment magnitude 7.0 event at $10 \mathrm{~km}$. These results were suspect at best and the entire study was conducted a second time rather than report such unlikely findings and it is the results from the second study that are presented in this dissertation.

After an extensive review of the input response spectra of the actual records for the CEUS events used in the pilot study and those for similar scenarios from the attenuation models, it became evident that there is a relatively large amount of high frequency content requested by the attenuation models. The response spectra from several of the synthetic motions that were checked appeared to match the overall shape of the target spectrum but fell below their target amplitudes. It was also noted that the synthetic motions generated in the first attempt had Fourier spectra that tended toward randomness [a noise condition].

Truncating the input spectra on the high frequency end was considered at this point; however, because each attenuation model provides a different number of spectral values at different periods, developing a method to determine which spectral 
accelerations to eliminate seemed counter to the intent of this study to be fair to all models. In the review just mentioned of the response spectra from actual records it was noticed that many of the actual records were provided using a 0.02 second discretization interval.

It was decided, therefore, to conduct the study the second time using a discretization interval of 0.02 seconds. The results of this attempt at the study gave more realistic responses as well as more realistic Fourier spectra. However, it was once again discovered that the spectra from the synthetic motions fell well below their targets from the attenuation models especially on the low-period end of the spectra. This result was both disappointing and mysterious especially in light of the fact that all of the synthetic motions generated using the same methodology for the pilot study including the two events recorded within the CEUS matched their targets very well. Moreover, motions generated from all models appeared to vary from their targets in a similar fashion and that the same process was used for all of the models in the main study. It was also recognized that the situation improved when the discretization interval was increased and that it was not possible to check the spectra of every model for every event at that point.

The results from the second study show that there is wide variation in the responses from the synthetic motions developed from the attenuation models. In order to verify that this variation is not due to the response spectra for the synthetic motions being below the target spectra and therefore not representative of the ground 
motions described by the attenuation models, a study investigating the truncation of the input spectra from the attenuation models of spectral accelerations at frequencies well above the natural frequencies of most practical structures was considered. An explanation and results of this truncation study is provided in the following pages.

\subsubsection{Truncation Study}

This truncation study was conducted using the magnitude and distance combinations that caused the greatest variation among those investigated in the main study. The input spectra for the two models that produced the highest and lowest responses for these scenarios were truncated and their structural responses were compared both to each other and to the response without truncation.

To be concise and in consideration of the fact that this study was conducted as an addendum to the main study, three representative events showing variation were chosen to be studied. The intent was to select these events to form bounds enclosing all of the events in the study.

Early studies showed that two events with some of the most variation in response among all of the models were the moment magnitude 5.5 at $10 \mathrm{~km}$ and the moment magnitude 7.5 at $50 \mathrm{~km}$ events. The models with the lowest and highest response for these events were used to investigate truncating the input spectra at the high frequency end. Also as pointed out earlier, the magnitude 6.5 at $25 \mathrm{~km}$ was among 
the most uncertain in ductility demands produced from the synthetic motions and therefore it was also included in the truncation study.

In further interest of conciseness, the attenuation models whose synthetics produced the extremes in ductility demand were only considered in the truncation study for the Magnitude 5.5 at $10 \mathrm{~km}$ and 7.5 at $50 \mathrm{~km}$ events. Again, the hope was that these responses formed the boundaries for the other responses within the scenarios included in the study. Finally, situated firmly within these boundaries is the magnitude 6.5 at $25 \mathrm{~km}$ scenario and for this scenario, the synthetic motions from all eight of the attenuation models were used in the truncation study. Figure 7.26 shows the ductility demands for the synthetics from the two attenuation models for the magnitude 5.5 at $10 \mathrm{~km}$ event used in the truncation study. Figures7.27 and 7.28 show the target response spectra from these two attenuation models along with those from their synthetics generated using 0.01 and 0.02 second discretization intervals and with their input spectral accelerations truncated below 0.1 and 0.2 second periods. Notice that the spectra progressively approach their targets as the short period accelerations are truncated from the input spectra. 


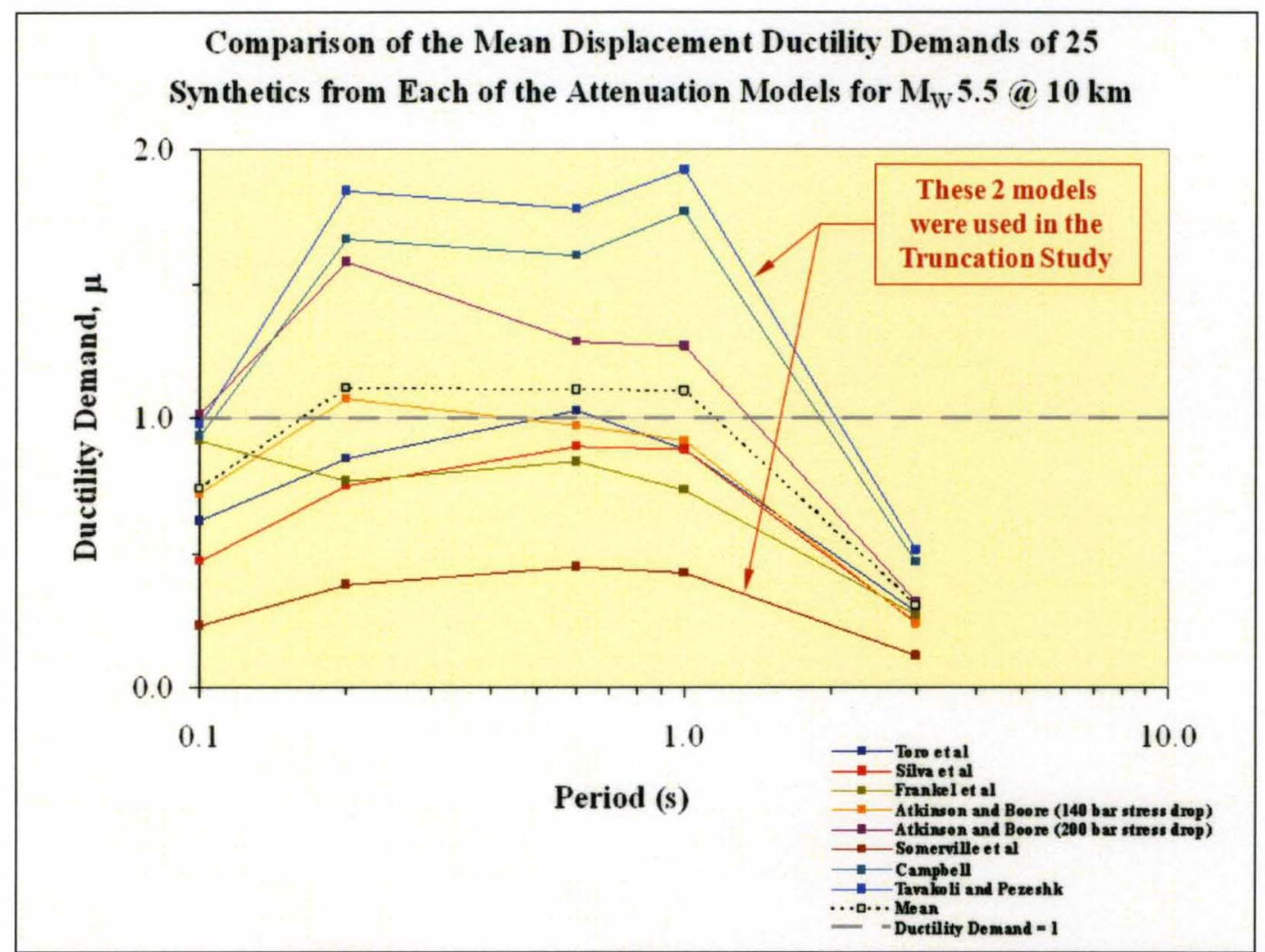

Figure 7.31 Selection of Models for Magnitude 5.5 at $10 \mathrm{~km}$ to be used in the Truncation Study 


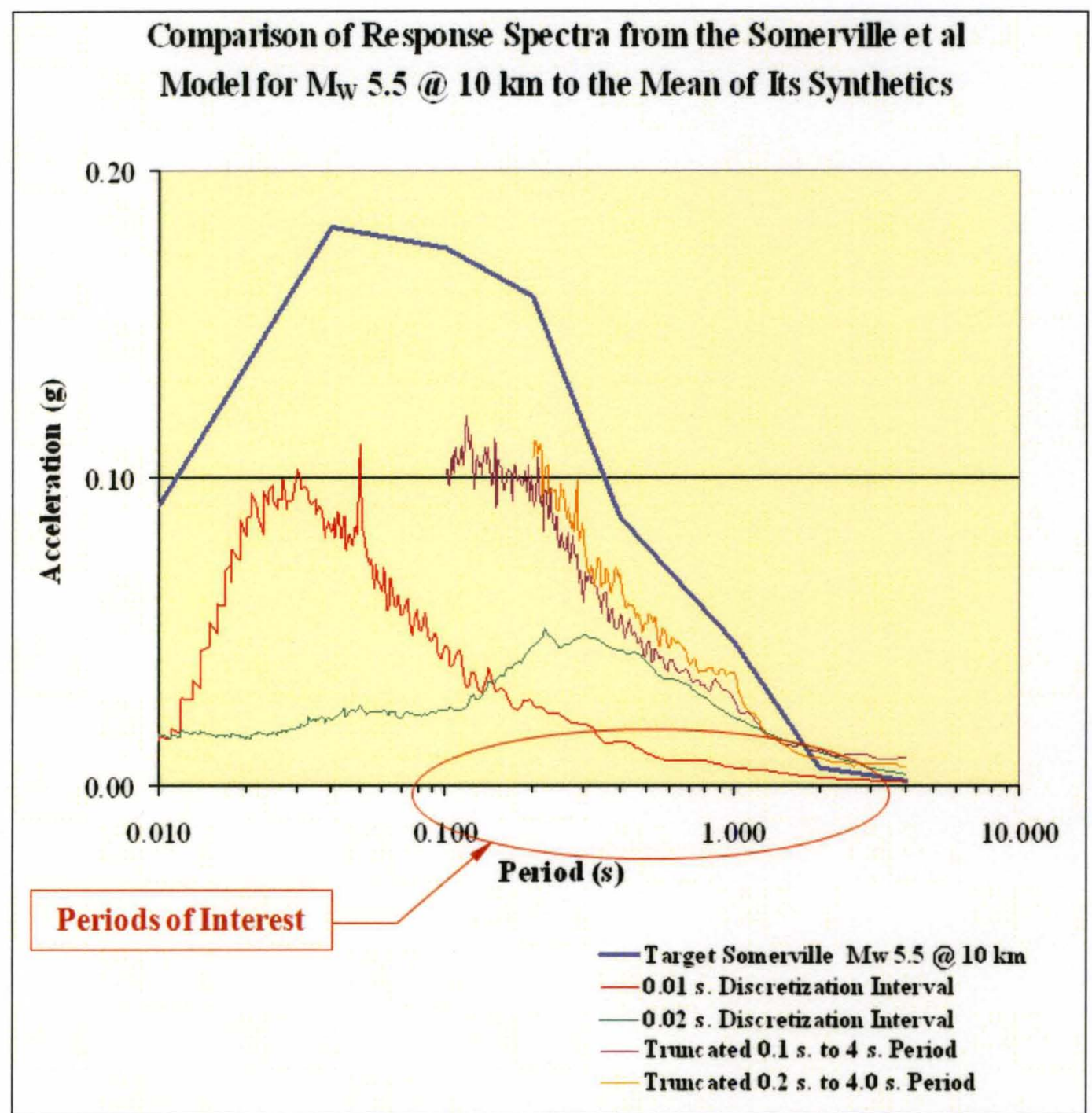

Figure 7.32 Comparison of Magnitude 5.5 at $10 \mathrm{~km}$ Target Spectrum from Somerville Model and Mean Spectra of Synthetics after Truncation

The red curves indicate the response spectra from the original study using a discretization interval of 0.01 seconds and the entire spectra as provided by the attenuation models as the targets (i.e. using all points provided in the attenuation models). The green curves indicate response spectra from the second study whose results are presented in the appendices. As explained earlier, the study was conducted using a 0.02 second discretization interval with all of the spectral values as provided 
from the attenuation models. The purple and gold curves represent the mean response spectra for the synthetics generated from the truncated input target spectra at the 0.1 second and 0.2 second spectral values, respectively. All of the synthetic motions in the truncation study were generated using a 0.01 second discretization interval.

Also, the periods of interest (i.e. the periods of most structures) are indicated. It can be seen clearly that the response spectra for the synthetic motions approached their target with increasing truncation. 


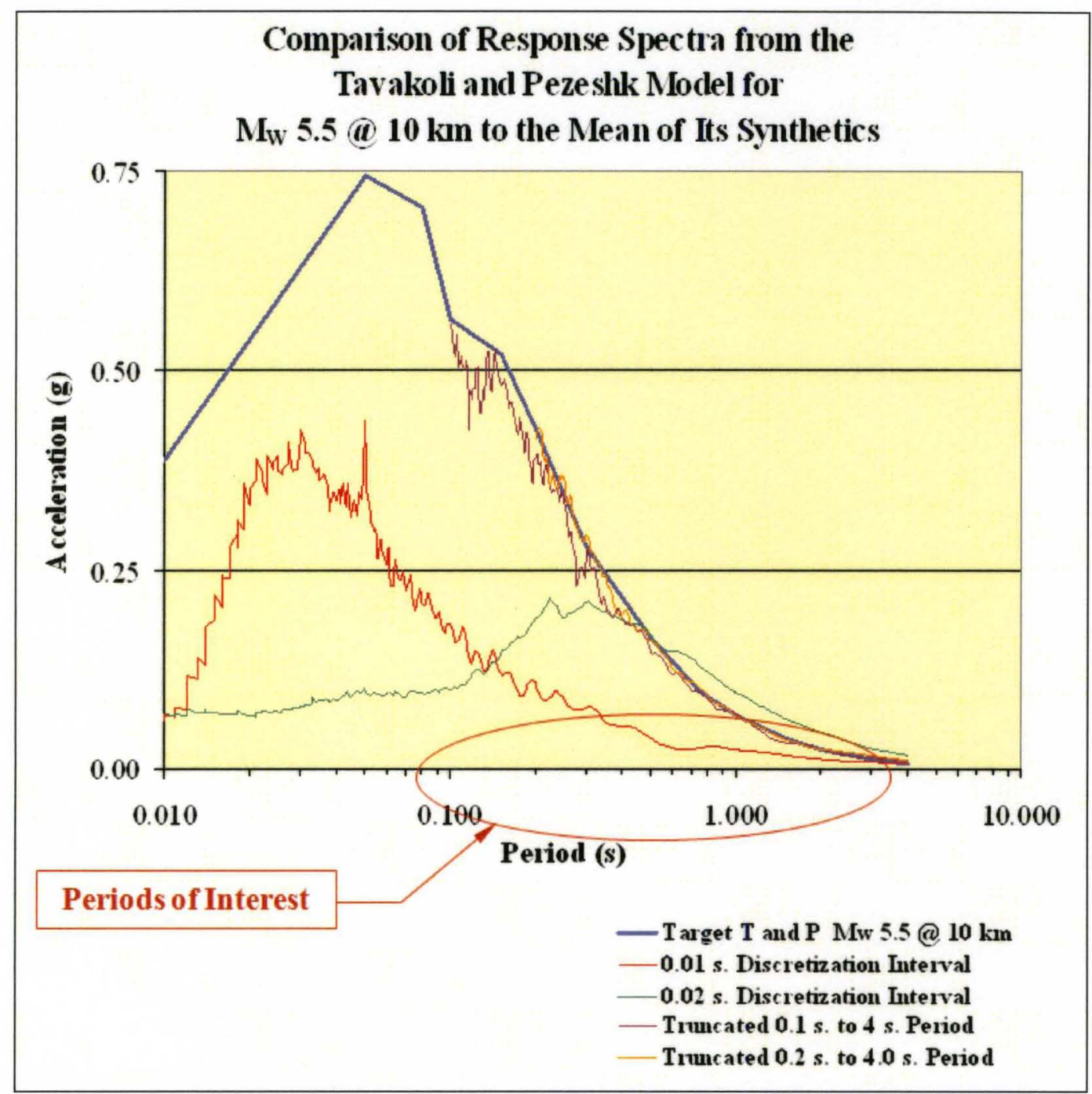

Figure 7.33 Comparison of Magnitude 5.5 at $10 \mathrm{~km}$ Target Spectrum from Tavakoli-Pezeshk Model and Mean Spectra of Synthetics After Truncation

Figure 7.29 shows the ductility demands for this scenario after the truncation up to the 0.2 second spectral value. Even though the synthetic motion spectra are much closer to their targets, discrepancies in the response are still present and in fact have actually increased slightly at the higher frequencies. 


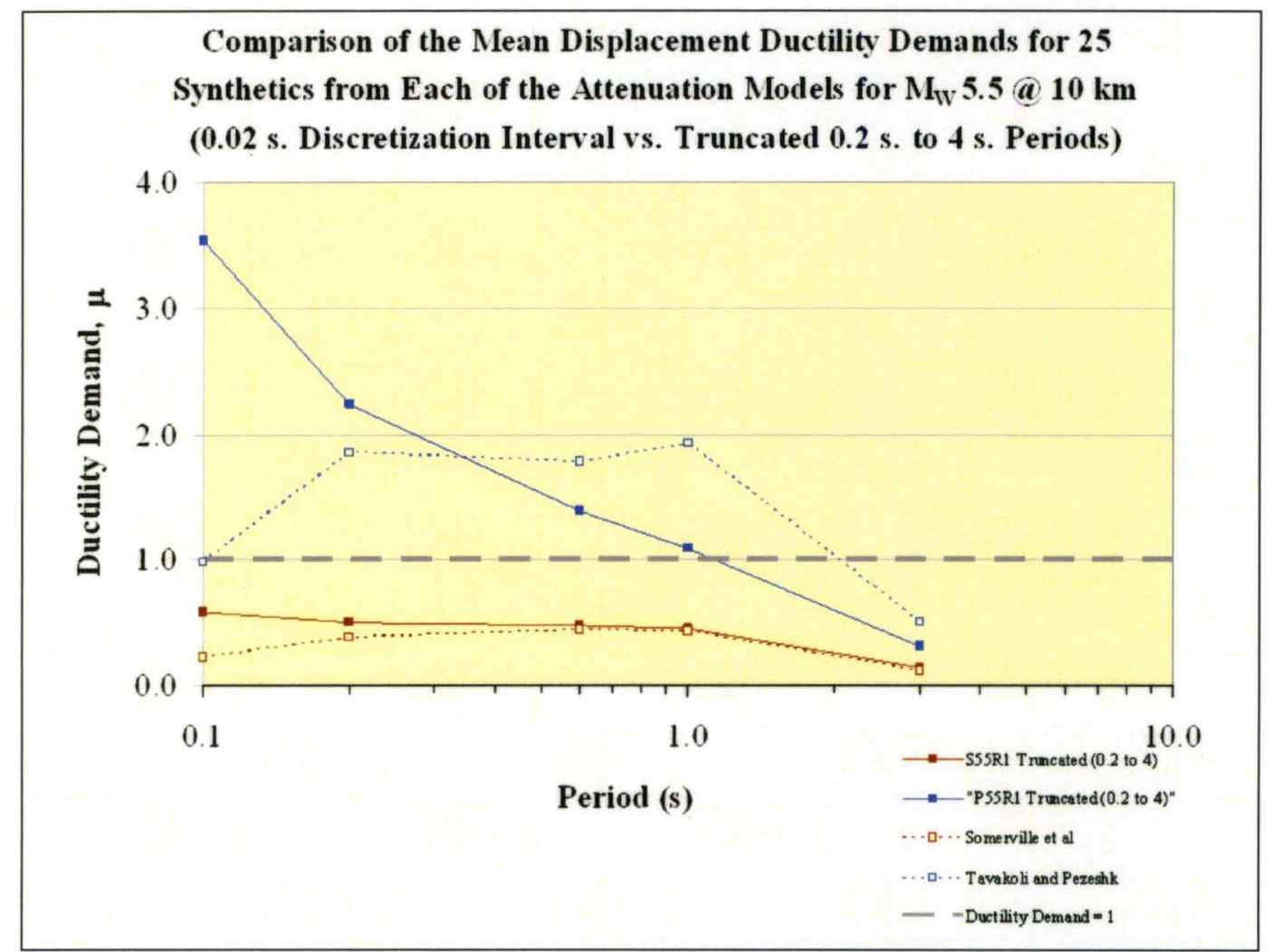

Figure 7.34 Change in Ductility Demands for Magnitude 5.5 at $10 \mathrm{~km}$ after Truncation of Target Spectra

As seen in Figure 7.30, there is very little change in the estimated magnitude for the synthetics from the Somerville model however those from the Tavakoli and Pezeshk have moved lower and closer to their target magnitude after truncating spectral accelerations for periods below 0.2 seconds. 


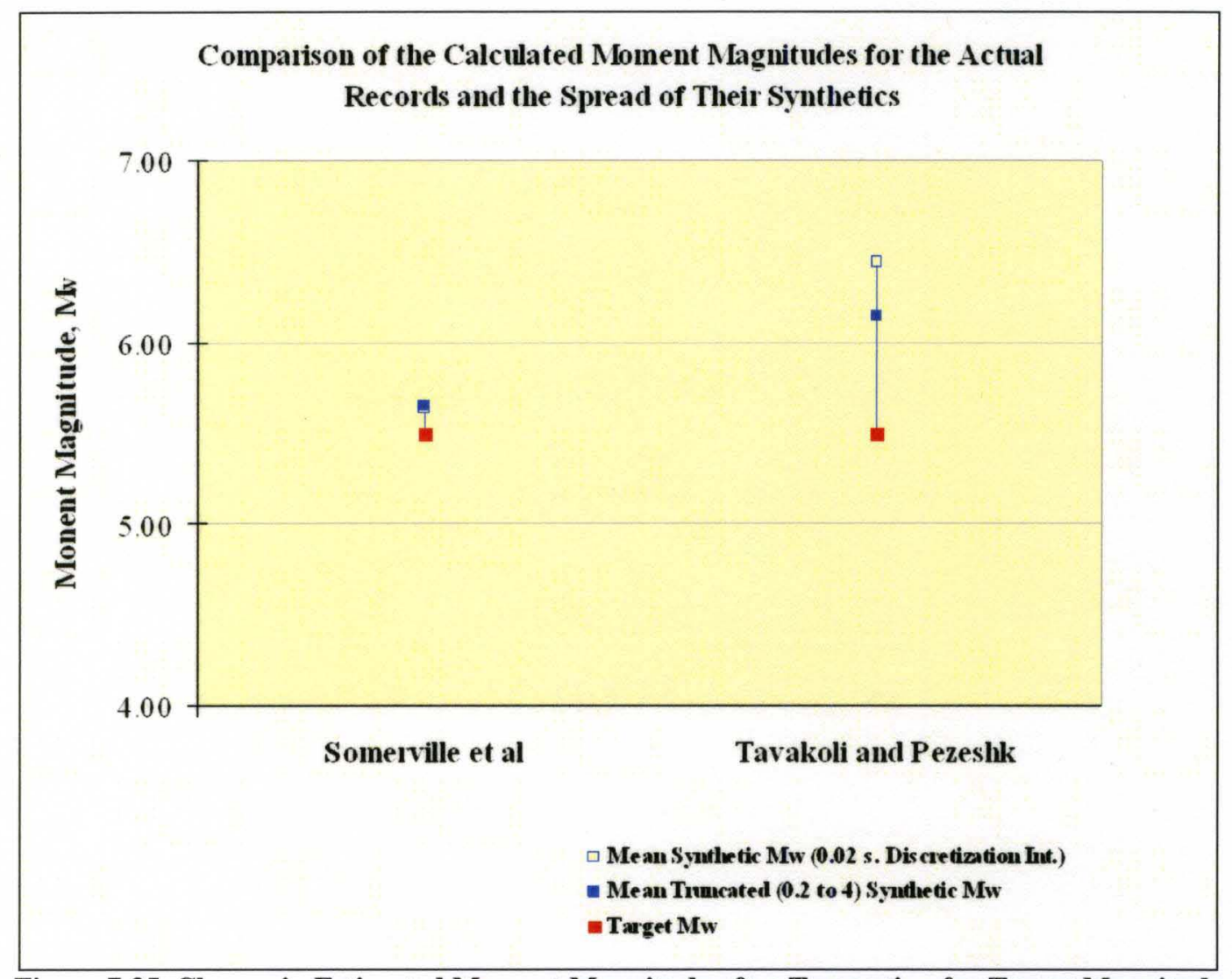

Figure 7.35 Change in Estimated Moment Magnitude after Truncation for Target Magnitude 5.5 at $10 \mathrm{~km}$ 


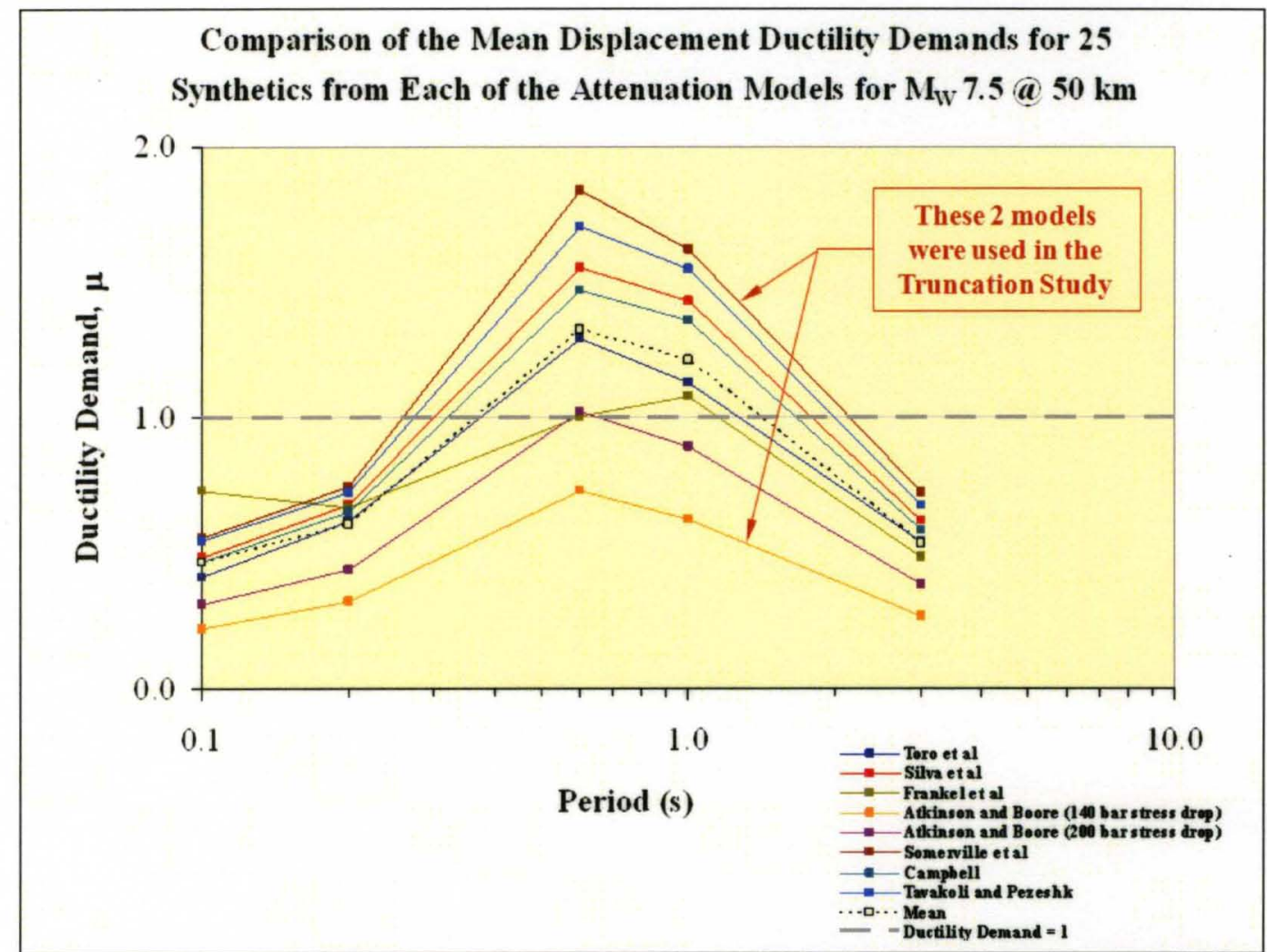

Figure 7.36 Selection of Models for Magnitude 5.5 at $10 \mathrm{~km}$ to be used in the Truncation Study 


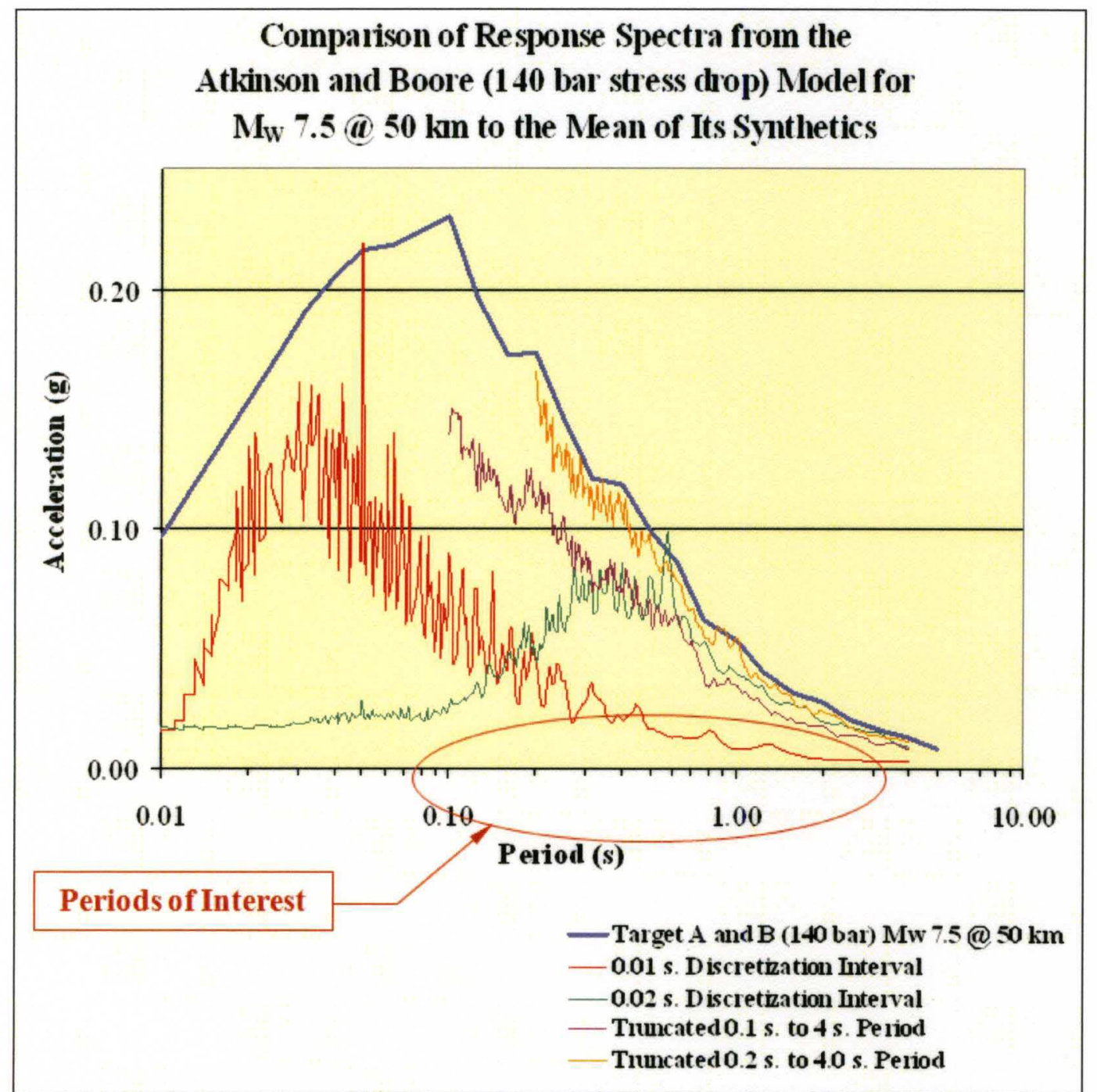

Figure 7.37 Comparison of Magnitude 7.5 at $50 \mathrm{~km}$ Target Spectrum from Atkinson-Boore Model with 140 bar Stress Drop and Mean Spectra of Synthetics after Truncation 


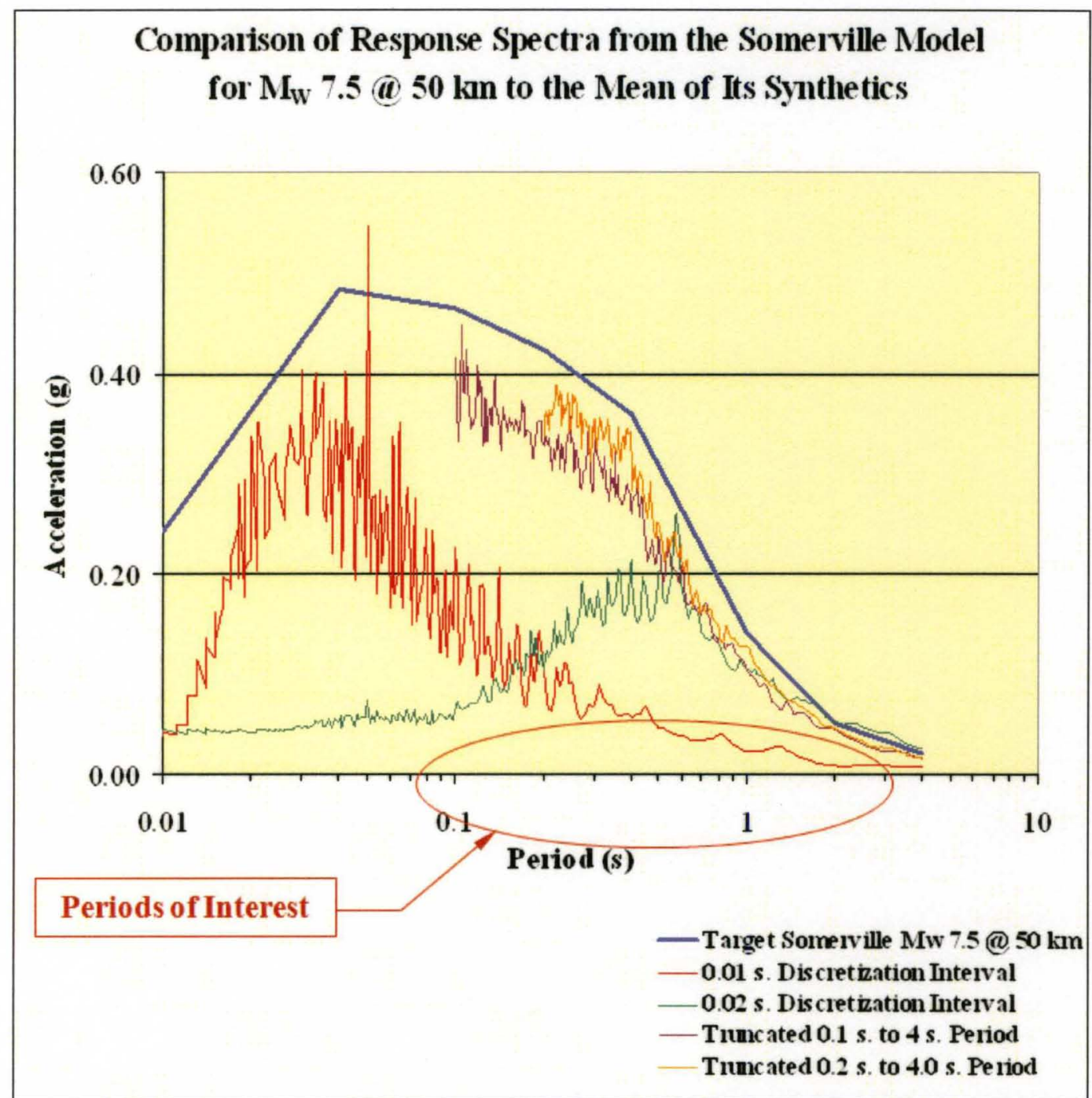

Figure 7.38 Comparison of Magnitude 7.5 at $50 \mathrm{~km}$ Target Spectrum from Somerville Model and Mean Spectra of Synthetics after Truncation

Again as shown in Figure 7.34, the discrepancies in the ductility demands from the synthetics are still present and once again, they have increased significantly at the high frequency shorter period structures. Note that the ductility demand at the 0.2 second response from synthetic motions for the Somerville model now indicates yielding in the structure where no damage for these structures was indicated by the synthetic motions from this model prior to truncation. 


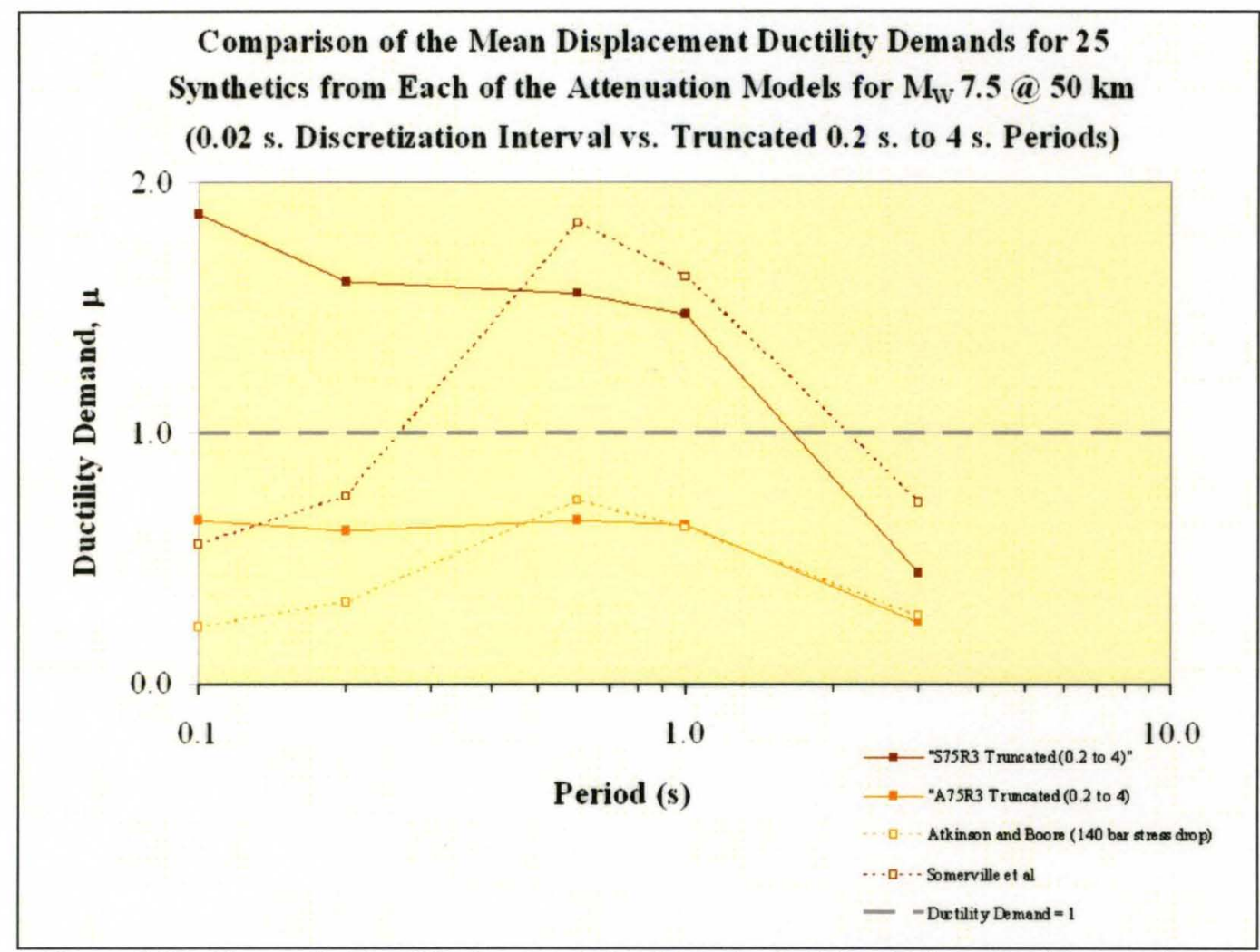

Figure 7.39 Change in Ductility Demands for Magnitude 7.5 at $50 \mathrm{~km}$ after Truncation of Target Spectra

Figure 7.35 shows that the estimated magnitudes for synthetics from both models for this scenario moved lower with those from the Somerville model moving closer towards their target magnitude. 


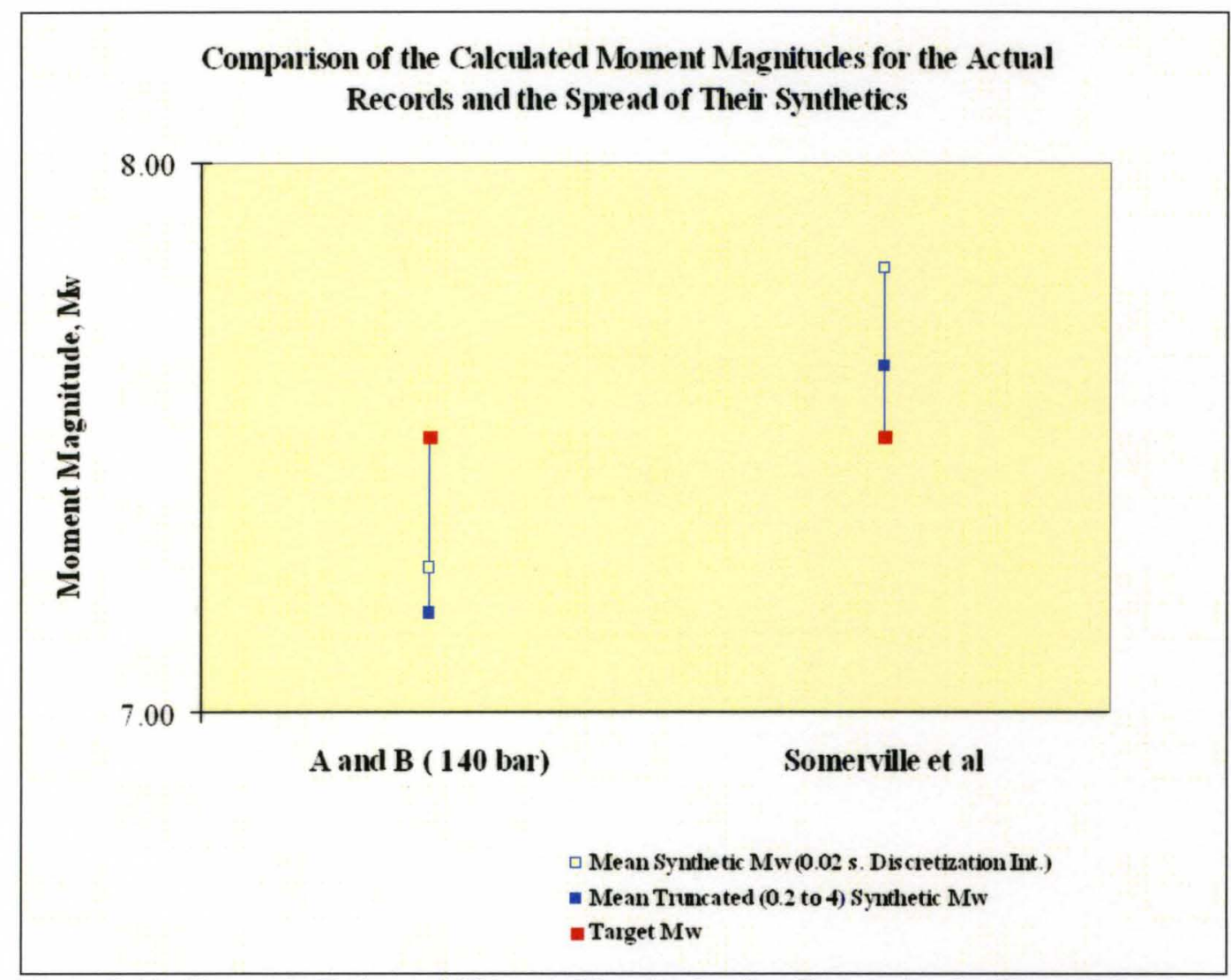

Figure 7.40 Change in Estimated Moment Magnitude after Truncation for Target Magnitude 7.5 at $50 \mathrm{~km}$

Finally, Figure 7.36 shows the ductility demands for the synthetics from all eight attenuation models for the magnitude 6.5 at $25 \mathrm{~km}$ scenario as produced using the entire response spectra from each model and a 0.02 discretization interval. The response spectra for all eight models after truncation are included in the appendices. It can be seen that again the spectra for the synthetics from all of the models moved closer towards their targets after truncation. 


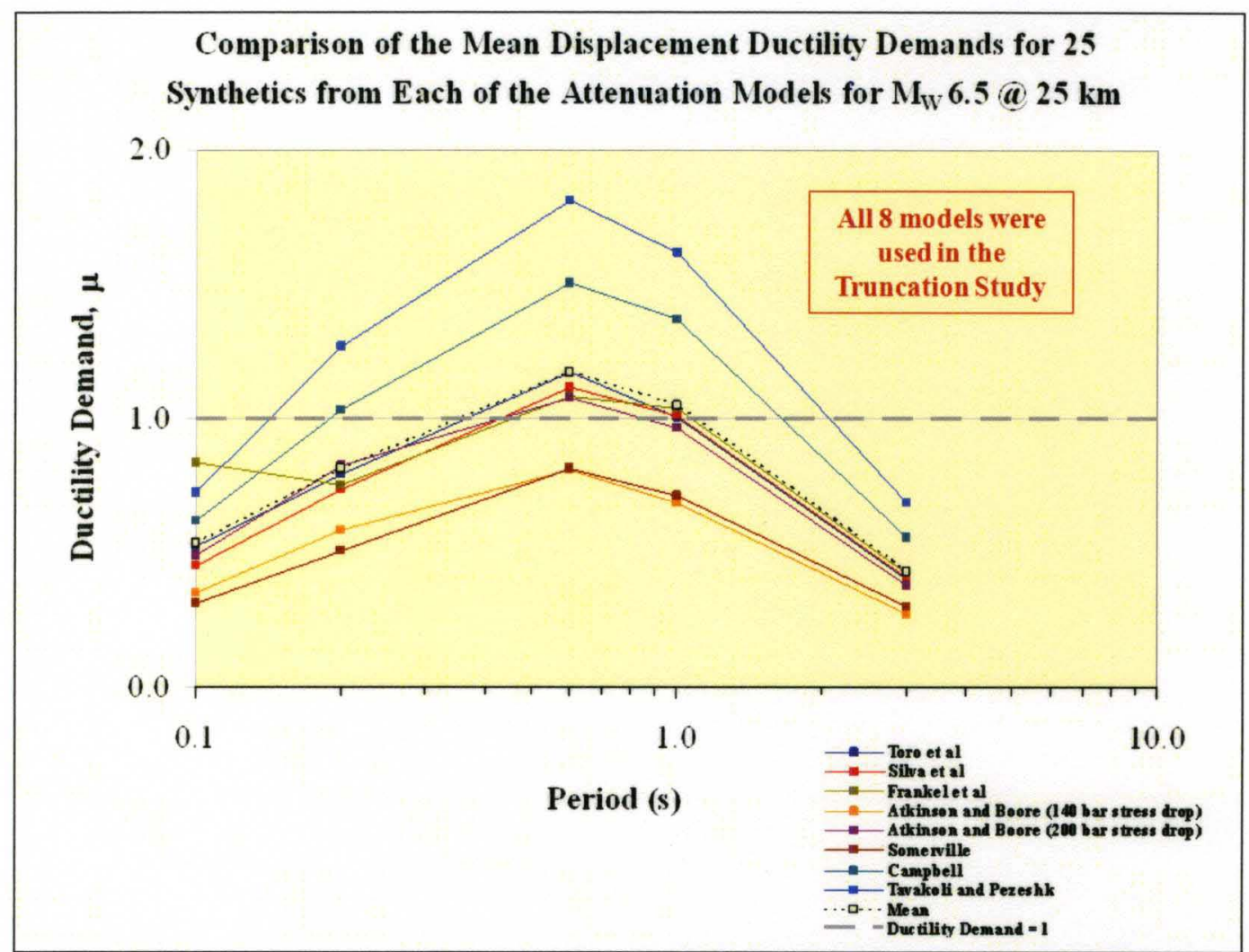

Figure 7.41 Selection of All Eight Models for Magnitude 6.5 at $25 \mathrm{~km}$ to be used in the Truncation Study

Only plots showing the target response spectra and the mean spectra for the synthetic motions generated from them with truncation of spectral values below 0.2 seconds for the two models whose synthetics produced the extreme values in ductility demand are shown here for conciseness, in Figures 7.37 and 7.38. Plots for the remaining attenuation models with the same truncation applied for this event are included in the appendix. 


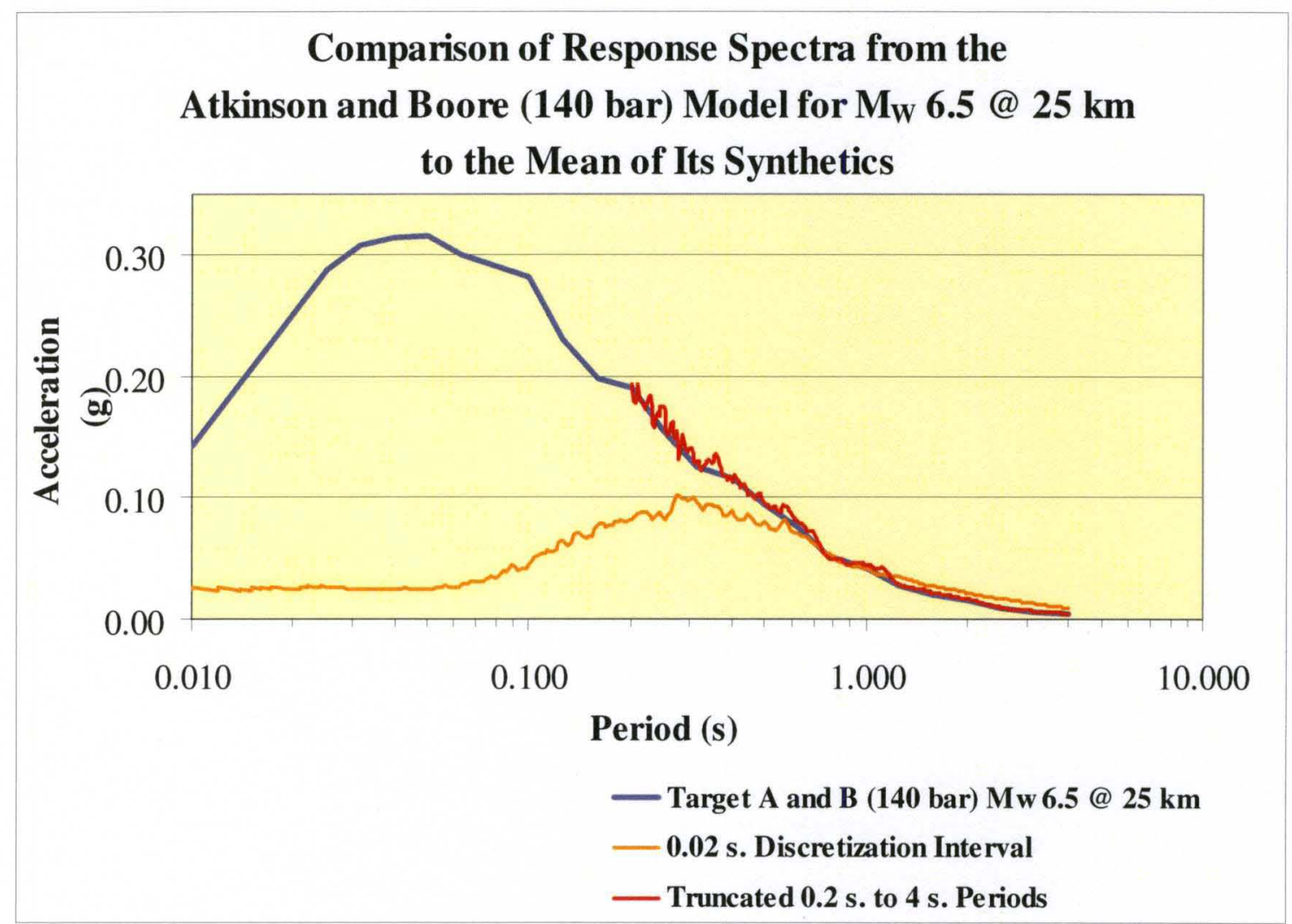

Figure 7.42 Comparison of Magnitude 6.5 at $25 \mathrm{~km}$ Target Spectrum from Atkins-Boore Model with 140 bar Stress Drop and Mean Spectra of Synthetics after Truncation 


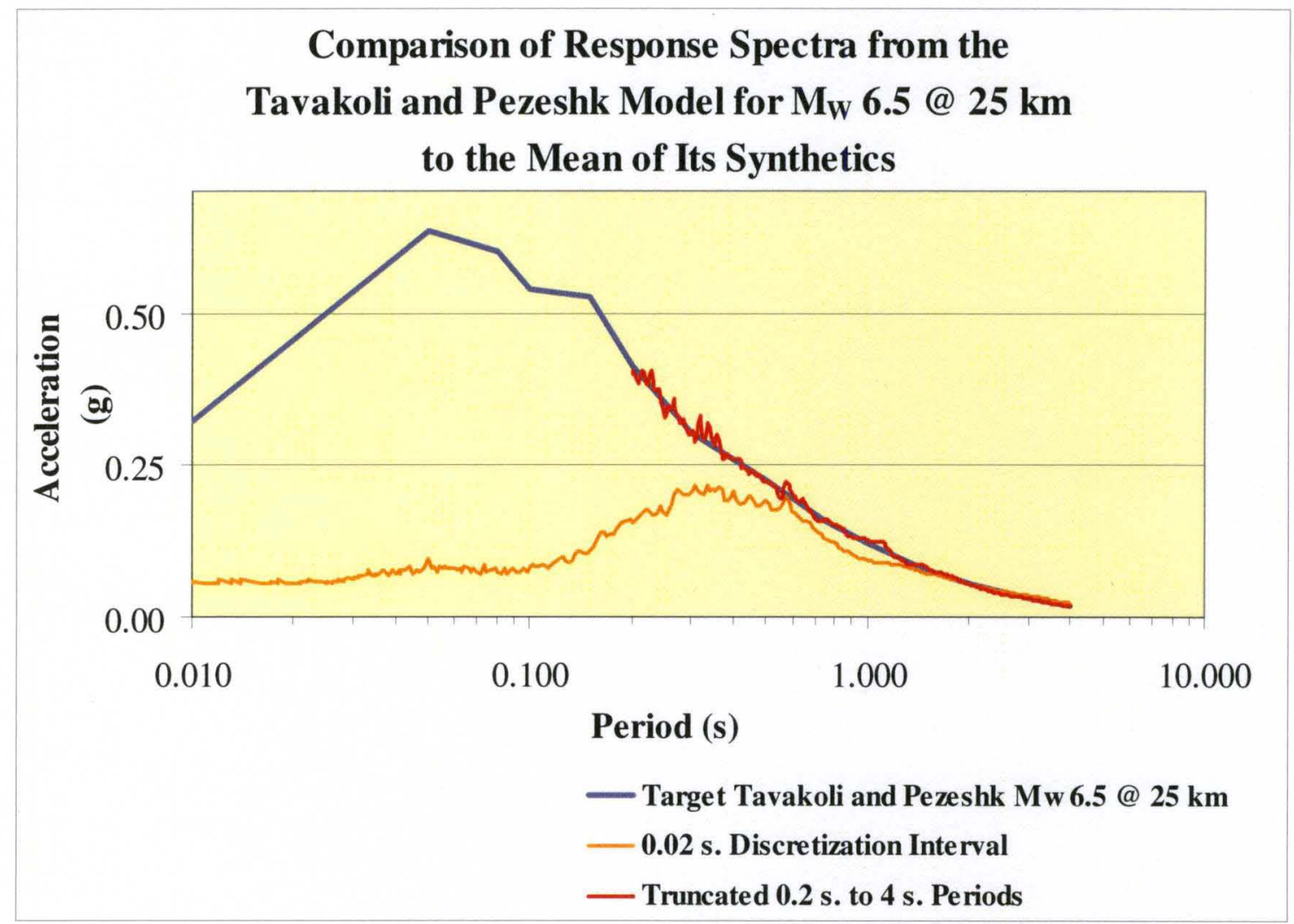

Figure 7.43 Comparison of Magnitude 6.5 at 25 km Target Spectrum from Tavakoli-Pezeshk Model and Mean Spectra of Synthetics after Truncation

Figure 7.39, shows that the discrepancies in ductility demand from the synthetic motions generated from all eight of the attenuation models remained and once again the response to all of the synthetics increased at the high frequency shorter period structures causing the discrepancies in ductility demand to actually increase. 


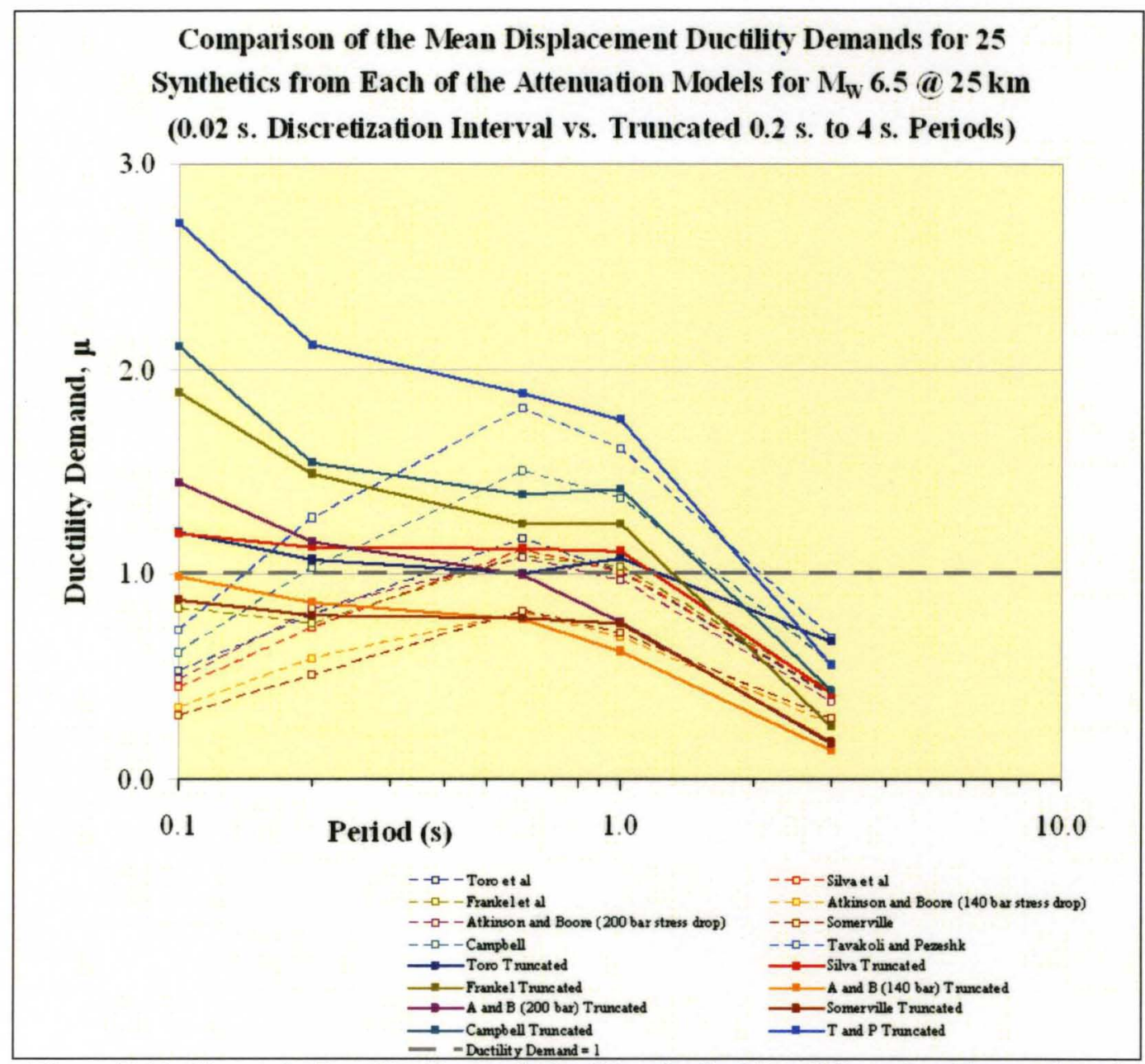

Figure 7.44 Change in Ductility Demands for Magnitude 6.5 at $25 \mathrm{~km}$ after Truncation of Target Spectra

It is obvious from Figure 7.40 that despite the response spectra from the synthetics now matching their targets very well, the discrepancies in the estimated ductility demands they produce are still prevalent. For this scenario the synthetics compatible with two of the models still induce elastic response across the spectrum even though they now match their targets rather well due to the truncation. Likewise, the synthetics from one model still indicate appreciable ductility demand on most structures. 


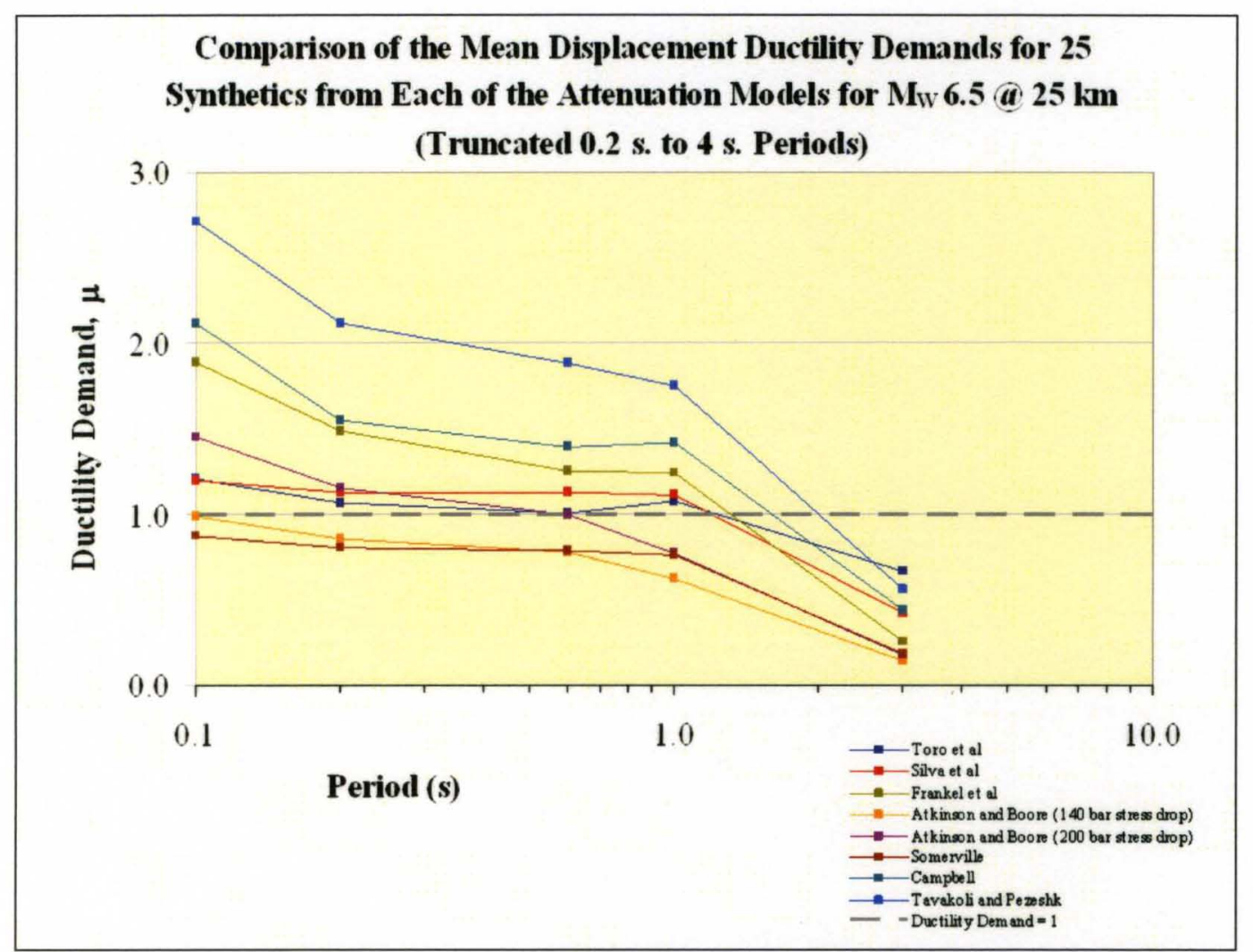

Figure 7.45 Ductility Demands from Magnitude 6.5 at $25 \mathrm{~km}$ after Truncation

The estimated magnitudes for the synthetic motions from all eight of the attenuation models before and after truncation for this event are shown in Figure 7.41. It can be seen that the mean magnitude of all synthetic spectra moved lower except for those of the Toro model. After truncating the spectral values for periods below 0.2 seconds, synthetics from the Silva model produced a mean estimated magnitude that is the closest to the target of 6.5 at this distance. Also, those of the Atkinson and Boore model with the 200 bar stress drop produced a mean estimated magnitude close but slightly below the target magnitude for this event. 


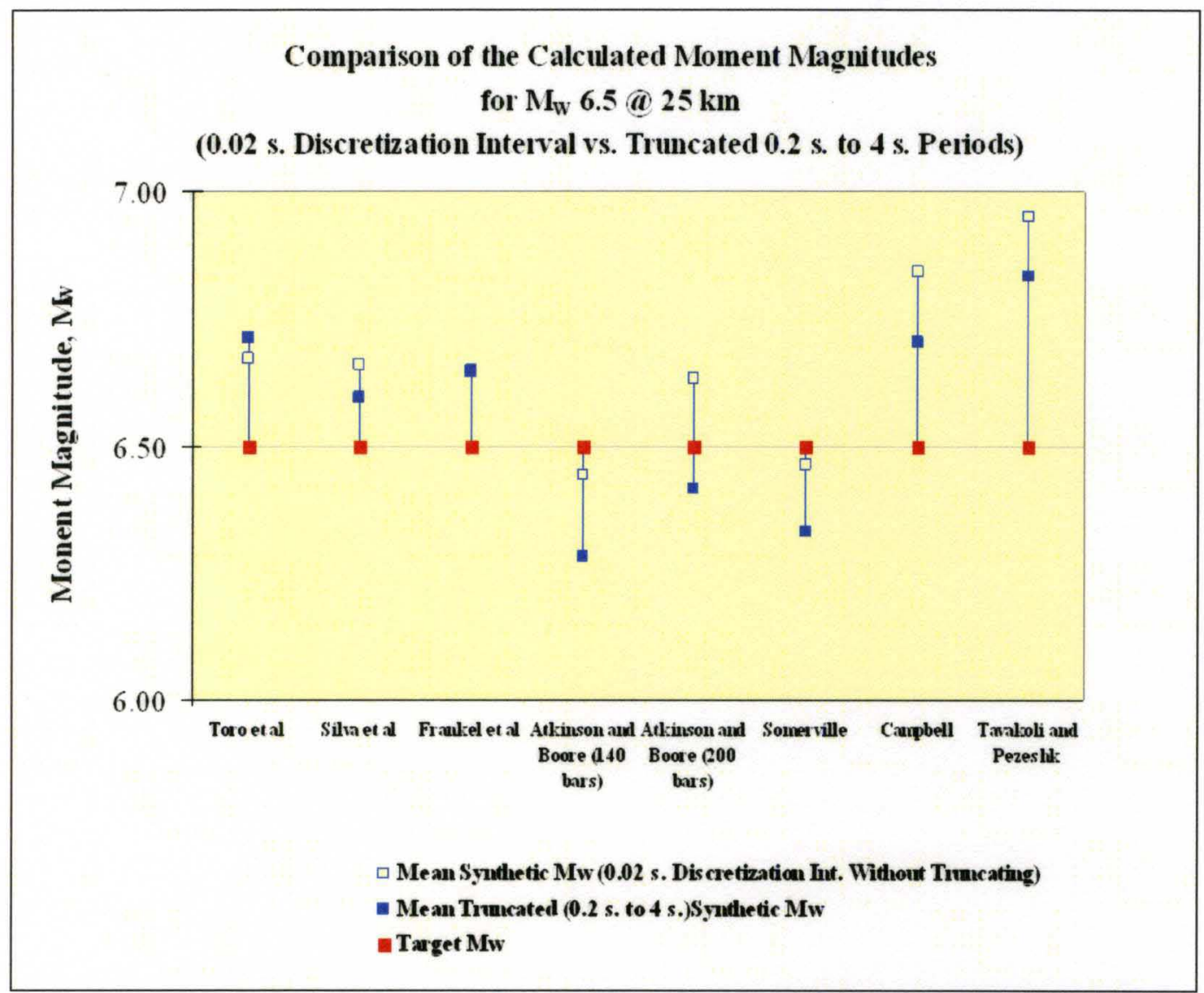

Figure 7.46 Change in Estimated Moment Magnitudes for Magnitude 6.5 at $25 \mathrm{~km}$ after Truncation

Following a rigorous inspection of both the response spectra developed from the attenuation models and the computer code used by the SIMQKE program as well as the results obtained from the truncation study, the following explanation of the response spectra for the synthetic motions in the original studies not meeting their targets is offered. 


\subsubsection{Hypothesis for Errors}

Recall the main equation used by the SIMQKE algorithm to estimate the spectral density function from the input target response spectrum starts at the lowest period/highest frequency (as in the order in which the input target spectra is provided) to calculate the spectral density function. It then moves to the next point and calculates a spectrum value again but subtracts the area under the curve developed up to that point to obtain the new value and all of those that follow.

The response spectra from the CEUS attenuation models have relatively high spectral values at high frequencies that increase as the period increases up to a maximum point. As a result, the spectral accelerations start by contributing substantially to the spectral density function because the first spectral values are relatively high. The next value increases with the area under the curve up to that point being subtracted but because it is early in the process, the area is small so the next spectral ordinate increases. The spectral density function continues to increase until at the maximum point, the calculated value in the first part of the equation is high but due to the area under the function having grown to the point that when it is subtracted it produces values that are not as high as the previous values. Because the previous values were greater [because the method subtracted large amounts of high frequencies], a substantial amount is subtracted reducing the value of the spectral density function. The generated function can not meet its target. The next point is still relatively high but not as high as the previous point and again because the previous values are substantial overall, quite a bit is subtracted. This process 
continues and the frequency content continues to fall below the target even as the frequency decreases. In other words, because of the relatively large values of high frequencies requested in the low period range, the algorithm reaches its maximum early in the process and is therefore not able to produce values that reach those requested at higher periods and consequently throughout the spectrum.

It was also noticed when comparing the spectra for the actual records in the CEUS with those from the attenuation models, that in addition to a relatively large amount of high frequency, the models also predict a relatively high amount of low frequency. Taken with the 0.01 second interval, this process produced a very wide band of frequency content which led to the unrealistically benign responses.

In addition, it is suspected that the large amounts of high frequency content along with large amounts of low frequency content as prescribed by the attenuation models for the CEUS did not mesh well with the analysis and approach used by SIMQKE. In fairness to the program, it should be pointed out that SIMQKE processes the input spectrum based on the response of a linear SDOF system to a base excitation using a random vibration approach to structural dynamics. SIMQKE uses this random vibration approach to obtain the squared amplification function for a linear single degree of freedom system whose input is support acceleration and response is relative displacement. The squared amplification function acts as a filter that attenuates the high frequency components. The overall result provides spectral density functions that take on a form similar to the Kanai-Tajimi model. This is a sound approach; 
however, it seems to fail at producing synthetic ground motions containing the amount of high frequency requested by these attenuation models without sacrificing the amount of low frequency also requested.

An explanation of why the situation improved when the discretization interval was increased from 0.01 to 0.02 seconds in the second study is that this in itself had the same effect as removing some of the high frequency content. The use of a 0.02 second discretization interval precludes the existence of smaller periods/higher frequencies. Recall that most of the models provide their first (and highest frequency) spectral acceleration value at 0.01 seconds and in fact this value was taken as the peak ground acceleration for the models in this study. Increasing the interval to 0.02 seconds is equivalent to truncating these values and others higher than this frequency from the target spectra.

By truncating the target spectra, the response spectra for the synthetic motions rose because some of the high frequency component was eliminated. As a result, the spectral density function itself was shortened. However, the specified number of partitions to calculate the areas under the curve was kept the same and a smaller amount was subtracted at each increment, causing it to decrease not as quickly. The progression of ductility demand with an increased discretization interval and then by truncation of the input spectra seems to imply there exists a "saturation" of high frequency in the predicted spectra from the CEUS attenuation models used in this study that leads to a benevolence in the structural response to synthetics generated to 
be compatible with them. This is not to question the validity of the attenuation models but it does validate truncating the high end of the input spectrum up to frequencies relative to the natural frequencies of most structures and demands proper consideration when applying these CEUS attenuation models as target spectra to generate synthetic ground motions using the SIMQKE program.

Unfortunately, the study conducted indicates that even after applying the truncation described earlier which moved the mean spectra of the synthetic motions much closer to their targets, there is still a large amount of variation among the responses and in fact, the variation increases at short periods as compared to that shown in the original study. Finally, the truncation study also showed that the response from synthetic motions of all models increased at short periods ( 0.1 and 0.2 seconds) despite truncating these periods from the input target spectra. This may be another indication that there is a large amount of high frequency in the target spectra from the attenuation models used in the SIMQKE program causing it to produce synthetics whose response spectra are below their targets in all periods. 


\section{Chapter 8}

\section{CONCLUSIONS}

\subsection{Summary}

The first step in any structural analysis is to determine the loading that will be applied to the structure. This process is essential to properly size and design the elements that make up the structure and nowhere is this process more critical, and at the same time elusive, than in the area of seismic design.

The purpose of this study was to determine how the variation in the response spectra compatible with the eight attenuation models developed for the CEUS and used to create the 2008 National Seismic Hazard Maps translated into variation among the structural responses in the form of displacement ductility demand. This study also sought to determine if this variation in structural response is too great to allow synthetic ground motions compatible with these attenuation models to be used for time history analyses and design of structures in the region.

As indicated by the analysis of the results compiled from this study there are many instances where the synthetic motions compatible with at least one model 
produce displacement ductility demands that indicate no yielding would occur to a particular structure with a given natural period and at the same time, the synthetic motions generated from other models indicate that yielding would occur for the same structure exposed to the same magnitude of event at the same distance.

In still other instances, even though the synthetic motions generated from all of the attenuation models give displacement ductility demands that indicate yielding, their values vary so widely that they may lead to extremely different seismic design approaches being taken.

The existence of instances of discrepancies as cited above indicates that there is in fact "too much" variation among the ductility demands induced by synthetic motions generated to be compatible with the CEUS attenuation models investigated in this study to provide a viable approach to design. Unfortunately, the structural engineer seeking to analyze the response of a particular structure due to seismic loading from a magnitude and distance combination that produces ductility demands that indicate yielding for one model and no damage for another has ultimately learned nothing in the end. The question now becomes which of the attenuation models is to be believed. Depending on one's agenda, the argument could go either way. Of course, the diligent structural engineer will tend to err on the side of conservatism. On the other hand, however, structural engineers often also must contend with an owner whose goal is to hold down costs as much as possible. 
Furthermore, the results of this study indicate that there are instances where synthetic motions generated from and compatible with all of the CEUS attenuation models produce ductility demands implying failure but with very widely varying values. It should be kept in mind that an accurate description of the ductility demand placed on a structure by a particular event is crucial in the seismic design in order for the structure to perform adequately during that event. As pointed out in the introduction, the main emphasis in earthquake engineering is to introduce ductility into the structure being designed. It is usually not the intent of the seismic design engineer to prevent failure but rather to control it. This prevention often is accomplished by introducing weaker portions of members in the structure that are allowed to yield in order to preserve the overall functionality of the structure. In order to produce these types of designs, structural engineers need the ability to model structural behavior accurately under seismic loading. Even though the ability to develop complex models of the structure exists through finite element techniques, this study has shown that there can be a wide variation among the responses to synthetic ground motions developed from attenuation models available for the CEUS.

Unfortunately, the results from this study indicate that the variation in structural response from synthetic ground motions compatible with attenuation models developed for the CEUS does not make this a feasible approach for the deterministic site specific seismic design of structures in the CEUS. The variation is simply too great and it is not possible to discern which synthetic motions compatible with which attenuation relationships provide the best approximation to the structural response 
that would be produced by actual ground motions from a particular magnitude event at a particular distance.

\subsection{Further Research}

As noted at the end of the analysis of results, the synthetic motions generated from the attenuation models unexpectedly did not match their target spectra. At this time, it is not understood if this discrepancy was due to the frequency contents predicted by the attenuation models themselves, the algorithm used by the SIMQKE program to process the input spectra, a combination of the two, or something altogether different. Further research needs to be conducted to explain why the response spectra from the generated synthetic motions did not match their target spectra from the attenuation models especially in light of the fact that the synthetic motions generated using the same methodology on actual records for the pilot study including two events within the CEUS did agree. An investigation also is needed into why using the entire spectra from the attenuation models with a 0.01 second discretization interval leads to unrealistically benign responses in the SDOF systems.

Further statistical analyses should be conducted to investigate multiple pair-wise comparisons of the ductility demands from the synthetic motions used in this study. This type of analysis could provide information useful in conducting these types of studies in the future. For example, of the eight models investigated in this study, if four produced ductility demands shown to have no significant difference statistically, 
it would only be necessary to use one of the four in the future investigation. This modification could reduce time and effort considerably.

This study investigated only the responses of structures assumed to be founded on bedrock. Future studies should consider local soil and site conditions. One way to accomplish this extension would be to process the motions produced from this study through a ground response analysis using one of the many computer programs available. Also, many of the attenuation models themselves contain coefficients that can be applied directly to simulate differing site conditions.

As was mentioned in the analysis of results, a marked increase in ductility demand was not shown at long distances with increasing magnitude as would be expected based on the decreased attenuation associated with the CEUS. It is hypothesized that by introducing soil/site response characteristics into the analysis, higher ductility demands would be seen at further distances.

Also this study was carried out on simple single degree of freedom systems characterized by an elasto-plastic stress-strain relationship. A logical progression would be to conduct this type of study on more sophisticated and elaborate finite element models that would provide a more accurate portrayal of the true structural response. 
Further research could also be conducted to determine stochastic distributions that could be used to describe the variations displayed in the ductility demands. These distributions could be used with Monte Carlo simulation techniques to conduct site specific risk analyses.

Also much more work could be done to investigate the effect the intensity envelope has on the output synthetic ground motion as generated by the SIMQKE program. Early investigations indicate that the intensity envelope used to describe the shape of the synthetic ground motion plays a role in the structural response.

Future work could also be done using the results from this study to make comparisons of the base shear forces developed using pseudo static procedures and those developed from time history analysis of the SDOF systems investigated in this study. This research could provide some indication of the differences (e.g. whether one procedure is over predicting or under predicting response, etc.) for the design of certain structures.

In a similar light, the results from this study could be used to investigate the differences in design effort and costs due to the variations in ductility requirements as determined by this study. This research could be done using both pseudo static procedures and time history analysis by using two models that produce extreme values for a particular event and distance. 
Finally, the methodology for this study could be applied once again to SDOF systems with different strength parameters to investigate the role those parameters play in structure response.

\subsection{Final Thoughts}

As explained at the end of Chapter 7, problems were encountered during this study, but those difficulties were addressed by truncating the short period spectral accelerations from the target spectra below values that are not applicable to most practical structures. In spite of these corrections which were shown to significantly improve the compatibility of the generated synthetic motions with their targets from the attenuation models, large variations in structural response persisted and there were many instances with conflicting predictions of ductility demand whether in terms of uncertainty if yielding would occur or if so, the amount of ductility required. In fact, in some cases, the variation was seen actually to increase even after making the corrections by applying the truncation to the target spectra.

As pointed out in the first chapter, it is obvious by merely viewing the response spectra generated from the attenuation relations for the CEUS used to develop the latest version (2008) of the National Seismic Hazard Maps that there is variation among the results obtained from the models. What is not so obvious from viewing these spectra is how this variation in spectral values translates into variation in the actual response of structures. This study has taken the first steps at trying to answer this question and provides indications that this variation is indeed great in many 
cases. From a probabilistic point of view, variation may be seen as a good thing; indicating a sample space covering a wide possibility of representations. However, to the practicing engineer, this variation in structural response indicated by the results of this study can be perplexing. The situations in which time histories compatible with one attenuation model for a particular scenario indicate no damage to a particular structure while time histories compatible with another model for the same scenario indicate significant yielding within that same structure forces the structural engineer performing the design to make some rather difficult decisions without any gain in direction as to how to do so. 


\section{REFERENCES}

Abrahamson, N.A. and Silva, W.J. (1997). "Empirical Response Spectral Attenuation Relations for Shallow Crustal Earthquakes”, Seismological Research Letters 68, 94127.

Abrahamson, N.A. (1998). Non-stationary Spectral Matching Program RSPMATCH, Pacific Gas \& Electric Internal Report.

Algermissen, S. T., Leyendecker, E. V., Bollinger, G. A., Donovan, N. C., Ebel, J. E., Joyner, W. B., Luft, R. W., Singh, J. P., (1991). "Probabilistic Ground-motion Hazard Maps of Response Spectral Ordinates for the United States", Proc. 4th Int'l. Conf. on Seismic Zonation, EERI.

Anderson, J. A. and H. O. Wood (1925). "Description and Theory of the Torsion Seismometer”, Bull. Seism. Soc. Am. 15, 1-72.

Anderson, J. S. Hough (1984). "A Model for the Shape of the Fourier Amplitude Spectrum of Acceleration at High Frequencies”, Bull. Seis. Soc. Am. 74, 1969-1993.

ASCE Standard ASCE/SEI 7-05. "Minimum Design Loads for Buildings and Other Structures", American Society of Civil Engineers.

ATC 10, (1982). "An Investigation of the Correlation between Earthquake Ground Motion and Building Performance", Applied Technology Council.

Atkinson, G.M. (1984). "Attenuation of Strong Ground Motion in Canada from a Random Vibrations Approach." Bull. Seism. Soc. Am., 74(5), 2629-2653.

Atkinson, G.M. (1993). "Source Spectra for Earthquakes in Eastern North America." Bull. Seism. Soc. Am., 83(6), 1778-1798.

Atkinson, G.M. and D.M. Boore (1995). "Ground Motion Relations for Eastern North America." Bull. Seism. Soc. Am., 85(1), 17-30.

Atkinson, G. M. and Boore, D. M., (1998). "Evaluation of Models for Earthquake Source Spectra in Eastern North America”, Bull. Seismol. Soc. Am. 88, 917-934. 
Atkinson, G. M. and Silva, W., (2000). "Stochastic Modeling of California Ground Motions", Bull. Seismol. Soc. Am. 90, 255-274.

Atkinson, G.M., and Boore, D.M., (2006). "Earthquake Ground Motion Prediction Equations for Eastern North America", Bulletin of the Seismological Society of America, v. 96, p. 2181-2205.

Bathe, K. J. and Wilson, E. L. (1976). "Numerical Methods in Finite Element Analysis", Prentice-Hall Inc., Englewood Cliffs, NJ.

Benz, H.M., Frankel, A., and Boore, D.M. (1997). "Regional Lg Attenuation for the Continental United States.” Bull. Seism. Soc. of Am., 87(3), 606-619.

Beresnev, I.A., Atkinson, G.M. (1998). "FINSIM - A FORTRAN Program for Simulating Stochastic Acceleration Time Histories from Finite Faults," Seism. Res. Lett., 69, pp. 27-52.

Boatwright, J. and Choy, G., (1992). "Acceleration Source Spectra Anticipated for Large Earthquakes in Northeastern North America." Bull. Seism. Soc. Am., 82, 660 682.

Boore, D.M., (1983). "Stochastic Simulation of High-frequency Ground Motions Based on Seismological Models of the Radiated Spectra," Bull. Seism. Soc. Am. 73, 1865-1894.

Boore, D.M. and Atkinson, G.M., (1987). "Stochastic Prediction of Ground Motion and Spectral Response Parameters at Hard-rock Sites in Eastern North America", Bull. Seism. Soc. Am., 77(2), 440-467.

Boore, D.M. and G.M. Atkinson (1992). "Source Spectra for the 1988 Saguenay, Quebec, Earthquakes." Bull. Seism. Soc. Am., 82(2), 683-719.

Boore, D.M. (1996). SMSIM- Fortran Programs for Simulating Ground Motions from Earthquakes: Version 1.0, Open File Report 96-80-A, U.S. Geological Survey.

Boore, D. M., (2003). "Simulation of Ground Motion Using the Stochastic Method", Pure and Applied Geophysics 160, 635-676.

Boore, D. and Joyner, B. (2004). "Stochastic Methods of Estimating Ground Motion”. Seismic Risk and Hazard Analysis. Ed. McGuire, R. K., Earthquake Engineering Research Institute.

Boore, D. M., (2005). "SMSIM - Fortran Programs for Simulating Ground Motions from Earthquakes: Version 2.3 - A Revision of OFR 96-80", U.S. Department of the Interior, U.S. Geological Survey. 
Brown, W. M. III, Perkins, D. M., . Leyendecker, E. V., Frankel, A. D., Hendley, J. W. II, and Stauffer, P. H., (2001). USGS Fact Sheet 183-96, U.S. Department of the Interior, U.S. Geological Survey.

Brune, J.N. (1970). "Tectonic Stress and the Spectra of Seismic Shear Waves from Earthquakes." J. Geophys. Res. 75, 4997-5009.

Brune, J.N. (1971). “Correction,” J. Geophys. Res., 76, 5002.

Campbell, K.W., (2002). "Prediction of Strong Ground Motion Using the Hybrid Empirical Method: Example Application to Eastern North America", Bull. Seism. Soc. Am.

Campbell, K.W. and Bozorgnia, Y., (2003). "Updated Near-source Ground-motion (Attenuation) Relations for the Horizontal and Vertical Components of Peak Ground Acceleration and Acceleration Response Spectra", Bulletin of the Seismological Society of America, v. 93, p. 314-331.

Carballo, J. E., and Cornell, C. A., (2000). "Probabilistic Seismic Demand Analysis: Spectrum Matching and Design", Department of Civil and Environmental Engineering, Stanford University, Report No. RMS-41.

Clough, Ray.W., and Penzien, J., Dynamics of Structures, Volume 2, McGraw Hill, New York, N.Y., 1993.

Chopra, A.K., Dynamics of Structures, Prentice Hall, Upper Saddle River, N.J., 1995.

Department of the Army, (2000). "Dynamic Analysis of Concrete Hydraulic Structures", U. S. Army Corps of Engineers, Circular No. EC 1110-2-6051, Washington, D.C.

DRAIN, (2003). "A General Purpose Computer Program for Static and Dynamic Analyses of Inelastic 2D Structures", National Information Service for Earthquake Engineering, Pacific Earthquake Engineering Research Center, University of California.

Electric Power Research Institute (1993). "Guidelines for Determining Design Basis Ground Motions", Palo Alto, Calif: Electric Power Research Institute, vol. 1-5, EPRI TR-102293.

Elnashai, A. S., Cleveland, L. J., Jefferson, T., Harrald, J., (2008). "Impact of Earthquakes on the Central USA", New Madrid Seismic Zone Catastrophic Earthquake Response Planning Project Final Phase I Report, Mid-America Earthquake Center Report No. 08-02. 
Federal Emergency Management Agency, (2008). "New Madrid Seismic Zone Catastrophic Earthquake Planning", U.S. Department of Homeland Security, Release Number: HQ-08-250.

Frankel, A., McGarr, A., Bicknell, J., Mori, J., Seeber, L. and Cranswick, E. (1990). "Attenuation of High-frequency Shear Waves in the Crust: Measurements from New York State, South Africa, and Southern California", J. Geo. Research, 95(B11), $17,441-17,457$.

Frankel, A. (1994). Implications of Felt Area-magnitude Relations for Earthquake Scaling and the Average Frequency of Perceptible Ground Motion", Bull. Seism. Soc. Am., 84(2), 462-465.

Frankel, A.D., Mueller, C., Barnhard, T., Perkins, D., Leyendecker, E.V., Dickman, N., Hanson, S., and Hopper, M. (1996). "National Seismic Hazards Maps Documentation," USGS Open File Report 96-532, U.S. Department of the Interior, U.S. Geological Survey.

Frankel, A. D., Petersen, M. D., Mueller, C. S., Haller, K. M., Wheeler, R. L., Leyendecker, E. V., Wesson, R. L., Harmsen, S. C., Cramer, C. H., Perkins, D. M., and Rukstales, K. S., (2002). "Documentation for the 2002 Update of the National Seismic Hazard Maps", USGS Open File Report 02-420, U.S. Department of the Interior, U.S. Geological Survey.

Gasparini, D., and Vanmarcke, E. H. (1976). "SIMQKE: A Program for Artificial Motion Generation", Department of Civil Engineering, Massachusetts Institute of Technology, Cambridge, MA.

Gomberg, J., Schweig, E., (2007). "Understanding Earthquake Hazards in the Central United States, Earthquake Hazard in the Heart of the Homeland", U.S. Geological Survey Fact Sheet 2006-3125, U.S. Department of the Interior, U.S. Geological Survey.

Gutenberg, B. and Richter, C. F. (1942). "Earthquake Magnitude, Intensity, Energy, and Acceleration, Bull. Seism. Soc. Am. 32, 163-192.

Hanks, T.C., and Kanamori, H. (1979). “A Moment Magnitude Scale,” J. Geophy. Res., 84, 2348-2350.

Hanks, T.C., and McGuire, R.K. (1981). "The Character of High-frequency Strong Ground Motion."Bull. Seism. Soc. Am., 71(6), 2071-2095

Hanks, T.C. and Johnston, A.C., (1992). "Common Features of the Excitation and Propagation of Strong Ground Motion for North American Earthquakes", Bull.

Seism. Soc. Am., 82(1), 1-23. 
Hartzell, S. (1978). “Earthquake aftershocks as Green's functions," Geophysical Research Letter, 5, 1-4.

Hartzell, S. (1985). “The Use of Small Earthquake as Green's Functions," Strong Motion Simulation and Earthquake Engineering Applications, Publication 85-02, R.E. Scholl and J.L. King, ed., Earthquake Engineering Research Institute, El Centro, CA.

Hays, W. W., (1980). "Procedures for Estimating Earthquake Ground Motions", Geological Survey Professional Paper No. 114, U. S. Geological Survey, Washington, DC.

Herrmann, R.B. and O.W. Nuttli (1982). "Magnitude: the Relation of ML to mbLg.", Bull. Seism. Soc. Am., 72(2), 389-397.

Herrmann, R. B., (2006). "Computer Programs in Seismology, A Overview of Synthetic Seismogram Computation", Department of Earth and Atmospheric Sciences, Saint Louis University.

Hildenbrand,T. G., Langenheim, V. E., Schweig, E., Stauffer, P. H., Hendley J. W. II, (1996). "Uncovering Hidden Hazards in the Mississippi Valley", U.S. Geological Survey Fact Sheet 200-96, U.S. Department of the Interior, U.S. Geological Survey.

Hughes, T., (1987). "The Finite Element Method - Linear Static and Dynamic Finite Element Analysis”, Prentice Hall, Inc.

IDARC, (2008). “A Computer Program for Seismic Inelastic Structural Analysis”, Dept. of Civil, Structural, and Environmental Engineering, State University of New York at Buffalo.

Illinois State Geological Survey, (1995). “1895 Halloween Earthquake”, Earthquake Facts 1995-1, Illinois Department of Natural Resources.

International Code Council, (2006). "International Building Code”, Falls Church, VA.

Kanamori, H., (1977). "The Energy Release in Great Earthquakes”, J. Geophys. Res., 82, 2981-2987.

Kim, T. W., (1998). "The ML Scale in Eastern North America", Bulletin of the Seismological Society of America, Vol. 88, No. 4, pp. 935-951.

Kramer, S.L. (1996). "Geotechnical Earthquake Engineering”, Prentice Hall, Upper Saddle River, NJ.

Leyendecker, E.V., Algermissen, S.T., and Frankel, A.D., (1994), "Use of Spectral Response Maps and Uniform Hazard Response Spectra in Building Codes", Proceedings of the 5th U.S. Conference on Earthquake Engineering, v. IV, p. 379387, Earthquake Research Institute. 
Leyendecker, E.V., Perkins, D.M., Algermissen, S.T., Thenhaus, P.C., and Hanson, S.L., (1995). "USGS Spectral Response Maps and Their Relationship with Seismic Design Forces in Building Codes", U.S. Geological Survey, Open-File Report 95596.

Motazedian, D. and G.M. Atkinson (2005). "Stochastic Finite-fault Model Based on Dynamic Corner Frequency", Bulletin of the Seismological Society of America 95, 995-1010.

Mueller, C., Hopper, M., and Frankel A., (1997). "Preparation of Earthquake Catalogs for the National Seismic-Hazard Maps: Contiguous 48 States", Open-File Report 97-464, U.S. Department of the Interior, U.S. Geological Survey.

Naeim, F. (1989). "Seismic Design Handbook", Van Nostrand Reinhold, New York, NY.

Naeim, F., Alimoradi, A., Pezeshk, S. (2004). "Selection and Scaling of Ground Motion Time Histories for Structural Design Using Genetic Algorithms", Earthquake Spectra, Volume 20, No. 2, pages 413-426, Earthquake Engineering Research Institute

National Earthquake Hazards Reduction Program, (1992). "Recommended Provisions for the Development of Seismic Regulations for New Buildings," Report Nos. FEMA 222 and 223, Federal Emergency Management Agency, Publications Division, Washington, DC.

NEHRP, (2003). "Recommended Provisions for Seismic Regulations for New Buildings and Other Structures (FEMA 450)", Building Seismic Safety Council of the National Institute of Building Sciences.

Newmark, N. M., (1959). “A Method of Computation for Structural Dynamics”, ASCE

Journal of the Engineering Mechanics Division, Vol. 85 No. EM3.

Newmark, N. M. and Hall, W. J. (1982). "Earthquake Spectra and Design”. Earthquake Engineering Research Institute, Berkeley, CA.

NONLIN, (1996). "Nonlinear Dynamic Time History Analysis of Single Degree of Freedom Systems", Federal Emergency Management Agency Training Center, Emmitsburg, Maryland, and Advanced Structural Concepts, Inc., Golden, Colorado.

Novikova, E. I., and Trifunac, M. D.(1994). "Duration of Strong Ground Motion in Terms of Earthquake Magnitude, Epicentral Distance, Site Conditions and Site Geometry."Earthquake Engrg. and Struct. Dyn., 23, 1023-1043. 
Nuttli, O. W., (1974). "Historic Earthquakes: New Madrid Earthquakes 1811-1812 Overview", Earthquake Information Bulletin, Volume 6, Number 2, U.S. Department of the Interior, U.S. Geological Survey.

Nuttli, O. W. (1981). "Similarities and Differences between Western and Eastern United States Earthquakes, and their Consequences for Earthquake Engineering." In Proceedings of the Conference on Earthquakes and Earthquake Engineering: the Eastern United States, edited by Beavers, J. E., vol. 1, Ann Arbor Science Publishers, Inc., Ann Arbor, Michigan, 25-27.

Nuttli, O. W. and Herrmann, R. B. (1987). "Ground Motion Relations for Eastern North American Earthquakes", Ground Motion and Engineering Seismology. Ed. Gakmak, A. S. Elsevier Computational Mechanics Publications.

OpenSees, (2006). “Open System for Earthquake Engineering Simulation”, Pacific Earthquake Engineering Research Center, University of California, Berkeley.

Papageorgiou, P., Haldorsson, B., Dong, G., (2004). "Target Acceleration Spectra Compatible Time Histories, TRSCTH User's Manual, Version 1.0”, Department of Civil, Structural, and Environmental Engineering, State University of New York at Buffalo, Engineering Seismology Laboratory.

Paz, M., (1991). “Structural Dynamics: Theory and Computation”, Van Nostrand Reinhold, New York, NY.

Petersen, M. D., Frankel, A. D., Harmsen, S. C., Mueller, C. S., Haller, K. M., Wheeler, R. L., Wesson, R. L., Zeng, Y., Boyd, O. S., Perkins, D. M., Luco, N., Field, E. H., Wills, C. J., and Rukstales, K. S., (2008). “2008 USGS Seismic Hazard Maps: Documentation for the 2008 Update of the United States National Seismic Hazard Maps", USGS Open-File Report 2008-1128, U.S. Department of the Interior, U.S. Geological Survey.

Preumont, (1994), "Random Vibration and Spectral Analysis", Kluwer Acedemic Publishers, Norwell, MA.

Reid, H.F., (1911). “The Elastic-rebound Theory of Earthquake," University of California Publications in the Geological Sciences, 6, 13-44.

Richter, C. F. (1935). “An Instrumental Earthquake Magnitude Scale”, Bull. Seism. Soc. Am. 25, 1-32.

Richter, C. F. (1958). Elementary Seismology, W. H. Freeman and Co., San Francisco.

SAP2000, (2000). "Integrated Finite Element Analysis and Design of Structures", Computers and Structures, Inc., Berkeley, California. 
Saragoni, G. R. and Hart, G. C. (1974), Simulation of Artificial Earthquakes, Earthq. Eng. Struct. Dyn.2, 249-267.

Scherbaum, F. (1996). Of Poles and Zeros, Fundamentals of Digital Seismology, Kluwer Academic Publishers.

Schweig, E., Gomberg, J., Hendley, J. W. II, (1995). "The Mississippi Valley Whole Lotta Shakin' Goin' On", U.S. Geological Survey Fact Sheet-168-95, U.S. Department of the Interior, U.S. Geological Survey.

Silva, W., and Lee, K. (1987). "State-of-the-art for Assessing Earthquake Hazards in the United States", Report 24, WES RASCAL Code for Synthesizing Earthquake Ground Motions, Miscellaneous Paper S-73-1, U.S. Army Engineer Waterways Experiment Station, Vicksburg, MS.

Silva, W., Gregor, N., and Darragh, R., (2002). "Development of Hard Rock Attenuation Relations for Central and Eastern North America", Internal Report from Pacific Engineering.

Singh, S. and Hermann, R.B., (1983). "Regionalization of Crustal Coda Q in the Continental United States", J. Geophys. Res., 88, 527-538.

Somerville, P.G., Irikura, K., Graves, R., Sawada, S., Wald, D., Abrahamson, N.A., Iwasaki, Y., Kagawa, T., Smith, N., and Kowada, A. (1999). "Characterizing Crustal Earthquakes Slip Models for Prediction of Strong Ground Motion,” Seism. Research Letters, 70(1), 59-80.

Somerville, P., Collins, N., Abrahamson, N., Graves, R., and Saikia, C., (2001). Ground Motion Attenuation Relations for the Central and Eastern United States, Final Report to U.S. Geological Survey.

Stein, S. and Wysession, M., (2003). "An Introduction to Seismology, Earthquakes, and Earth Structure", Blackwell Publishing Ltd.

Stewart, J. P., Chiou, S. J., Bray, J. D., Graves, R. W., Somerville, P. G., and Abrahamson, N. A., (2001). "Ground Motion Evaluation Procedures for Performance-Based Design", PEER Report 2001/09, Pacific Earthquake Engineering Research Center, College of Engineering, University of California, Berkeley.

Street, R., Kiefer, J. D., Raisor, J., (2008). "Assessing the Felt Reports of the 181112 New Madrid Earthquakes in the Central United States", Kentucky Geological Survey Report of Investigations 20 Series XII. 
Tavakoli, B., and Pezeshk, S., (2005). "Empirical-stochastic Ground-motion Prediction for Eastern North America", Bulletin of the Seismological Society of America, v. 95, p. 2283-2296.

Toro, G.R. and McGuire, R.K. (1987). "An Investigation into Earthquake Ground Motion Characteristics in Eastern North America." Bull. Seism. Soc. Am., 77(2), 468-489.

Toro, G.R., Abrahamson, N.A. and J.F. Schneider (1997). "Model of Strong Ground Motions from Earthquakes in Central and Eastern North America: Best Estimates and Uncertainties." Seismological Research Let., 68(1), 41-57.

Uhrhammer, R. A. and Collins, E. R.(1990). "Synthesis of Wood-Anderson Seismograms from Broadband Digital Records", Bull Seism. Soc. Am. $80,702-716$.

Uhrhammer, R. A., Loper, S. J. and Romanowicz, B. (1996). "Determination of Local Magnitude Using BDSN Broadband Records", Bulletin of the Seismological Society of America, Vol. 86, No. 5, pp. 1314-1330.

US Army Corps of Engineers (2003). "Time History Dynamic Analysis of Concrete Hydraulic Structures”. Engineer Manual EM1110-2-6051.

U.S. Geological Survey Earthquake Hazards Program, (2009). "Historic Earthquakes: Charleston, South Carolina", Abridged from Seismicity of the United States, 1568-1989 (Revised), by Carl W. Stover and Jerry L. Coffman, U.S. Geological Survey Professional Paper 1527, United States Government Printing Office, Washington: 1993.

U.S. Geological Survey Earthquake Hazards Program, (2009). "Historic Earthquakes: New Madrid Earthquakes 1811-1812 Earthquake Summary", Abridged from Seismicity of the United States, 1568-1989 (Revised), by Carl W. Stover and Jerry L. Coffman, U.S. Geological Survey Professional Paper 1527, United States Government Printing Office, Washington: 1993.

Vanmarcke, E. H. (1976). "Structural Response to Earthquakes, Seismic Risk and Engineering Decisions", C. Lomnitz C. and Rosenblueth, E. eds., Elsevier Science Publishers BV, Amsterdam, the Netherlands, 287-337.

Wheeler, R. L., Trevor, N. K., Tarr, A. C. and Crone, A. J., (2001). "Earthquakes In and Near the Northeastern United States", 1638-1998, U.S. Geological Survey Fact Sheet FS-0006-01, U.S. Department of the Interior, U.S. Geological Survey.

Williams, A., (2005). Seismic and Wind Forces, Structural Design Examples, International Code Council. 
Wilson, E. L., (1962). "Dynamic Response by Step-By-Step Matrix Analysis", Proceedings, Symposium On The Use of Computers in Civil Engineering, Lisbon, Portugal.

Wilson, E. L., Farhoomand, I., and Bathe, K. J. (1973). “Nonlinear Dynamic Analysis of Complex Structures", Earthquake Engineering and Structural Dynamics, 1, 241-252. 


\section{APPENDIX A}

This appendix provides plots of the mean displacement ductility demands as a function of natural period for the single degree of freedom (SDOF) systems investigated in this study. These SDOF systems have natural periods of $0.1,0.2,0.6$, 1.0 and 3.0 seconds. The curves shown are made up of the mean ductility demands from 25 synthetics from each of the attenuation models for each of the magnitude and distance combinations considered in this study. 
Comparison of the Mean Displacement Ductility Demands of 25 Synthetics from Each of the Attenuation Models for $M_{W} 5.5$ @ 10 km

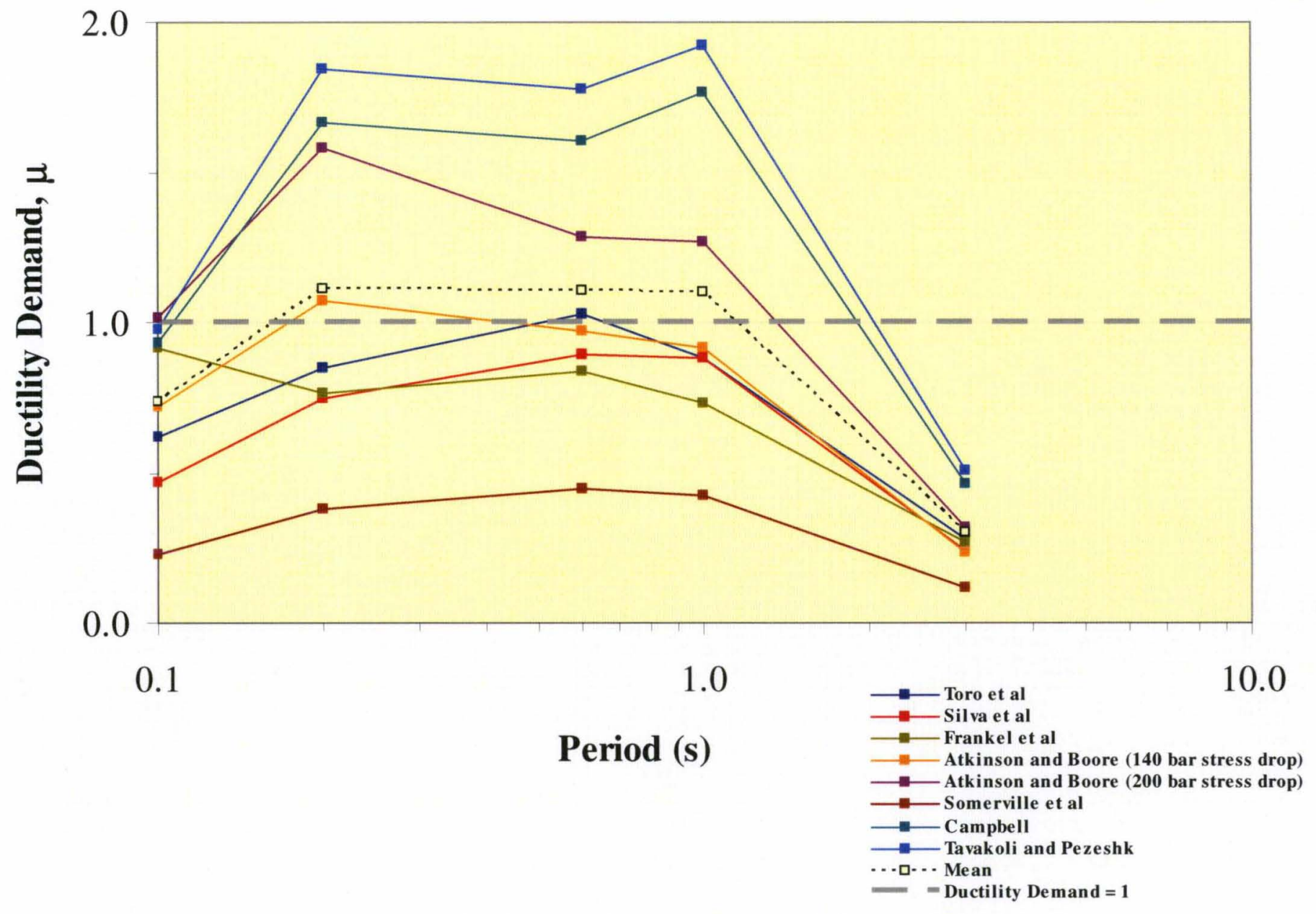




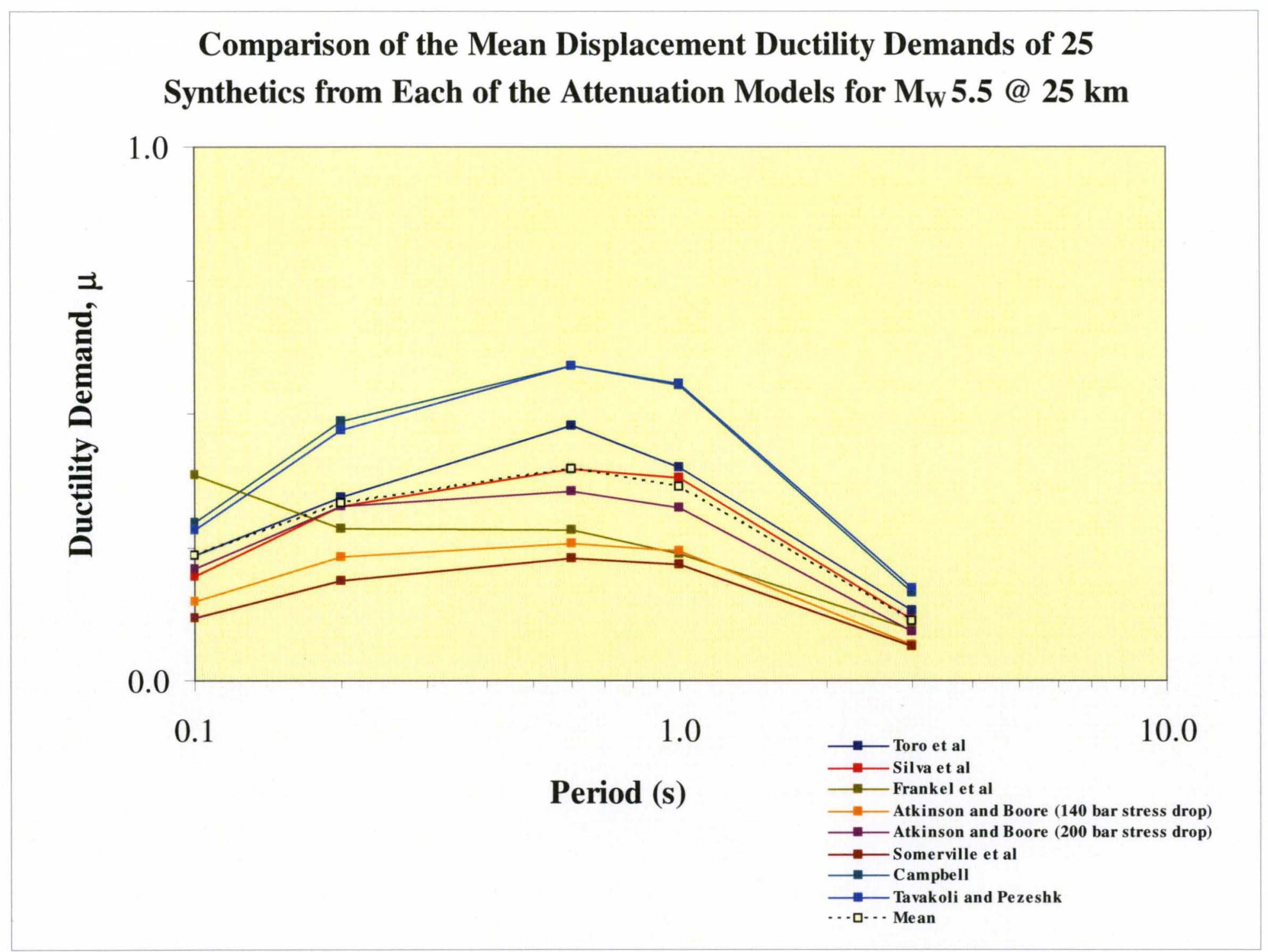




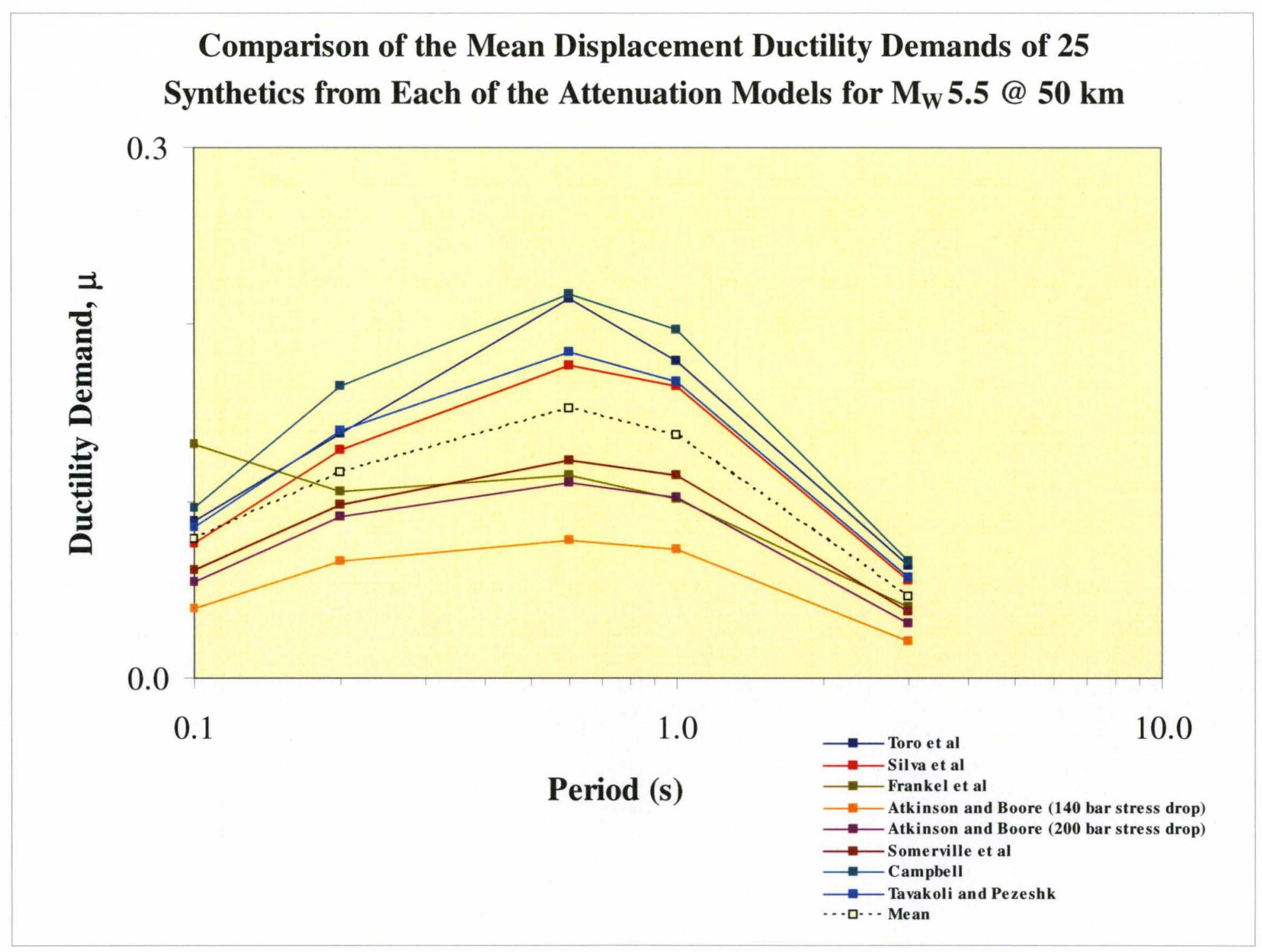




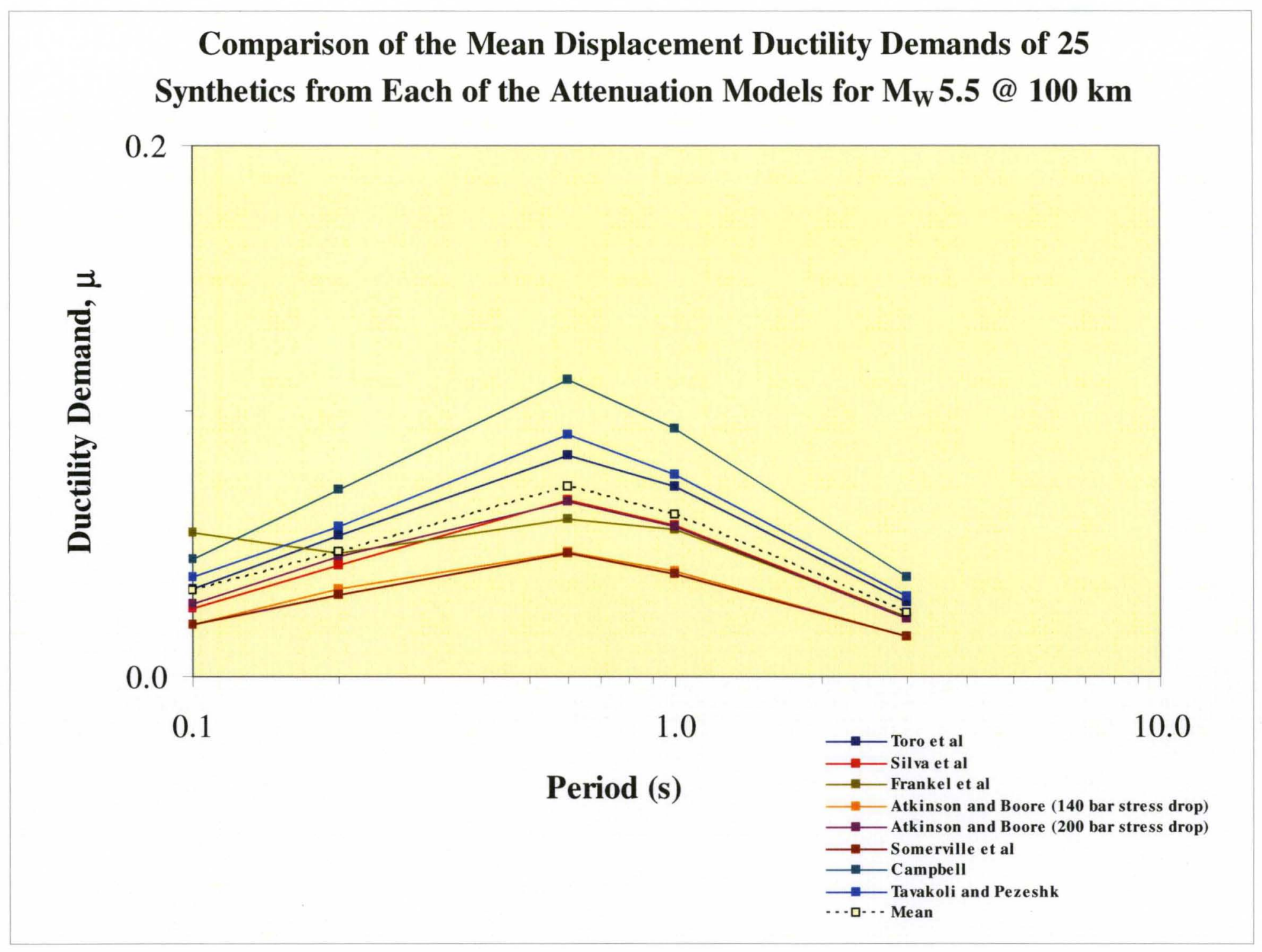




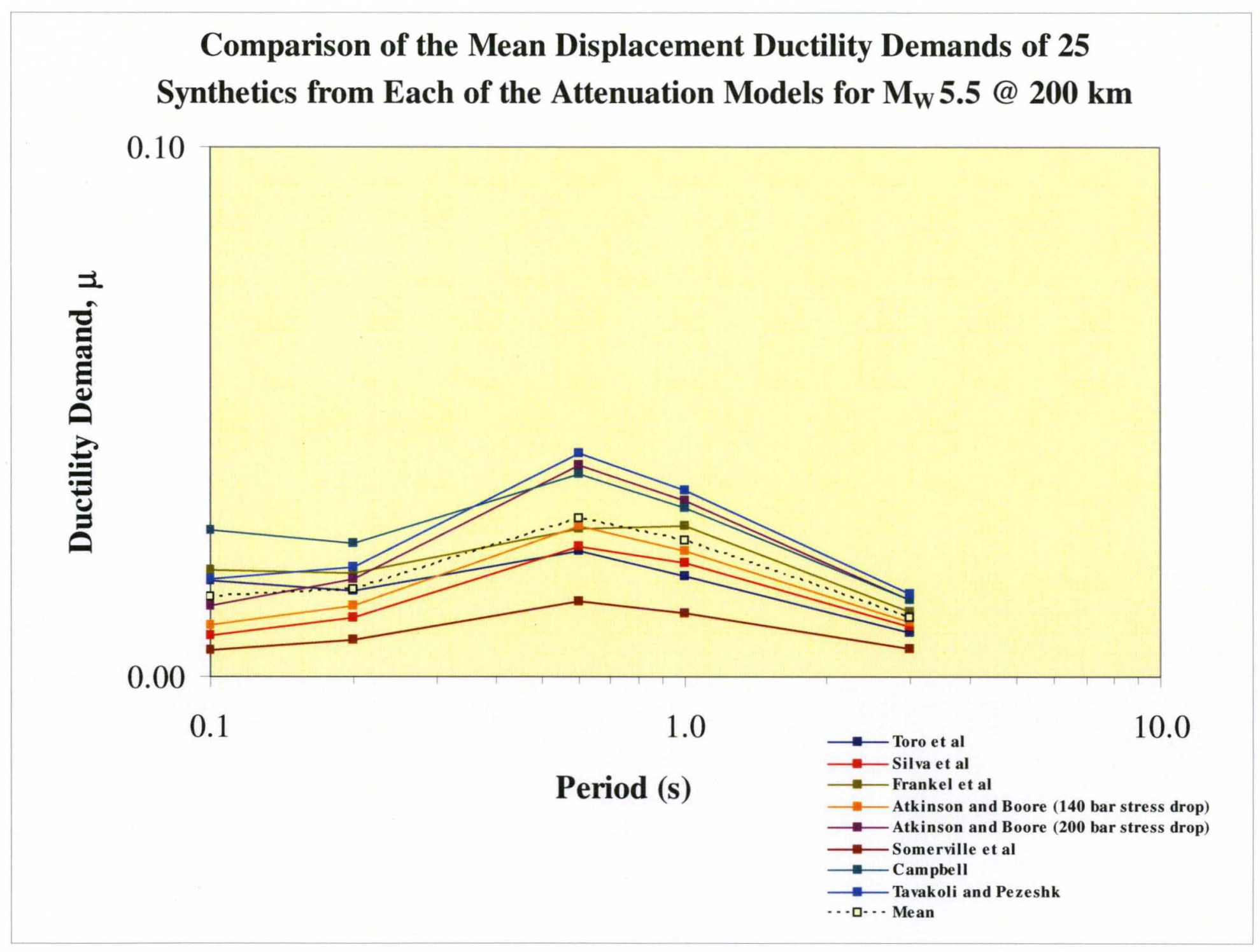




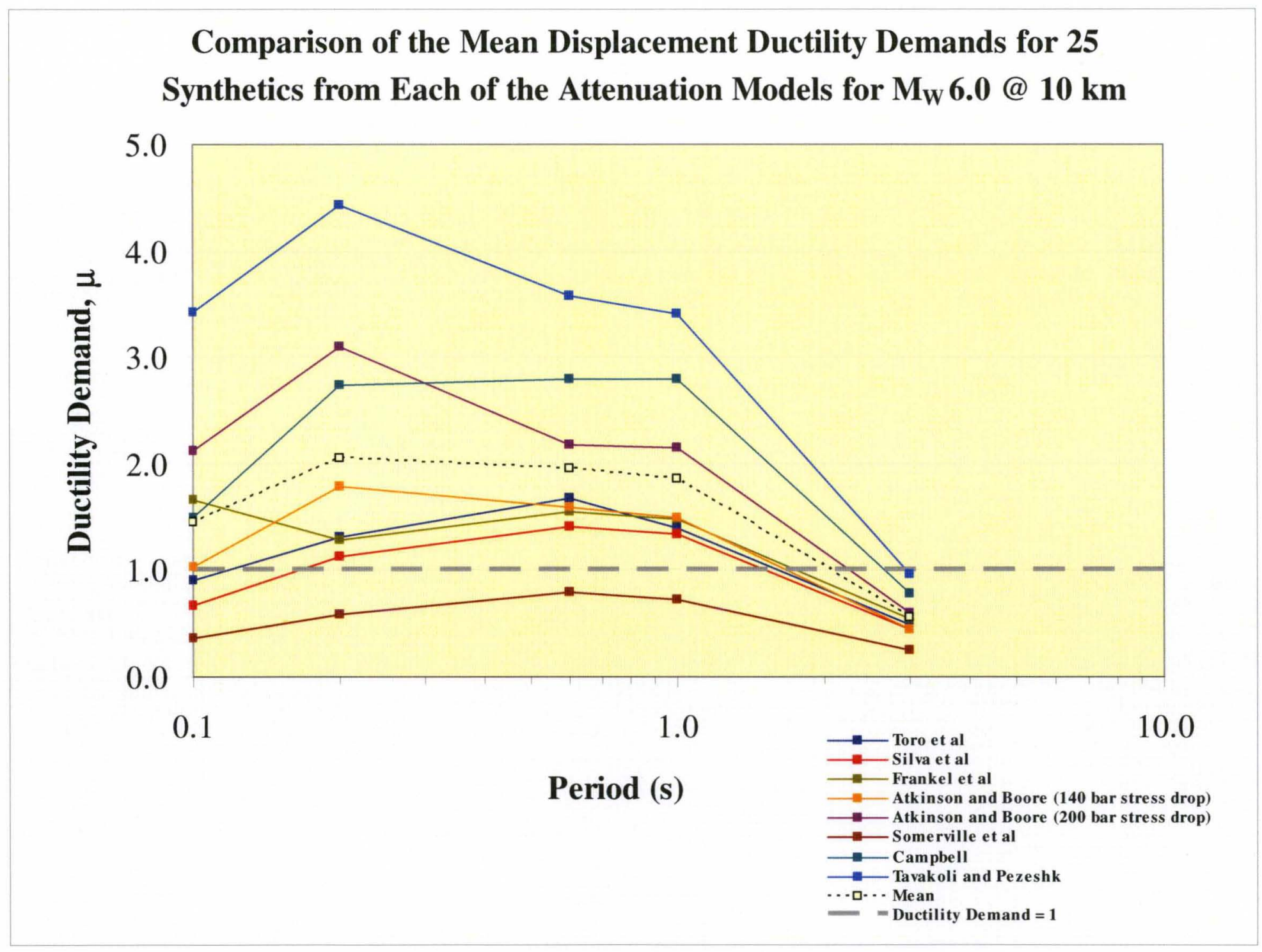




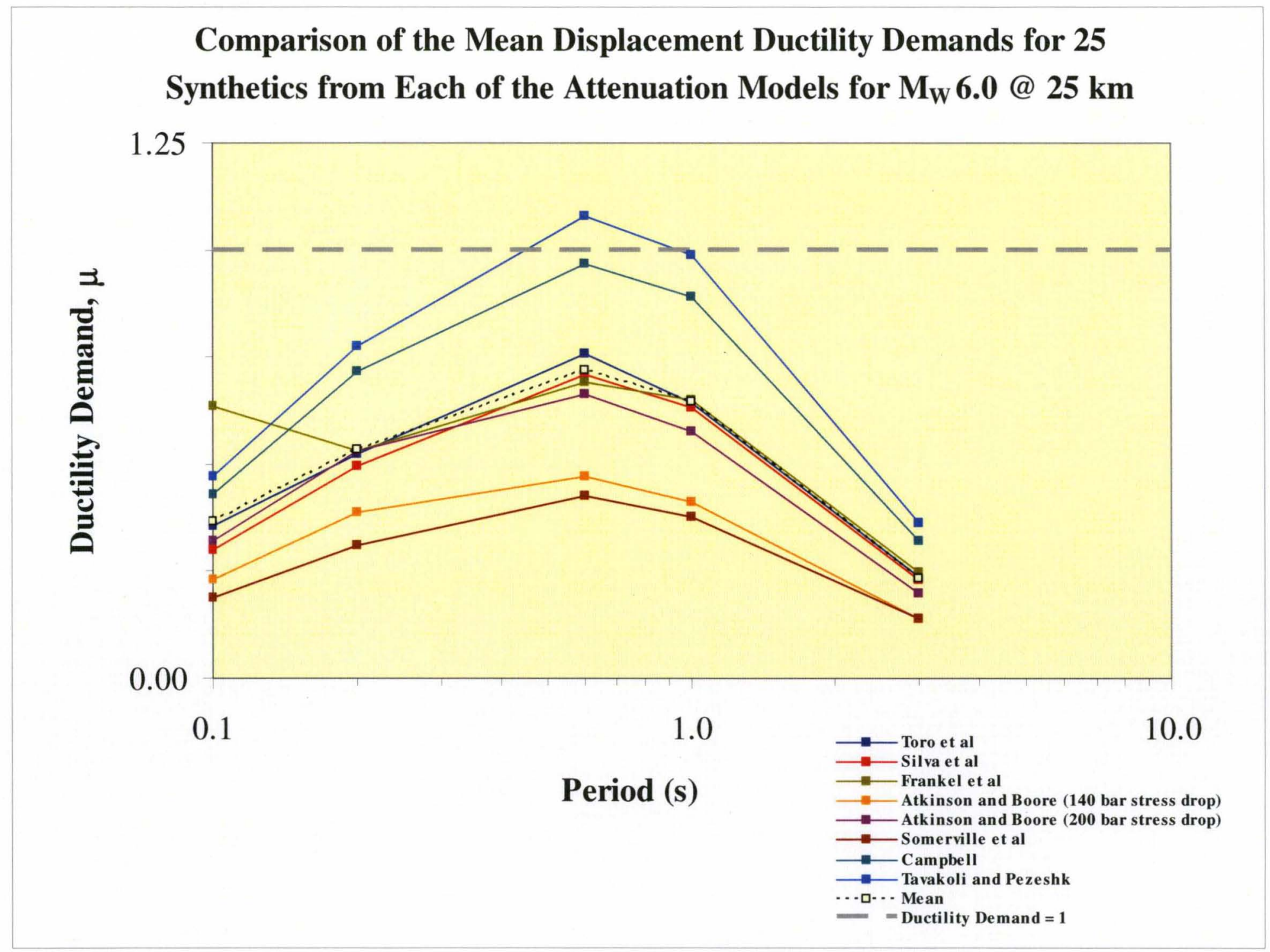




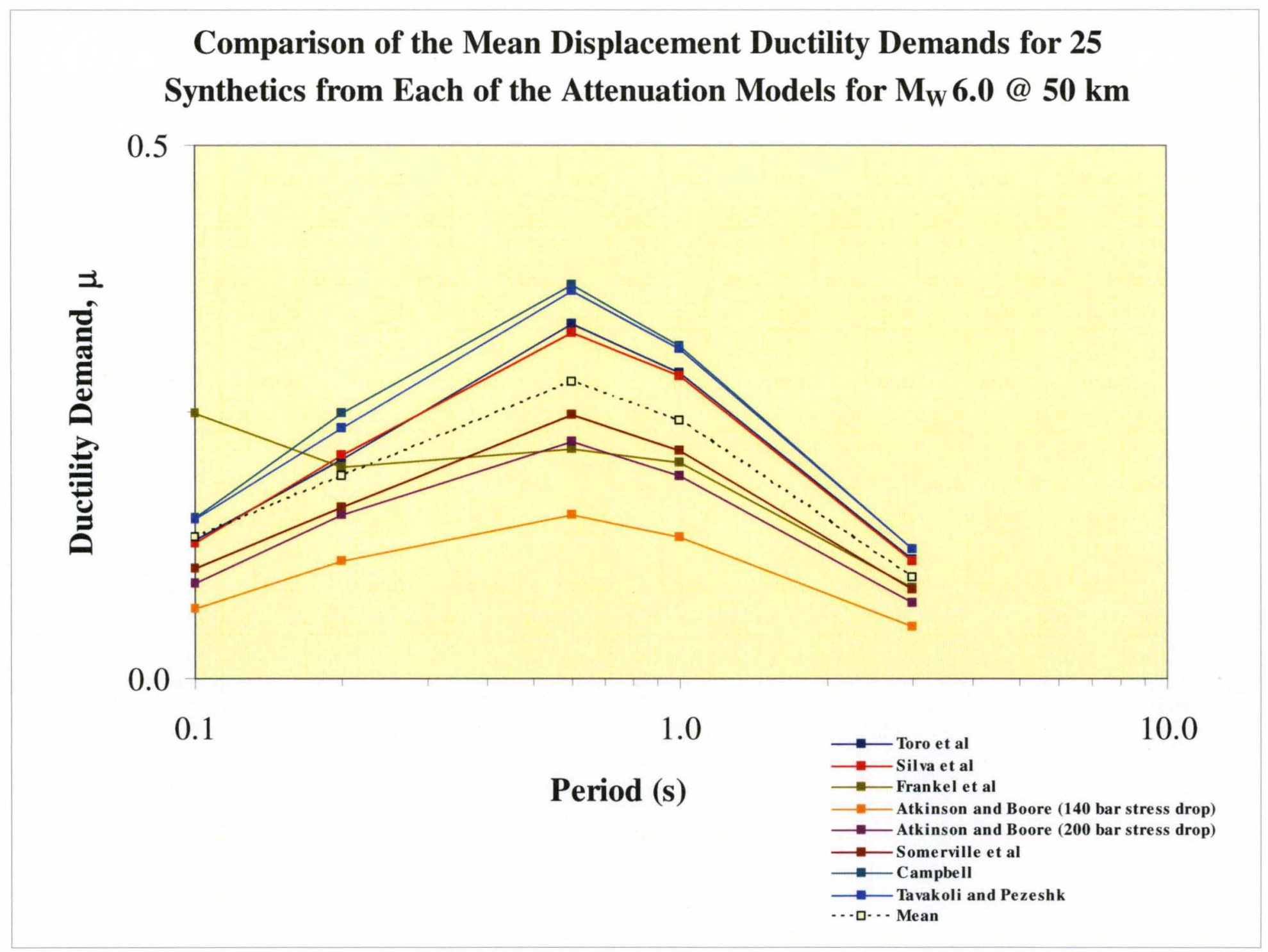


Comparison of the Mean Displacement Ductility Demands for 25

Synthetics from Each of the Attenuation Models for $M_{W} 6.0 @ 100$ km

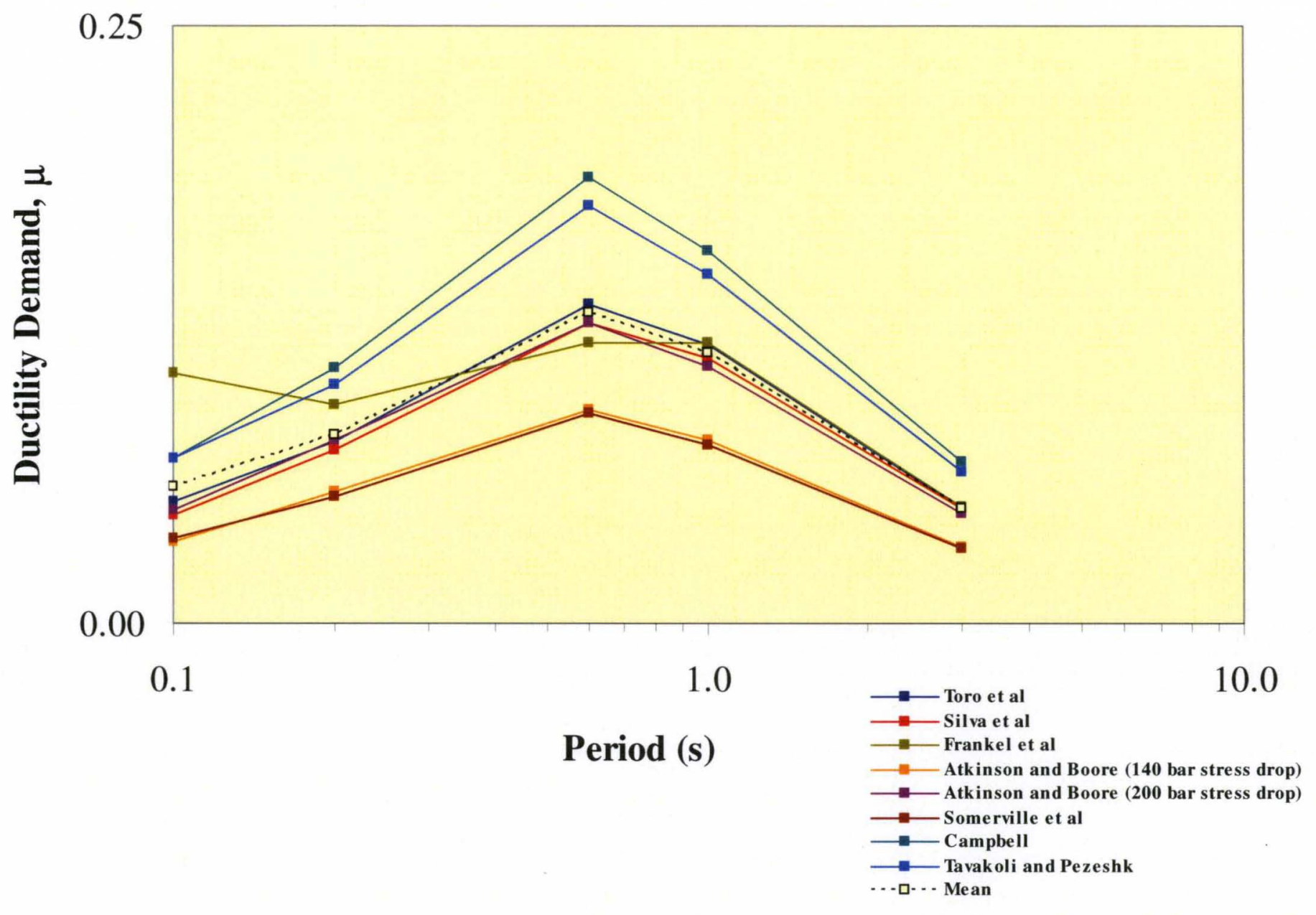




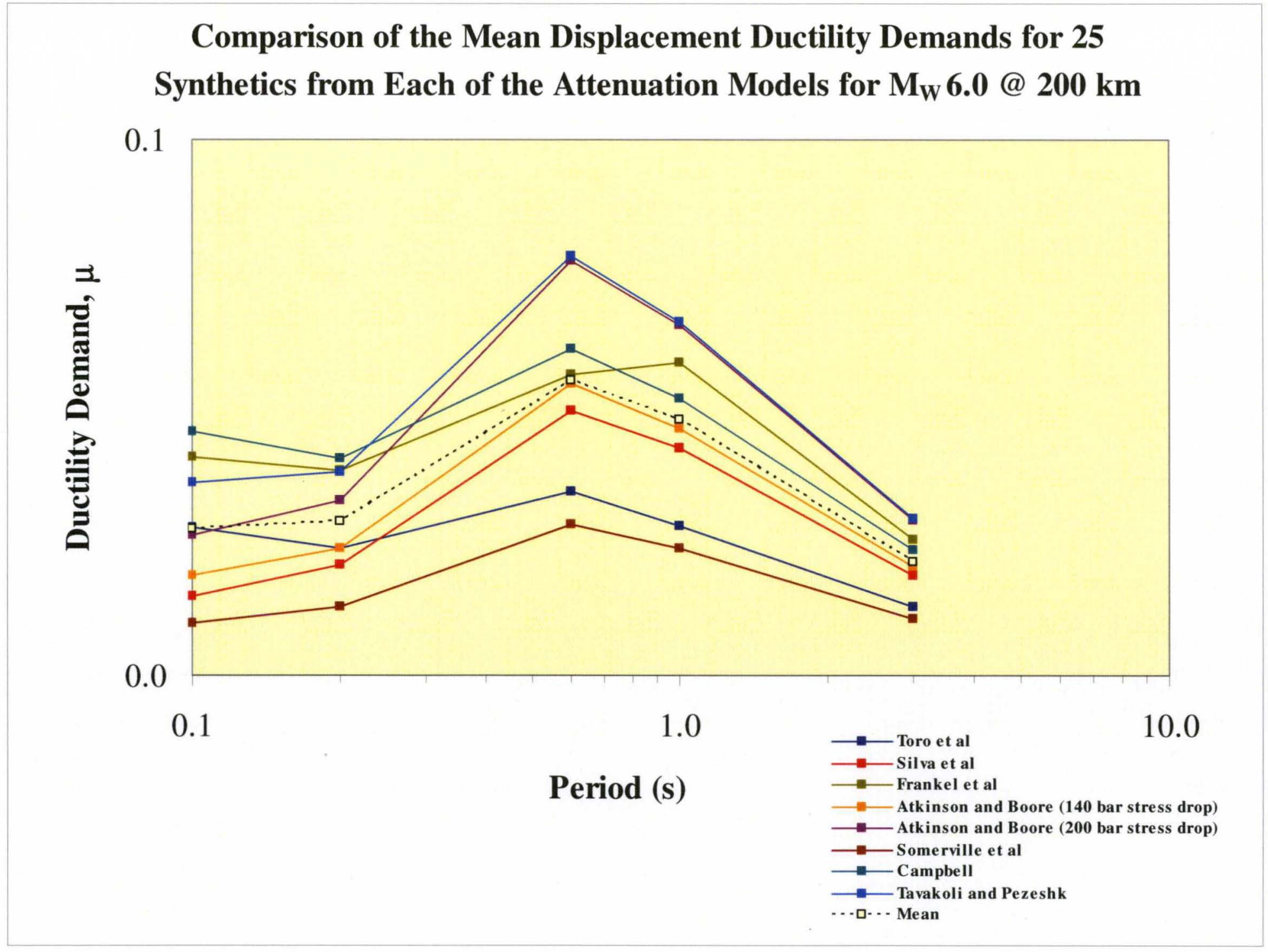




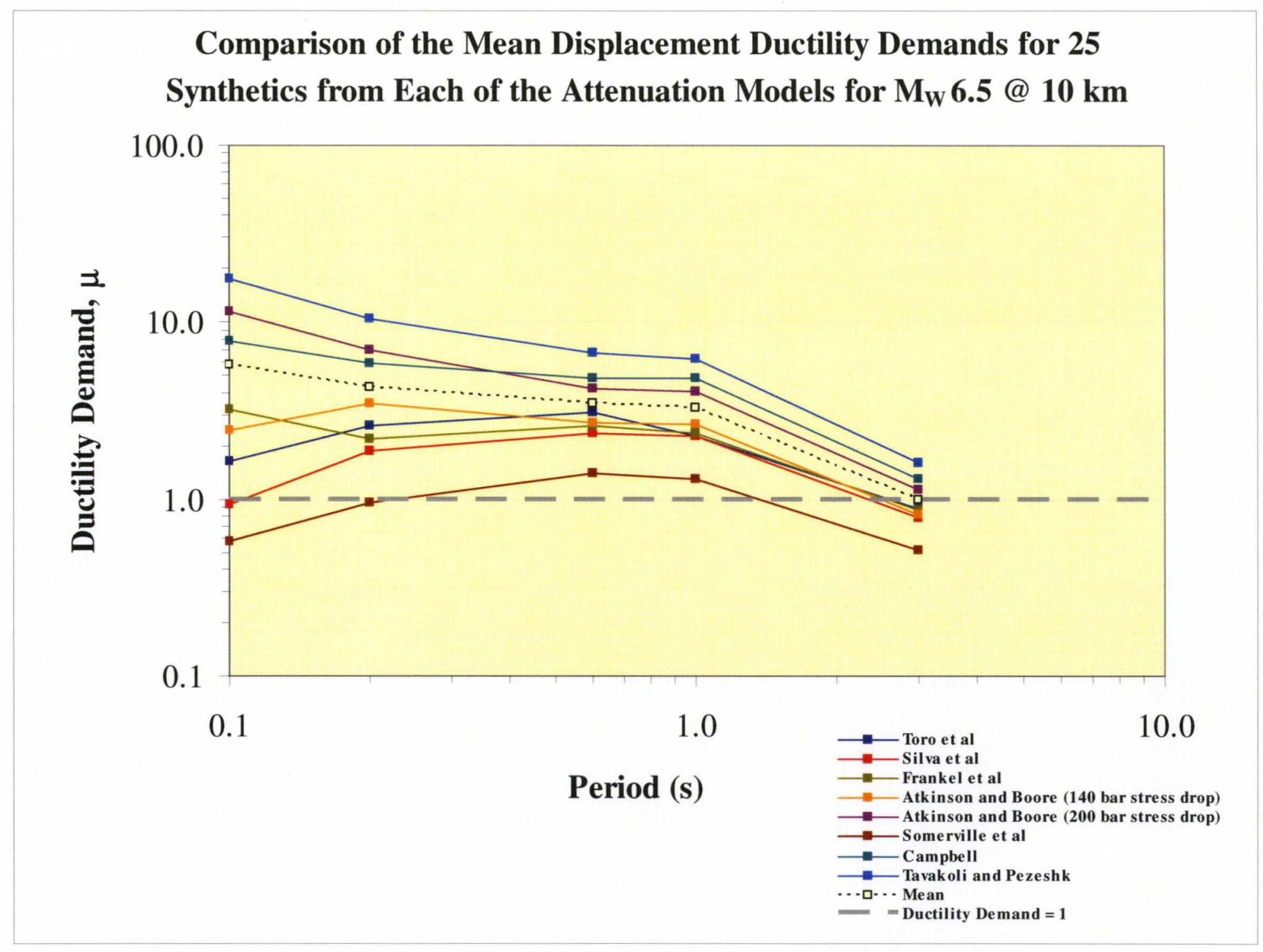




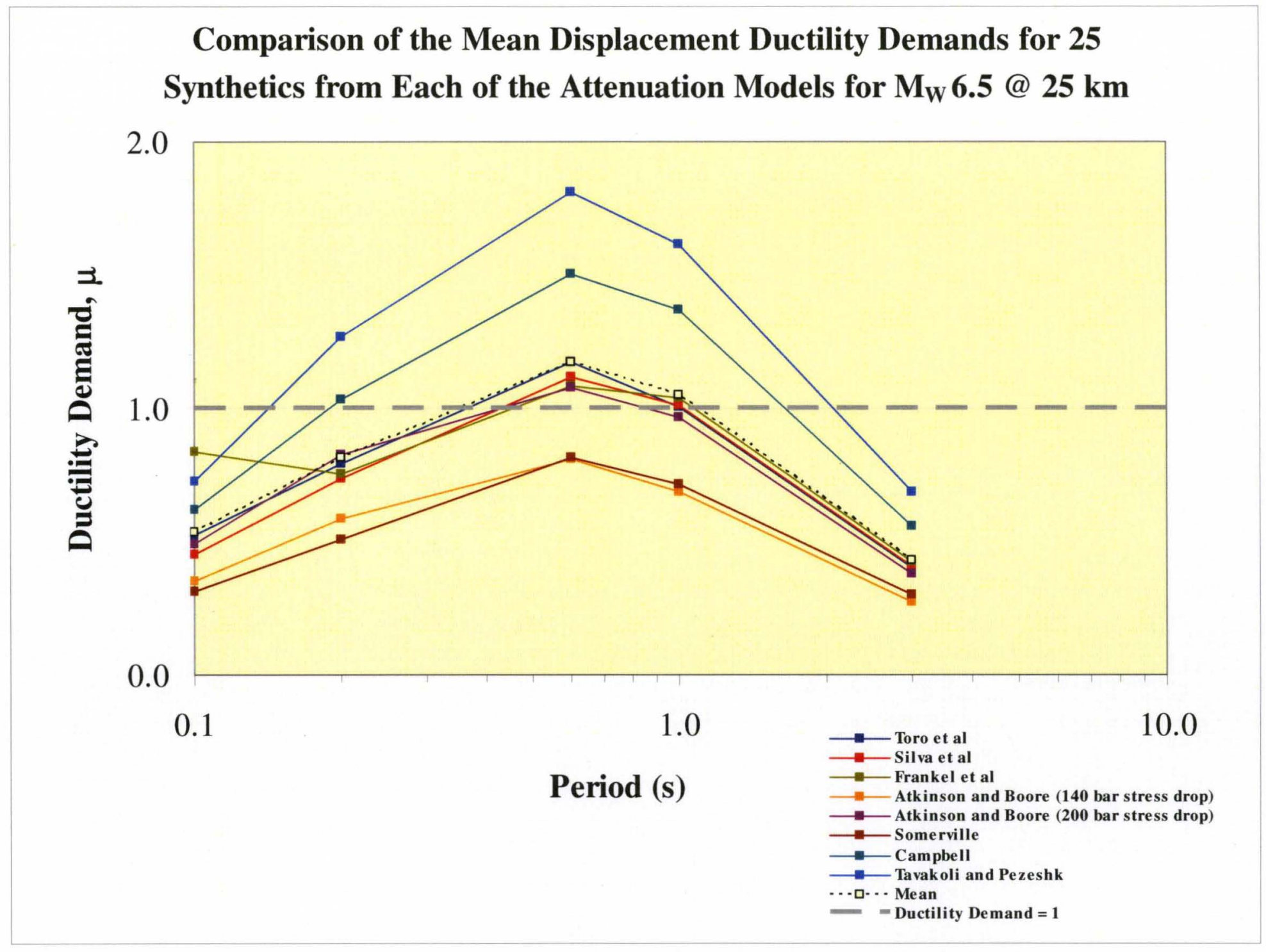




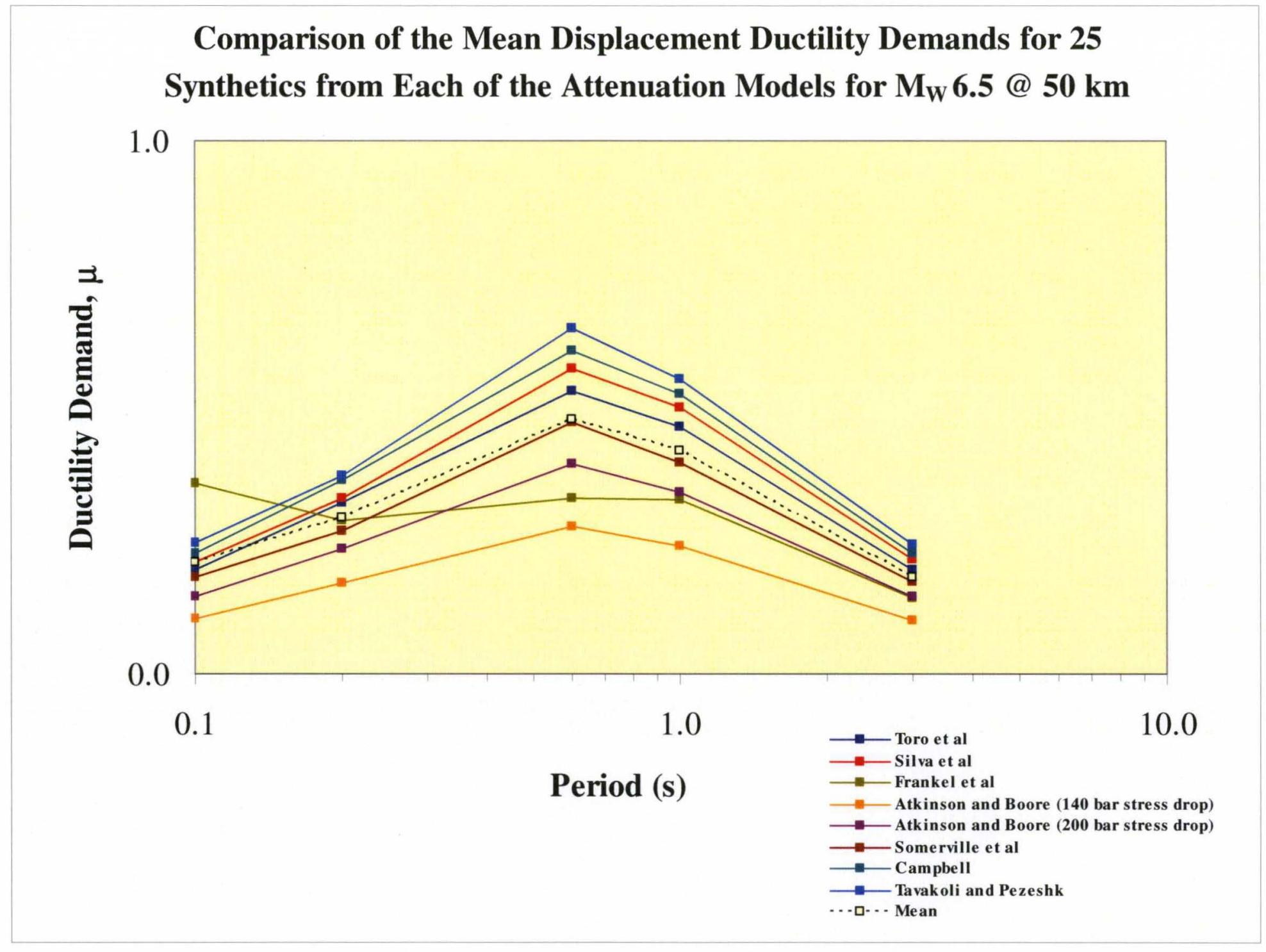




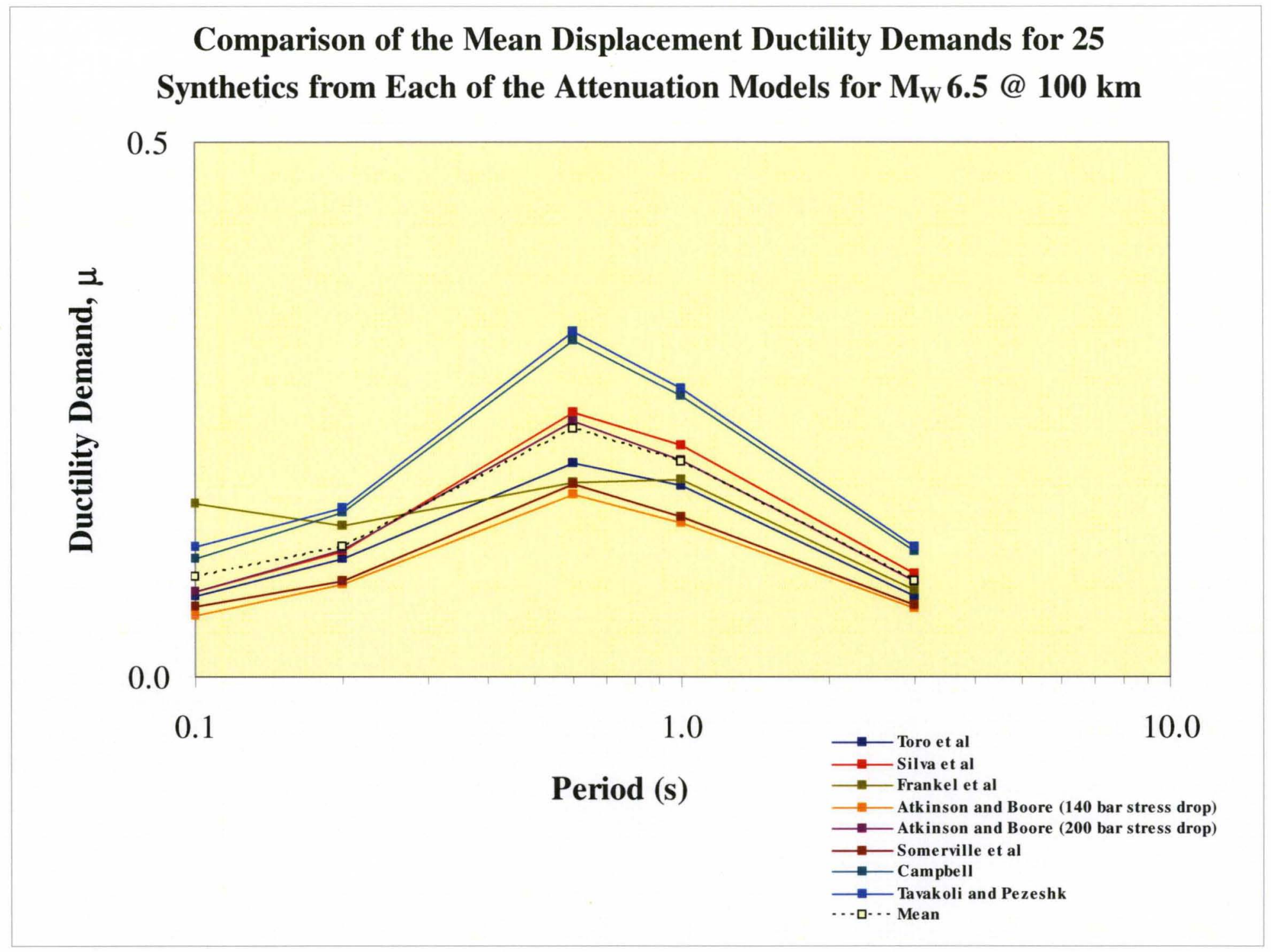




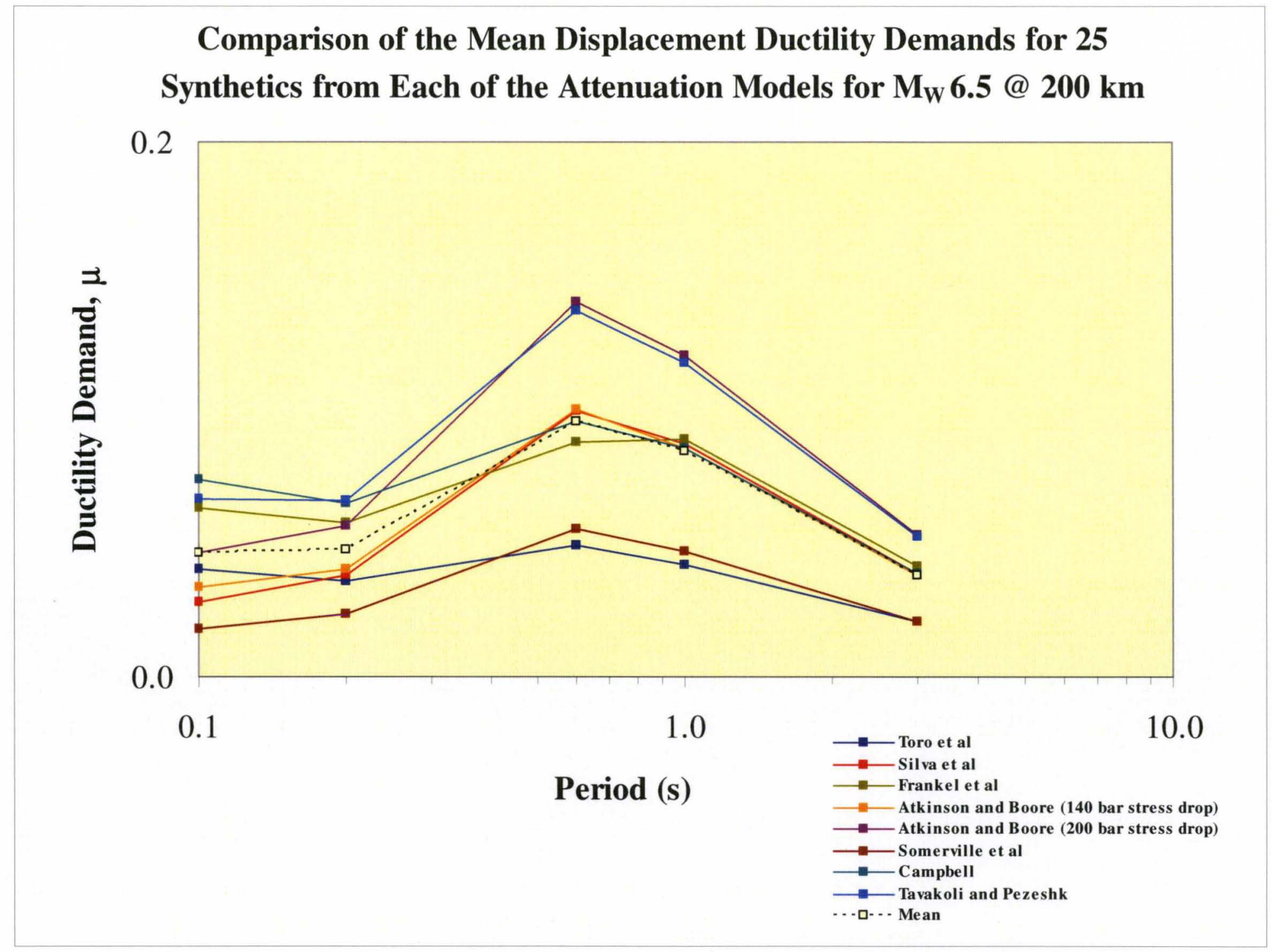




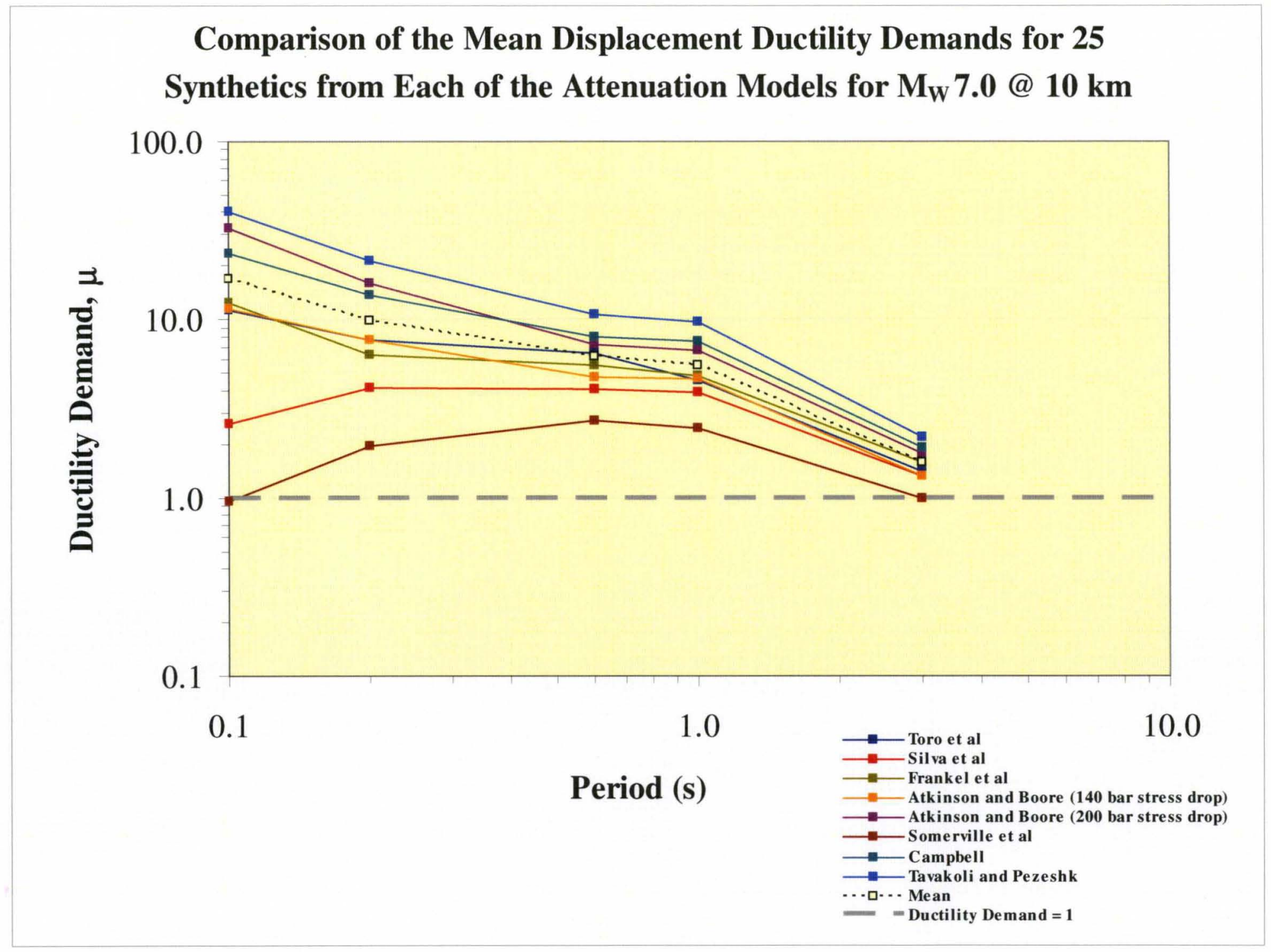




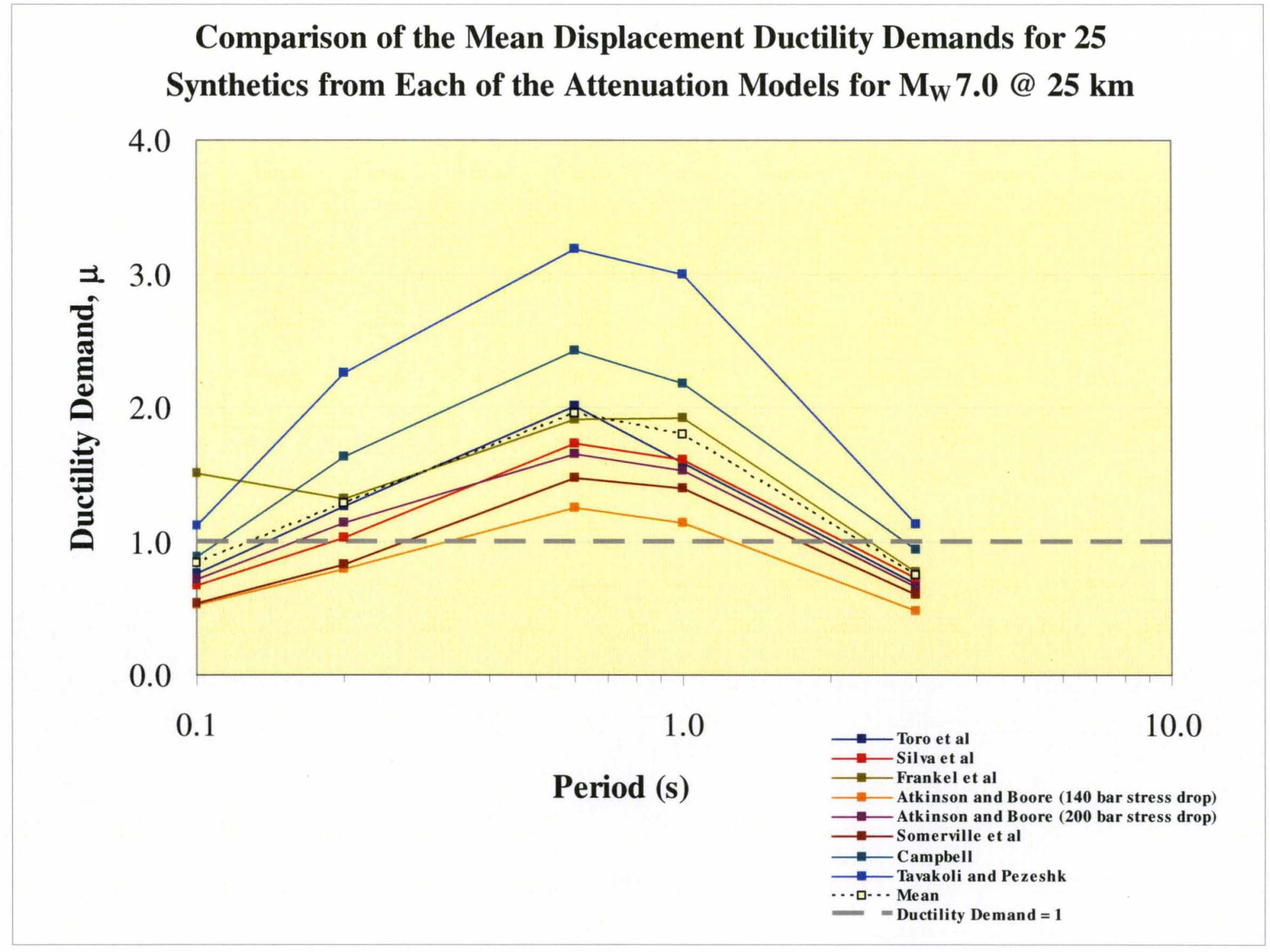




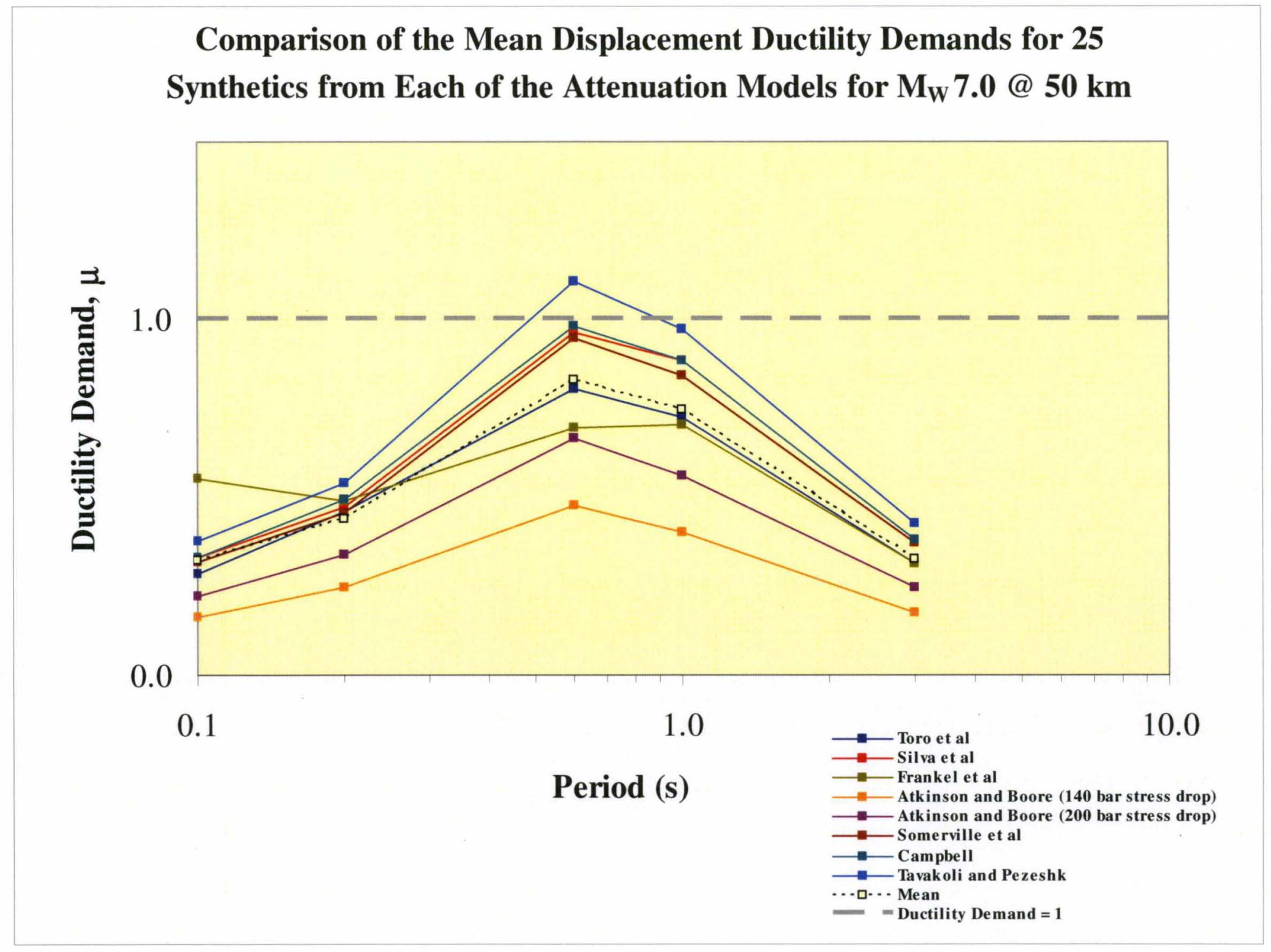




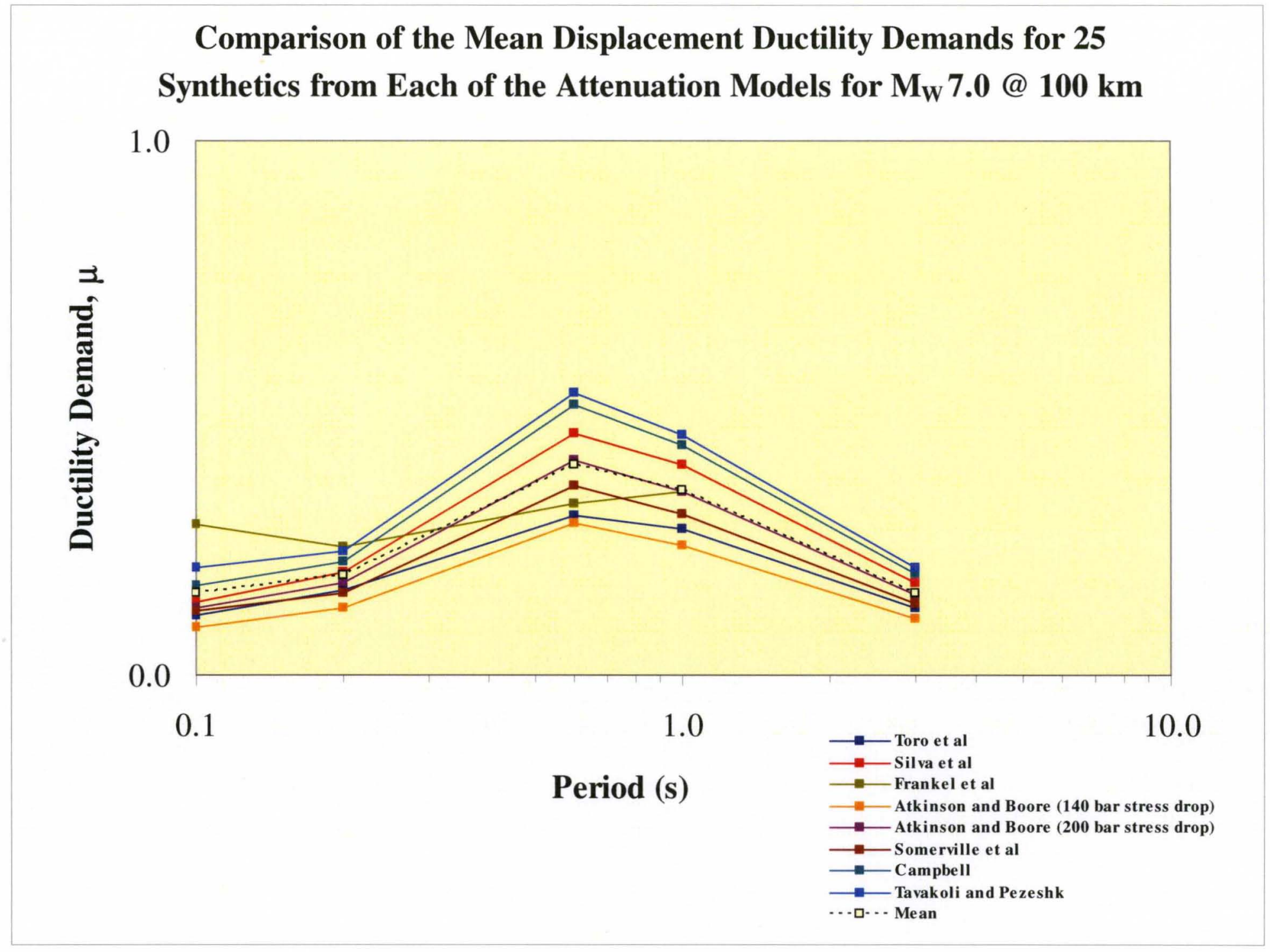




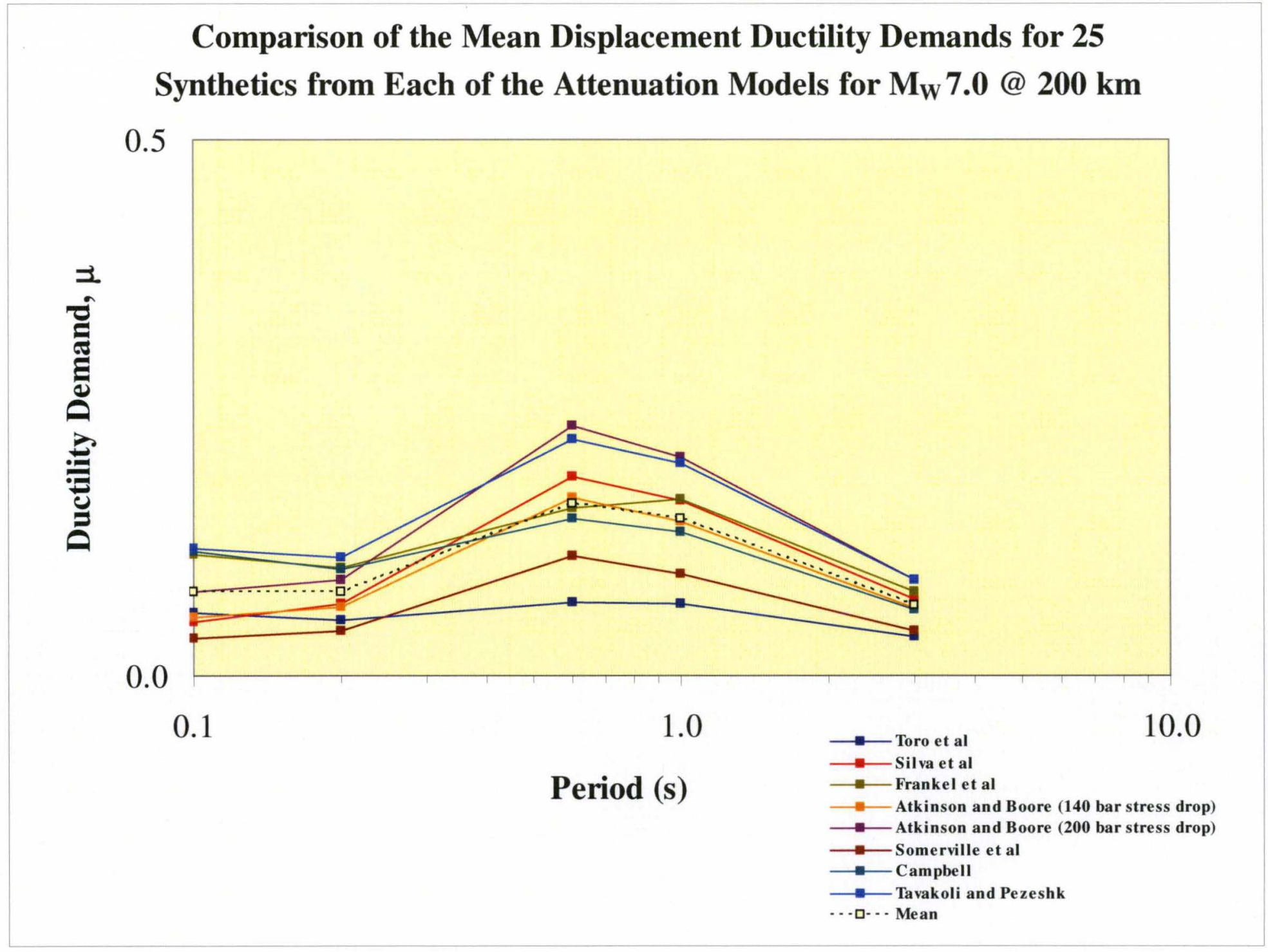


Comparison of the Mean Displacement Ductility Demands for 25

Synthetics from Each of the Attenuation Models for $M_{W} 7.5$ @ 10 km

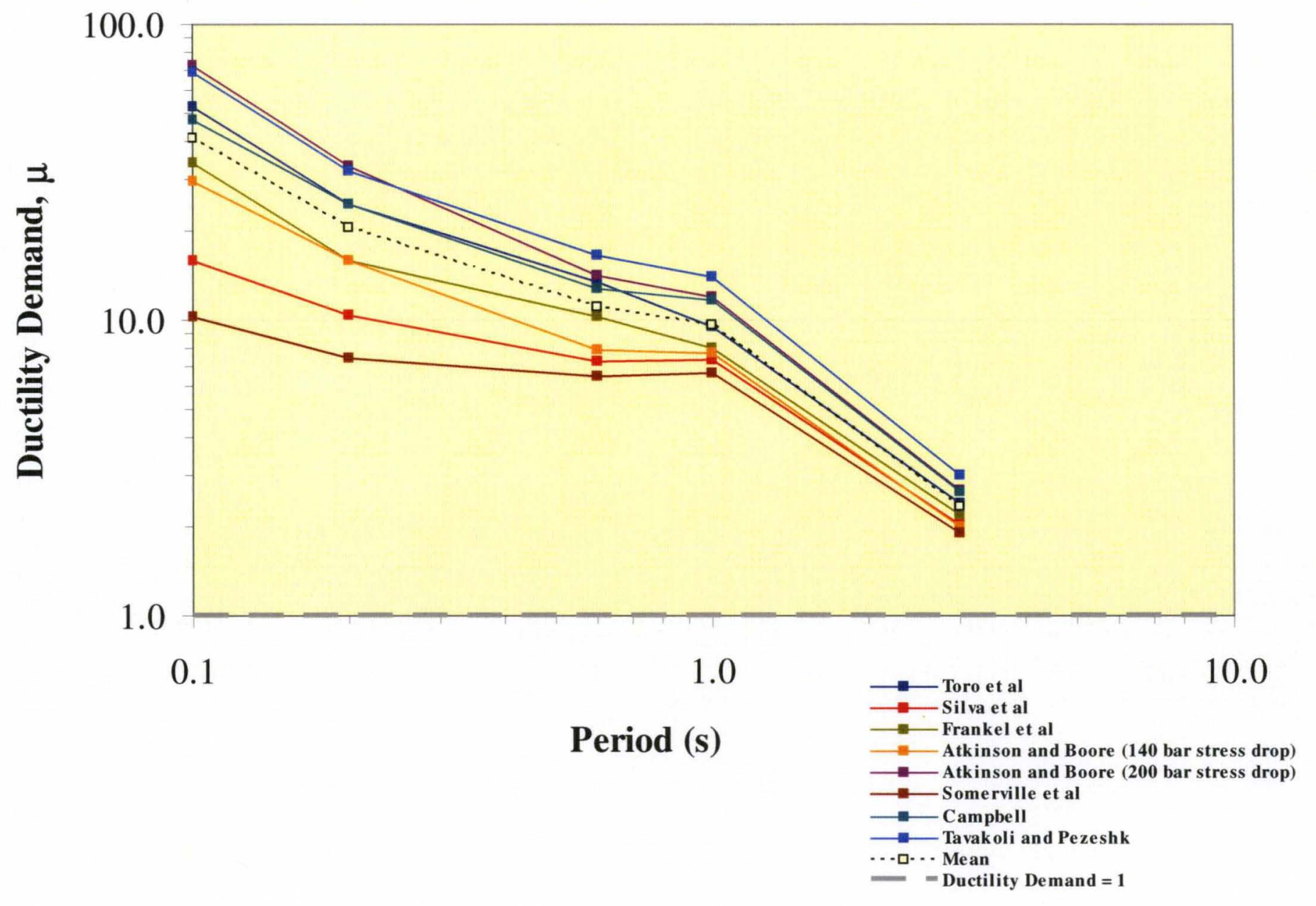


Comparison of the Mean Displacement Ductility Demands for 25

Synthetics from Each of the Attenuation Models for $M_{W} 7.5$ @ 25 km

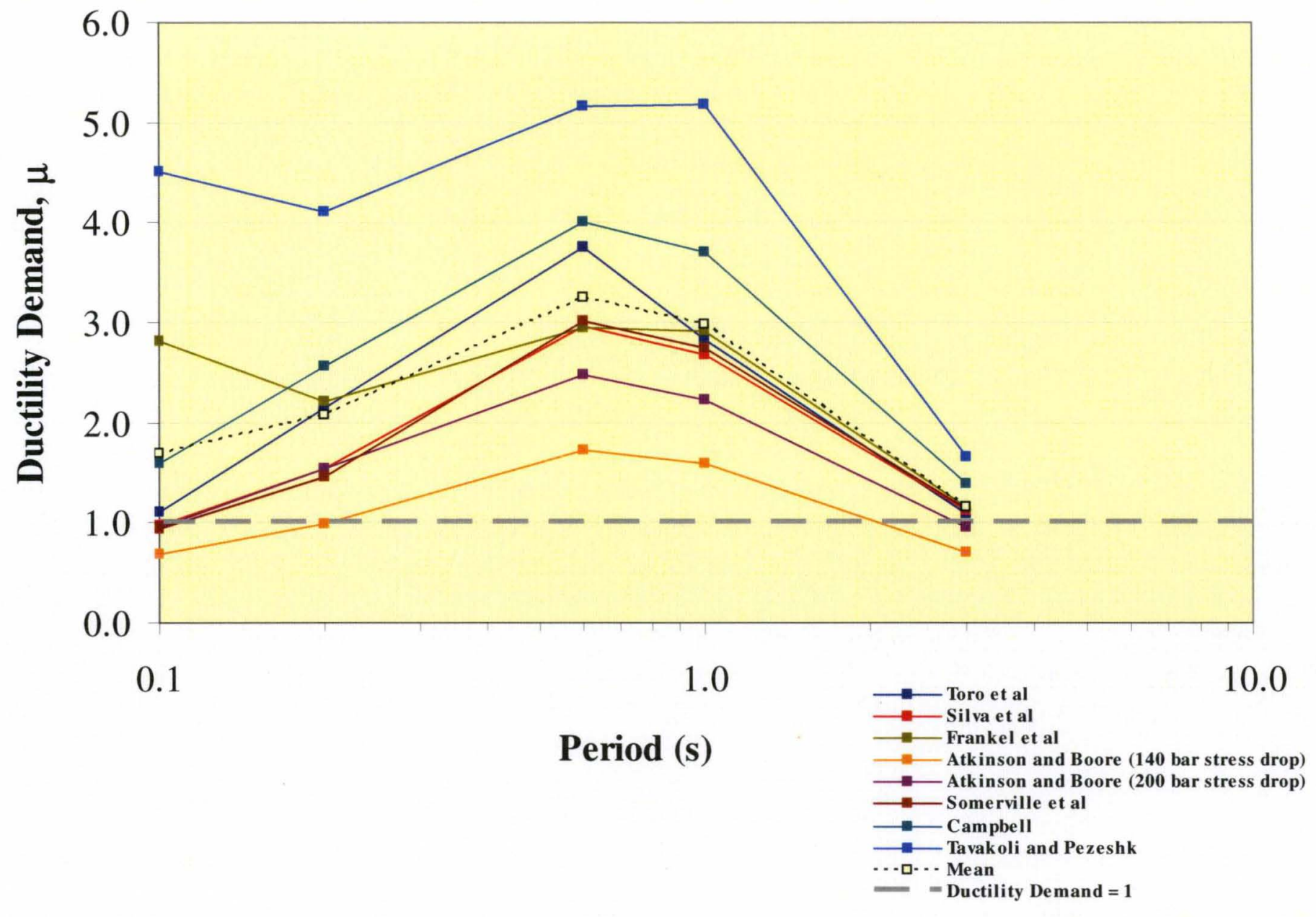


Comparison of the Mean Displacement Ductility Demands for 25

Synthetics from Each of the Attenuation Models for $M_{W} 7.5$ @ 50 km

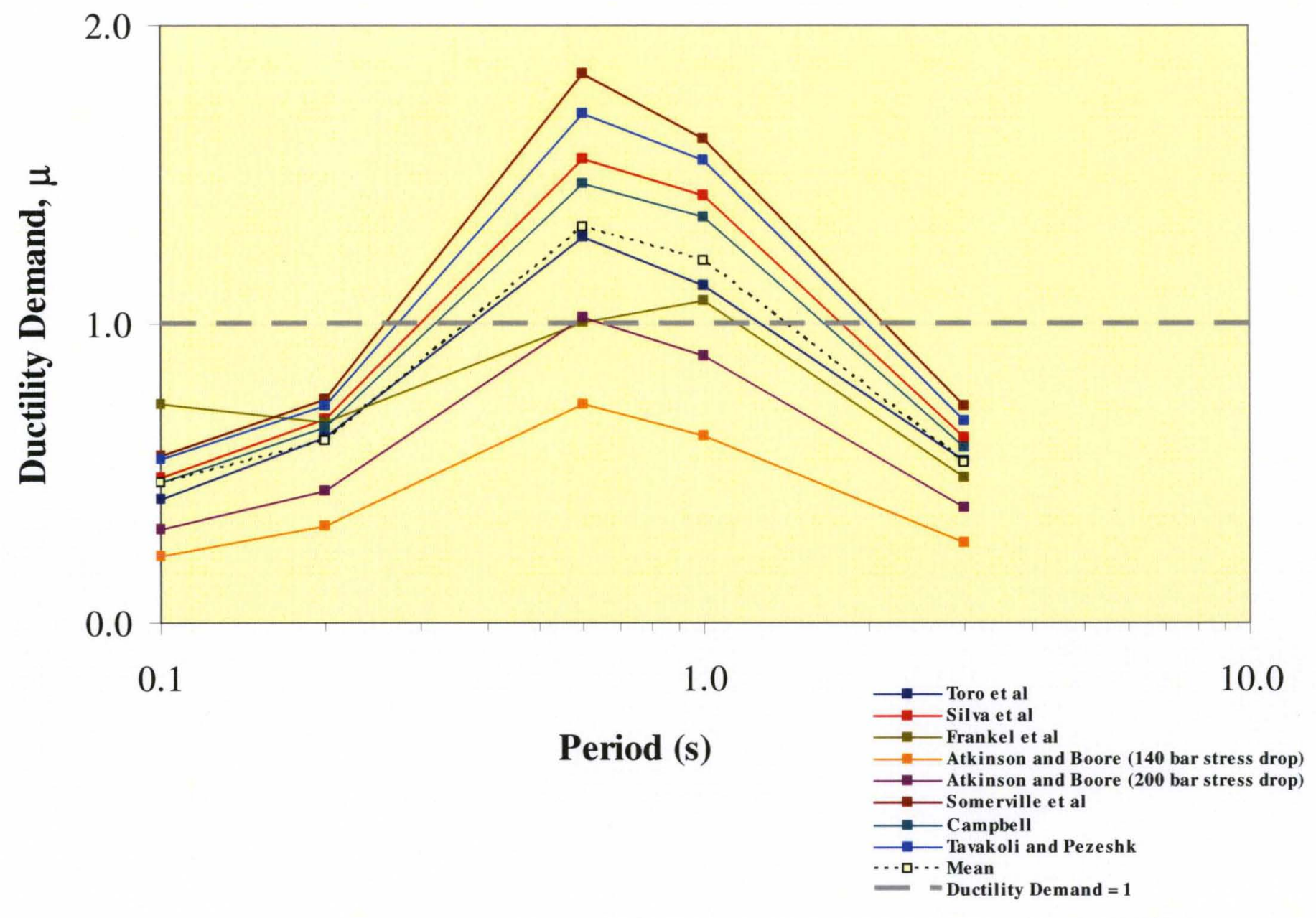




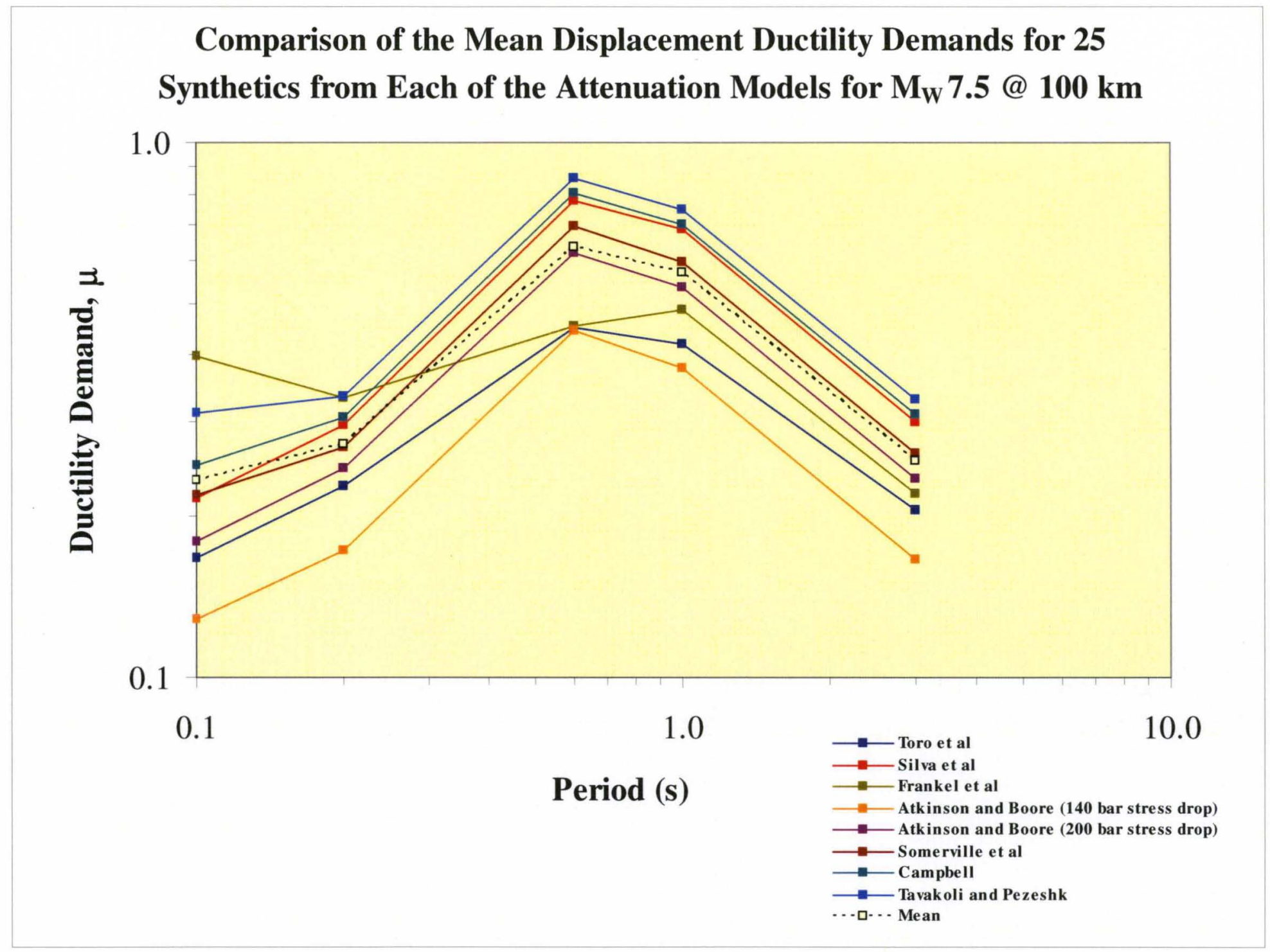


Comparison of the Mean Displacement Ductility Demands for 25

Synthetics from Each of the Attenuation Models for $M_{W} 7.5$ @ 200 km

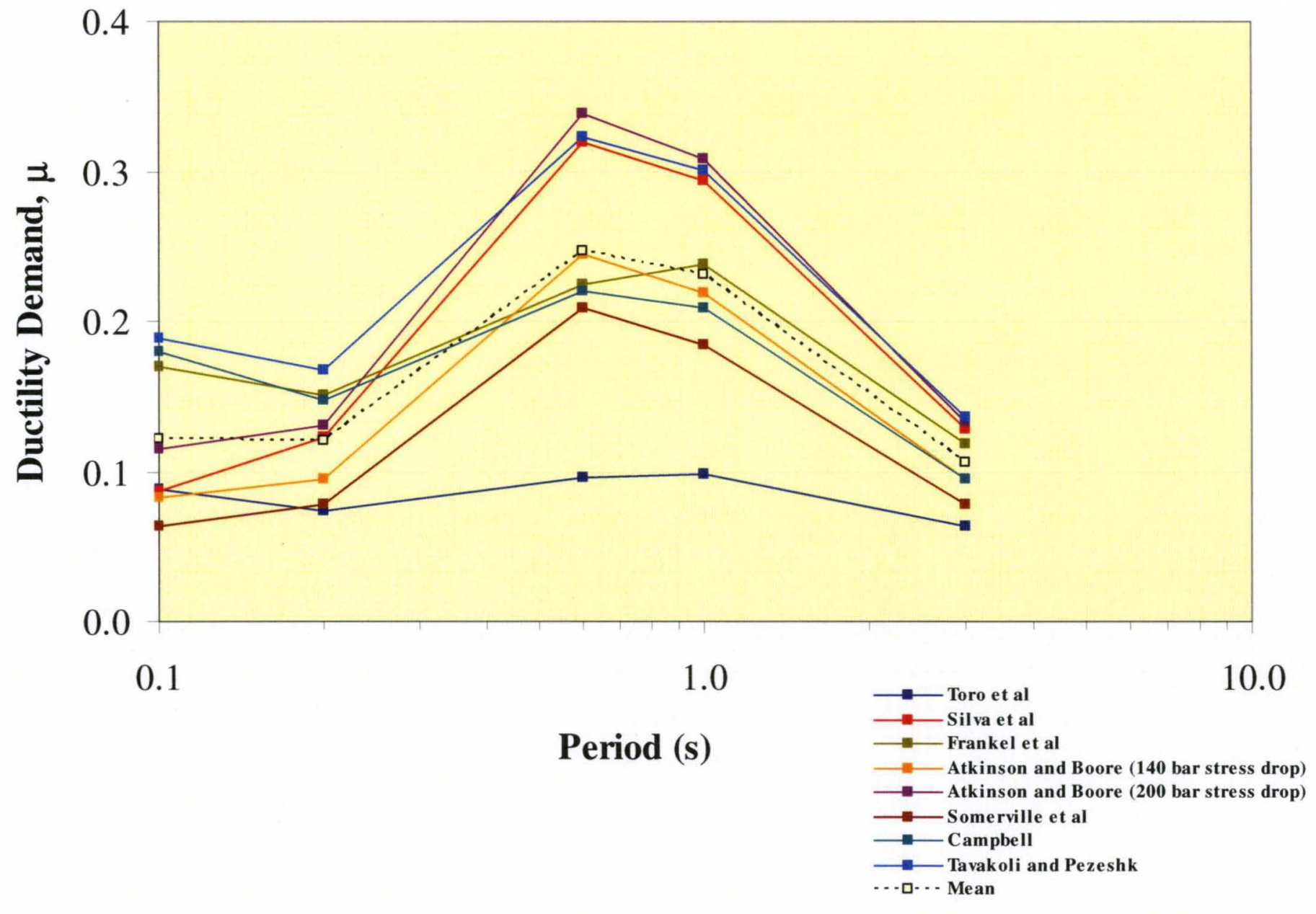




\section{APPENDIX B}

This appendix provides charts containing $95 \%$ confidence intervals for displacement ductility demand from nonlinear time history analyses with 25 synthetics for each attenuation model, each magnitude and distance combination, and each SDOF system. These confidence intervals were obtained from individual onesample t-tests on the 25 observations from each model at a $95 \%$ confidence level.

This appendix also provides charts containing the $95 \%$ confidence intervals of the calculated moment magnitudes for each of the attenuation models at each of the five distances investigated in this study. The moment magnitudes were calculated using the maximum displacements as determined from the nonlinear time history analyses with parameters set to simulate those of the Wood-Anderson seismograph. Corrections were applied for distances of $100 \mathrm{~km}$ and $200 \mathrm{~km}$ for Eastern North America. The confidence intervals are based upon 25 observations and individual one-sample t-tests on each model at each magnitude for each of he five distances investigated in the study. 


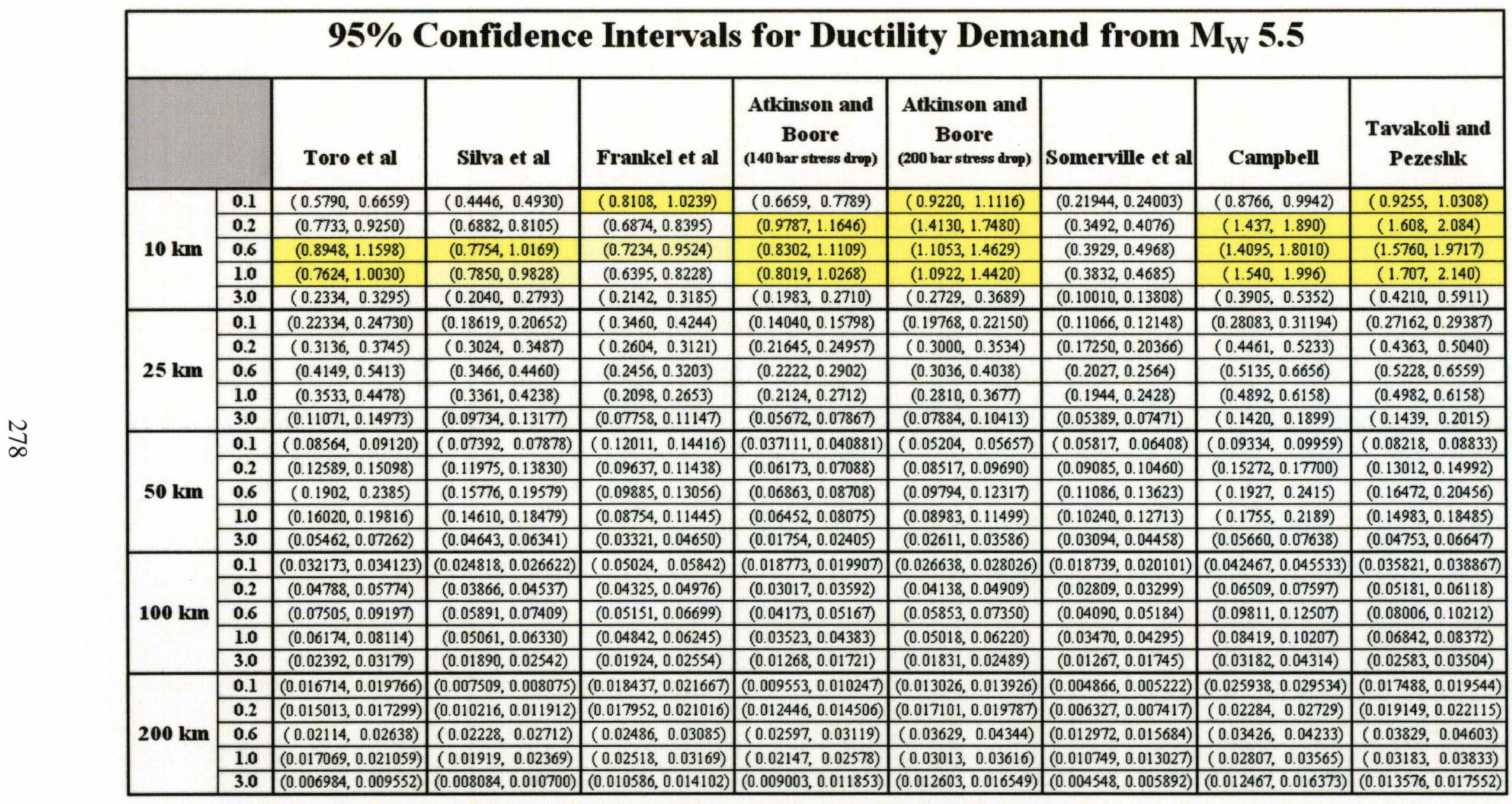

Based on 25 Observations per Model on each SDOF and a One-Sample $t$-Test with a 95\% Confidence Level.

Indicates that the Confidence Interval Contains a Ductility Demand of One or Above. 


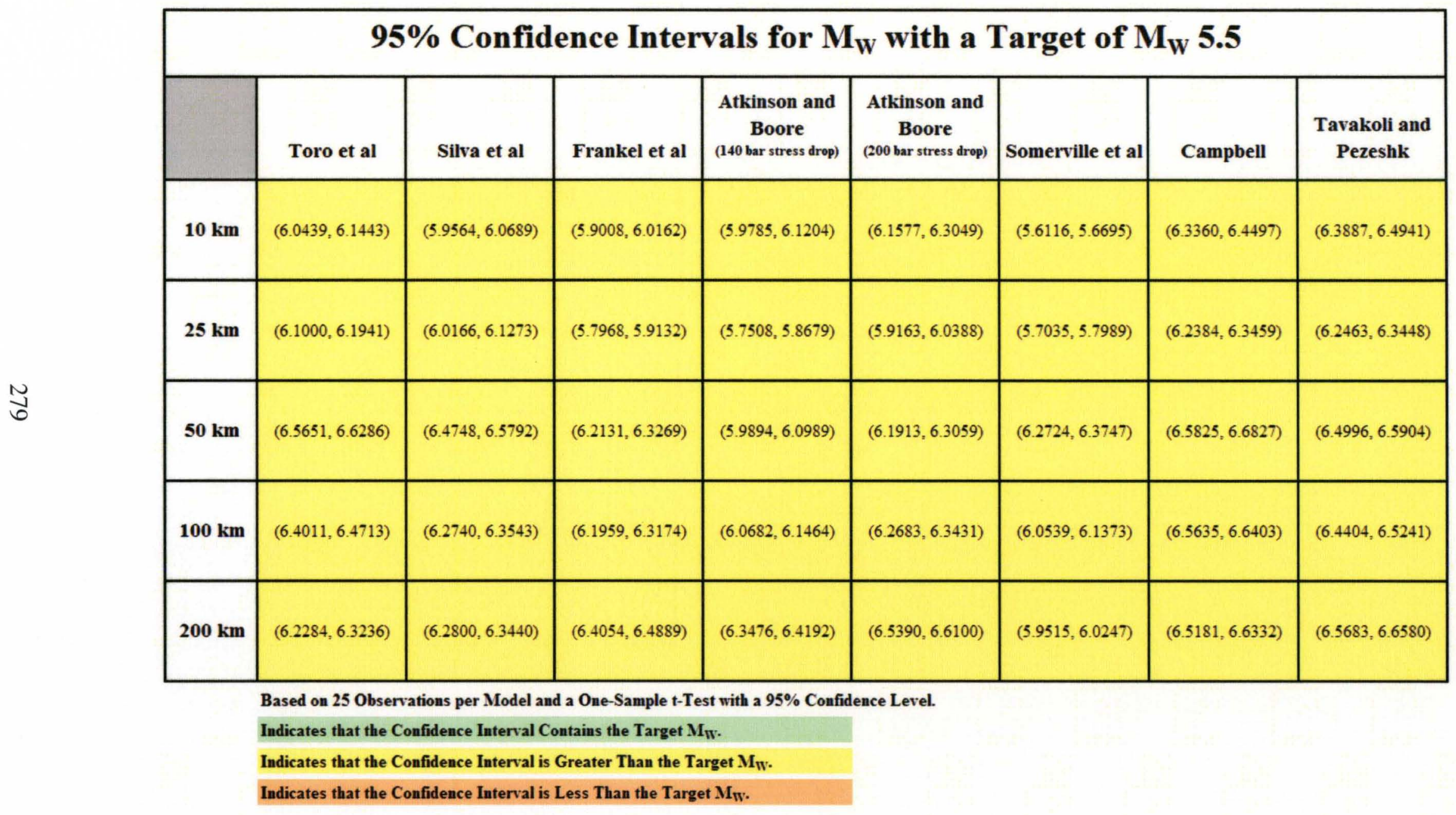




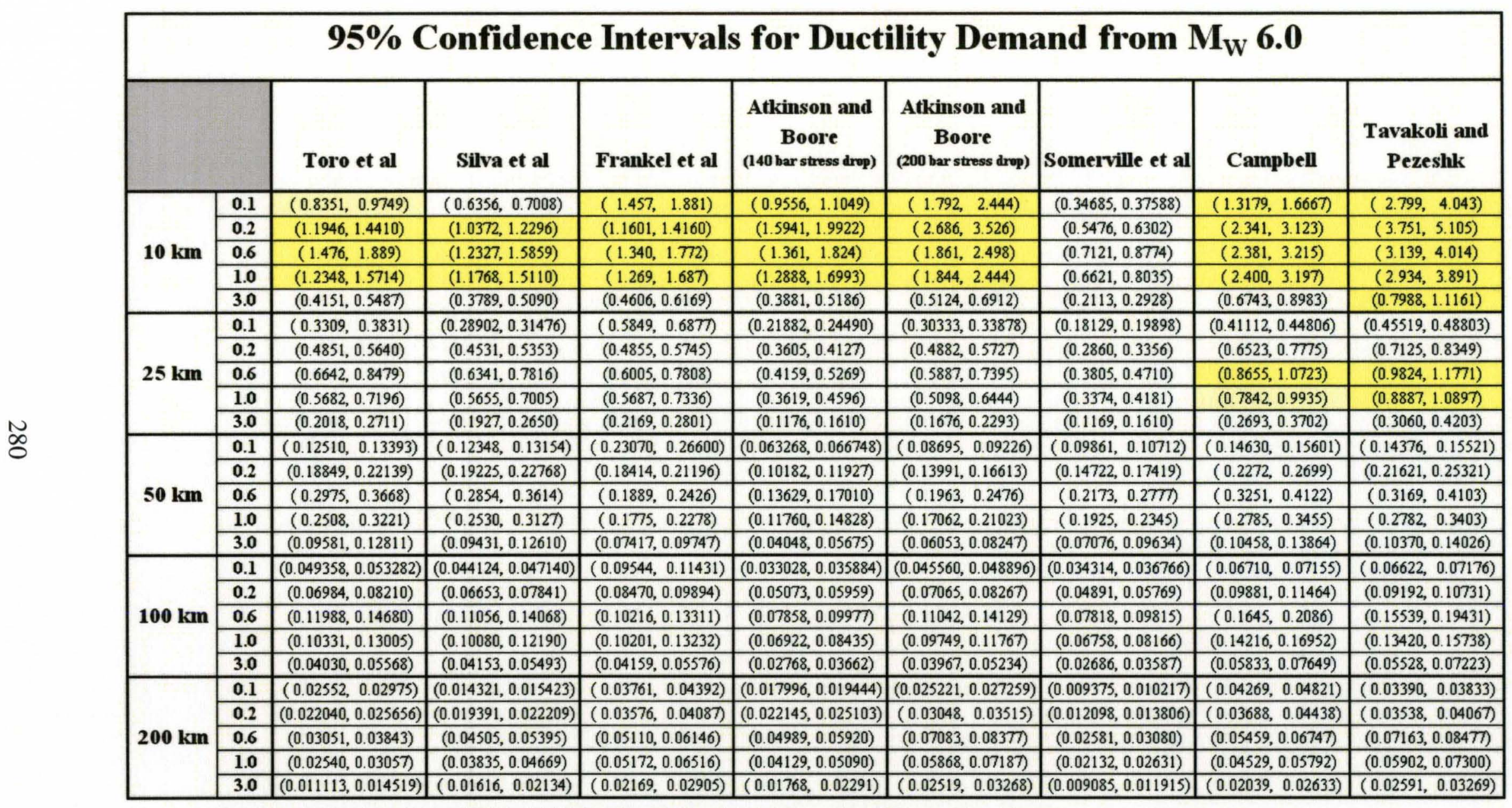

Based on 25 Observations per Model on each SDOF and a One-Sample t-Test with a 95\% Confidence Level.

Indic ates that the Confidence Interval Contains a Ductility Demand of One or Above. 


\begin{tabular}{|c|c|c|c|c|c|c|c|c|}
\hline \multicolumn{7}{|c|}{$95 \%$ Confidence Intervals for $\mathbf{M}_{W}$ with a Target of $\mathbf{M}_{W} \mathbf{6 . 0}$} \\
\hline & Toro et al & Silva et al & Frankel et al & $\begin{array}{c}\text { Atkinson and } \\
\text { Boore } \\
(140 \text { bar stress drop) }\end{array}$ & $\begin{array}{c}\text { Atkinson and } \\
\text { Boore } \\
(200 \text { bar stress drop) }\end{array}$ & Somerville et al & Campbell & $\begin{array}{c}\text { Tavakoli and } \\
\text { Pezeshk }\end{array}$ \\
\hline $\mathbf{1 0} \mathbf{~ k m}$ & $(6.2931,6.3898)$ & $(6.2306,6.3311)$ & $(6.2740,6.3742)$ & $(6.2865,6.4148)$ & $(6.4594,6.5887)$ & $(5.8895,5.9765)$ & $(6.5752,6.6786)$ & $(6.6794,6.7611)$ \\
\hline $\mathbf{2 5} \mathbf{~ k m}$ & $(6.3536,6.4439)$ & $(6.3397,6.4285)$ & $(6.3292,6.4386)$ & $(6.0752,6.1893)$ & $(6.2717,6.3809)$ & $(6.0531,6.1311)$ & $(6.5296,6.6304)$ & $(6.6054,6.6869)$ \\
\hline $\mathbf{5 0} \mathbf{~ k m}$ & $(6.8313,6.9033)$ & $(6.8180,6.8972)$ & $(6.5945,6.7104)$ & $(6.3751,6.4667)$ & $(6.5925,6.6685)$ & $(6.6591,6.7387)$ & $(6.8862,6.9658)$ & $(6.8758,6.9591)$ \\
\hline $\mathbf{1 0 0} \mathbf{~ k m}$ & $(6.6562,6.7382)$ & $(6.6491,6.7235)$ & $(6.6191,6.7251)$ & $(6.4429,6.5158)$ & $(6.6350,6.7077)$ & $(6.4250,6.5043)$ & $(6.8515,6.9205)$ & $(6.8144,6.8870)$ \\
\hline $\mathbf{2 0 0} \mathbf{~ k m}$ & $(6.4574,6.5519)$ & $(6.6699,6.7287)$ & $(6.8199,6.8944)$ & $(6.7157,6.7931)$ & $(6.9170,6.9901)$ & $(6.3381,6.4122)$ & $(6.7846,6.8987)$ & $(6.9244,7.0150)$ \\
\hline
\end{tabular}

Based on 25 Observations per Model and a One-Sample t-Test with a 95\% Confidence Level.

Indicates that the Confidence Interval Contains the Target $\mathbf{M}_{W}$.

Indicates that the Confidence Interval is Greater Than the Target $\mathbf{M}_{\mathbf{W}}$.

Indicates that the Confidence Interval is Less Than the Target $\mathbf{M}_{\mathrm{W}}$. 


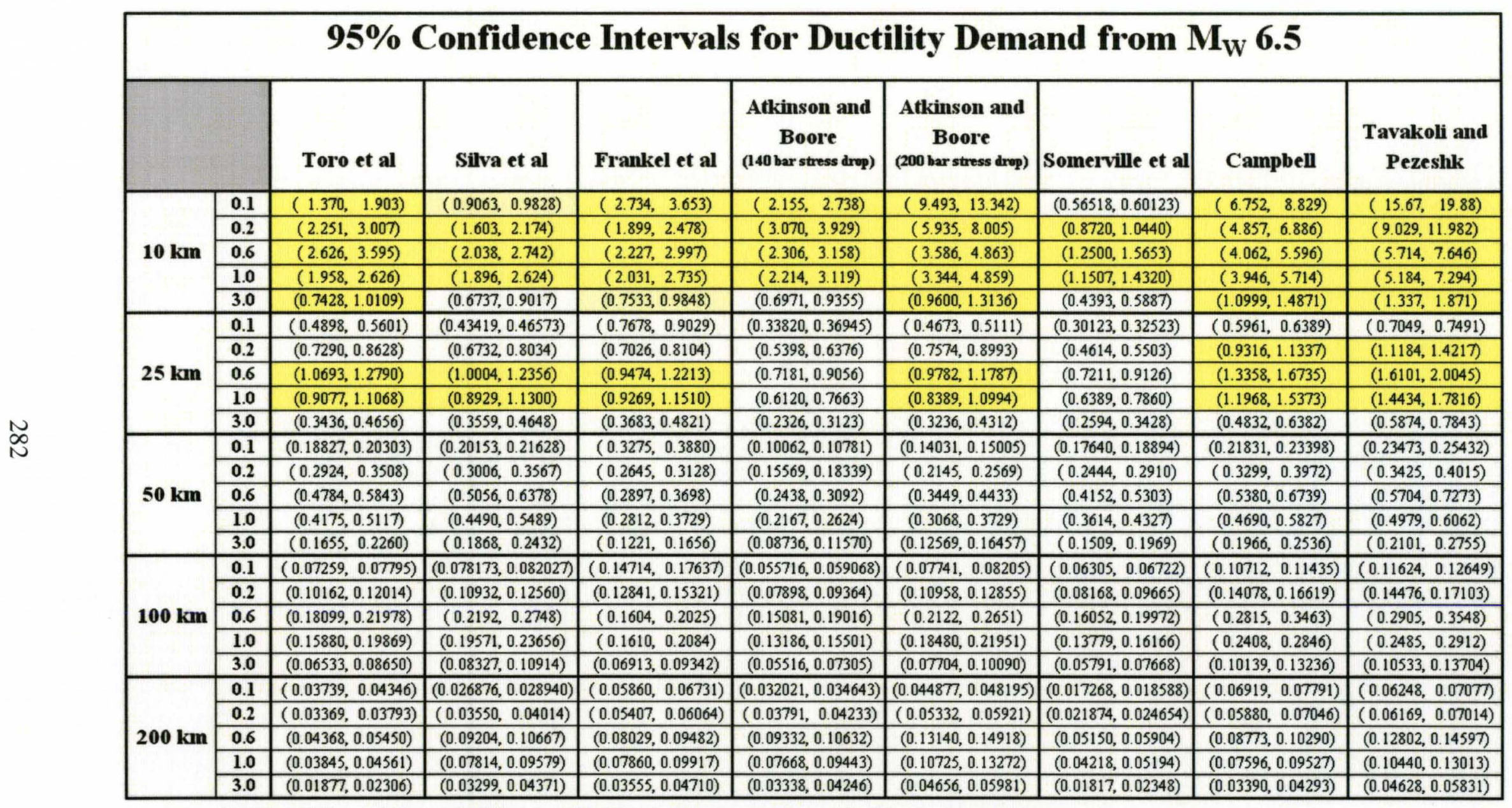

Based on 25 Observations per Model on each SDOF and a One-Sample t-Test with a 95\% Confidence Level.

Indicates that the Confidence Interval Contains a Ductility Demand of One or Above. 


\begin{tabular}{|c|c|c|c|c|c|c|c|c|}
\hline \multicolumn{9}{|c|}{$95 \%$ Confidence Intervals for $M_{W}$ with a Target of $M_{W} 6.5$} \\
\hline & Toro et al & Silva et al & Frankel et al & $\begin{array}{l}\text { Atkinson and } \\
\text { Boore } \\
\text { (140 har stress drop) }\end{array}$ & $\begin{array}{l}\text { Atkinson and } \\
\text { Boore } \\
\text { (200 bar stress drop) }\end{array}$ & Somerville et al & Campbell & $\begin{array}{c}\text { Tavakoli and } \\
\text { Pezeshk }\end{array}$ \\
\hline $10 \mathrm{~km}$ & $(6.5685,6.6494)$ & $(6.5010,6.5779)$ & $(6.4955,6.6035)$ & $(6.5557,6.6642)$ & $(6.7455,6.8534)$ & $(6.2330,6.3162)$ & $(6.7991,6.8786)$ & $(6.9240,6.9984)$ \\
\hline $25 \mathrm{~km}$ & $(6.6361,6.7060)$ & $(6.6205,6.6971)$ & $(6.5940,6.6963)$ & $(6.3983,6.4860)$ & $(6.5923,6.6746)$ & $(6.4260,6.5002)$ & $(6.8003,6.8843)$ & $(6.9109,6.9902)$ \\
\hline $50 \mathrm{~km}$ & $(7.0972,7.1705)$ & $(7.1406,7.2148)$ & $(6.8404,6.9630)$ & $(6.7233,6.7957)$ & $(6.9221,6.9970)$ & $(7.0248,7.0935)$ & $(7.1758,7.2482)$ & $(7.2114,7.2789)$ \\
\hline $100 \mathrm{~km}$ & $(6.8975,6.9798)$ & $(7.0184,7.0744)$ & $(6.8681,6.9781)$ & $(6.7926,6.8589)$ & $(6.9862,7.0449)$ & $(6.8173,6.8934)$ & $(7.1334,7.1987)$ & $(7.1411,7.2212)$ \\
\hline $200 \mathrm{~km}$ & $(6.7017,6.7900)$ & $(7.0575,7.1278)$ & $(7.0928,7.1529)$ & $(7.0592,7.1364)$ & $(7.2508,7.3294)$ & $(6.7185,6.7918)$ & $(7.0403,7.1539)$ & $(7.2481,7.3358)$ \\
\hline
\end{tabular}

Based on 25 Observations per Model and a One-Sample t-Test with a 95\% Confidence Level. Indicates that the Confidence Interval Contains the Target $\mathbf{M}_{\mathbf{w}}$.

Indicates that the Confidence Interval is Greater Than the Target $M_{W}$. Indicates that the Confidence Interval is Less Than the Target $\mathbf{M}_{\mathrm{W}}$. 


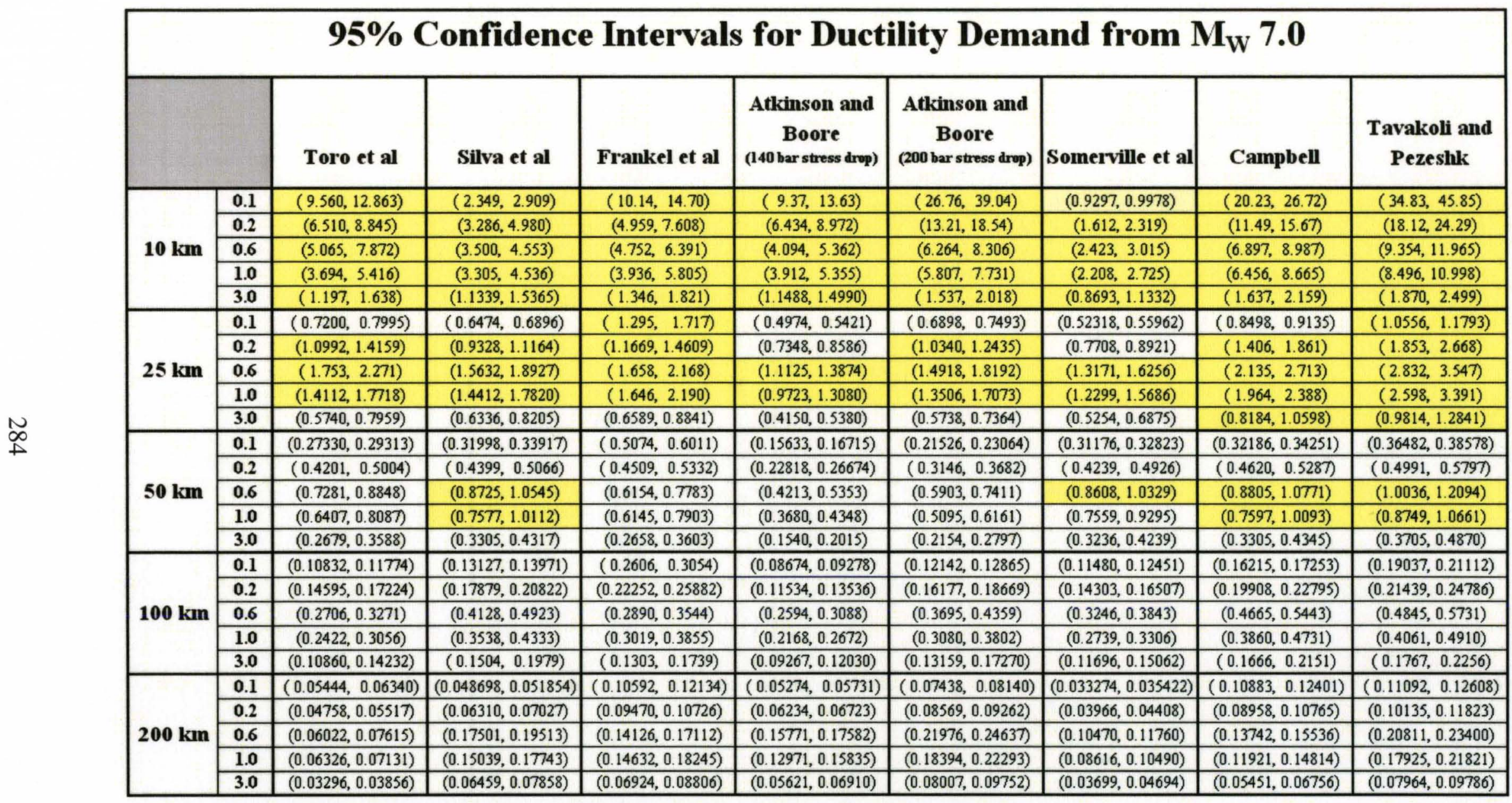

Based on 25 Observations per Model on each SDOF and a One-Sample t-Test with a 95\% Confidence Level.

Indicates that the Confidence Interval Contains a Ductility Demand of One or Above. 


\begin{tabular}{|c|c|c|c|c|c|c|c|c|}
\hline \multicolumn{9}{|c|}{$95 \%$ Confidence Intervals for $M_{W}$ with a Target of $M_{W} 7.0$} \\
\hline & Toro et al & Silva et al & Frankel et al & $\begin{array}{l}\text { Atkinson and } \\
\text { Boore } \\
\text { (140 har stress drop) }\end{array}$ & $\begin{array}{l}\text { Atkinson and } \\
\text { Boore } \\
\text { (200 bar stress drop) }\end{array}$ & Somerville et al & Campbell & $\begin{array}{c}\text { Tavakoli and } \\
\text { Pezeshk }\end{array}$ \\
\hline $10 \mathrm{~km}$ & $(6.8410,6.9167)$ & $(6.7350,6.8079)$ & $(6.7852,6.8939)$ & $(6.8014,6.8836)$ & $(6.9850,7.0648)$ & $(6.5728,6.6404)$ & $(6.9859,7.0558)$ & $(7.0830,7.1525)$ \\
\hline $25 \mathrm{~km}$ & $(6.8778,6.9611)$ & $(6.8971,6.9655)$ & $(6.8850,6.9896)$ & $(6.6743,6.7490)$ & $(6.8619,6.9341)$ & $(6.7973,6.8590)$ & $(7.0510,7.1257)$ & $(7.1682,7.2365)$ \\
\hline $50 \mathrm{~km}$ & $(7.3407,7.4238)$ & $(7.4349,7.5030)$ & $(7.2590,7.3672)$ & $(7.0110,7.0722)$ & $(7.2028,7.2618)$ & $(7.4222,7.4867)$ & $(7.4391,7.5037)$ & $(7.5102,7.5715)$ \\
\hline $100 \mathrm{~km}$ & $(7.1555,7.2369)$ & $(7.3317,7.3969)$ & $(7.2199,7.2987)$ & $(7.0631,7.1254)$ & $(7.2577,7.3263)$ & $(7.1896,7.2584)$ & $(7.3889,7.4586)$ & $(7.4141,7.4930)$ \\
\hline $200 \mathrm{~km}$ & $(6.9552,7.0564)$ & $(7.4317,7.4961)$ & $(7.4447,7.4991)$ & $(7.3626,7.4319)$ & $(7.5638,7.6284)$ & $(7.1150,7.1951)$ & $(7.3120,7.4013)$ & $(7.5440,7.6225)$ \\
\hline
\end{tabular}

Based on 25 Observations per Model and a One-Sample t-Test with a 95\% Confidence Level.

Indicates that the Confidence Interval Contains the Target $\mathrm{M}_{\mathrm{WW}}$.

Indicates that the Confidence Interval is Greater Than the Target $\mathbf{M}_{W}$.

Indicates that the Confidence Interval is Less Than the Target $\mathbf{M}_{W}$. 


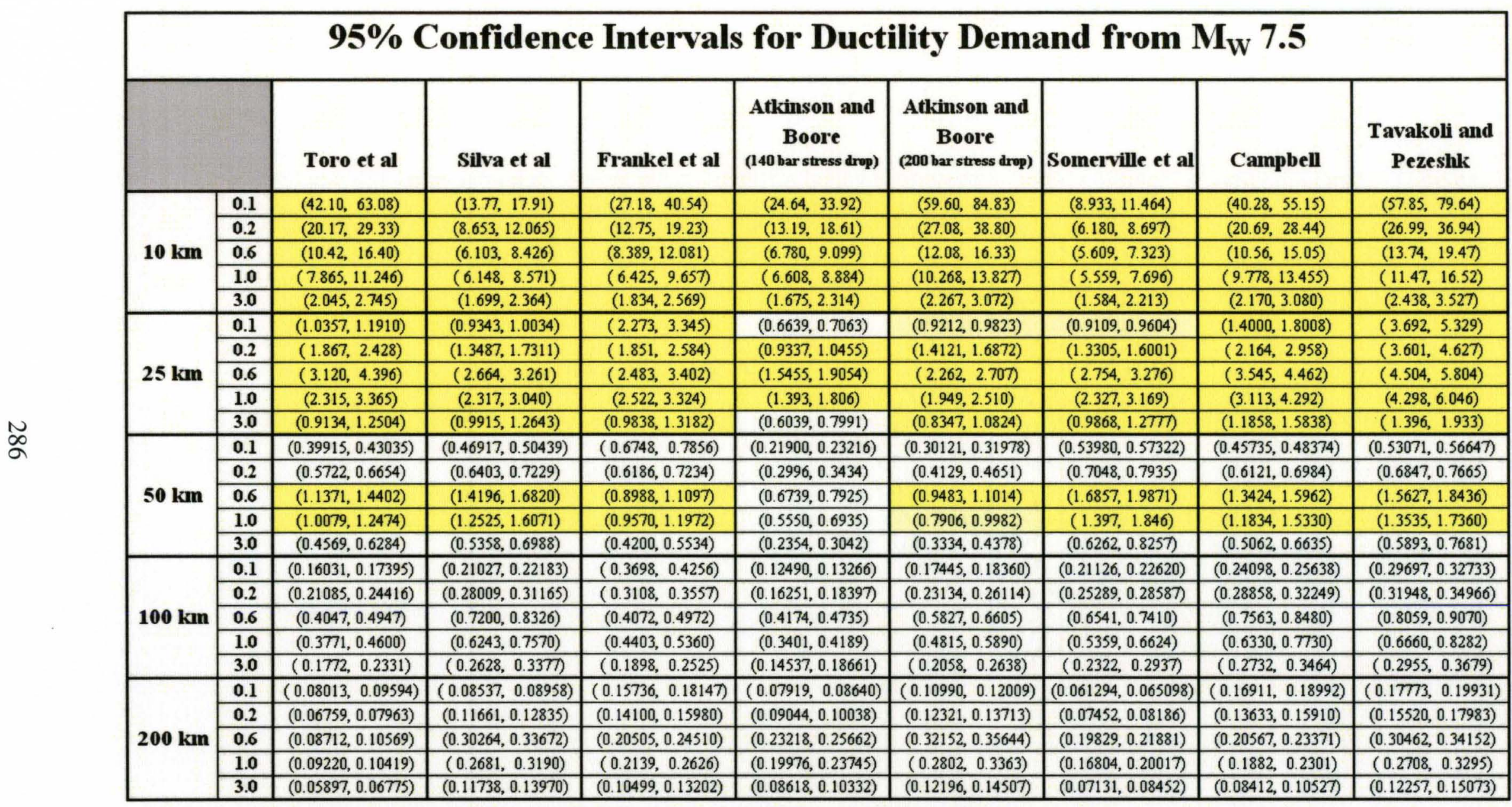

Based on 25 Observations per Model on each SDOF and a One-Sample t-Test with a 95\% Confidence Level.

Indicates that the Confidence Interval Contains a Ductility Demand of One or Above. 


\begin{tabular}{|c|c|c|c|c|c|c|c|c|}
\hline \multicolumn{7}{|c|}{$95 \%$ Confidence Intervals for $\mathbf{M}_{\mathbf{W}}$ with a Target of $\mathbf{M}_{\mathbf{W}} \mathbf{7 . 5}$} \\
\hline & Toro et al & Silva et al & Frankel et al & $\begin{array}{c}\text { Atkinson and } \\
\text { Boore } \\
(140 \text { bar stress drop) }\end{array}$ & $\begin{array}{c}\text { Atkinson and } \\
\text { Boore } \\
(200 \text { bar stress drop) }\end{array}$ & Somerville et al & Campbell & $\begin{array}{c}\text { Tavakoli and } \\
\text { Pezeshk }\end{array}$ \\
\hline $\mathbf{1 0} \mathbf{~ k m}$ & $(7.0868,7.1788)$ & $(6.9518,7.0195)$ & $(6.9747,7.0753)$ & $(6.9969,7.0661)$ & $(7.1846,7.2521)$ & $(6.9118,6.9697)$ & $(7.1297,7.1986)$ & $(7.1948,7.2588)$ \\
\hline $\mathbf{2 5} \mathbf{~ k m}$ & $(7.1319,7.2251)$ & $(7.1377,7.2004)$ & $(7.0797,7.1665)$ & $(6.8702,6.9353)$ & $(7.0587,7.1262)$ & $(7.1398,7.1997)$ & $(7.2561,7.3241)$ & $(7.3545,7.4189)$ \\
\hline $\mathbf{5 0} \mathbf{~ k m}$ & $(7.6111,7.6981)$ & $(7.6909,7.7492)$ & $(7.5139,7.5836)$ & $(7.2285,7.2940)$ & $(7.4240,7.4900)$ & $(7.7760,7.8405)$ & $(7.6636,7.7258)$ & $(7.7393,7.8062)$ \\
\hline $\mathbf{1 0 0} \mathbf{~ k m}$ & $(7.4063,7.4823)$ & $(7.6201,7.6935)$ & $(7.4360,7.4904)$ & $(7.2925,7.3621)$ & $(7.4889,7.5554)$ & $(7.5564,7.6306)$ & $(7.6439,7.7149)$ & $(7.6882,7.7639)$ \\
\hline $\mathbf{2 0 0} \mathbf{~ k m}$ & $(7.2243,7.3209)$ & $(7.7652,7.8371)$ & $(7.6578,7.7154)$ & $(7.6096,7.6680)$ & $(7.8073,7.8649)$ & $(7.5030,7.5673)$ & $(7.5712,7.6525)$ & $(7.7836,7.8568)$ \\
\hline
\end{tabular}

Based on 25 Observations per Model and a One-Sample t-Test with a 95\% Confidence Level.

Indicates that the Confidence Interval Contains the Target $\mathbf{M}_{\mathrm{WW}}$.

Indicates that the Confidence Interval is Greater Than the Target $\mathbf{M}_{\mathrm{W}}$.

Indicates that the Confidence Interval is Less Than the Target $\mathbf{M}_{\mathrm{W}}$. 


\section{APPENDIX C}

This appendix contains plots showing comparisons of the mean estimated moment magnitudes for 25 synthetics from each of the attenuation models and their target moment magnitudes at the five distances investigate in this study. The moment magnitudes were calculated using the maximum displacements as determined from the nonlinear time history analyses with parameters set to simulate those of the Wood-Anderson seismograph. Corrections for Eastern North America were applied at distances of $100 \mathrm{~km}$ and $200 \mathrm{~km}$. 


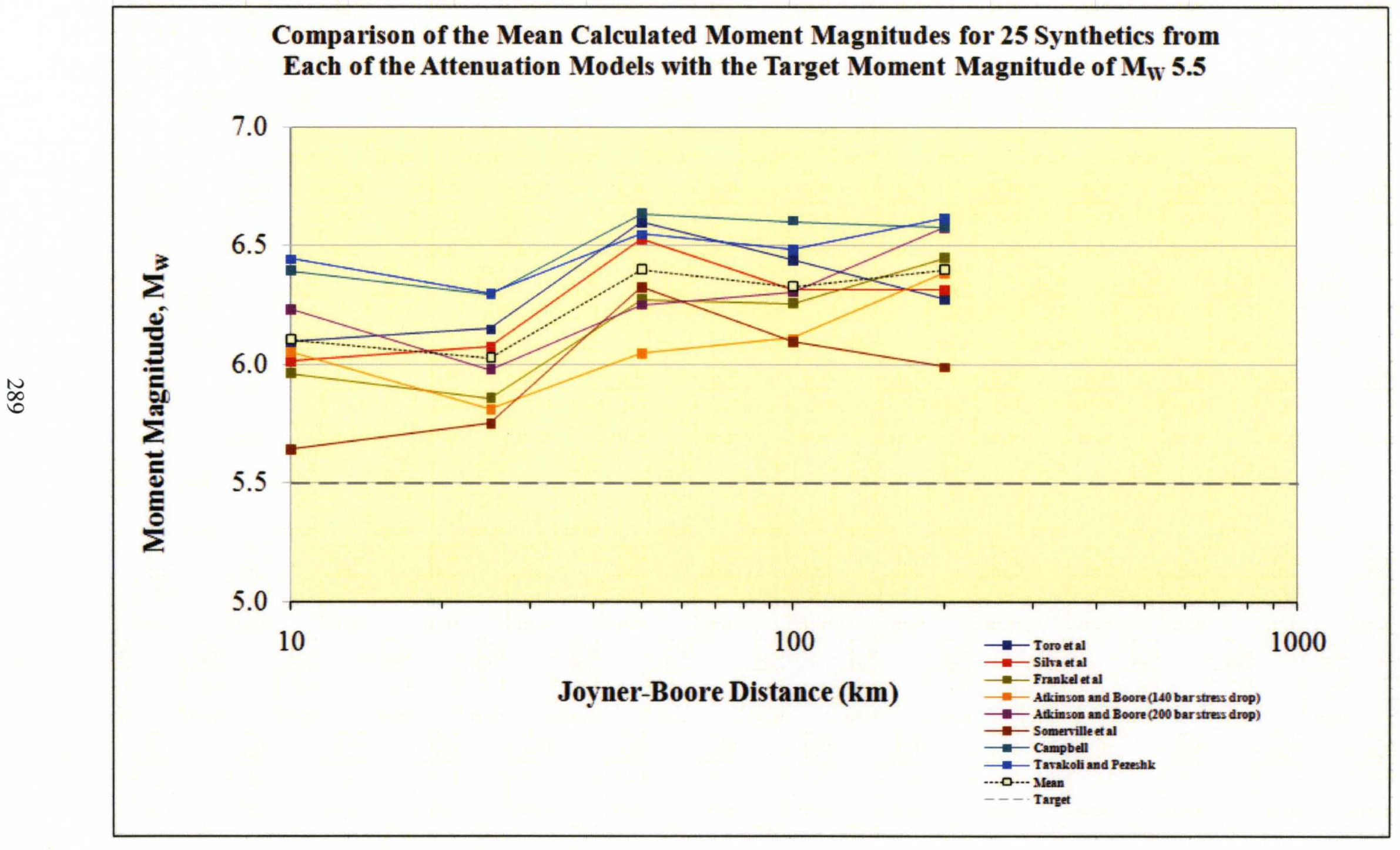




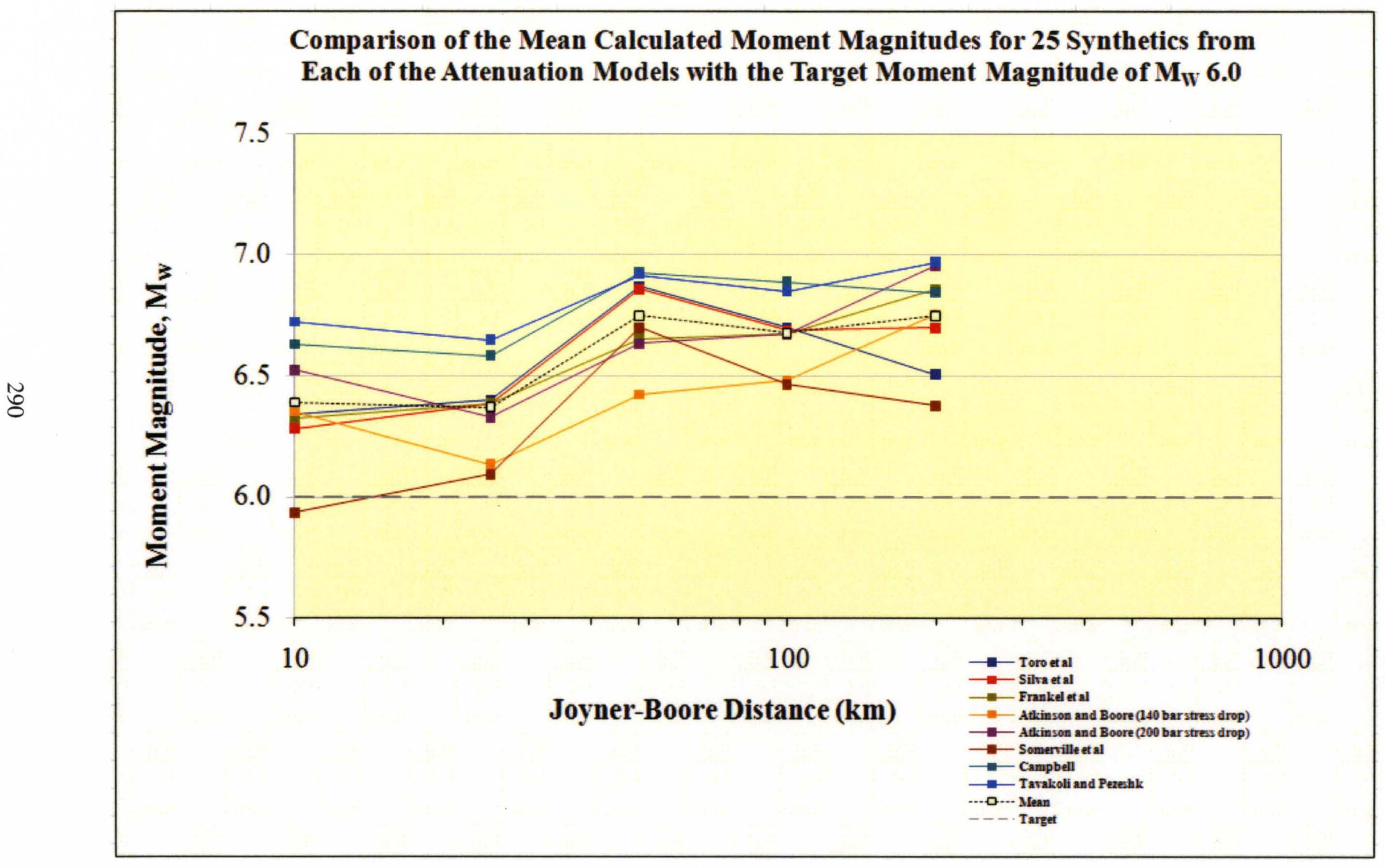




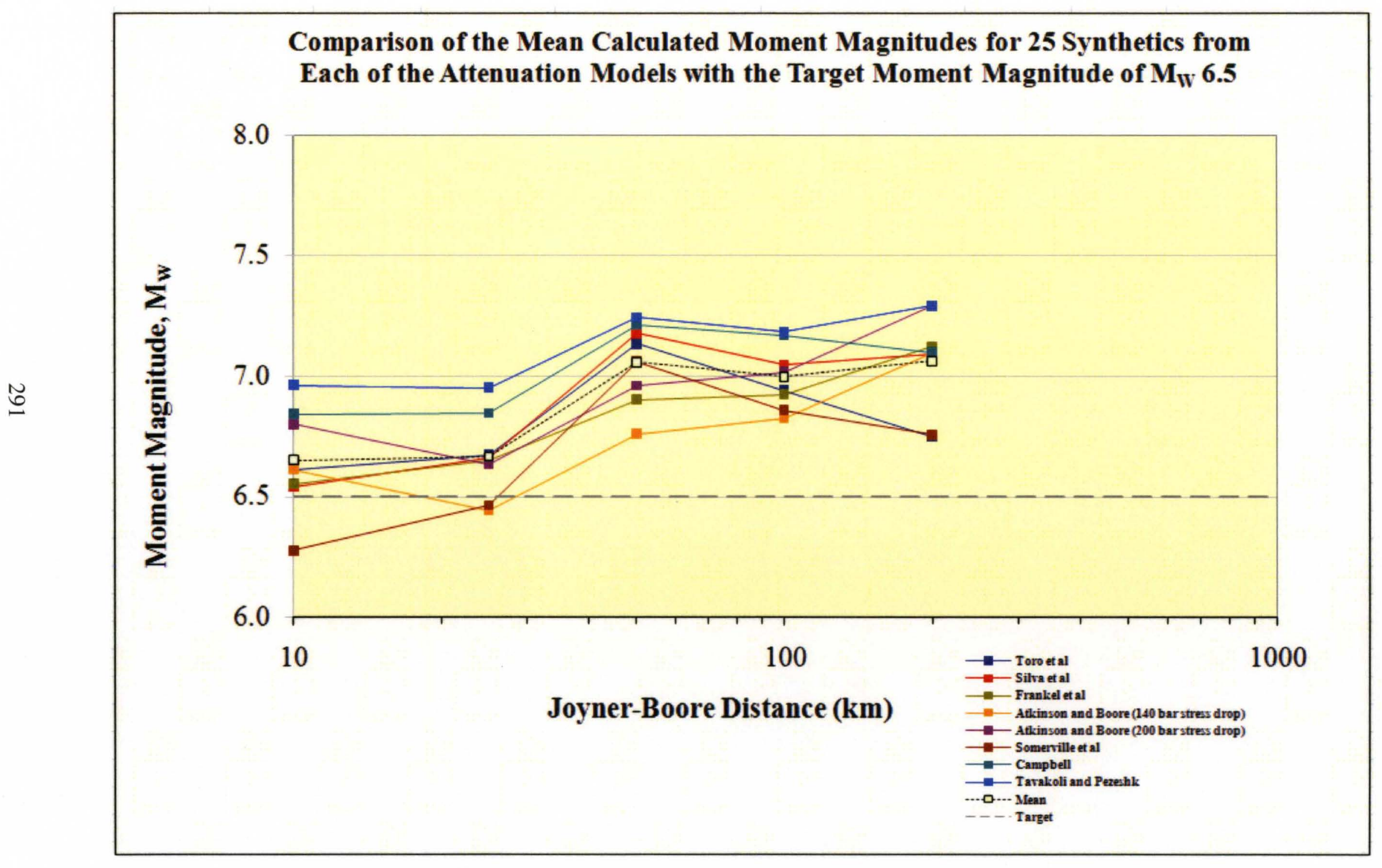




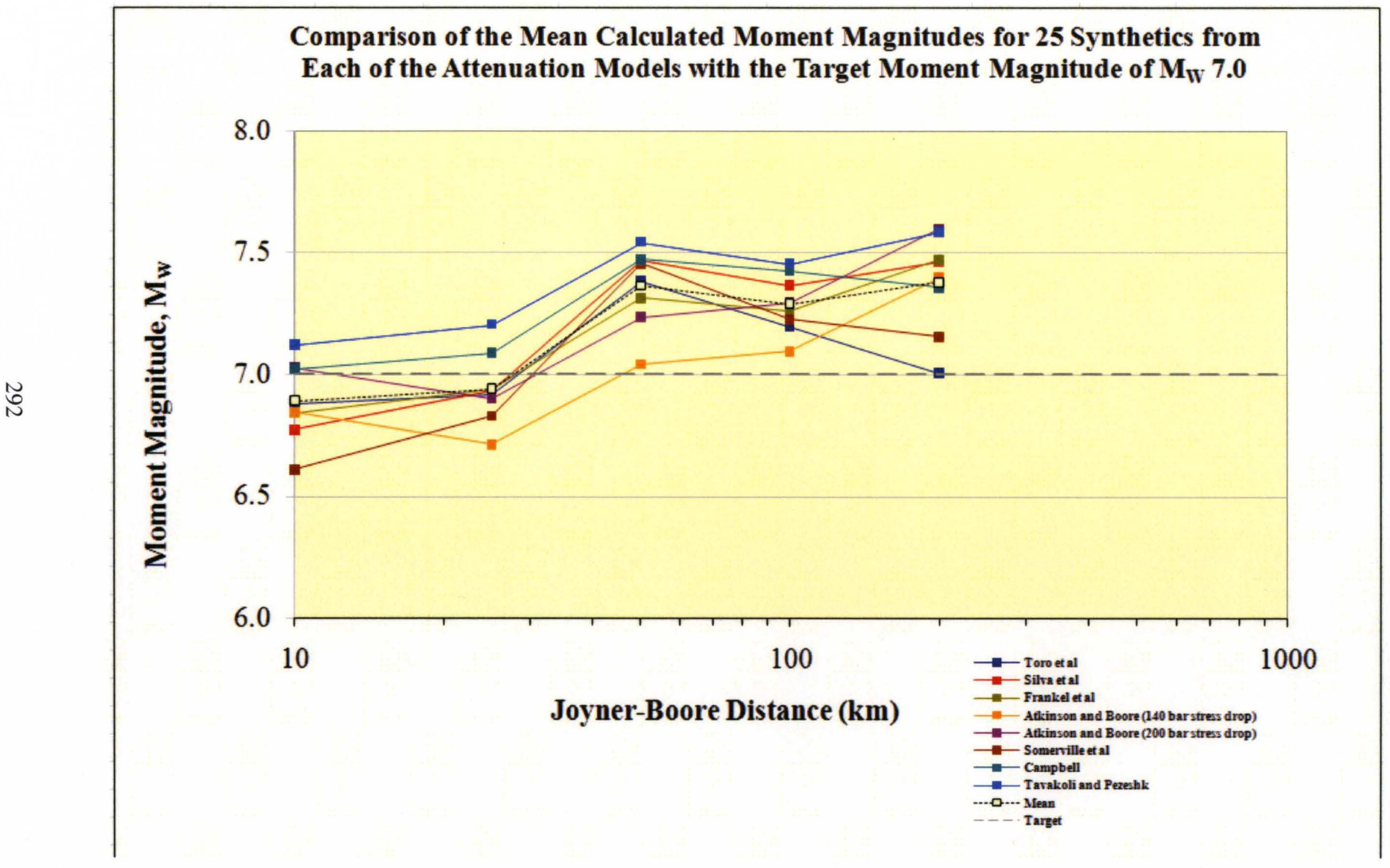




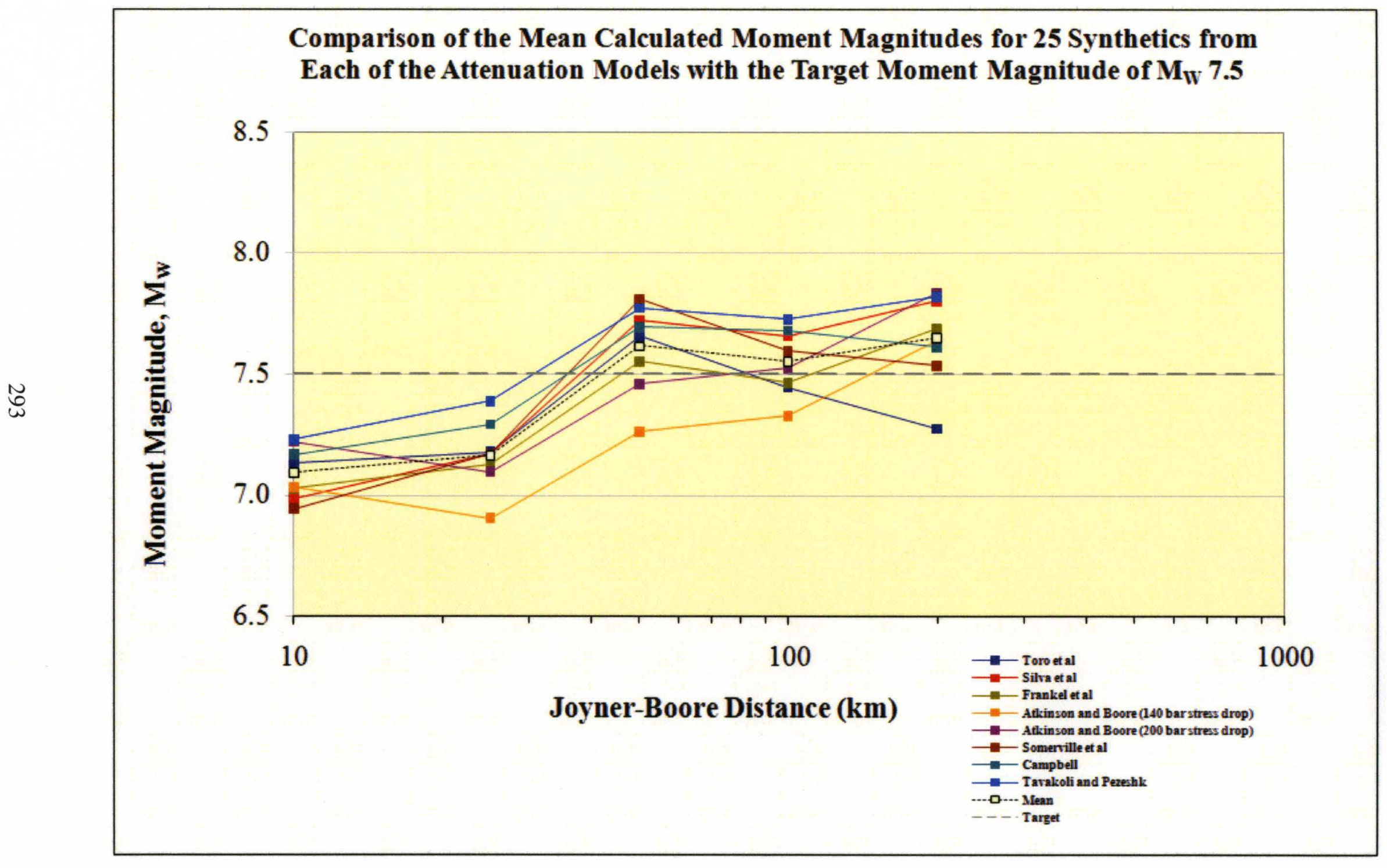




\section{APPENDIX D}

This appendix provides plots of the mean displacement ductility demands for each moment magnitude and single degree of freedom (SDOF) system as a function of distance. The distances investigated in this study are $10 \mathrm{~km}, 25 \mathrm{~km}, 50 \mathrm{~km}, 100 \mathrm{~km}$ and $200 \mathrm{~km}$. The curves shown are made up of the mean ductility demands from 25 synthetics from each of the attenuation models for each of the magnitude and distance combinations applied as loading on each of the SDOFs. 


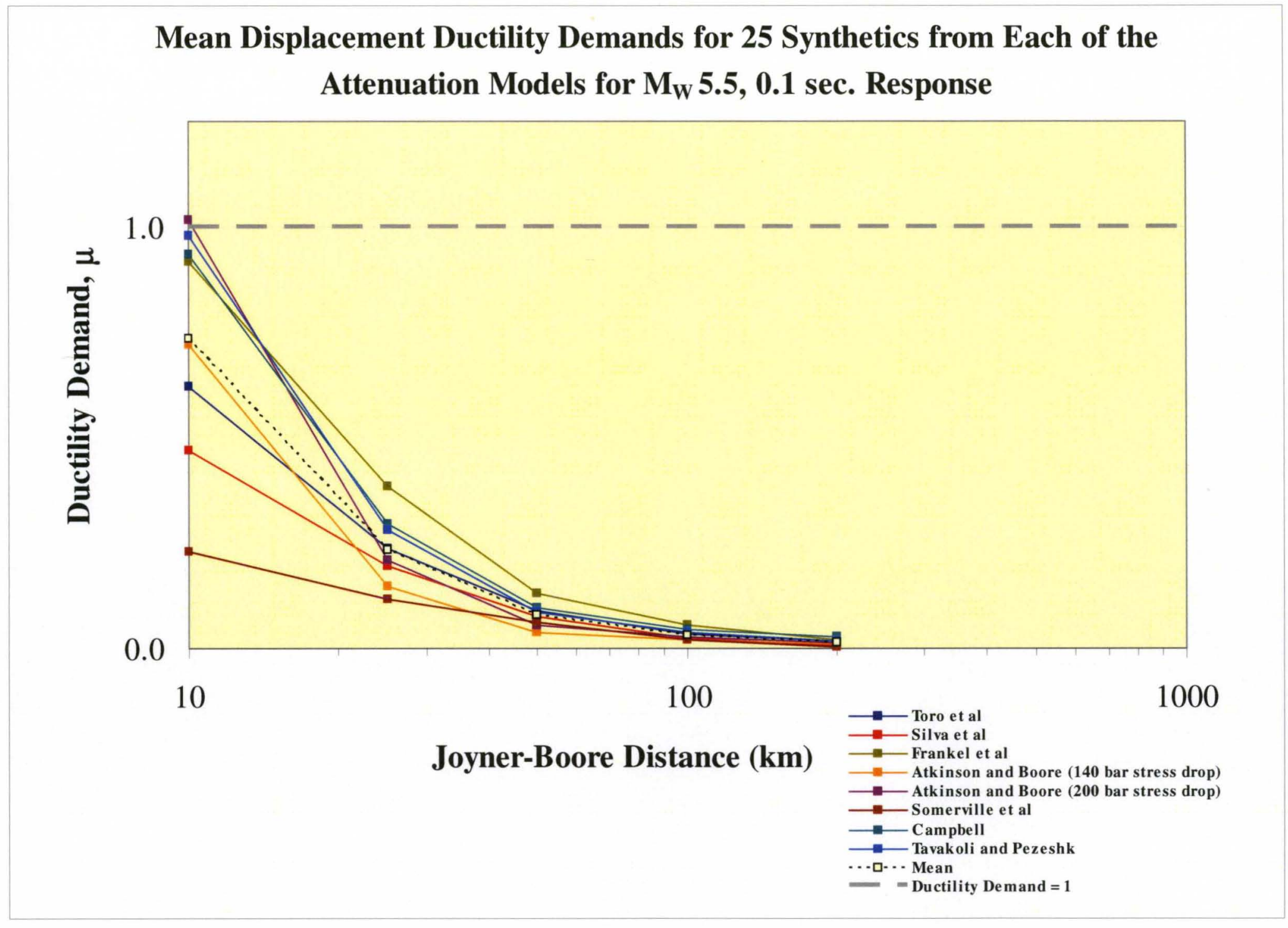




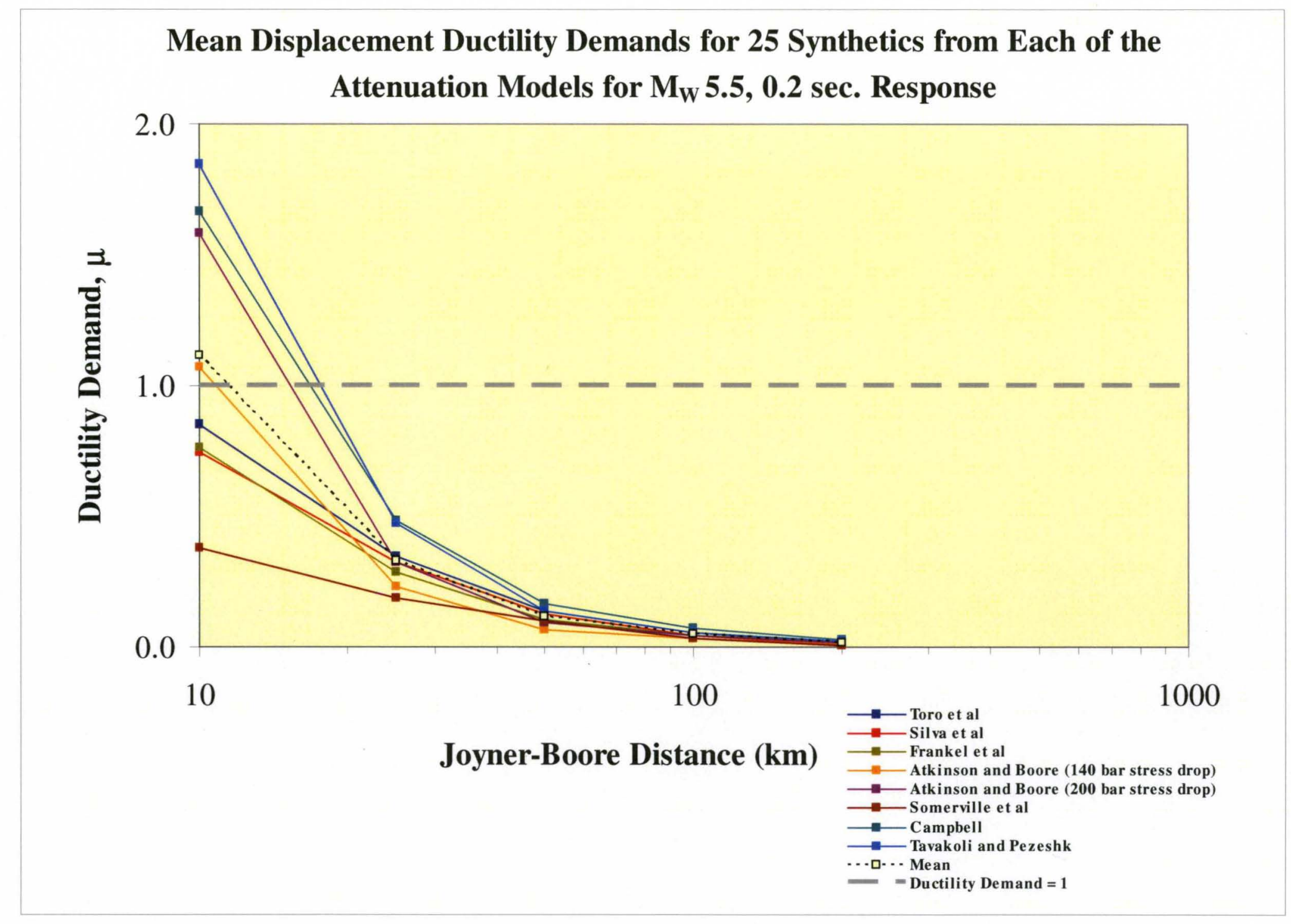




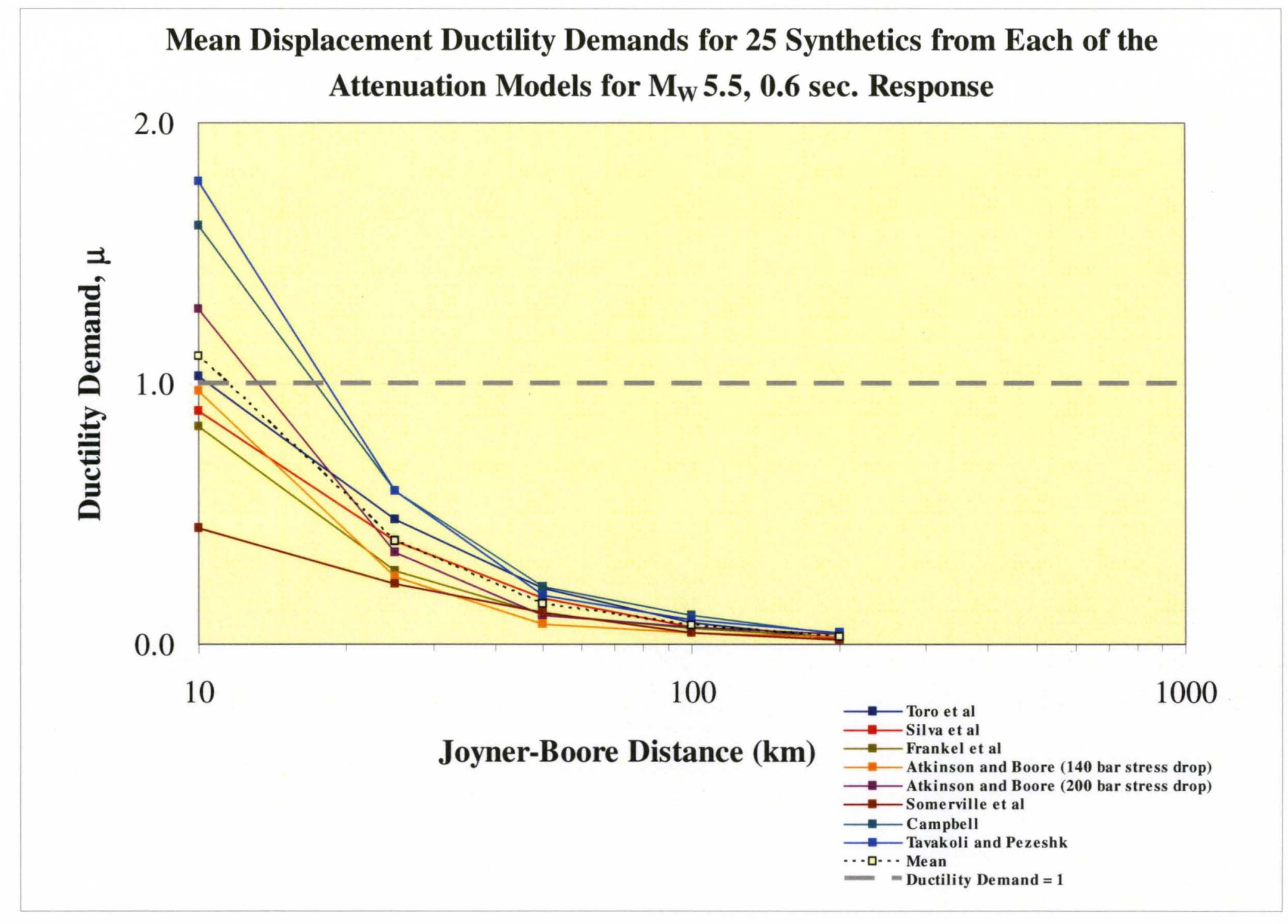




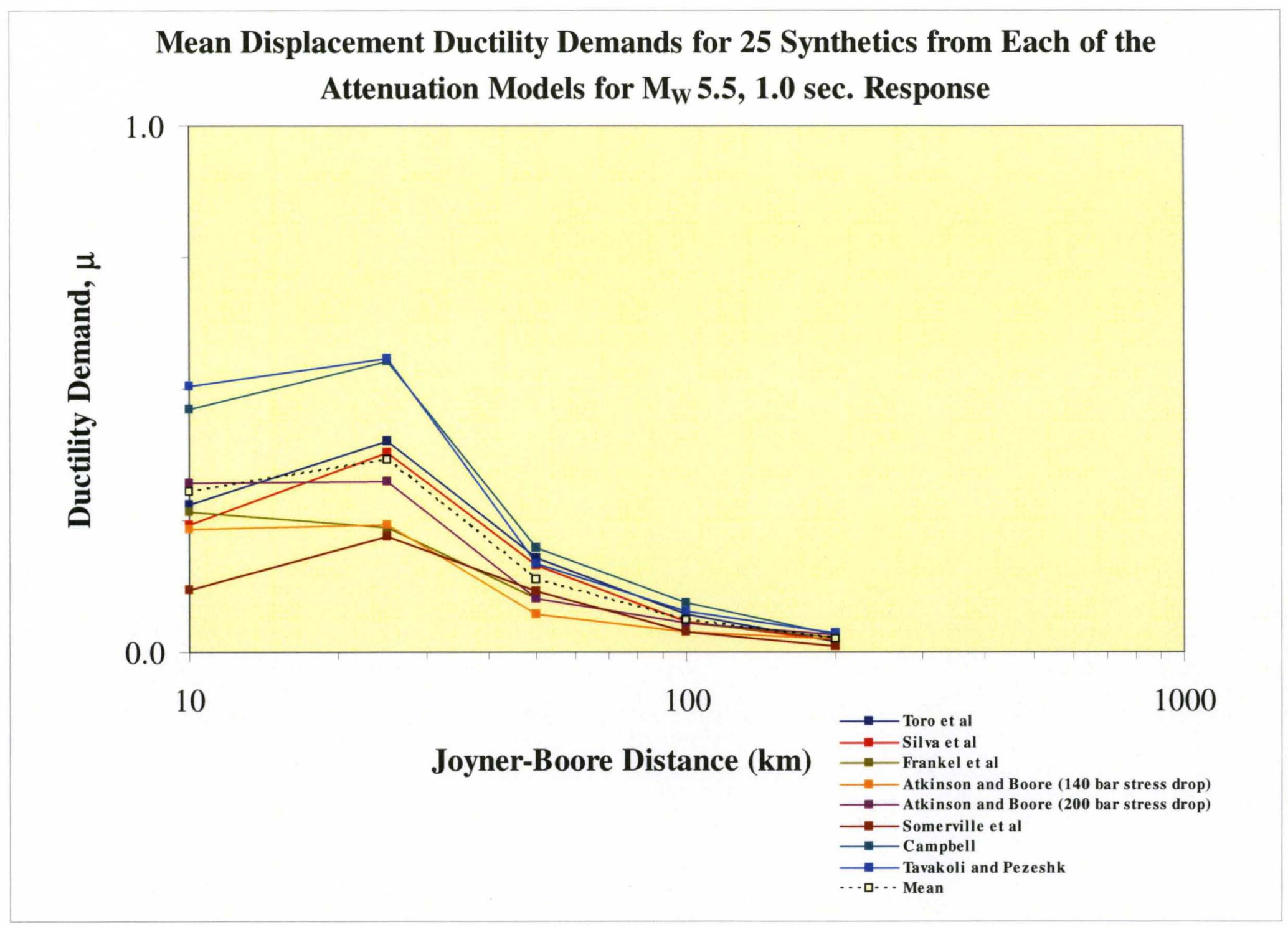




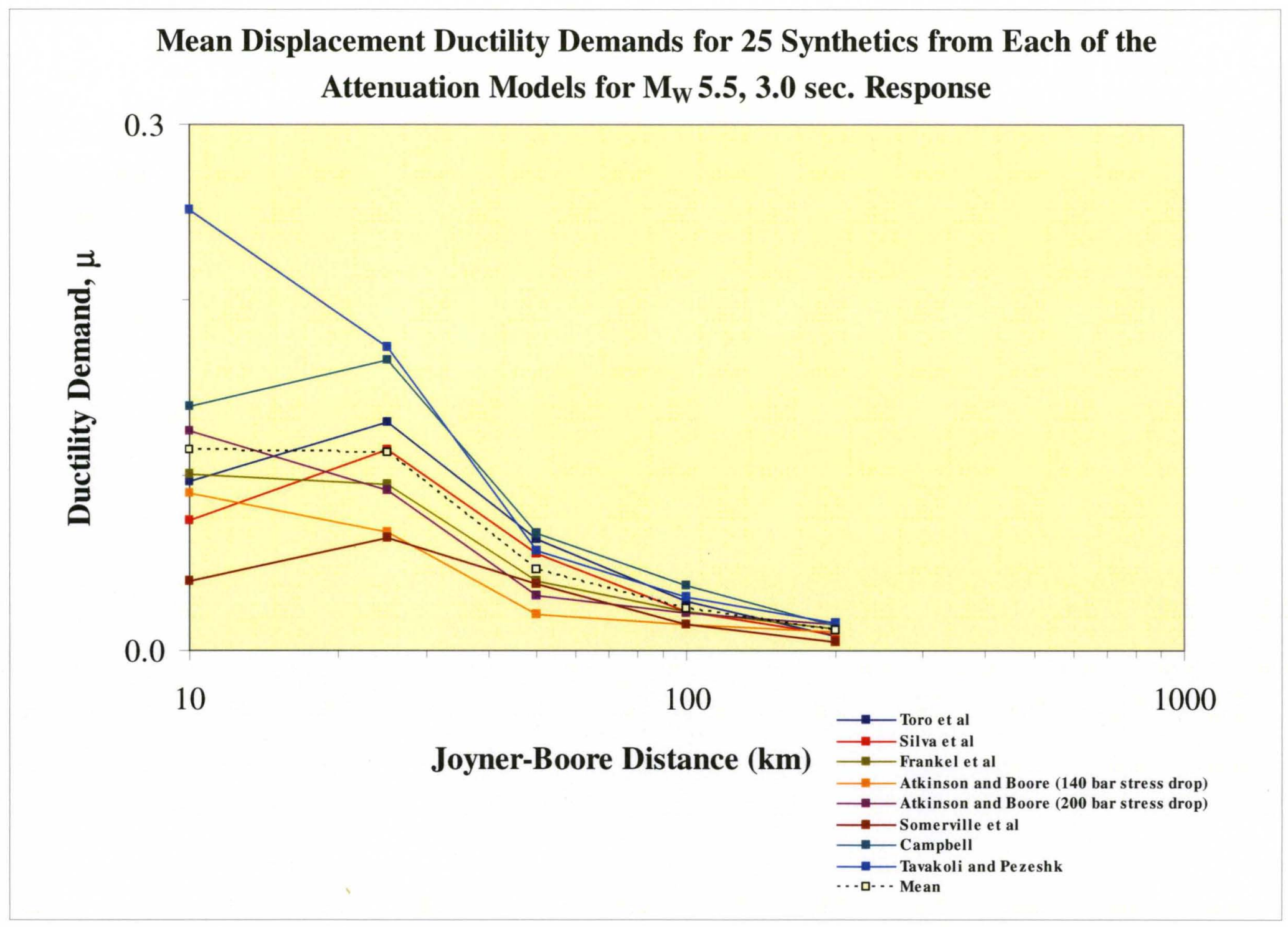




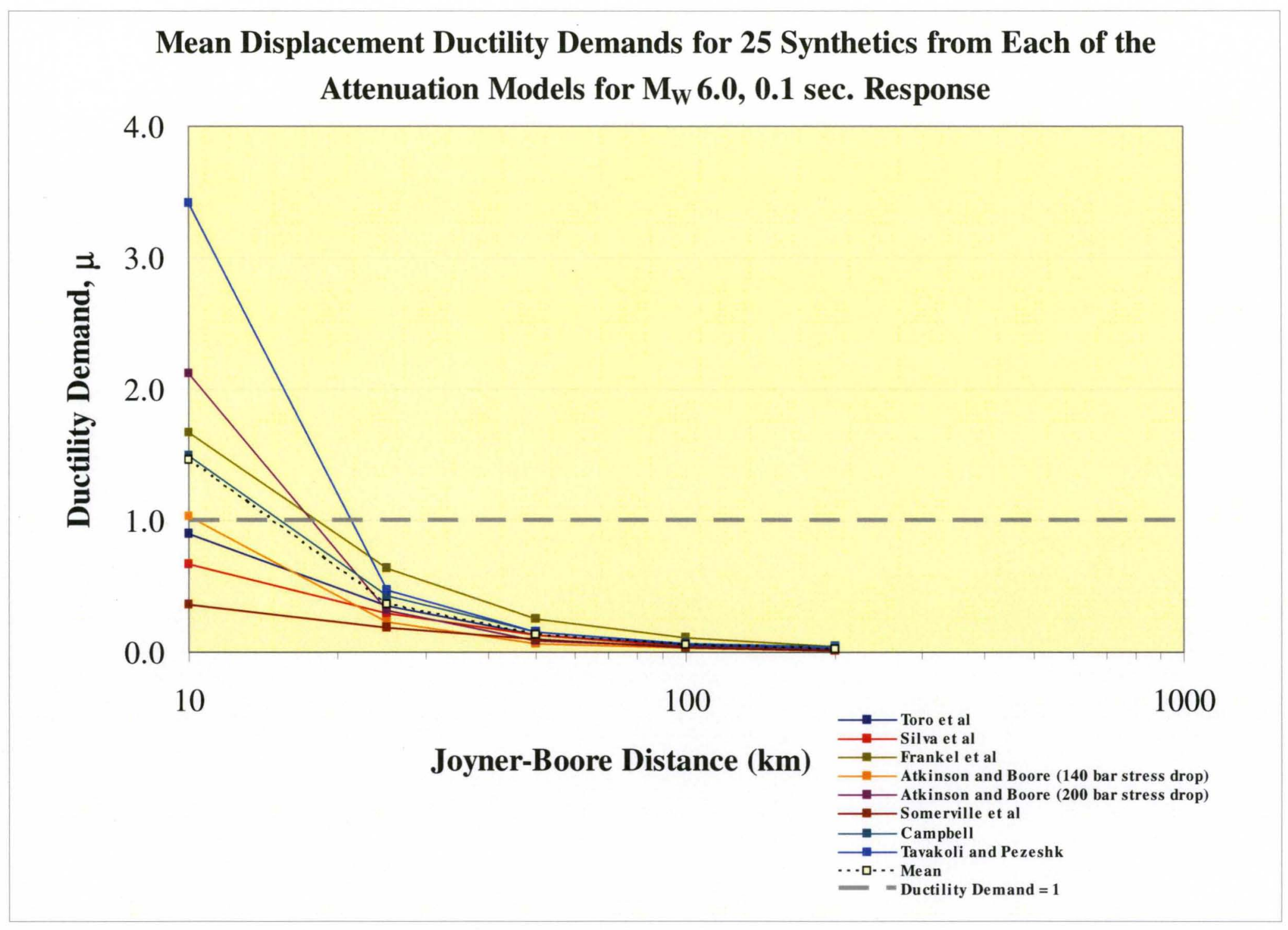




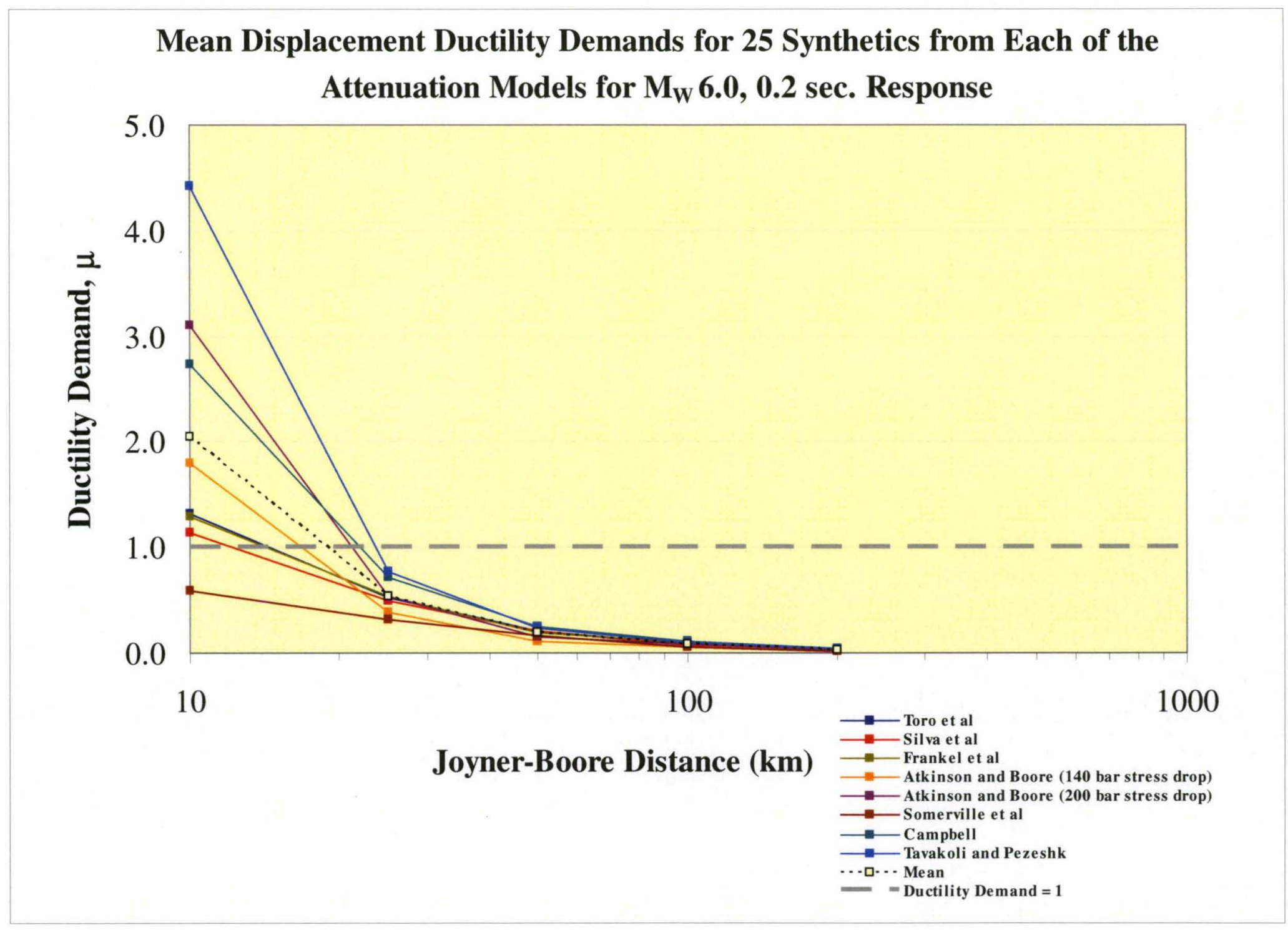




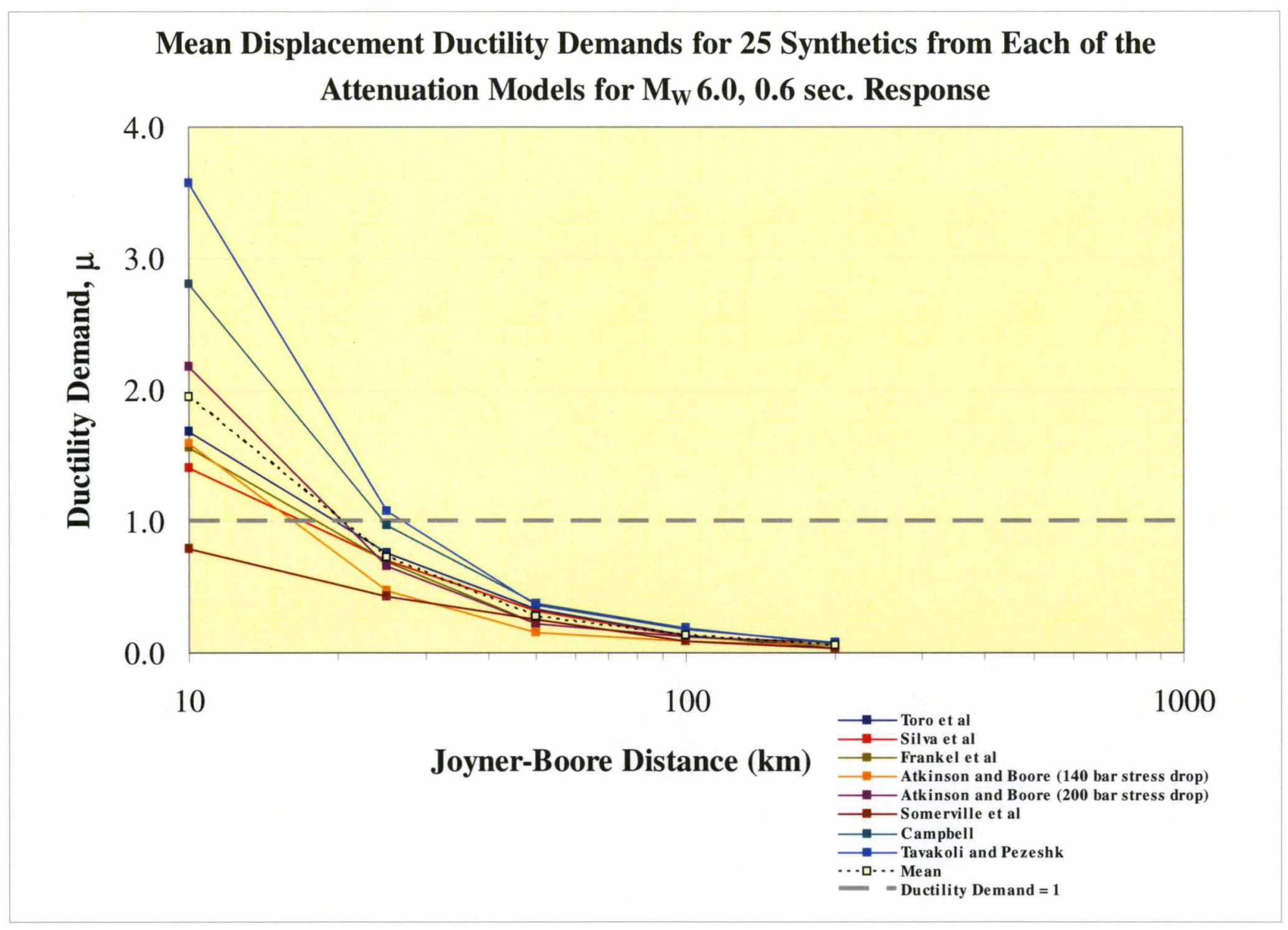




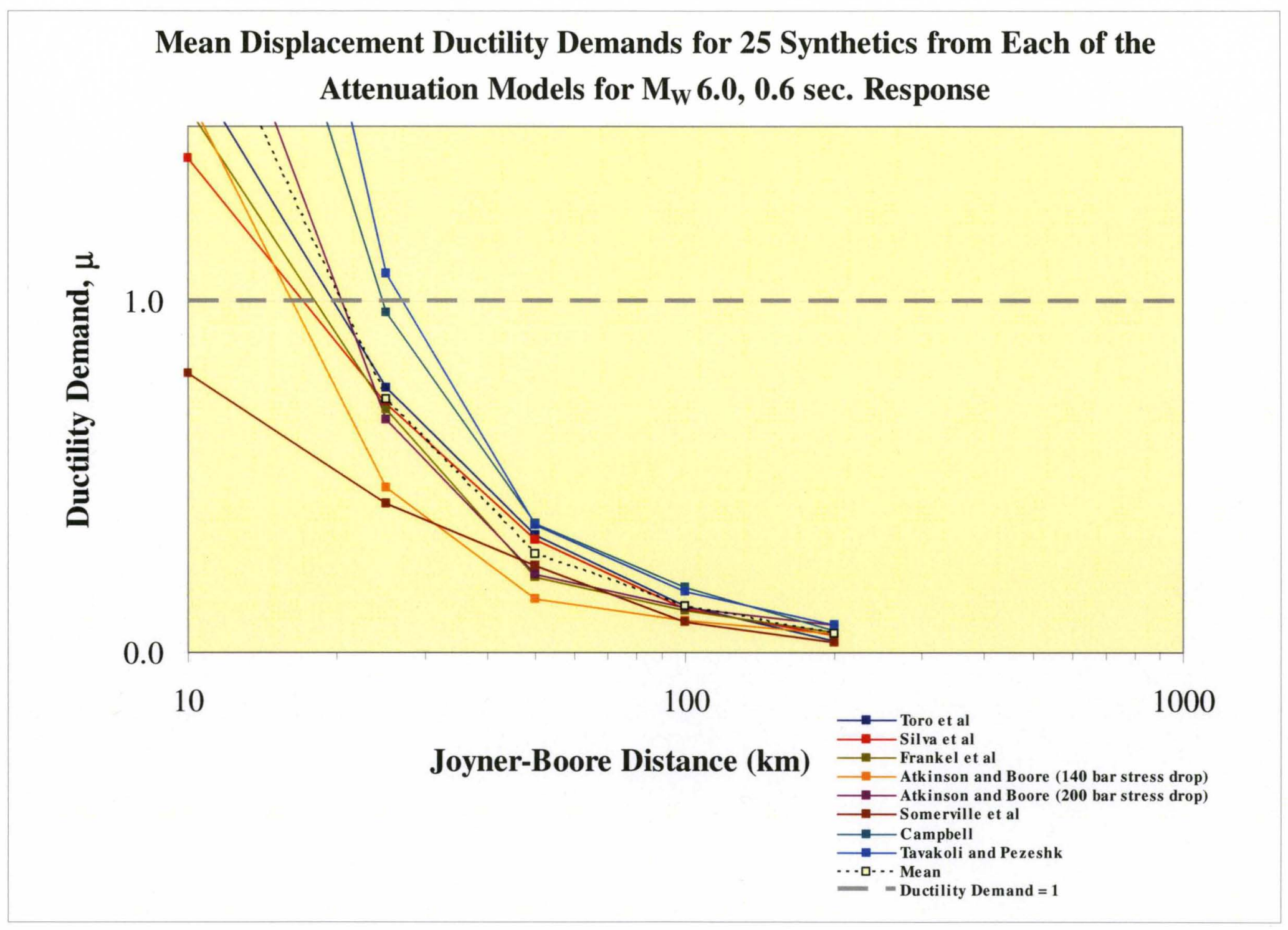




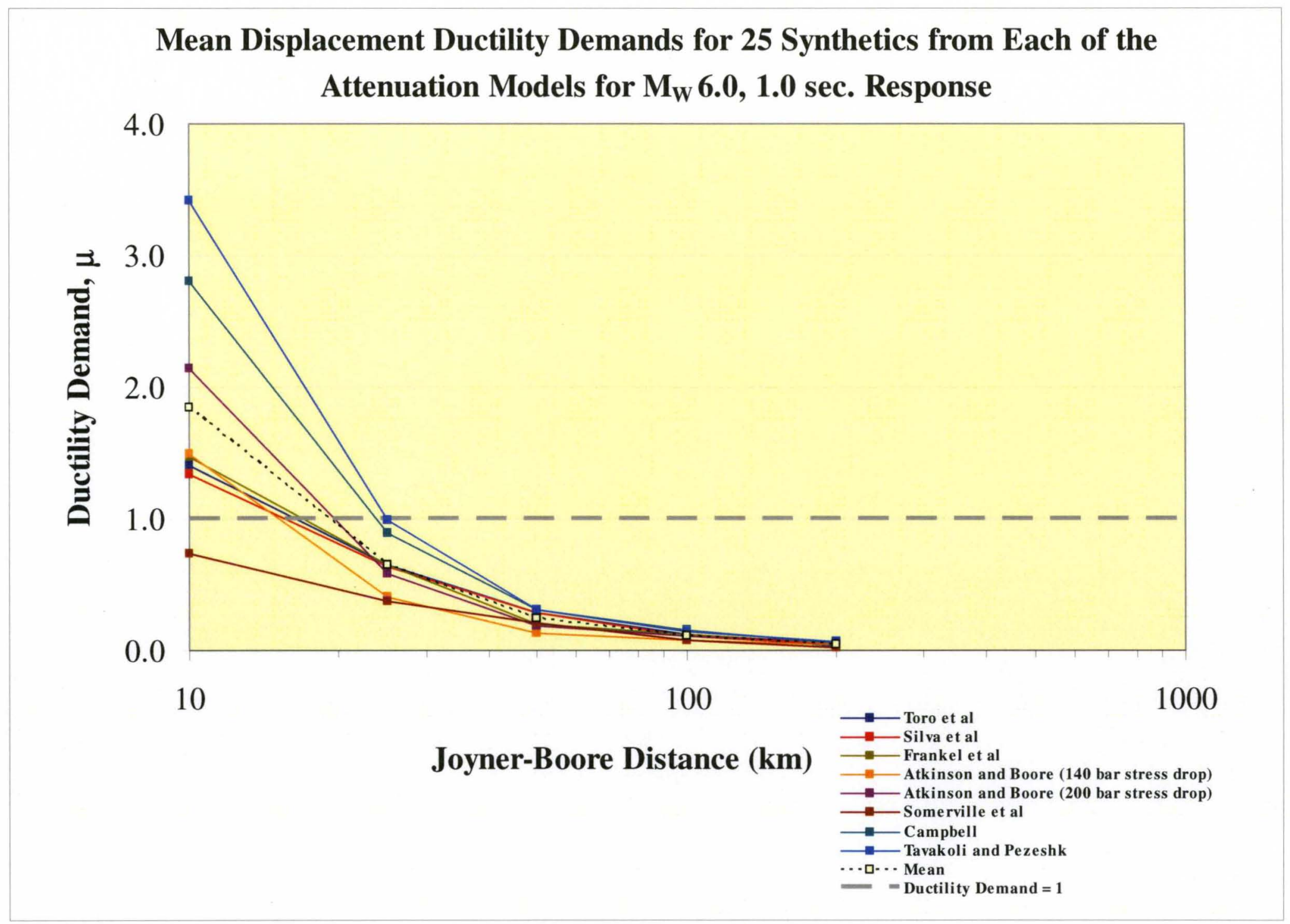




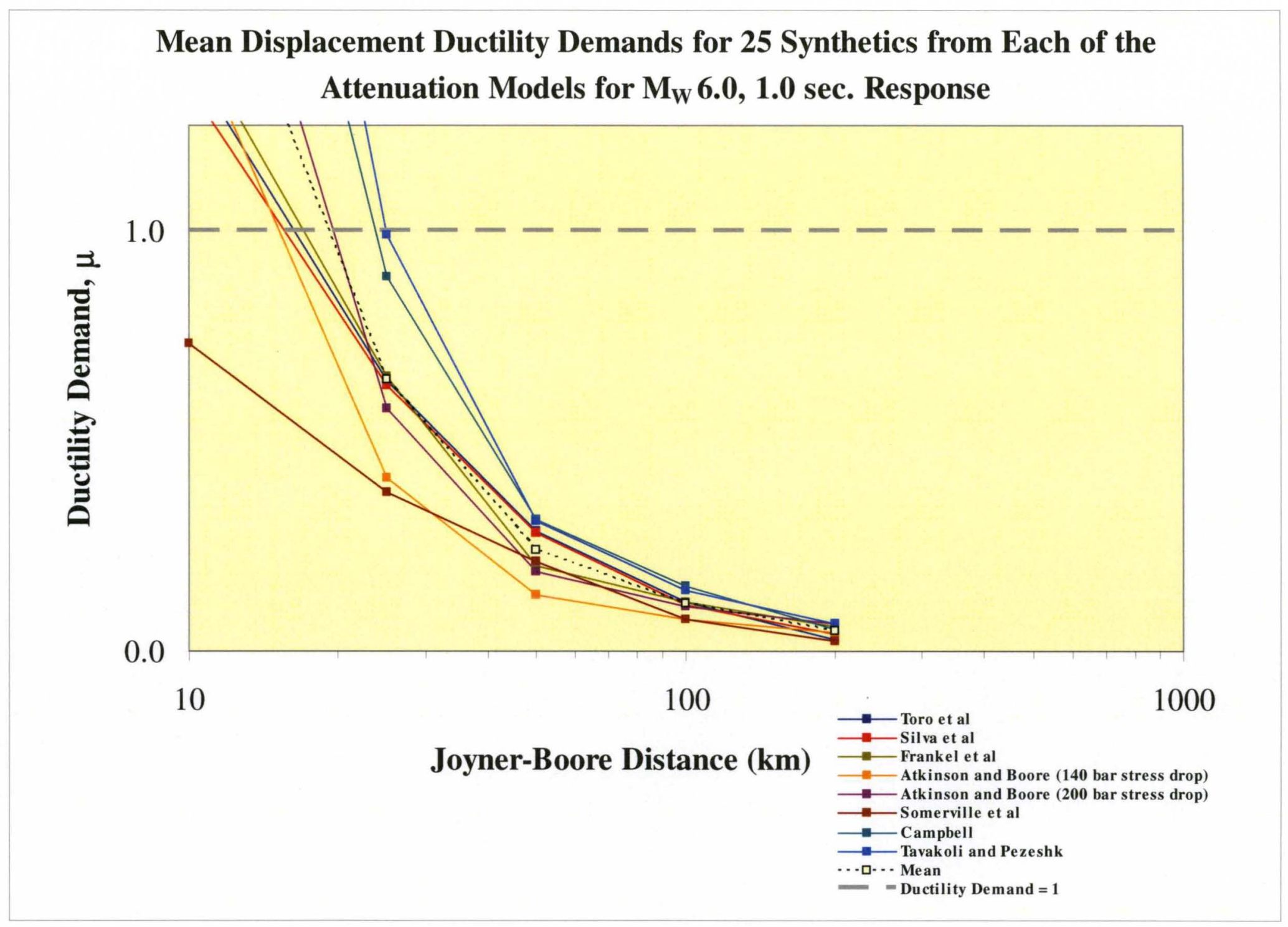




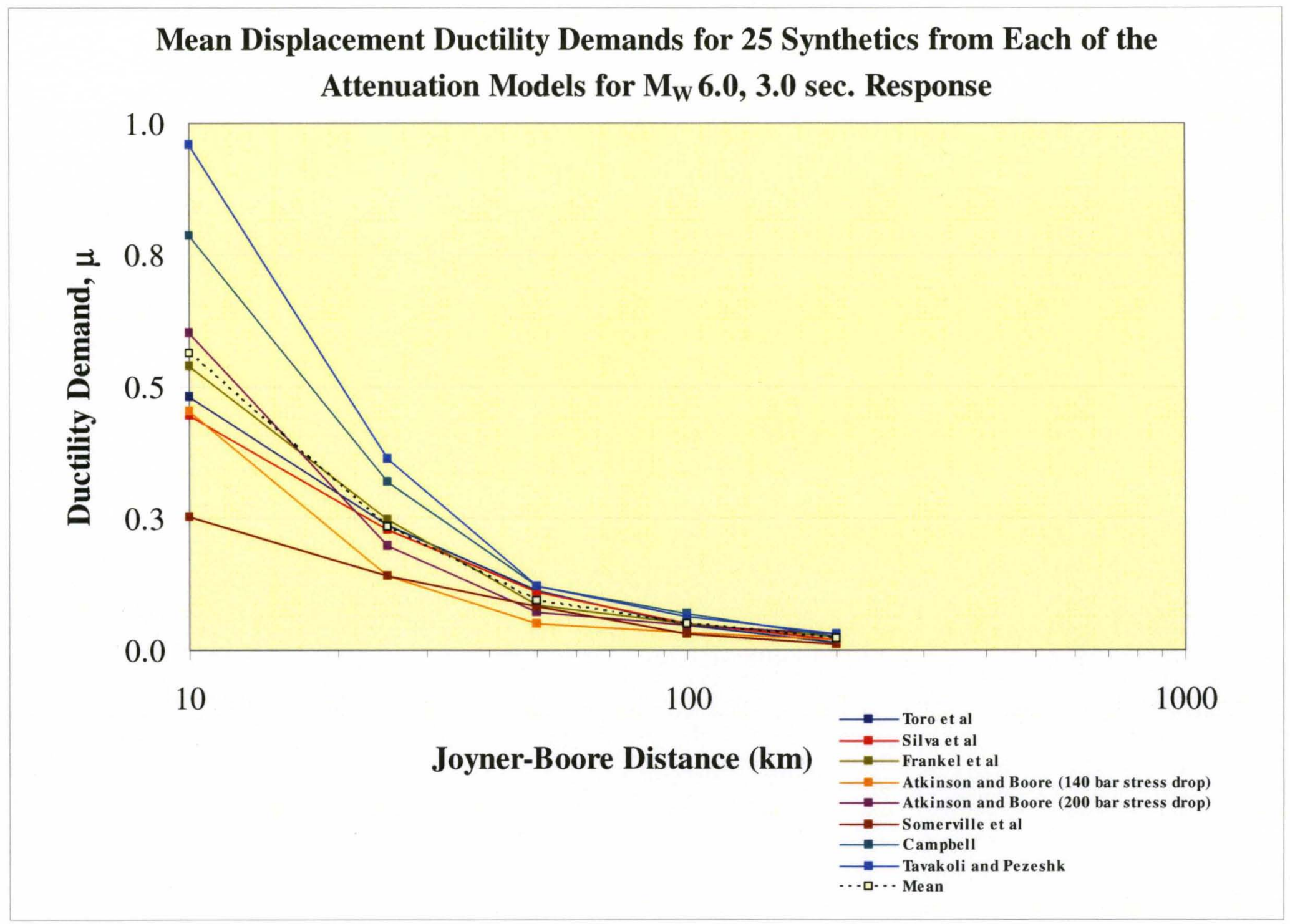




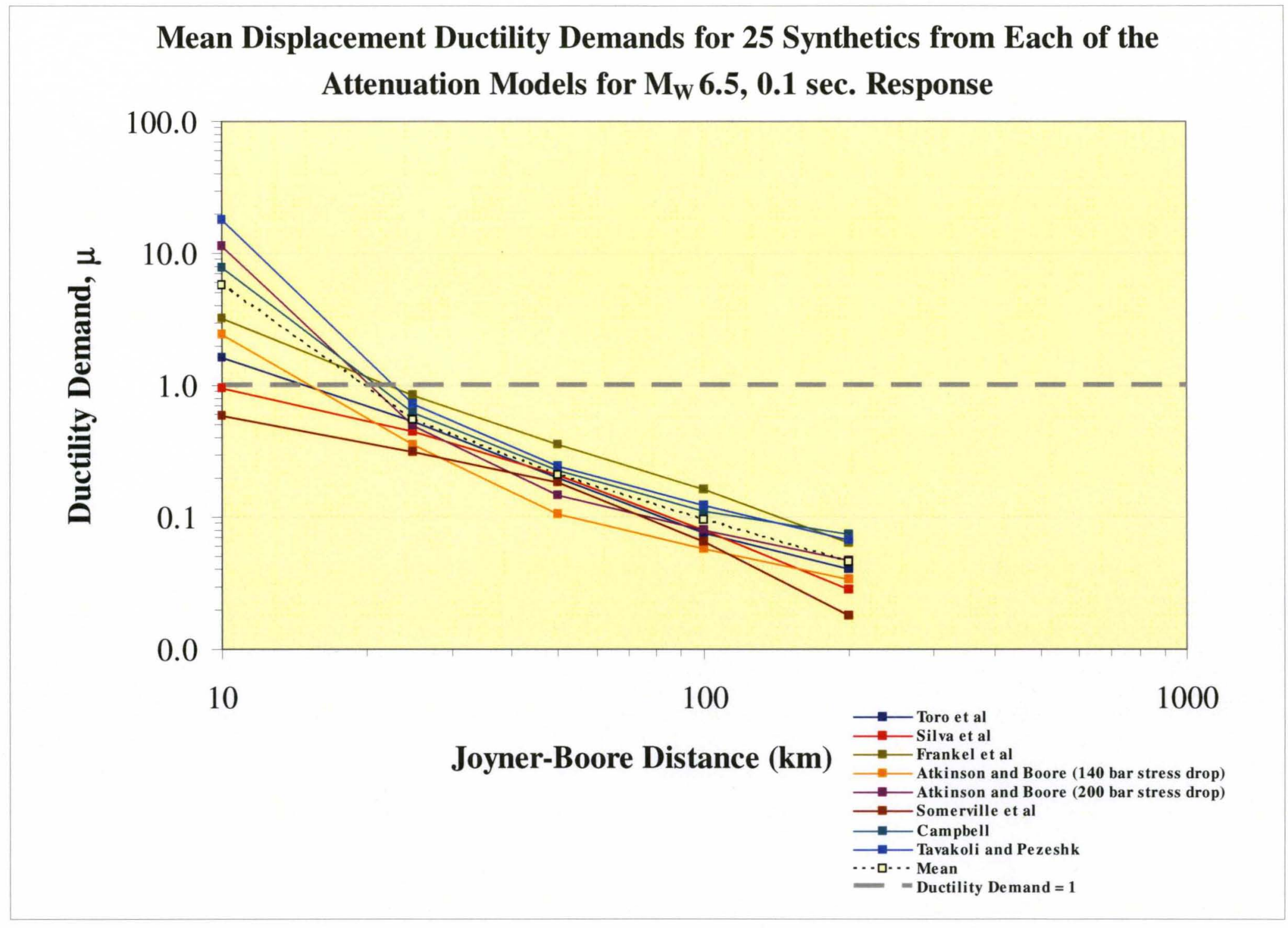




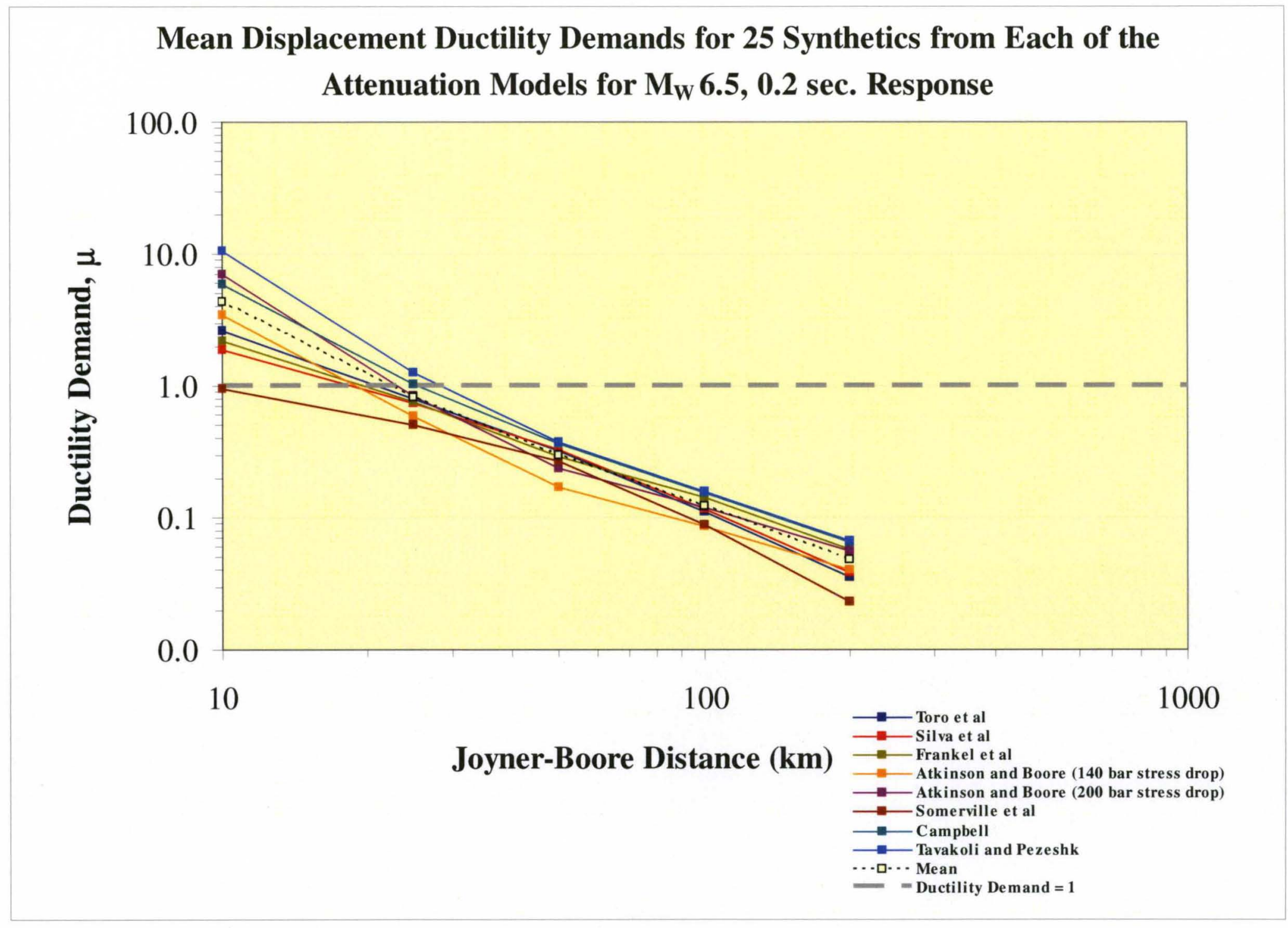




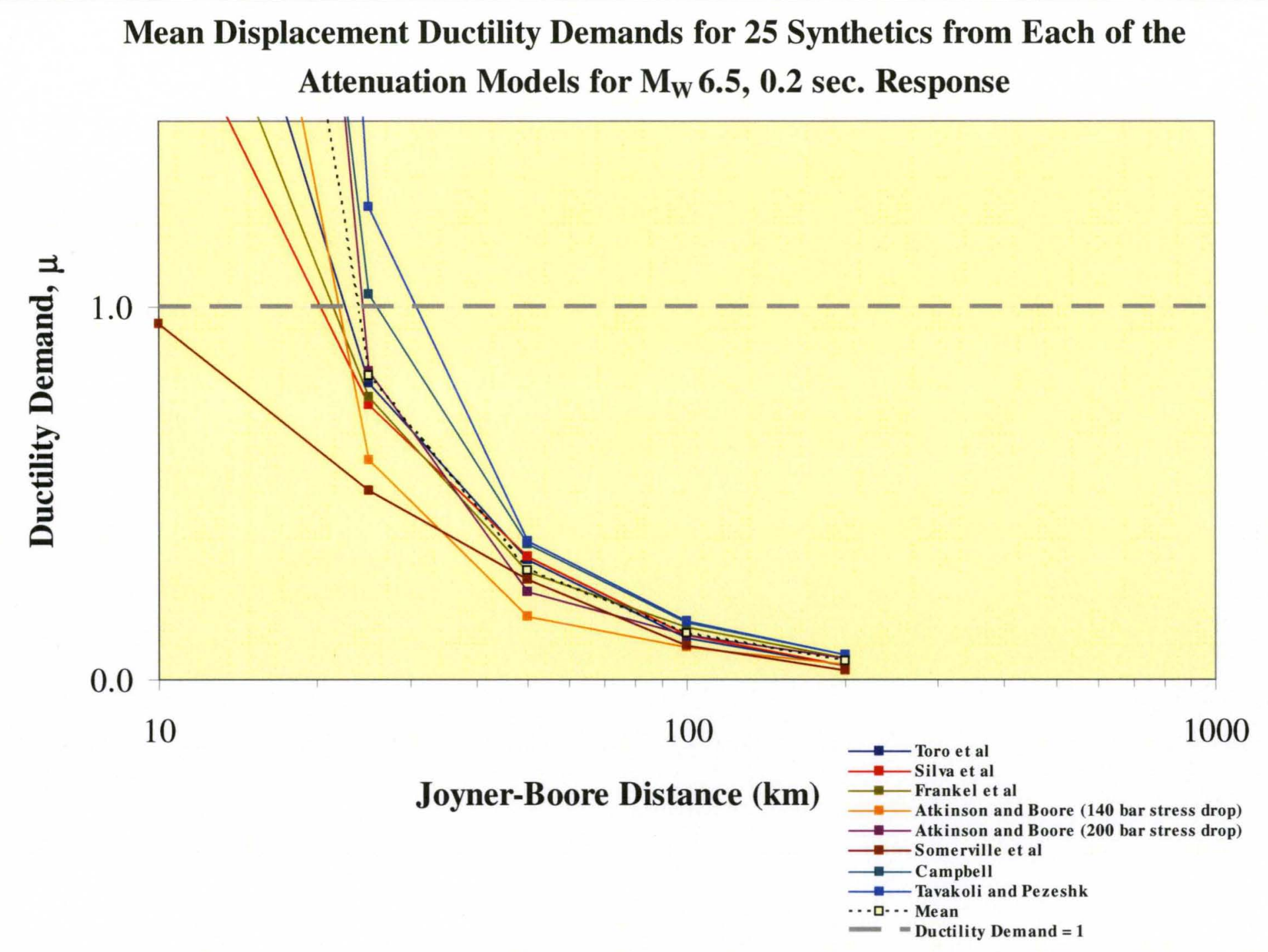




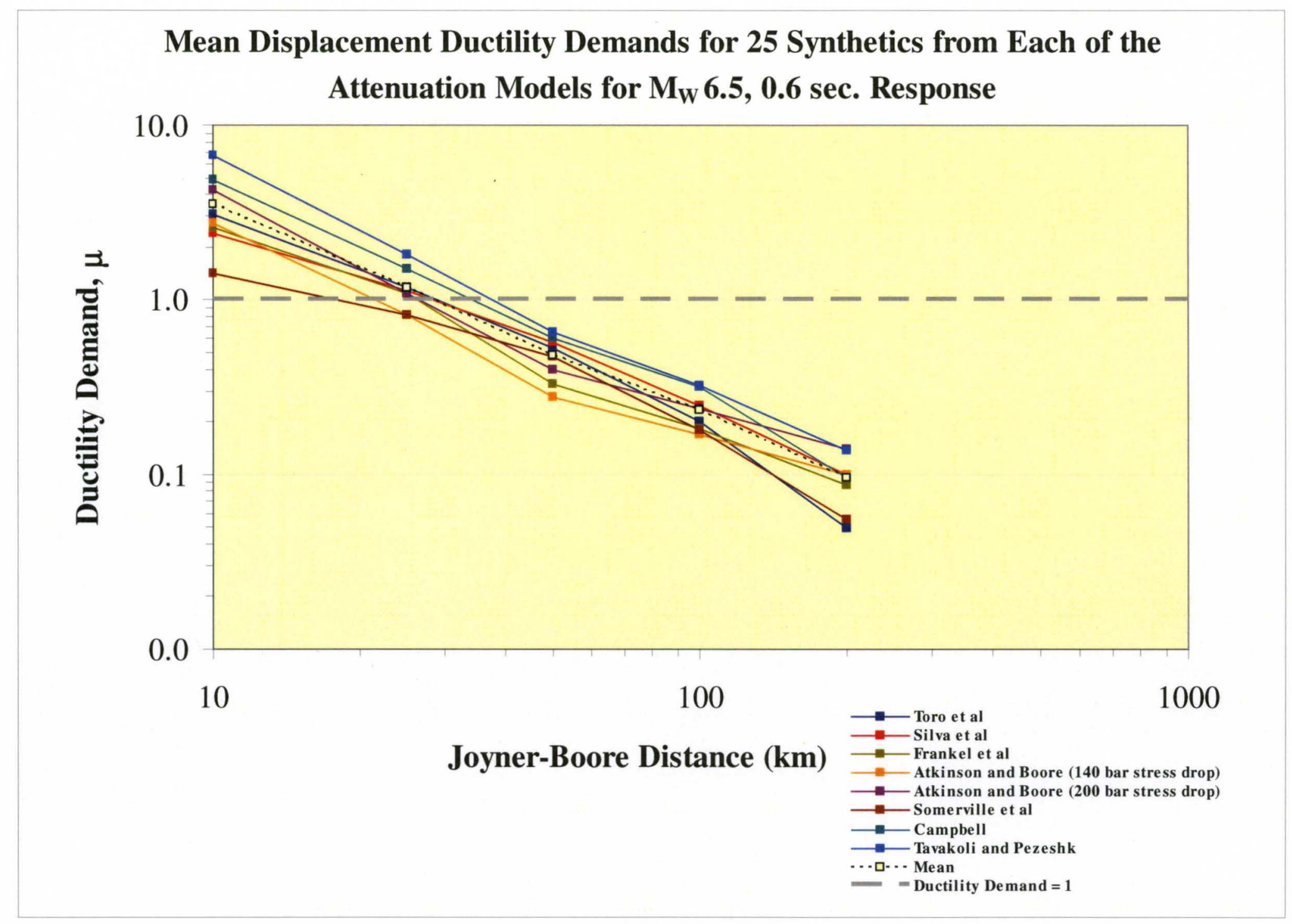




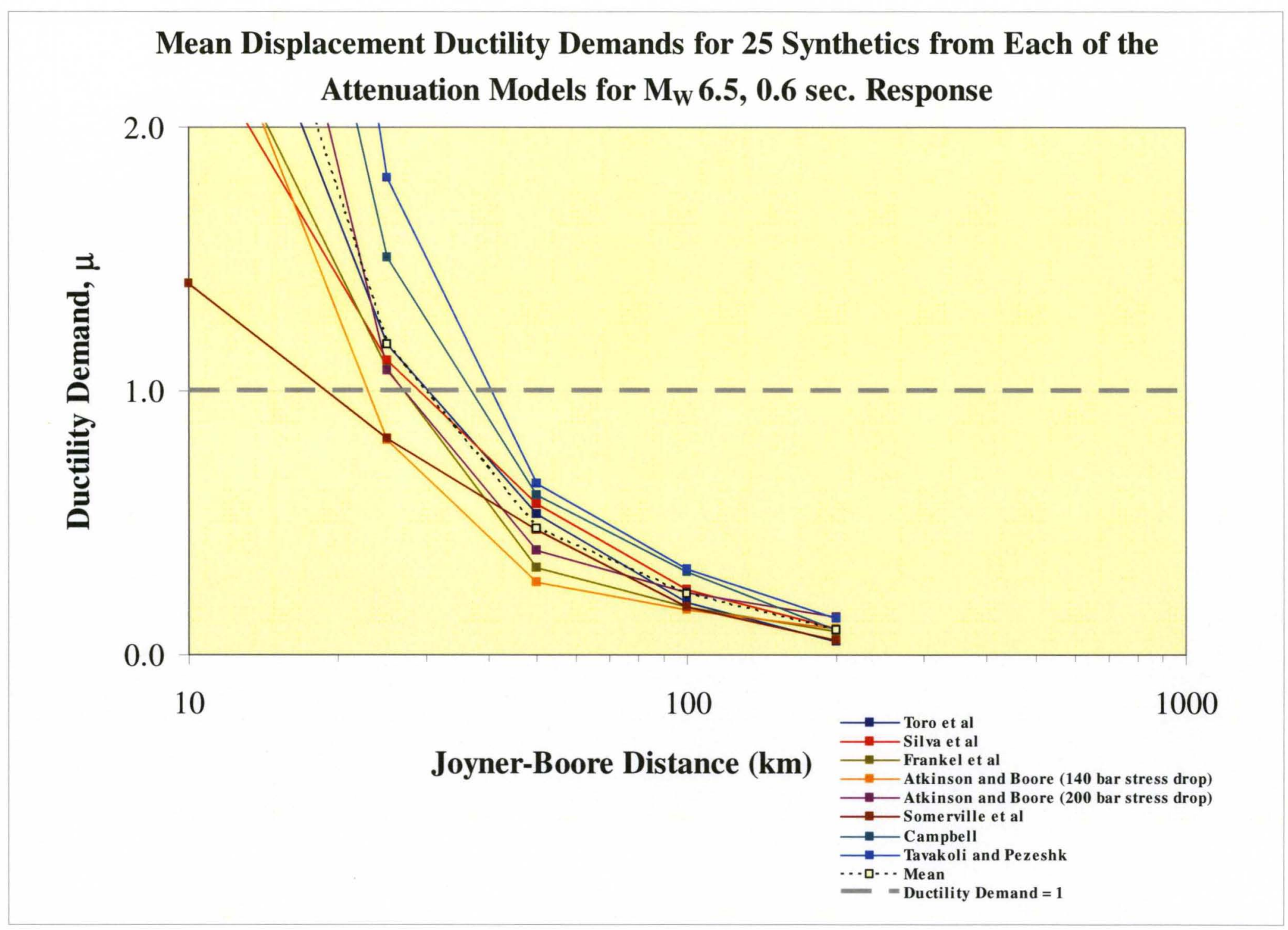




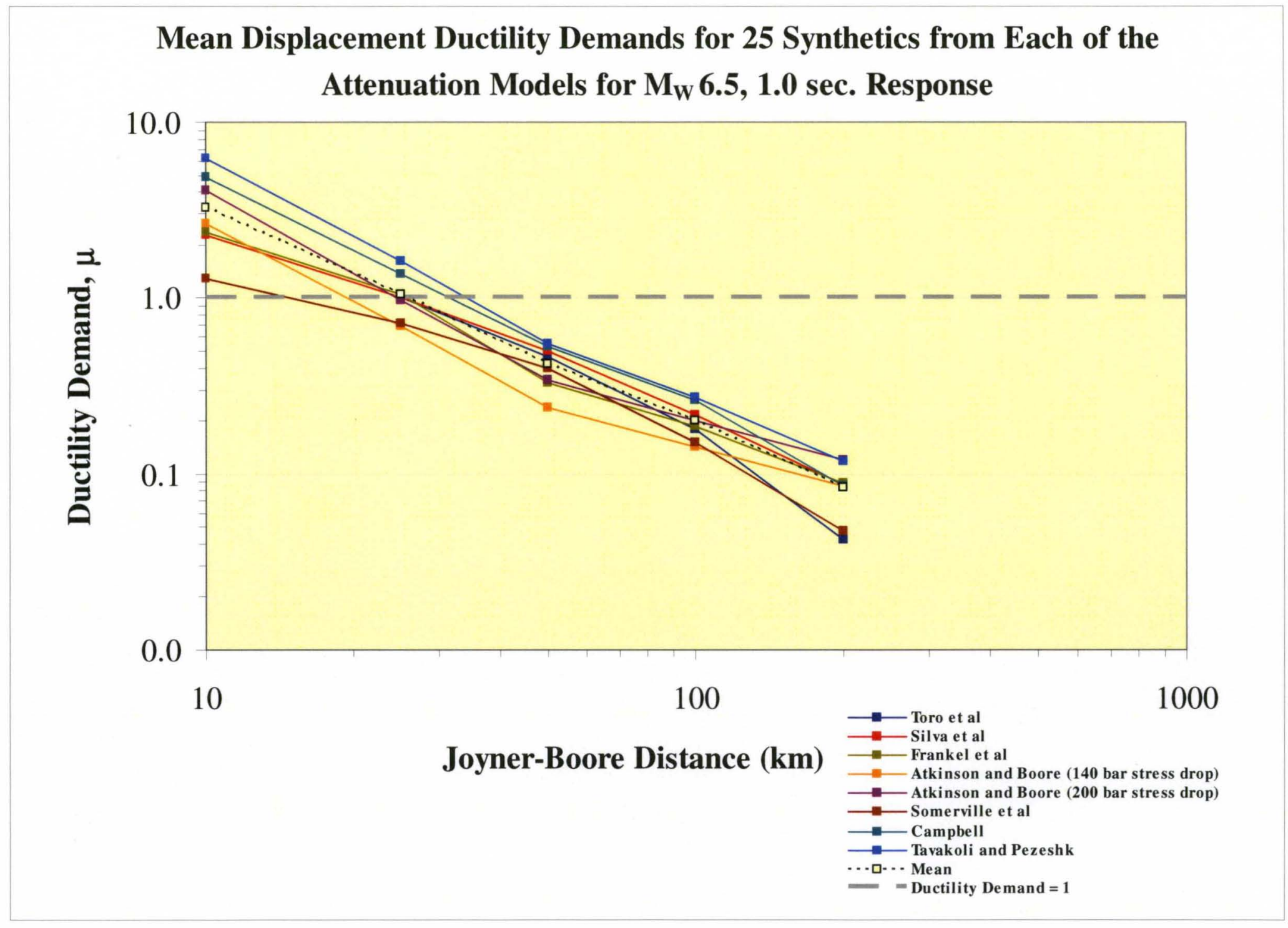




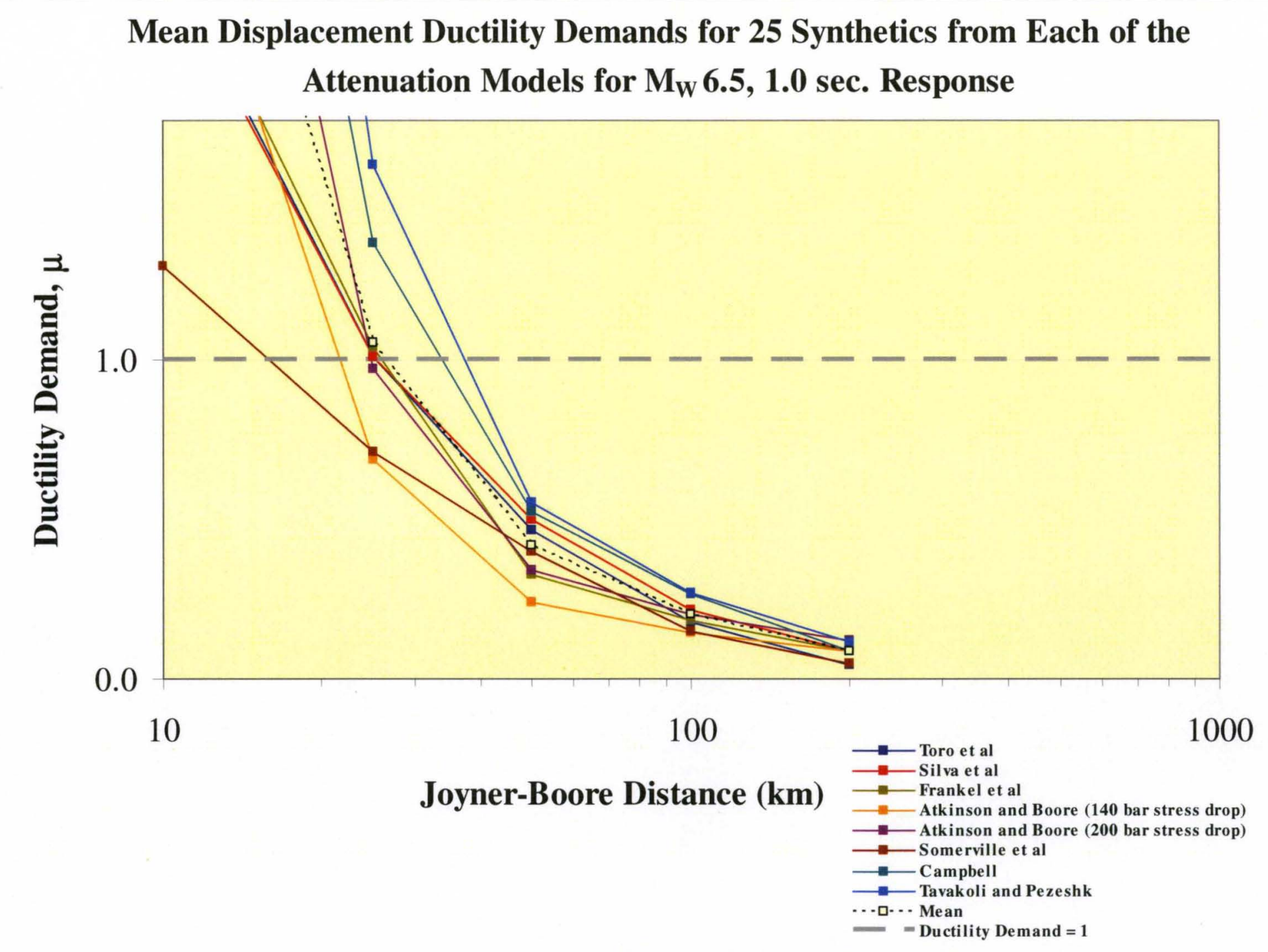




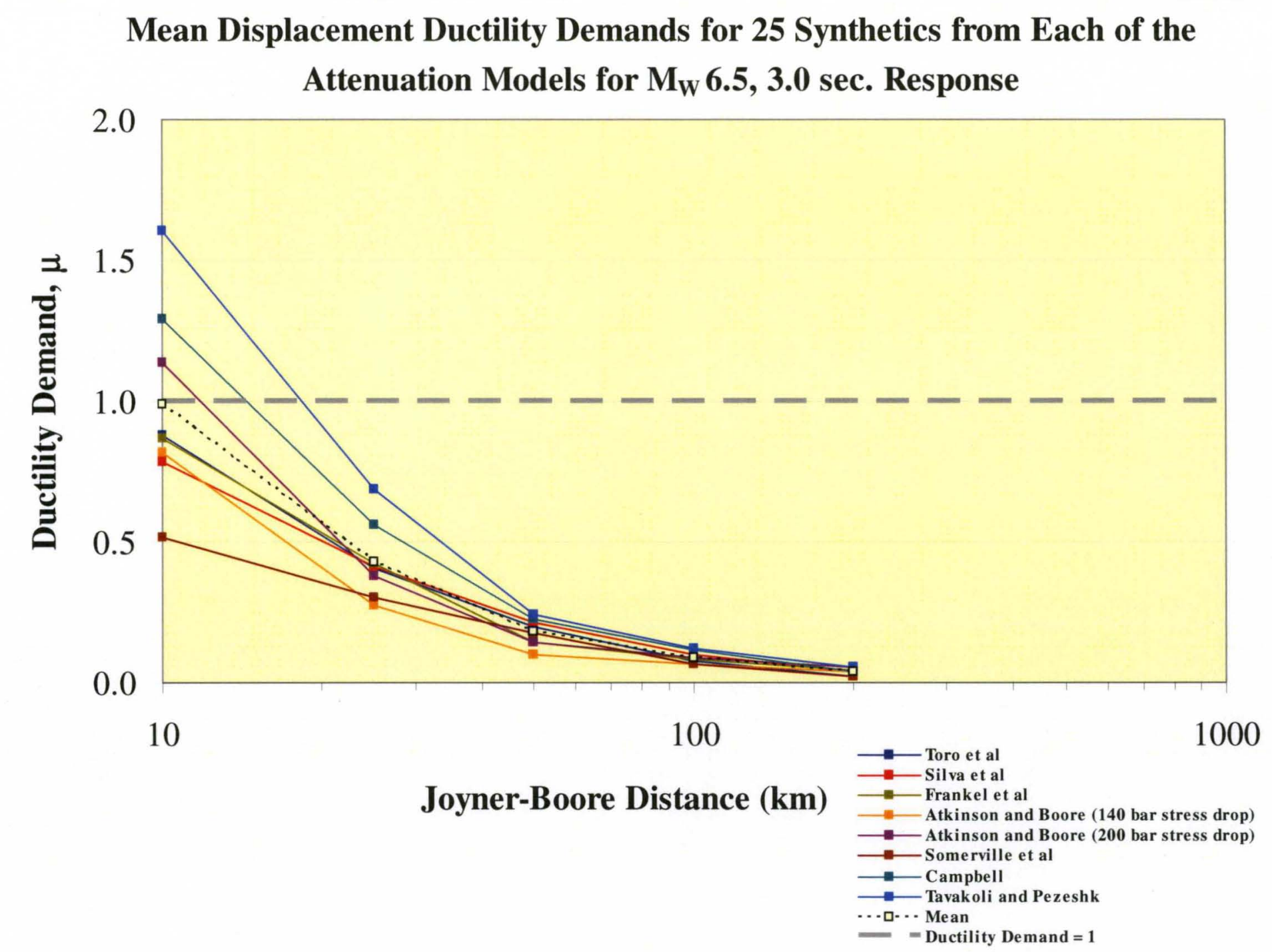




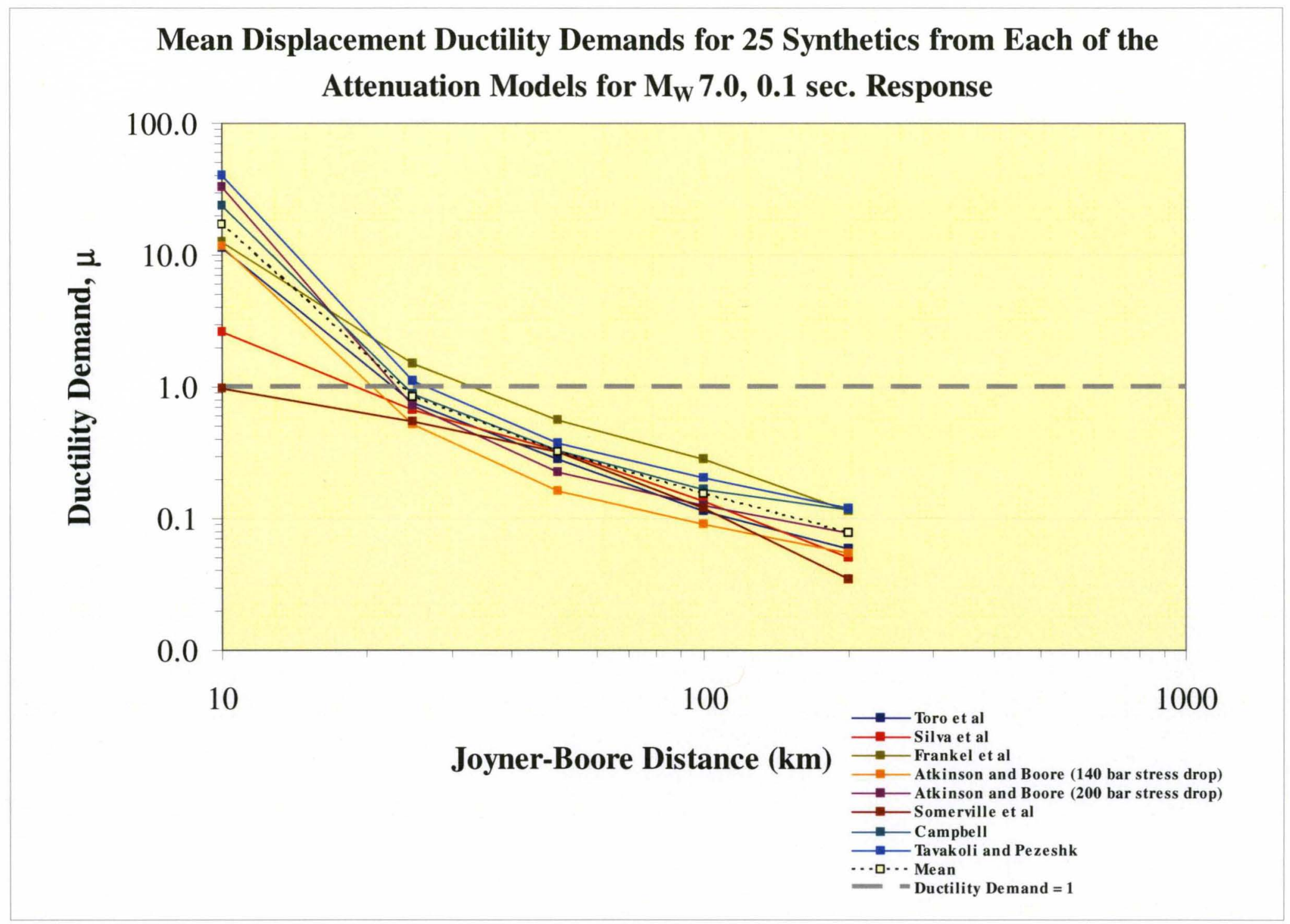




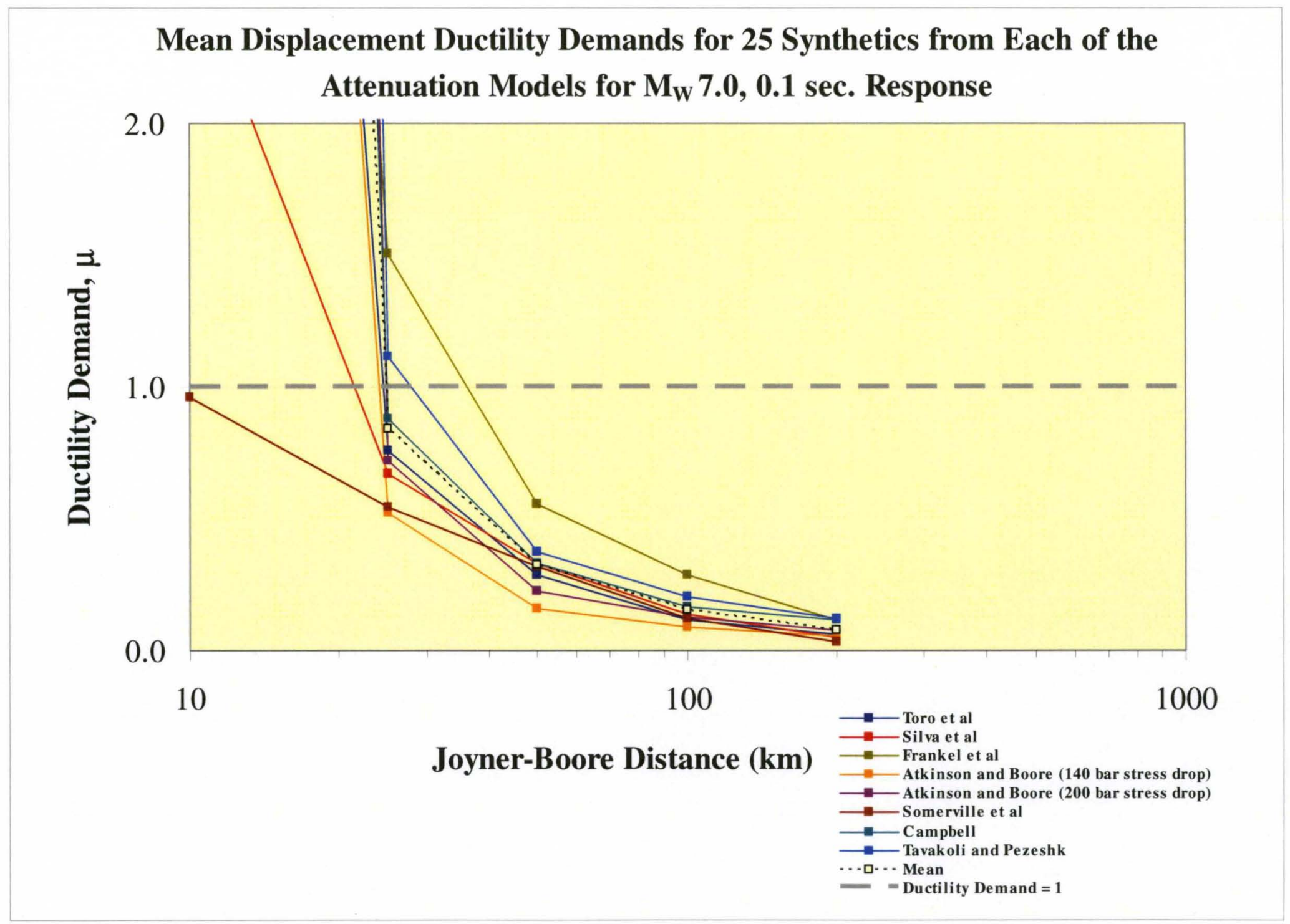




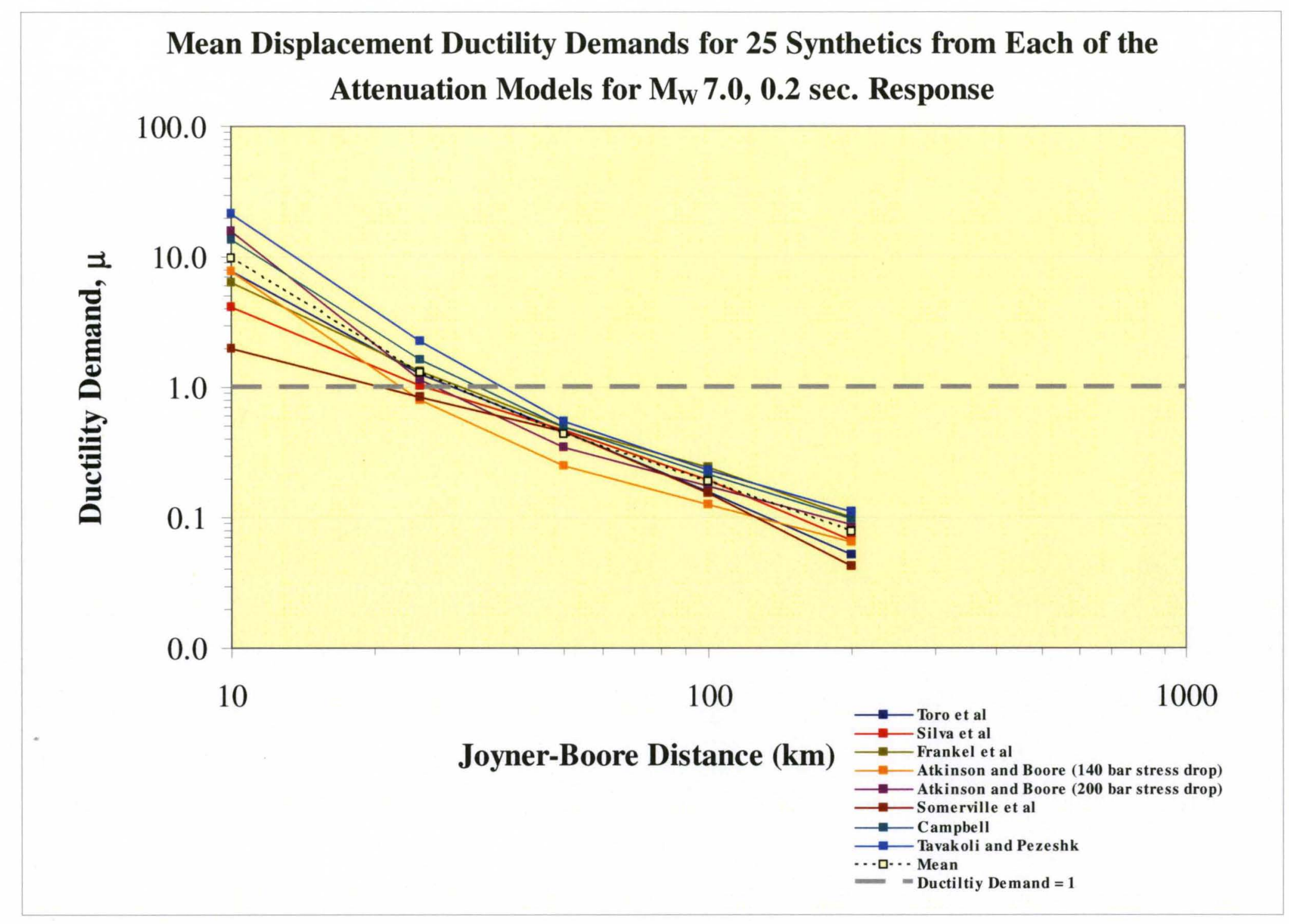




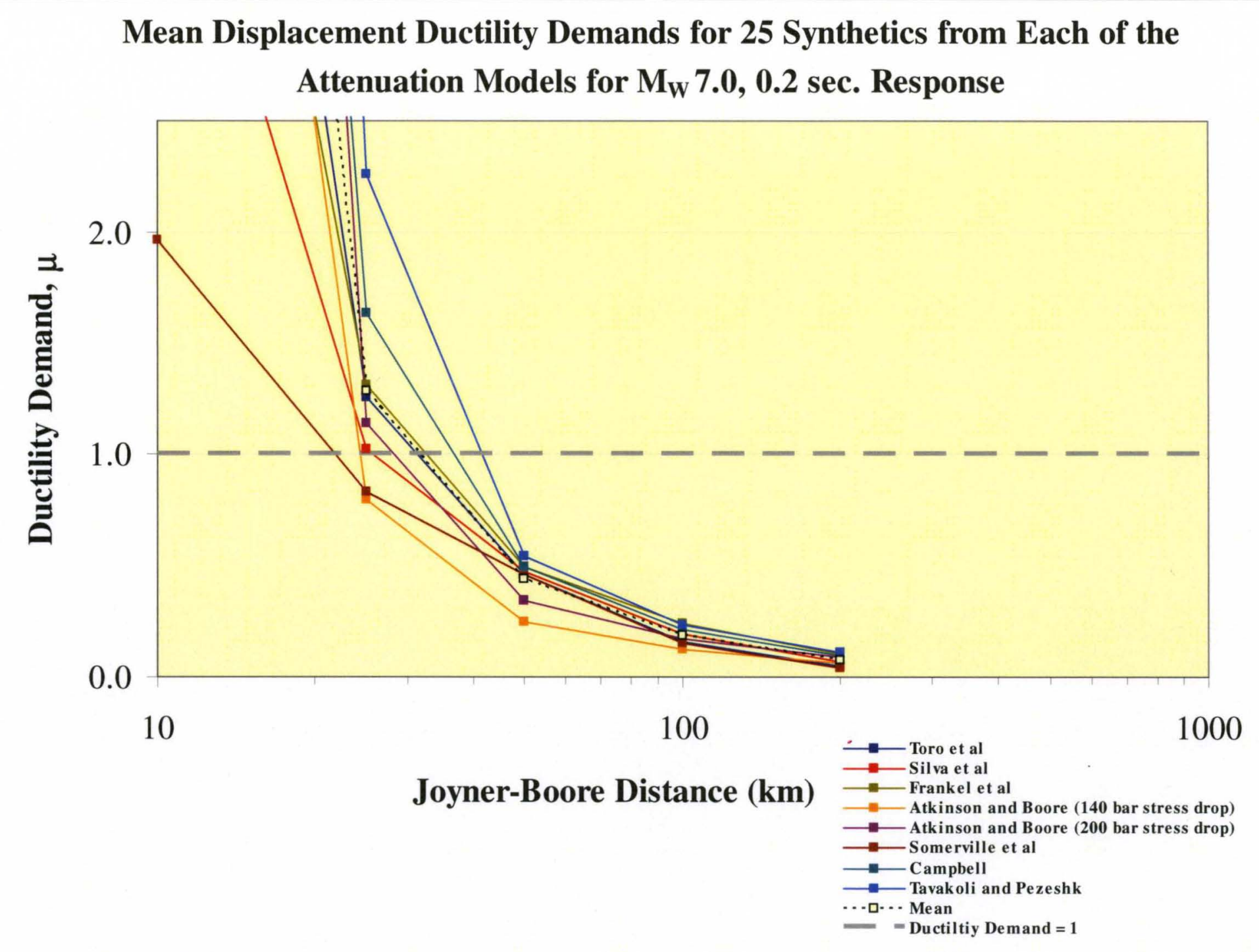




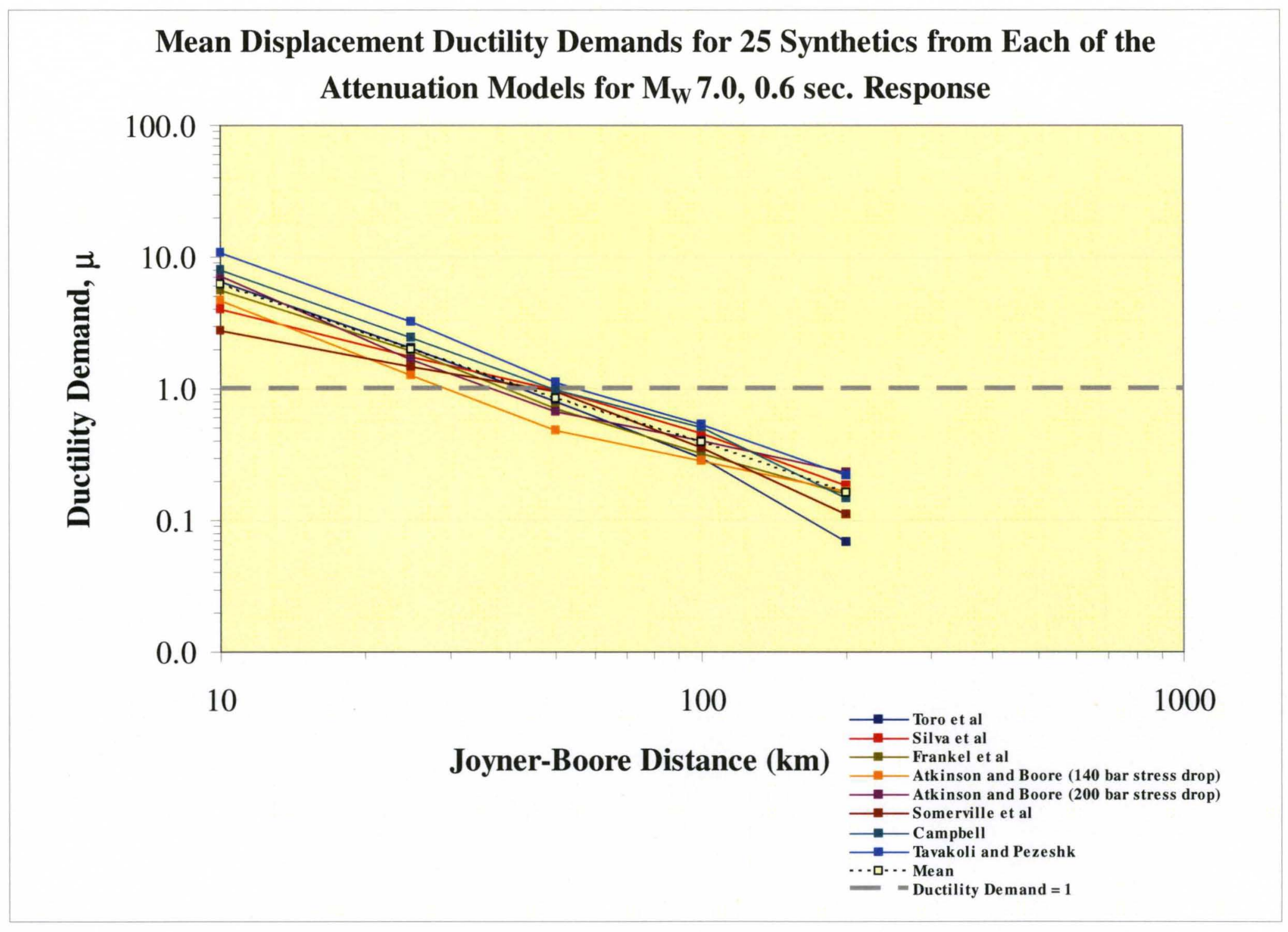




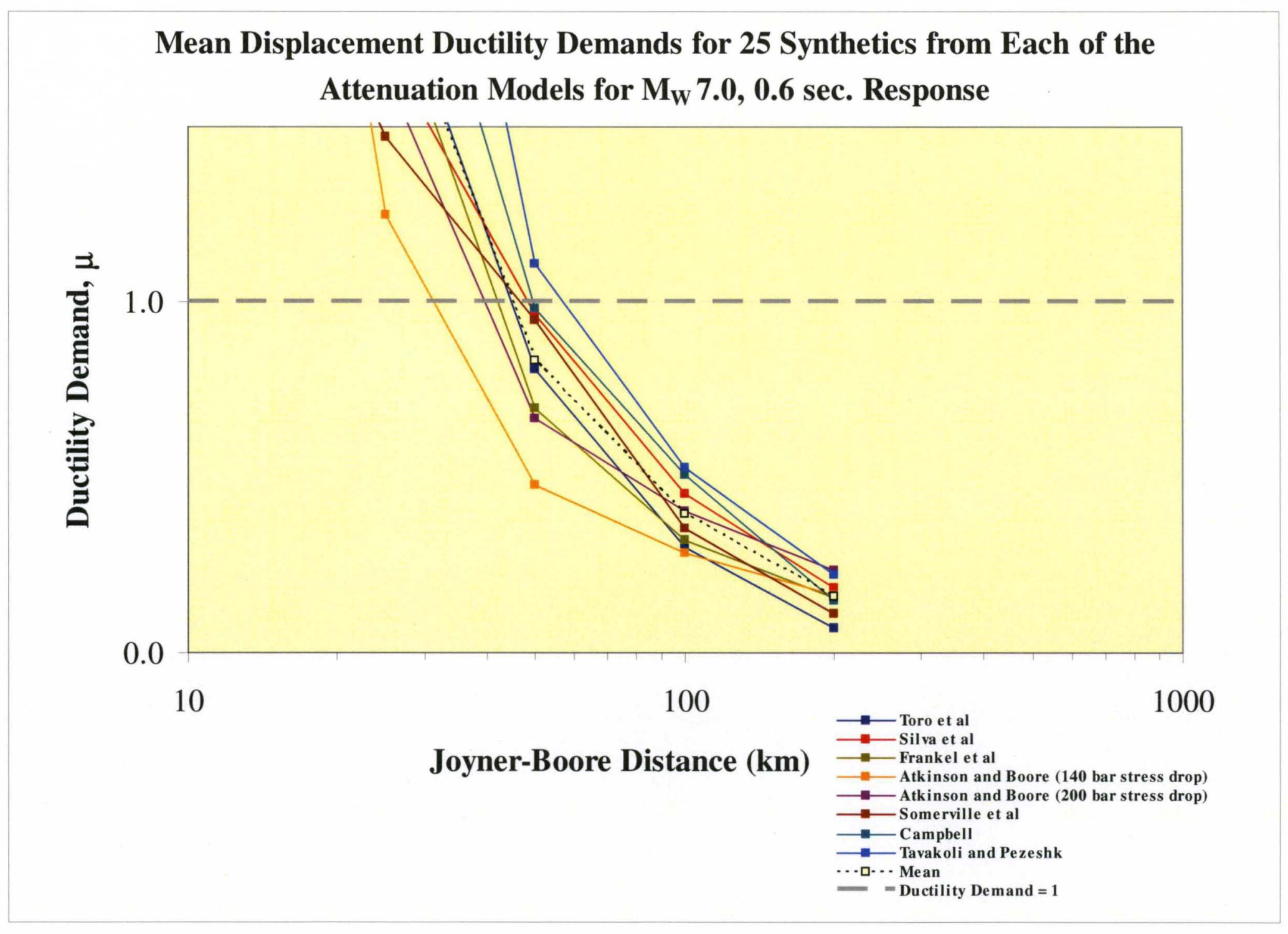




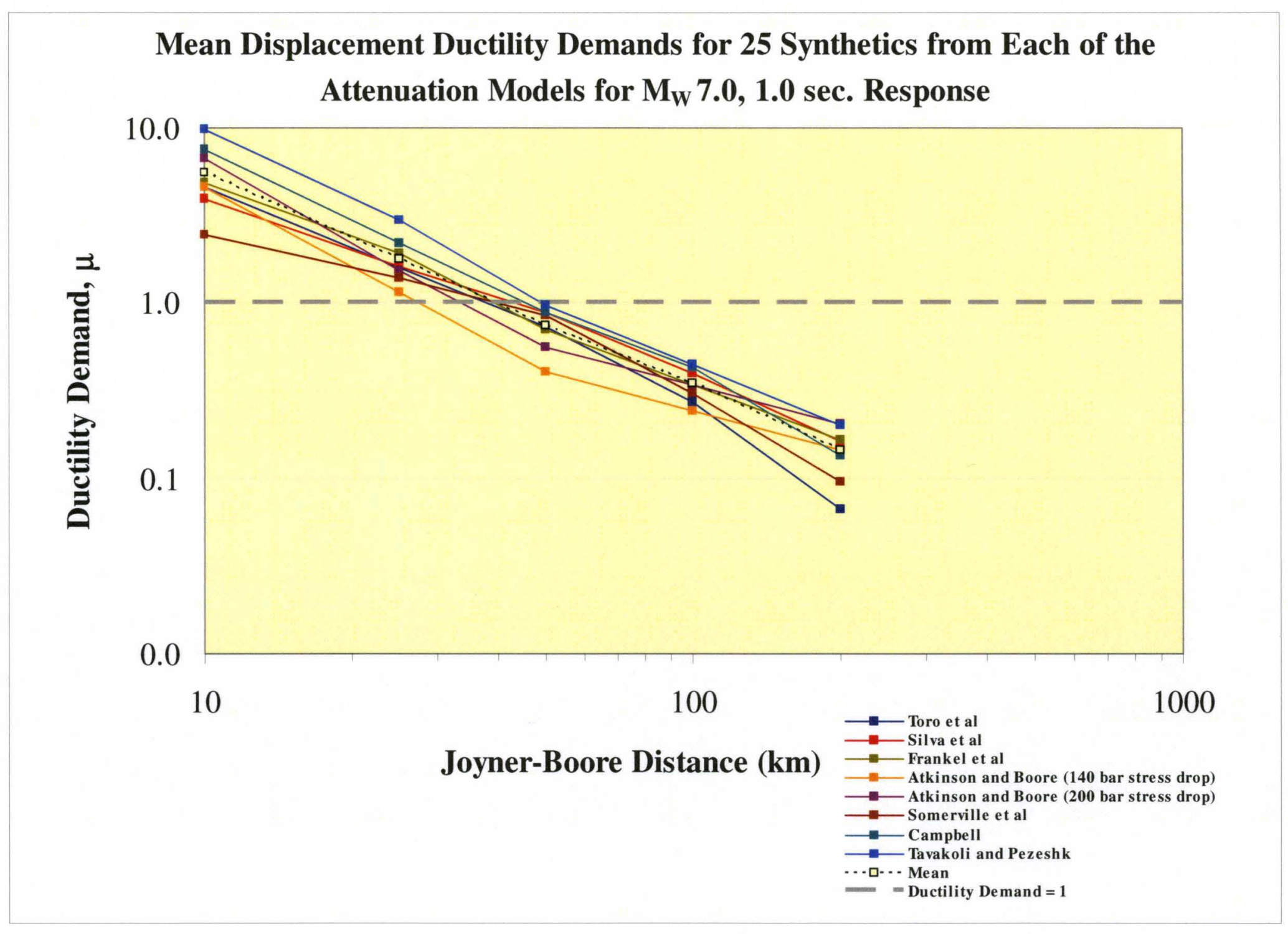




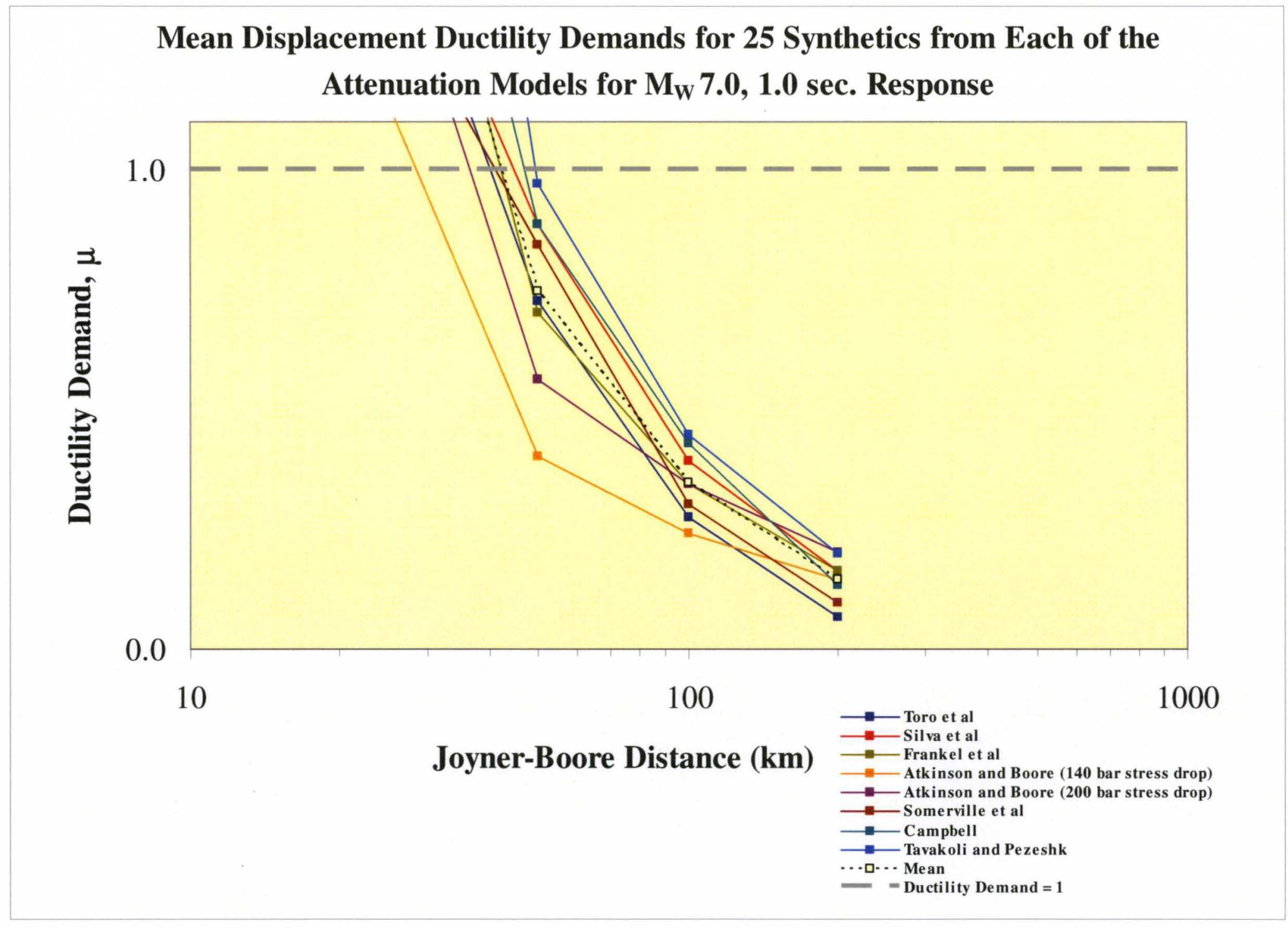




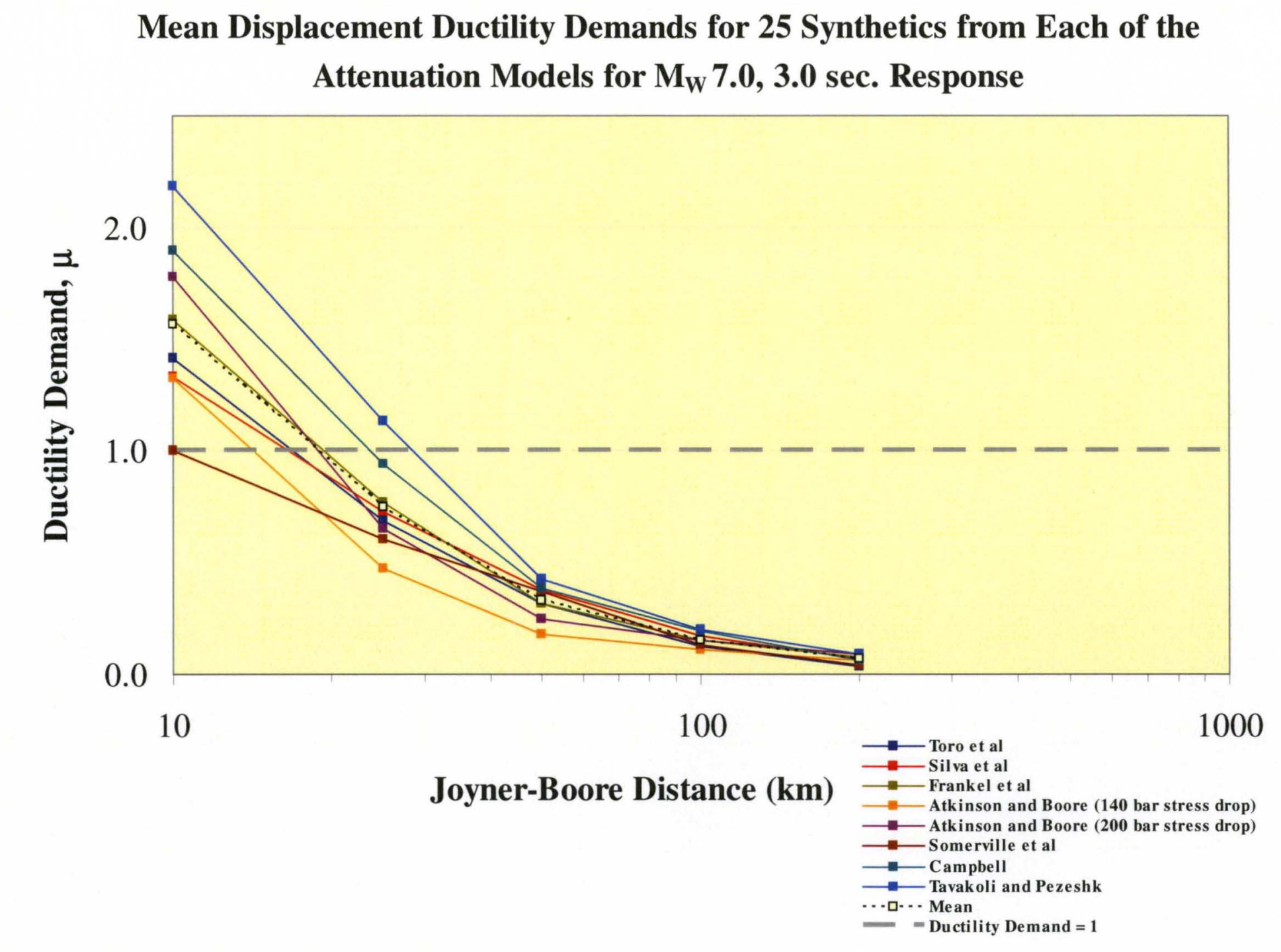




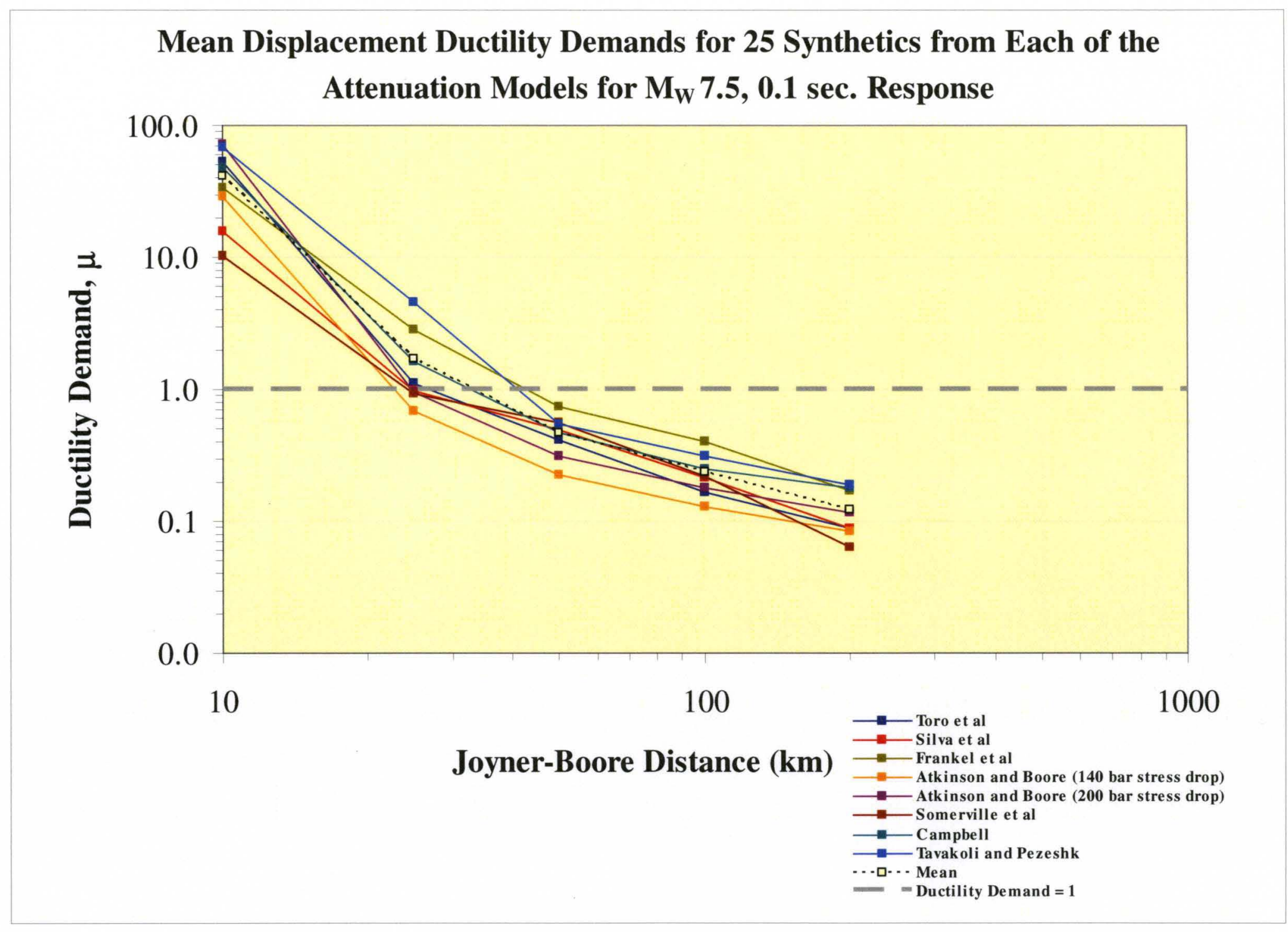




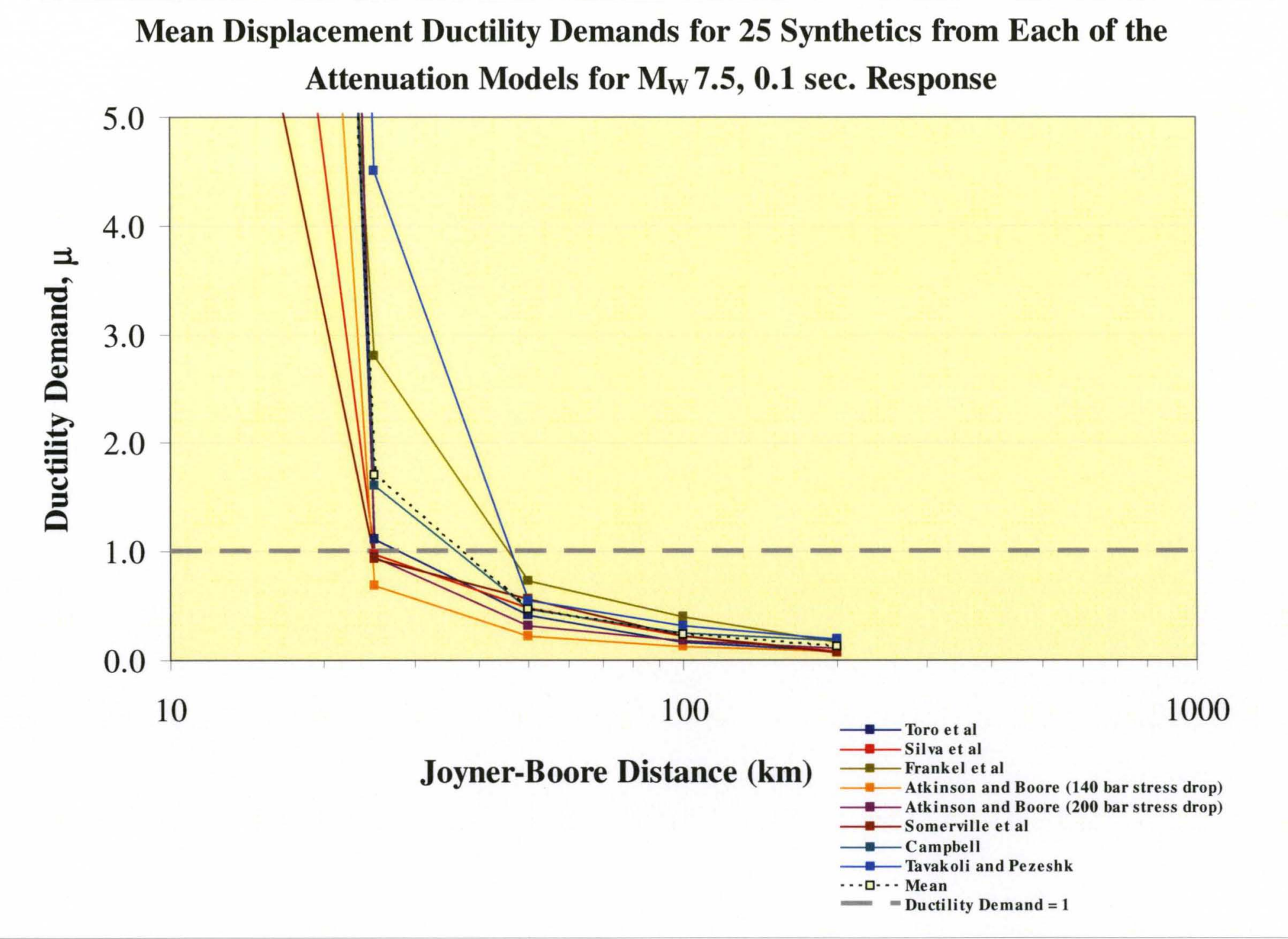




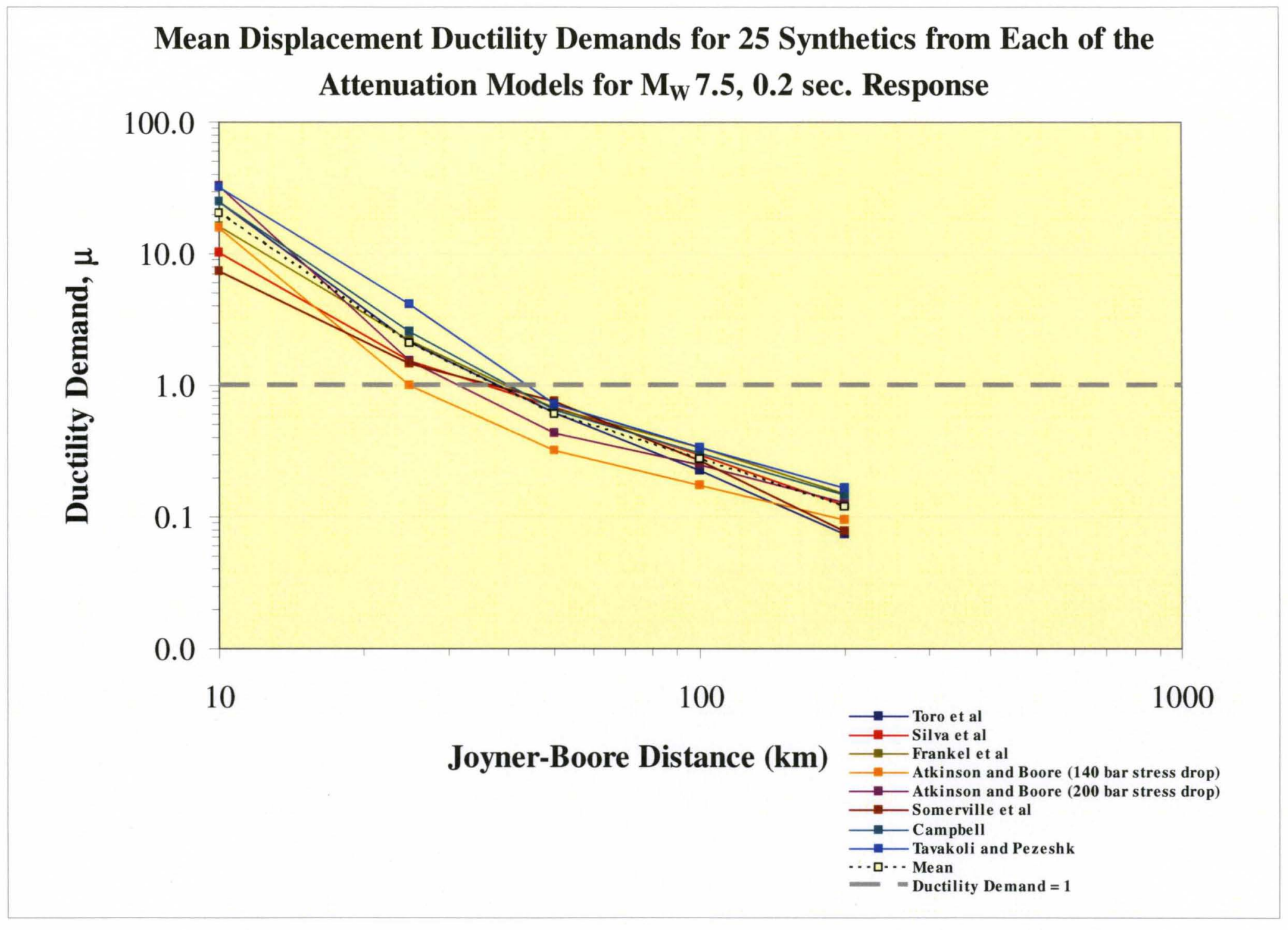




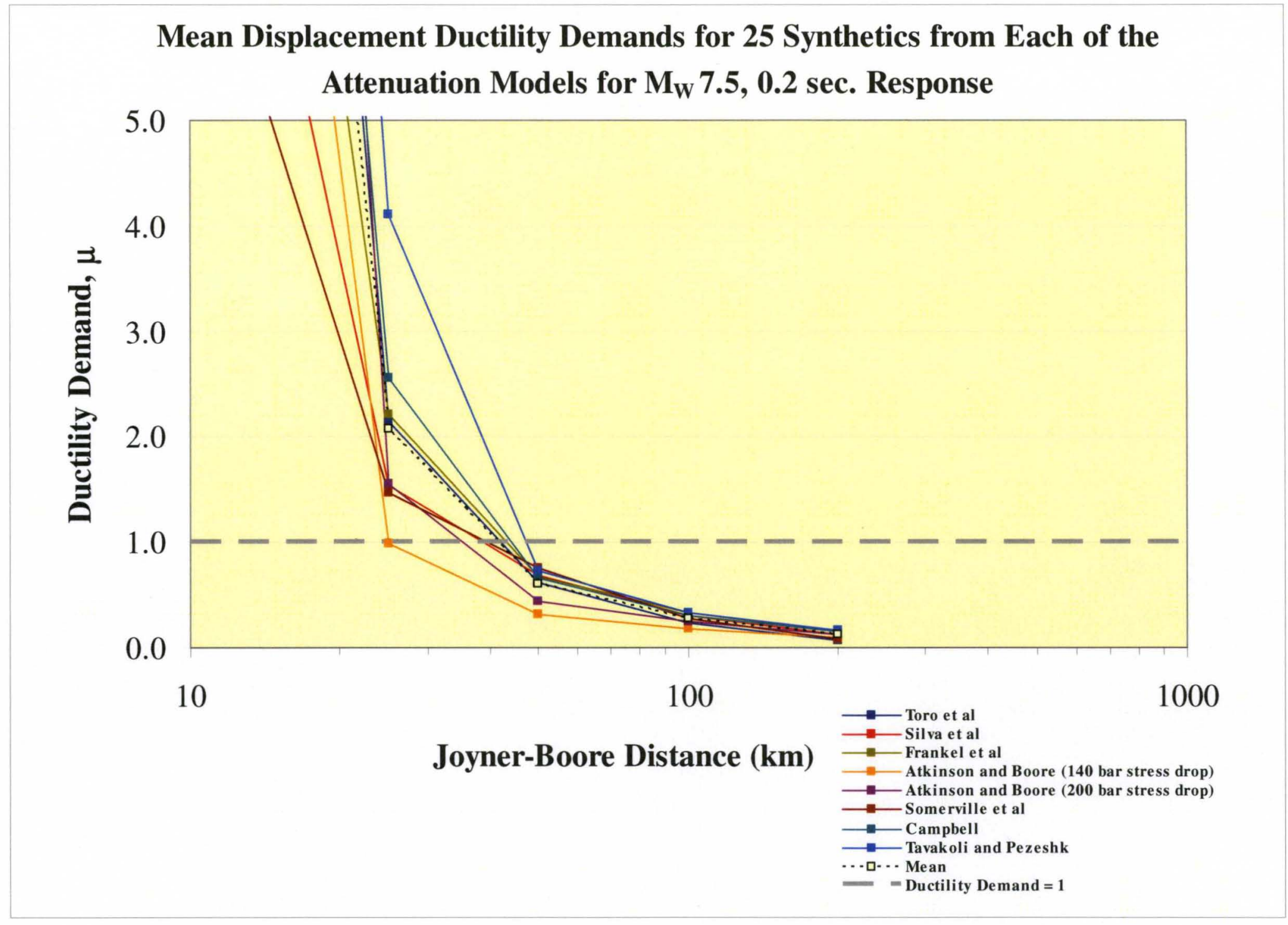




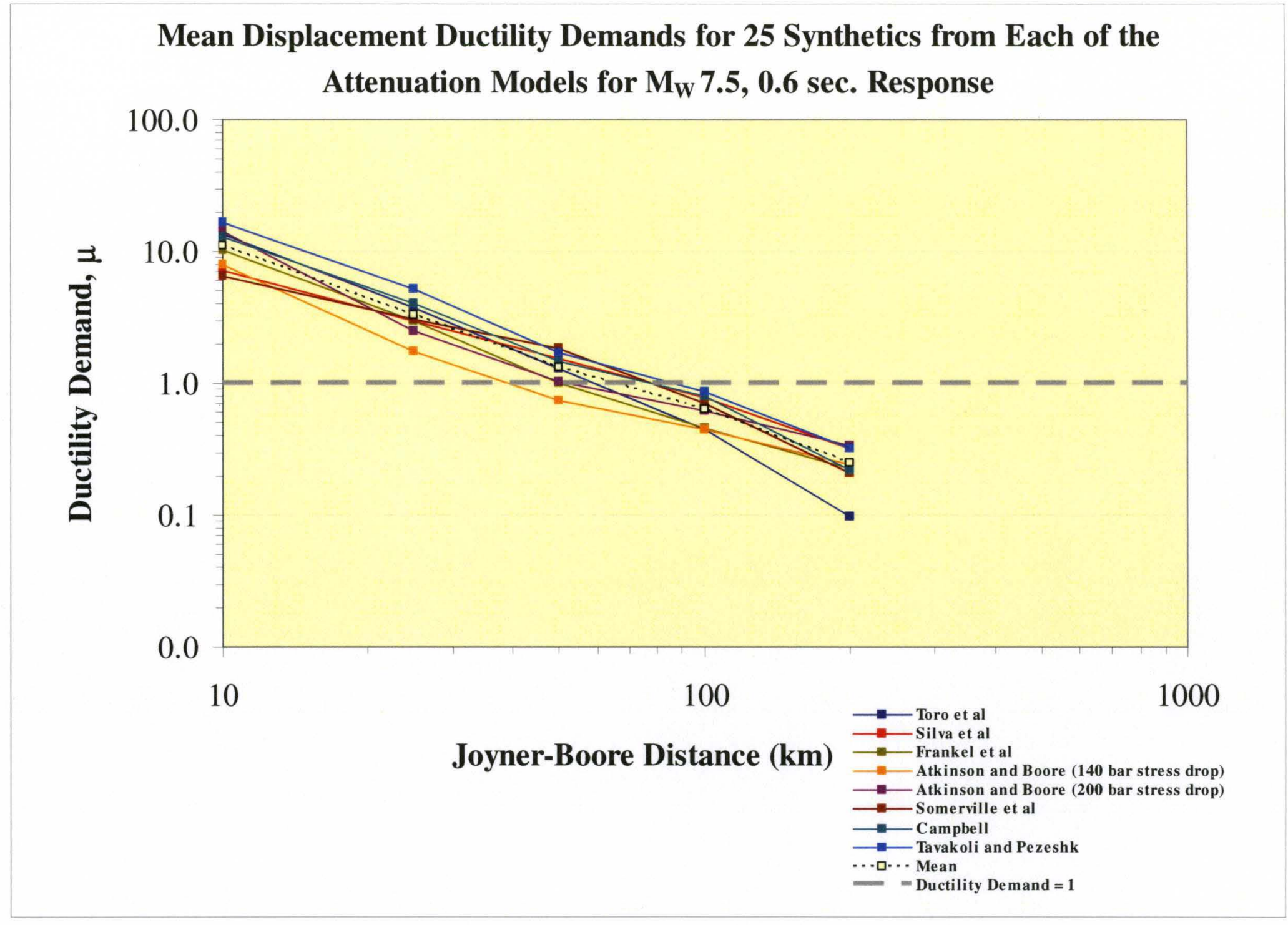




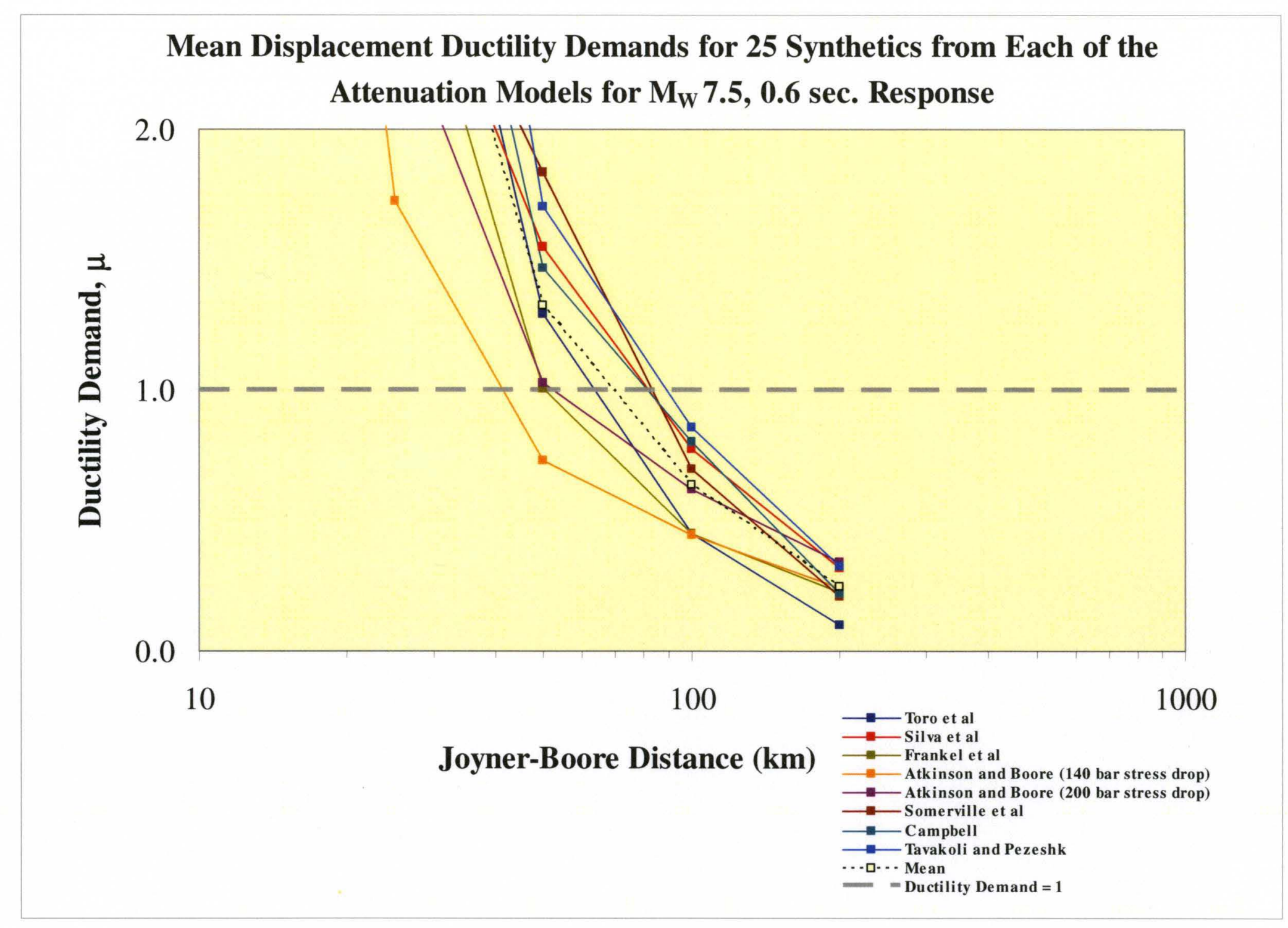


Mean Displacement Ductility Demands for 25 Synthetics from Each of the Attenuation Models for $M_{W} 7.5,1.0$ sec. Response

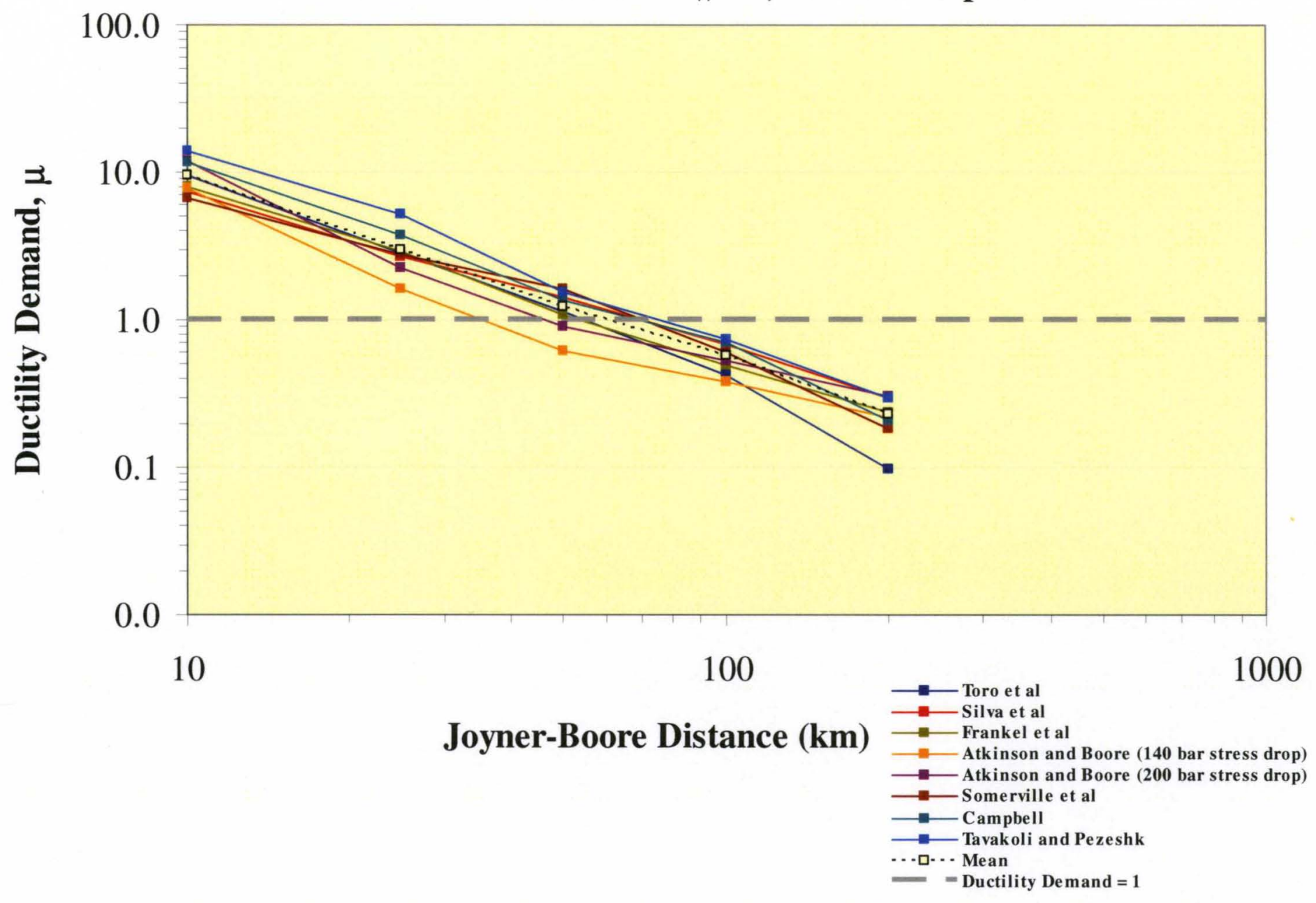




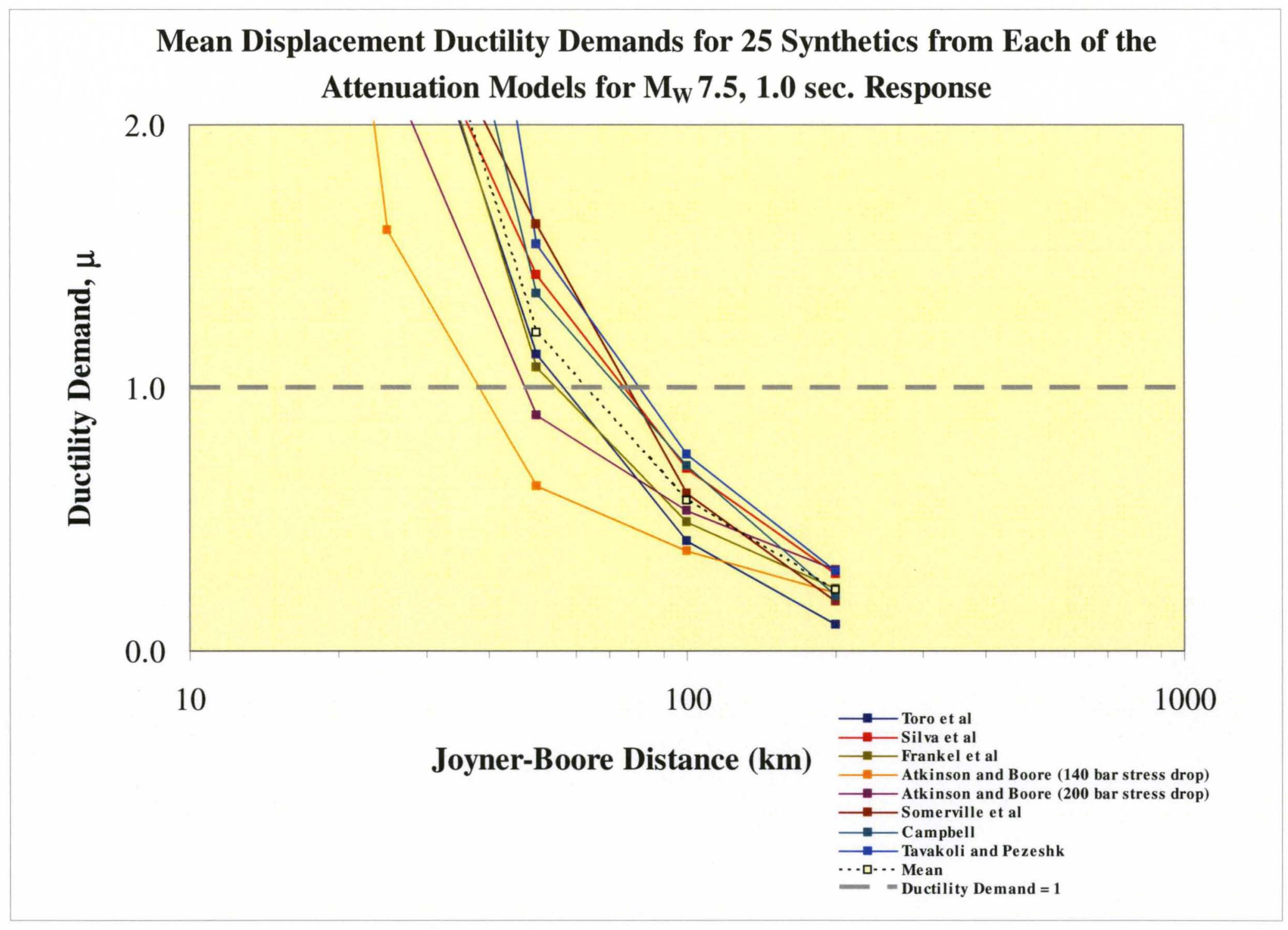




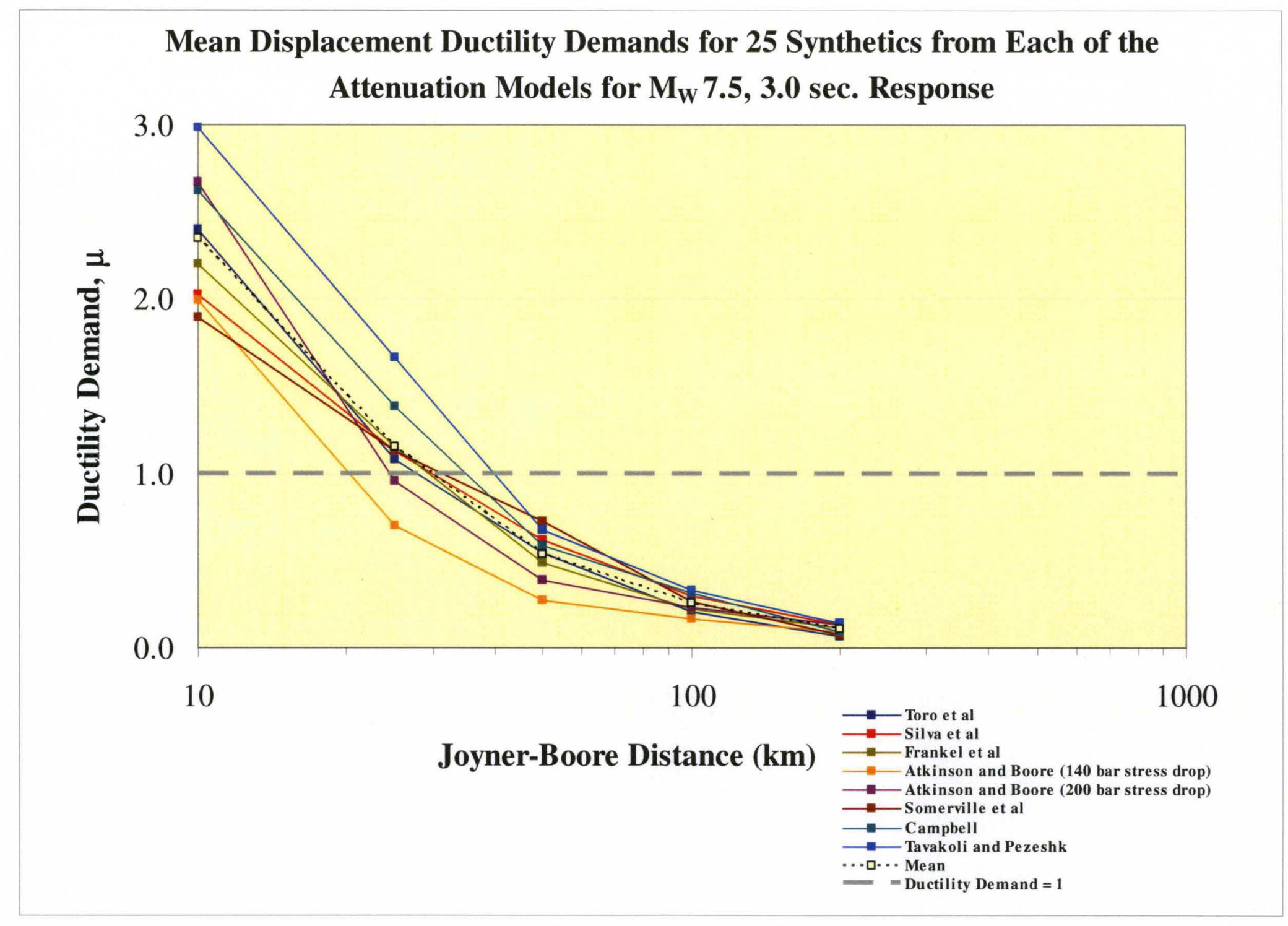




\section{APPENDIX E}

This appendix contains 2-dimensional surface plots showing the trends in the standard deviations of the displacement ductility demands for each moment magnitude as a function of natural period and distance. It also contains the same surface plots for trends in the coefficient of variation at each moment magnitude taken to be as the ratio of the standard deviation to the mean. Finally, this appendix also contains these same surface plots showing the trends in the mean displacement ductility demands at each moment magnitude as a function of natural period and distance. 


\section{Trends in the Means of Ductility Demands from a $\mathrm{M}_{\mathrm{W}} \mathbf{5 . 5}$}

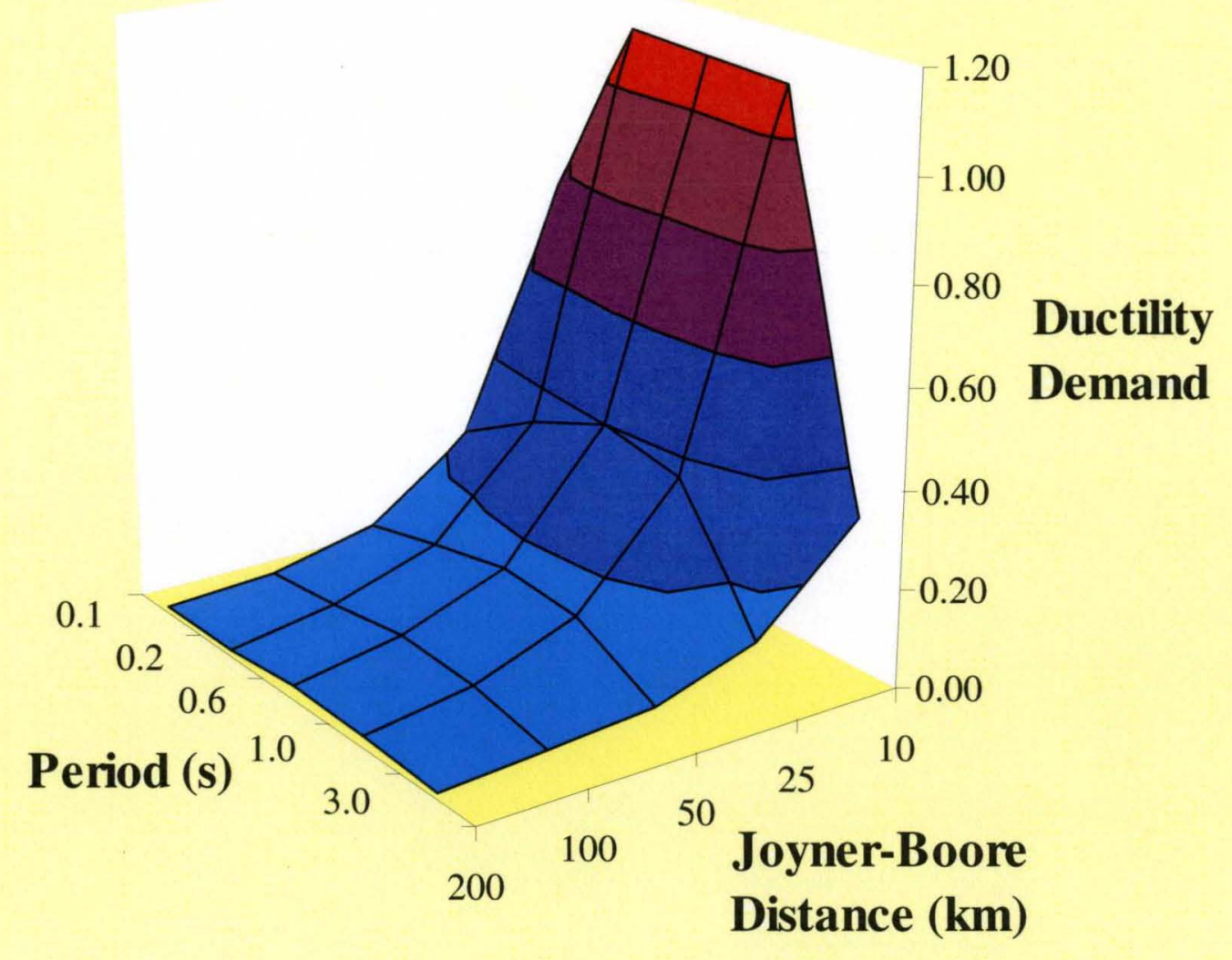




\section{Trends in Standard Deviation for Ductility Demands from a $M_{W} 5.5$}

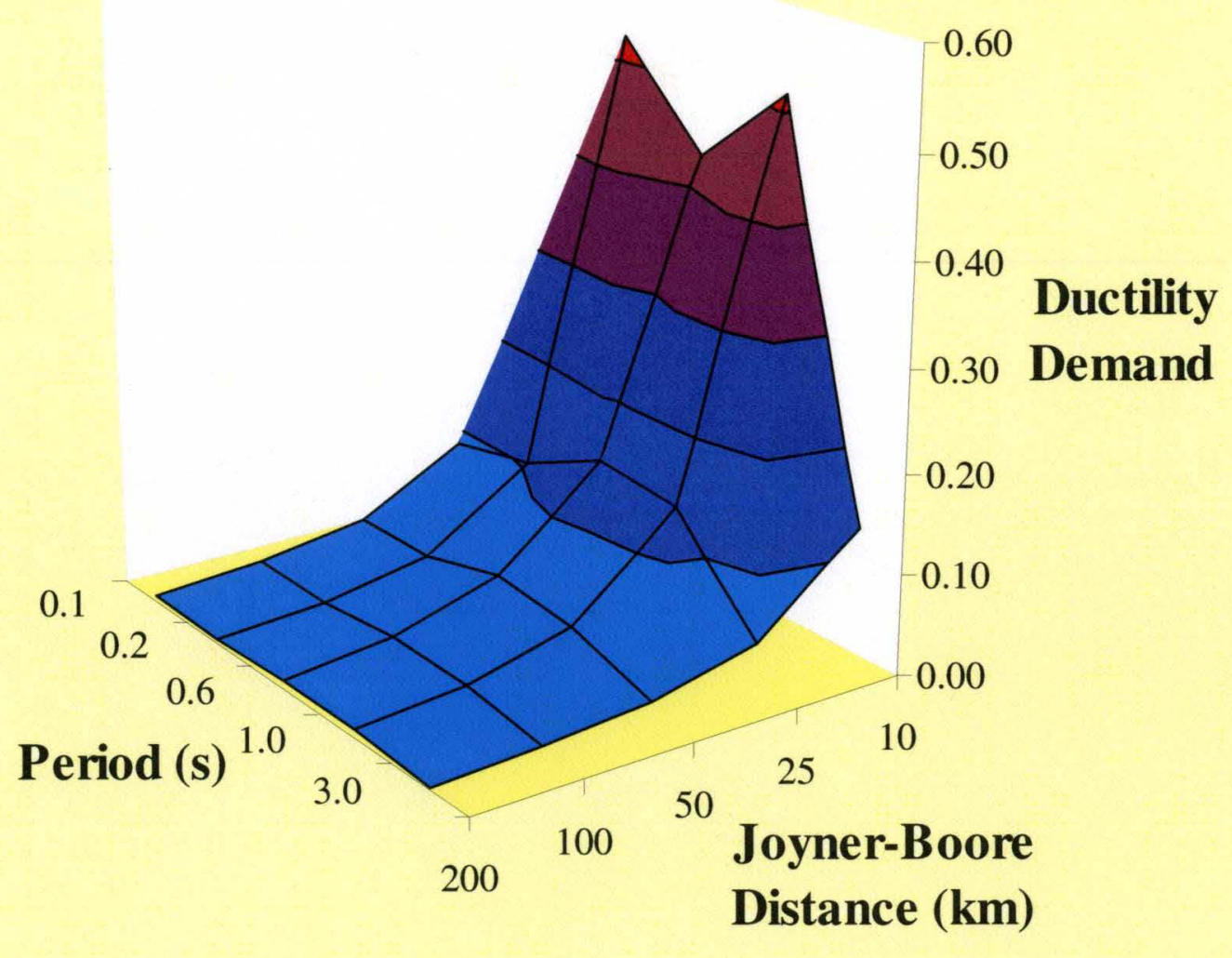




\section{Trends in Coefficient of Variation for Ductility Demands from a $\mathrm{M}_{\mathrm{W}} \mathbf{5 . 5}$}

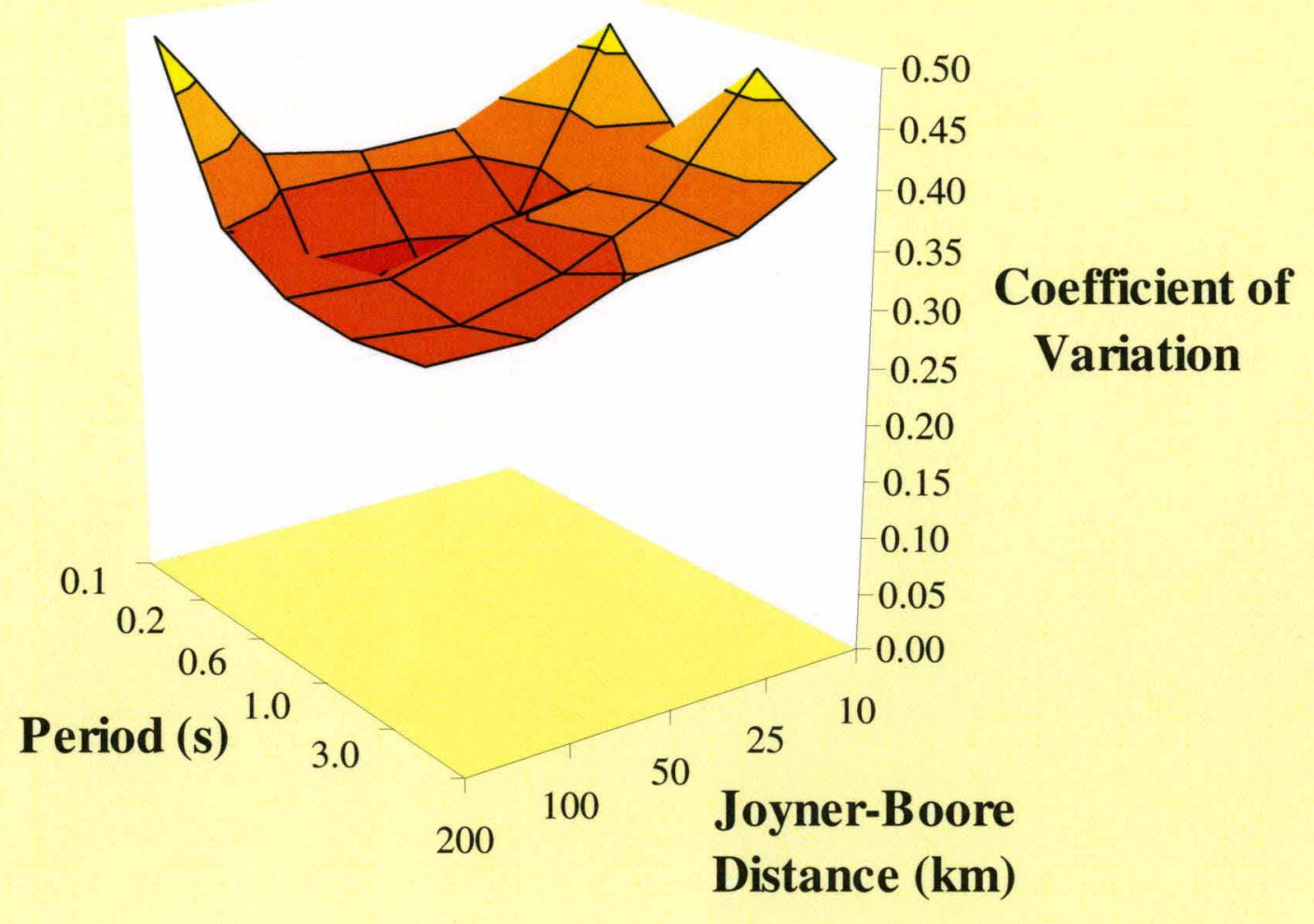




\section{Trends in the Means of Ductility Demands from a $M_{W} 6.0$}

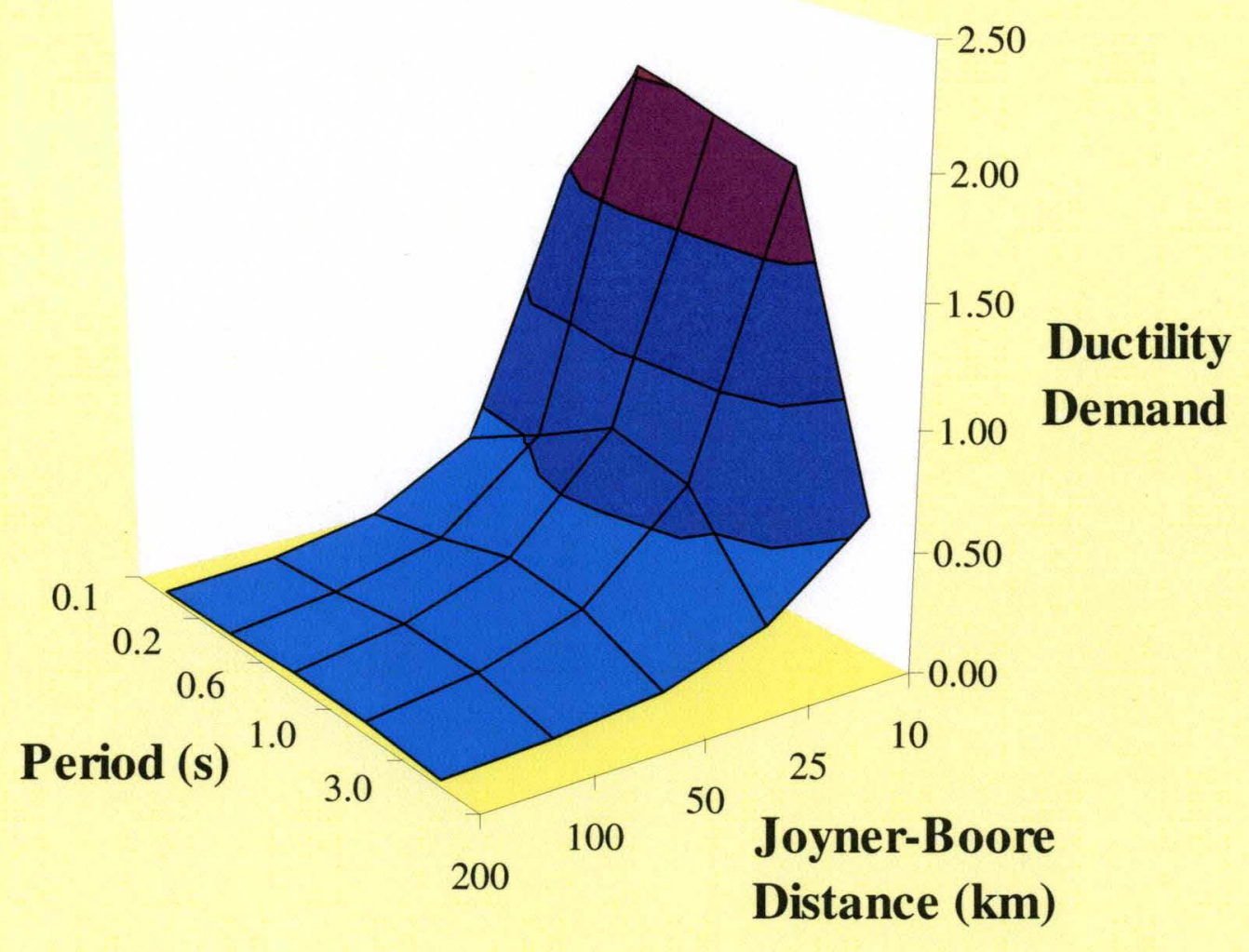




\section{Trends in Standard Deviation for Ductility Demands from a $M_{W} 6.0$}

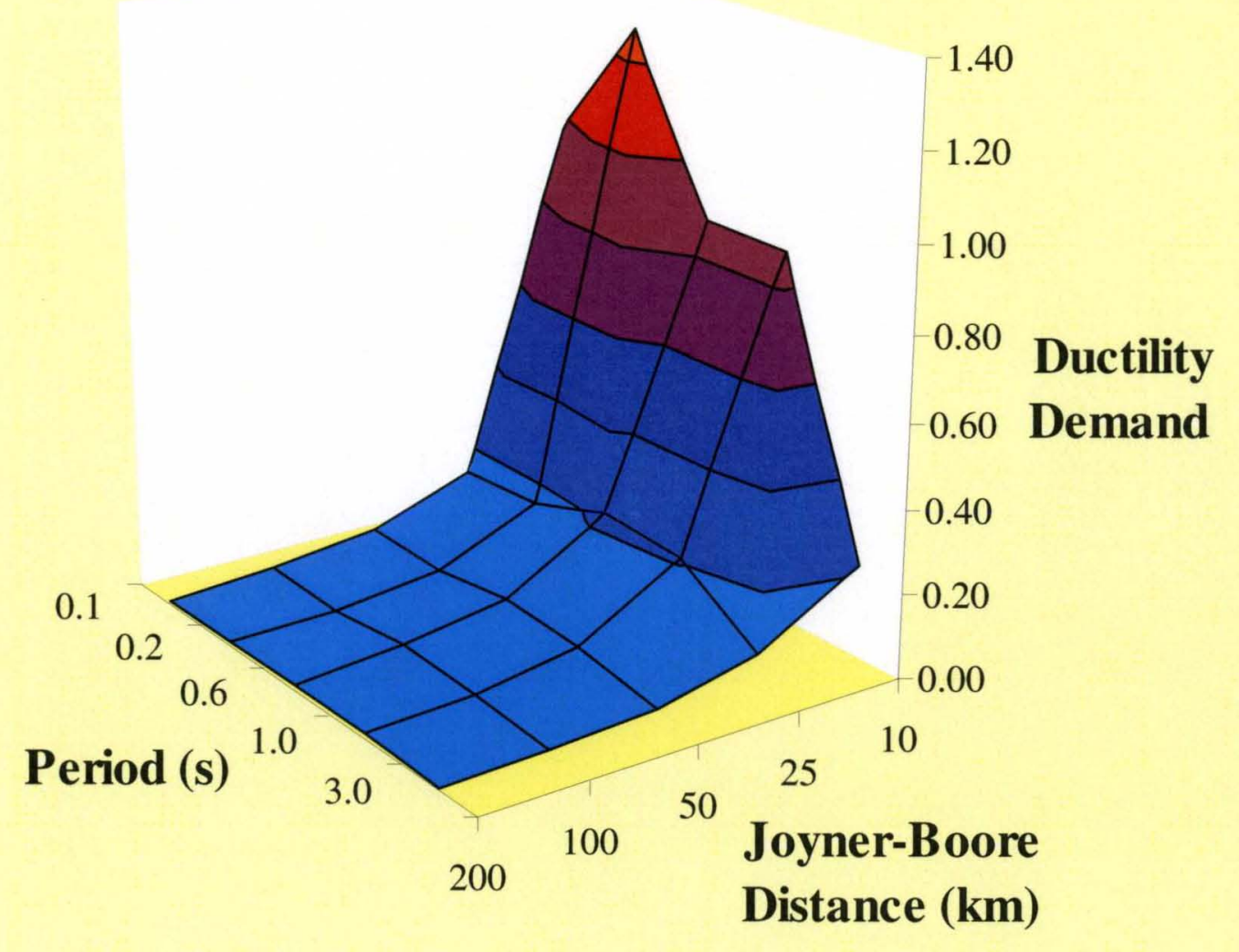




\section{Trends in Coefficient of Variation for Ductility Demands from a $M_{W} 6.0$}

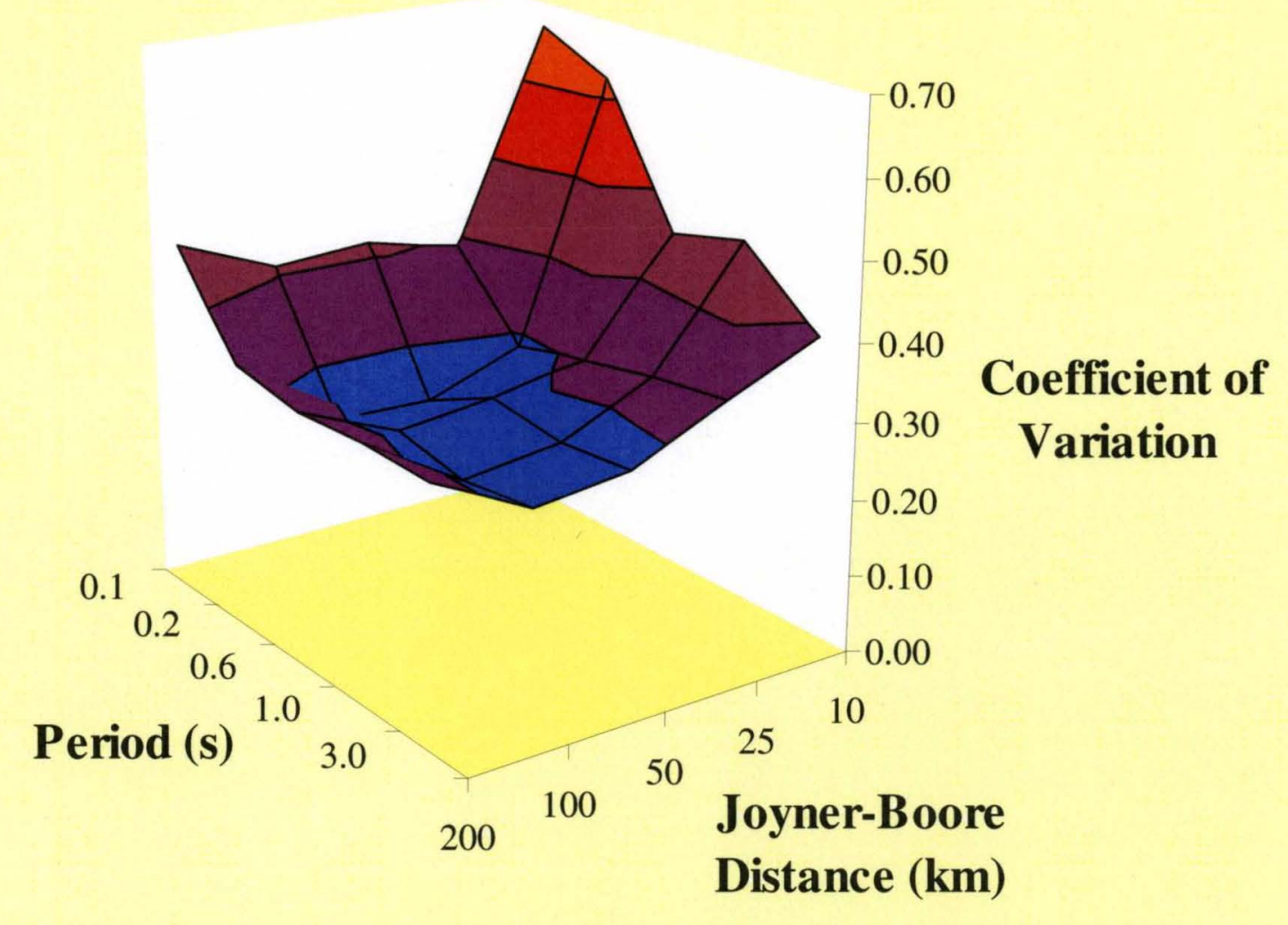




\section{Trends in the Means of Ductility Demands from a $M_{W} 6.5$}

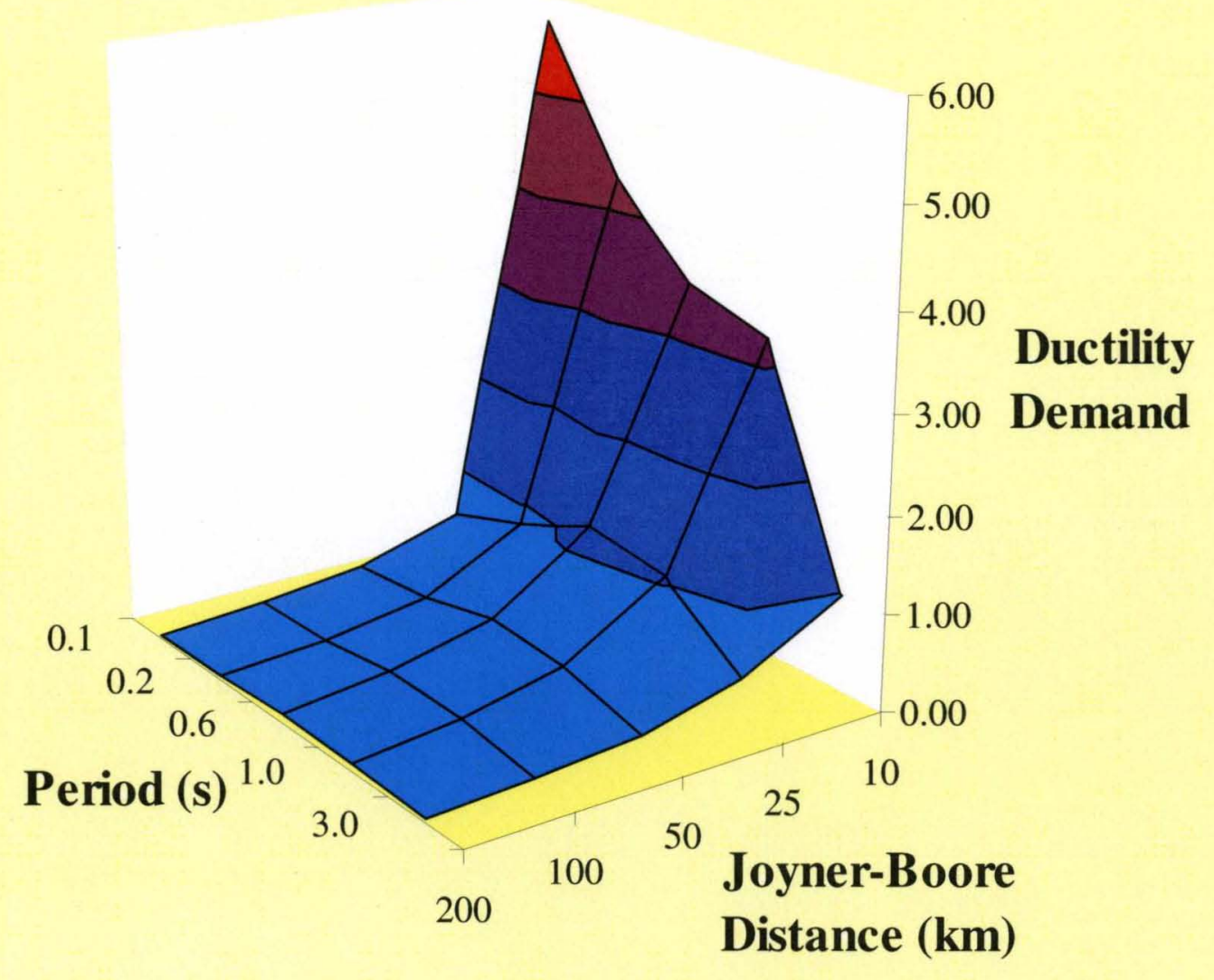




\section{Trends in Standard Deviation for Ductility Demands from a $M_{W} 6.5$}

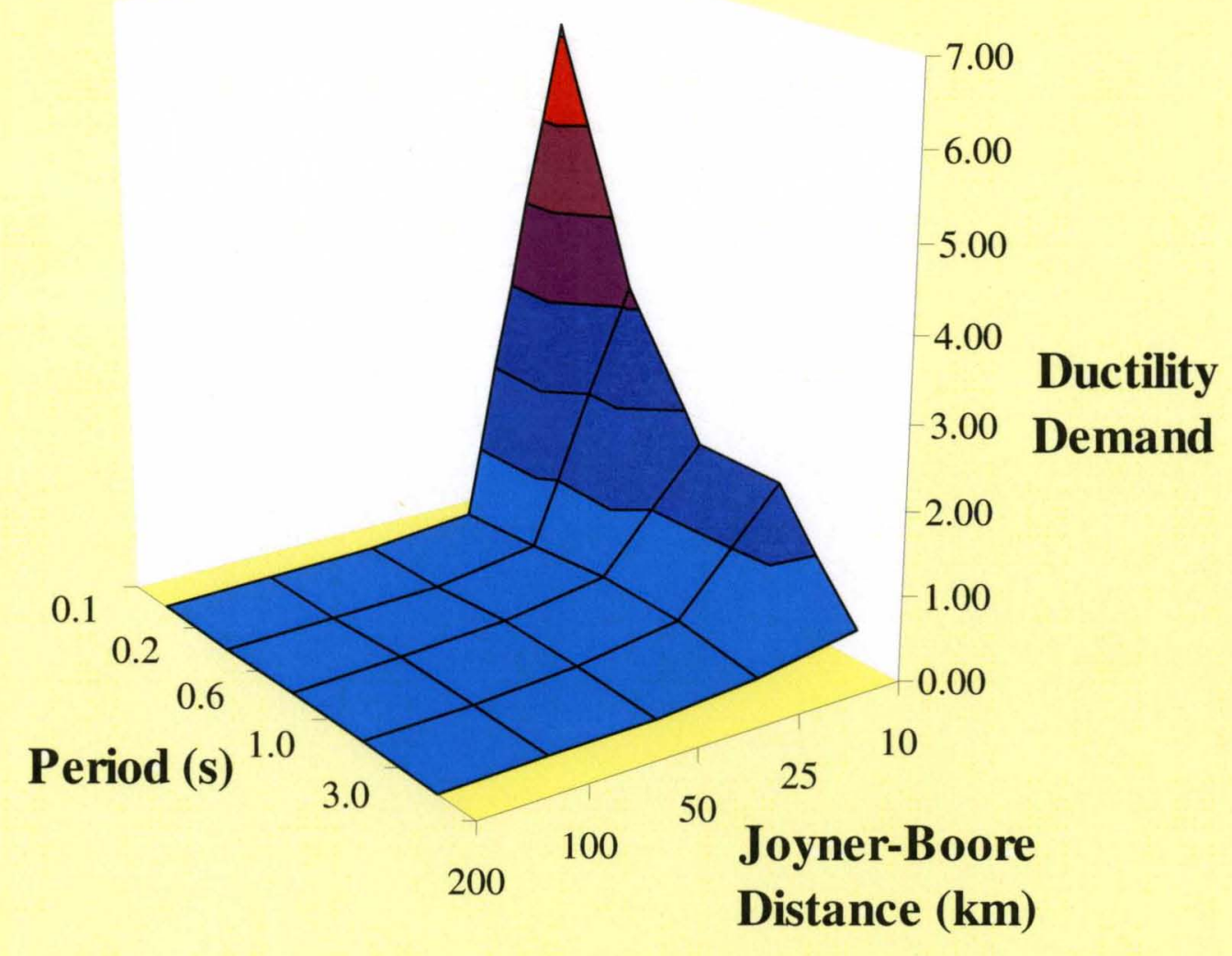




\section{Trends in Coeffecient of Variation for Ductility Demands from a $M_{W} 6.5$}

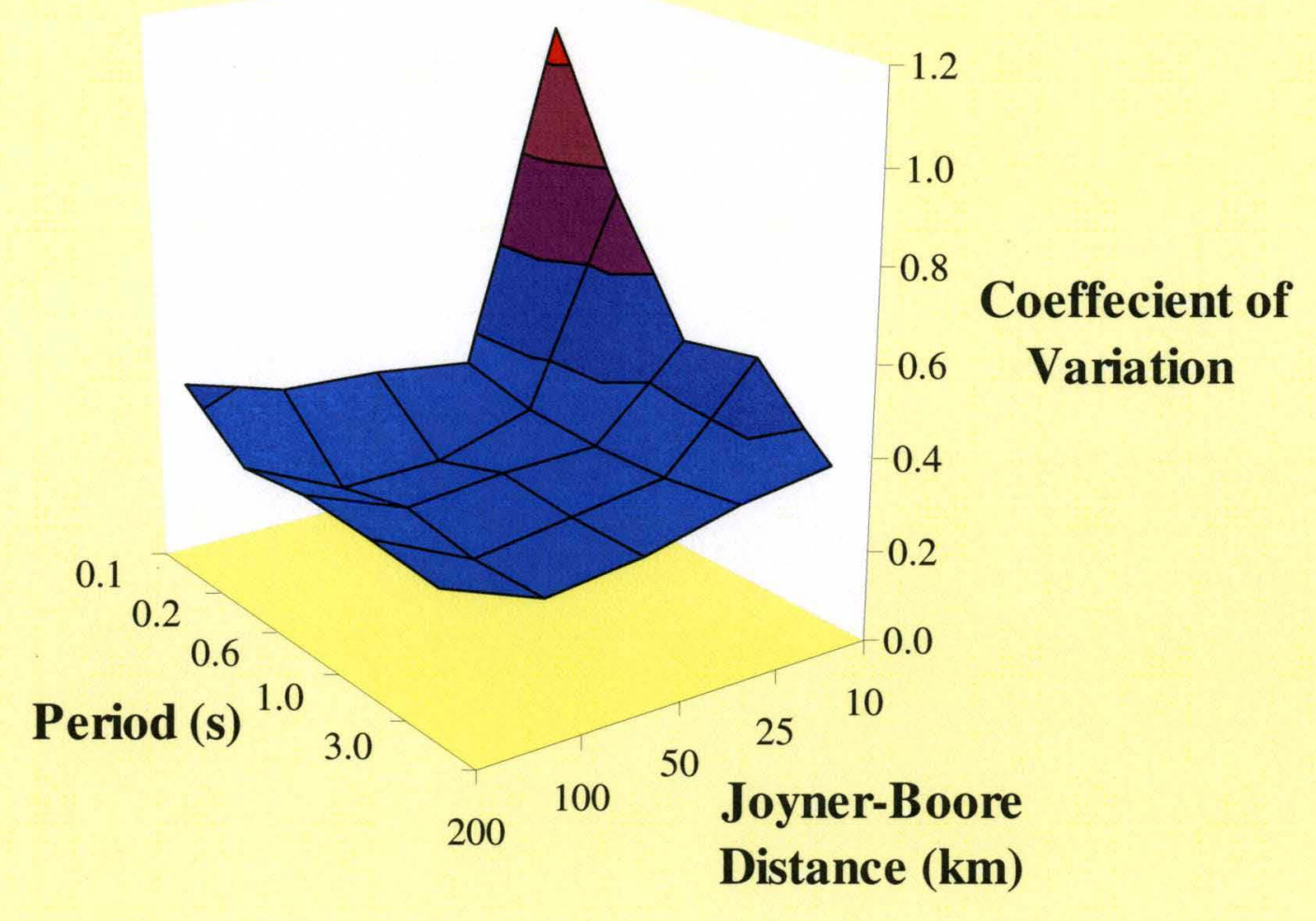




\section{Trends in the Means of Ductility Demands from a $M_{W} 7.0$}

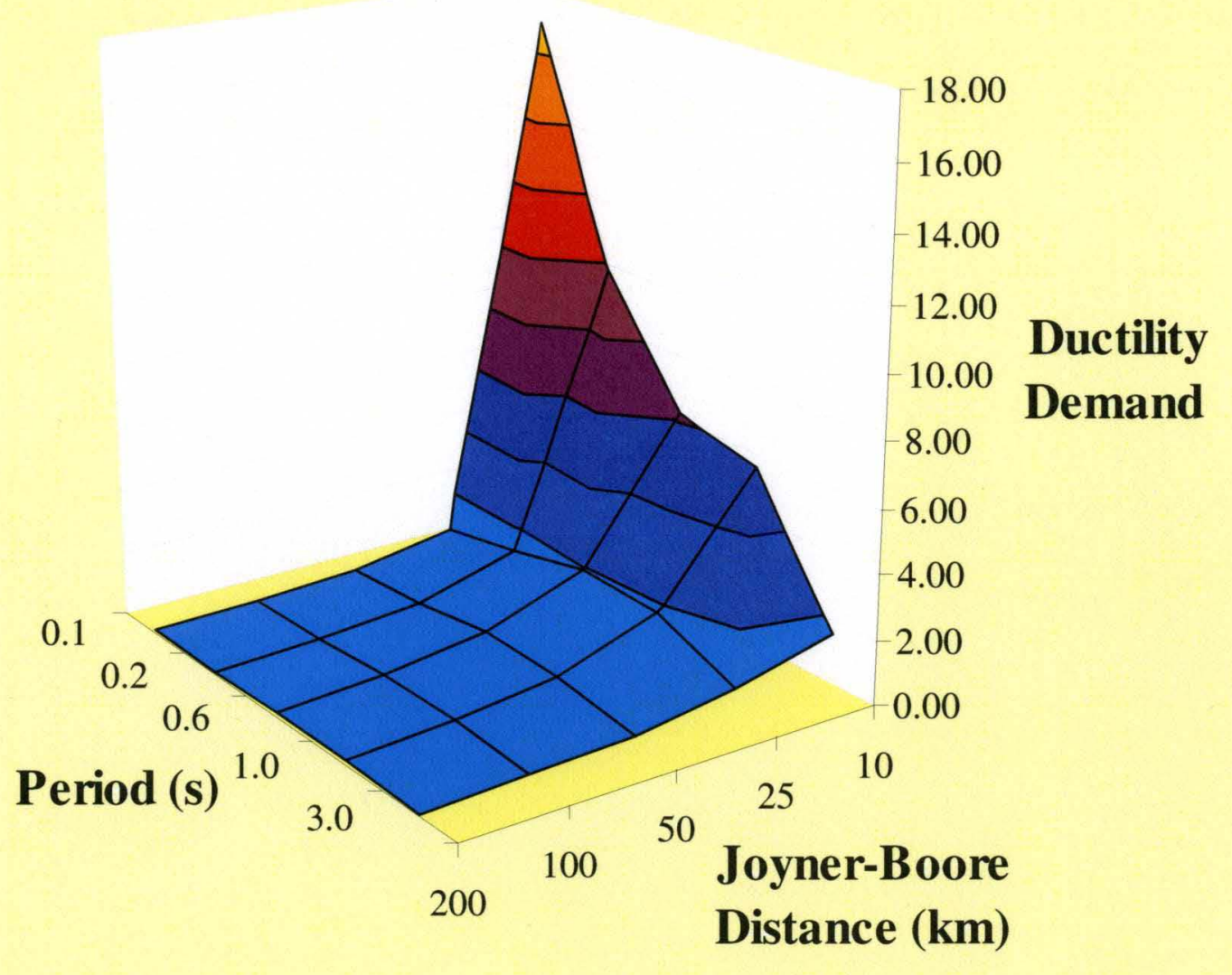




\section{Trends in Standard Deviation for Ductility Demands from a $M_{W}$ 7.0}

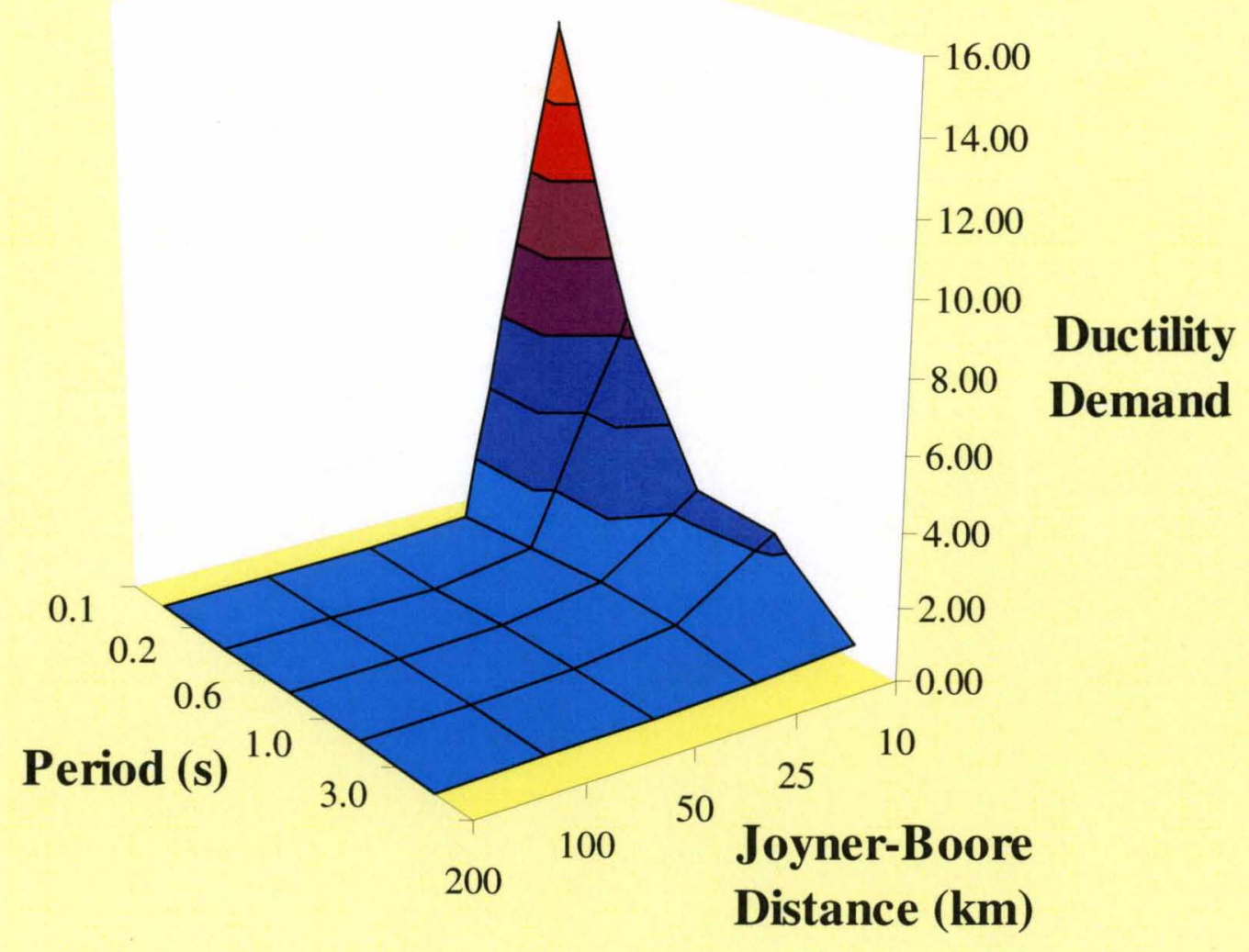




\section{Trends in Coefficient of Variation for Ductility Demands from a $M_{W}$ 7.0}

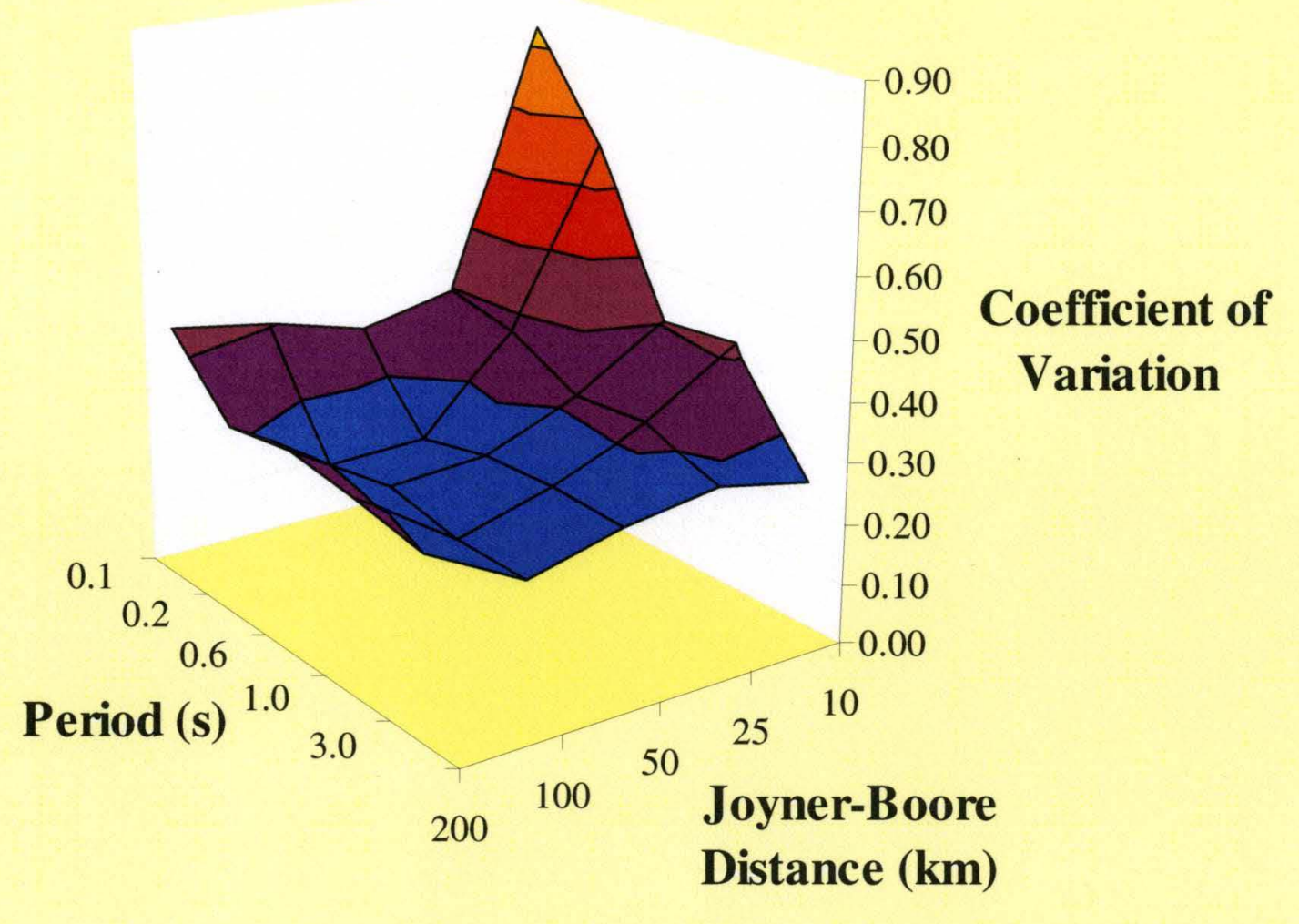




\section{Trends in the Means of Ductility Demands from a $M_{W} 7.5$}

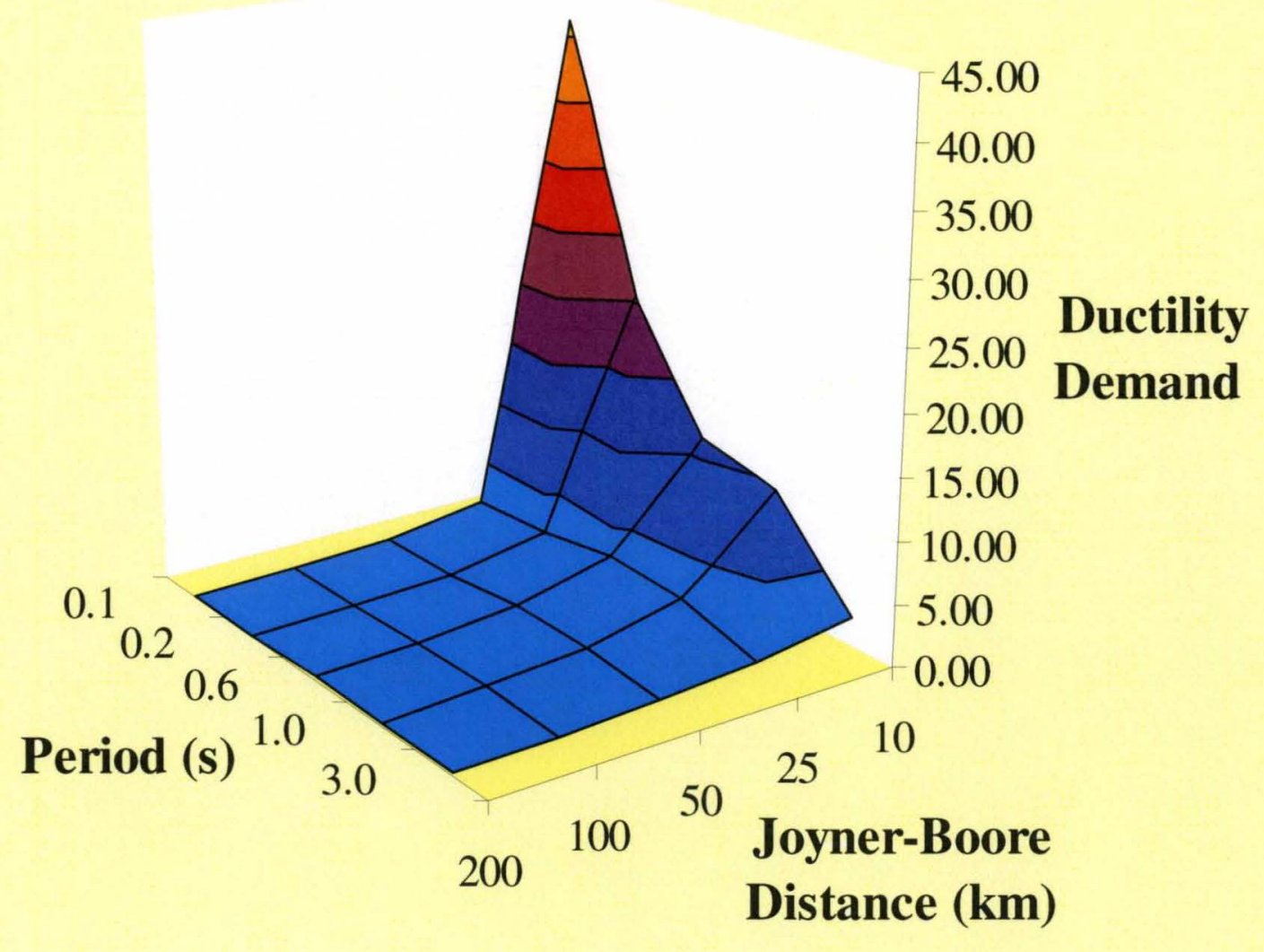




\section{Trends in Standard Deviation for Ductility Demands from a $M_{W} 7.5$}

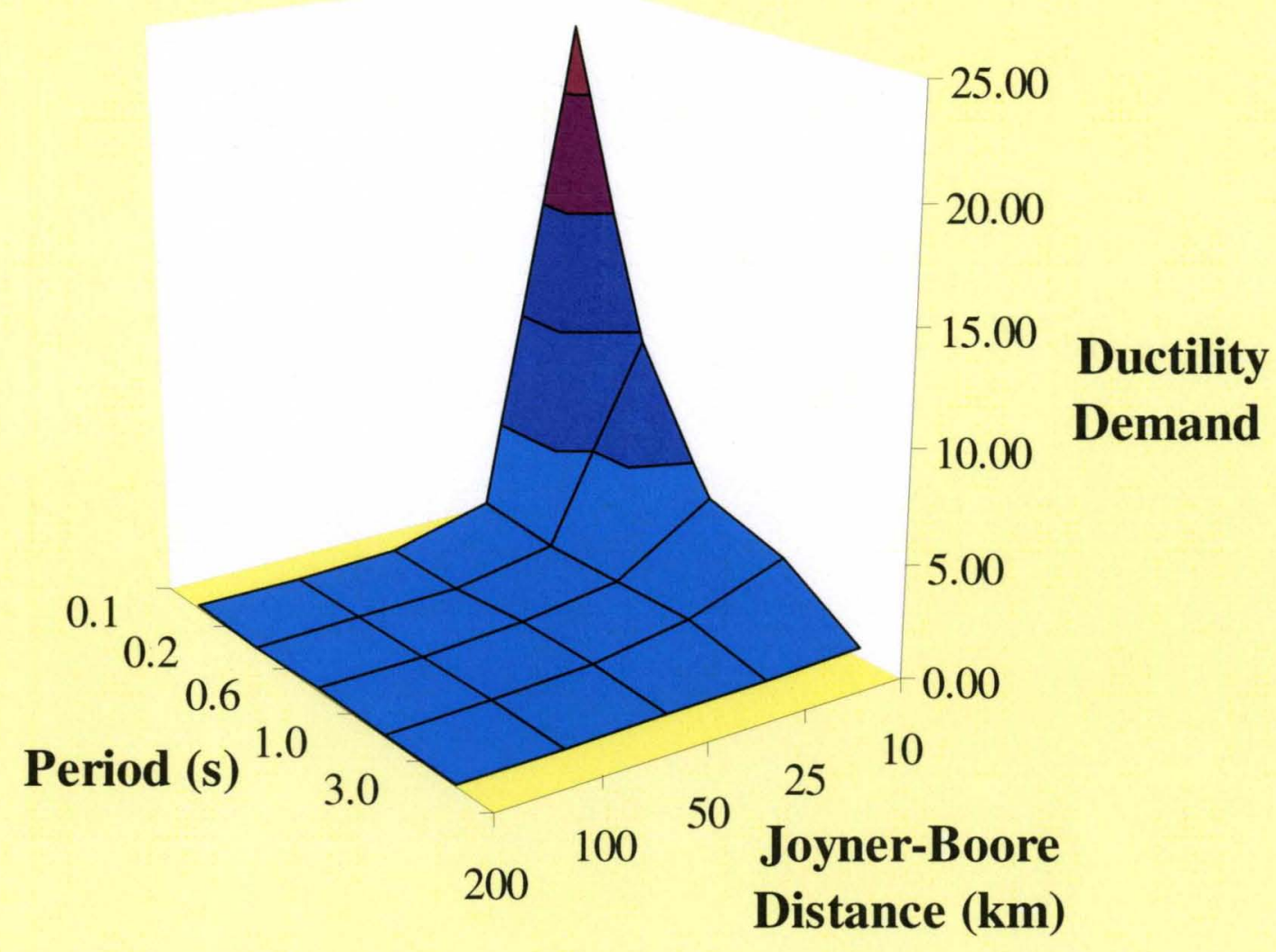




\section{Trends in Coefficient of Variation for Ductility Demands from a $M_{W} 7.5$}

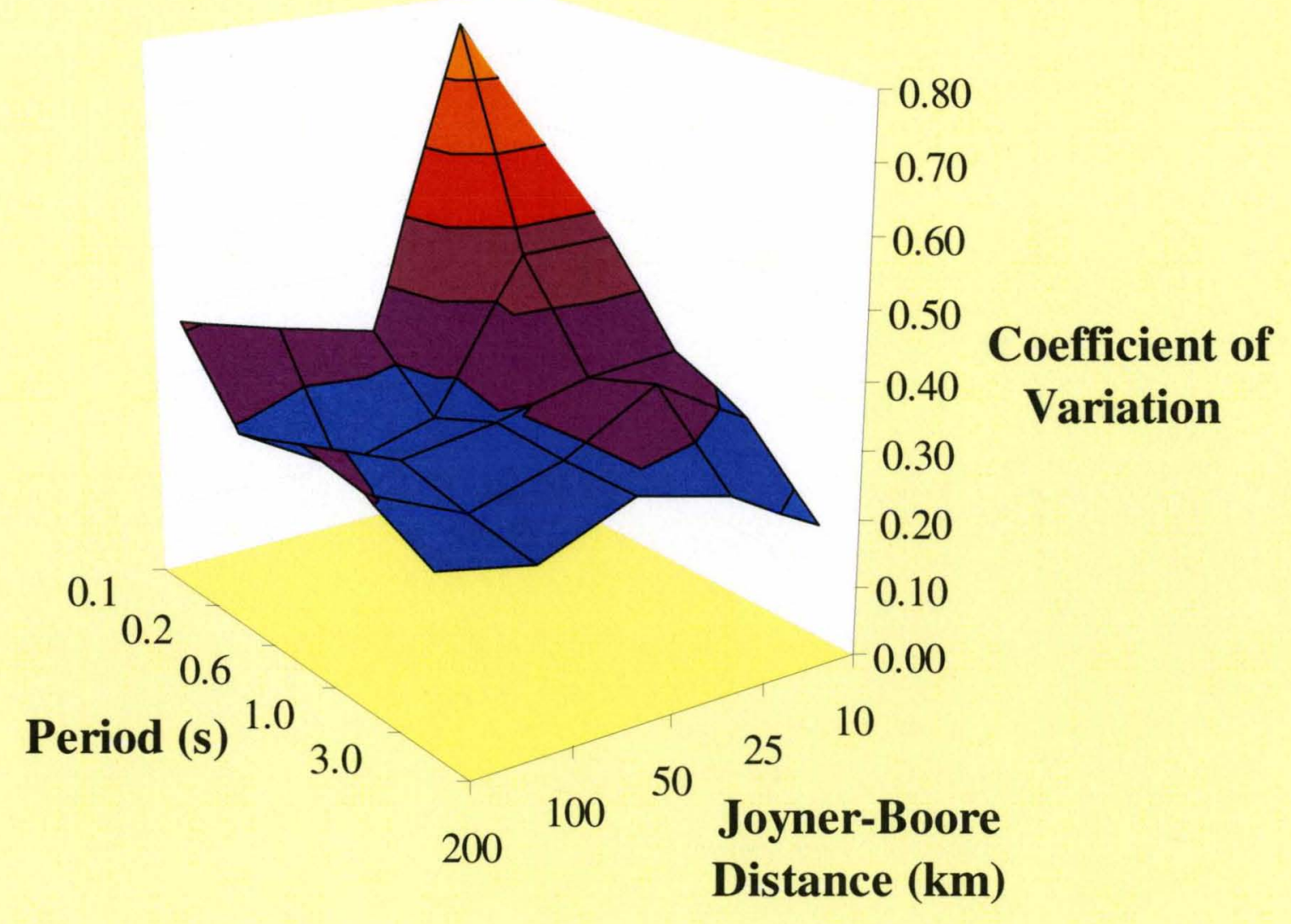




\section{APPENDIX F}

This appendix provides example plots showing the displacement ductility demands for the magnitude 7.0 at $10 \mathrm{~km}$ and $25 \mathrm{~km}$ scenarios as determined from the initial final study using a discretization interval of 0.01 seconds in generating the synthetic records. Note the relatively benign response despite this large event at close distances. The entire study was repeated using a discretization interval of 0.02 seconds producing more realistic displacement ductility demands which are provided as the result for the final study. Also shown are comparisons of the mean for synthetics from the two attenuation models that produced the highest and lowest ductility demands for this scenario. The mean spectra from these attenuation models are shown compared to their target spectra for the 7.0 at $25 \mathrm{~km}$ scenario. Note that the mean spectra of the synthetic records are below their targets. 


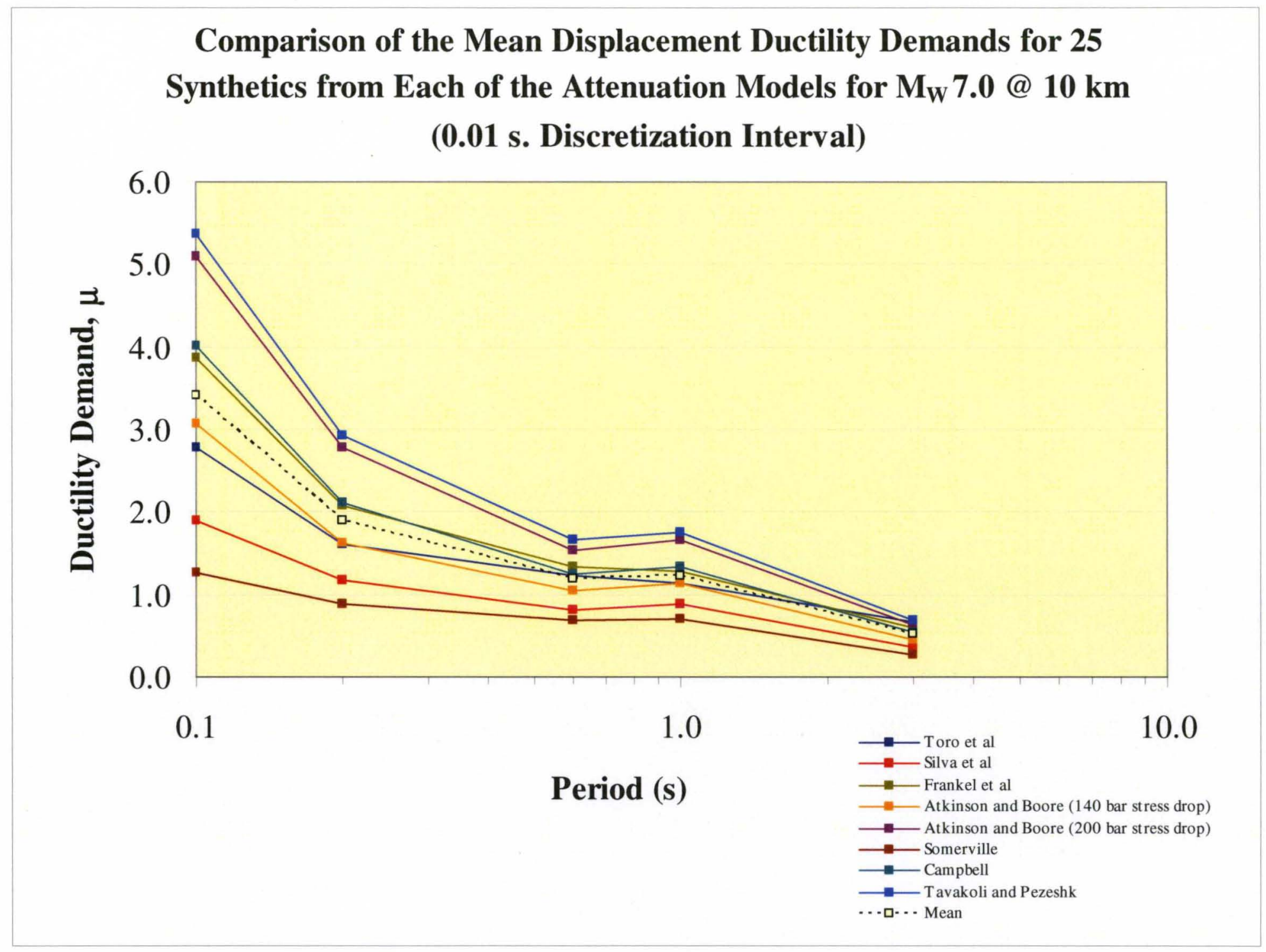




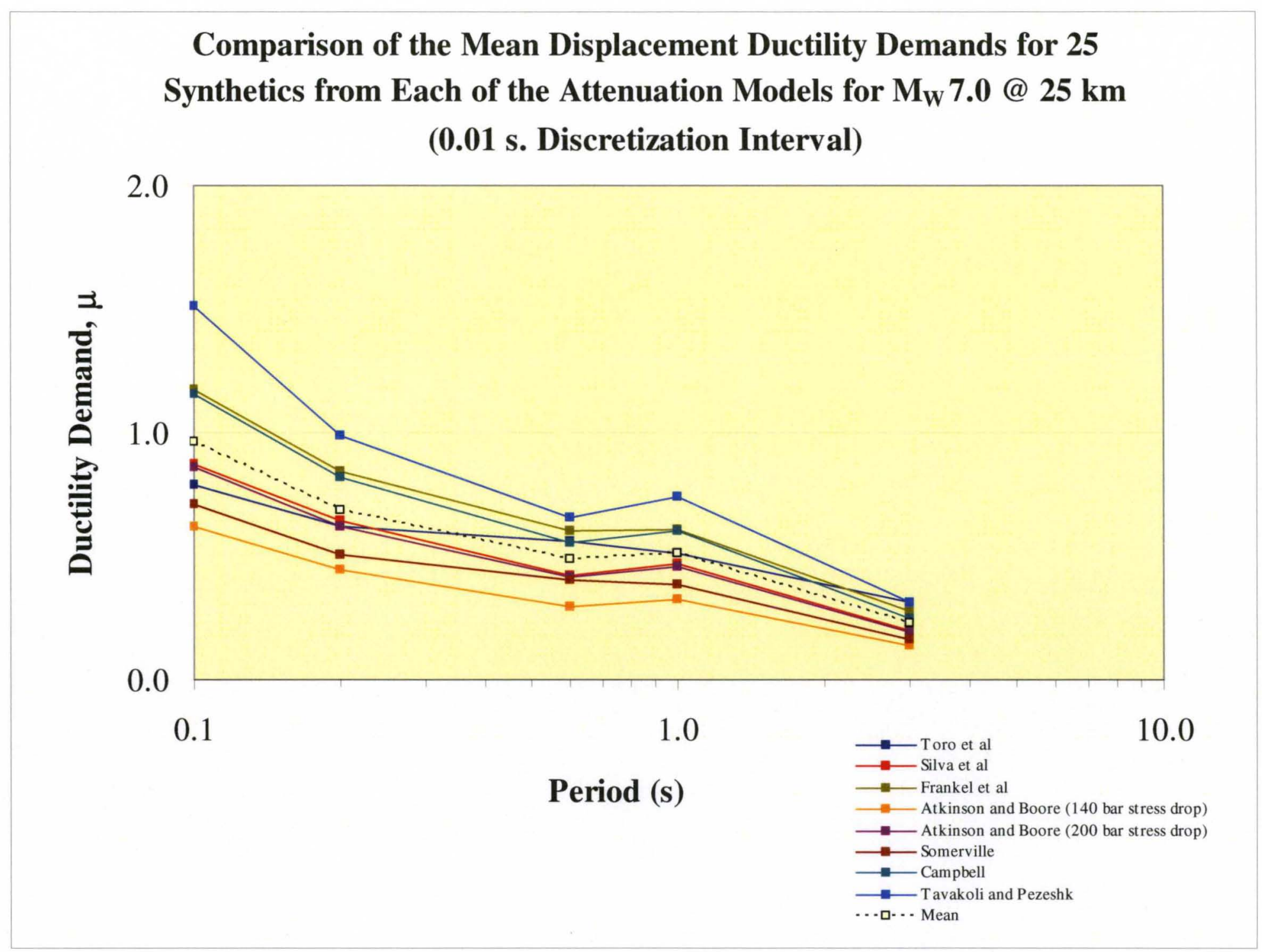




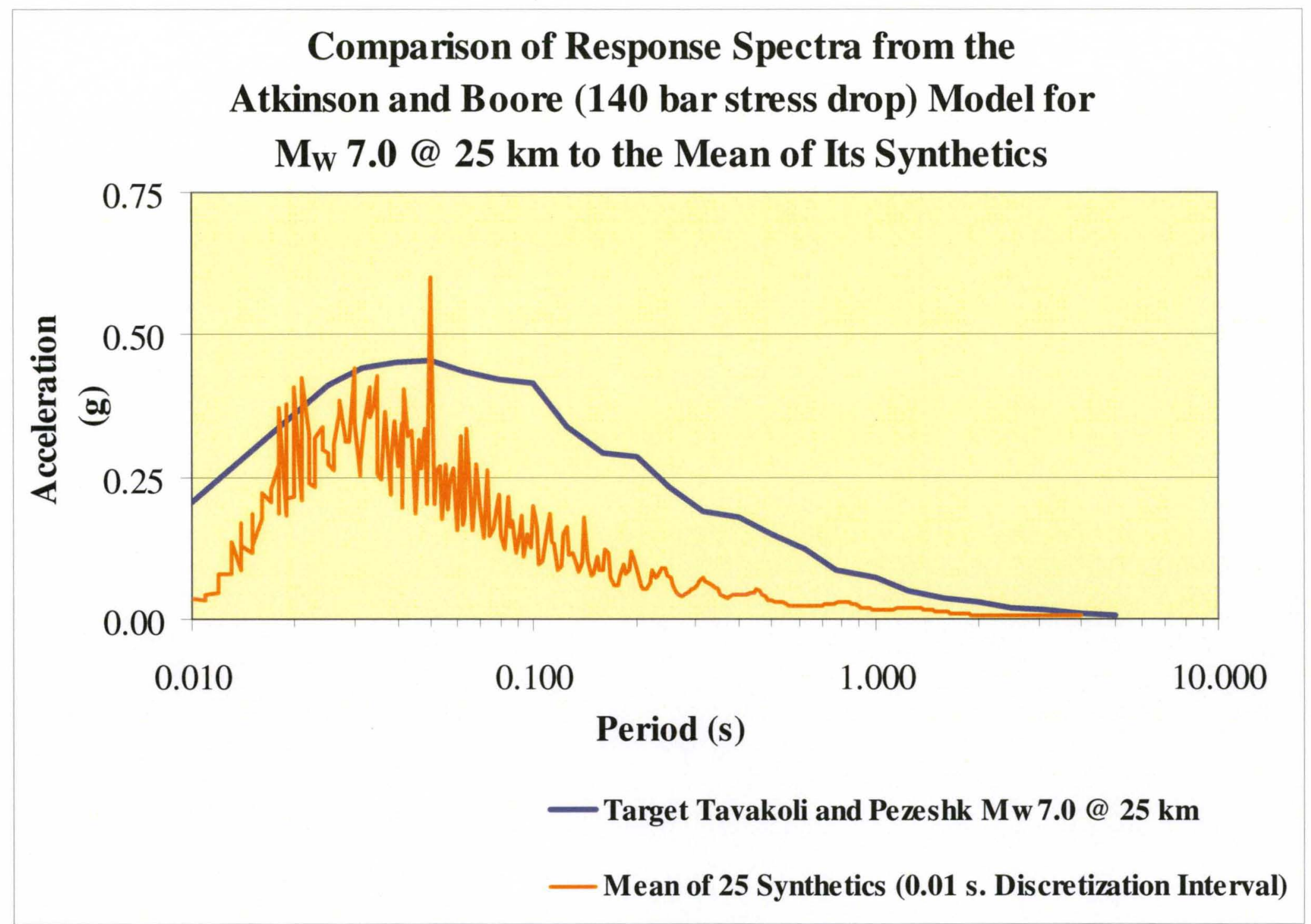




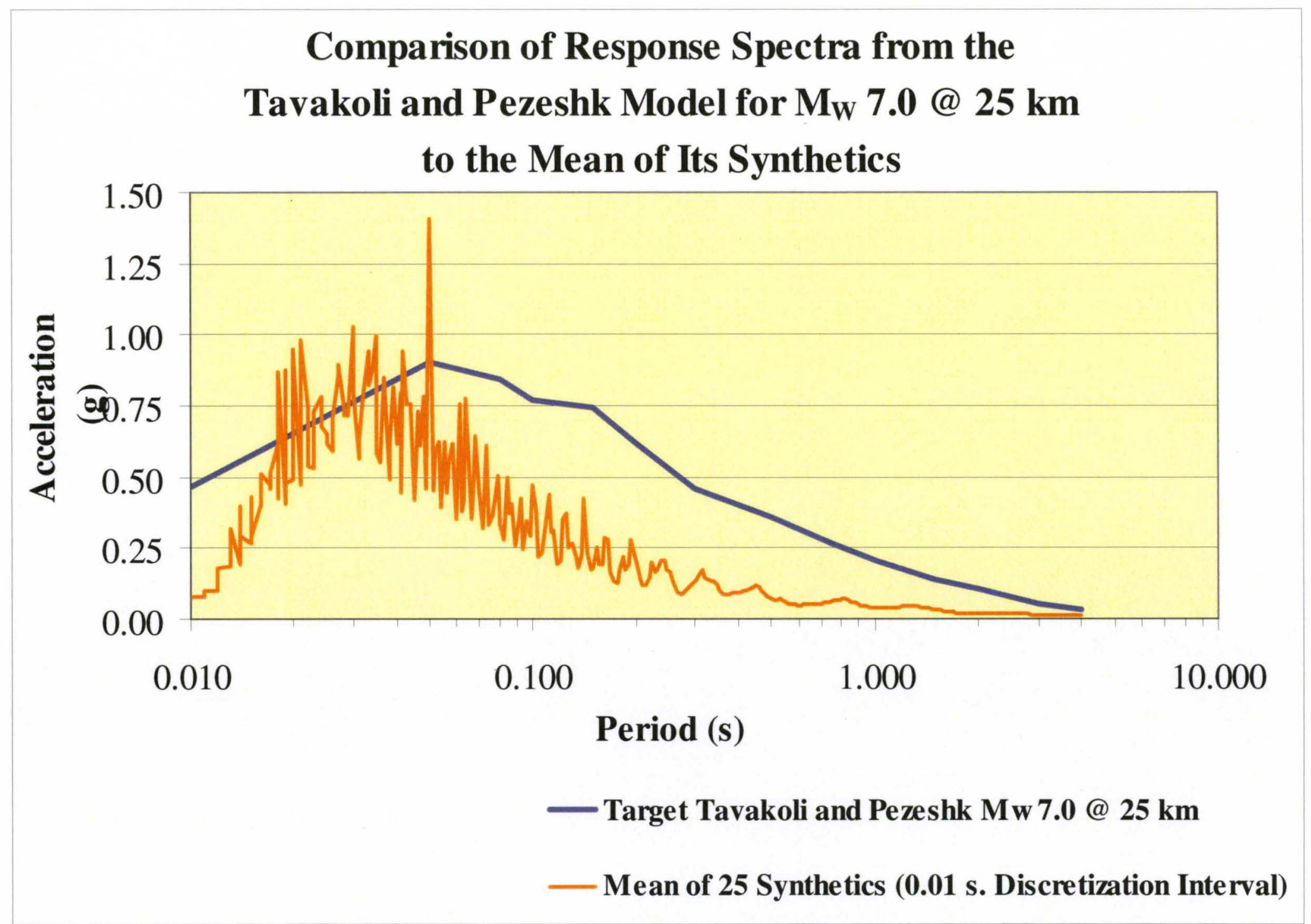




\section{APPENDIX G}

This appendix contains plots showing the remaining spectra comparisons for the actual records used in the pilot study to the mean of the synthetics generated from them. This appendix also contains plots showing the ductility demands for the five SDOFs from the actual records along with mean displacement ductility demands from their respective synthetics. The plots also include the individual ductility demands for each synthetic indicating the variation in the displacement ductility demand among the twenty five replicates. Finally, this appendix contains a plot showing the dispersion of the estimated moment magnitudes for the synthetic ground motions developed from the actual events used in the pilot study compared to their respective target magnitudes estimated from the actual records. 


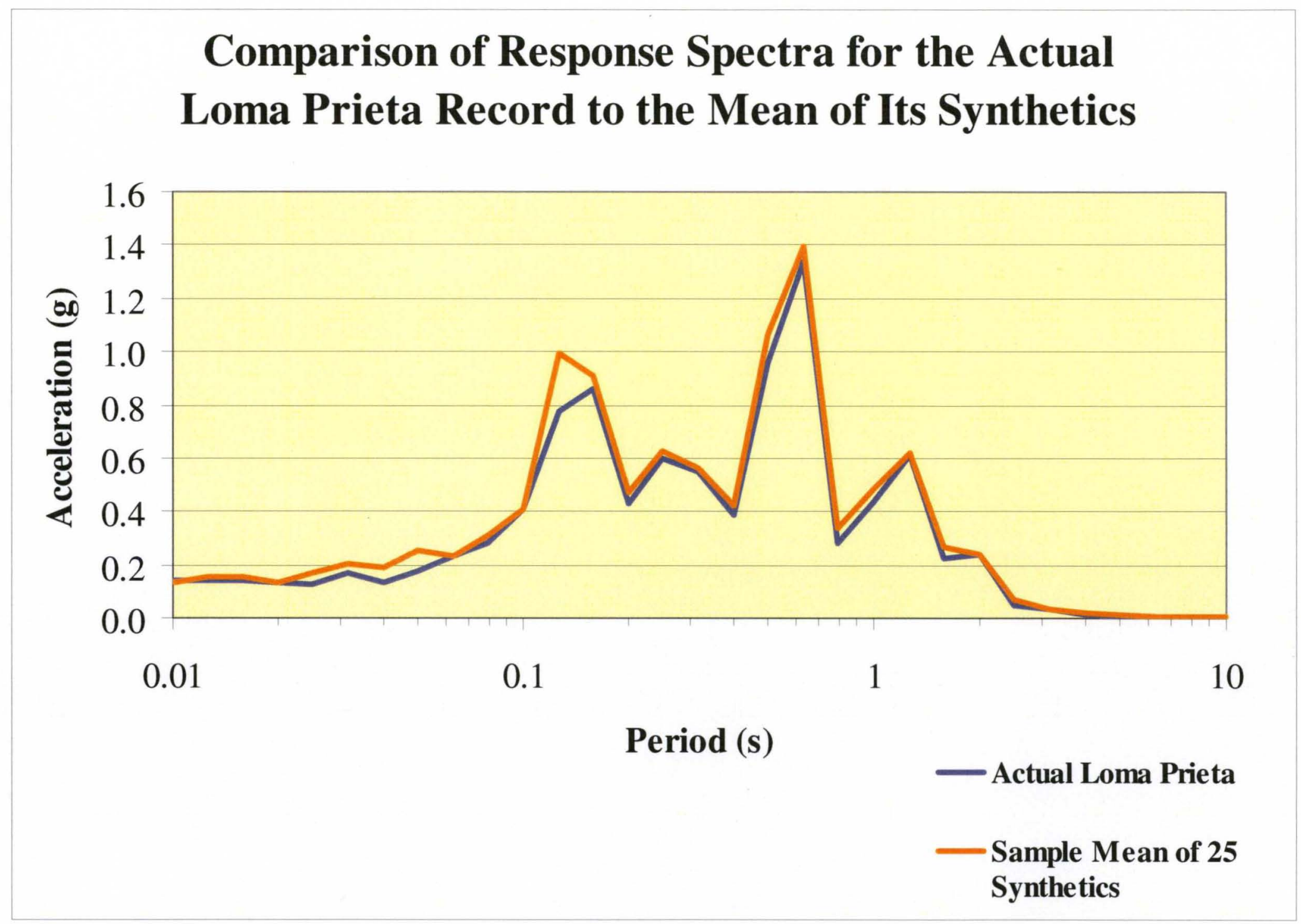


Comparison of Response Spectra for the Actual NE Ohio Record to the Mean of Its Synthetics

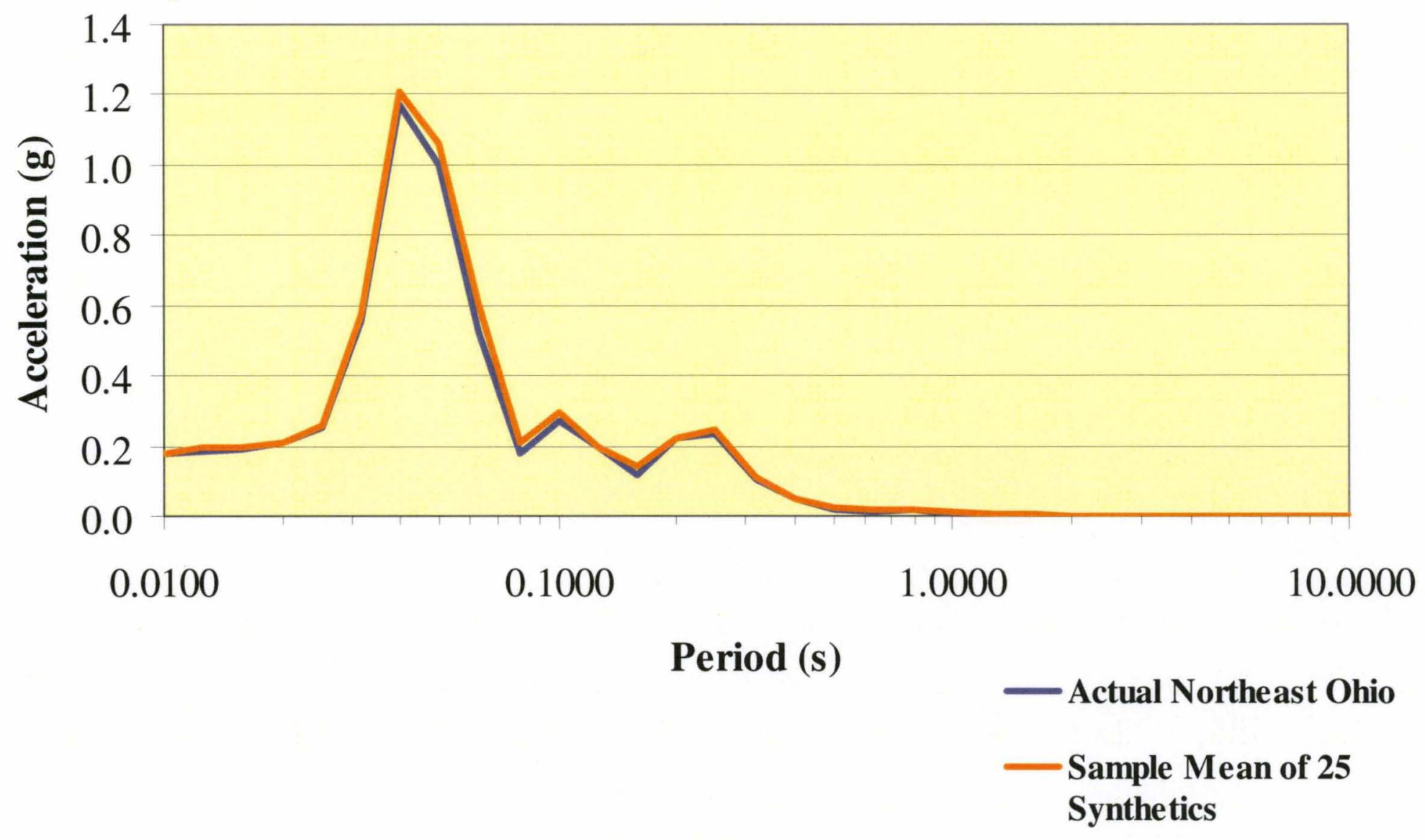




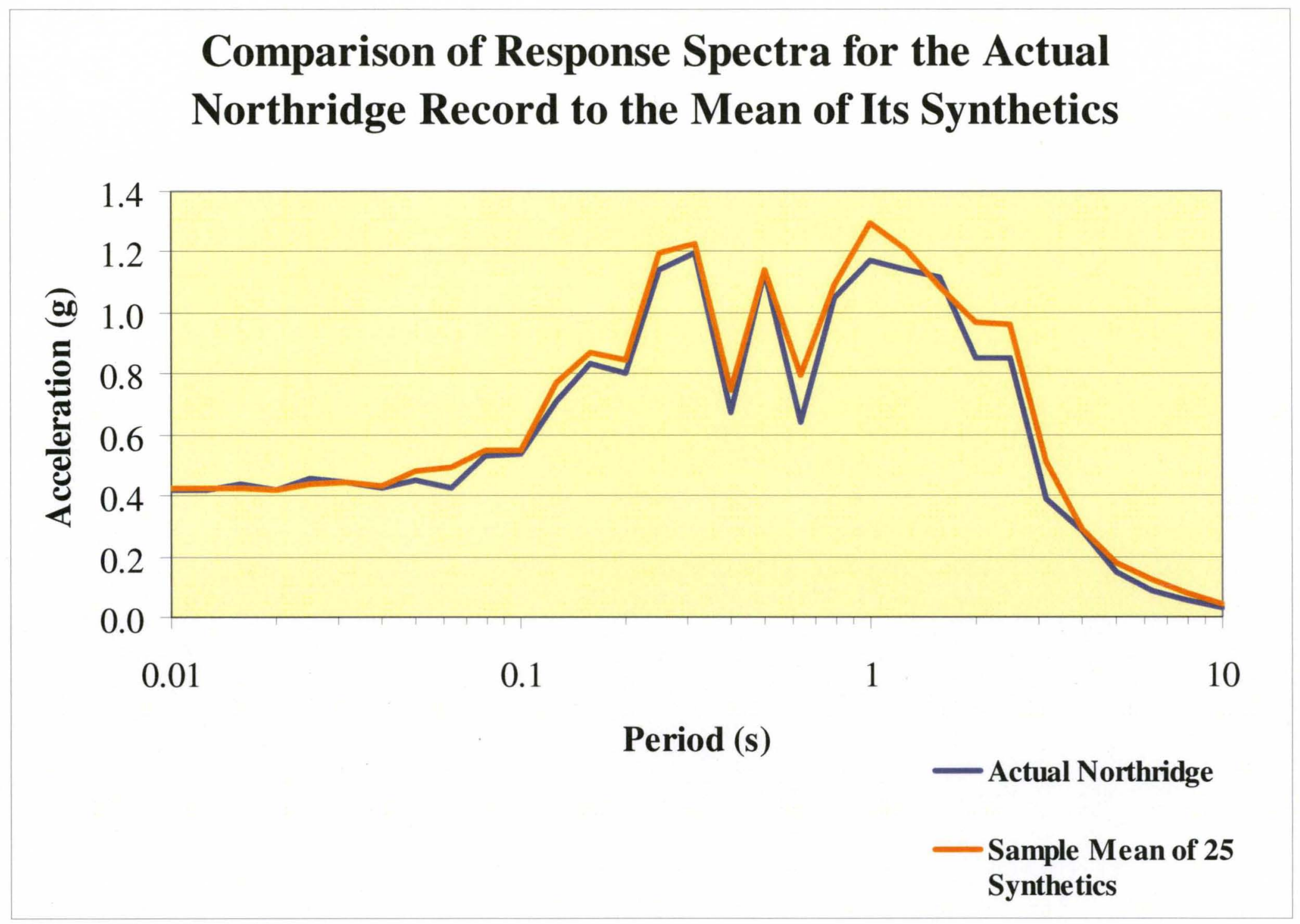


Comparison of Response Spectra for the Actual Saguenay Record to the Mean of Its Synthetics

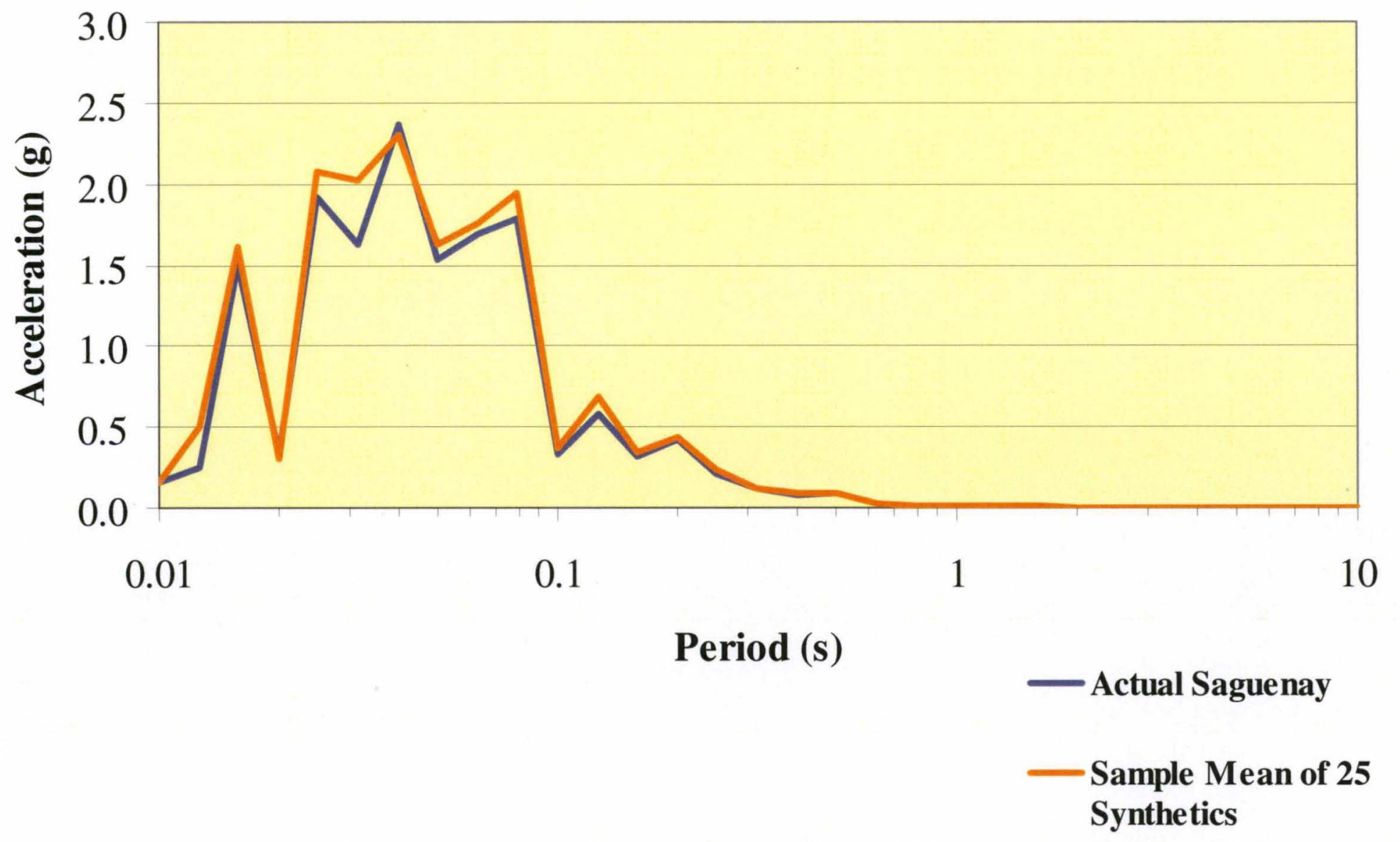


Comparison of Displacement Ductility Demands from the Actual El Centro Record and the Means of Its Synthetics

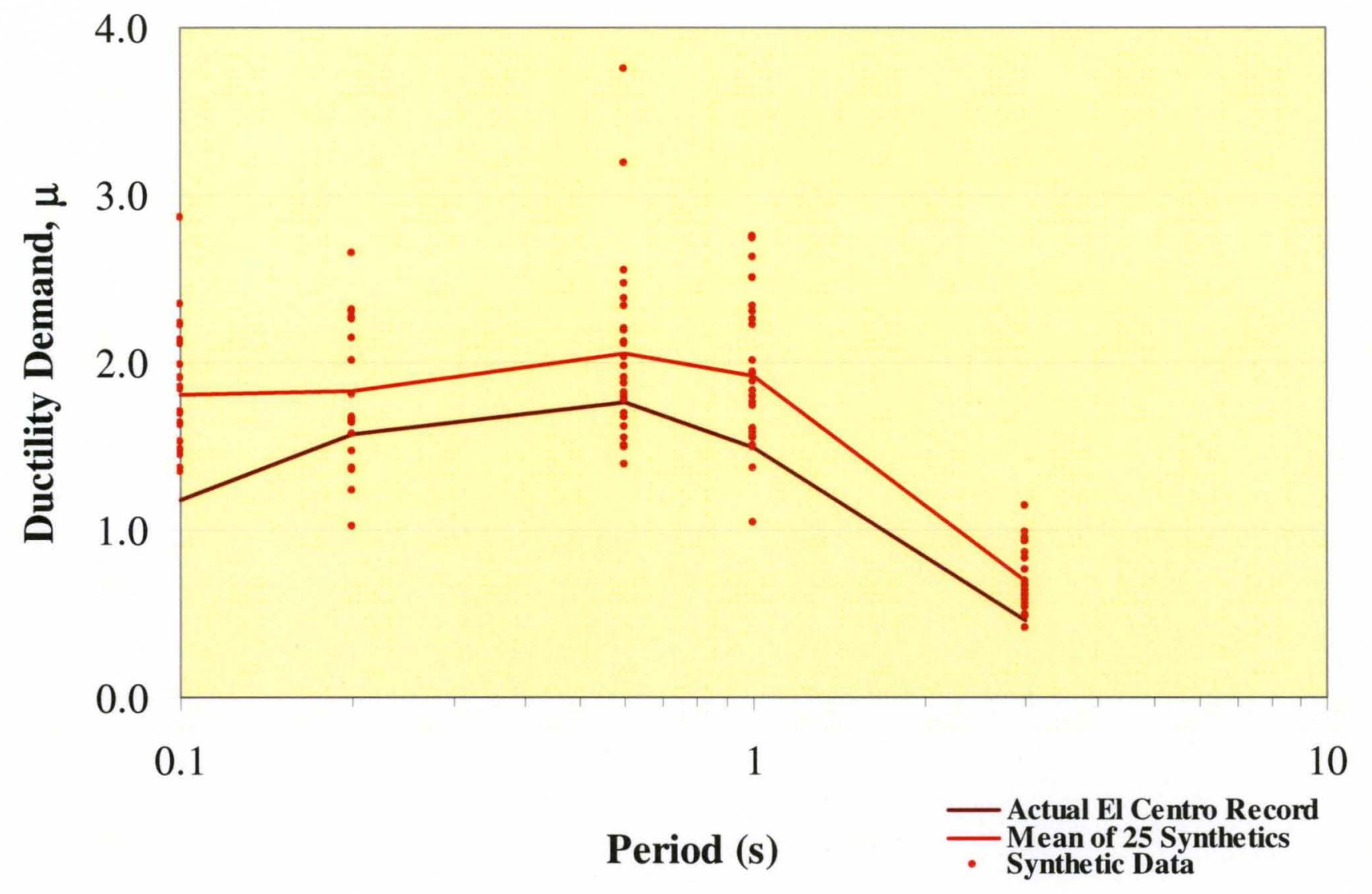


Comparison of Displacement Ductility Demands from the Actual Loma Prieta Record and the Means of Its Synthetics

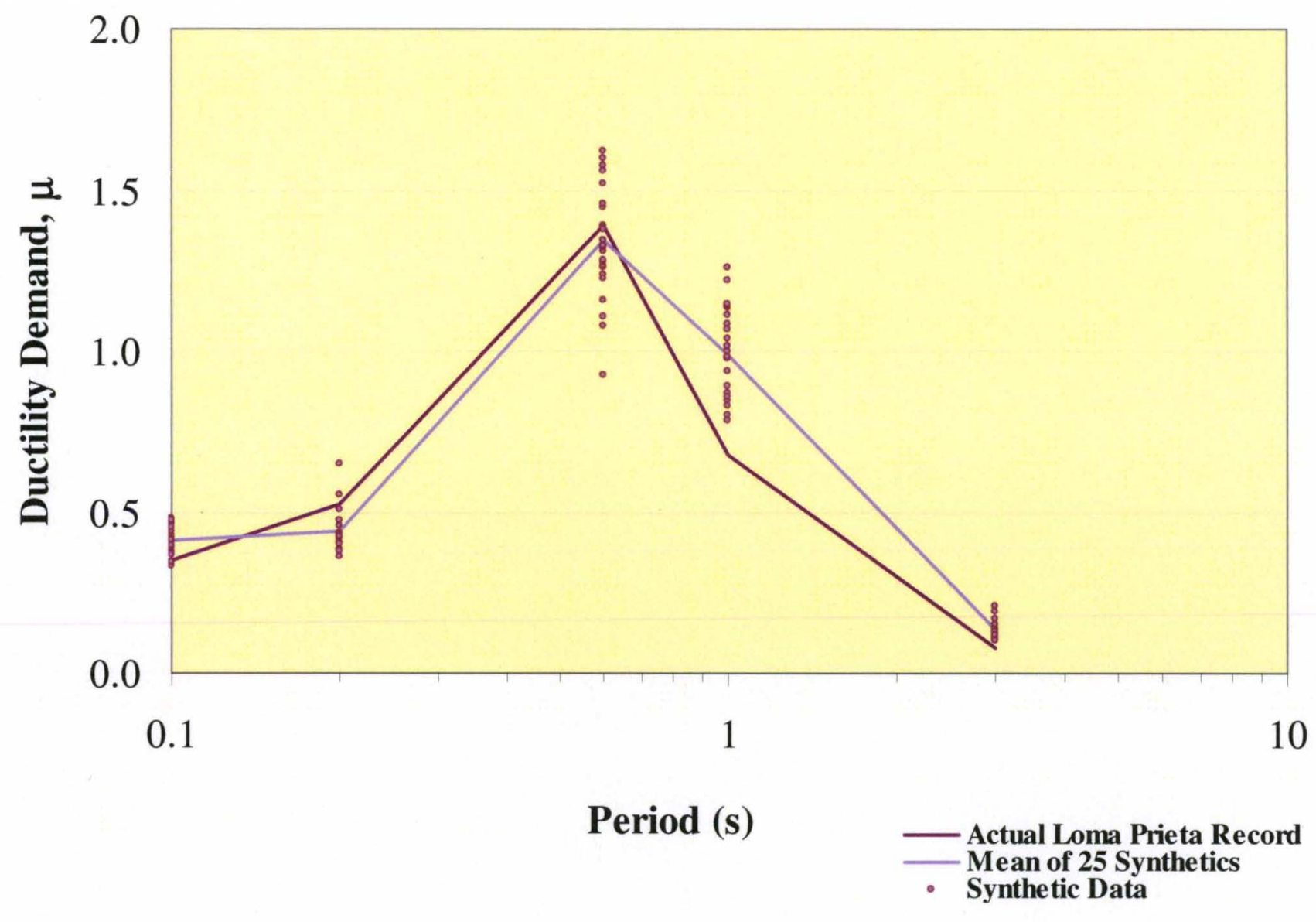


Comparison of Displacement Ductility Demands from the Actual Loma Prieta Record and the Means of Its Synthetics

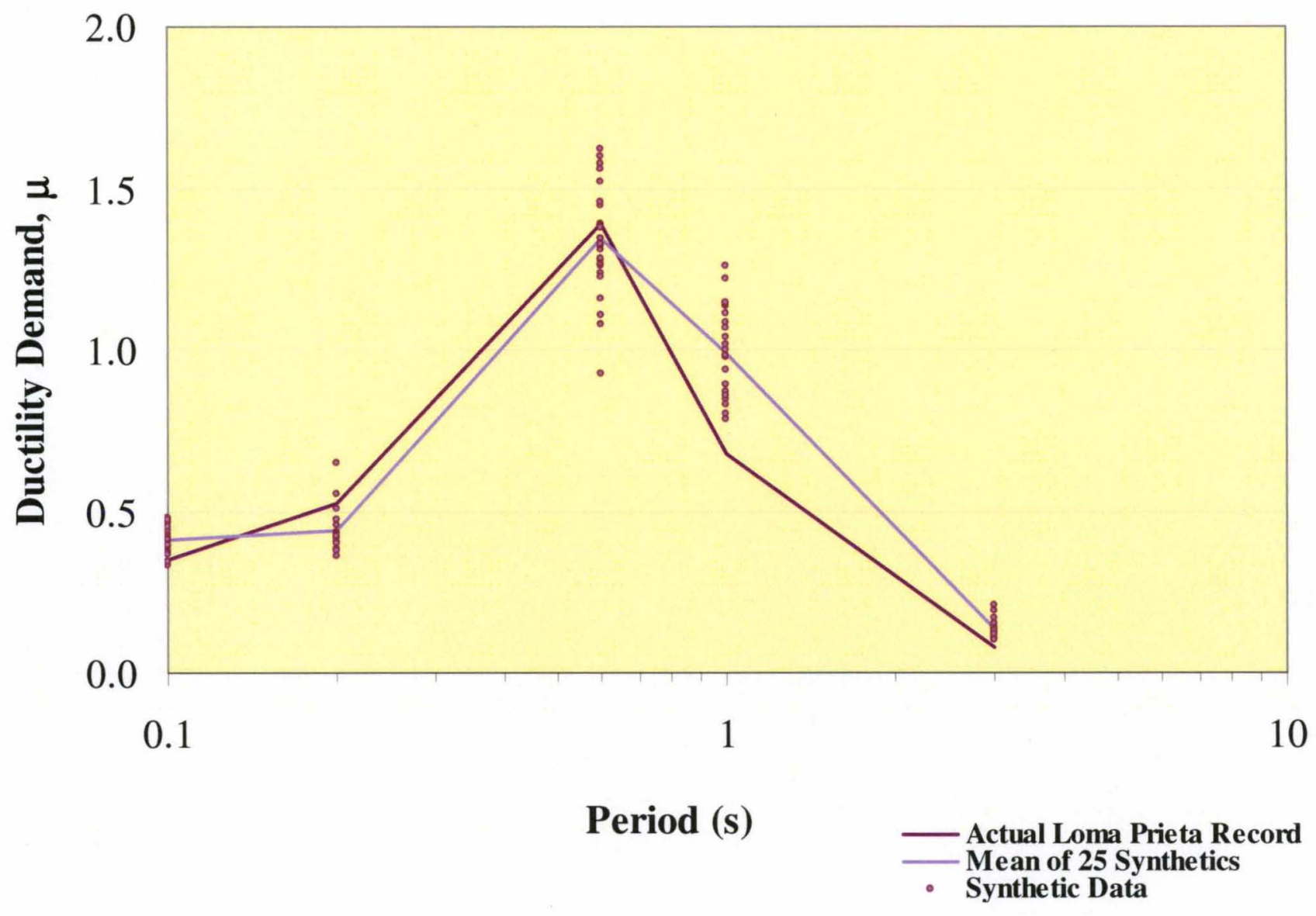


Comparison of Displacement Ductility Demands from the Actual NE Ohio Record and the Means of Its Synthetics

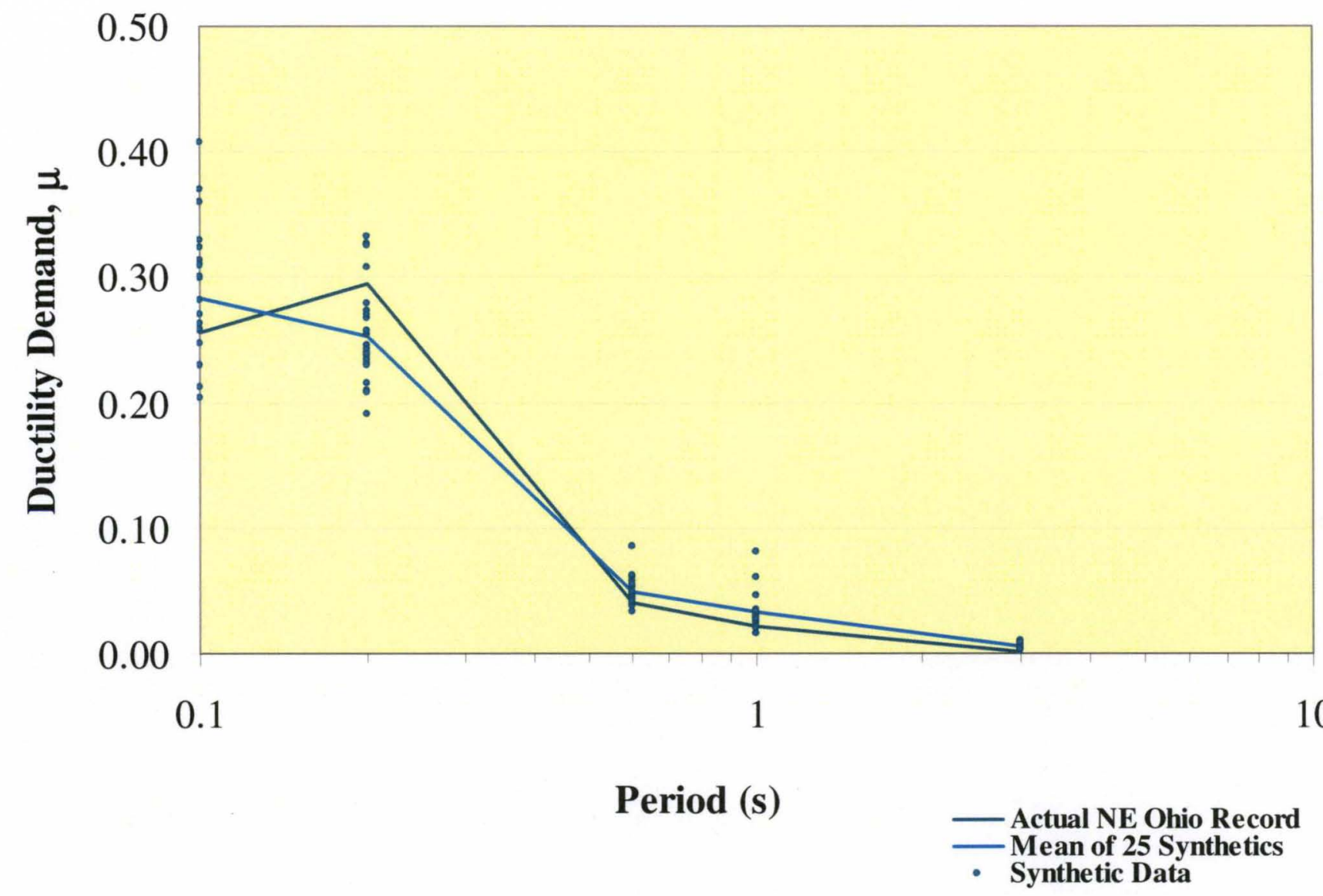


Comparison of Displacement Ductility Demands from the Actual Northridge Record and the Means of Its Synthetics

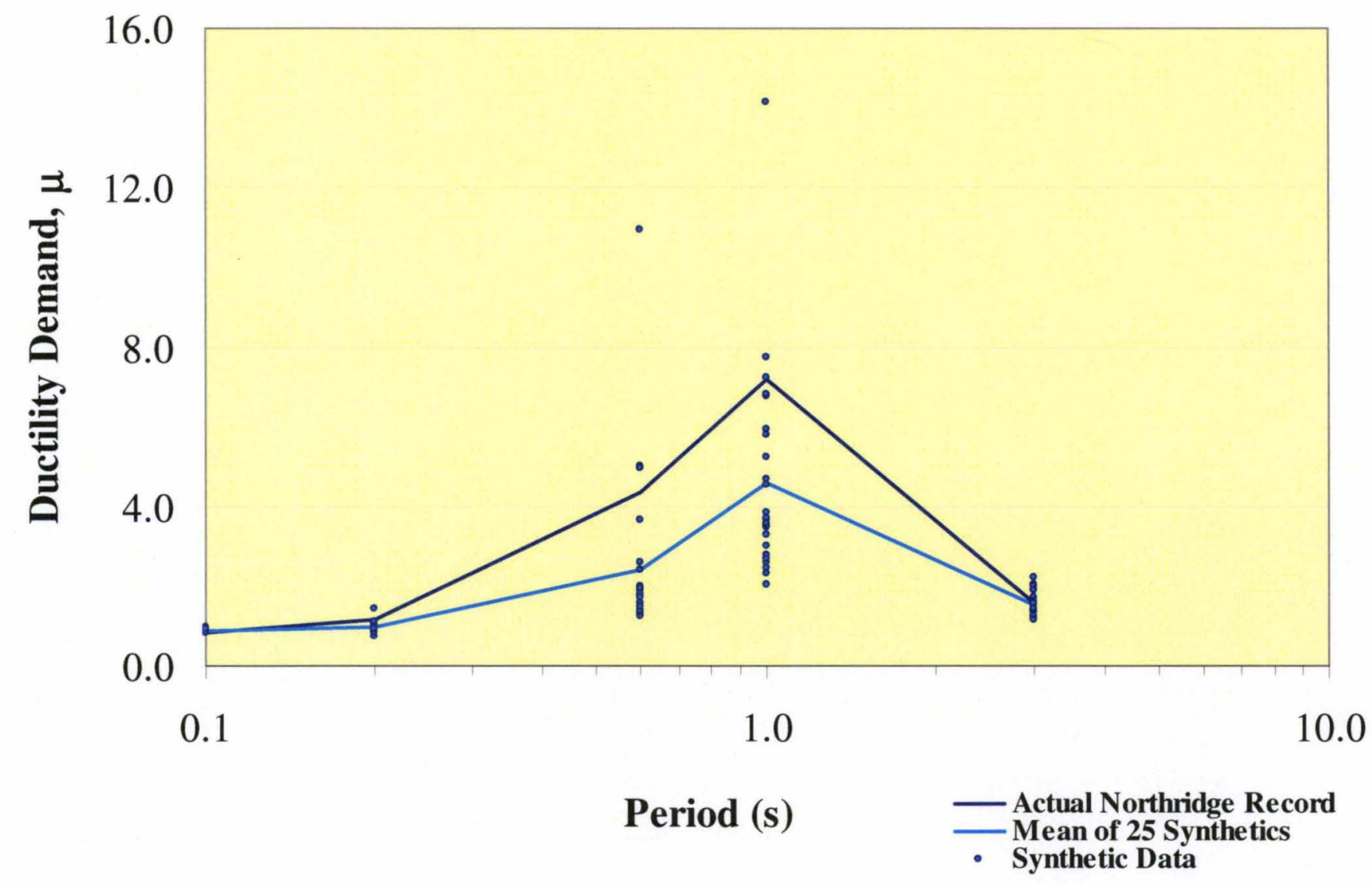


Comparison of Displacement Ductility Demands from the Actual Saguenay Record and the Means of Its Synthetics

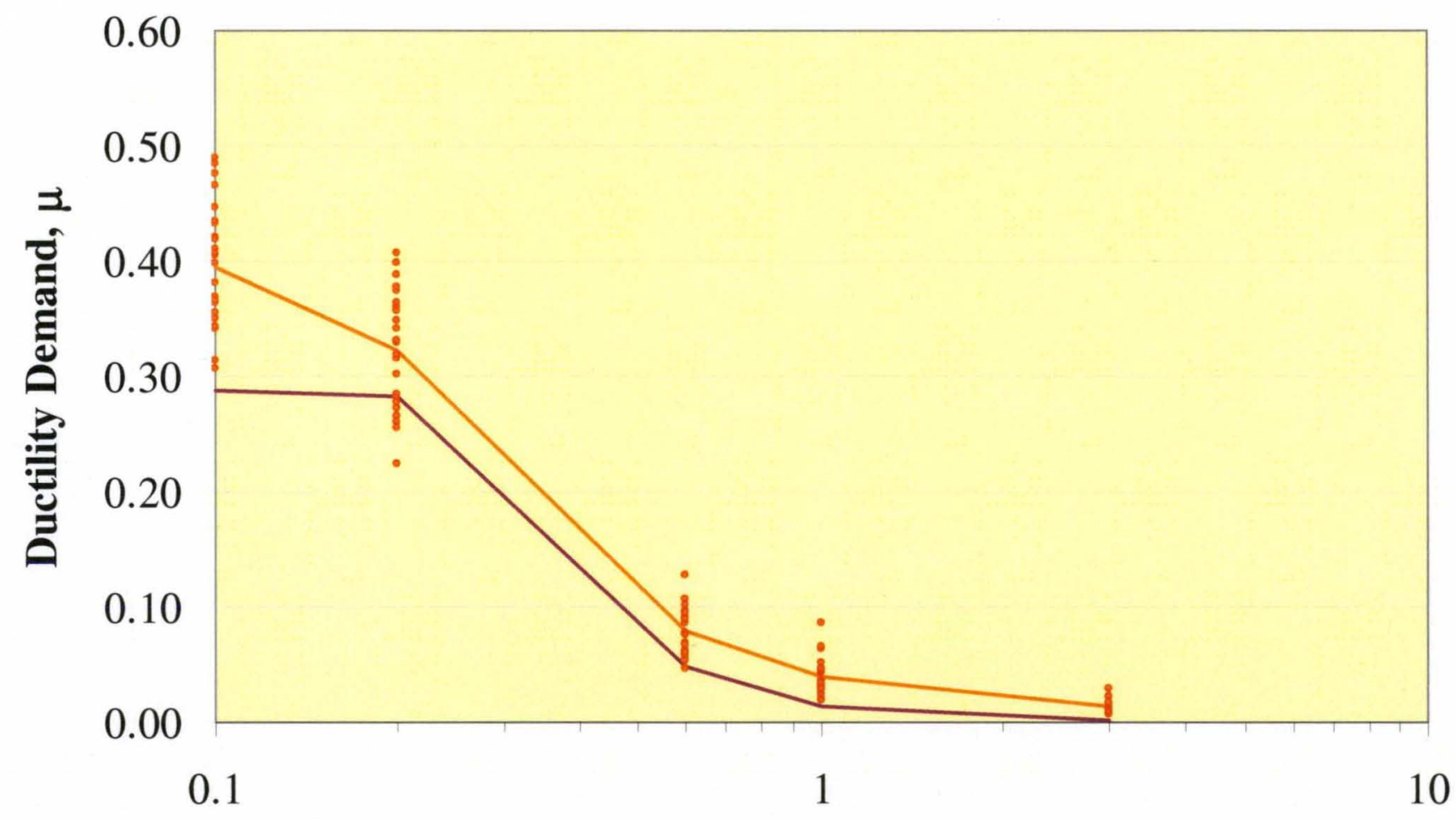

Period (s) 


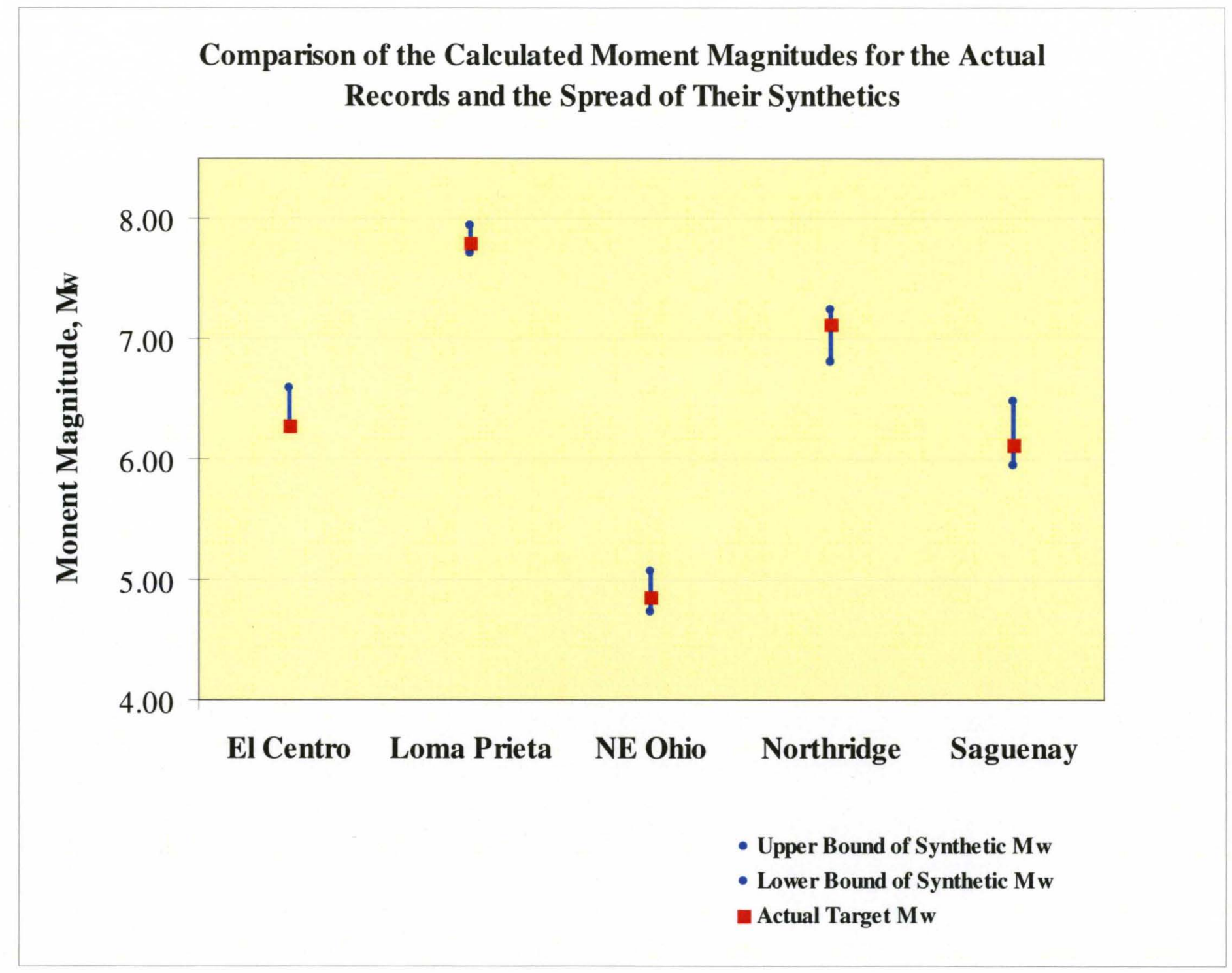




\section{APPENDIX H}

This appendix contains plots showing comparisons of the full target response spectra from each attenuation model for the $\mathrm{Mw} 6.5$ at $25 \mathrm{~km}$ event and synthetics developed from them using a 0.02 second discretization interval and using the input target spectra truncated to remove spectral acceleration values below the 0.2 second period. Notice that the synthetic records developed using the truncated spectra provide much better matches with their targets. Also shown are typical synthetic records developed using the truncated spectra from each attenuation model representing this scenario event. 

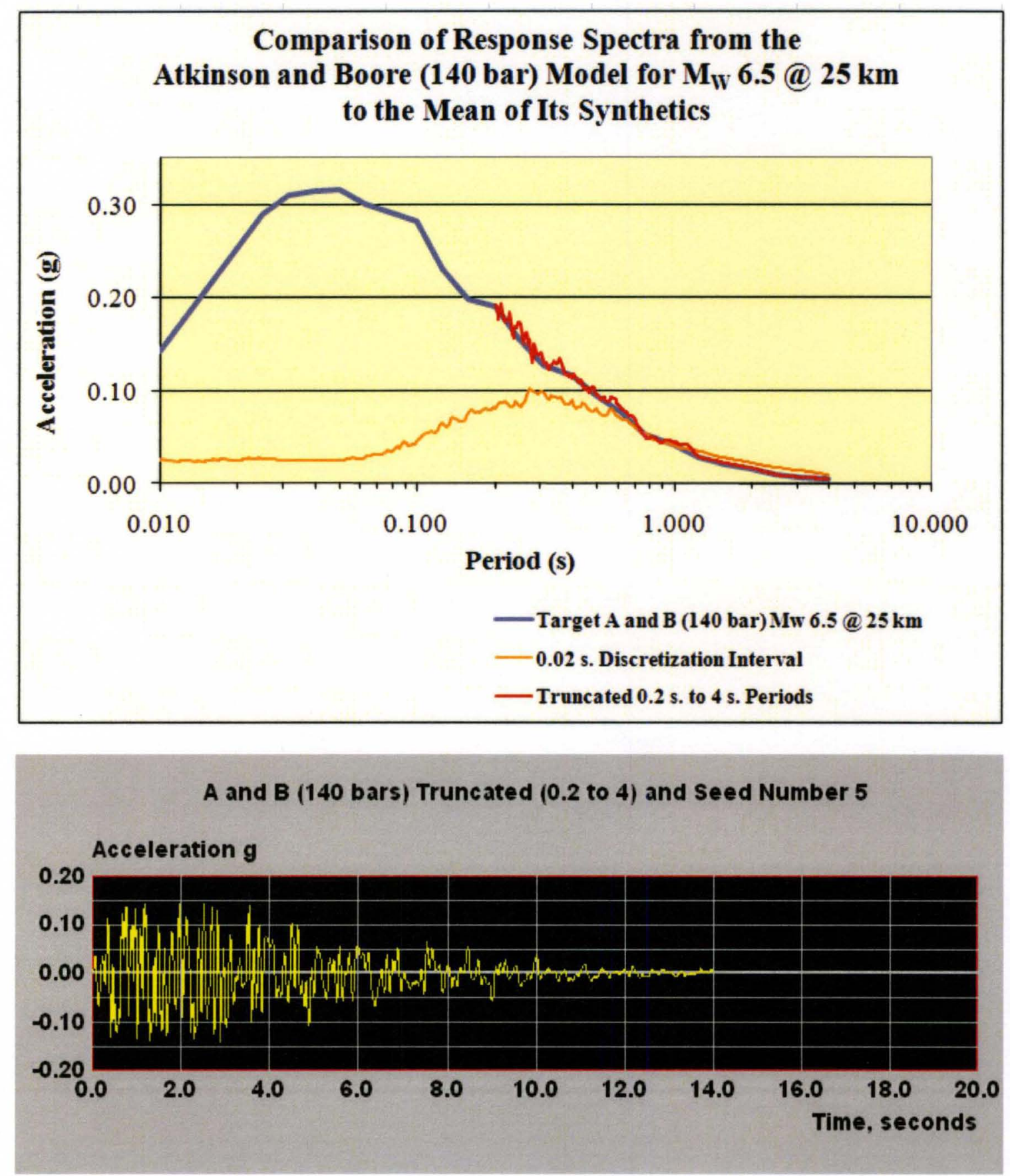


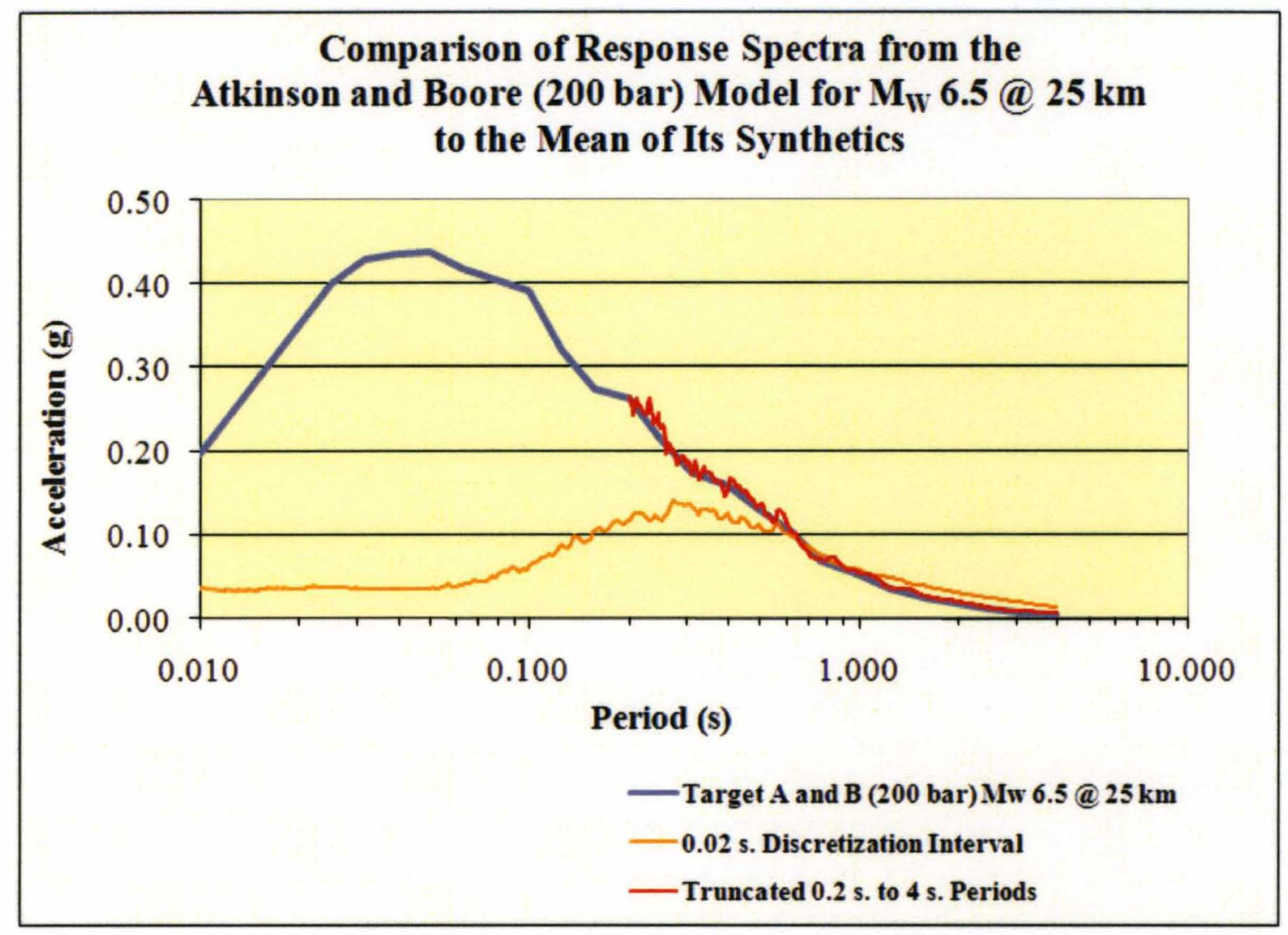

$A$ and $B(220$ bars) Truncated $(0.2$ to 4$)$ and Seed Number 5

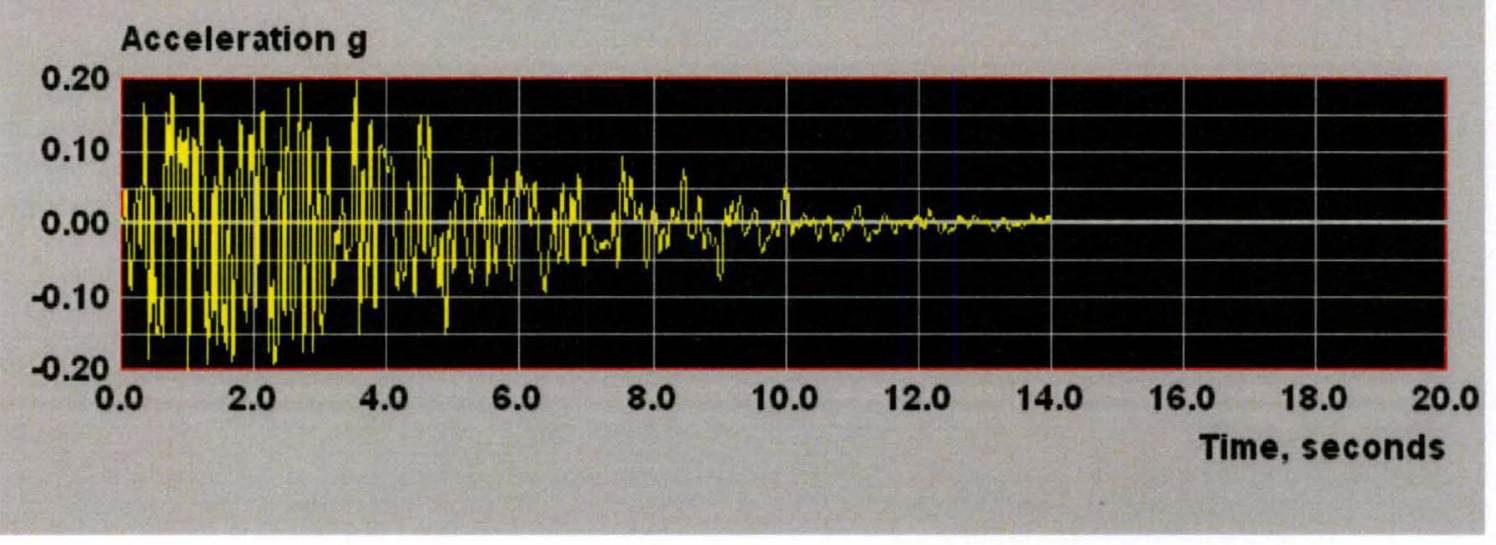



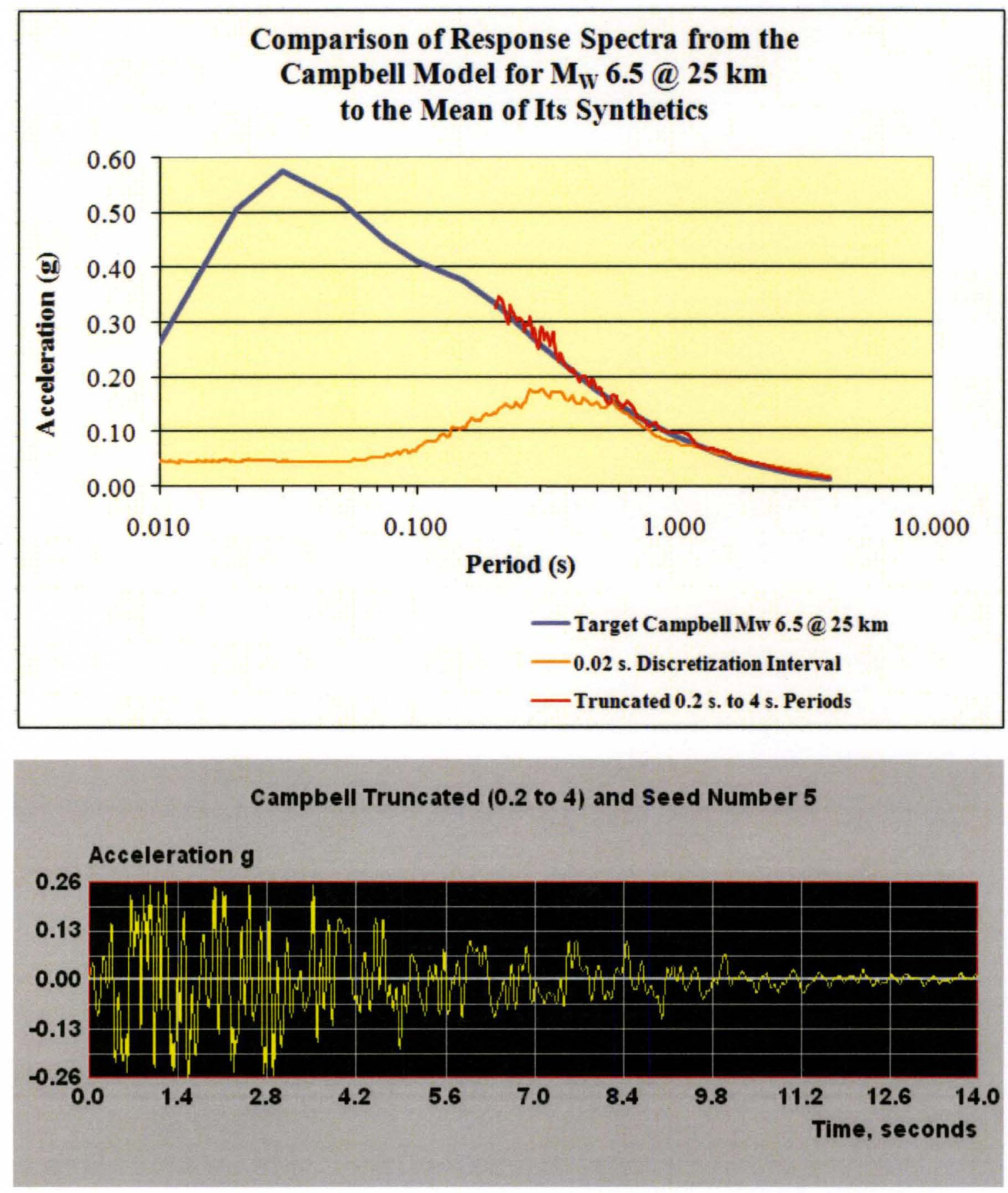

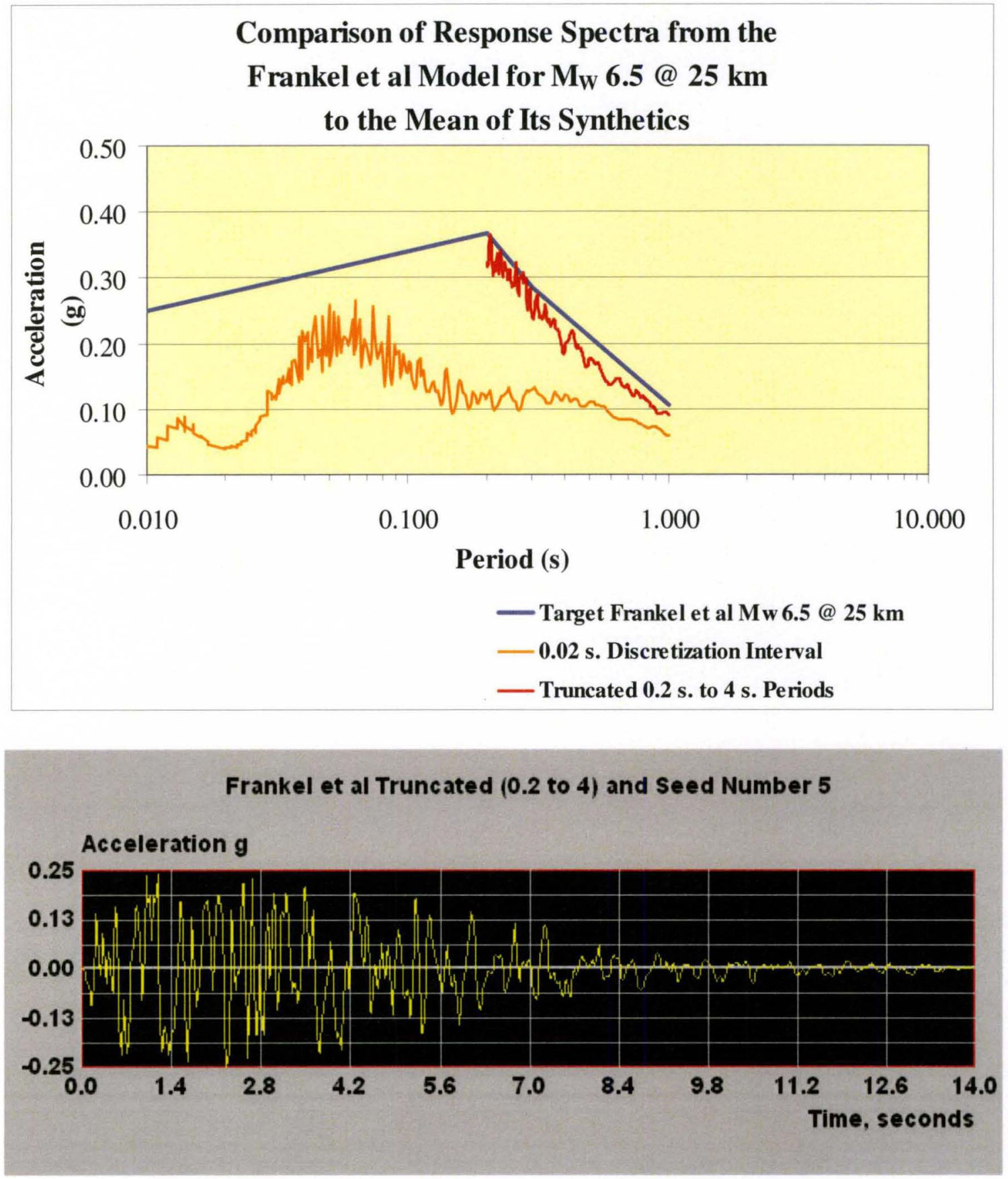

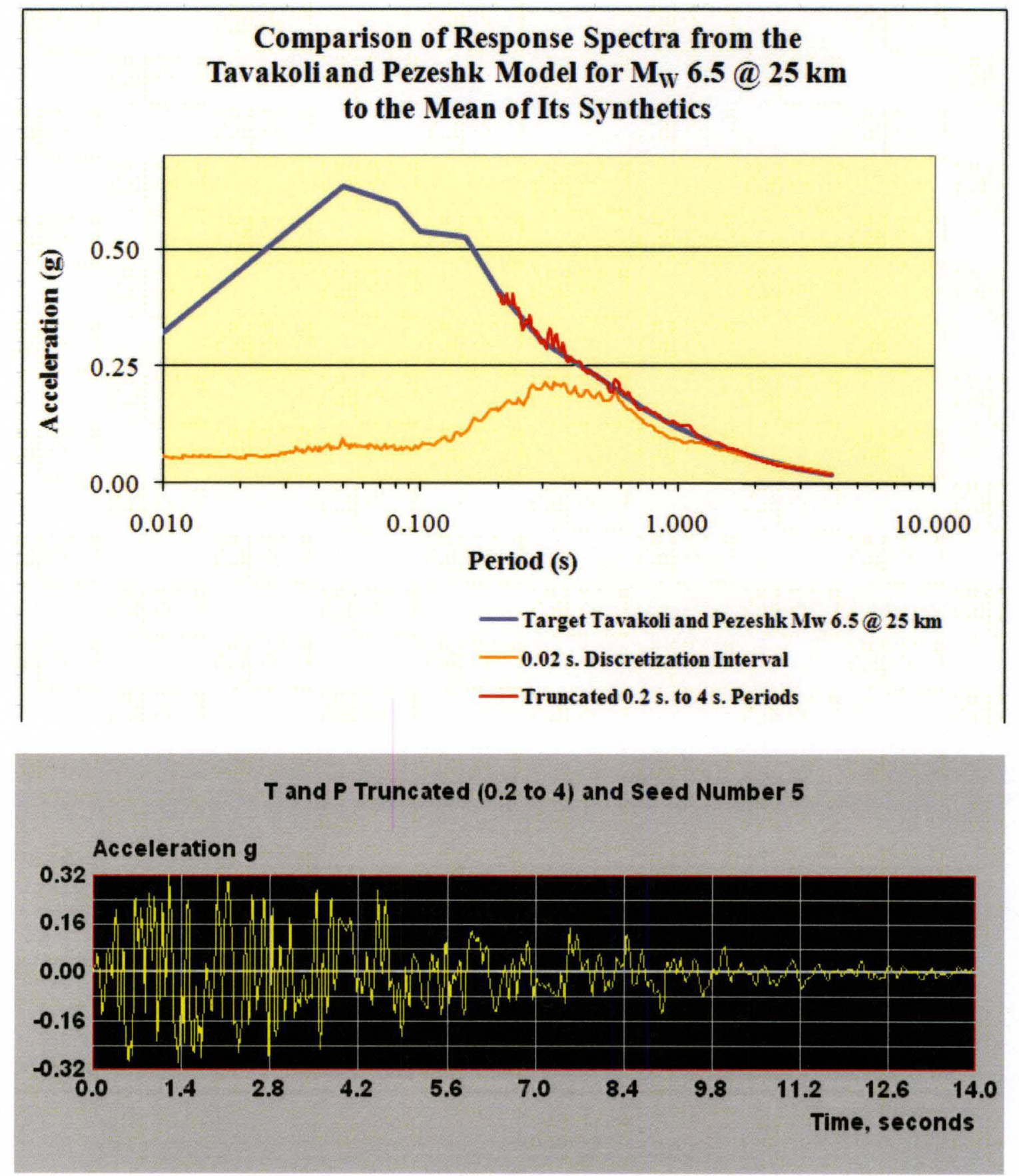

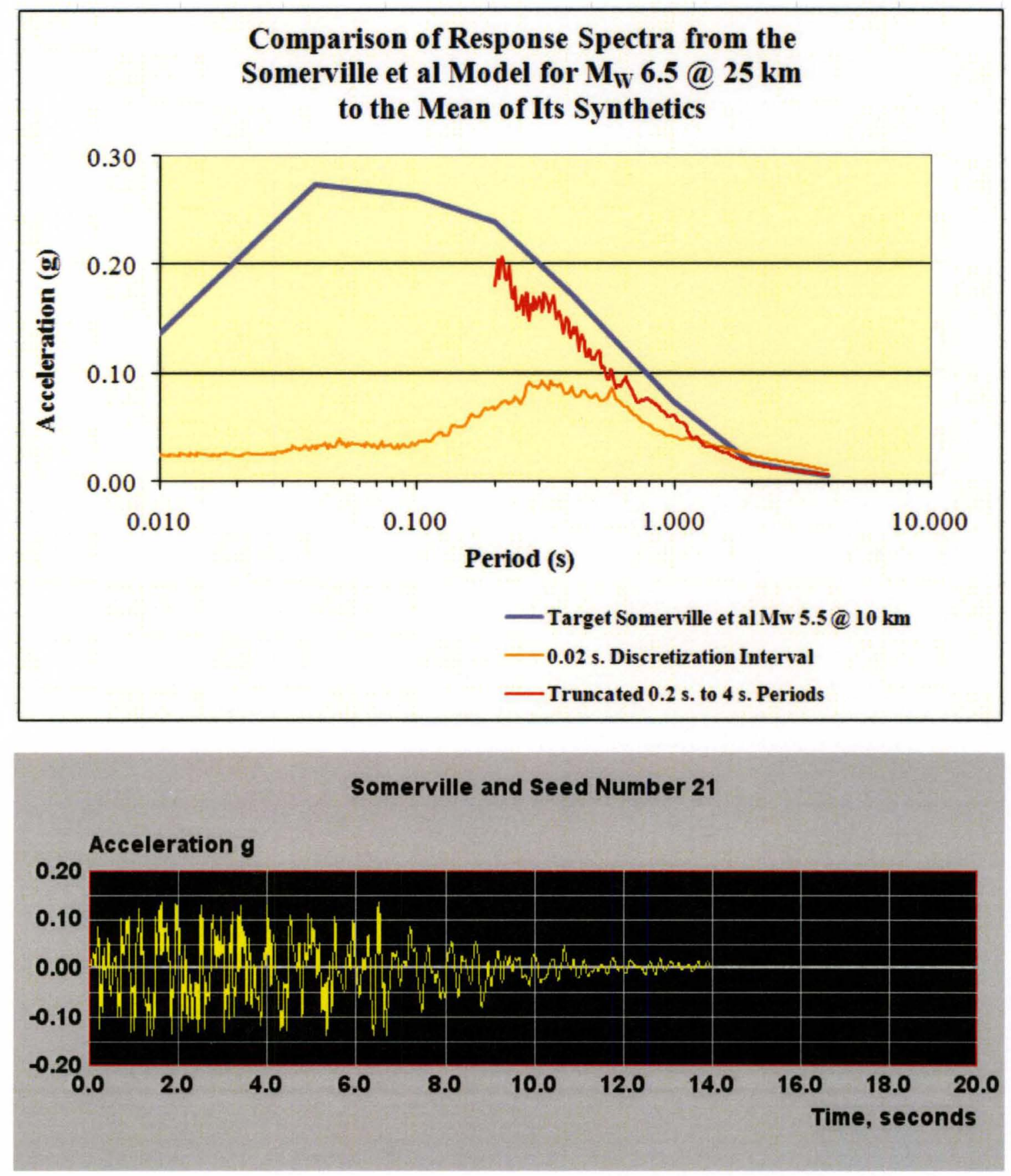

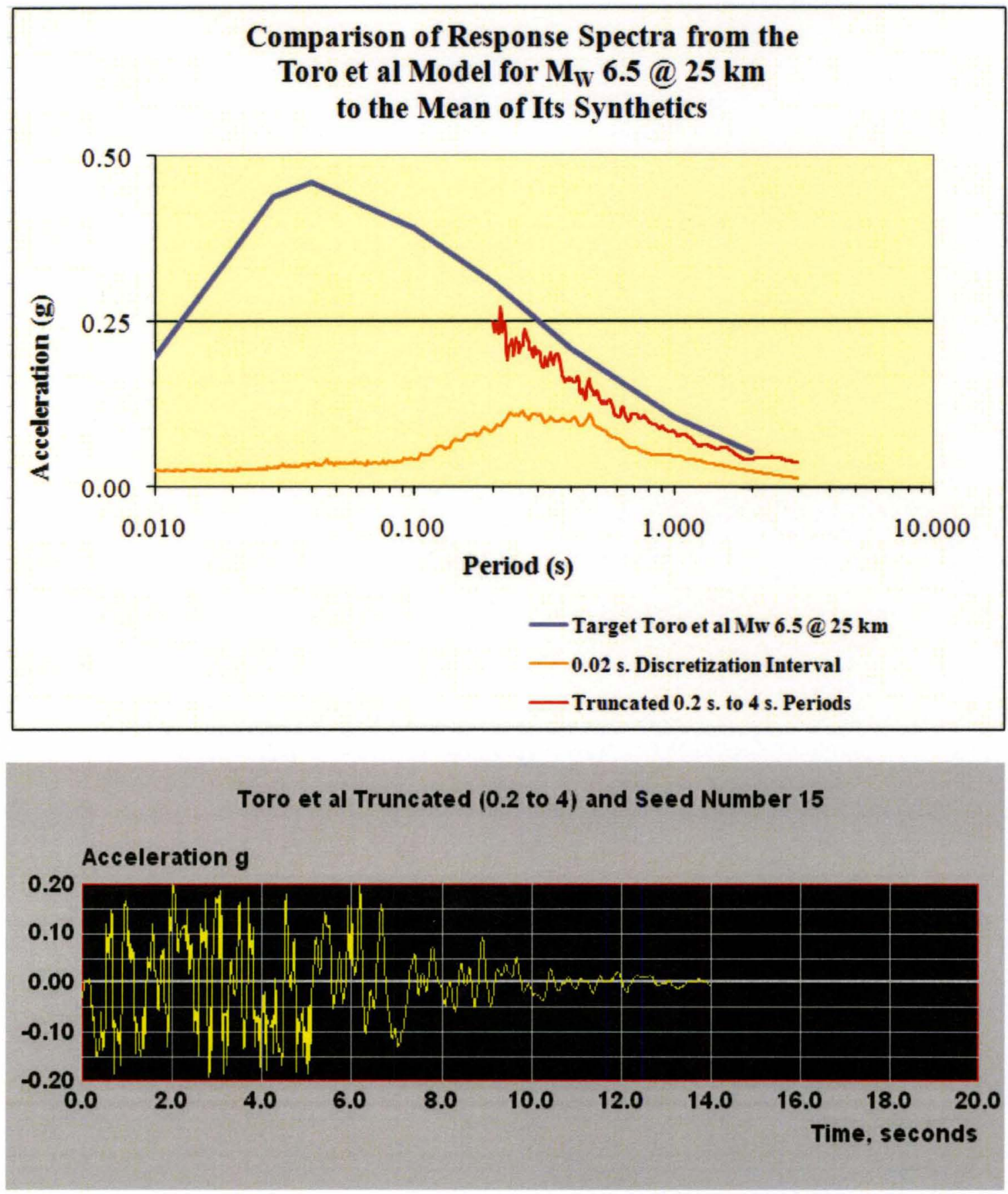

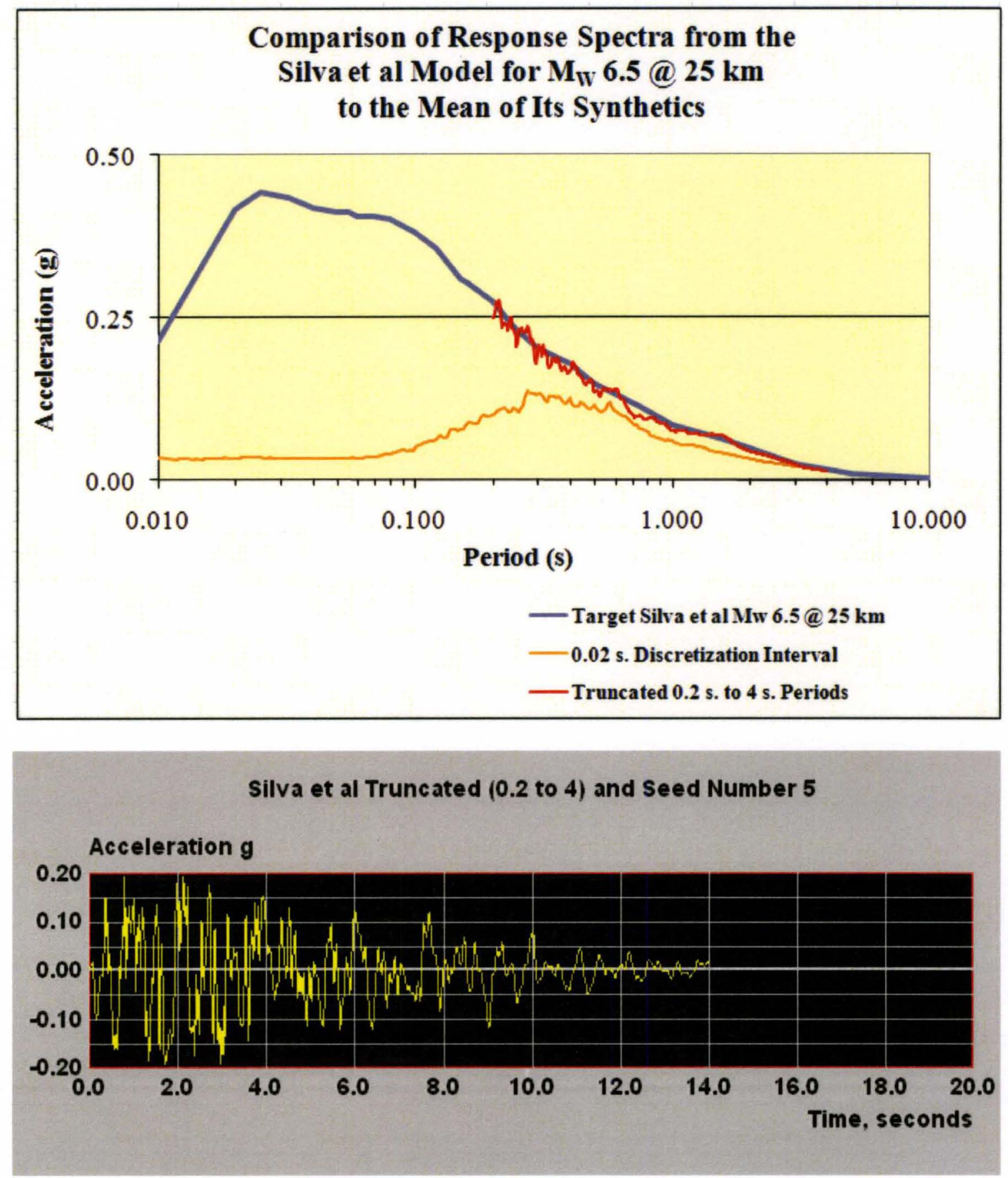


\section{APPENDIX I}

This appendix provides simultaneous plots of the Sargoni and Hart shape function and the exponential function as available in the SIMQKE program used to develop parameter values for the intensity envelope used in this study. It also shows the functions developed as fit to the three parameter values required to define the exponential intensity envelope available in the SIMQKE program as determined by

adjusting the parameters to overlay the Saragoni and Hart model at the duration times of $5,10,20,30,40,50$, and 60 seconds. 

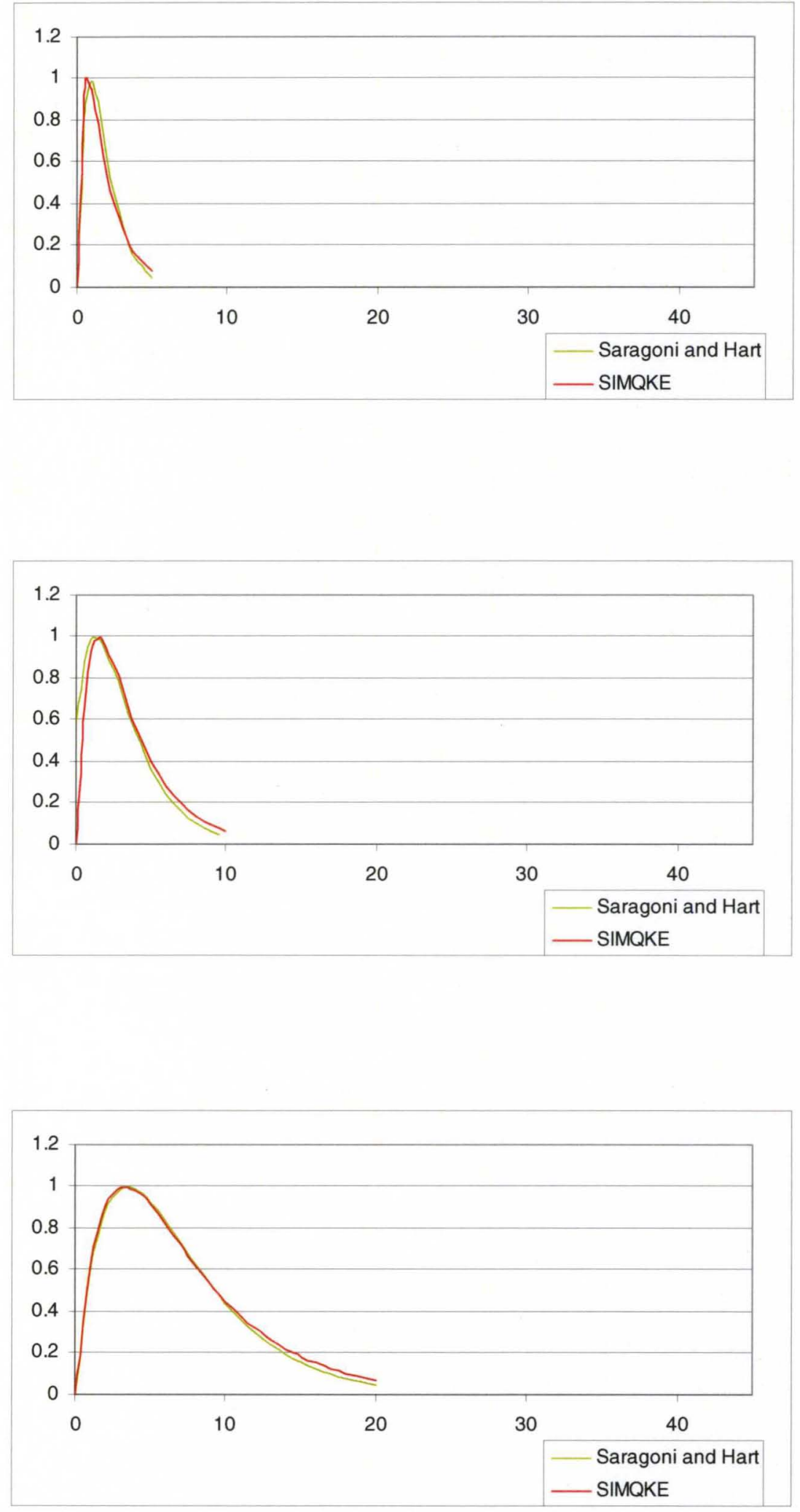

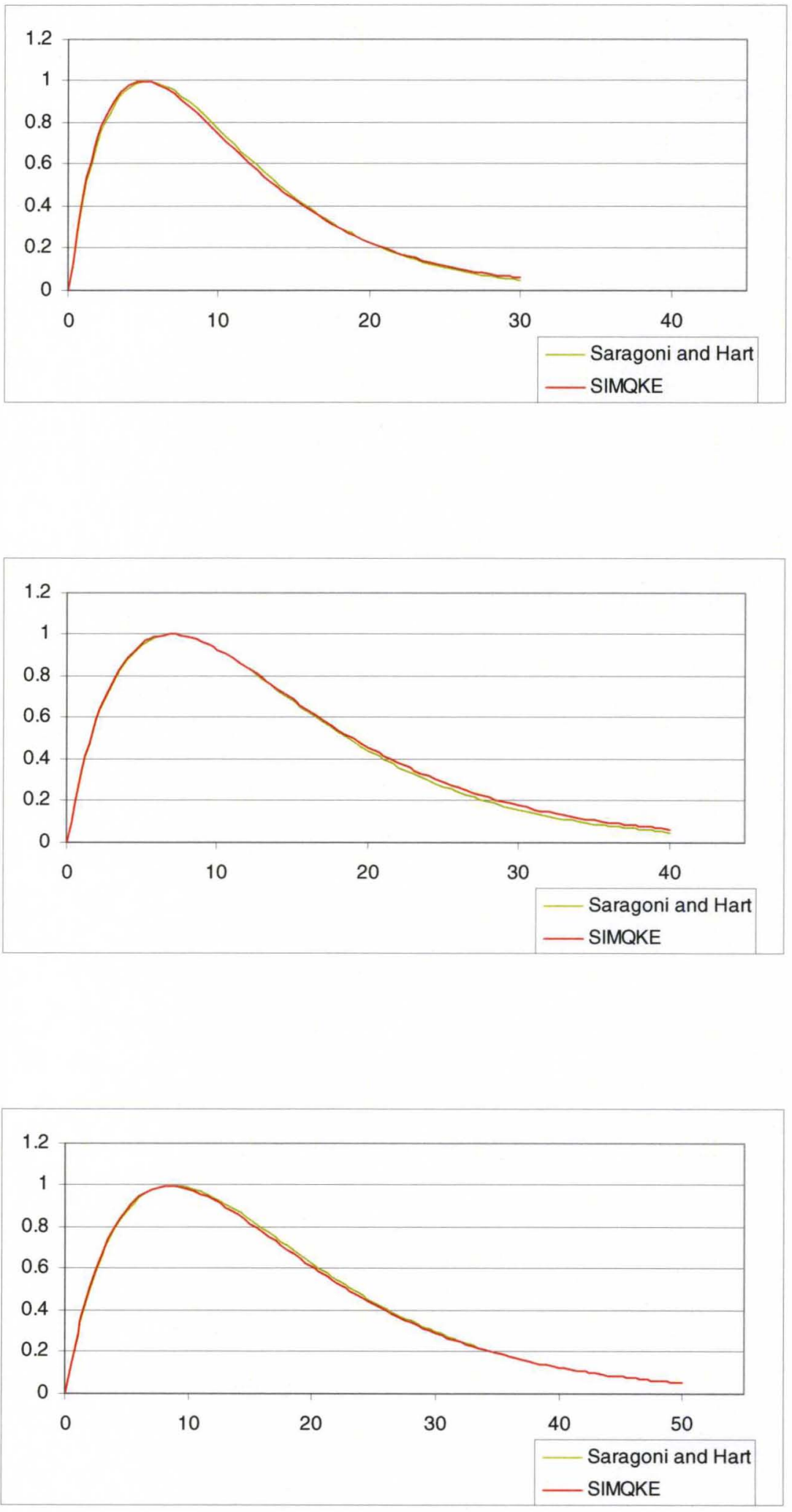


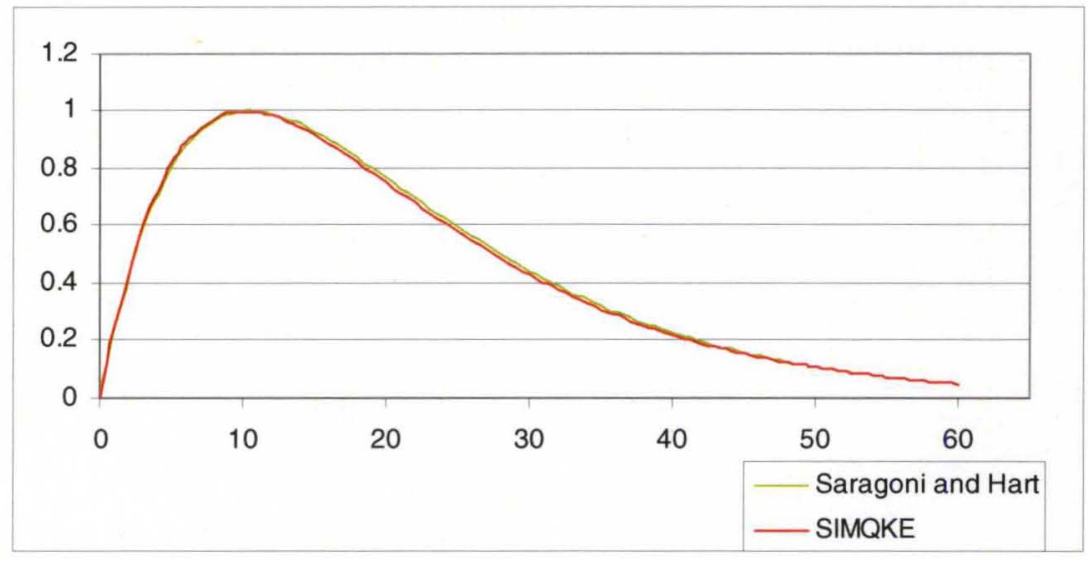

\section{$A_{0}$ Power Fit}

$\operatorname{corr}(\overrightarrow{\mathrm{H}(\mathrm{X})}, \mathrm{Y})=0.998$

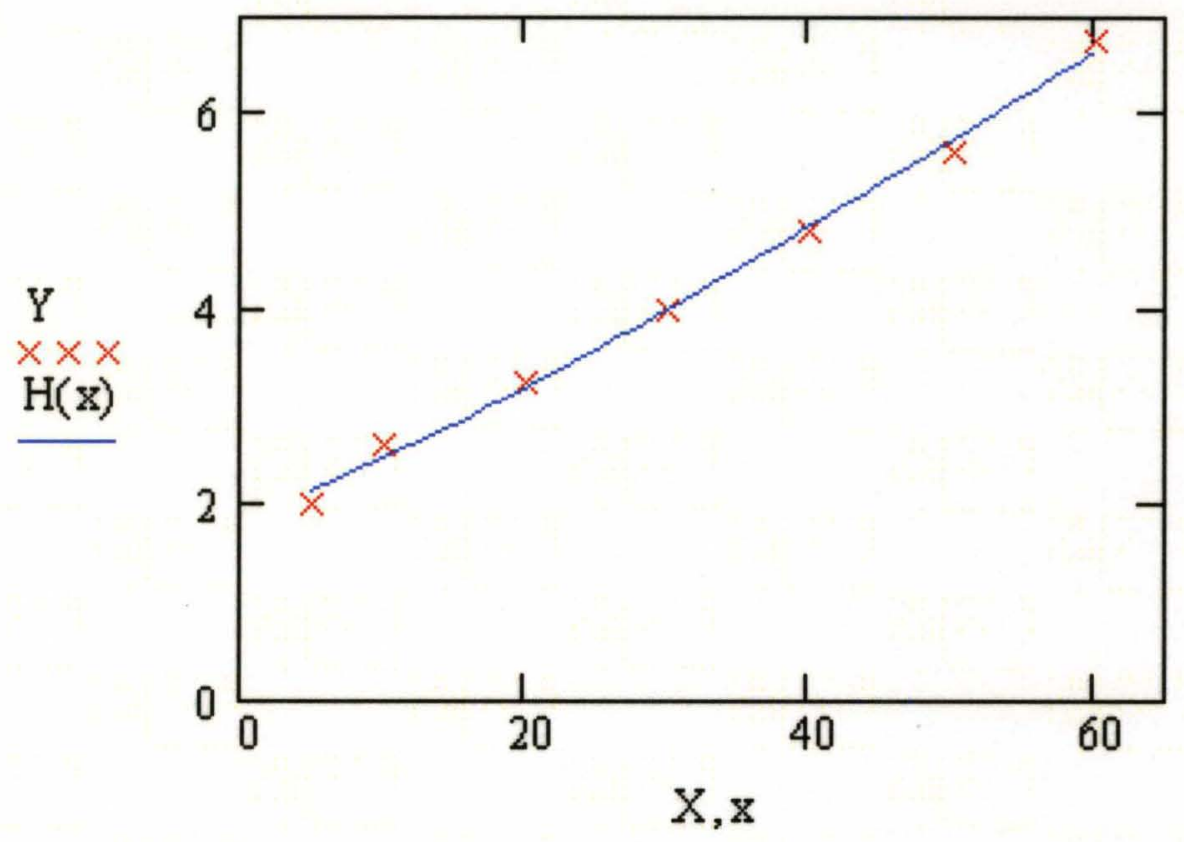




\section{$\beta_{0}$ General Fit}

$$
\operatorname{corr}(\overrightarrow{\mathrm{h}(\mathrm{X})}, \mathrm{Y})=1
$$

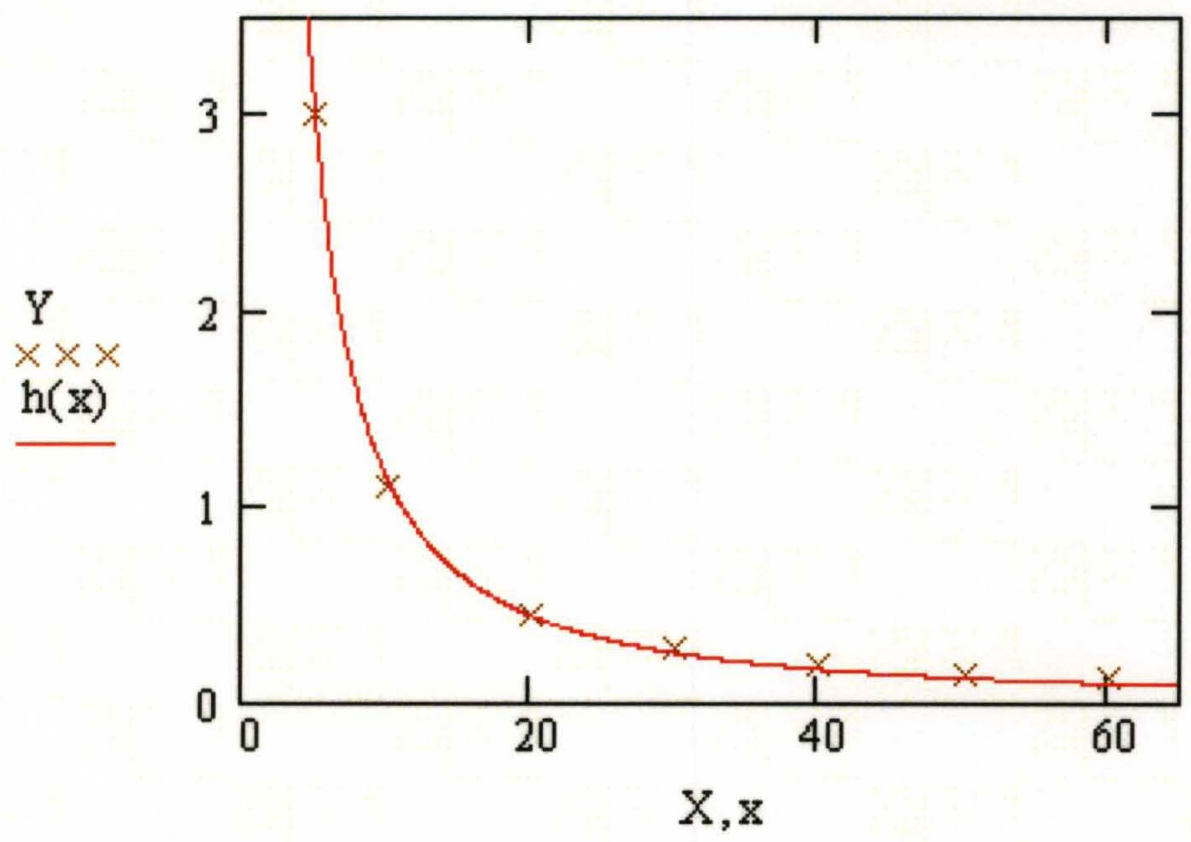




\section{$\alpha_{0}$ General Fit}

$\operatorname{corr}(\overrightarrow{\mathrm{h}(\mathrm{X})}, \mathrm{Y})=1$

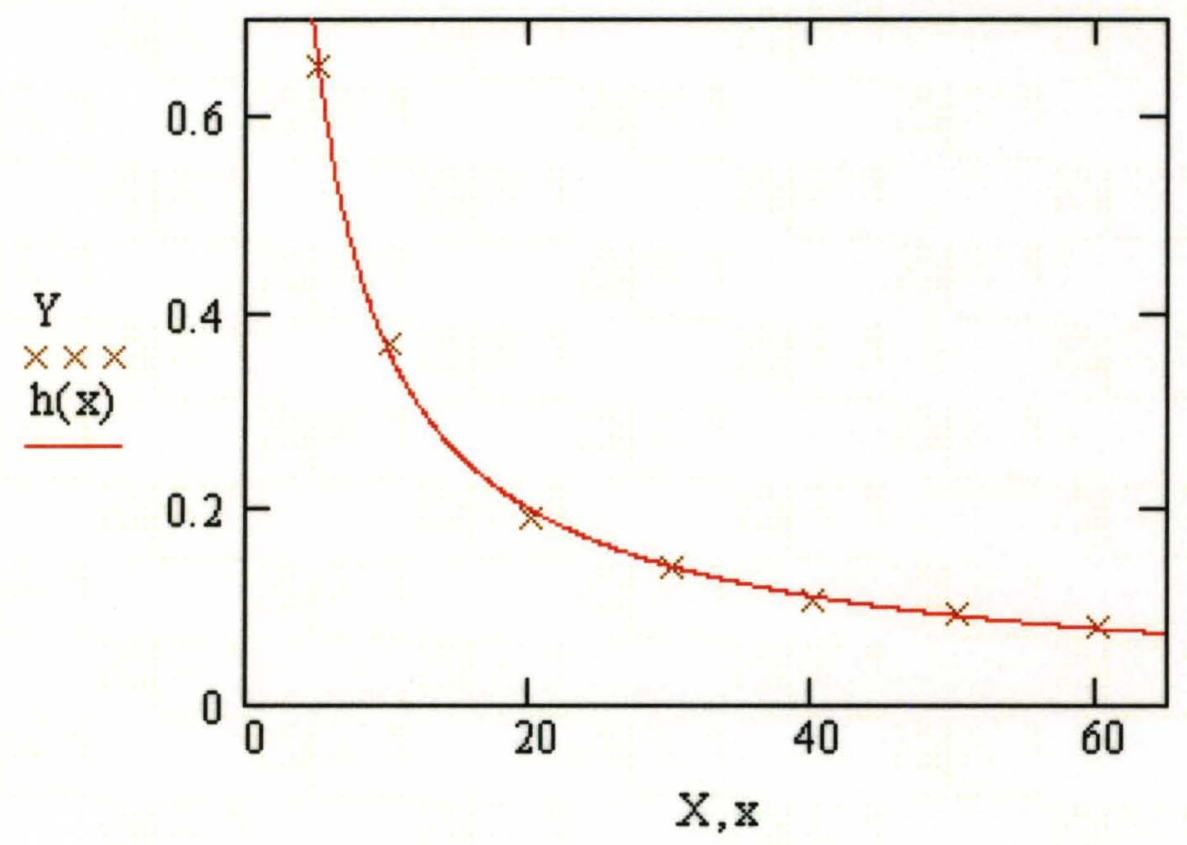




\title{
CURRICULUM VITAE
}

\author{
Troy W. Milburn
}

6420 Silver Mesa Dr. Unit D

Highlands Ranch, CO 80130

\section{Education}

University of Louisville, Graduate School, Louisville, KY

Doctor of Philosophy in Civil and Environmental Engineering,

Dissertation Defended and Approved on September 25, 2008

To be Conferred May 9, 2009

University of Louisville, Speed Scientific School, Louisville, KY

Civil and Environmental Engineering,

Master of Engineering with specialization in the field of Civil Engineering,

Conferred August 8, 2003

With High Honors

University of Louisville, Speed Scientific School, Louisville, KY

Civil and Environmental Engineering,

Bachelor of Science in Civil Engineering, Conferred May 11, 2002

With High Honors

\section{Work History}

9/08 - Present, Research Structural Engineer GS-0810-12, U. S. Army Corps of Engineers, Louisville District, Engineering Division, Office of the Chief

Currently serving on a Reimbursable Interagency Detail Agreement from the U. S. Army Corps of Engineers to the U. S. Geological Survey Geologic Hazards Team in Golden, Colorado. Duties are to plan and conduct original and creative research, both 
fundamental and applied, in earthquake engineering in order to asses earthquake hazards and reduce earthquake risks to the built environment. Conduct fundamental research studies for the development of vulnerability models for critical structures subjected to earthquake ground shaking. Develop vulnerability models for common structures via nonlinear dynamic structural analysis and/or statistical data analysis. Enhance vulnerability models recently developed for the HAZUS model building types, including the use of more realistic representations of the model building types and of vector and/or alternative ground motion amplitude measures. Expecting three journal article submissions during fiscal year 2009 based on dissertation and USGS work.

\section{9/07 - 4/08, Structural Engineer GS-0810-12, U. S. Army Corps of Engineers, Afghanistan Engineer District, Engineering Branch}

Performed quality assurance reviews for geotechnical reports, design analyses, construction submittals, plans, drawings, and specifications as presented by engineering contractors for construction projects of the U.S. and Afghan governments as part of the overall rebuilding effort for the country of Afghanistan. Ensured the sufficiency, practicability, and compliance with design requirements and established policies. Utilized specialized knowledge to pay particular attention to address seismic issues for the region and rendered advice concerning the design of structural features especially those involving seismic concerns.

\section{2/06 - 9/08, Structural Engineer GS-0810-11, U. S. Army Corps of Engineers, Louisville District, Engineering Division, Design Branch, Structural Section}

Promoted to next grade after successful completion of Engineering Division Intern Program. Continued assignment in the Design Branch, Structural Engineering Section.

Worked independently to develop a highly complex and detailed probabilistic based risk model for seismic stability and internal stress of dams and their intake towers under seismic loads incorporating Monte Carlo simulation. Assisted in the Corps' recent movement towards probabilistic risk assessment and played a key role in the development of both methodology and tools to conduct structural seismic risk analysis towards this effort. Contributed in establishing standard operating procedures for the development of seismic hazard modules of future dam safety periodic assessments using risk analysis.

Utilized the theory and principles of structural engineering to develop original plans, design analyses, and specifications of structures for civil and military projects consisting of components for use in navigation locks, flood control, and an assortment of military facilities. Prepared structural design calculations, drawings, and supporting documentation for various structural systems subjected to static and 
dynamic loads including blast loads and seismic ground motions. Wrote engineering reports presenting design premises and assumptions, basic data, structural characteristics, analytical

procedures utilized, and recommended solutions for project structures.

Reviewed drawings, calculations, and design plans of other structural engineers to ensure they conform to applicable criteria and prepared recommendations for clarification and corrections. Provided technical guidance in applications of engineering theory to lower graded engineers and technicians.

Performed site visits and made field inspections of existing facilities to evaluate the adequacy of their structural design and to ensure their compliance with existing requirements. Prepared inspection evaluations and applied district policies, and knowledge of operating characteristics of structures to develop inspection reports based on individual participation in periodic inspections. Furnished technical advice, assistance, and engineering support to field elements and a variety of district officials.

Applied a variety of computer software applications to perform structural engineering duties and accomplish assigned tasks including: finite element analysis, computeraided design and drafting, spreadsheet development, and other approaches to making, evaluating, and documenting design decisions.

Participated in professional meetings and workshops on earthquake hazard. Also participated in technical conferences with project managers, other structural engineers, the customer, contractors, technical representatives of industry and other specialists in associated engineering disciplines in a team effort to develop integrated designs.

\section{8/04 - 2/06, Civil Engineer GS-0810-9, U. S. Army Corps of Engineers, Louisville District, Engineering Division, Design Branch, Structural Section}

Realigned for rotation in Design Branch, Structural Engineering Section while continuing Engineering Division Intern Program.

\section{7/04 - 8/04, Civil Engineer GS-0810-9, U. S. Army Corps of Engineers, Louisville District, Engineering Division, Civil Branch, Geotechnical and Dam Safety Section}

Promoted to next grade while successfully continuing Engineering Division Intern Program. Designed and supervised geotechnical field investigations and soil laboratory testing. Prepared geotechnical reports defining foundation design parameters such as bearing capacity and footing size, and fully-developed pavement designs. Utilized software for nonlinear seismic soil response analyses as well as 
others for finite element slope stability analyses. Assignments included field activities relating to inspection of existing facilities as well as site investigations of special conditions for engineering studies. Prepared drawings for preliminary design and final plans. Developed layouts for engineering studies and project plans for construction and renovation/repair projects. Participated in pre-design conferences with project team personnel.

2/04 - 7/04, Civil Engineer GS-0810-7, U. S. Army Corps of Engineers, Louisville District, Engineering Division, Civil Branch, Geotechnical and Dam Safety Section

Accepted into Engineering Division Intern Program. Continued assignment to Civil Engineering Branch, Geotechnical and Dam Safety Section.

8/03 - 2/04, Student Trainee (Engineering) GS-0899-7, U. S. Army Corps of Engineers, Louisville District, Engineering Division, Civil Branch, Geotechnical and Dam Safety Section

Realigned for rotation to Civil Engineering Branch, Geotechnical and Dam Safety Section.

\section{3/03 - 8/03, Student Trainee (Engineering) GS-0899-7, U. S. Army Corps of Engineers, Louisville District, Engineering Division, Civil Branch, Civil Section}

Applied engineering principles and methods to solve problems for both civil works and military projects. Civil works projects included various tasks for developing flood protection projects including proposed wall and levy alignments. Military projects included working with post/installation security officials to implement antiterrorism/force protection security measures including the design and establishment of security perimeters and check points around cantonment and parking facilities. Prepared drawings for preliminary design and final plans for construction and renovation/repair projects. Made field reconnaissance and site investigations to obtain first-hand data on topography, existing facilities or special conditions. Utilized various computer software to make, evaluate, and document design decisions. Compiled and prepared technical data for use by senior technical and other directorate personnel.

\section{9/03 - 12/03, Instructor, University of Louisville, Dept. of Civil and} Environmental Engineering

Responsible for all aspects of teaching two sections of the course Mechanics I: Statics including development and presentation of lectures, syllabus, course content, exams, 
quizzes, etc., and assigning grades. Also responsible for teaching a civil-structural FE/EIT review course as well as several lectures in mechanics of materials and structural analysis.

5/01 - 9/03, Graduate Teaching Assistant, University of Louisville, Dept. of Civil and Environmental Engineering

Responsible for all aspects of teaching the course Mechanics I: Statics including development and presentation of lectures, syllabus, course content, exams, quizzes, etc., and assigning grades. Also responsible for teaching a civil-structural FE/EIT review course as well as several classes in mechanics of materials and structural analysis.

\section{0/98 - 5/01, Student Assistant, University of Louisville, Dept. of Transitional} Studies -Mathematics

Provided students with additional supplementary instruction in all levels of mathematics. Assisted students in utilizing math software.

\section{5/00 - 8/00, Undergraduate Research Assistant, Mid-America Earthquake Center, University of Illinois at Urbana-Champaign Dept. of Civil and Environmental Engineering}

Responsible for the development of a database and conducted research analysis under a National Science Foundation fellowship grant to participate in the ST-13 project which studied the effectiveness of seismic retrofitting of bridge columns within the New Madrid Seismic Zone.

\section{5/99 - 9/99, Co-op. Intern, Presnell and Associates, Inc., Louisville, KY}

Produced and revised calculations, specifications, and drawings utilized in highway bridge design.

9/96 - 10/98, Asst. Designer, Asst. Plant Eng., Jefferson Smurfit / Container Corp. of America, Louisville, KY

Assistant Structural Designer: Assisted with the design and development of corrugated packaging, produced and revised specification drawings, built prototypes. 
Assistant to Plant Engineer: Responsible for all maintenance department ordering, monitored monthly budget and reported directly to plant manager.

\section{9/94 - 9/96, Service Quality Assurance Rep., Metropolitan Lincoln-Mercury, Louisville, KY}

Maintained Service Dept. records and files. Contacted customers to follow-up on service performance.

\section{4/88 - 9/94, Owner/Operator, Handcrafted Sign Co., Louisville, KY}

Responsible for sales, layout, production, and installation of commercial signage. Obtained Contractor's License.

9/81 - 4/88, Journeyman Pictorial Artist, Naegele Outdoor Advertising, Louisville, KY

Utilized scale drawings to hand-paint lettering and illustrations onto outdoor bulletins (billboards). Solely responsible for the rigging of all necessary scaffolding as well as the supervision of an assistant. Served 4.5 year apprenticeship to obtain journeyman status.

\section{Awards Received}

U. S. Army Corps of Engineers, Great Lakes and Ohio River Division, Commanders Special Emphasis Award, June 2008

Department of the Army, Commanders Award for Civilian Service Medal (Operation Enduring Freedom), April 2008

North Atlantic Treaty Organization, Non Article 5 NATO Medal (International Security Assistance Force, Afghanistan), April 2008

U. S. Army Corps of Engineers, Afghanistan Engineer District Civilian Combat Service Pin, October, 2007

U. S. Army Engineer District, Louisville Engineering Division FY 07 Toolbox Performance Award (Monroe and Brookeville Dams Periodic Assessment Seismic Evaluation)

U. S. Army Engineer District, Louisville Engineering Division FY 06 Toolbox Performance Award (J. T. Myers Locks and Dam Periodic Inspection) 
Nominated and selected for inclusion in Who's Who Among America's Teachers, 2004

U. S. Army Engineer District, Louisville Engineering Division FY 03 Toolbox Performance Award (Fort Knox Cantonment Security)

Graduated 2002 and 2003 Magna Cum Laude, University of Louisville

2001 Graduate Teaching Assistantship, University of Louisville

2000 REU Fellowship, National Science Foundation

1998 Recipient of the W. B. Wendt Award for First Honors in Civil Engineering, University of Louisville

Chi Epsilon National Civil Engineering Honor Society member, served two consecutive terms as Chapter President at the University of Louisville

Tau Beta Pi National Engineering Honor Society member

Golden Key National Honor Society member

\section{Additional Training}

Risk Analysis in Engineering and Planning (06/01/2007)

40 hrs., Huntington District, U. S. Army Corps of Engineers

Soil-Structure Interaction (03/31/2006)

40 hrs., U. S. Army Corps of Engineers, Engineer Research and Development Center, Vicksburg, Miss.

Action Officer Development Course (09/21/2005)

40 hrs., The Army Institute of Professional Development, U. S. Army Training

Support Center

Intern Leadership Development Course (04/22/2005)

40 hrs., Department of the Army, Civilian Leadership Training Division

\section{List of Publications and Reports}

Milburn, T. W., In Preparation, A Comparison of the Structural Response to Synthetic Ground Motions Compatible with Central Eastern United States 
Attenuation Models, PhD Dissertation, The Graduate School of the University of Louisville, Louisville, Kentucky.

Milburn, T. W., November 2007, Notes on the Structural Response to Synthetic Ground Motions, Abstract submitted to Ohio River Valley Soils Seminar XXXVIII, Louisville, Kentucky.

Milburn, T. W., May 2007, Seismic Hazard Evaluation for Monroe Lake Dam, Module 10 of the Periodic Assessment Report, U. S. Army Engineer District, Louisville Engineering Division.

Milburn, T. W., May 2007, Seismic Hazard Evaluation for Brookville Lake Dam, Module 10 of the Periodic Assessment Report, U. S. Army Engineer District, Louisville Engineering Division.

Structural Periodic Inspection Reports: Markland Locks and Dam (2007), Brookville Dam and Monroe Dam (2007), J. T. Myers Locks and Dam (2006), C.J. Brown Dam and West Fork Dam (2005), U. S. Army Engineer District, Louisville Engineering Division.

Milburn, T. W., February 2007, Bulkhead Inspection after Barge Removal Effort at Lower Dam of McAlpine Locks and Dam, Memorandum for Record, U. S. Army Engineer District, Louisville Engineering Division.

Milburn, T. W., May 2007, Finite Element Analysis of Monolith L24 with Loading from Proposed Miter Gate Storage Pier, Markland Locks and Dam, Memorandum for Record, U. S. Army Engineer District, Louisville Engineering Division.

Milburn, T. W., Werncke, G. A., November 2006, Seismic Stability Risk Analysis of J. T. Myers Dam, Uniontown, Kentucky, (as a portion of the Major Rehabilitation Study), U. S. Army Engineer District, Louisville Engineering Division.

Milburn, T. W., Cassaro, M. A., July 2005, Exploration of Attenuation Relationships in the Development of Synthetic Ground Motions to be Used for Global Seismic Design, International Conference on Energy, Environment, and Disasters (INCEED 2005), Charlotte, North Carolina.

Sullivan, T. M., Milburn, T. W., December 2004, An exploration into Water Seepage at the Nose of Shaft Enclosure DS-19 Lower Land Wall, Lock and Dams 52 and 53 Replacement, Olmsted, Illinois, U. S. Army Engineer District, Louisville Engineering Division.

Milburn, T. W., June 2004, Geotechnical Report for Renovations to the Arthur MacArthur UCARC/OMS Installation, Springfield, Massachusetts; U. S. Army Engineer District, Louisville Engineering Division. 
Milburn, T. W., Zhong, Q., and Hawkins, N. M., Preliminary Development of Database and Analysis to Study the Effectiveness of Seismic Retrofitting Bridge Columns within the New Madrid Seismic Zone, Summer 2000, National Science Foundation, Research Experience for Undergraduates, Mid-America Earthquake Center, University of Illinois at Urbana-Champaign.

\section{Memberships}

American Society of Civil Engineers

Society of American Military Engineers

Kentucky Society of Professional Engineers

American Concrete Institute

Earthquake Engineering Research Institute

Applied Technology Council

\section{License}

Commonwealth of Kentucky EIT No. 12038 and approved to sit for the PE Examination. 